U. S. DEPARTMENT OF COMMERCE Vatienal Burean of Stamlards FUNDAMENTAL PHYSICAL CONSTANTS

These constants were compiled by E. R. Colien and B. N. Taylur under llie auspices of the COD ATA Task Group on Fundaniental Constants, officially adopred 1973), CODAT A Bulletin No. 11 (Dec. 1973), and DIMENSIO VS/NBS (Jan. 1974).

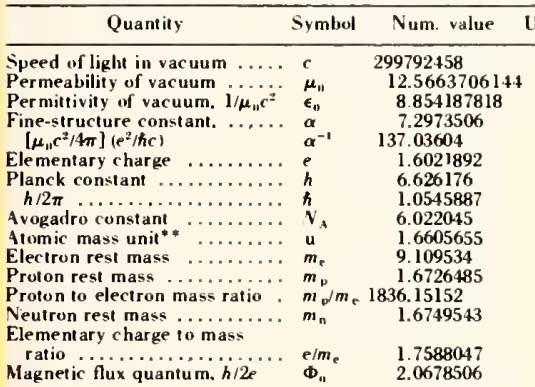

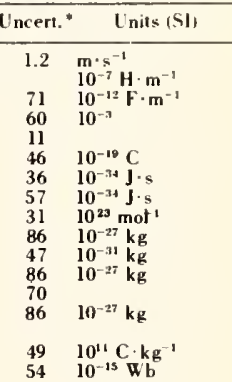

\begin{tabular}{|c|c|c|c|c|}
\hline 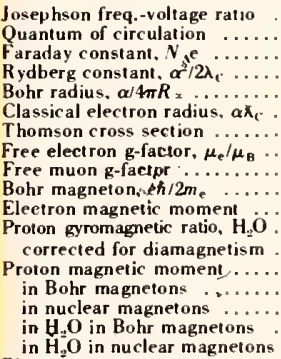 & 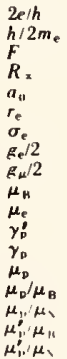 & $\begin{array}{l}4.835989 \\
3.6369455 \\
9.648456 \\
1.097373177 \\
5.2917706 \\
2.8179380 \\
0.6652448 \\
1.0011596567 \\
1.00116616 \\
9.274078 \\
9.284832 \\
2.6751301 \\
2.6751987 \\
1.4106171 \\
1.521032209 \\
2.7928456 \\
1.52099322 \\
2.7927740\end{array}$ & $\begin{array}{l}13 \\
60 \\
27 \\
83 \\
44 \\
70 \\
33 \\
35 \\
31 \\
36 \\
36 \\
75 \\
75 \\
55 \\
16 \\
11 \\
10 \\
11\end{array}$ & $\begin{array}{l}10^{14} \mathrm{~Hz} \cdot \mathrm{V}^{-1} \\
10^{-4} \mathrm{~J} \cdot \mathrm{s} \cdot \mathrm{kg}^{-1} \\
10^{4} \mathrm{C} ; \mathrm{mot}^{\prime} \\
10^{7} \mathrm{~m}^{-1} \\
10^{-11} \mathrm{~m} \\
10^{-15} \mathrm{~m} \\
10^{-2 m} \mathrm{~m}^{2}\end{array}$ \\
\hline Electron-proton mag. mom. & & & & \\
\hline $\begin{array}{l}\text { ratio ................. } \\
\text { Nuclear magneton, ehi } 2 m_{n} \\
\text { Compton wavelengih: }\end{array}$ & $\begin{array}{l}\mu_{e} / \mu_{p} \\
\mu_{l}\end{array}$ & $\begin{array}{c}658.21068800 \\
5.050824\end{array}$ & $\begin{array}{l}66 \\
20\end{array}$ & $10^{-2 \pi} \mathrm{J} \cdot \mathrm{T}^{-1}$ \\
\hline $\begin{array}{l}\text { electron, } h / m_{\mathrm{e}} c=\alpha^{\ddot{z}} / 2 R \times \ldots \ldots \\
\text { proton, } h / m_{\nu} c \ldots \ldots \ldots \ldots \ldots \\
\text { neutron, } h / m_{\mathrm{n}} c \ldots \ldots \ldots \ldots \ldots \\
\text { N uon-proton mag. mom. ratio }\end{array}$ & $\begin{array}{l}\lambda_{c} \\
\lambda_{C, p} \\
\lambda_{C, p} \\
\mu_{p, p} / \mu_{p}\end{array}$ & $\begin{array}{l}2.4263089 \\
1.3214099 \\
1.3195909 \\
3.1833402\end{array}$ & $\begin{array}{l}40 \\
22 \\
22 \\
72\end{array}$ & $\begin{array}{l}10^{-12} \mathrm{~m} \\
10^{-13} \mathrm{~m} \\
10^{-15} \mathrm{~m}\end{array}$ \\
\hline
\end{tabular}




\begin{tabular}{|c|c|c|c|c|}
\hline Quantity & Symbol & Num. value & Uncert. & Units (SI) \\
\hline 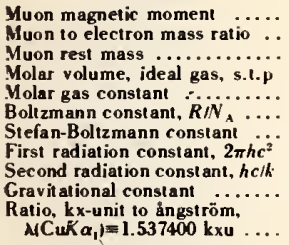 & $\begin{array}{l}\mu_{\mu} \\
m_{\mu} / m_{e} \\
m_{\mu} \\
V_{m} \\
R \\
k \\
\sigma \\
c_{1} \\
c_{2} \\
G\end{array}$ & $\begin{array}{c}4.490474 \\
206.76865 \\
1.883566 \\
22.41383 \\
8.31441 \\
1.380662 \\
5.67032 \\
3.741832 \\
1.438786 \\
6.6720\end{array}$ & $\begin{array}{l}18 \\
47 \\
11 \\
70 \\
26 \\
44 \\
71 \\
20 \\
45 \\
41\end{array}$ & $\begin{array}{l}10^{-2 h} \mathrm{~J} \cdot \mathrm{T}^{-1} \\
10^{-2 m} \mathrm{~kg} \\
10^{-11} \mathrm{~m}^{3} \cdot \mathrm{mol}^{-1} \\
\mathrm{~J} \cdot \mathrm{mol}^{-1} \cdot \mathrm{K}^{-1} \\
10^{-23} \mathrm{~J} \cdot \mathrm{K}^{-1} \\
10^{-\mathrm{K}} \mathrm{W} \cdot \mathrm{m}^{-2} \cdot \mathrm{K}^{-1} \\
10^{-16} \mathrm{~W} \cdot \mathrm{m}^{-2} \\
10^{-2} \mathrm{~m} \cdot \mathrm{K} \\
10^{-11} \mathrm{~m}^{3} \cdot \mathrm{s}^{-2} \cdot \mathrm{kg}^{-1}\end{array}$ \\
\hline
\end{tabular}

ENERGY CONVERSION FACTORS AND EQUIVALENTS

\begin{tabular}{|c|c|c|c|}
\hline Quantity & Num. value & Uncertainty* & Units \\
\hline 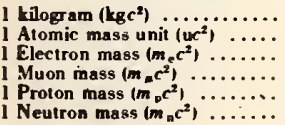 & $\begin{array}{c}5.609545 \\
931.5016 \\
0.5110034 \\
105.65948 \\
938.2796 \\
939.5731\end{array}$ & $\begin{array}{l}16 \\
26 \\
14 \\
35 \\
27 \\
27\end{array}$ & $\begin{array}{l}10^{2 y} \mathrm{MeV} \\
\mathrm{MeV} \\
\mathrm{MeV} \\
\mathrm{MeV} \\
\mathrm{MeV} \\
\mathrm{MeV}\end{array}$ \\
\hline
\end{tabular}

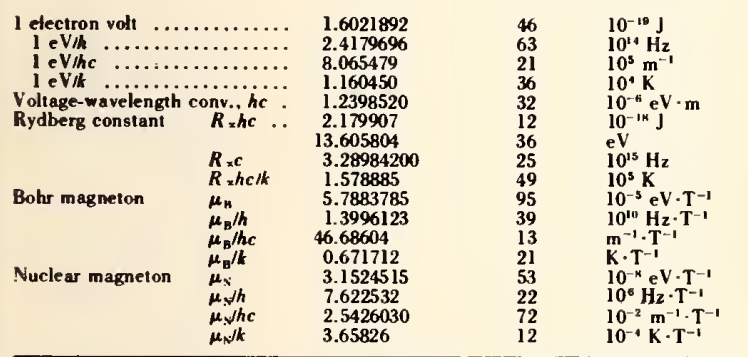

- Uncertainty (1 std. dev.) applies to last digits of preceeding column; $\mu_{0}$ is exactly $\pi \times 10^{-7} \mathrm{H} \cdot \mathrm{m}^{-1}$. For some entries, energies are given in non-Si units. For values in $\mathrm{S}$ nits, see revised reprint of the Janu

NBS Special Publication 398, issued August 1974, supersedes NBS Spec. Publ. 344. For sale by Superintendent of Documents, U.S. Government Printing Office, Washington, D. C 20402: order by SD Catalog No. C13.10:39, 25 cents each. 6.35 per 100 . Stock Number 0303-01331.

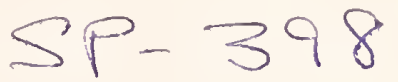




\section{NBS SPECIAL PUBLICATION 399 \\ Volume 1}

U.S. DEPARTMENT OF COMMERCE / National Bureau of Standards

NBS FORTRAN Test Programs

Volume 1-Documentation for Versions 1 and 3 
The National Bureau of Standards ${ }^{1}$ was established by an act of Congress March 3, 1901. The Bureau's overall goal is to strengthen and advance the Nation's science and technology and facilitate their effective application for public benefit. To this end, the Bureau conducts rcsearch and provides: (1) a basis for the Nation's physical measurement system, (2) scientific and technological services for industry and government, (3) a technical basis for equity in trade, and (4) technical services to promote public safety. The Bureau consists of the Institute for Basic Standards, the Institute for Materials Research, the Institute for Applied Technology, the Institute for Computer Sciences and Technology, and the Office for Information Programs.

THE INSTITUTE FOR BASIC STANDARDS provides the central basis within the United States of a complete and consistent system of physical measurement; coordinates that system with measurement systems of other nations; and furnishes essential services leading to accurate and uniform physical measurements throughout the Nation's scientific community, industry, and commerce. The Institute consists of a Center for Radiation Research, an Office of Measurement Services and the following divisions:

Applied Mathematics - Electricity - Mechanics - Heat - Optical Physics - Nuclear Sciences $^{2}$ - Applied Radiation ${ }^{2}$ - Quantum Electronics ${ }^{3}$ - Electromagnetics ${ }^{3}$ - Time and Frequency ${ }^{3}$ - Laboratory Astrophysics ${ }^{3}$ - Cryogenics ${ }^{3}$.

THE INSTITUTE FOR MATERIALS RESEARCH conducts materials research leading to improved methods of measurement, standards, and data on the properties of well-characterized materials needed by industry, commerce, educational institutions, and Government; provides advisory and research services to other Government agencies; and develops, produces, and distributes standard reference materials. The Institute consists of the Office of Standard Reference Materials and the following divisions:

Analytical Chemistry - Polymers - Metallurgy - Inorganic Materials - Reactor

Radiation - Physical Chemistry.

THE INSTITUTE FOR APPLIED TECHNOLOGY provides technical services to promote the use of available technology and to facilitate technological innovation in industry and Government; cooperates with public and private organizations leading to the development of technological standards (including mandatory safety standards), codes and methods of test; and provides technical advice and services to Government agencies upon request. The Institute consists of a Center for Building Technology and the following divisions and offices:

Engineering and Product Standards - Weights and Measures - Invention and Innovation - Product Evaluation Technology - Electronic Technology - Technical Analysis - Measurement Engineering - Structures, Materials, and Life Safety ${ }^{*}$ - Building Environment ${ }^{4}$ - Technical Evaluation and Application ${ }^{4}$ - Fire Technology.

THE INSTITUTE FOR COMPUTER SCIENCES AND TECHNOLOGY conducts research and provides technical services designed to aid Government agencies in improving cost effectiveness in the conduct of their programs through the selection, acquisition, and effective utilization of automatic data processing equipment; and serves as the principal focus within the executive branch for the development of Federal standards for automatic data processing equipment, techniques, and computer languages. The Institute consists of the following divisions:

Computer Services - Systems and Software - Computer Systems Engineering - Information Technology.

THE OFFICE FOR INFORMATION PROGRAMS promotes optimum dissemination and accessibility of scientific information generated within NBS and other agencies of the Federal Government; promotes the development of the National Standard Reference Data System and a system of information analysis centers dealing with the broader aspects of the National Measurement System; provides appropriate services to ensure that the NBS staff has optimum accessibility to the scientific information of the world. The Office consists of the following organizational units:

Office of Standard Reference Data - Office of Information Activities - Office of Technical Publications - Library - Office of International Relations.

${ }_{1}^{1}$ Headquarters and Laboratories at Gaithersburg, Maryland, unless otherwise noted; mailing address Washington, D.C. 20234.

2 Part of the Center for Radiation Research.

"Located at Boulder, Colorado 80302.

- Part of the Center for Building Technology. 


\section{NBS FORTRAN Test Programs \\ Volume 1-Documentation for Versions 1 and 3}

Frances E. Holberton and

Elizabeth G. Parker

Institute for Computer Sciences and Technology

National Bureau of Standards

Washington, D.C. 20234

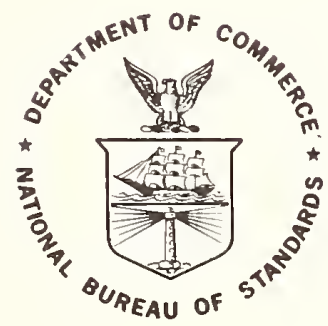

U.S. DEPARTMENT OF COMMERCE, Frederick B. Dent, Secretary NATIONAL BUREAU OF STANDARDS, Richard W. Roberts, Director 


\section{Library of Congress Cataloging in Publication Data}

Holberton, Frances E

NBS FORTRAN test programs.

National Bureau of Standards Special Publications 399.

CONTENTS: V. 1. Documentation for versions 1 and $3 .-\mathrm{V} .2$. Listings for version 1.-V. 3. Listings for version 3.

Supt. of Docs. No.: C 13.10:399.

1. Computer programs-Testing. 2. FORTRAN (Computer program language) I. Parker, Elizabeth G., joint author. II. United States. National Bureau of Standards. III. TitIe. IV. Series: United States. National Bureau of Standards. Special Publication 399.

QC100.U57 no. 399 [QA76.6]

$389^{\prime} .08$ s [001.6'425] 74.12314

\section{National Bureau of Standards Special Publication 399}

Nat. Bur. Stand. (U.S.), Spec. Publ. 399, 171 pages (Oct. 1974)

CODEN: XNBSAV 


\section{FOREWORD}

It has now become imperative, because of the multitude of computers on the market and the corresponding multitude of FORTRAN compilers, to develop a means of testing the overall quality of these compilers, thereby making meaningful comparisons possible. The National Bureau of Standards has sponsored a project to develop methods and tools to assist in the evaluation process. Before the evaluation process can be undertaken, it is necessary to develop a primary tool, such as a set of FORTRAN programs which can validate whether a FORTRAN compiler is in compliance with the FORTRAN specification as described in the American Standard FORTRAN document X3.9-1966.

In 1966 the National Bureau of Standards formulated the design criteria and specifications for the development of such a set of FORTRAN programs. The initial implementation of this design was performed, under contract, by the Advanced Computer Techniques Corporation in 1967. Since this time, these programs have been desk checked, computer checked, revised, extended, many test units replaced, and the system reorganized to improve the tests and decrease the difficulty of performing the actual validation process.

Version 2 of these test programs was prepared by NBS, under contract, for the Joint Technical Support Activity of the Defense Communications Agency.

The purpose of these FORTRAN programs is to assist in the validation of FORTRAN compilers. There is no attempt to measure the performance of the compiler or the object program efficiency.

Currently, the FORTRAN Standand, ASA X3.9-1966, is undergoing revision and the FORTRAN language is being extended by the X3J3 technical committee of the American National Standards Institute (formerly identified as the American Standards Association). The revised FORTRAN Standand will be considered for Federal adoption. This will, if approved, require that the test programs be revised accordingly.

The National Bureau of Standards wishes to thank the Bell Telephone Laboratories for the preparation of the camera-ready copy of the program listings derived from the NBS FORTRAN Test Program Distribution Tape. These listings appear in Volumes 2 and 3 of this report. 


\title{
NBS FORTRAN TEST PROGRAMS
}

The NBS FORTRAN test programs, written in Standand FORTRAN, are designed to test whether a FORTRAN compiler accepts the forms and interpretations of the FORTRAN language as described in the American National Standard FORTRAN document X3.9-1966. The test programs, comprised of 116 test units, are structured into two versions, each containing approximately 14,500 punch card images. The test units may be used as separate executable FORTRAN programs; or may be linked end to end with other test units, with a minimum of user effort, to improve operating efficiency. Version 1 is structured into 116 executable FORTRAN programs, and Version 3, containing the same 116 test units, iș structured into 14 executable FORTRAN programs for use on large FORTRAN processors.

The test program design criteria was to:

- Constrain all test programs to the FORTRAN Standard X3.9-1966.

- Reduce the effect of those areas in which the FORTRAN Standard does not prescribe a method or solution, e.g., range, precision, size of computer, etc.

- Simplify the use of the FORTRAN test programs.

- Test FORTRAN language elements before they are used in support of other tests.

- Maintain an open ended system so that tests may be changed or added.

The test programs require the use of a card reader, printer and one intermediate tape unit.

During the development of the test systems ten different computing systems were used, and the current set of tests were run on five major systems. The largest test unit required less than 3,000 words of memory and when structured into 14 executable programs the largest program required less than 6,000 words of memory to execute the compiled programs. The test units, for the most part, are straight line programs and during the debugging of the tests, less than 15 minutes was required to compile and execute the set of 14 structured FORTRAN programs, excluding card read and printer time.

The magnetic tape, containing the NBS FORTRAN Test Programs, Version 1 and Version 3, together with the documentation ( 3 volumes) is available from:

\author{
National Technical Information Service \\ Department of Commerce \\ 5285 Port Royal Road \\ Springfield, Virginia 22151
}

The magnetic tape is available in $800 \mathrm{cpi}$ recording density in the following forms:

7 track even parity BCD, recorded from the FORTRAN $\mathrm{H}$ set punch card code (see FORTRAN Standard, Appendix D X3.9-1966)

9 track odd parity EBCDIC code

9 track odd parity ASCII code

Key words: Computer programming language; FORTRAN: FORTRAN validation; language validation; standards FORTRAN; test program design. 
This document, Volume 1 of three volumes, contains the documentation, including the systems design, operating procedures, expected test results and distribution tape organization for a set of FORTRAN Test Programs developed by the Institute for Computer Sciences and Technology, National Bureau of Standards. These test programs are written in ASA Standard FORTRAN and test the language elements described in the ASA Standard FORTRAN document X3.9-1966.

The NBS FORTRAN Test Programs, Version 1, contain 116 test units, each structured as an executable FORTRAN program.

The NBS FORTRAN Test Programs, Version 3, containing the same 116 test units, structured into 14 executable FORTRAN Programs, have been organized for use on large FORTRAN Processors for the purpose of reducing the number of systems control cards needed to perform the tests.

The NBS FORTRAN Test Programs, recorded on magnetic tape as approximately 14,500 punch card images for each version, contain the FORTRAN source language programs and data. Extensive FORTRAN comment lines are interspersed throughout the programs to enable the user to both run the programs and determine the nature of the tests without the need for additional documentation. The test results contain information related to the expected results.

Volume 1, Section I describes the system design, the programing techniques and conventions used in the program development and should enable the user to extend, alter or reorganize the test programs.

Volume I, Section II defines the organization and operating procedure for performing the tests and contains a set of representative results obtained from actual muning of the test programs on several FORTRAN processors.

Volume 1, Section III describes the onder and location of each test unit and data as recorded on magnetic tape for distribution.

Volume 2 contains the program listings for the NBS FORTRAN Test Programs, Version 1 .

Volume 3 contains the program listings for the NBS FORTRAN Test Programs, Version 3. 
Table of Contents

Foreword

Abstract

Introduction

Section I SYSTEMS MANUAL

A FORTRAN Test Program Design

A1. Objective

$\mathrm{I}-\mathrm{A}-1$

A2. Design Criteria

$\mathrm{I}-\mathrm{A}-1$

A3. Design Considerations

$\mathrm{I}-\mathrm{A}-1$

A4 。 Design Implementation

$\mathrm{I}-\mathrm{A}-4$

A5. FORTRAN Concepts Excluded from the Test Programs

$\mathrm{I}-\mathrm{A}-6$

A6. Interpretations Made to the FORTRAN Standard

$\mathrm{I}-\mathrm{A}-8$

B. Description of Each Segment

I-B-1

C. Test Unit Segments Indexed to the FORTRAN Standard

$\mathrm{I}-\mathrm{C}-1$

Document ASA X3.9-1966

D. Program Information

D1. Conventions Used in the Test Programs

I-D- 1

D2。 Assumed Levels for Non-specified FORTRAN Areas

$\mathrm{I}-\mathrm{D}-4$

D3. Names and Statement Numbers Used in the Test

$\mathrm{I}-\mathrm{D}-6$

E。 Structuring, Restructuring and Extending the Test Programs

E1. Program Structure

$\mathrm{I}-\mathrm{E}-1$

E2. Consolidating Test Program Units Using Version 1

$\mathrm{I}-\mathrm{E}-1$

E3. Deleting a Section of a Test Unit

$\mathrm{I}-\mathrm{E}-4$

E4. Deleting an Entire Test Unit

$\mathrm{I}-\mathrm{E}-4$

E5. Adding to a Test Unit

I-E-4

E6. Adding New Test Units

$\mathrm{I}-\mathrm{E}-5$

F。 Difficulties Encountered During the Test Program Development I-F-1

F1。 Interpreting the FORTRAN Standard

$\mathrm{I}-\mathrm{F}-1$

F2. Precision, Conversion and Maximum Value of

Numeric Data

F3. Meaningful Tests and Comprehensible Results

I-F- 1

F4. FORTRAN Compilers with Language Extensions

$\mathrm{I}-\mathrm{F}-3$

F5. Performing the Tests

I-F-3 
G. References

Section II USERS MANUAL

A. Operating Procedures

Al. Organization of Tests and Facilities Requirements II $-\mathrm{A}-1$

A2. Input Data Preparation II $-\mathrm{A}-3$

A3. List of Test Programs for Version 1 II $-\mathrm{A}-4$

A4. List of Test Units by Parts for Version 3

II $-\mathrm{A}-11$

B. Procedures for Isolating Test Unit Failures from Version 3 II-B-1

B1. Deleting a Test Unit

II $-B-1$

B2. Creating a Single Test from a Deleted Unit

II $-B-1$

C. Sample Test Results

I I-C-1

C1. Interpreting the Test Results

II $-\mathrm{C}-1$

C2. Test Results

Section III DISTRIBUTION TAPE ORGANIZATION

A. General Description

III-A- I

A1. FORTRAN Test Programs and Data Version 1

II I-A-3

A2. FORTRAN Test Programs and Data Version 3

III $-\mathrm{A}-5$ 
NBS FORTRAN Test Program Results

Table of Contents

Page

008 - FMTRW

009 - AFRMT

010 - DATA2

011 - AASGN

013 - DASGN

015 - CASGN

016 - LASGN

017 - INTRL

020 - UGOTO

021 - AGOTO

022 - CGOTO

030 - ARBAD

031 - ARFAD

032 - ARBSB

033 - ARFSB

034 - ARBAS

035 - ARFAS

036 - ARBMI

037 - ARBMR

038 - ARFMD

039 - ARBDV

040 - ARFDV

041 - ARBEX

042 - ARFEX

043 - ARBHI

050 - SBB67

051 - SBB45

052 - SBB13

$053-\mathrm{SBF} 17$

054 - SIMIF

055 - IFABS

056 - IFFLT

057 - IFFIX

058 - IFSGN

059 - IFDAB

060 - IFTRN

061 - IFMOD

062 - IFMAX

063 - IFMIN

064 - IFDSG
Formatted Input/Output

A-Conversion

DATA Statement Use

Real and Integer Arith Assignment. Stmnts.

Simple D.P. Assignment Statements

Simple Complex Assignment Statements

Logical Assignment Statements

Arithmetic Assignment Statements

Unconditional GO TO Statements

GO TO Assignment Statements

Computed GO TO Statements

Basic Addition

Double Precision Addition

Basic Subtraction

Double Precision Subtraction

Basic Addition and Subtraction

Addition and Subtraction of D.P. Values

Multiplication of Integer Values

Multiplication of Real Values

Multiplication of D.P. Values

Division of Integer and Real Values

Division of D.P. Values

Exponentiation of Integer and Real Values

Exponentiation of D.P. Values

Hierarchy of Operators and Parentheses

Subscripts of Integer, Real Arrays $v, k$

Subscripts of Int., Real Arrays $v+k, v-k$

Subscripts of Int., Real Arrays $c^{*} v, c^{*} v+k$, $c^{*} \mathrm{v}-\mathrm{k}$

Subscripts of D.P. Arrays $v, k, c^{*} v, c^{*} v+k$, $c^{*} \mathrm{v}-\mathrm{k}, \mathrm{v}+\mathrm{k}, \mathrm{v}-\mathrm{k}$

Arith. IF, Logical IF followed by GO TO

Intrinsic Functions ABS, IABS

Intrinsic Function FLOAT

Intrinsic Function IFIX

Intrinsic Functions SIGN, ISIGN

Intrinsic Function DABS

Intrinsic Functions AINT, INT, IDINT

Intrinsic Functions AMOD, MOD

Intr. Funct. AMAX0, AMAX1, MAX0, MAX1', DMAX1

Intr. Funct. AMINO, AMIN1, MINO, MIN1, DMIN1

Intrinsic Function DSIGN
II- C- 3

II- C- 7

II- C- 8

II- C- 9

II- C- 11

II- C- 15

II- C- 19

II-C- 20

II- C- 22

II- C- 22

II- C- 23

II- C- 23

II- C- 24

II- C- 24

II- C- 24

II- C - 24

II- C - 25

II- C- 25

II-C- 25

II- C- 25

II- C- 26

II-C- 26

II-C- 26

II- C- 26

II- C- 27

II- C- 27

II- C- 27

II- C- 28

II- C- 28

II-C- 28

II- C- 29

II- C - 29

II- C- 29

II-C- 29

II- C- 29

II-C- 30

II-C- 30

II- C- 31

II-C- 32

II- C- 32 
NBS FORTRAN Test Program Results

Table of Contents

Page

065 - IFDIM

066 - IFSGL

067 - IFREL

068 - IFIMG

069 - IFDBL

070 - IFCPX

071 - IFCJG

072 - IFBMS

073 - IFFMS

080 - EXPON

081 - DEXPO

082 - CEXPO

083 - LOGTM

084 - DPLOG

085 - CXLOG

086 - COLOG

087 - DCLOG

088 - SINUS

089 - DPSIN

090 - CSICO

091 - COSNS

092 - DPCOS

094 - TANGH

095 - SQROT

096 - DSQRO

097 - CSQRO

098 - ARCTG

099 - DACTG

100 - ACTG2

101 - DATN2

102 - DMODA

103 - CABSA

110 - BSFTS

111 - FSFTS

140 - CPXAD

141 - CPXMU

142 - CPXDV
Intrinsic Functions DIM, IDIM

Intrinsic Function SNGL

Intrinsic Function REAL

Intrinsic Function AIMAG

Intrinsic Function DBLE

Intrinsic Function CMPLX

Intrinsic Function CONJG

Integer and Real Intrinsic Functions

Int., Real and D.P. Intrinsic Functions

Basic External Function EXP

Basic External Function DEXP

Basic External Function CEXP

Basic External Function ALOG

Basic External Function DLOG

Basic External Function CLOG

Basic External Function ALOG10

Basic External Function DLOG10

Basic External Function SIN

Basic External Function DSIN

Basic External Functions CSIN, CCOS

Basic External Function CoS

Basic External Function DCOS

Basic External Function TANH

Basic External Function SQRT

Basic External Function DSQRT

Basic Externa1 Function CSQRT

Basic External Function ATAN

Basic External Function DATAN

Basic External Function ATAN2

Basic Externa1 Function DATAN2

Basic External Function DMOD

Basic External Function CABS

Statement Functions - Integer and Real

Statement Funct. - D.P., Complex, Logical

Addition and Subtraction of Complex

Multiplication of Complex Numbers

Division of Complex Numbers
II- C- 33

II- C -33

II- C- 34

II- C- 34

II-C- 35

II- C- 35

II- C 35

II- C- 36

II- C- 36

II- C- 37

II- C- 38

II- C- 38

II- C- 40

II- C- 40

II- C- 41

II- C- 42

II- C- 43

II- C- 43

II- C- 44

II- C- 44

II- C- 45

II- C- 45

II- C- 46

II-C- 46

II- C- 47

II- C- 47

II- C- 48

II- C- 48

II- C- 49

II- C- 49

II-C- 50

II- C- 50

II- C- 51

II- C - 51

II- C- 52

II- C- 52

II-C- 53 
Table of Contents

Page

143 - CPXEX

144 - CPXOP

145 - CREAD

146 - CREMU

147 - CREDV

148 - CREOP

149 - MISC3

150 - MISC4

160 - BRFCP

161 - BIFCP

162 - FRFCP

163 - FIFCP

164 - CFCCP

165 - DPFCP

166 - BFCCP

167 - SBRTN

168 - FSBRT

169 - BLKDT

179 - BLKDA

180 - UNFRW

182 - BACUP

190 - DOTRM

191 - DOLMT

192 - DONSC

193 - DONSI

194 - DONSX

195 - DONML

196 - DONIO

197 - MORDO

200 - SUBRI

300 - LOGIF

301 - BARIF

302 - FARIF

310 - IOFMT

312 - RDFMT

350 - MISC 5

351 - FUNMX

352 - NAMES

360 - SPEC 2
Exponentiation of Complex Numbers

Arithmetic Operations on Complex

Add and Subtract Coinplex and Real Numbers

Multiply Complex by Real Numbers

Divide Complex by Real and the Reverse

Combined Operations on Complex and Real

Blanks in, Cont. of Statement to Max Lines

Special Characters for Continuations

Real External Functions

Integer External Functions

Real External Functions

Integer External Functions

Complex External Function

Double Precision External Functions

Logical External Functions

Subroutine Subprogram

Subroutine Subprogram

BLOCK DATA Test

BLOCK DATA Test

Unformatted WRITE and READ

BACKSPACE Tape

DO Loops - Terminal Statements

Do Loops - Parameters as Variable Names

DO Loops - Completely Nested Nest

DO Loops - Incomplete DO, Exit by GO TO

DO Loops - Extended Range

DO Loops - Nested Nest

DO Loops - I/O Terminal Statements

DO Loops - I/O, Statmt. Ft., Intr Ft., CALL

Subroutine - Operations Done at Sub Level

Logical IF Statements

Arithmetic IF Statements - Integer, Real

Arithmetic IF Statements - D.P.

Formatted READ/WRITE - Additional Features

Formats in Arrays

Specifications for Program Form

Basic External Functions - Trig Formulae

Names Resemble FORTRAN Verbs, Functions

COMMON, DIMENSION, EQUIVALENCE
II- C - 53

II- C- 54

II- C- 54

II- C- 54

II- C- 54

II- C- 55

II- C- 55

II- C -55

II- C -55

II- C- 56

II-C- 56

II- C- 56

II- C- 56

II- C- 57

II- C - 57

II- C- 57

II-C 58

II- C- 58

II-C - 59

II-C- 59

II-C - 60

II- C- 60

II-C- 60

II- C - 60

II- C - 60

II-C- 61

II- C- 61

II- C- 61

II- C- 62

II- C- 62

II-C- 62

II- C- 63

II- C - 63

II- C- 63

II-C- 65

II-C- 66

II- C- 67

II- C- 67

II- C - 67 
A. FORTRAN TEST PROGRAM DESIGN

A1. Objective: To develop a set of FORTRAN test programs, available to a wide range of FORTRAN processors with a minimum of user effort required to perform the tests. These tests shall conform to the ASA FORTRAN Standard X3.9-1966.[1]

A2. Design Criteria

a) To constrain all test programs to the FORTRAN language described in the ASA FORTRAN Standard X3.9-1966.

b) To reduce the effect of those areas in which the FORTRAN standard does not prescribe a method or solution; the programs must be adaptable to differing environments such as:

- Size of computer and I/O facilities.

- Power of the FORTRAN compiler as reflected in the size and complexity of a FORTRAN program.

- Variations in the range and precision of numeric values.

- Differences in form and media for submitting a program and data.

- Differences in procedures for compiling and muning a FORTRAN program.

c) To simplify the use of the FORTRAN test programs.

- The cost of computer time for compiling and running must be kept to a minimum.

- The cost of human resources for the analysis of test results, determination of test failures and the comprehension of the test design must be taken into consideration in the system design.

d) To test FORTRAN language elements before they are used in support of other tests.

e) To maintain an open ended system so that tests may be changed or added.

A3. Design Considerations

It is recognized that any set of programs which is designed to test a complex set of specifications, such as ASA FORTRAN (X3.9-1966) can never test every interaction of every FORTRAN statement, with all permissible forms, in all permissible positions in an executable program. However, it is desirable to design a system such that those parts of the Ianguage which have been tested are relatively easy to determine and at the same time permit extensions to the system without extensive knowledge of the entire system.

The test programs must be designed with the realization that a FORTRAN processor might not accept various elements of the language and the action could be identified at one or more of the following times or conditions: 
a) Compile time.

- The compiler might terminate without completion of the compilation and with insufficient information for the user to determine the cause.

- The compilation may be completed with diagnostic messages on the program listing, which as a general rule (although outside of the FORTRAN standard specification) assist in locating the trouble.

b) Link Edit and Load Time.

- The executable program may fail to meet the loader, etc., requirements-which may or may not be identified in the program listing.

c) Execution Time.

Conditions in the computer or compiler may produce improper machine code which causes the test progran to be aborted before completion. (Any one or more of these conditions could occur prior to obtaining the test program results.)

d) Unexpected Test Results.

The running of the test programs could produce printed results which were different from the expected results. This can occur if:

- Some well defined element of the Standard FORTRAN language was implemented in the compiler in a variant way.

- Some ill defined part of the language was interpreted by the compiler writer different from the test program writers.

- An improper interpretation of the standard by the test program writers.

- An actual bug in the test programs.

- An actual bug in the compiler.

Because many unforseen difficulties can occur during the muning of the test programs, where it will be necessary for the user to refer to the program listing to determine what elements of the language are being tested as distinct from those elements which must be used to support the test, it is imperative that the program listing be liberally interspersed with FORTRAN comment lines to assist the reader.

Because the FORTRAN standard document is a semi-technical specifications document without a rigid definition of the semantics of the language, the document is subject to interpretation differently by different individuals.

The ASA FORTRAN Standard is a reference standard and does not address the medium or its coded characteristics, so that the form of the FORTRAN program on a medium such as punched cards is outside the scope of the standard. However, because a common medium is punched cards, and the H-set punch card code was designed for FORTRAN, the H-set is deemed the most universally 
accepted card code on which to prepare the FORTRAN test programs and data for a processor. If a processor does not accept this card code it is reasoned that a conversion routine probably does exist which could convert this set to the processor punch card code.

If the programs are to be available to both large and small FORTRAN processors the I/O facilities must be kept to a minimum. If the processor has a card reader then most likely a printer and either one tape unit or a disc would also be available, so that the test programs could be confined to these I/O devices.

In order to determine what capabilities existed for FORTRAN or FORTRAN-like compilers in 1966 when this project was initiated, a survey of the literature was made and specifications for forty compilers were compared. From this unpublished study, a "FORTRAN processor" was defined to contain the minimum range and precision of numeric values and the most limited program size which could be found among the forty compilers examined. This lead to the constraints used in the test programs which are described under Program Information Section I-D.

The assumption was made, because of the nature of FORTRAN, that all processors probably had something akin to "Compile-Load-and Go" as a form of operations.

Each test program, if it were to run on a small computer, must be limited in size. It is theoretically possible to test almost all characteristics of the language in a single executable program if a processor were large enough. However, it might be desirable to test a new compiler on a large computer for the first time with small test elements, so that any difficulties might be recognized more rapidly, while any later running of the test programs or updated versions of the compiler could be performed more economically if the test elements were combined into larger executable programs.

In order for the test units to be run independently and later combined into larger executable programs, as well as changed or expanded it was necessary to consider the following:

- The required positioning of certain FORTRAN statements such as specification statements and statement functions.

- The choice of symbolic names, such that they did not constrain the testing of elements of the language, and at the same time would not require the knowledge by the user of symbolic names which had been used when changes or expansion of the test were necessary.

- The allocation of statement labels so that duplication would not result.

- The handling of those aspects of a FORTRAN program which are not covered by the standard such as precision, size of program, number of arguments, depth of DO nesting, the number of subprograms, etc. 
The FORTRAN test programs are not designed for use in debugging of a FORTRAN compiler. In fact, the assumption has been made that the compiler, for the most part, is working but may not have all of the FORTRAN language features available in the system.

Those elements of the language which are used in support of test units are limited to what can be considered "defacto FORTRAN". That is, language features which were not universally implemented in 1966 but which appear in the standard are tested but are never used in support of other tests. Therefore, such features as: extended range of a DO, the GW.d format field descriptor, a constant of the form $26 \mathrm{E} 1$ containing no decimal point, etc., are not used after their appearance in a test unit.

The test program units, for the most part, are small main programs with straight line logic. Each test unit is implemented to be run as a separate test or linked end to end with another test. All data used within a program test unit is defined within that unit, except the tests for the FORMAT statement which require external input data to be read.

The selected order of the test units is dictated by the need for testing the basic fundamentals of the language so that these features may be used to support later tests. Certain elements of formatted READ and WRITE are tested first, so that test results can be written out.

The initial test of the DATA statement appears as an early test sequence because a constraint would be placed upon the use of symbolic names in other test units prior to the occurrence of the DATA statement test if the test appeared later in the set. Other appearances of the DATA statement are in a subprogram and as a format specification. These are for the purpose of the tests and no further use is made of this statement.

A11 testing is performed at the main program level except those concepts and associations which are unique to a subprogram. One test unit which is performed at the main program level containing a variety of FORTRAN statements is basically duplicated in a test unit which performs the same statements at the subprogram level. Other appearances of subprograms in the test set are basically for the purpose of argument association testing and for those FORTRAN statements which may occur only in a subprogram.

The FORTRAN statements used in the test units may appear, at first glance, to be nonsense operations. To comprehend the true meaning of the statement in a test unit, it is necessary to read the statement transforming the variable name or constant used into its attributes and utilization associated with an operator. Such an example might be: A one dimensional array element appearing in a common block is raised to the power of an unsigned integer constant.

To assist the test program implementors as well as the reader of the test programs, naming conventions described in Program Information, 
Section I-D, have been used throughout the programs to convey the attributes of the name, which appear in specification statements, directly in the name itself, so that no reference need be made to the specification statements to comprehend this information. In addition, comment cards have been used freely in the test units to convey the nature of the test and the operations being performed.

The design of a computer program system for automatic insertion of operating system control cards and the linking of test program units was initiated. Further analysis into the problems has brought to light the potential difficulties of using the output of such a system and its doubtful economics. For the following reasons, this system has not been implemented:

- The lack of common terminology for similar functions among various operating systems control languages would cause difficulty in communicating with a wide audience the information required to be inserted into an automated system for producing the desired effect. For example, what is called a JOB card in one system is called a RUN card in another, while what is called a RUN card in another system may be called an EXECUTE card in the first system. Because similar terminology for operating system control functions is used for functions of the system at different levels of control, it would be necessary to describe levels of functions to a user, who might not be aware of this logic.

- The FORTRAN standard does not define the order of presentation to a compiler of program units, so that this becomes an additional burden to the user to comprehend when this order may not affect the majority of FORTRAN processors.

- Operating systems control cards may require special control punch codes which are outside the codes defined for data use. For example, a control card which contains a code containing the digits $6,7,8$ and 9 in a single column on the card can be obtained only by a keypunch device with provisions for over striking in a single column.

- To produce punched cards from an automated system with special codes outside the normal punch card character set would require the software-hardware system to permit column binary cards to be punched。 This facility, although available in the hardware of some systems is not available to the user because of software constraints. Of the computer systems surveyed, only one system permitted column binary cards to be produced and this facility is available only to the assembly language programming system.

- If cards can be produced by the column binary operations from the system, the device which interprets and prints on the card would not necessarily print the appropriate symbols, because codes for certain FORTRAN characters and the control card codes may have different graphic associations or no valid association. 
- If the test programs with their interpersed operating systems control cards were placed on tape, there is no assurance that the receiving installation has provisions for using or even obtaining punched cards from such a tape. Although the images on tape would be in coded character representation for the receiving installation, the operating system may not permit the reading of control card sensitive information and the passing of it to an applications program for the purpose of producing punch cards. If the tape is read as a binary tape, the parity bit, record size, pulse code and blocking characteristics might not conform to the receiving computer's requirements。

Because of these numerous difficulties which may affect the user and the potential additional costs which may be encountered in preparing the test program information at the receiving installation, these test programs have been prepared for use without the inclusion of systems control cards。

To simplify the task of grouping test units together into larger programs for testing, and thus eliminate the need for an abundance of systems control cards to operate each test unit as a separate computer run, those cards which must be revised are identified in the test units as comment cards containing the characters " $\mathrm{C}=$ " in the first two columns. The FORTRAN specification statements taken from different test units require the elimination of duplicate names to conform to the language definition. To simiplify this task, symbolic names appearing in a " $\mathrm{C}=$ " specification statement will always appear in the same type of specification statement throughout the entire program test set, so that elimination of duplicate names is achieved by inspection of a collection of a similar type of specification statement. That is, if an array declarator in one program test unit is contained in an INTEGER statement, all other occurrences of that symbolic name in a specification statement will be in an INTEGER statement and not in a DIMENSION statement. See Structuring, Restructuring and Extending Test Programs, Section I-E.

A5. FORTRAN Concepts Excluded from the Test Programs.

Because the FORTRAN Clarification Reports [2, 3] do not have the status of updating the current ASA FORTRAN document X3.9-1966, extreme caution was exercised in making use of some of the interpretations in the FORTRAN test programs. The following FORTRAN Statements and concepts have been excluded from the FORTRAN Test Programs:

a) An I/O unit number specified by an unsigned integer constant. All I/O statements express the unit numbers as integer variable names which are assigned values in the first executable statements. This increases program portability.

b) PAUSE and PAUSE $n$. These statements are excluded from the test because many systems do not permit them and, action by an operator would be required to resume the program test. 
c) The name of a Basic External Function specified as a user subprogram name. This action would not permit the inclusion of a Basic External Function so defined to appear in any test unit which was combined with other test units.

d) An external procedure written in a language other than FORTRAN. Unless Basic External Functions can be considered in this class of procedures no test is made of this facility.

e) As currently structured in Version 3, with test units 169 and 179 in Parts 11 and 12, respectively, a single labeled common block does not receive initialized data from more than one Block Data Subprogram. The proposed revised FORTRAN Standard tentatively places such a restriction upon Block Data Subprograms.

Combining these test units would test the ability to initialize data from more than a single Block Data Subprogram to a specified labeled common block. Individual data elements, however, are not initialized more than once.

f) Formatted and Unformatted records on the same I/O device within the same test unit. This concept is the subject of a FORTRAN clarification. Because a unit may be declared by the implementor not to contain this property, because this concept conflicts with the Magnetic Tape Label for Information Interchange Standard (X3.27-1969) and because this concept does not enhance program interchange, this feature was excluded from a single test unit. However, when test units 196 and 197 are combined in an executable program as in Version 3 Part 12 this feature is tested.

g) A Formatted external output field whose width does not contain enough character positions to include a positive sign and a leading zero. This concept is the subject of a FORTRAN clarification. Because these optional character positions are described in the FORTRAN Standard in the same paragraph which describes the optional external exponent form (implementor option), it is unclear whether the optional character positions are an implementor or a user option.

h) A subprogram name passed as an actual argument, and then a corresponding dummy name appearing in an argument list of a function reference or CALL to a lower level subprogram. The rules of the FORTRAN standard are incomplete. Because a dummy subprogram name may not appear in an EXTERNAL statement it is unclear how a subprogram name may be passed more than one level and maintain a proper association as a subprogram.

i) A labeled FORMAT statement which is not referenced in an I/O statement. It is unclear in the FORTRAN Standard whether a standard conforming FORTRAN program may contain such a statement which is not referenced. 
j) Hollerith constants are constrained to the FORTRAN character set, and therefore the character set is a subset of the characters capable of representation by the processor. This increases program portability.

k) The ENDFILE statement appears in a test unit but cannot be tested, because the action is undefined when an endfile record is encountered during execution of a READ statement.

A6. Interpretations Made to the FORTRAN Standard

The following interpretations have been made to the FORTRAN Standard:

a) Those items identified in the FORTRAN Clarification Reports as "Correction to Typographical and Transcription Errors" and "Corrections to Mistakes" in the FORTRAN document X3.9-1966 have been recognized and the interpretation to the standard is as if these items had actually been corrected in the original document.

b) A relational operator is not immediately followed by a signed constant. A left parenthesis appears between the relational operator and the signed constant. The FORTRAN standard does not appear to permit two adjacent operators.

c) Hollerith data does not appear "under the guise" of a complex or double precision type.

d) The word "range" may not be broadened to include "extended range" and therefore a GO TO or arithmetic IF statement in an "extended range" may not reenter the DO nest at a common terminal statement.

e) The FORTRAN Standard does not state how a Hollerith constant is positioned in a storage unit. In order for a Format Specification to be introduced into an array by way of a DATA Statement, the following assumption has been made based upon the Aw Format field descriptor, "Let ' $g$ ' be the number of characters represented in a storage unit", and " $w$ " be the value of $n$ in the $n H$ form of a Hollerith constant, then:" If the field width is less than $g$, the $w$ characters will appear left justified with $g-w$ trailing blanks in the internal representation [1, page 22L22].

f) There are no separate class rules for Basic External Functions and therefore referencing of these is handled under Class $V$, an external function. By these rules a Basic External Function may be passed as the actual argument of an external procedure reference provided the symbolic name appears also in an EXTERNAL statement.

g) The unit of angular measurement for the trigonometric functions is assumed to be expressed in radians. 
h) "The value zero is considered neither positive nor negative", does not constrain the appearance as a constant to be an unsigned zero, but may appear with either a plus (+) or minus (-) sign.

i) The FORTRAN Standard does not describe the condition of non-nested DO loops contained in an outer DO loop, nor is this condition described in earlier FORTRAN implementation manuals. However, this concept is fundamental to the DO loop and is considered defacto.

In the following picture each bracket is considered to be a DO loop:

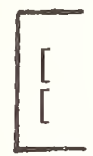



The FORTRAN Test Programs are made up from 185 segments containing sequences of FORTRAN statements. There are 116 main program sequences, whose segment number and name are each printed with the test program results, 63 subprograms which are each associated with a single test, and 6 sequences, one of which (segment 007) is always associated with each executable program. Elements from the other five sequences (segments 000, 001, 003, 005, 006) are included when appropriate. The FORTRAN Test Programs Version 1 and 3 are structured to include the necessary elements from these segments. The following is a brief description of each segment:

000, (non-executable) contains a Directory of Test Programs introduced by comment lines before the first executable program on Version 1. In Version 3, the Directory appropriate to each of the 14 parts is inserted before each of the 14 executable programs.

001, SPECS, (non-executable) declares variable types, function types, and array sizes and types for use in later segments of the test programs. This segment is not executable since it contains only specification statements, but statements from this segment are included in other segments, as required, to furnish the necessary specifications for an executable program.

003, DATA1, (non-executable) examines the format of the DATA statement, which causes variables and array elements to be initially defined. It is run with segment 010 , DATA2.

005, BSFDF, (non-executable) defines arithmetic statement functions of type integer and real. Segment 005 is run with segment 110 and 197.

006, FSFDF, (non-executable) defines statement functions of type double precision, logical, and complex.

The expressions contain constants, variables and intrinsic function references, references to previously defined statement functions and to external functions. Segment 006 is run with segment 111.

007, IODEF, (included in all executable programs) defines the system input, system output and a work unit to be used in the testing programs. Three integer variables are given values in simple assignment statements, to be associated with those units, which must be included with each program that requires such definitions. However, the values assigned to these variables may be changed to satisfy specific computer systems. These units are referred to by the following variable names:

NUVI - for results, usually a line printer defined as unit 6 .

IRVI - for input, usually a card reader defined as unit 5.

INVI - for intermediate input/output data, usually a magnetic tape defined as unit 9. This unit is used only in Segments $180,182,196,197$, and 200. 
In Versions 1 and 3, 6 input cards (three of which are prepared by the user) are associated with this segment but run with segment 008. In Version 3 also each one of the executable programs (14 Parts) contains these cards so that the user can identify the environment of the execution of the tests.

008, FMTRW, (executable) tests the FORMAT and formatted I/O statements. Under control of the FORMAT statements in the segment, 40 data cards are read in from the system input unit, and written to the system output unit. The reading into and writing from a FORMAT specification as well as the symmetry of the terminal slash in a FORMAT specification is inserted into segment 007 but executed as part of segment 008. Also written to the output unit, are lines of data produced by Hollerith information showing how the data should appear. Additional tests are performed in Segment 310 .

009, AFRMT, (executable) tests FORMAT and formatted I/0 statements as related to A-conversion. It tests that the Aw descriptor causes $w$ Hollerith characters to be read into or written from a single list item, provided $w$ does not exceed the number of characters representable in a single storage unit. The last line of the test results should print the last letters of the alphabet equal to the number of Hollerith characters contained in a storage unit. If the number of characters is less than 4, the first three test lines will contain missing characters, but the corresponding Hollerith information should be aligned.

010, DATA2, (executable) tests the contents of variables and array elements which were initialized by way of the DATA statement, in segment 003 . Via formatted output, the contents of the initialized variables and array elements are written out. The values are integer, real, double precision, complex and Hollerith. The FORMAT statements are varied, and contain descriptors, repeated by parentheses and constants.

011, AASGN, (executable) tests simple arithmetic assignment statements with the formation of integer and real constants.

013, DASGN, (executable) tests the formation of double precision constants, the referencing of double precision array elements and the assignment of values to this type in arithmetic assignment statements. The proper application of the unary sign to double precision is also tested.

015, CASGN, (executable) tests the formation of complex constants, the referencing of complex variables and array elements and the assignment of values to this type in arithmetic assignment statements. The proper application of the unary sign to complex types is also tested.

016, LASGN, (executable) tests logical assignment statements. Values are assigned to integer variables used in relational expressions of logical assignment statements. Variables and array elements are declared logical in type statements, then used in mixtures of relational expressions and logical expressions which are assigned to variables and array elements. Logical values are either true or false. 
017, INTRL, (executable) tests arithmetic assignment statements in which each side of the equation is of a different type. Integer values are assigned to real and double precision variables and arrays; real values are assigned to integer and to double precision variables and arrays .

020, UGOTO, (executable) tests the unconditional GOTO statement. Branching into labeled executable statements, in both a forward direction and a backward direction and to statements immediately following the GOTO. Each set of statements causes an integer to be generated. The test is designed to cause the unconditional transfers to be executed in such an order as to produce a consecutive set of integer values.

021, AGOTO, (executable) tests the GOTO assignment statement. The integer variable used in an ASSIGN statement is referenced only in an assigned GOTO statement, while defined as a statement label. Assigned GOTO statements branch only to excutable statements; they have a maximum of nine branches, though the ANSI standard does not specify a maximum. The value of the integer variable after the execution of the ASSIGN statement is designed to correspond to a statement labe 1 in the list of the assigned GOTO statement.

022, CGOTO, (executable) tests the computed GOTO statement. Lists in the statements have nine or fewer statement labels, which are within the same program unit. The integer variable referenced is always greater than zero and does not exceed the number of statement labels in the list.

030, 031, 032, 033, examine the formation of expressions with the addition or subtraction operator. Expressions involve variables, array elements and constants in varying orders, such as:

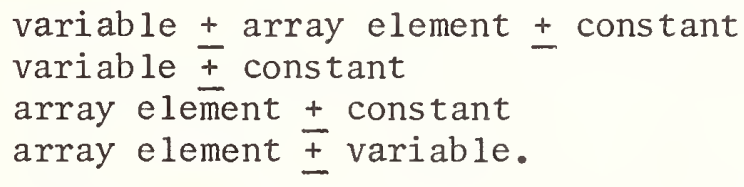

In each of these segments, numeric values are assigned to the variables and array elements which are then referenced in simple arithmetic statements.

030, ARBAD, (executable) forms expressions in which real values or integer values are added together. Expressions contain two to eight terms. One expression contains only variables, one contains only array elements and an other contains only constants.

031, ARFAD, (executable) combines double precision values with the addition operator. Values are positive or negative variables and array elements. Two, four or five terms make up each expression. 
032, ARBSB, (executable) forms expressions in which real or integer values are subtracted. Values are positive or negative variables and array elements. Expressions contain two to four variables, array elements and constants.

033, ARFSB, (executable) examines expressions involving the subtraction of double precision values. Values are positive and negative. Elements are variables, array elements and constants. Statements contain two to four variables, array elements and constants.

034, ARBAS, (executable) combines both addition and subtraction in expressions containing real or integer values. Variables, array elements and constants appear in various combinations and orders. Numeric values which are assigned are positive and negative. Expressions contain two to six elements.

035, ARFAS, (executable) combines subtraction and addition in expressions with double precision values. Some expressions contain parenthesized expressions within parenthesized expressions, others contain variables, array elements and constants without parentheses.

036, ARBMI, (executable) tests the multiplication of integer values, which are both positive and negative. One to six multiplication operations occur within a single expression.

037, ARBMR, (executable) tests the multiplication of real values. Expressions contain two to seven terms. Values are positive and negative.

038, ARFMD, (executable) tests expressions which involve the multiplication of double precision values. Variables, array elements and constants occur in various orders in expressions which contain from two to seven terms.

039, ARBDV, (executable) tests expressions of type real or integer in which variables and constants are divided by variables and constants. Some expressions contain successive division operations, in order to examine the order of evaluation of the terms.

040, ARFDV, (executable) tests the division of double precision variables, array elements and constants. Within an expression, values are of the same type and divisors are never zero. Expressions contain one to four division operations.

041, ARBEX, (executable) tests expressions in which integer or real values are raised to integer or real powers. The exponent assumes values which include zero and a negative one. Successive exponentiation occurs in some expressions so that the order of evaluation might be examined.

$$
\begin{aligned}
& A * * B \\
& (A * * B) * * C \\
& (A * * B) * *(C * * D)
\end{aligned}
$$


042, ARFEX, (executable) tests expressions in which double precision values are raised to real and double precision powers. Exponentiated values are raised to exponentiated values. Expressions contain variables, array elements, and constants.

043, ARBHI, (executable) tests the hierarchy of operators and parentheses. Only integer expressions are used in this segment which also tests that the laws of association and commutation may be applied. Integer terms containing division, do not follow these laws. The order of evaluation, generally, is according to the following hierarchy:
1. exponentiation
2. multiplication/division
3. addition/subtraction.

The elements of the expressions are then regrouped, using parentheses, to cause new orders of evaluation.

050, SBB67, (executable) tests the formation of subscripts for integer and real arrays, where the form of the subscript is either an integer variable, $v$, or an integer constant, $k$. Arrays are one, two or three dimensions, and the variables in the subscripts are given values in simple arithmetic assignment statements.

051, SBB45, (executable) tests the formation of subscripts for integer and real arrays, where the form of the subscript is either a variable plus a constant, $v+k$, or a variable minus a constant, $v-k$. Expressions also contain array elements with constant subscripts. Variables and constants in subscripts are of integer type.

052, SBB13, (executable) tests the formation of subscripts for integer and real arrays where the form of the subscript is a variable multiplied by a constant, $c^{*} v$, or a variable multiplied by a constant plus a constant, $\mathrm{c}^{*} \mathrm{v}+\mathrm{k}$, or a variable multiplied by a constant minus a constant, $\mathrm{c}^{*} \mathrm{v}-\mathrm{k}$. Through simple arithmetic statements, real and integer values are assigned to variables and array elements. Integer values are assigned to the variables occuring in subscripts of array elements, which are then computed; the array elements are then used in the evaluation of the expression in which they occur.

053, SBF17, (executable) tests the formation of subscripts for double precision arrays using the allowable subscript constructs: $v, k, v+k$, $\mathrm{v}-\mathrm{k}, \mathrm{c}^{*} \mathrm{v}, \mathrm{c}^{*} \mathrm{v}+\mathrm{k}, \mathrm{c}^{*} \mathrm{v}-\mathrm{k}$, where $\mathrm{c}$ and $\mathrm{k}$ are integer constants and $\mathrm{v}$ is an integer variable. Arrays are one, two or three dimensional; subscript expressions are of integer type and the values assigned to array elements are of double precision type.

054, SIMIF, (executable) tests simple forms of expressions in an arithmetic IF statement and a logical IF statement followed by a GOTO, so that these statements may be used in subsequent tests, the logical IF is further tested in segment 300, and the arithmetic IF in segments 301 and 302 . 
055, IFABS, (executable) references the intrinsic functions, ABS, and IABS, which obtain the value of the argument, disregarding the sign. The arguments are integer, real variable names, and expressions.

056, IFFLT, (executable) references the intrinsic function, FLOAT, which is to convert an integer to the real form. Arguments are integer variable names and expressions.

057, IFFIX, (executable) references the intrinsic function, IFIX, which is to convert a real value to the integer form. Arguments are real variable names and expressions.

058, IFSGN, (executable) references the intrinsic functions, SIGN and ISIGN which are to transfer the sign of the second argument to the first argument. Arguments are integer or real variable names or expressions.

059, IFDAB, (executable) references the intrinsic function, DABS, which obtains the value of a double precision argument, disregarding the sign. Arguments are double precision variable names and expressions.

060, IFTRN, (executable) references the intrinsic functions, AINT, INT, and IDINT which are to truncate real and double precision values. Arguments are variable names.

061, IFMOD, (executable) references the intrinsic functions AMOD and MOD, defined as remaindering. The arguments are real and integer variables, respectively.

062, IFMAX, (executable) references the intrinsic functions AMAXO, AMAX1, MAX0, MAX1, DMAX1, which are to choose the largest argument of a set of arguments. Arguments are real, integer, and double precision variables. There are two to five arguments in each argument list, though the ANSI standard does not set a limit on the number of arguments.

063, IFMIN, (executable) references the intrinsic functions AMINO, AMIN1, MINO, MIN1, DMIN1, which are to choose the smallest value of a set of arguments Arguments are integer, real, or double precision variables. There are two to five arguments in each list.

064, IFDSG, (executable) references the intrinsic function DSIGN, which is the transfer of sign from the second argument to the first. The two arguments are double precision variables.

065, IFDIM, (executable) references the intrinsic functions DIM and IDIM which are to obtain the positive difference. Arguments are real and integer variables, resp.

066, IFSGL, (executable) references the intrinsic function SNGL, which is to obtain the most significant part of a double precision value. Arguments are variables and expressions. The first and the last result should be the same value.

$$
I-B-6
$$


067, IFREL, (executable) references the intrinsic function REAL which is to obtain the real part of a complex quantity. Arguments are variables.

068, IFIMG, (executable) references the intrinsic function AIMAG, which obtains the imaginary part of a complex value. Arguments are constants and variables.

069, IFDBL (executable) references the intrinsic function DBLE, which expresses a single precision argument in double precision form. Arguments are variables and intrinsic function references.

070, IFCPX, (executable) references the intrinsic function CMPLX, which is to form a complex value from two real arguments. Arguments are constants and variables.

071, IFCJG, (executable) references the intrinsic function CONJG, which is to obtain the conjugate of a complex value. Arguments are constants and variables.

072, IFBMS, (executable) tests the use of arithmetic expressions of several terms or containing references to intrinsic functions as arguments to other intrinsic functions.

073, IFFMS, (executable) references many of the intrinsic functions. The arguments to them consist of all the primaries.

080, EXPON, (executable) references Basic External Function, EXP, the exponential function of type real. The arguments which are powers of 2, are real variables and expressions containing intrinsic functions.

The expected results printed to a precision greater than the computed results in the Basic External Function tests, are obtained from Table values. [4]

081, DEXPO, (executable) references Basic External Function, DEXP, the double precision exponential function. Arguments are powers of 2 , ranging from $-16.0 \mathrm{D} 0$ to $+16.0 \mathrm{D} 0$. Some arguments are expressions containing intrinsic functions.

082, CEXPO, (executable) references Basic External Function, CEXP, the complex exponential function. The testing range extends from 0 to 16 by steps of $\mathrm{PI} / 3$.

083, LOGTM, (executable) references Basic External Function, ALOG, the natural logarithm function of type real. Arguments are real variables and expressions containing intrinsic functions.

084, DPLOG, (executable) tests Basic External Function, DLOG, the double precision natural logarithm function. Arguments are double precision variables and expressions containing intrinsic functions.

085, CXLOG, (executable) references Basic External function, CLOG, the complex logarithm function. The testing range extends from 0 to 5. .E 7 by steps of $\mathrm{PI} / 3$. 
086, COLOG, (executable) references Basic External Function, ALOG10, the common logarithm function of type real. Arguments are real variables and expressions containing intrinsic functions.

087, DCLOG, (executable) references Basic External Function, DLOG10, the double precision logarithm function. Arguments are double precision variables and expressions containing intrinsic functions.

088, SINUS, (executable) references Basic External Function, SIN, the trigonometric sine function of type real. The arguments which range from 0 to 2 PI, are real variables and expressions containing intrinsic functions.

089, DPSIN, (executable) references Basic External Function, DSIN, the double precision trigonometric sine function. The arguments which range from 0 to 2 PI are double precision variables and expressions containing intrinsic functions.

090, CSICO, (executable) references Basic External Functions, CSIN and CCOS, the complex trigonometric sine and cosine functions. Arguments are complex variables.

091, CoSNS, (executable) references Basic External Function, Cos, the trigonometric cosine function of type real. The arguments range from 0 to 2 PI, and are real variables and expressions.

092, DPCOS, (executable) references Basic External Function, DCOS, the trigonometric cosine function of type double precision. Arguments are double precision variables and expressions which range from 0 to 2 PI.

094, TANGH, (executable) references Basic External Function, TANH, the hyperbolic tangent function of type real. Arguments are real variables and expressions containing intrinsic functions.

095, SQROT, (executable) references Basic External Function, SQRT, the square root function of type real. Arguments are real variables and expressions whose values are prime numbers.

096, DSQRO, (executable) references Basic External Function, DSQRT, the double precision square root function. Arguments are double precision variables and expressions whose values are prime numbers.

097, CSQRO, (executable) references Basic External Function, CSQRT, the complex square root function. Arguments are complex expressions.

098, ARCTG, (executable) references Basic External Function, ATAN, the trigonometric arctangent function of type real. Arguments are real variables and expressions containing intrinsic functions and whose values are powers or sums of 2 .

099, DACTG, (executable) references Basic External Function, DATAN, the single argument trigonometric arctangent of type double precision. Arguments are real variables and simple arithmetic expressions containing intrinsic functions, whose values are powers or sums of 2 . 
100, ACTG2, (executable) references Basic External Function, ATAN2, the two argument trigonometric arctangent function of type real. Arguments are real variables and expressions containing intrinsic functions, whose values are powers or sums of 2 .

101, DATN2, (executable) references Basic External Function, DATAN2, the two argument trigonometric arctangent function of type double precision. Arguments are double precision variables and expressions containing intrinsic functions, whose values are powers or sums of 2 .

102, DMODA, (executable) references Basic External Function, DMOD, the remaindering function of type double precision. Arguments are double precision variables.

103, CABSA, (executable) references Basic External Function, CABS, the modulus function. Arguments are the elements of an array of type complex.

110, BSFTS, (executable) references statement functions defined in an earlier segment, 005. The arguments are integer or real constants, variables and arithmetic expressions. Type statements are used to reaffirm the type of some intrinsic functions.

111, FSFTS, (executable) references statement functions in which the arguments are logical, double precision or complex constants, variables, and logical or arithmetic expressions. The statement functions were defined in segment 006. Type statements are used to reaffirm the type of some intrinsic functions.

140, CPXAD, (executable) tests expressions in which complex values are added or subtracted. Complex variables and constants occur in various orders and combinations, with two to nine elements in each expression.

141, CPXMU, (executable) tests expressions in which complex values are multiplied by complex values. Expressions contain from two to ten terms in various orders and combinations of complex variables and complex constants.

142, CPXDV, (executable) contains expressions in which complex values are divided by complex values. Variables and constants appear both as dividends and divisors. Some expressions involve only complex variables, some only complex constants, and others a combination of both.

143, CPXEX, (executable) involves the exponentiation of complex values. The value of the integer power varies from 3 to 100. Expressions contain variable and constant values raised to variable or constant powers. Each expression contains a single term.

144, CPXOP, (executable) performs several arithmetic operations within an expression containing complex values. Each of the arithmetic statements performs addition, subtraction, multiplication, division, and exponentiation. Only the exponents are of integer type. 
145, CREAD, (executable) performs addition and subtraction within an expression containing complex and real values. Other than in exponentiation, complex values may only be combined with real values。

146, CREMU, (executable) performs multiplication of real and complex values within an expression. The number of terms in an expression varies from two to four.

147, CREDV, (executable) performs division of complex values by real values and of real values by complex values. Expressions contain terms in which values are variables or constants.

148, CREOP, (executable) performs, within an expression, addition, subtraction, multiplication and division of complex and real values, and exponentiation of complex values. Exponents are integer values, only. The hierarchy rules determine the order of evaluation.

149, MISC3, (executable) contains arithmetic assignment statements in which the statements are continued for several lines and are interspersed with many blanks. Blanks occur within variable names and throughout the statements which are one to twenty lines in length. The statements involve real and integer values only. The digits, the letters and the special FORTRAN characters make up the list of continuation characters for the multiple line statements. The digit, zero, and the character, blank, are not legitimate continuation characters, but are used in the initial line of a statement.

150, MISC4, (executable) has interspersed blanks within arithmetic assignment statements containing complex values. Statements are one to twenty lines with letters and special characters to indicate the continuation. Statements occur which have a single character on a line; others have one or two terms of the expression on a line. Uncounted blanks do not appear in the midst of Hollerith information. Continuation lines of both a FORMAT statement and an assignment statement contain non space characters in columns 2 through 5. The arithmetic assignments used in this segment are similar to those used in segment 148 .

160, BRFCP, (executable) references REAL functions, contained in segments 400, $420,430,440,450,460$. The arguments of the functions are either integer or real variable names, array names, array element names, and arithmetic expressions. Arguments are given numerical values in arithmetic assignment statements, and their names, values, or expressions appear in the argument list of the function reference. Function references contain one or two arguments in the argument list with only one list containing many arguments.

161, BIFCP, (executable) references INTEGER functions contained in segments $401,421,431,441,451,461$. Arguments are integer or real variable names, array names, array element names and arithmetic expressions. Argument lists contain as few as one argument and as many as twenty arguments, though no limit is imposed by the ANSI standard. The expression in which the references occur are of the same type as the function value to be returned. 
162, FRFCP, (executable) references REAL functions; the arguments are the types integer, real, double precision, complex and logical, and are variable names, array names, array element names, and external procedure names. The functions referenced are contained in segments 402,422 , $432,442,452$. Reference is also made to two intrinsic functions, REAL and AIMAG, which return the real part and the imaginary part of complex values, resp. to the expressions in which they occur. Common storage is shared by the referencing program and a function.

163, FIFCP, (executable) references INTEGER functions with arguments of types integer, real, double precision, complex and logical. Variable names, array names, array element names and external procedure names appear in the argument lists. Common storage is shared by the referencing program and a function. The functions referenced are in segments 403,423,433,443,453. One argument list contains twentyone arguments; all others contain one or two arguments.

164, CFCCP, (executable) references COMPLEX functions with arguments of types integer, real, double precision, complex, and logical. The argument lists include variable names, array names, array element names and external procedure names. The functions referenced are contained in segments $404,414,424,434,444,454,464$. Common storage is shared by the referencing program and a function.

165, DPFCP - (executable) references DOUBLE PRECISION functions with arguments of types integer, real, double precision, complex and logical. Variable names, array names, array element names, and external procedure names appear in the argument lists. Common storage is shared by the referencing program and a function. The functions referenced are in segments $405,415,425,435,445,455,465,475$. These functions return a value which is of the same type as the expressions in which they occur within the calling program.

166, BFCCP, (executable) references LOGICAL functions with arguments of types integer, real, double precision, complex and logical. The argument lists include variable names, array names, array element names, and external procedure names. Referenced functions are in segments 406, 416, 426, 436, $446,456,466,476$; the value of the function returned from each reference is of type logica1. Common storage is shared by the referencing program and a function.

167, SBRTN, (executable) calls subroutine subprograms. Arguments are the types integer and real and include variable names, array names, expressions and a Basic External Function. A CALL from a subroutine is made to another subroutine. One subroutine CALL contains no argument list. Subroutines called are in segments 407,417,427, and one of them shares common storage with the calling program.

168, FSBRT, (executable) calls subroutine subprograms. Arguments are the types integer, real, double precision, complex and logical and include variable names, array names, and expressions. A CALL from one subroutine is made to another subroutine; one subroutine CALL contains no argument 
1ist. Subroutines called are in segments 408, 418, 428, and share common storage with the calling program. Values are returned via the argument list of the CALL。

169, BLKDT, (executable) uses a block data subprogram. Labeled common blocks contain variable names and dimensioned arrays. Implicit types of variables and arrays are overridden by double precision, complex and logical statements. The block data subprogram used to supply the initial values of the labeled common blocks is contained in segment 409. This segment writes out the values which are contained in the labeled common blocks.

179, BLKDA, (executable) uses three block data subprograms, which contain six labeled common blocks with elements to be initialized. Elements of any block are initialized through only one of the block data subprograms contained in segments 419, 429, 439. Implicit typing is sometimes overridden by double precision, complex, and logical statements. This segment writes out the values which are contained in the labeled common blocks. They correspond to the labeled common blocks of the block data subprogram.

180, UNFRW, (executable) tests the unformatted WRITE statement and the unformatted READ statement with and without a list. Included in the segment is an ENDFILE statement. This segment uses an intermediate tape.

182, BACUP, (executable) examines the backspace statement. Data is created in memory, written to tape, then changed in memory. The tape is then backspaced, and the data read to memory in a forward direction. The data block is 1024 words in length and is written and read by way of unformatted input/output statements. This segment uses an intermediate tape 。

190, DOTRM, (executable) examines DO statements and DO ranges which terminate with a CONTINUE, ASSIGl, or logical IF statement. DO statements meet the requirements that parameters of the DO must be greater than zero, and must not be redefined during the execution of the range of that DO. In some DO statements, the incrementation parameter appears; in others, it does not appear and has an implied value of one.

191, DOLMT, (executable) examines a DO statement and its range, in which the parameters are integer variable names. Numerical values are given for them in arithmetic assignment statements occurring before the DO statement. The DO range consists of an arithmetic assignment statement involving the induction variable and the terminal statement which is a CONTINUE.

192, DONSC, (executable) examines DO ranges contained within other DO ranges, the parameters of which are integer constants and variables. Each range of a DO within the nest has its own terminal statement. Another nest of DO's has a single terminal statement. Nests contain two to five DO statements and the DG range includes arithmetic IF statements and GO TO statements。 
193, DONSI, (executable) examines a DO statement and its associated range, in which an exit is made from the range of a DO before the DO has been satisfied. The induction variable is used both within and outside of the range of the DO.

194, DONSX, (executable) examines a DO nest which has an extended range. Exit from the innermost DO is by way of an unconditional transfer, reentry is by way of an arithmetic IF statement.

195, DONML, (executable) examines the ranges of DO's which are within the range of another DO, but are not nested. All parameters are integer constants and the ranges contain arithmetic assignment statements.

196, DONIO, (executable) examines the ranges of DO's which have input or output statements as the terminal statement. The terminal statements include a READ, a REWIND and a WRITE statement, each of which is also the only statement within the range of that DO. This segment uses an intermediate tape.

197, MORDO, (executable) examines DO ranges which have within, references to statement functions and intrinsic functions, CALLS to subprograms, and DO's with extended ranges. Input, output and rewind statements are also within these DO ranges. This segment uses an intermediate tape.

200, SUBR1, (executable) passes the I/O assignments through common then cal1s a subroutine subprogram without an argument list, and returns to an unlabeled CONTINUE statement. The subroutine called is contained in segment 410 . This segment uses an intermediate tape.

300, LOGIF, (executable) examines the logical IF statement. Variables and array elements, declared logical, are assigned values of true or false. These values are then used in the logical IF statement, which includes an executable statement which is not a DO statement nor an other logical IF statement. A signed zero constant is tested in a relational expression.

301, BARIF, (executable) examines the arithmetic IF statement which contains integer or real values and references to intrinsic functions. The effect of the sign of zero is tested.

302, FARIF, (executable) examines arithmetic IF statements in which the expressions contain double precision values and references to intrinsic functions.

310, IOFMT, (executable) examines the formatted READ and WRITE statements and FORMAT statements as they relate to fields of input card images. There are 38 card images read as input to this segment; the formats under which the variables and array elements are read and written include each of the descriptors. Formats occur in which there is a one to one correspondence between elements in the 1ist and descriptors; other formats occur which do not have the same number of descriptors as there are elements in the lists. Segment 310 examines additional features not contained in segment 008 . 
312, RDFMT, (executable) examines formatted READ and WRITE statements in which the format specifications are contained within arrays. Reference is to an array name, in place of the reference to a format statement labe1. The format specifications contained in arrays do not have nH field descriptors. FORMAT specifications are defined in DATA statements, read in as elements of an array, and passed as an argument to a subroutine. There are 13 card images read in this segment.

350, MISC5, (executable) examines the specifications of the program form. This includes verifying that comments are not executed, that every statement within the unit, can be reached, that all characters in a line are accepted, that 1 abels can be one to 5 characters 1 ong and may be placed anywhere in columns one to five. Other features of program form are also examined.

351, FUNMX, (executable) further tests some Basic External Functions by using trigonometric formulas.

352, NAMES, (executable) determines whether the compiler can distinquish predefined function names and data names from FORTRAN verbs. The names of intrinsic functions and FORTRAN verbs appear as variable names and array names in a program unit. In other units of the same program, these names appear as intrinsic function names and as FORTRAN verbs. Subprogram units are segments $413,463,473,483$.

360, SPEC2, (executable) examines the use of integer variables and arrays and real variables and arrays, which are either in COMMON, or appear in EQUIVALENCE statements, or both. All array names are in DIMENSION statements; some have two or three dimensions, which are written as one dimensional arrays in the EQUIVALENCE statement. The array element successor function defines a relation by which a multi-dimensional array can be made equivalent to a one dimensional array. The order of the specifications is DIMENSION, COMMON, EQUIVALENCE and no dummy arguments appear in COMMN or EQUIVALENCE statements. Numeric values are assigned to variables and array elements to which other variables and array elements have been equivalenced. The associated variables and array elements are then used in arithmetic assignment statements, DO statements, IF statements and computed GOTO statements. A special blank common arrangement is used in this segment and this segment may not be combined with other segments requiring blank common.

Segments beginning with segment 400 are subprograms.

400, AFS, to be run with main program segment 160 , is a real function of one real argument. The value of the function is the result of multiplying the dummy argument by a constant.

420, BFS, to be run with main program segment 160 , is a real function of two real arguments which are added together to produce the value of the function. 
430 , CFS, to be run with segment 160 , is a real function of an integer argument, which is the power to which a constant is raised, to produce the value of the function.

440 , DFS, is a real function of two integer arguments, one of which is subtracted from the other producing the power to which a real constant is raised. The result is the value of the function. This function is referenced in segment 160 .

450, EFS, is a real function of a real array, the size of which is declared in a DIMENSION statement. The value of the function is the sum of the elements of the array. This function is referenced in segment 160 .

460, FFS, is a real function with twenty arguments of integer and real variables and integer and real arrays. The expression defining the function contains addition, subtraction, multiplication and exponentiation of arguments. This subprogram is referenced in segment 160 .

401, IAFI, is an integer function of a single real argument. The value of the function is the product of a constant and the argument. This subprogram is referenced in the main program contained in segment 161.

421, IBFI, is an integer function of two real variables. The value of the function is the sum of the two arguments. This subprogram is referenced in the main program contained in segment 161.

431, ICFI, is an integer function of an integer variable. The value of the function is obtained by exponentiating a real constant. This segment is referenced in the main program contained in segment 161.

441, IDFI, is an integer function of two integer arguments. The value of the function is obtained by raising a real value to the power which is the difference between the two arguments. The real variable is defined in a DATA statement. Segment 441 is referenced in segment 161.

451, IEFI, is an integer function with a single argument consisting of an integer array. The size of the array is declared in a DIMENSION statement and the elements of the array are added together to produce the value of the function. Segment 451 is referenced in connection with segment 161 .

461, IFFI, is an integer function with twenty arguments of real variables and arrays and integer variables and arrays. The dimensionality of each array is declared within the subprogram. The value of the function is obtained by evaluating the equation which contains addition, subtraction, multiplication and exponentiation, of variables and array elements. This segment is referenced in segment 161.

402, GFS, is a real function of a double precision argument. The argument is assigned to the function name. This subprogram name is passed as an argument in segment 162 to segment 442, JRFS, which references it. 
422, HFS, is a real function of two complex variables. The value of the function is obtained by assigning the imaginary part of the product of the complex values to the function name. This segment is referenced in segment 162 .

432, IRFS, is an explicitly typed real function of a logical variable. The function value is defined by one of two logical IF statements, depending upon the value of the argument. This segment is referenced twice in segment 162 .

442, JRFS, is an explicitly typed real function of an external procedure (segment 402) and a double precision variable. The value of the function is the value of the external procedure of which the double precision value is the argument. This segment is referenced in segment 162 .

452, RFS, is a real function with twenty-one arguments of all the types of variables and arrays and an external procedure which is not referenced. Array and variable types are declared in logical, complex and double precision statements. Adjustable arrays appear in this subprogram. This segment is referenced in segment 162 .

403, IFI, is an integer function of a double precision variable. The variable is assigned to the function name to produce the value of the function. This segment is referenced in segment 163 and also passed as an argument from segment 163 to segment 453 and segment 443 .

423, JFI, is an integer function of two complex arguments. The value of the function is the imaginary part of the product of the two arguments. This segment is referenced in segment 163.

433, KFI, is an integer function of a logical argument. The value of the function is determined by one of two logical IF statements, depending upon the value of the argument. This segment is referenced twice in segment 163.

443, LFI, is an integer function of the external procedure IFI (segment 403) and a double precision variable. The value of the function is the value of the external procedure of which the variable is the argument. This segment is referenced in segment 163.

453, MFI, is an integer function with twenty-one arguments of all the types of variables and arrays and an external procedure. An adjustable array and its adjustable dimensions are dummy arguments of this subprogram. This segment is referenced in segment 163, and is similar to segment 452 except for function type, and the dummy function is referenced.

404, AFC, is a complex function, explicitly typed, of a real variable. The sum of the real variable and a complex value is the value of the function. This segment is referenced in segment 164. 
414, BFC, is a complex function of an integer argument. A complex value is raised to an integer power to produce the function value. This segment is referenced in segment 164.

424, CFC, is a complex function of a real array. The elements of the array are subtracted from a complex constant to produce the function value. This segment is referenced in segment 164 .

434, DFC, is a complex function of a double precision variable. The value of the function is obtained by subtracting a complex constant from the product of a complex constant and a real variable. This segment is referenced in segment 164.

444, EFC, is a complex function of a complex variable. The function value is the complex argument minus a constant. This segment is referenced in segment 164 。

454, FFC, is a complex function of a logical variable. The value of the function is determined by one of two logical IF statements, depending upon the value of the argument. This segment is referenced twice in segment 164 .

464, HFC, is a complex function with twenty-one arguments of all the types of variables and arrays and a complex function which is not referenced. Variable and array types are declared in type statements in the subprogram. Adjustable arrays are arguments in this subprogram. A value is passed through common and is redefined within the subprogram。 This segment is referenced in segment 164 and is similar to segment 452 。

405, AFD, is a double precision function of a real argument. The value of the function is set equal to the argument. This subprogram is referenced in segment 165 and also passed as an argument from segment 165 to segment 455 .

415, BFD, is a double precision function of an integer variable. A double precision constant is raised to the power of the integer variable. This segment is referenced in segment 165.

425, CFD, is a double precision function of a double precision argument. The value of the function is the value of the argument. This segment is referenced in segment 165 .

435, DFD, is a double precision function of two complex variables. The value of the function is the imaginary part of the product of the two complex variables. This segment is referenced in segment 165 .

445, EFD, is a double precision function of a logical variable. The value of the function is determined by one of two logical IF statements, depending upon the value of the argument. This segment is referenced twice in segment 165 .

455, FFD, is a double precision function of an external procedure (segment 405) and a double precision variable. This segment is referenced in segment 165 . 
465, GFD, is a double precision function of a double precision array. The elements of the array are added together to produce the value of the function. This segment is referenced in segment 165 .

475, HFD, is a double precision function with twenty-one arguments of all the types of variables and arrays and a double precision function which is not referenced. Adjustable arrays are arguments in this segment. A value is passed through common and redefined in the function subprogram. This segment is similar to segment 452 and is referenced in segment 165.

406, AFB, is a logical function of a real variable. This function is referenced in segment 166.

416, BFB, is a logical function of an integer argument. This segment is referenced in segment 166.

426, CFB, is a logical function of a double precision argument. This segment is referenced in segment 166 .

436, DFB, is a logical function of a logical variable. The value of the function is the value of the argument. This segment is referenced in segment 166 .

446, EFB, is a logical function of a complex variable. This segment is referenced in segment 166.

456, FFB, is a logical function of a real array. This segment is referenced in segment 166 .

466, GFB, is a logical function of a real variable and a logical external procedure (segment 406). This segment is referenced in segment 166.

476, HFB, is a logical function with twenty-one arguments of all the types of variables and array elements and an external function which is referenced. This segment is referenced in segment 166.

407, AAQ, is a subroutine subprogram with integer and real variable and array names and a function in the argument list. This subprogram, called in segment 167, calls another subprogram (segment 417), whose argument list cuntalns integer and real array names.

417, $A B Q$, is a subroutine subprogram called from another subroutine subprogram (segment 407) which is called in segment 167.

427, ACQ, is a subroutine subprogram which has no argument list. Variables and arrays are passed through common; some are redefined within the subprogram. This segment is referenced in segment 167.

408, $\mathrm{ADQ}$, is a subroutine subprogram with twenty-four arguments of type integer, real, double precision, complex, and logical variables and arrays. This subprogram, called in segment 168, calls another subprogram (segment 418), whose arguments are integer and real variables and arrays. 
418, AEQ, is a subroutine subprogram called from another subroutine subprogram (segment 408). The arguments are integer and real variables and arrays. This subroutine is used with segment 168 .

428, AFQ, is a subroutine subprogram which has no arguments. Variables and arrays are passed through common; some are redefined within the subprogram. This segment is referenced in segment 168.

409, BLOKD, is a block data subprogram, which contains type, EQUIVALENCE, DATA, DIMENSION, and COMMON statements. These are the allowable statements in a block data subprogram, in which data statements assign values to variables and array elements which are in labeled common blocks. Hollerith data is assigned to each type of array, which are one, two, and three dimensional. This segment is to be run with segment 169.

410, SUBRQ, is a subroutine subprogram which contains no argument list and returns no values to the calling program. Arguments are passed through blank common. The subprogram contains FORTRAN statements, including input/output statements and references to intrinsic functions. This subroutine is called in segment 200. This segment is similar to main program segment 197.

Segment 419 BLAKD,

Segment 429 BLBKD,

Segment 439 BLCKD, are three block data subprograms, each of which, through data statements, assigns values to a different labeled common block. Each of these subprograms contains all of the statements allowed in a block data subprogram and each contains arrays of one, two, and three dimensions. These segments are run with segment 179 .

411, SMCQ, is a subroutine subprogram called from a logical IF statement in the calling program, segment 300 .

412, MDQ, is a subroutine subprogram called from within a DO of the calling program. It is called from segment 197.

462, FMTQ, is a subroutine subprogram called by segment 312 . FORMAT specifications and Hollerith constants are passed as arguments of the subroutine. An empty FORMAT specification is also tested.

413, MAQQ, is a subroutine subprogram in which an intrinsic function name is used as a variable name and a second intrinsic function name is referenced。 This subroutine is called from segment 352 .

463, MBQQ, is a subroutine subprogram in which an intrinsic function name is used as a variable name. It is called from segment 352 .

473, AMQQ, is a subroutine subprogram in which an intrinsic function name is used as a variable name. This subroutine is called from segment 352 .

483, BMQQ, is a subroutine subprogram in which several intrinsic function references are nested and one intrinsic function name is used as a variable name. This subroutine is called from segment 352. 

C. TEST UNIT SEGMENTS INDEXED TO THE FORTRAN STANDARD DOCUMENT ASA X3.9-1966

The following is the table of contents to the FORTRAN document X3,9-1966 with the corresponding FORTRAN Test Program Segments identified.

ASA $\times 3.9-1966$

Section Number and Title

FORTRAN Test Program Segment

1. Purpose and Scope

2. Basic Terminology

3. Program Form

3.1 The FORTRAN Character Set

3.1.1 Digits

3.1.2 Letters

3.1.3 Alphabetic Characters

3.1.4 Special Characters

3.1.4.1 Blank Character

3.2 Lines

3.2.1 Comment Line

3.2 .2 End Line

3.2.3 Initial Line

3.2.4 Continuation Line

3.3 Statements

3.4 Statement Labe 1

3.5 Symbolic Names

3.6 Ordering of Characters

008,149

008,360

008,009

008,009

008,009

$008,009,149,150$

150,350

ALL, 350

ALL, 441

ALL

ALL , 149,150

ALL , 149, 150

ALL , 150, 350

ALL , 350, 352, 463, 473, 483

(ASSUMED)

4. Data Types

4.1 Data Type Association

4.2 Data Type Properties

4.2.1 Integer Type

4.2.2 Real Type

4.2.3 Double Precision Type

4.2.4 Complex Type

4.2 .5 Logical Type

4.2.6 Hollerith Type

$003,010,149, \mathrm{ALL}$

$054,301,302$

011

011

013

015

016

003,010

5. Data and Procedure Identification

5.1 Data and Procedure Names

5.1.1 Constants

5.1.1.1 Integer Constant

5.1.1.2 Real Constant

5.1.1.3 Double Precision

Constant

5.1.1.4 Complex Constant

5.1.1.5 Logical Constant

5.1.1.6 Hollerith Constant

017

$003,010,011$

$003,010,011$

$003,010,013$

$003,010,015,067$

$003,010,016$

$003,010,312,462$ 
5.1.2 Variable

5.1.3 Array

5.1.3.1 Array Element

5.1.3.2 Subscript

5.1.3.3 Subscript Expressions

5.1.4 Procedures

5.2 Function Reference

5.3 Type Rules for Data and

Procedure Identifiers

5.4 Dummy Arguments

6. Expressions

6.1 Arithmetic Expressions

6.2 Relational Expressions

6.3 Logical Expressions

6.4 Evaluation of Expressions

7. Statements

7.1 Executable Statements

7.1.1 Assignment Statements

7.1.1.1 Arithmetic Assignment

Statement

7.1.1.2 Logical Assignment

Statement

7.1.1.3 GO TO Assignment

Statement

7.1.2 Control Statement

7.1.2.1 GO TO Statements

7.1.2.1.1 Unconditional GO TO

Statement

7.1.2.1.2 Assigned GO TO

Statement

7.1.2.1.3 Computed GO TO

Statement

7.1.2.2 Arithmetic If Statement

7.1.2.3 Logical If Statement

7.1.2.4 CALL Statement

7.1.2.5 RETURN Statement

7.1.2.6 CONTINUE Statement

7.1.2.7 Program Control

Statements

\subsection{STOP Statement}

7.1.2.7.2 PAUSE Statement

7.1.2.8 DO Statement

7.1.3 Input/Output Statements

7.1.3.1 (initial record, next

and preceding record)

7.1.3.2 READ and WRITE Statements

7.1.3.2.1 Input/Output Lists

7.1.3.2.2 Formatted READ

7.1.3.2.3 Formatted WRITE

7.1.3.2.4 Unformatted READ

7.1.3.2.5 Unformatted WRITE
$003,010,312$

003,010

003,010

003,010

$050,051,052,053$

(See Section 8)

(See Section 8)

$003,010,110,111$, (Tables 3 \& 4 See

Section 8)

(See Section 8)

$030-043,140-148$

016,300

016,300

$011,013,015,017,043$
$011,013,015,017,030-043,140-148$

016

021

020

021

022

$054,301,302$

054,300

$167,168,200,312,352,408$

400-483

$150,190-197,200$

ALL, 360

(Omitted)

190-197

180,182

008,310

$008,009,196,310,312$

$008,009,196,310,312$

180,182

180,182 
7.1.3.3 Auxiliary Input/Output

Statements

7.1.3.3.1 REWIND Statement

7.1.3.3.2 BACKSPACE Statement

7.1.3.3.3 ENDFILE Statement

180,196

182

180

7.1.3.4 Printing of Formatted 008

Records

7.2 Nonexecutable Statements

7.2.1 Specification Statements

7.2.1.1 Array-Declarator

7.2.1.1.1 Array Element

Successor Function

and value of a

subscript

7.2.1.1.2 Adjustable Dimension

7.2.1.2 DIMENSION Statement

7.2.1.3 COMMON Statement

7.2.1.4 EQUIVALENCE Statement

7.2.1.5 EXTERNAL Statement

ALL

$003,008,180,192$

7.2.1.6 Type-Statements

7.2.2 Data Initialization Statement

7.2.3 FORMAT Statement

7.2.3.1 Field Descriptors

7.2.3.2 Field Separators

7.2.3.3 Repeat Specifications

7.2.3.4 Format Control Interaction with Input/Output List

7.2.3.5 Scale Factor

7.2.3.5.1 Scale Factor Effects

7.2.3.6 Numeric Conversions

7.2.3.6.1 Integer Conversion

7.2.3.6.2 Real Conversions

7.2.3.6.3 Double Precision Conversions

7.2.3.6.4 Complex Conversion

7.2.3.7 Logical Conversion

7.2.3.8 Hollerith Field

Descriptor

7.2.3.9 Blank Field Descriptor

$162-166,452,453,464,475,476$

$003,008,169,409,360$ (a].1 parts)

$162-169,200,360,409$

$169,409,360$

162-167

$003,022,110,111$

$003,010,312,441$

$008,009,310,312,410,462$

$008,009,312,462$

008,009

008,009

008,310

7.2.3.10 Format Specification in Arrays

008,310

008,310

008,310

008,011

008,011

008,013

008,015

008,016

009

008

312,462

8. Procedures and Subprograms

8.1 Statement Functions

8.1.1 Defining Statement Functions

8.1.2 Referencing Statement Functions

$005,006,410$

$110,111,197,410$

$055-073,352$

8.2 Intrinsic Functions and Their References 
8.3 External Functions

8.3.1 Defining Function Sub- 400,420,430, etc. programs

8.3.2 Referencing External 160-166

Functions

8.3.3 Basic External Functions

$080-103,351$

8.4 Subroutine

8.4.1 Defining Subroutine Subprograms

$408,410,418,427,428,462$

8.4.2 Referencing Subroutines

$167,168,200,312$

8.5 Block Data Subprogram

$169,179,409,419,429,439$

9. Programs

Rules stated are inciuded under

10. Intra-and Inter-Program Relationships

tests related to Section 3 through

Section 8 of the FORTRAN Standard 
D. PROGRAM INFORMATION

The following points describe the organization of FORTRAN test programs:

The programs are divided into a number of small segments.

Most segments, except for specification statements, I/O assignment statements, statement functions, subprograms and DATA statements, are completely self-contained.

Most segments are very simply written with the testing devoted to related features described in the ASA standard. The number of FORTRAN statement types is minimized in order to make each test less dependent on other language features.

Every segment begins with a heading of comment lines which gives the segment name, segment number, pertinent ASA references, purpose of the segment and restrictions observed in the segment.

The last line in every segment is marked by a comment line with the message "END OF TEST SEGMENT xxx."

Comments, throughout each segment, give detailed ASA references and additional explanations of the coding.

\section{D1. Conventions Used in the Test Programs}

Certain conventions have been adopted and are used throughout the document, the program code and the test results. These conventions provide the user with a means to:

identify types of data,

determine the number of dimensions associated with a given array,

distinguish program elements,

correlate references between the ASA FORTRAN standard document and the pertinent test segments.

The conventions are described below.

\section{a) Segment Identification}

Each segment is identified in the following two ways:

By a 3- to 5- character (A-Z, 0-9) descriptive name (e.g., DPLOG, SBRTN).

By a unique 3-digit (0-9) number.

Both the segment name and number appear in the program listing, the documentation and the generated test results. 
b) Line Numbers

Line numbers, columns 73-80, are outside of the standard, but are usually available in an implementation of the FORTRAN Standard, when the source statements are introduced to the processor from punched cards.

The scheme used to identify FORTRAN lines is a compromise between the ability to associate the program listing with this document and the card handling problem. The FORTRAN test program listing represents both a statement of the program for the processor and a document for the user. The program listing also assists in the consolidation and isolation of test units. Although each line number is unique, a test program unit may contain FORTRAN lines with columns 74-76 (segment number) with segment numbers 001-007 inserted within the test units. Columns 73-80 are coded in the following fashion:

\begin{tabular}{|c|c|c|}
\hline Column 73 contains & $\begin{array}{l}\mathrm{P} \\
\mathrm{H}\end{array}$ & $\begin{array}{l}\text { for FORTRAN Test Version } 1 \text {. } \\
\text { for FORTRAN Test Version } 3 \text {. }\end{array}$ \\
\hline Columns $74,75,76$ contain & nnn & $\begin{array}{l}\text { where 'nnn' are } 3 \text { unique } \\
\text { digits }(0-9) \text { which identify } \\
\text { the program segment. (The } \\
\text { greatest segment number } \\
\text { allowed is } 699) \text {. }\end{array}$ \\
\hline Columns $77,78,79$ contain & $\mathrm{mmm}$ & $\begin{array}{l}\text { where'mmm' are } 3 \text { digits }(0-9) \\
\text { representing a line number } \\
\text { within the program segment. }\end{array}$ \\
\hline Column 80 contains & $x$ & $\begin{array}{l}\text { where } x \text { is either zero or five } \\
\text { and allows for the insertion of } \\
\text { lines at a later time. }\end{array}$ \\
\hline
\end{tabular}

In Version 1, the sequence numbers (columns 77-80 for segments 001 and 007 start with 0010 and are incremented by 5, with each new segment number (columns 74-76) forcing the beginning sequence number to be even.

In Version 3, the sequence numbers (columns 77-80) for segments 000, 001 , and 007 are increased by 5 in column 80 and each Part is initiated by the following sequence number: Part 1, 0010; Part 2, 0400;

Part 3, 0700; Part 4, 1200; Part 5, 1800; Part 6, 2300; Part 7, 2700; Part 8, 3200; Part 9, 3700; Part 10, 4300; Part 11, 4800; Part 12, 5400; Part 13, 6000; Part 14, 6400; and the statement function definition segment 005 imbedded in segment 197 begins at 0500 .

c) Statement Labels

Each statement 1 abel is a string of four digits (0-9)。 To avoid duplicate labels in the test program, the first three digits of the string contains the number of the segment in which the statement label is found. (See the description of columns 74-76 above). The fourth digit is used to make the string unique within that particular segment. 
This convention provides ten unique labels per program segment. When more than ten labels are needed in any segment, digits 1-3 of the extra labels contain a unique number between 700 and 999, instead of the program segment number. For this reason, the greatest program number allowed is 699. A table of currently used additional statement numbers is contained in Section I-D3e.

d) Format of Comments

Every comment line contains ' $\mathrm{C}$ ' in column 1, followed by five asterisks $\left(^{*}\right)$ or a " $\mathrm{C}=$ ": in columns 1 and 2 .

Each segment is preceded by a heading of comment cards which give the segment name, segment number, purpose of the segment, restrictions observed, ASA references and miscellaneous comments.

Additional comment lines, interspersed with the actual coding describe the specific purpose of the coding which follows and give pertinent ASA references.

Comment lines containing " $\mathrm{C}=$ " in columns 1 and 2 denote the required Specification statements, I/O Assignment statements, STOP statement and END line needed to construct a FORTRAN program if each main program segment is to be run as a separate test unit.

e) Format of the Generated Test Results

The generated test results of every segment start on a new page and are headed by several lines which give the segment name, segment number, purpose of the segment (very briefly stated), and ASA references. The printed area is constrained to an $81 / 2$ by 11 inch page, with a maximum of 57 lines printed per page.

f) Naming Conventions

A unique 3- to 5-character designation is used to identify a variable, array, function or subprogram. The combination of the last two characters in the name indicates the type and category. The character preceding the last two flags items which appear in COMMON or EQUIVALENCE statements. One or two optional characters may begin each name to make it unique. The conventions are as follows:

If character 5 is (or last character)
I the type is integer;

$S$ the type is real;

D the type is double precision;

C the type is complex;

B the type is logical;

$\mathrm{H}$ the actual argument is Hollerith;

Q the string represents a subroutine. 


\section{If character 4 is \\ (or next to last \\ character)}

If character 3 is
Characters 1 and 2 are the string represents a variable; the string represents a function; where ' $n$ ' is a digit (1-3), the string represents an array with $n$ dimensions; where ' $a$ ' is any other letter (A-E, $\mathrm{G}-\mathrm{U}, \mathrm{W}-\mathrm{Z}$ ) for cases in which none of the other codes are applicable.

W the name is a dummy argument;

$X$ the name appears in a COMMON statement;

$Y$ the name appears in an EQUIVALENCE statement;

$Z$ the name appears in both COMMON and EQUIVALENCE statements;

a where ' $a$ ' is any other letter (A-V), the name appears neither in COMMON nor in an EQUIVALENCE statement.

aa where 'aa' are any letters (A-Z) associated with the string. These two characters are used only to insure that each name is unique. Either or both of them may be omitted, if desired.

Examples of this convention are A3I, BBXVD, CBFS, PAAQ where the strings represent a 3 -dimensional integer array, a double precision variable (used in a COMMON statement), a real function name and a subroutine name, respective1y.

D2. Assumed Levels for Non-specified FORTRAN Areas

The ASA standard does not impose specifications in many areas that are clearly subject to limitations in actual FORTRAN compilers. Therefore, in order to design meaningful tests, some additional specifications have been established. These limits are described below.

a) Level of Nesting

The DO loop segments of the program contain a maximum of FIVE nested loops.

b) Number of Arguments

The test program contains subprograms with up to TWENTY-FIVE arguments.

c) Size of Arrays

The size of arrays is generally very small, i.e., usually less than TWENTY words . 
d) FORMAT Standards

FORMAT statements never cause more than FORTY characters on a line to be generated in the output.

e) Number of Parentheses

Expressions in the test program never exceed TEN levels of parentheses.

f) GO TO Branches

The number of branches in assigned and computed GO TO statements never exceeds TWELVE branches.

g) Constant Length

Constants are kept small in order not to exceed the storage unit length capacity of some computers. The limits on constant

length are set as follows:

\begin{tabular}{|c|c|}
\hline Integer constants & - 5 digits \\
\hline Real constants & - 7 digits \\
\hline Double precision & \\
\hline constants & - 14 digits \\
\hline $\begin{array}{c}\text { Complex constants } \\
\text { (each half) }\end{array}$ & - 7 digits \\
\hline Hollerith constants & $\begin{array}{l}\text { - } 2 \text { characters except in segment } 009 \\
\text { which tests A-conversion for } 1 \text { to } 4 \\
\text { characters and } 26 \text { characters for the } \\
\text { truncation test. }\end{array}$ \\
\hline
\end{tabular}


D3. Names and Statement Numbers Used in the Test Programs

Only those names which are used as array names, external function and subroutine names, common block names, and variable names appearing in a DATA statement appear in the following lists. The list of array declarators appearing in type statements and COMMN statements is supplied to assist the user when he wishes to extend or revise the test programs.

a) Subprogram Names Used in the Test Program Set and the Number of Arguments

Integer Functions

$\begin{array}{rr}\text { IAFI } & 1 \\ \text { IBFI } & 2 \\ \text { ICFI } & 1 \\ \text { IDFI } & 2 \\ \text { IEFI } & 1 \\ \text { IFFI } & 20 \\ \text { IFI } & 1 \\ \text { JFI } & 2 \\ \text { KFI } & 1 \\ \text { LFI } & 2 \\ \text { MFI } & 21\end{array}$

Double Precision

Functions

$\begin{array}{lr}\text { AFD } & 1 \\ \text { BFD } & 1 \\ \text { CFD } & 1 \\ \text { DFD } & 2 \\ \text { EFD } & 1 \\ \text { FFD } & 2 \\ \text { GFD } & 1 \\ \text { HFD } & 21\end{array}$

Subroutines

$\begin{array}{rr}\text { AAQ } & 9 \\ \text { ABQ } & 3 \\ \text { ACQ } & 0 \\ \text { ADQ } & 24 \\ \text { AEQ } & 8 \\ \text { AFQ } & 0 \\ \text { MDQ } & 2 \\ \text { SMCQ } & 1 \\ \text { FMTQ } & 22 \\ \text { SUBRQ } & 0 \\ \text { MAQQ } & 2 \\ \text { MBQQ } & 2 \\ \text { AMQQ } & 2 \\ \text { BMQQ } & 2\end{array}$

\section{Real Functions}

$\begin{array}{cr}\text { AFS } & 1 \\ \text { BFS } & 2 \\ \text { CFS } & 1 \\ \text { DFS } & 2 \\ \text { EFS } & 1 \\ \text { FFS } & 20 \\ \text { GFS } & 1 \\ \text { HFS } & 2 \\ \text { IRFS } & 1 \\ \text { JRFS } & 2 \\ \text { RFS } & 21\end{array}$

Complex Functions

Block Data Subprograms-No Names Permitted

In FORTRAN Language But Identified by Comment Cards As:

$\begin{array}{lr}\text { AFC } & 1 \\ \text { BFC } & 1 \\ \text { CFC } & 1 \\ \text { DFC } & 1 \\ \text { EFC } & 1 \\ \text { FFC } & 1 \\ \text { HFC } & 21\end{array}$


b) Array Declarators in Type Statements and COMMON Statements

Double Precision

$\operatorname{AC1D}(10)$

A1D (4)

$\mathrm{A} 2 \mathrm{D}(2,2)$

A3D $(2,2,2)$

$\mathrm{BC} 2 \mathrm{D}(7,4)$

CC 3D $(7,2,2)$

$\operatorname{DPA1D}(5)$

$\operatorname{DPA} 2 \mathrm{D}(2,2)$

EP1D (43)

FC2D $(5,5)$

$\operatorname{MCA} 3 \mathrm{D}(1,4,2)$

$\operatorname{RC} 3 \mathrm{D}(3,3,3)$

AX1D

AX2D

AX3D

DX1D

DX2D

DX 3D

$\underline{\text { Logica1 }}$

$\mathrm{AlB}(2)$

$\mathrm{A} 2 \mathrm{~B}(2,2)$

$A 3 B(2,2,2)$

$\mathrm{GG} 1 \mathrm{~B}(2)$

$\operatorname{GH} 2 B(1,2)$

$\operatorname{GI} 3 B(1,1,2)$

$\operatorname{MCA} 1 B(7)$

$\operatorname{L1B}(10)$

$\mathrm{AX} 1 \mathrm{~B}$

$\mathrm{AX} 2 \mathrm{~B}$

AX3B

DX1B

DX2B

DX3B
Complex

$\mathrm{A} 1 \mathrm{C}(12)$

A2C $(2,2)$

$\mathrm{A} 3 \mathrm{C}(2,2,1)$

B1C (8)

$\operatorname{B2C}(4,2)$

B3C $(2,2,2)$

LL1C (32)

$\operatorname{LM2C}(8,4)$

$\operatorname{LN3C}(9,2,2)$

EP1C (30)

AX1C

$\mathrm{A} \times 2 \mathrm{C}$

AX3C

DX1C

DX2C

DZ3C

Integer

I II (5)

I2I $(2,2)$

I3I $(2,2,2)$

$\operatorname{MCA3I}(2,3,3)$

IU2 I $(4,2)$

IT3I $(4,2,2)$

IU3I $(2,3,3)$ 


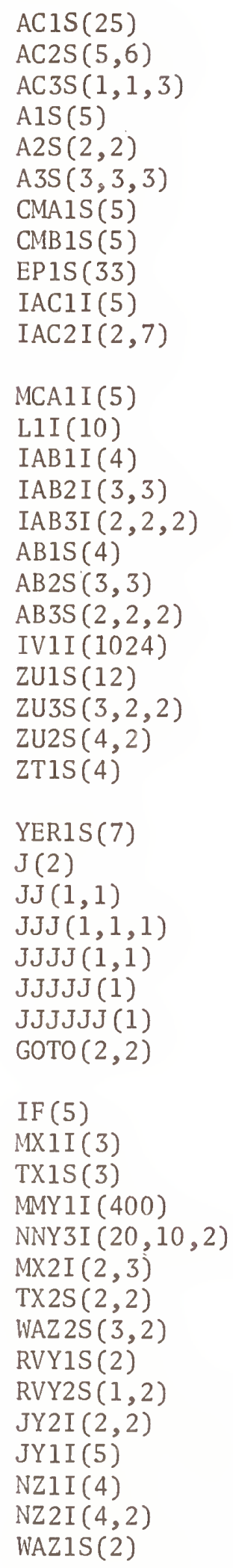


c) Blank Common Organization and Block Names

There are two separate mappings of COMMN in the Program Set. Segment 360 , the last test in the program set, tests COMMON, EQUIVALENCE, and DIMENSION using a special organization of blank COMMN not associated with any other program segment. For this reason segment 360 may not be combined with any of the segments listed below which make use of a different arrangement.

The following ordering of blank COMMON is used in Segments 162, $163,164,165,166,167,168$ and 200.

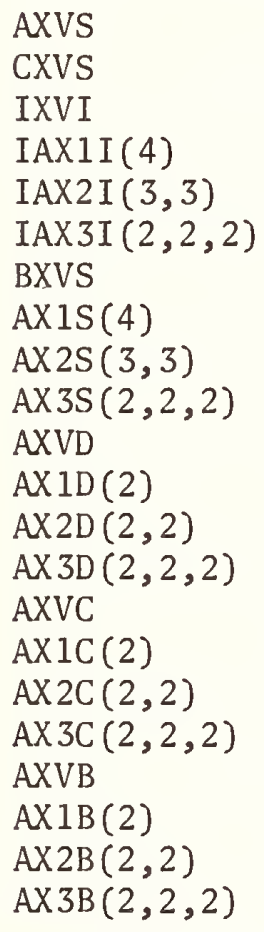

The six labeled COMMON blocks are identified by the names:

$$
\text { BLKn where } \mathrm{n} \text { is } 1 \text { to } 6
$$

The organization of the data in the labeled COMMON blocks is specified in Segment 179. 
d) Variables and Array Elements Defined in DATA Statements

Symbolic names of variables and array elements with their corresponding values are defined in DATA statements in segment 003 and tested in segment 010 . When augmenting the test programs the following variable names and array element names may not appear in subsequent DATA statements nor be redefined in tests which precede segment 010 (e.g., 008 or 009). No restriction is placed upon the redefinition of these variables or array elements in test segments which follow segment 010 .

DATA Statement 1

Symbolic Name

Form and Value of the Entry

$\operatorname{III}(1)$

$\operatorname{MCA} 3 \mathrm{I}(1,2,1)$

0

$\operatorname{MCA} 3 \mathrm{I}(2,2,2)$

IAC $21(2,5)$

$\operatorname{IAC} 2 \mathrm{I}(2,6)$

$\operatorname{MCA} 31(2,1,1)$

DATA Statement 2

Symbolic Name

$\operatorname{EP} 1 S(8)$
$\operatorname{EP} 1 S(10)$
$\operatorname{EP} 1 S(12)$
$\operatorname{AC} 2 S(5,5)$
$\operatorname{EP} 1 S(11)$
$\operatorname{AC} 2 S(5,3)$
$\operatorname{AC} 2 S(5,2)$

DATA Statement 3

Symbolic Name

BVD

$\operatorname{DPA} 2 \mathrm{D}(2,1)$

CVD

$\operatorname{DPA} 2 \mathrm{D}(1,2)$

DVD

DPA2D $(2,2)$

DATA Statement 4

Symbolic Name

ADSVC

$\operatorname{LN} 3 \mathrm{C}(9,1,2)$

LL1C (30)

$\operatorname{LN} 3 \mathrm{C}(8,2,2)$

$\operatorname{LM} 2 \mathrm{C}(8,3)$

$\operatorname{LN} 3 \mathrm{C}(9,1,1)$

LL1C (32)

$\operatorname{LN} 3 \mathrm{C}(8,1,2)$
Form and Value of the Entry

$2 * 10$

$3 * 246$

$2 * 0.0$

$2 *-750.05$

$.24615 \mathrm{E} 3$

$2.4615 \mathrm{E} 2$

$3.54674 \mathrm{E}+3$

Form and Value of the Entry

$+34567890.1 \mathrm{D}-3$

$345.678901 \mathrm{D}+2$

$112233.5 \mathrm{D}-08$

$11.22335 \mathrm{D}-4$

3. $4 \mathrm{D} 12$

$0.34 \mathrm{D} 13$

Form and Value of the Entry

$2 *(11.1,22.22)$

$(-3.45 \mathrm{E} 1,-67.8 \mathrm{E}-1)$

$(-34.5 \mathrm{E} 0,-6.78 \mathrm{E} 0)$

(10.E0, -20.E0)

$(1.0 \mathrm{E} 1,-2.0 \mathrm{E} 1)$

$(-20.0 \mathrm{E} 1,+4 . \mathrm{E} 3)$

$(-200 . \mathrm{E} 0,+4000 . \mathrm{E} 0)$ 
DATA Statement 5

Symbolic Name

MAVB

$\operatorname{MCAlB}(6)$

MBVB

DATA Statement 6 Symbolic Name

$\operatorname{GI} 3 B(1,1,2)$

GG1B(1)

EP1S (15)

DATA Statement 7

Symbolic Name

III (2)

IAC2 I $(1,5)$

$\operatorname{IAC} 2 \mathrm{I}(1,3)$

III (5)

$\operatorname{IAC} 2 \mathrm{I}(2,4)$

$\operatorname{MCA} 3 \mathrm{I}(1,1,2)$

AVI (Integer type)

EP1S (13)

$\operatorname{AC} 2 S(2,6)$

$\operatorname{AC} 2 S(1,6)$

$\operatorname{AC} 3 \operatorname{S}(1,1,1)$

$\operatorname{AC} 2 \mathrm{~S}(3,6)$

$\operatorname{AC} 3 S(1,1,2)$

$\operatorname{AC} 2 S(4,6)$

AVD

AlD (1)

$\operatorname{DPA} 2 \mathrm{D}(1,1)$

$\operatorname{MCA3D}(1,1,1)$

$\operatorname{AlD}(2)$

$\operatorname{MCA3D}(1,1,2)$

LL1C (29)

$\operatorname{LN3C}(8,2,1)$

$\mathrm{BCVC}$

$\operatorname{LM} 2 \mathrm{C}(8,4)$

$\operatorname{GH} 2 \mathrm{~B}(1,1)$

$\operatorname{GI} 3 B(1,1,1)$

MCVB

$\operatorname{III}(3)$

III (4)

MCA3I $(1,2,2)$

$\operatorname{AC} 2 S(5,6)$

JVS (type REAL)

EPIS (14)

$\operatorname{AC} 3 \mathrm{~S}(1,1,3)$

$\operatorname{IAC} 2 \mathrm{I}(1,4)$

CHEVC
Form and Value of the Entry

$2 *$.TRUE.

.FALSE.

Hollexith Data Form

$2 \mathrm{HNO}$

$2 * 2 \mathrm{HAD}$

Form and Value of the Entry

$3 * 0$

$4 *-750$

$2 * 0$ 。

$2 * 246.15$

$354674 \cdot E-2$

$354.674 \mathrm{E}+1$

$35467.4 \mathrm{E}-01$

$3 *-.295 \mathrm{D} 5$

$-29.5 \mathrm{D}+3$

$3456.78901 \mathrm{D}+01$

$0.345678901 \mathrm{D}+5$

$2 *(1.11 \mathrm{E} 1,+222.2 \mathrm{E}-1)$

$(-34.5,-6.78)$

$(-.345 E 2,-678 . E-2)$

$2 *$.TRUE.

-FALSE.

$2 * 10$

$+246$

$-.75005 \mathrm{E} 03$

$-7.5005 \mathrm{E}+02$

$2 \mathrm{HBC}$

$2 \mathrm{H}^{*}=$

$2 \mathrm{H} \mathrm{P}$

$2 *(10,,-20$. 


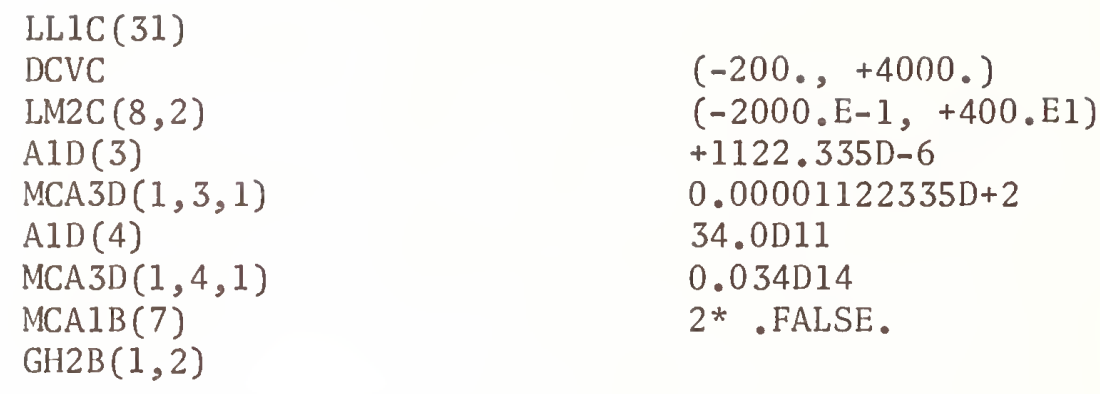

e) Statement Numbers Used (and Not Used) Between 7000-9999 With the Segment Numbers Associated

\begin{tabular}{|c|c|c|c|c|c|}
\hline Statement Labe 1 & Segment \# & Not Used & Statement Labe 1 & Segment & Not Used \\
\hline $7000-7001$ & 160 & & $8863-8864$ & 410 & 8865 \\
\hline $7002-7003$ & 161 & $7004-7006$ & $8866-8869$ & 410 & \\
\hline $7007-7009$ & 163 & & $8870-8873^{*}$ & $015 \&$ & $8874-8875$ \\
\hline $7010-7012$ & 162 & 7013 & & 410 & \\
\hline $7014-7022$ & 165 & $7023-7029$ & $8876-8878$ & 410 & 8879-8899 \\
\hline $7030-7034$ & 166 & $7035-7079$ & $8900-8909$ & 190 & $8910-8919$ \\
\hline $7080-7105$ & 008 & $7106-7107$ & $8920-8929$ & 192 & $8930-8939$ \\
\hline $7108-7112$ & 008 & $7113-7117$ & 8940 & 194 & 8941 \\
\hline $7118-7120$ & 008 & 7121 & 8942 & 194 & 8943 \\
\hline $7122-7124$ & 008 & 7125 & 8944 & 194 & 8945 \\
\hline $7126-7135$ & 008 & $7136-7137$ & $8946-8954$ & 194 & $8955-9189$ \\
\hline $7138-7156$ & 008 & $7157-7169$ & $9190-9198$ & 197 & $9199-9300$ \\
\hline $7170-7173$ & 017 & 7174-7199 & $9301-9308$ & 302 & $9309-9319$ \\
\hline $7200-7201$ & 020 & $7202-7209$ & $9320-9349$ & 310 & $9350-9901$ \\
\hline $7210-7219$ & 021 & & $9902-$ & 190 & $9903-9904$ \\
\hline $7220-7229$ & 022 & $7230-7359$ & 9905 & 190 & $9906-9907$ \\
\hline $7360-7369$ & 360 & $7370-753.9$ & 9908 & 190 & $9909-9919$ \\
\hline $7540-7546$ & 054 & $7547-7849$ & $9920-9921$ & 192 & 9922 \\
\hline $7850-7852$ & 085 & $7853-7879$ & 9923 & 192 & $9924-9929$ \\
\hline 7880 & 088 & $7881-7889$ & $9930-9931$ & 312 & $9932-9938$ \\
\hline 7890 & 089 & $7891-7899$ & $9939-9960$ & 300 & \\
\hline $7900-7909$ & 190 & & 9961 & 197 & 9962 \\
\hline 7910 & 091 & $7911-7919$ & $9963-9964$ & 197 & 9965 \\
\hline $7920-7929$ & 192 & $7930-7939$ & $9966-9969$ & 197 & \\
\hline $7940-7947$ & 194 & 7948 & $9970-9975$ & 302 & $9976-9979$ \\
\hline 7949 & 194 & & $9980-9989$ & 301 & $9990-9993$ \\
\hline $7950-7956$ & 300 & $7957-7991$ & $9994-9995$ & 073 & \\
\hline 7992 & 092 & $7993-8094$ & $9996-9997$ & 063 & \\
\hline $8095-8123$ & 350 & 8124-8209 & $9998-9999$ & 062 & \\
\hline $8210-8216$ & 021 & $8217-8219$ & $1-14$ & 350 & \\
\hline $8220-8226$ & 022 & $8227-8299$ & 22 & 350 & \\
\hline $8300-8337$ & 301 & $8338-8359$ & 333 & 350 & \\
\hline $8360-8366$ & 360 & $8367-8859$ & 22255 & 350 & \\
\hline 8860 & 410 & $8861-8862$ & & & \\
\hline
\end{tabular}

*These statement numbers appear in a main program and a subprogram. 
E. STRUCTURING, RESTRUCTURING AND EXTENDING THE TEST PROGRAMS

E1. Program Structure

Version 1 has been structured as 116 executable FORTRAN programs with provisions for linking test units end to end. Version 3 has been structured into 14 executable FORTRAN programs.

Every main program test unit contains at least two segment numbers, the first executable statements which assign the I/O unit numbers, identified as segment 007 in columns 74-76, and the test segment identified by the 3-digit identification 008 to 360 .

An executable program includes some of the following segment numbers:

Specification Statements

DATA Statements

Statement Function Definitions

I/O Assignment Statements

Main program segments

Subprograms

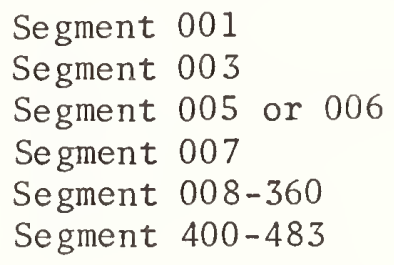

Because test units may be linked end to end, the segment numbers 001 to 007 are identified by these numbers within the test unit in which they are embedded to facilitate the identification and location of these elements in a FORTRAN program and to aid in the elimination of duplicate elements when test units are consolidated.

Each test unit, even when consolidated with other test units, can be viewed from the program listing as an independent test because the necessary Specification statements, I/O assignment statements, STOP statements, and END lines are inserted as specially structured comment lines in their appropriate locations. Lines beginning with the characters " $\mathrm{C}=$ " identify these otherwide FORTRAN statements.

E2. Consolidating Test Program Units Using Version 1

Version 1 contains a directory of the test segments as a set of 342 comment lines before the first test segment. These are identified as segment 000 and may be used to create a directory to head any consolidation of the test programs. (A directory of only those test units appearing in a specific part heads each executable program in Version 3.)

In both versions, comment lines have been inserted to ease the burden of coupling test units together or isolating them.

Specification statements and END lines have unique position requirements in the FORTRAN standard. Specification statements must precede Statement Function definitions and the first executable statement, and the END line must be the last line of a program unit. Comment lines may be anywhere before the END line. 
Each main program unit in Version 1 has been created as if it had been developed from Version 3. That is, the comment lines inserted into each test unit which directs the user how to create a single test program from a consolidated set has actually been performed to create Version 1, leaving the comment lines in place. This permits the user who has consolidated the test programs to later isolate individual test units as needed with directions for the process contained in the program. For example, in segment 008 test unit, the FORTRAN text contains the following message:

C***** WHEN EXECUTING ONLY SEGMENT 008, THE SPECIFICATION STATEMENTS C***** WHICH APPEAR AS COMMENTS MUST HAVE THE C $=$ IN COLUMNS

$\mathrm{C} * * * * * 1$ AND 2 REMOVED

Below this message is a set of comment lines which, except for columns 1 and 2, look like Specification statements with the segment number 001 in columns 74-76. In Version 1, this action has already been performed leaving the $\mathrm{C}=$ comment lines in the program and inserting the actual Specification statements below these comment lines but with the segment number changed from 001 to the test segment number, in this case 008 . The four digit sequence number, columns 77-80 is unique for these inserted lines, and is assigned characters and digits which will facilitate the location of these lines. Similar messages appear before the I/O assignment statements and the STOP statement and END line. The following identification code has been assigned for columns 74-80:

Specification Statements

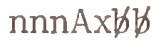

I/0 Assigment Statements

$\mathrm{nnn} B \mathrm{~d} \not \not \not b$

STOP and END

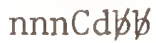

Where nnn is the test segment number in which the statements are embedded, $\mathrm{x}$ is 1 to 9 and $\mathrm{A}$ to $\mathrm{F}$, and $\mathrm{d}$ is 1 or 2. The last two character positions are blank. Specification statements may contain continuation lines, so that the sequence number is significant.

In order to link test units end to end into a single executable program, it is necessary to eliminate duplicate specifications, STOP and END lines, and I/O assignment statements (if the unit numbers are changed by the user). These appear only in the main program test units. Elimination of duplicate symbolic names from the Specification statements is performed on each of the nine (DIMENSION, COMMON, EQUIVALENCE, EXTERNAL, REAL, INTEGER, DOUBLE PRECISION, COMPLEX, and LOGICAL) statements independently. That is, if dimension information is expressed in a type statement instead of a DIMENSION statement, all test programs which require this specification information for a particular symbolic name will be consistent.

The appropriate directory and the consolidated specifications, identified as segment 001, should be placed in front of the first test unit of the consolidated set, the I/O assignments placed as the first executable statements (segment 007) within the first test unit, and a single STOP statement and END line must appear as the last lines of the main program unit. If Statement Function definitions are a part of a test unit, these 
must be placed before the first executable statement. Segments 110 , 111, and 197 contain Statement Function definitions. If segments 110 and 197 are combined into the same executable program, one copy of segment 005 must be removed. Test units should be performed in the order of the directory, particularly segment 010, Data Statement Use, must appear in order, because the potential reuse of data names appearing in a DATA statement in other program test units cannot be guaranteed.

If during the consolidation process, an attempt is made to include more test units than the FORTRAN processor will accept into a single executable program, it will be necessary to return the specification statements, I/O assignment statements and STOP and END lines to the appropriate test units not included with the finally consolidated set for later use in another consolidation. The segment number associated with these lines identified by the letters " $A$ ", "B", and "C" in column 77 is contained in columns 74-76.

When test programs are consolidated into larger executable programs, it is desirable to have some means of identifying the test results with some additional information related to the environment of performing the tests, such as computer name, compiler version, operating system version, date, and any additional information which would distinguish successive running of the test programs. This can be achieved by incorporating the FORTRAN lines, identified as segment 007-which are embedded in segment 008 starting with the comment line "IDENTIFY THE SOURCE OF THE TEST PROGRAMS", into the first test of each consolidated test set following the I/O assignment statements. The last continuation line of the FORMAT statement at Statement Label 0071 should be altered to reflect a unique means of identifying each executable program. In Version 3, this has been done by identifying each executable program as a PART, numbered from 1 to 14. The first six input cards associated with segment 008 will then be required for running each of the consolidated test sets。Cards 1, 3 and 5 are prepared by the user, replacing the dummy information on the card images supplied, with the environmental information. See Section II-A2 Input Data Preparation.

The number of test segments which may be linked end to end is a function of the power of the FORTRAN processor with the following exceptions:

a) Segment 360 may not be linked with any other test segment which uses blank common.

b) Segments 169 and 179 when consolidated into a single program will cause different elements of a specific labeled common block to be initialized from DATA statements in different BLOCK DATA subprograms 。 While the current FORTRAN standard does not exclude this, it is anticipated that the future revised FORTRAN standard may prohibit the user from so doing. 
E3. Deleting a Section of a Test Unit

If certain test elements fail to perform on a system because some elements of the FORTRAN language have not been implemented and the test unit cannot be executed, it will be necessary to inspect the test unit to determine what statements together with the corresponding WRITE statements are affected. When a section of a test unit is altered it is recommended that those statements which are changed or deleted have appropriate comment cards inserted to identify the change. This can be achieved by making the current statements into comment lines with a character other than blank, *, or $=$ as the second digit and a comment card containing the number of lines which follow in the replacement. If a statement which is deleted contains a statement label, it will be necessary to repunch the card with the four digit statement label right justified and replace column 1 with a "C".

If a program test unit is too large for running as a single test unit it may be separated into smaller units for testing. This may be necessary for test segment 008, Formatted READ and WRITE, because of its current size and the number of FORMAT statements included in this test unit. The sample Result Output should be inspected. The breaks in the program should conform to locations where a new page indicator is detected at the beginning of a FORMAT statement. Data cards are identified in the program listing and the card number is given at the point of the appropriate READ statement.

E4. Deleting an Entire Test Unit

All test units are identified by segment numbers in columns 74-76. Test units contain " $\mathrm{C}=$ " comment cards for specification statements and I/O assignment statements with segment numbers 001 and 007 , respectively. STOP and END cards appear at the end of each test unit as " $\mathrm{C}=$ " comment cards with the sequence number the same as the test unit number. All cards related to a test unit may be removed by inspection of the program listing. Any subprogram which is associated with that program test unit will not be assoicated with any other program test unit and may be removed. Distinctive comment cards separate test units.

E5. Adding to a Test Unit

Any program test unit may be extended by appending statements after the last executable statement in a program test unit. See Program conventions for symbolic name and statement label use Section I-D. Result output pages have been limited to $81 / 2$ by 11 inch pages with a new page indicator for each page. All variables must have their values initialized in the test unit. Any new specifications must be introduced into "C=" cards within the test unit and a check made of the specifications contained at the beginning of the Part in Version 3. Array declarators used in the test set are identified in Section I-D-3. 
E6. Adding New Test Units

Be sure that the programming conventions used in this test set are followed. A segment number which has not been used less than 399 may be used for a main program test. Numbers 400-699 which have not been used are available for subprograms. In general, the number chosen should be high enough so that those elements of the language which must be used in the test have already been tested.

Make sure that each new test is self contained. Initialize all values within the new segment itself. Use the same comment line structure to separate the new test unit, and intersperse comments to describe the test. Update the directory, specification section and the comment cards at the beginning of the Part in Version 3 to reflect any new program test units added. The listing of the program is supposed to contain enough comments to permit the programs to be used if additional documentation is not available. 

During the implementation of the program design, a number of difficulties other than the normal program debugging arose which required resolution. These difficulties have been classified under the following five categories.

\section{F1. Interpreting the FORTRAN Standard}

In interpreting the FORTRAN Standard document X3.9-1966, there was a conscious effort to glean from the document only that which was stated, and not to be influenced by earlier implementations of the FORTRAN language. This lead to a long list of questions which needed resolution. The ASA FORTRAN technical committee, X3J3, reconvened to address these and other questions of interpretation of the Standard. The committee published two clarification reports [2,3] concerning the interpretation of the standard. Those questions which could not be resolved without actually revising the standard have been deferred and will be handled in the future revision and extension to the FORTRAN standard. Some of the questions did not arise until some initial test units were run on different processors and the different interpretations of the standard could be asserted and appeared to be justified by the wording in the standard.

F2. Precision, Conversion and Maximum Value of Numeric Data

The choice of the actual values used in arithmetic expressions presented considerable difficulty. The range of the exponent, which is not covered by the FORTRAN standard was kept small so that the variation on different processors would not be reflected in the test results. To overcome some of the precision problems, small fractional powers of two were used in the hope that the conversion of these values would be exact. Recognizing that the FORTRAN standard defines a real constant to be an "approximation to the digit string interpreted as a decimal numeral" the equation $1.3+1.3=2.6$ may not be true if the result were compared to the constant 2.6 because the constant when converted and when doubled may not have the same internal representation as the constant 2.6. Rather than attempt to apply an error tolerance to the results, it was decided to subtract the expression result from the expected result and rely upon the rounding under the Fw.d format field descriptor on output to compensate for a small difference in values. Because the Fw.d format field descriptor cannot be applied to results derived from a double precision operation, it was necessary to stipulate in the test results a reasonable error factor to be applied to the value if the result was not zero.

F3. Meaningful Tests and Comprehensible Results

The development of meaningful test programs of the FORTRAN Standard language cannot be separated from the presentation of the results of the test. If it can be considered that any test result value printed from a specific application of the test programs on a FORTRAN processor could be in error, the means to determine the statements involved in the test result must be readily discernible. This lead to examining, on a case basis, how to present the results. Where ever possible the results obtained from 
arithmetic operations were subtracted from the expected result and the expected value of zero printed, so that the user could quickly scan a page of results and determine any errors. When this was not possible, Hollerith information is printed directly above the expected value so that the eye can quickly scan the results for discrepancies, or some appropriate means such as the test number for the value in error, so that reference back to the program listing could be made.

Various elements of the FORTRAN language presented some difficulties in displaying the results.

The effect of the scale factor both on READ and WRITE is such an example. If the FORTRAN processor does not perform this conversion properly, and only the expected result is printed with the processor result, it is tedious to determine from the program listing what actual data and format field descriptor is associated with a value. Because of this, the information which a user would need to determine the operation being performed is presented in a tabular form with the expected result and the actual result.

The Intrinsic Functions SNGL and DBLE presented the problem of storage unit size and how can it be determined whether these functions are actually performed when the maximum real and double precision constant length established for the programs is 7 and 14 digits respectively. The FORTRAN Standard does not define these functions to operate under the same rules as the corresponding assignment statement operation identified in Table 1 of the FORTRAN Standard. The FORTRAN Standard does not address the precision of a value, so that it cannot be determined from the document if a standard conforming program may READ or WRITE values which express a precision in excess of the processor capability. Because of these factors, for FORTRAN processors which can express a REAL value of 14 or more digits in a single storage unit, the printed results may not display the value to a precision large enough to encompass the actual function result. Increasing the number of decimal digits expressed in the format field descriptor should eliminate the difficulty.

The Basic External Functions presented a significant problem for devising test programs because the units of the arguments are not specified in the FORTRAN Standard and there was to be no attempt to address the unspecified range of arguments, precision or accuracy of the function results. This lead to using the "defacto standard" practice for the units of arguments and to select arguments which reduced the probability of variation due to conversion and for which there were also published table values. It is hoped that the arguments selected with their expected results, although not necessarily representative of normal usage, would constitute a basis for the user determining whether the function referenced is, in fact, the function obtained. Table values were not readily available for the complex functions so that a different method had to be employed. 
F4. FORTRAN Compi lers with Language Extensions

The difficulty of assuring that a FORTRAN program is confined to that which is defined in the FORTRAN Standard X3.9-1966 is substantial.

Because a program produces the same correct results on many FORTRAN processors does not in itself substantiate the program to be standard conforming. Even though the test programs were desk checked, not all non-standard usage was picked up by this method. Moving from one processor to another brought to light the differences in the extensions or relaxations permitted on various FORTRAN processors. Those programming errors which persisted undetected through many FORTRAN processors where they were treated as extensions are:

- Missing type declarations for dummy arguments of statement functions

- Missing commas after an $\mathrm{nH}$ format field description in FORMAT statements

- Non agreement between format field description and the type of the list element

- Missing decimal point in a real constant in a real expression

- Lack of agreement of type between actual and dummy arguments of a subprogram where the dummy argument is not referenced.

F5. Performing the Tests

The initial running of the completed test programs on various FORTRAN processors was performed from punched cards. The punch card code used was the BCD-H set which is identified in Appendix D of the FORTRAN Standard X3.9-1966. It was assumed that any computer installation would have a conversion routine for this code to its own, if it were not an option of the compiler. For the most part the testing was performed on the consolidated test set which reduced the number of executable programs from 116 to 14. This was done to minimize the number of control cards needed to be inserted around the programs. In spite of assistance from systems personnel at the test site in every initial running on a different processor one or more programs had to be resubmitted to the computer because of operating systems control card errors. The kinds of errors were:

- Failure to identify the FORTRAN Programs as BCD card code producing errors in scanning the FORTRAN statements.

- Failure to identify the data as BCD H set causing the execution to be aborted on improper symbols on input cards.

- Missing or mispunched control cards.

- Inproper sequencing of control cards.

At no time was the alloted time on the computer or the maximum number of pages of printing exceeded. Because many of the test programs may require the same set of control cards, special care must be taken for those programs requiring data, an auxiliary tape unit and subprograms. 
The differences in capability of operating systems did not present a difficulty but the lack of standard terminology and definition of functional capability presented barriers in human communications.

When a compiler had an option to check the programs for conformance to the FORTRAN standard and no diagnostic messages resulted, the test program writers were lulled into the belief that the programs met the standard, which later running on a different compiler proved not to be the case. Not all non-standard usage even within a single FORTRAN program unit were detected. 
G. REFERENCES

1. American Standard FORTRAN X3.9-1966 - since the original publication of the FORTRAN Standard, the standardizing organization has changed its name from American Standards Association to United States of America Standards Institute and recently to American National Standards Institute. Therefore, documents identified as ASA X3.9-1966, USASI X3.9-1966 and ANS $\times 3.9-1966$ all refer to the same document.

2. Clarification of FORTRAN Standards - Initial Report. Communications of the ACM Vol. 12, No. 5, May 1969.

3. Clarification of FORTRAN Standards - Second Report. Communications of the ACM Vol. 14, No. 10, October 1971.

4. Handbook of Mathematical Functions with Formulas, Graphs, and Mathematical Tables. NBS. M. Abramowitz and Irene A. Stegun, editors, Applied llathematical Series 55, 1966. 

SECTION II USERS MANUAL

\section{A. OPERATING PROCEDURES}

The NBS FORTRAN Test Programs are designed to test the acceptance of the ASA FORTRAN Standard X3.9-1966 language definition by a FORTRAN processor.

The Test Programs are comprised of 116 test units and approximately 14,500 card images。

\section{Al. Organization of Tests and Facilities Requirements}

The FORTRAN Test Programs are presented in two forms, one for execution on small FORTRAN processors identified as Version 1, and the other for large FORTRArJ processors identified as Version 3.

The tests make use of a maximum of $3 \mathrm{I} / 0$ units. These I/O units are identified as integer variable names which are assigned values in the first executable statements in each executable program and the statements may be altered by the user. No subprogram directly references these variable names or values.

The variable names and their current values are:

IRVI - for input, usually a card reader, is defined as unit 5. NUVI - for test results, usually a line printer is defined as unit 6.

INVI - for intermediate input/output data, usually a magnetic tape, is defined as unit 9.

The test programs should be run in numeric order. Test sequences contained in later test units may depend upon the successful execution of earlier test units.

\section{a) Program Order}

The FORTRAN Standard does not define the order of presentation of a main program, BLOCK DATA subprograms, FUNCTION or SUBROUTINE subprograms to a FORTRAN processor. This order is prescribed by the implementor and may vary from system to system. Systems also vary on the need for systems control cards or special cards preceding each subprogram. The test programs have been arranged with no intervening control cards but contain the necessary STOP and END cards as follows:

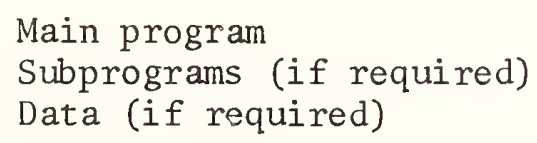

Some systems may require a specific order for BLOCK DATA subprograns distinct from FUNCTION or SUBROUTINE subprograms. 
The subprograms appear after the corresponding main program unit and before the data in the order listed in Section II-A3 for Version 1 and Section II-A4 for Version 3.

Each program is set up (except for the system control cards) for a FORTRAN compile-load-and-go execution.

The user is assumed to be familiar with the operating system control requirements necessary to perform a FORTRAN compilation.

These steps should be followed:

- Choose the appropriate control cards for a FORTRAN compile.

- Check the format and ordering of control cards carefully.

- In particular, check if any control cards are necessary for FUNCTION, BLOCK DATA, and SUBROUTINE subprograms.

- Check the particular FORTRAN system documentation for any special requirements for ordering of subprograms which may differ from the order of the test program.

- Check if the test program requires input data. Version 1 requires data for test segments $008,009,310$ and 312; Version 3, for all parts. Cards 1,3 and 5 of segment 008 for Version 1 and all parts for Version 3 may be prepared by the user and replace the sample cards supplied with the programs. Section II-A2.

Sections II-A3 contains the list of test programs for Version 1. The accompanying table identifies the I/O facilities requirements and other related information.

Sections II-A4 contains the list of test programs for each of the 14 Parts for Version 3 and identifies the $\mathrm{I} / 0$ facilities requirements as well as a summary sheet related to all Parts.

b) Memory Requirements to Execute the Test Programs

During the development of the test systems ten different computing systems were used and the current set of tests were run on five major systems. Although no requirements for memory can be determined without experimentation, the largest test unit in Version 1 required less than 3,000 words of memory. When structured into 14 executable programs as Version 3, the largest program required less than 6,000 words of memory.

c) Time

The time to compile and execute the test programs varies with the power of the computer and the compiler. The test units, for the most part are straight line programs. During the debugging of the test program set of Version 3 on different large scale systems less than 30 seconds was required to compile and execute any one of the 14 Parts excluding card read and print time. 
All data card images associated with the FORTRAN Test Programs are included with the program distribution. It is not essential to the performance of the test programs to prepare any input data, however, provisions have been made to facilitate the identification of the test program results for a given FORTRAN processor.

In Version 1 , test units $008,009,310$, and 312 require input data which is supplied with the programs. The first six (6) cards associated with test unit 008 cause a heading page to be produced for the program set.

In Version 3, all test Parts 1 to 14 include six (6) input cards as the total input data to that part, except Parts 1 and 13 which include additional input data cards supplied with the test programs.

These six cards permit information to be introduced by the user to identify: the computer, FORTRAN compiler identification, operating system level, date, etc., which describe the environment in which the test is performed. Cards 1,3 and 5 must be replaced and prepared to introduce three (3) lines of print which precedes test unit 008 in Version 1 or is appended to the initial output page of each test part in Version 3.

The first 40 characters from each of three cards (cards 1, 3 and 5) are read and replace the Hollerith information supplied in each of three FORMAT statements. The first character of each card must be blank (for print carriage control) and the other 39 characters must be from the FORTRAN character set. Cards 2, 4, and 6 must remain as prepunched. These six cards are part of the first test unit (008) in Part 1, testing the replacement of Hollerith information in a FORMAT statement by a formatted READ, and the symmetry of interpretation of a terminal slash (/) in a FORMAT statement used for READ and WRITE, causing cards 1,3 , and 5 to be read and written, and cards 2, 4 and 6 to be skipped on input and blank lines to be produced on output.

These six cards are not part of the test in parts other than Part 1 but are included for user output documentation only.

WARNING: The following four characters should be avoided in preparation of the three cards, because these characters differ in the punch card code for input preparation devices:

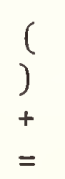


A3. List of Test Programs for Version 1

The I/O Unit numbers used in the Test Programs are:

$\begin{array}{ll}\text { Input (card reader) } & 5 \\ \text { Output (printer) } & 6 \\ \text { Intermediate } & 9\end{array}$

The following table identifies each of the 116 Test Programs for Version 1 and the associated subprograms.

Codes Used to Describe the Information in the Table

Column

$1 \quad M \quad$ Main Program

F External Function

S Subroutine

B BLOCK DATA

2 I Input Required

3 No. of Pages of Output
Column

$4 \quad X$ Intermediate Tape Required

5 C Blank Common Block

/ Special Blank Common

6 D DATA Statement Defined

7 No. of Cards per Segment

\begin{tabular}{|c|c|c|c|c|}
\hline Seg. Name & Test & Table & & \\
\hline 000 & Directory of Test Programs & -- & - & -342 \\
\hline 008 - FMTRW & $\begin{array}{l}\text { Formatted Input/Output } \\
6 \text { Identification Cards and } 40 \text { Data Cards }\end{array}$ & $\begin{array}{l}M I 8 \\
--\end{array}$ & $\begin{array}{l}- \\
-\end{array}$ & $\begin{array}{r}-529 \\
-\quad 46\end{array}$ \\
\hline $009-\mathrm{AFRMT}$ & $\begin{array}{l}\text { A-Conversion } \\
3 \text { Data Cards }\end{array}$ & $\begin{array}{l}\text { M I } 1 \\
--\end{array}$ & $\begin{array}{l}- \\
-\end{array}$ & $\begin{array}{rr}- & 115 \\
- & 3\end{array}$ \\
\hline $\begin{array}{l}010-\text { DATA2 } \\
003 \text { - DATA1 }\end{array}$ & $\begin{array}{l}\text { DATA Statement Use } \\
\text { Test Format of DATA Statement }\end{array}$ & $\begin{array}{l}M-3 \\
M--\end{array}$ & $\begin{array}{l}- \\
-\end{array}$ & $\begin{array}{ll}- & 74 \\
\mathrm{D} & 84\end{array}$ \\
\hline 011 - AASGN & Real and Integer Arith Assignmt. Stmnts. & $M-3$ & - & -268 \\
\hline 013 - DASGN & Simple D.P. Assignment Statements & $M-8$ & - & -420 \\
\hline 015 - CASGN & Simple Complex Assignment Statements & $M-9$ & - & -469 \\
\hline 016 - LASGN & Logical Assignment Statements & $M-1$ & - & -106 \\
\hline 017 - INTRL & Arithmetic Assignment Statements & $M-4$ & - & -185 \\
\hline 20 - UGOTO & Unconditional GO TO Statements & $M-1$ & - & 69 \\
\hline
\end{tabular}




\begin{tabular}{|c|c|c|c|c|c|}
\hline 021 & - AGOTO & GO TO Assignment Statements & $M-1$ & - & - - \\
\hline 022 & - CGOTO & Computed GO TO Statements & $M-1$ & - & - \\
\hline 030 & - ARBAD & Basic Addition & $M-1$ & - & - \\
\hline 031 & - ARFAD & Double Precision Addition & $M-1$ & - & - \\
\hline 032 & - ARBSB & Basic Subtraction & $M-1$ & - & - \\
\hline 033 & - ARFSB & Double Precision Subtraction & $M-1$ & - & - \\
\hline 034 & - ARBAS & Basic Addition and Subtraction & $M-1$ & - & - \\
\hline 035 & - ARFAS & Addition and Subtraction of D.P. Values & $M-1$ & - & - \\
\hline 036 & - ARBMI & Multiplication of Integer Values & $M-1$ & - & - \\
\hline 037 & - ARBMR & Multiplication of Real Values & $M-1$ & - & - \\
\hline 038 & - ARFMD & Multiplication of D.P. Values & $M-1$ & - & - - \\
\hline 039 & - ARBDV & Division of Integer and Real Values & $M-1$ & - & - \\
\hline 040 & - ARFDV & Division of D.P. Values & $M-1$ & - & - \\
\hline 041 & - ARBEX & Exponentiation of Integer and Real Values & $M-1$ & - & - \\
\hline 042 & - ARFEX & Exponentiation of D.P. Values & $M-1$ & - & - \\
\hline 043 & - ARBHI & Hierarchy of Operators and Parentheses & $M-1$ & - & - \\
\hline 050 & $-\mathrm{SBB} 67$ & Subscripts of Integer, Real Arrays $v, k$ & $M-1$ & - & - \\
\hline 051 & $-\mathrm{SBB} 45$ & Subscripts of Int., Real Arrays $v+k, v-k$ & $M-1$ & - & - \\
\hline 052 & $-\mathrm{SBB} 13$ & $\begin{array}{l}\text { Subscripts of Int., Real Arrays } c^{*} v, c^{*} v+k \text {, } \\
c^{*} v-k\end{array}$ & $M-1$ & - & - \\
\hline 053 & - SBF17 & $\begin{array}{l}\text { Subscripts of D.P. Arrays } v, k, c^{*} v, c^{*} v+k \text {, } \\
c^{*} v-k, v+k, v-k\end{array}$ & $M-1$ & - & - \\
\hline 054 & - SIMIF & Arith. IF, Logical IF followed by GO TO & $M-1$ & - & - \\
\hline 055 & - IFABS & Intrinsic Functions ABS, IABS & $M-1$ & - & - \\
\hline 056 & - IFFLT & Intrinsic Function FLOAT & $M-1$ & - & - \\
\hline 057 & - IFFIX & Intrinsic Function IFIX & $M-1$ & - & - \\
\hline 058 & - IFSGN & Intrinsic Functions SIGN, ISIGN & $M-1$ & - & - \\
\hline 059 & - IFDAB & Intrinsic Function DABS & $M-1$ & - & - \\
\hline
\end{tabular}




\begin{tabular}{|c|c|c|c|c|c|c|c|}
\hline 060 & - IFTRN & Intrinsic Functions AINT, INT, IDINT & M & -1 & - & - - & 107 \\
\hline 061 & - IFMOD & Intrinsic Functions AMOD, MOD & M & -1 & - & - - & 84 \\
\hline 62 & - IFMAX & Intr. Funct. AMAX0, AMAX1, MAX0, MAX1, DMAX 1 & M & -2 & - & -- & 248 \\
\hline 63 & - IFMIN & Intr. Funct. AMIN0, AMIN1, MIN0, MIN1, DMIN1 & M & -2 & - & - & 225 \\
\hline 64 & - IFDSG & Intrinsic Function DSIGN & M & -1 & - & - & 58 \\
\hline 065 & - IFDIM & Intrinsic Functions DIM, IDIM & M & -1 & - & - & 69 \\
\hline 066 & - IFSGL & Intrinsic Function SNGL & M & -1 & - & - & 80 \\
\hline 067 & - IFREL & Intrinsic Function REAL & & -1 & - & - & 102 \\
\hline 068 & - IFIMG & Intrinsic Function AIMAG & M & -1 & - & - & 129 \\
\hline 069 & - IFDBL & Intrinsic Function DBLE & M & -1 & - & - & 57 \\
\hline 070 & - IFCPX & Intrinsic Function CMPLX & M & -1 & - & - & 61 \\
\hline 071 & - IFCJG & Intrinsic Function CONJG & M & -1 & - & - & 66 \\
\hline 072 & - IFBMS & Integer and Real Intrinsic Functions & M & -1 & - & - & 129 \\
\hline 073 & - IFFMS & Int., Real and D.P. Intrinsic Functions & M & -2 & - & - & 181 \\
\hline 080 & - EXPON & Basic External Function EXP & M & -1 & - & - & 60 \\
\hline 081 & - DEXPO & Basic External Function DEXP & M & -1 & - & - - & 68 \\
\hline 082 & - CEXPO & Basic External Function CEXP & M & -3 & - & - & 98 \\
\hline 083 & - LOGTM & Basic External Function ALOG & M & -1 & - & - & 57 \\
\hline 084 & - DPLOG & Basic Extemal Function DLOG & M & -1 & - & - & 67 \\
\hline 085 & - CXLOG & Basic Extemal Function CLOG & M & -3 & - & - & 106 \\
\hline 086 & - COLOG & Basic External Function ALOG10 & M & -1 & - & - - & 56 \\
\hline 087 & - DCLOG & Basic External Function DLOG10 & M & -1 & - & - & 66 \\
\hline 088 & - SINUS & Basic External Function SIN & $M$ & -1 & - & - - & 81 \\
\hline 089 & - DPSIN & Basic External Function DSIN & M & -1 & - & - - & 82 \\
\hline 090 & - CSICO & Basic Extemal Functions CSIN, CCOS & M & -1 & - & - - & 65 \\
\hline 091 & - COSNS & Basic External Function COS & M & -1 & - & - & 82 \\
\hline 092 & - DPCOS & Basic External Function DCOS & M & -1 & - & - - & \\
\hline
\end{tabular}




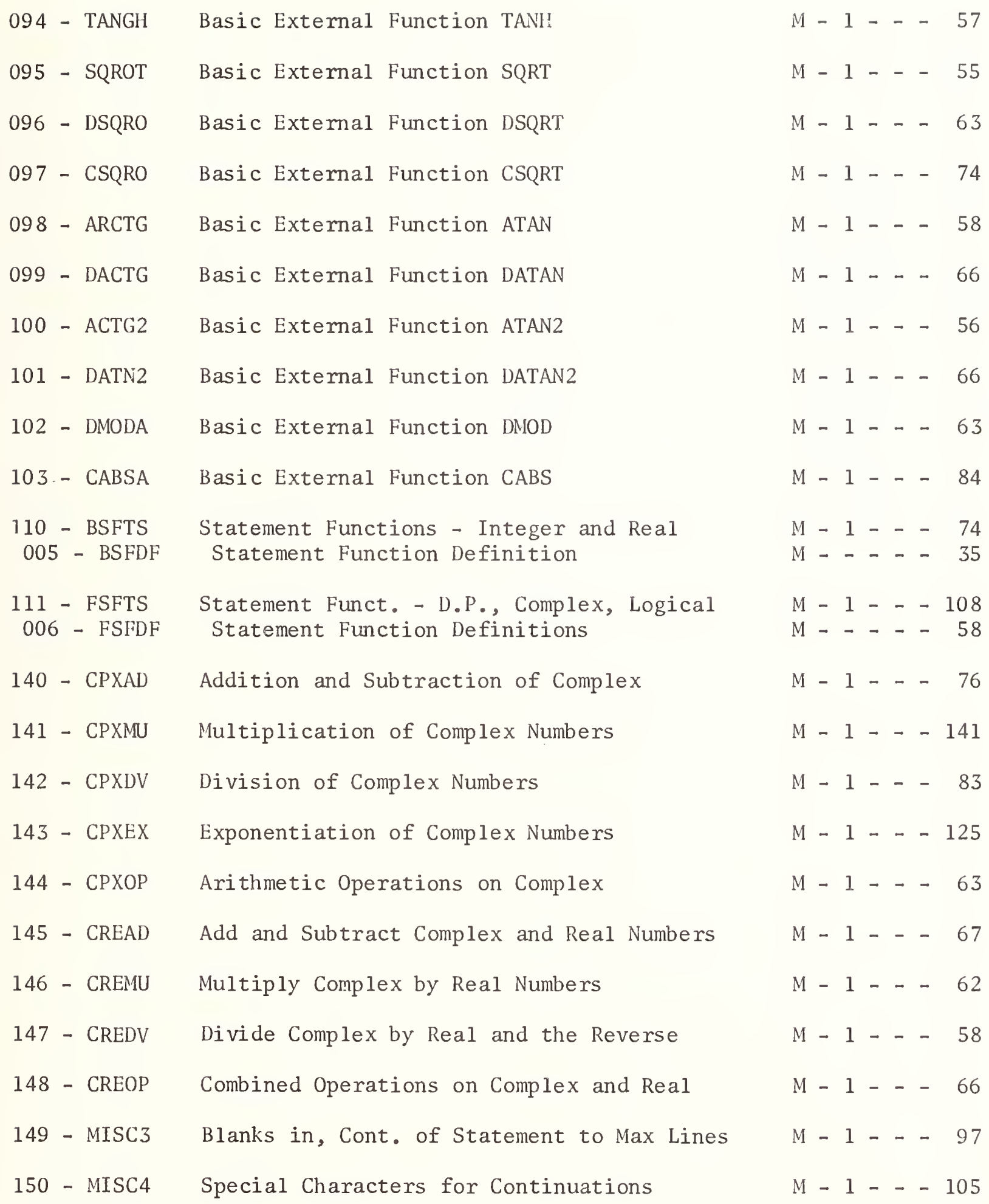




\begin{tabular}{|c|c|c|}
\hline 160 & - BRFCP & Real External Functions \\
\hline 400 & $-\quad$ AFS & Real Argument \\
\hline 420 & BFS & Real Arguments \\
\hline 430 & CFS & Integer Argument \\
\hline 440 & DFS & Integer Arguments \\
\hline 450 & EFS & Array Name as Argument \\
\hline 460 & FFS & Different Types of Arguments \\
\hline & - BIFCP & Integer External Functions \\
\hline 01 & $-\quad$ IAFI & Real Argument \\
\hline+21 & $-\quad$ IBFI & Real Arguments \\
\hline 31 & - ICFI & Integer Argument \\
\hline 41 & - IDFI & Integer Arguments \\
\hline 1 & - IEFI & Array Name as Argument \\
\hline 51 & $-\quad$ IFFI & Different Types of Arguments \\
\hline & - FRFCP & Real External Functions \\
\hline 02 & $-\quad$ GFS & D.P. Argument \\
\hline 422 & HFS & Complex Arguments \\
\hline 432 & - IRFS & Logical Argument \\
\hline+42 & - JRFS & External Procedure \\
\hline 452 & RFS & Different Types of Arguments \\
\hline-63 & - FIFCP & Integer External Functions \\
\hline & $-\quad$ IFI & D.P. Argument \\
\hline 23 & JFI & Complex Arguments \\
\hline 433 & KFI & Logical Argument \\
\hline 443 & LFI & External Procedure \\
\hline 453 & MFI & Different Types of Arguments \\
\hline 164 & - $\mathrm{CFCCP}$ & Complex External Functions \\
\hline 404 & $-\quad \mathrm{AFC}$ & Real Argument \\
\hline 414 & $\mathrm{BFC}$ & Integer Argument \\
\hline 24 & CFC & Array Name as Argument \\
\hline 434 & $\mathrm{DFC}$ & D.P. Argument \\
\hline 444 & EFC & Complex Argument \\
\hline 454 & FFC & Logical Argument \\
\hline 464 & $\mathrm{HFC}$ & Different Types of Arguments \\
\hline 165 & - DPFCP & Double Precision External Functions \\
\hline 4( & $-\quad A F D$ & Real Argument \\
\hline 41 & $B E D$ & Integer Argument \\
\hline & CFD & D.P. Arguments \\
\hline & DFD & Complex Argument \\
\hline & $\mathrm{EED}$ & Logical Argument \\
\hline & FED & External Procedure \\
\hline & GFD & Array Name as Argument \\
\hline & HFD & Different Types of Arguments \\
\hline
\end{tabular}

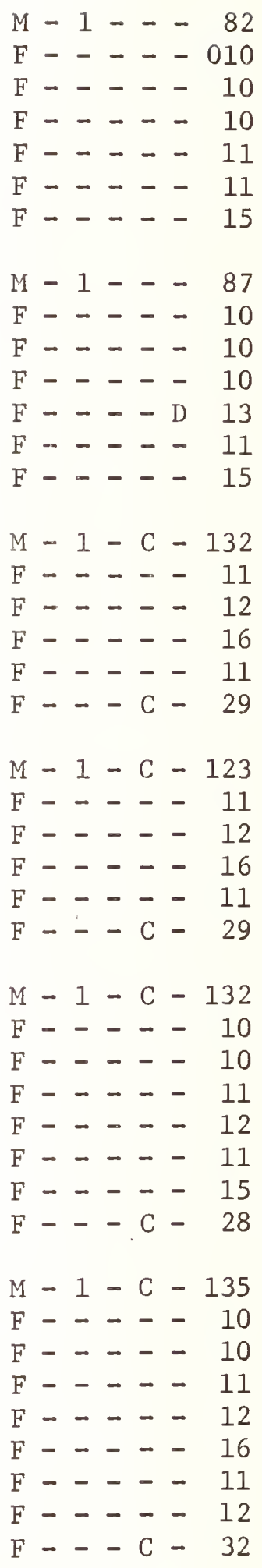


166 - BFCCP

406 - $A F B$

416 - BFB

426 - $\mathrm{CFB}$

436 - DFB

446 - EFB

456 - FFB

466 - GFB

476 - HFB

167 - SBRTN

407 - AAQ

417 - $\mathrm{ABQ}$

427 - ACQ

168 - FSBRT

408 - ADQ

418 - AEQ

428 - $A F Q$

169 - BLKDT

409 - BLOKD

179 - BLKDA

419 - BLAKD

429 - BLBKD

439 - BLCKD

180 - UNFRW

182 - BACUP

190 - DOTRM

191 - DOLMT

192 - DONSC

193 - DONSI

194 - DONSX

195 - DONML

196 - DONIO

197 - MORDO

005 - BSFDF

412 - MDQ

200 - SUBR 1

410 - SUBRQ
Logical External Functions

Real Argument

Integer Argument

D.P. Argument

Logical Argument

Complex Argument

Array Name as Argument

External Procedure

Different Types of Arguments

Subroutine Subprogram

Integer, Real Variables, Array Elements

Array Elements

No Argument List

Subroutine Subprogram

Different Types of Arguments

Array Names and Integer Arguments

No Argument List

BLOCK DATA Test

BLOCK DATA Subprogram

BLOCK DATA Test

BLOCK DATA Subprogram

BLOCK DATA Subprogram

BLOCK DATA Subprogram

Unformatted WRITE and READ

BACKSPACE Tape

DO Loops - Terminal Statements

DO Loops - Parameters as Variable Names

DO Loops - Completely Nested Nest

DO Loops - Incomplete DO, Exit by GO TO

DO Loops - Extended Range

DO Loops - Nested Nest

DO Loops - I/O Terminal Statements

DO Loops - I/O, Statml. Ft., Intr Ft., CAILL Statement Functions

Subroutine Subprogram

Subroutine - Operations Done at Sub Level

Subroutine Subprogram - No Arg. List
$M-1-C-144$

F - - - 10

$\mathrm{F}-\mathrm{F}^{-}-10$

F - - - 11

F - - - 11

F - - - 12

F - - - 12

F - - - 11

$F--C-25$

$M-1-C-103$

S - - - 23

S - - - 13

$\mathrm{S}--\mathrm{C}-21$

$M-1-C-153$

S - - - 39

S - - - 23

S - - C - 41

M - 1 - - 71

B - - D 36

M - 1 - - 70

B - - - D 24

B - - - D 17

B - - D 20

$M-1 X--133$

M - $1 X--74$

M - 1 - - 135

$M-1$ - - 62

M - 1 - - 166

$M-1$ - - 60

M - 1 - - 130

$M-1$ - - 65

$M-1 X--91$

M - 1 X - 143

M - - - 35

S - - - 13

$M-1 X C-52$

S - X C - 101 
300 - LOGIF Logical IF Statements

411 - SMCQ Subroutine Subprogram

301 - BARIF Arithmetic IF Statements - Integer, Real

302 - FARIF Arithmetic IF Statements - D.P.

310 - IOFMT Formatted READ/WRITE - Additional Features 38 Data Cards

312 - RDFMT Formats in Arrays

462 - FMTQ

$350-$ MISC5

351 - FUNMX

352 - NAMES

$413-\mathrm{MAQQ}$

$463-\mathrm{MBQQ}$

473 - AMQQ

483 - $\mathrm{BMQQ}$

360 - SPEC2

Total Cards
Subroutine Subprogram

13 Data Cards

Specifications for Program Form

Basic External Functions - Trig Formulae

Names Resemble FORTRAN Verbs, Functions

Sub routine (Intrinsic Function Names

Sub routine

Sub routine used as Variable Names in some Subrts. and as

Sub routine Functions in others)

COMMON, DIMENSION, EQUIVALENCE
M - 1 - - 275

S - - - 12

M - 1 - - 175

$M-1--99$

M I 5 - - 310

- - - - 38

M I 1 - D 201

S - - - 33

- - - - 13

M - $1--156$

$M-1--58$

M - I - - 79

S - - - 15

S - - - 15

S - . - D 21

S - - - 16

$M-1-/-169$ 
A4. List of Test Units by Parts for Version 3

FORTRAN TEST PROGRAMS SUMMARY INFORMATION FOR VERSION 3

\begin{tabular}{|c|c|c|c|c|c|c|}
\hline Part \# & $\begin{array}{c}\text { \# of } \\
\text { TEST UNITS }\end{array}$ & $\begin{array}{c}\# \text { of } \\
\text { SUBPROGRAMS }\end{array}$ & $\begin{array}{l}\text { INPUT } \\
\text { DATA* }\end{array}$ & $\begin{array}{l}\text { INTERMEDIATE } \\
\text { TAPE REOUIRED }\end{array}$ & $\begin{array}{l}\text { Pgs of } \\
\text { OUTPUT }\end{array}$ & $\#$ of CARDS \\
\hline 1 & 4 & & $x$ & & 16 & 1123 \\
\hline 2 & 2 & & & & 18 & 932 \\
\hline 3 & 10 & & & & 14 & 1076 \\
\hline 4 & 13 & & & & 14 & 1123 \\
\hline 5 & 11 & & & & 14 & 1153 \\
\hline 6 & 9 & & & & 11 & 912 \\
\hline 7 & 13 & & & & 18 & 997 \\
\hline 8 & 12 & & & & 13 & 951 \\
\hline 9 & 11 & & & & 12 & 971 \\
\hline 10 & 5 & 29 & & & 6 & 1031 \\
\hline 11 & 5 & 23 & & & 6 & 1090 \\
\hline 12 & 12 & 5 & & $\mathrm{x}$ & 13 & 1433 \\
\hline 13 & 5 & 2 & $\mathrm{x}$ & & 10 & 1190 \\
\hline 14 & 4 & 4 & & & 5 & 579 \\
\hline & 116 & 63 & & & 170 & 14561 \\
\hline
\end{tabular}

*Input data other than the 6 cards which are appended to each Part for user output documentation

input unit $\# 5=$ card reader

output unit \#6 = printer

intermediate unit \#9 
Segment \# and Name

000

001 SPECS

003 I)ATA1

1. 007 IODEF

2. 008 FMTRW*

3. 009 AFRMT *

010 DATA2*

011 AASGN*
Test

Special Documentation

Specifications needed for Part 1

Test Format of DATA Statement

I/O Unit Assignment Statements

Formatted Input/Output

A-Conversion

DATA Statement Test

Real and Integer Arithmetic Assignment Statements

Input 49 cards - prepare 3 cards (cards 1, 3, and 5) Unit \#5 Output - Print 16 pages

*Produce Output

Unit \#6

Note 1 The first 6 input cards (user prepared cards 1, 3, and 5) are associated with seg. 007 program element, however, performing tests under segment 008. See Data Preparation Section II-A-2. These 6 cards are part of the test for this part only. Inclusion of these cards in later part tests is for user output documentation on $1 \mathrm{y}$.

Note 240 input cards - for test of seg. 008

Note 303 input cards - for test of seg. 009 
Segment \# and Name

000

001 SPECS

1. 007 IODEF

013 DASGN*

015 CASGN*
Test

Special Documentation

Specifications needed for Part 2

I/O Unit Assignment Statements

Simple Double Precision Assignment Statements

Simple Complex Assignment Statements

Input 6 cards - prepare 3 cards $(1,3$, and 5) Unit \#5 Output Print 18 pages

*Produce Output

Note 1 Prepare replacement cards for cards 1,3 , and 5 as described in Data Preparation Section II-A-2. These cards in Part 2 are not part of the test, but are included for user output documentation only. 
Segment \# and Name

000

001 SPECS

1. 007 IODEF

016 LASGN *

017 INTRL*

020 UGOTO*

021 AGOTO*

022 CGOTO*

030 ARBAD*

031 ARFAD*

032 ARBSB*

033 ARFSB*

034 ARBAS*
Test

Special Documentation

Specifications needed for Part 3

I/O Unit Assignment Statements

Logical Assignment Statements

Arithmetic Assignment Statements

Unconditional GO TO Statements

GO TO Assignment Statements

Computed GO TO Statements

Basic Addition-Integer and Real

Double Precision Addition

Basic Subtraction-Integer and Real

Double Precision Subtraction

Basic Addition and Subtraction-Integer

and Real
Input 6 cards

Output Print 14 pages

*Produce Output
Unit \#5

Unit \#6

Note 1 Prepare replacement cards for cards 1, 3, and 5 as described in Data Preparation Section II-A-2. These cards in Part 3 are not part of the test, but are included for user output documentation only. 
Segment\# and Name

000

001 SPECS

1. 007 IODEF

035 ARFAS*

036 ARBMI *

037 ARBMR*

038 ARFMD*

039 ARBDV*

040 ARFDV*

041 ARBEX*

042 ARFEX*

043 ARBHI*

$050 \mathrm{SBB} 67 *$

051 SBB45*

052 SBB13*

$053 \mathrm{SBF} 17$ *

Input 6 cards

Output Print 14 pages

*Produce Output
Test

Special Documentation

Specifications needed for Part 4

I/O Unit Assignment Statements

Addition and Subtraction of Double Precision

Values

Multiplication of Integer Values

Multiplication of Real Values

Multiplication of Double Precision Values

Division of Integer and Real Values

Division of Double Precision Values

Exponentiation of Integer and Real Values

Exponentiation of Double Precision Values

Hierarchy of Operations and Parentheses

Subscripts of Integer and Real Arrays $v, k$

Subscripts of Integer and Real Arrays $v+k, v-k$

Subscripts of Integer and Real Arrays $c+v$, $c^{*} \mathrm{v}+\mathrm{k}, \mathrm{c}^{*} \mathrm{v}-\mathrm{k}$

Subscripts of Double Precision Arrays $v, k, c^{*} k$, $c^{*} v+k, c^{*} v-k, v+k, v-k$

Unit $\# 5$

Unit \#6

Note 1 Prepare replacement cards for cards 1, 3, and 5 as described in Data Preparation Section II-A-2. These cards in Part 4 are not part of the test, but are included for user output documentation only. 
Segment\# and Name

000

001 SPECS

1. 007 IODEF

054 SIMIF*

055 IFABS*

056 IFFLT*

057 IFFIX *

058 IFSGN*

059 IFDAB*

060 IFTRN*

061 IFMOD*

062 IFMAX *

063 IFMIN*

064 IFDSG*
Test

Special Documentation

Specification needed for Part 5

I/O Unit Assignment Statements

Arithmetic IF, logical IF followed by GO TO ABS, IABS (Absolute Value Functions)

FLOAT (Convert from Integer to Real)

IFIX (Convert from Real to Integer)

SIGN, ISIGN (Transfer of Sign)

DABS (Absolute Value)

AINT, INT, IDINT (Truncation)

AMOD, MOD (Remaindering)

AMAXO, AMAX1, MAX0, MAX1, DMAX1 (Choose Largest Value)

AMINo, AMIN1, MIN0, MIN1, DMIN1 (Choose

Smallest Value)

DSIGN (Transfer of Sign)

Input 6 cards

Output Print 14 pages

Unit \#5

*Produce Output

Unit \#6

Note 1 Prepare replacement cards for cards 1, 3, and 5 as described in Data Preparation Section II-A-2. These cards in Part 5 are not part of the test, but are included for user output documentation only. 


\section{VERSION 3 PART 6 MAIN PROGRAM \\ (Intrinsic Functions)}

Segment \# and Name

$\begin{array}{ll}000 & \\ 001 & \text { SPECS } \\ 007 & \text { IODEF } \\ 065 & \text { IFDIM* } \\ 066 & \text { IFSGL* } \\ 067 & \text { IFREL* } \\ 068 & \text { IFIMG* } \\ 069 & \text { IFDBL* } \\ 070 & \text { IFCPX* } \\ 071 & \text { IFCJG* } \\ 072 & \text { IFBMS* } \\ 073 & \text { IFFMS* }\end{array}$

Input 6 cards

Output Print 11 pages

*Produces Output
Test

Special Documentation

Specifications needed for Part 6

I/O Unit Assignment Statements

DIM, IDIM (Positive Differences)

SNGL (Obtain most Significant part)

REAL (Obtain Real Part of Complex Argument)

AIMAG (Obtain Imaginary Part of Complex Number)

DBLE (Express Real Argument in D.P. Form)

CMPLX (Express Two Real Arg. in Complex Form)

CONJG (Obtain Conjugate of a Complex Number)

All Intrinsic Functions-Real and Integer

A11 Intrinsic Functions-Real, Integer and D.P

Note 1 Prepare replacement cards for cards 1, 3, and 5 as described in Data Preparation Section II-A-2. These cards in Part 6 are not part of the test, but are included for user output documentation only. 
Segment \# and Name

000

001 SPECS

1. 007 IODEF

080 EXPON*

081 DEXPO*

082 CEXPO*

083 LOGTM*

084 DPLOG*

085 CXLOG*

086 COLOG*

087 DCLOG*

088 SINUS*

089 DPSIN*

090 CSICO*

091 COSNS*

092 DPCOS*
Test

Special Documentation

Specifications needed for Part 7

I/O Unit Assignment Statements

Basic External Function - EXP

Basic External Function - DEXP

Basic External Function - CEXP

Basic External Function - ALOG

Basic External Function - DLOG

Basic External Function - CLOG

Basic External Function - ALOG10

Basic External Function - DLOG10

Basic External Function - SIN

Basic External Function - DSIN

Basic External Function .. CSIN and CCSIN

Basic External Function - COS

Basic External Function - DCOS

Input 6 cards

Unit \#5

Output Print 18 pages

Unit \#6

*Produces Output

Note 1 Prepare replacement cards for cards 1, 3, and 5 as described in Data Preparation Section II-A-2. These cards in Part 7 are not part of the test, but are included for user output documentation only. 


\section{VERSION 3 PART 8 MAIN PROGRAM}

Segment \# and Name

000

001 SPECS

005 BSFDF

006 FSFDF

1. 007 IODEF

094 TANGH *

095 SQROT *

096 DSQRO*

097 CSQRO*

098 ARCTG*

099 DACTG*

100 ACTG2*

101 DATN2*

102 DMODA*

103 CABSA*

110 BSFTS*

111 FSFTS *
Test

Special Documentation

Specifications needed for Part 8

Statement Function Definitions for Segment 110

Statement Function Definitions for Segment 111

I/O Unit Assignment Statements

Basic External Function - TANH

Basic External Function - SQRT

Basic External Function - DSQRT

Basic Extermal Function - CSQRT

Basic External Function - ATAN

Basic External Function - DATAN

Basic External Function - ATAN2

Basic External Function - DATAN2

Basic Extermal Function - DMOD

Basic External Function - CABS

Statement Functions (Real and Integer)

Statement Functions (D.P., Logical and Complex)

Input 6 cards

Output Print 13 pages

Unit \#5

*Produce Output

Unit \#6

Note 1 Prepare replacement cards for cards 1, 3, and 5 as described in Data Preparation Section II-A-2. These cards in Part 8 are not part of the test, but are included for user output documentation only. 


\section{VERSION 3 PART 9 MAIN PROGRAM}

Segment \# and Name

000

001 SPECS

1. 007 IODEF

140 CPXAD*

141 CPXMU *

142 CPXDV*

143 CPXEX*

144 CPXOP*

145 CREAD*

146 CREMU*

147 CREDV*

148 CREOP *

149 MISC 3*

150 MISC4*
Test

Special Documentation

Specifications needed by Part 9

I/O Unit Assignment Statements

Addition and Subtraction of Complex Numbers

Multiplication of Complex Numbers

Division of Complex Numbers

Exponentiation of Complex Numbers

Arithmetic Operations on Complex Numbers

Addition, Subtraction of Complex, Real Numbers

Multiplication of Complex by Real Numbers

Division of Real, Complex by Complex, Real Numbers

Combined Operations on Complex and Real Numbers Blanks in, and Continuation of Statements to Maximum Lines

Special Characters for Continuation Lines

Input 6 cards

Output Print 12 pages

Unit \#5

*Produce Output

Unit \#6

Note 1 Prepare replacement cards for cards 1, 3, and 5 as described in Data Preparation Section II-A-2. These cards in Part 9 are not part of the test, but are included for user output documentation only. 
Segment \# and Name

000

001 SPECS

1. 007 IODEF

160 BRFCP*

161 BIFCP*

162 FRFCP*

163 FIFCP*

$164 \mathrm{CFCCP}^{*}$

Subprograms

400 AFS

420 BFS

430 CFS

440 DFS

450 EFS

460 FFS

Subprograms

401 IAFI

421 IBFI

431 ICFI

441 IDFI

451 IEFI

461 IFFI

Subprograms

402 GFS

422 HFS

432 IRFS

442 JRFS

452 RFS

Subprograms

403 IFI

423 JFI

433 KF I

443 LFI

453 MFI

Subprograms

404 AFC

414 BFC

424 CFC

434 DFC

444 EFC

454 FFC

464 HFC
Test

Special Documentation

Specifications needed for Part 10

I/O Unit Assignment Statements

External Function Test - Real

External Function Test - Integer

External Function Test - Real - All

Argument Types

External Function Test - Integer - A11

Argument Types

External Function Test - Complex

Used with Segment 160 - Real Function

Real Argument

Real Arguments

Integer Argument

Integer Arguments

Array Name

Integer and Real Arguments

Used with Segment 161 - Integer Function

Real Argument

Real Arguments

Integer Argument

Integer Arguments

Array Name

Integer and Real Arguments

Used with Segment 162 - Real Function

Double Precision Arguments

Complex Arguments

Logical Argument

Argument - External Procedure

Different Types of Arguments

Used with Segment 163 - Integer Function Double Precision Arguments

Complex Arguments

Logical Arguments

Argument - External Procedure

Different Types of Arguments

Used with Segment 164 - Complex Function

Real Argument

Integer Argument

Array Name

Double Precision Argument

Complex Argument

Logical Arguments

Different Types of Arguments 
Input 6 cards

Unit \#5

Output Print 6 pages

Unit \#6

*Produces Output

Note 1 Prepare replacement cards for cards 1, 3, and 5 as described in Data Preparation Section II-A-2. These cards in Part 10 are not part of the test, but are included for user output documentation only. 
Segment \# and Name

$$
\begin{array}{ll}
000 & \\
001 & \text { SPECS } \\
007 & \text { IODEF } \\
165 & \text { DPFCP* } \\
166 & \text { BFCCP* } \\
167 & \text { SBRTN * } \\
168 & \text { FSBRT * } \\
169 & \text { BLKDT * }
\end{array}
$$

Subprograms

405 AFD

$415 \quad B F D$

425 CFD

435 DFD

445 EFD

455 FFD

465 GFD

475 HFD

Subprograms

$\begin{array}{ll}406 & \text { AFB } \\ 416 & \text { BFB } \\ 426 & \text { CFB } \\ 436 & \text { DFB } \\ 446 & \text { EFB } \\ 456 & \text { FFB } \\ 466 & \text { GFB } \\ 476 & \text { HFB }\end{array}$

Subprograms

407 AAQ

$417 \quad \mathrm{ABQ}$

427 ACQ

Subprograms

408 ADQ

418 AEQ

428 AFQ

Subprogram

409 BLOKD
Test

Special Documentation

Specifications needed for Part 11

I/O Unit Assignment Statements

External Function Test - Double Precision

External Function Test - Logical

Subroutine Subprogram Test

Subroutine Subprogram Test

Block Data Subprogram Test

Used with Segment 165 - D.P. Function

Real Argument

Integer Argument

Doub le Precision Argument

Complex Argument

Logical Argument

Argument - External Procedure

Array Name

Different Types of Arguments

Used with Segment 166 - Logical Function

Real Arguments

Integer Arguments

Double Precision Argument

Logical Argument

Complex Argument

Array Name

Argument - External Procedure

Different Types of Arguments

Used with Segment 167 - Subroutine Subprogram Integer and Real variables and Array Elements Array Elements

No Argument List - Arguments passed thru Common

Used with Segment 168 - Subroutine Subprogram Different Types of Arguments

Array Names and Integer Arguments

No Argument List - Arguments Passed through Common

Used with Segment 169 - Block Data Test Block Data Subprogram
Input 6 cards

Output Print 6 pages

*Produces Output
Unit \#5

Unit \#6

Note 1 Prepare replacement cards for cards 1, 3, and 5 as described in Data Preparation II-A-2. These cards in Part 11 are not part of the list, but are included for user output documentation only. 
Segment \# and Name

000

001 SPECS

005 BSFDF

1. 007 IODEF

179 BLKDA*

180 UNFRW*

182 BACUP*

190 DOTRM*

191 DOLMT*

192 DONSC*

193 DONSI*

194 DONSX *

195 DONML *

196 DONIO*

197 MORDO*

200 SUBR $1 *$

Subprogram

410 SUBRQ

Subprogram

$412 \mathrm{MDQ}$

Subprograms

419 BLAKD

429 BLBKD

439 BLCKD
Test

Special Documentation

Specifications needed for Part 12

Statement Function Definitions used with

Segment 197

I/O Unit Assignment Statements

Block Data Test

Unformatted Read and Write

Backspace Tape

DoLoops - Terminal Statements

DoLoops - Parameters integer variable names

DoLoops - Completely Nested Nest

DoLoops - Incomplete Looping

DoLoops - Extended Range

DoLoops - Nested Nests

DoLoops - I/O Terminal Statements

DoLoops - I/O, Intrinsic Functions, CALL included Subroutine Called

Used with Segment 200

Used with Segment 197

Subroutine Subprogram

Used with Segment 179 - Block Data Test

Block Data Subprogram

Block Data Subprogram

Block Data Subprogram
Input 6 cards

Output Print 13 pages

Intermediate tape

*Produces Output
Unit \#5

Unit \#6

Unit \#9

Note 1 Prepare replacement cards for cards 1, 3, and 5 as described in Data Preparation Section II-A-2. These cards in Part 12 are not part of the test, but are included for user documentation only. 
Segment \# and Name

000

001 SPECS

1. 007 IODEF

300 LOGIF*

301 BARIF*

302 FARIF*

2. 310 IOFMT *

3. 312 RDFMT*

Subprogram

411 SMCQ

Subprogram

462 FMTQ
Test

Special Documentation

Specifications needed for Part 13

I/O Unit Assignment Statements

Logical If Statements

Arithmetic If Statements (Integer and Real

Expressions)

Arithmetic If Statements

Formatted Read and Write, additional properties of

Formats in Arrays

Used with Segment 300

Sub routine

Used with Segment 312

Sub routine

Input 57 cards - prepare 3 cards (cards 1, 3, and 5) Unit \#5 Output Print 10 pages

*Produce Output

Unit \#6

Note 1 The first 6 input cards in Part 13 (user prepared cards 1, 3, and 5) are not part of the test, but are included for output documentation only. See Data Preparation Section II-A-2.

Note 238 input cards - for test of seg. 310

Note 313 input cards - for test of seg. 312 
Segment \# and Name

000

001 SPECS

1. 007 IODEF

350 MISC5*

351 FUNMX*

2. 352 NAMES*

360 SPEC2*

Subprogram

413 MAQQ

$463 \mathrm{MBQQ}$

473 AMQQ

483 BMQQ
Test

Special Documentation

Specifications needed for Part 14

I/O Unit Assignment Statements

Specifications for Program Form (Test)

Basic External Functions using Trig Formula

Names resembling FORTRAN Verbs and Function Names

Common, Dimension and Equivalence

Used with Segment 352

Sub routine Called from NAMES

Subroutine Called from NAMES

Subroutine Called from NAMES

Sub routine Called from NAMES

Input 6 cards

Output Print 5 pages

Unit \#5

*Produce Output

Unit \#6

Note 1 Prepare replacement cards for cards 1, 3, and 5 as described in Data Preparation Section II-A-2. These cards in Part 14 are not part of the test, but are included for user output documentation only.

Note 2 This test may cause difficulties in some compilers and may have to be run independently of other tests. 
The following procedures assume the NBS FORTRAN Test Programs, Version 3, are being used with the programs on interpreted punch cards rather than from magnetic tape.

B1. Deleting a Test Unit

If any part fails to complete the execution of all the test units within the part, the printed results will probably contain at least the heading of the segment which failed and no test unit beyond this point will have been completed. If the test which failed is not the last one in a part, remove the cards which define the particular test and proceed with the test with this test unit deleted. Parts 10-14 contain subprograms which may have to be removed if a test failure occurs in these parts.

B2. Creating a Single Test from a Deleted Unit

Each test unit may be run independently by either of the following two methods.

a) Append the FORTRAN specification statements which appear at the beginning of the appropriate part to the beginning of the test unit to be retested. Include the one (or two) Input-Output assignment statements appearing as a segment 007 card within the first test unit of the part. This statement should be inserted into the test unit to be retested as the first executable statement, which can be located by the corresponding statement appearing in the test unit as a comment card with $\mathrm{C}=$ in the first two locations. Supply a STOP statement and an END card at the end of the test unit main program. Although specifications not used within this test unit may cause diagnostics to appear as warning messages to non referenced data names, the program test unit is still a standard conforming FORTRAN program.

b) Isolate the test unit. Check the initial comment lines in the listing related to the part containing the test unit. If any additional segments are required to run this test unit, they are identified. For every card in the isolated test unit containing a "C $="$ in columns 1 and 2, duplicate the cards with the "C $="$ changed to blanks and omit punching columns 73-80 of the card. Return the comment cards to their original locations in the deck with the corresponding FORTRAN created statements immediately below the comment card. These "C = " comment indicators have been appended to what otherwise would be FORTRAN specification statements, I/O assignment, STOP statements and END lines. Omitting the duplication of columns 73-80 will make it easier to remove these cards when the test unit is returned to its original state for reinsertion into its appropriate location in the test part.

Test units numbered 008, 009, 310 and 312 are the only units which require input data cards to perform the test. 

C1. Interpreting the Test Results

An attempt was made in the design of these tests to produce test results which were as much as possible self explanatory. Wherever a value of zero could not be created by the addition or subtraction of a constant from the calculated result, a Hollerith equivalent precedes the test result for comparison purposes.

The effects of conversion, precision, and exponent range are minimized by the use of values which are integer and fractional powers of 2 where the choice of values affected the test results. Other results are truncated to minimize the effects of differences in systems precision.

The ASA FORTRAN Standard does not prescribe the external output form for a Rea1 or Double Precision zero. Systems implementors have used a wide variety of forms with and without + or - signs. Some implementations

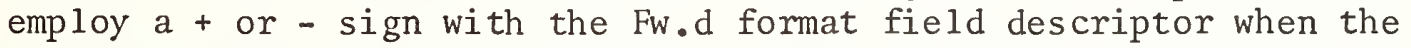
printed value is zero to denote a truncated value whose sign corresponds. to the sign of the original value. Expect variations in the form of zero.

The ASA FORTRAN Standard permits the implementor a choice of form for output.

A positive sign is not required.

A leading zero before the decimal point for $E$ and $D$ conversion is not required.

The following exponent forms are equivalent and correct for E conversion:

$\mathrm{E}+02$

E 02

$+002$

The following exponent forms are equivalent and correct for $D$ conversion:

$$
\begin{aligned}
& \mathrm{D}+13 \\
& \mathrm{D} 13 \\
& \mathrm{E}+13 \\
& \mathrm{E} 13 \\
& +013
\end{aligned}
$$

In the test program results where $D$ conversion is used on output and the expected output value is stipulated to be zero, any value containing a negative exponent of D-13 or mathematically less is considered to be zero. The test units containing the Basic External Functions do not attempt to test either the range or the precision of these functions. A selected set of arguments to these functions is presented for the purpose of determining only whether the function name referenced is actually the function delivered. 
The following limits have been set for constants in this test program set:

Integer 5 digits

Rea1 7 digits

Double Precision 14 digits

Complex 7 digits (each half)

Hollerith 2 characters except in segment 009 which tests A-conversion for 1 to 4 characters and 26 characters for the truncation test.

Where the precision of a FORTRAN processor for a REAL datum approaches the limit established for a Double Precision datum (14 digits) it will be necessary to increase the number of digits printed out for the test of the intrinsic function SNGL (test unit 066) to obtain meaningful test results.

\section{C2. Test Results}

The following test results were obtained from actual execution of Version 1 or Version 3 of the NBS FORTRAN Test Program set. These results are a composite set of output pages derived from five FORTRAN processors showing various forms for zero and differences in exponent form. 


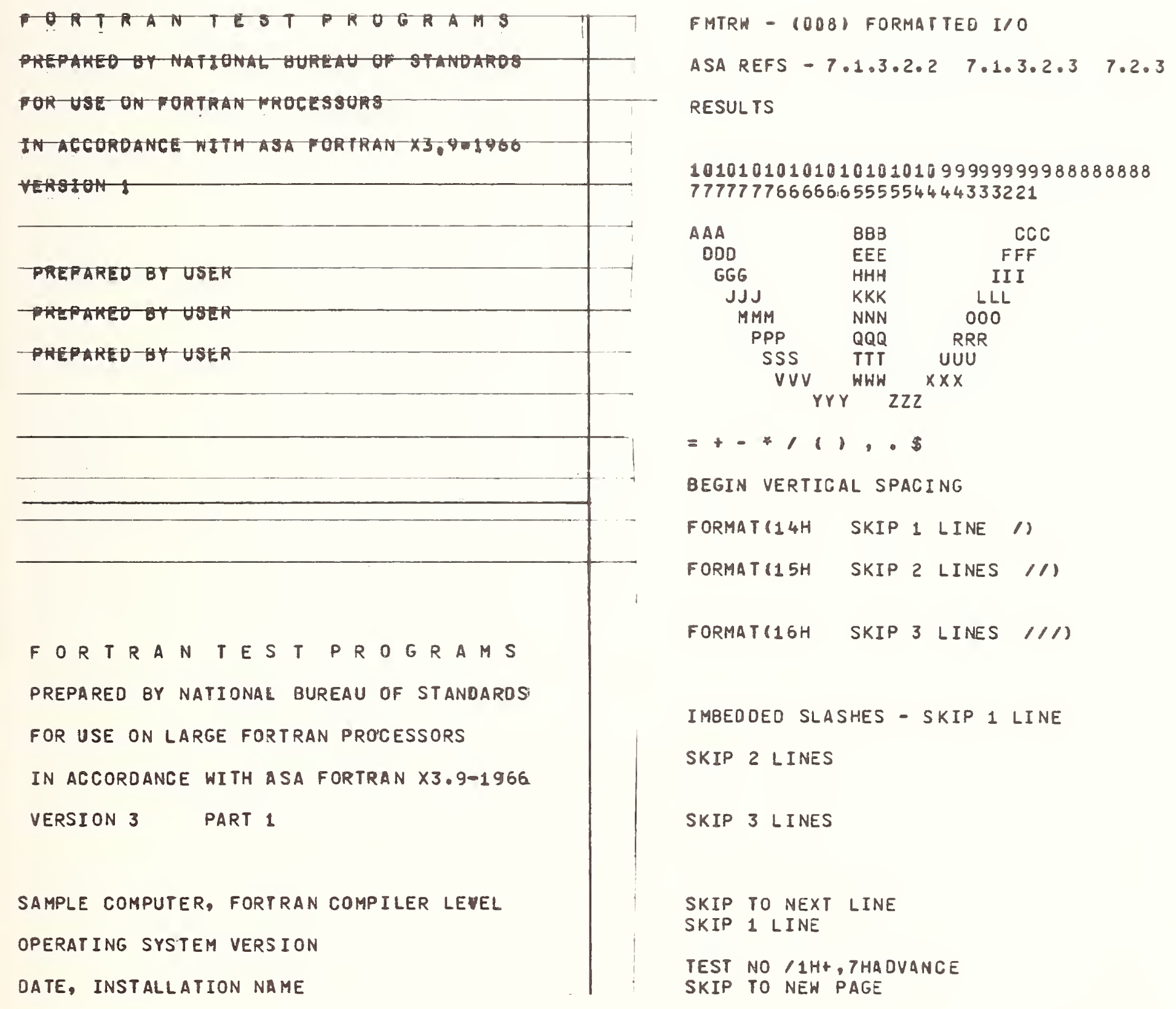


$0.123456789010+10$

$.123456789010+10$

$0.987654321098760-01$ $.987654321098760-01$ $.987654321098760-11$ - $987654321098760-01$

$-0.5555555420+03$

$-.5555555420+03$

$-.5555555420+03$

BEGIN L CONVERSION TEST

LINES BELOW SHOULD BE IDENTICAL

$$
\begin{array}{llllll}
T & F & F & T & T & F T F \\
T & F & F & T & T & F T F
\end{array}
$$

TEST UNSUBSGRIPTED ARRAY NAMES IN I/O LISTS. EACH GROUP OF LINES SHOULD BE IDENTICAL.

$9 \cdot 91 \cdot 19 \cdot 92 \cdot 29 \cdot 93 \cdot 39 \cdot 94 \cdot 4$

9.91 .19 .92 .29 .93 .39 .94 .4

$9.91 \cdot 19.92 \cdot 29 \cdot 93 \cdot 39 \cdot 94.4$

$-9.9-9.9-9.9-9.9$

$-9.9-9.9-9.9-9.9$

$-0.990+01-0.990+01-0.990+01-0.990+01$ $-.990+01-.990+01-.990+01-.990+01$

$-.990+01-.990+01-.990+01-.990+01$

9999999999

9999999999

$0.990+010.990+010.990+010.990+01$ $.990+01.990+01.990+01.990+01$

$\begin{array}{rrrrrrrrr}0.9 & 0.9 & 0.9 & 0.9 & 0.9 & 0.9 & 0.9 & 0.9 & 0.9 \\ .9 & .9 & .9 & .9 & .9 & .9 & .9 & .9 & .9 \\ .9 & .9 & .9 & .9 & .9 & .9 & .9 & .9 & .9 \\ .9 & .9 & .9 & .9 & .9 & .9 & .9 & .9 & .9\end{array}$

TF

TF

TFTFTFTF

TFTFTFTF

99999999

99999999

$$
\begin{array}{r}
0.990+01 \\
.99+001 \\
.99+001 \\
.99+001 \\
.99+001 \\
.99+001
\end{array}
$$

9.95 .59 .96 .69 .97 .79 .98 .8

9.95 .59 .96 .69 .97 .79 .98 .8

9.95 .59 .96 .69 .97 .79 .98 .8

9.95 .59 .96 .69 .97 .79 .98 .8

9999999999999999

9999999999999999

TFF T

TFF T

9.99 .99 .99 .99 .9

9.99 .99 .99 .99 .9

LEADING BLANK INSERTION TEST

EACH PAIR OF LINES SHOULD BE IDENTICAL

8

8

22

22

22

22

22

22

22

22

7. 7

$7 \cdot 7$

$8 \cdot 88$

$8.8 B$ 


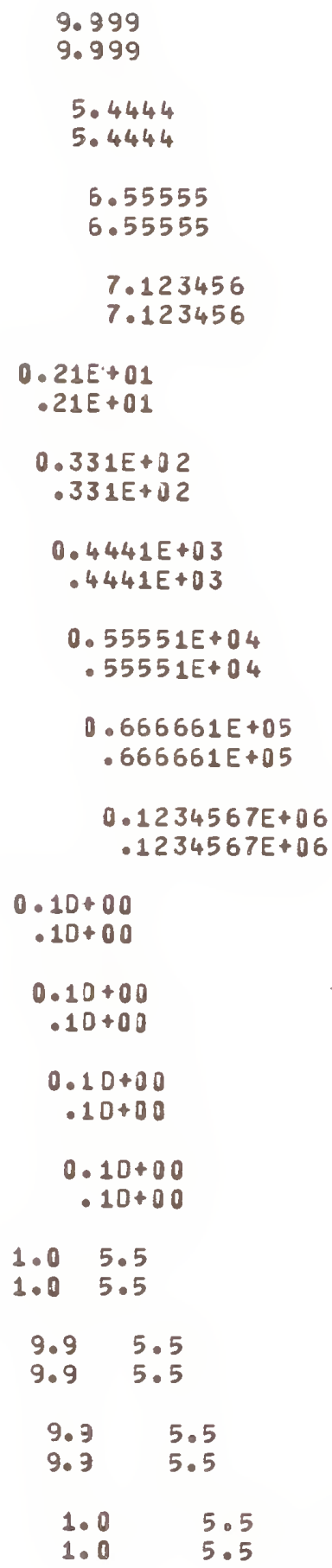

TEST LOGICAL FIELOS WITH BLANKS LINES BELOW SHOULO BE IOENTICAL

$$
\begin{array}{llll}
T & F & T & F \\
T & F & T & F
\end{array}
$$

TEST $D=0, W=0+1 \quad$ (PAIRS OF LINES BELOW SHOULO BE IDENTICAL)

\section{4.}

4444.

.55555

.55555

BEGIN G CONVERSION

EACH PAIR OF LINES SHOULD BE IDENTICAL

. 1235E+05 1235. 123.5

. $1235 E+05 \quad 1235$. 123.5

$12.35 \quad 1.235 \quad .1235$

$12 \cdot 35$

1.235

.1235 
SCALE FACTOR ON READ

IN ORDER OF FORMAT OCCURRENCE

$\begin{array}{lrrr}\text { CARD } & 9876.54 & 98.7654 E 2 & 9876.54 \\ \text { DESC } & 2 P F 8.3 & -2 P E 9.4 & \text { F9.4 } \\ \text { TO BE } & 98.7654 & .3877 E+04 & 987654.00 \\ \text { IS } & 98.7654 & .9877 E+04 & 987654.00 \\ \text { CARD } & 987.654 & 8647860-4 & 86.4786 E 2 \\ \text { DESC } & 0 P G 9.4 & 09.4 & -2 P E 9.4 \\ \text { TO BE } & 987.654 & .85480-02 & .8648 E+04 \\ \text { IS } & 987.654 & .86480-02 & .8648 E+04 \\ & & & \\ \text { CARD } & 86.4786 & 8557.8700 & 9876.54 \\ \text { DESC } & \text { F9.4 } & 09.4 & 2 P G 9.4 \\ \text { TO BE } & 8647.860 & .86580+04 & 98.77 \\ \text { IS } & 8647.860 & .85580+04 & 98.77\end{array}$

SCALE FACTOR ON WRITE

IN OROER OF FORMAT OCCURRENCE

$\begin{array}{lrrr}\text { CARD } & 9.87655 & 98.7654 E 2 & 9876.54 \\ \text { DESC } & 2 P F 12.2 & -2 P E 12.4 & \text { F12.4 } \\ \text { TO BE } & 987.65 & .0099 E+06 & 98.76154 \\ \text { IS } & 987.66 & .0099 E+06 & 98.7654 \\ & & & \\ \text { CARD } & 987.654 & 8647860-3 & 86.4786 E 2 \\ \text { DESC } & 1 P G 12.2 & 012.4 & -2 P E 12.4 \\ \text { TO BE' } & 9.88 E+02 & 8.64790+02 & .0086 E+06 \\ \text { IS } & 9.88 E+02 & 8.64790+02 & .0086 E+06 \\ & & & \\ \text { CARD } & 86.4786 & 8657.8600 & 9876.54 \\ \text { DESC } & 2 P F 12.2 & 1 P 012.4 & 2 P 616.4 \\ \text { TO BE } & 8647.86 & 8.65790+03 & 9877 . \\ \text { IS } & 8647.86 & 8.65790+03 & 987.0\end{array}$

THE LAST THO LINES OF EACH

SET SHOULD BE THE SAME

FORMAT RESCAN - THE SECONO GROUP OF

EACH SET SHOULO AGREE WITH THE FIRST

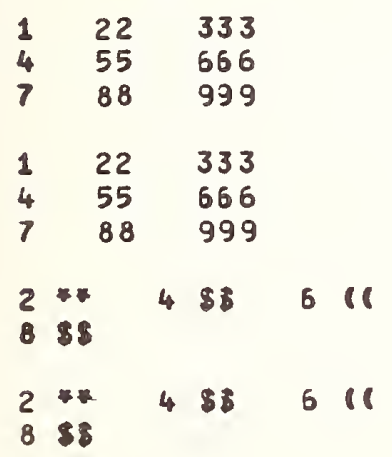

AFRMT - (009) A-CONVERSION

ASA REF 7.2 .3 .8
EACH PAIR OF LINES SHOULD BE IDENTICAL
FOR COMPUTERS STORING FOUR
OR MORE CHARACTERS PER WORD

ABCDEFGHIJKLMNOPQRSTUVWX YZ ABCDEF GHIJKLMINOPQRSTUVWX YZ

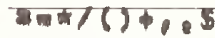

$=n /()+0$

$0123456789+A B 2 S(C)$

$01234567896402 S(C)$

TEST A CONVERSION - ADDING BLANKS

EACH PAIR OF LINES SHOULD BE IDENTIEAL

A

$A$

th

t

Q

0

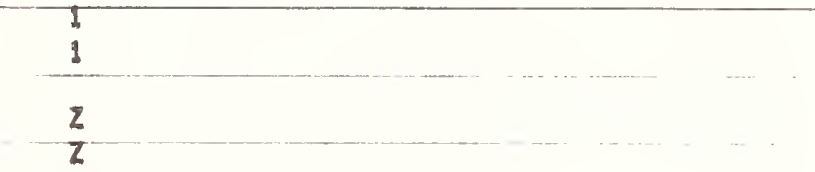

TEST AFIELD TRUNCATION

ZND LINE SHOULD PARTIALLY MATCH IST

ABCOEFGHIJKLMNOPQRSTUVWXYZ

VWXYZ 


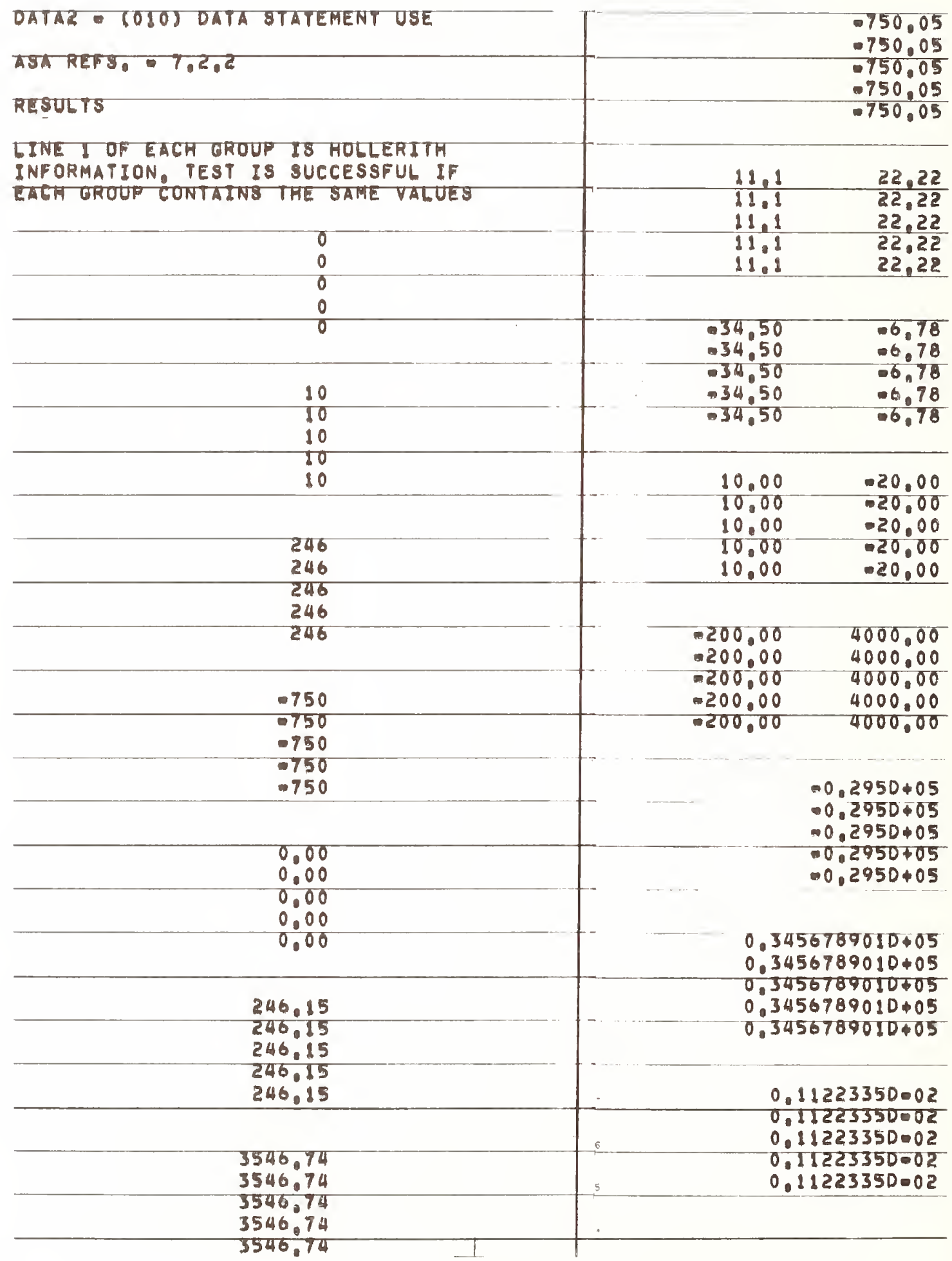

II- C - 8 


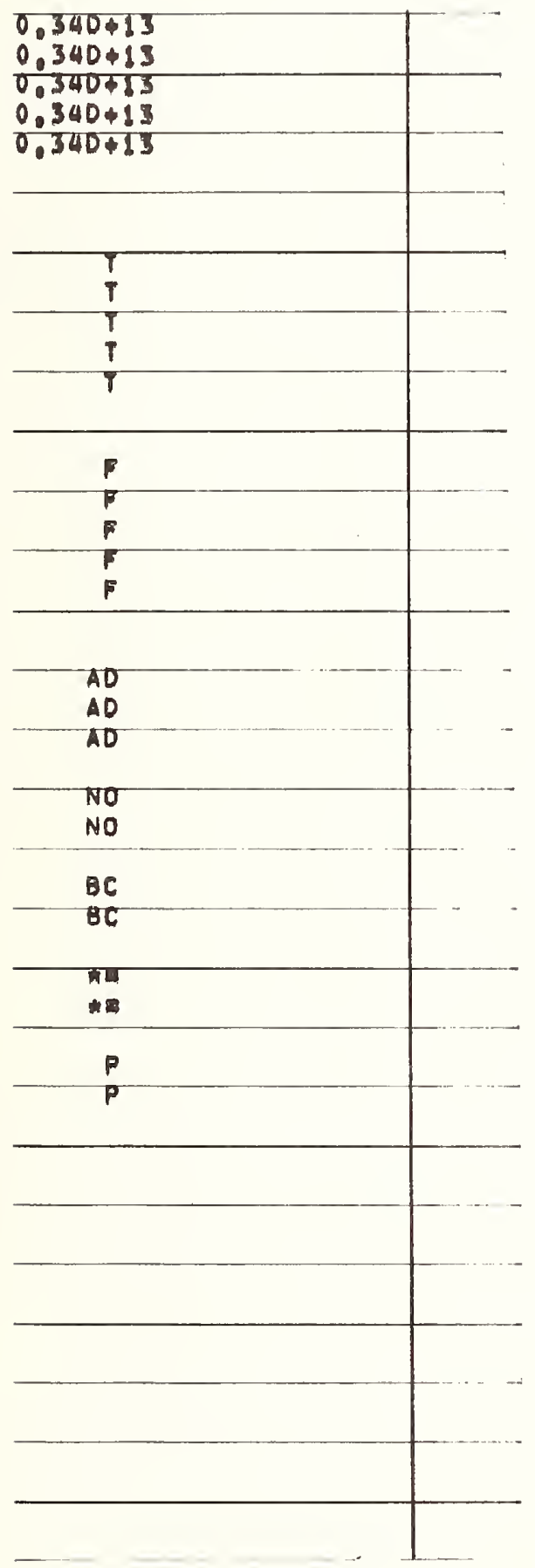

AASGN - 10111 SIMPLE REAL AND INTEGER ARITHMFTIC ASSIGNMENT STATEMENTS

ASA REF - $7.1 \cdot 1$

LINE I OF EACH PAIR IS HOLLERITH INFORMATION

INTEGER RESULTS

$\begin{array}{rrr}1 & 12345 & 0 \\ 1 & 12345 & 0 \\ 2 & -3 & -8765 \\ 2 & -3 & -8765 \\ 6912 & 0 & -23 \\ 6912 & 0 & -23 \\ 4444 & 4321 & 45 \\ 4444 & 4321 & 45 \\ 2468 & -3123 & 0 \\ 2468 & -3123 & 0\end{array}$

REAL RESULTS

\begin{tabular}{|c|c|c|}
\hline 1.0 & 358.6724 & $-2 \cdot 0$ \\
\hline 1.0 & 358.6724 & $-2 \cdot 0$ \\
\hline 3.0 & -2714.250 & 29.30542 \\
\hline 3.0 & -2714.250 & 29.30542 \\
\hline 86.27 & 1034.2 & 0.0 \\
\hline 86.27 & 1034.2 & .0 \\
\hline 0.0 & 345.678 & $-2 \cdot 5$ \\
\hline .0 & 345.678 & -2.5 \\
\hline-5.66 & 1.111111 & 1.0 \\
\hline-5.66 & 1.111111 & 1.0 \\
\hline$-2 \cdot 0$ & 3.0 & 4.0 \\
\hline$-2 \cdot 0$ & 3.0 & 4.0 \\
\hline $5 \cdot 0$ & $-6 \cdot 0$ & 0.0 \\
\hline 5.0 & -6.0 & .0 \\
\hline D. 23 & -0.716 & -0.7 \\
\hline .23 &. .716 & -.7 \\
\hline 0.81 & 0.9 & \\
\hline .81 & .9 & \\
\hline
\end{tabular}




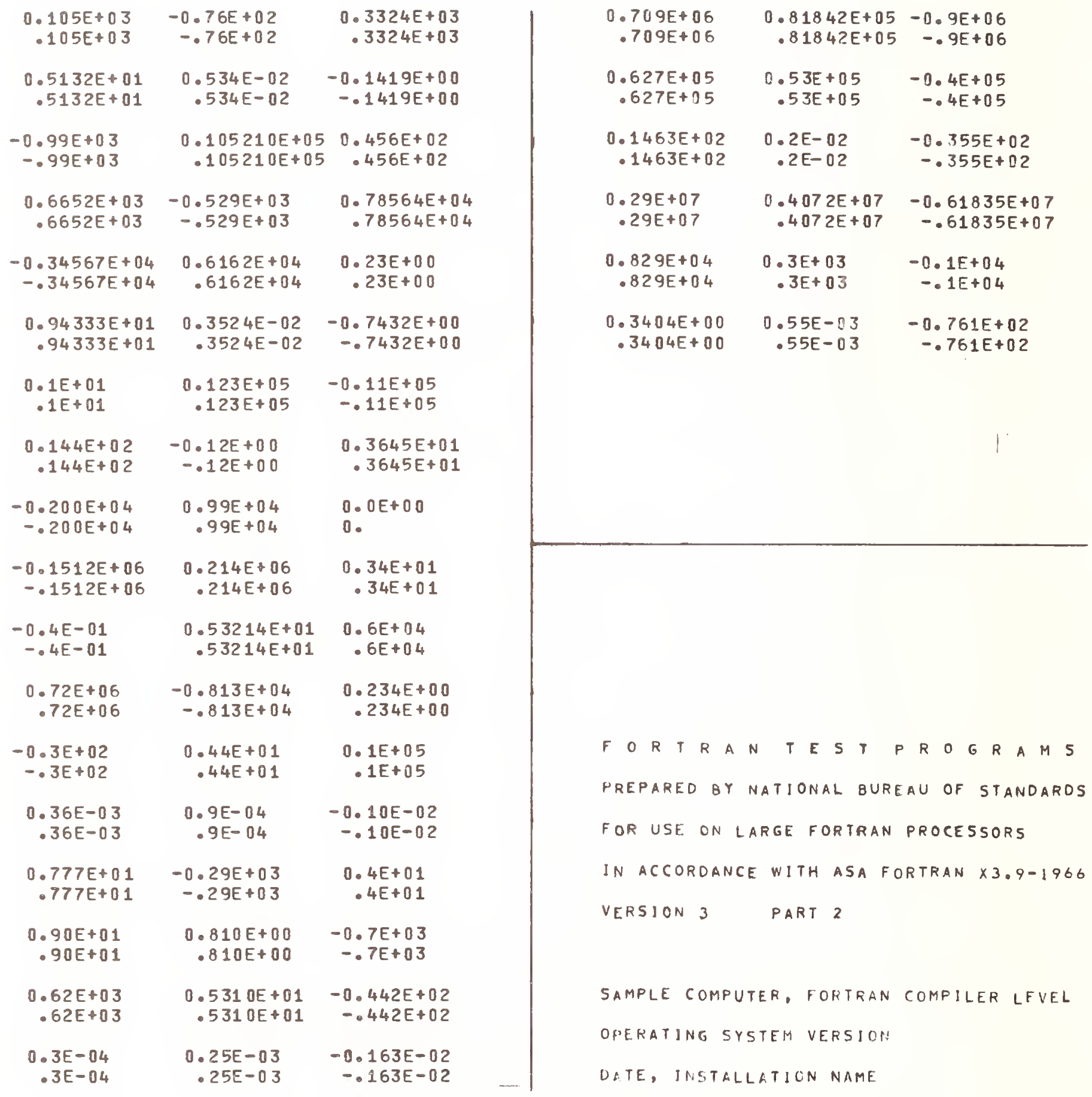




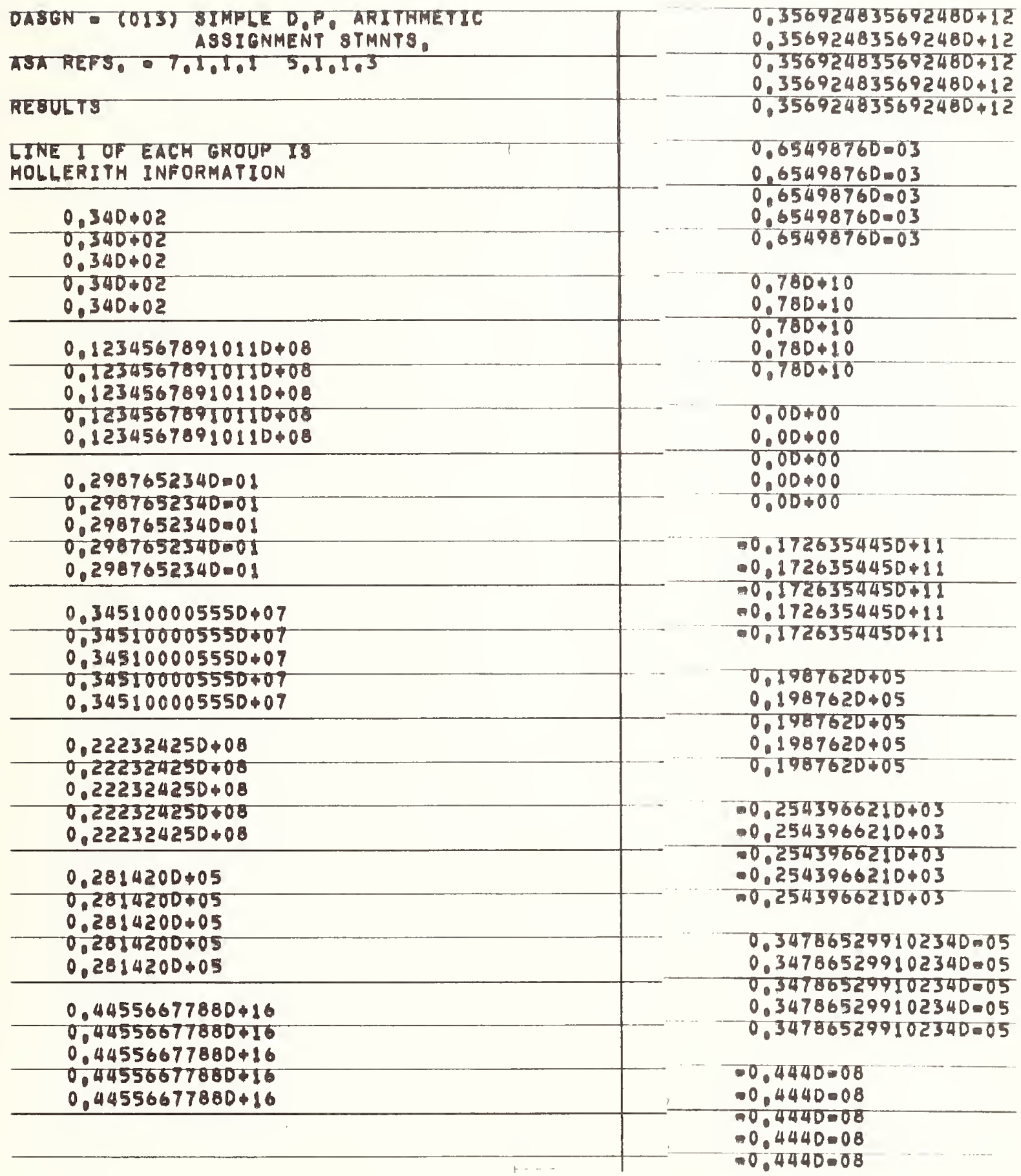




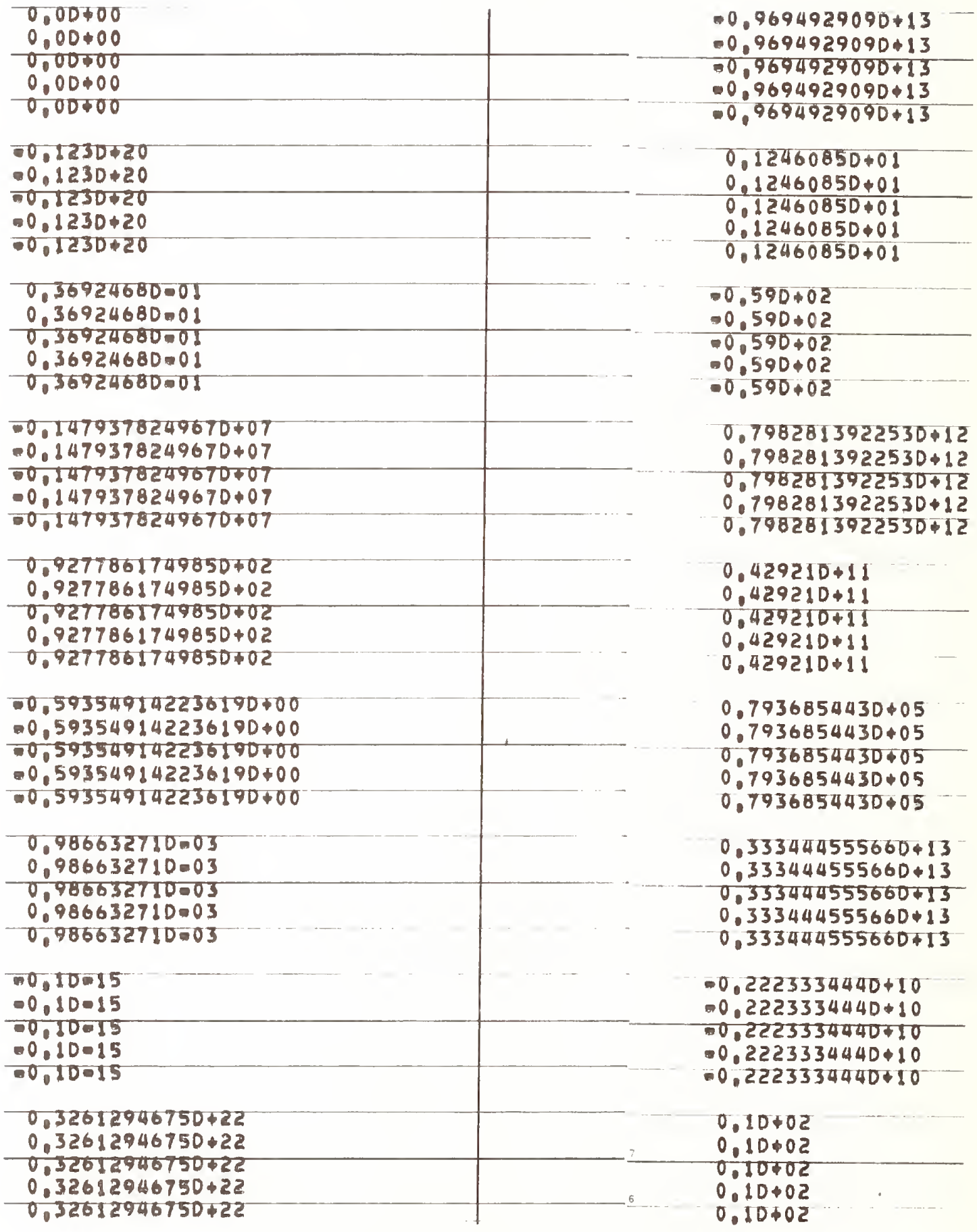




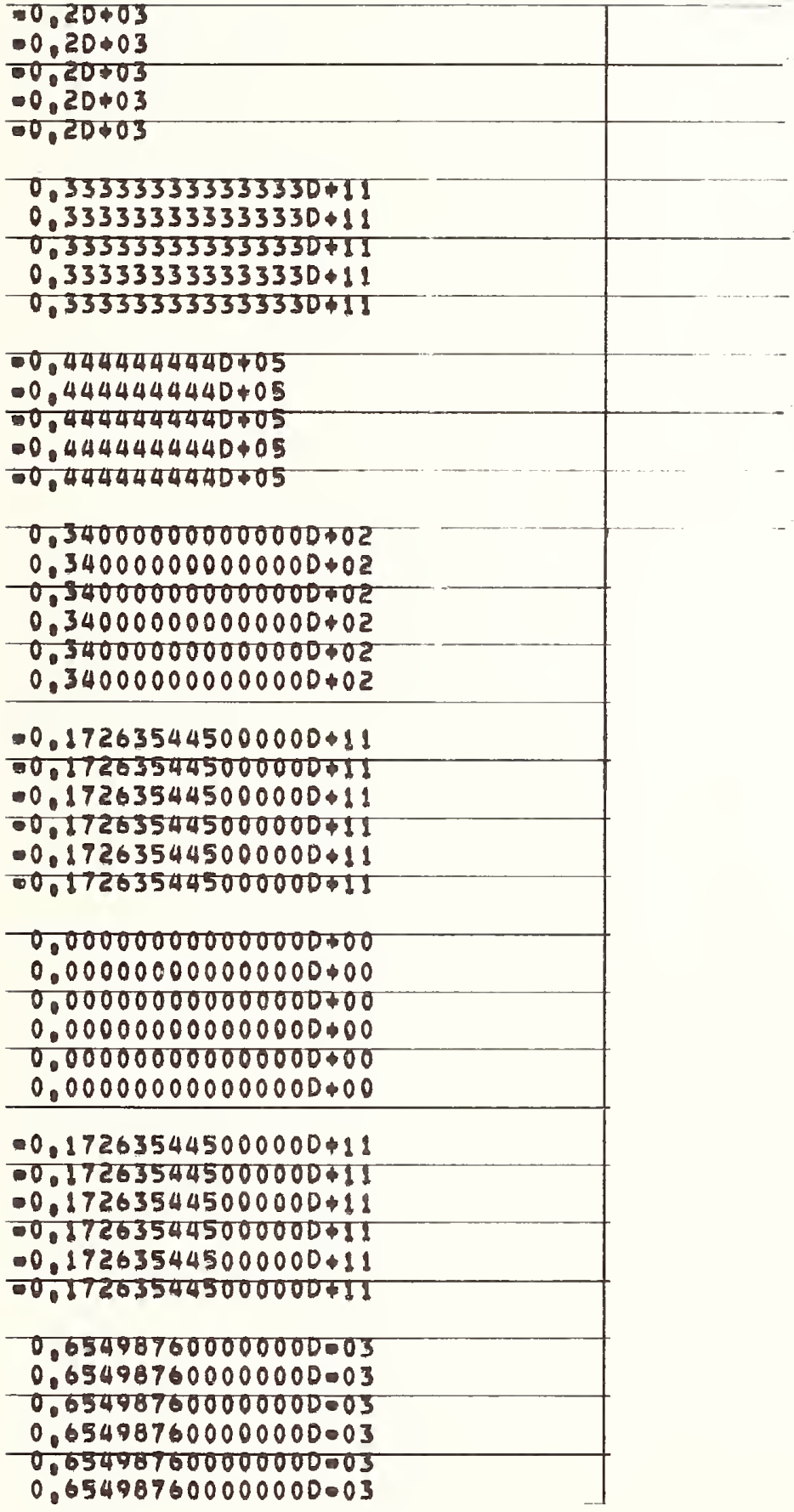


EACH GROUP SHOULD BE IDENTICAL EXCEPT FOK THE SIGNS OF THE FIRST TWO LINES

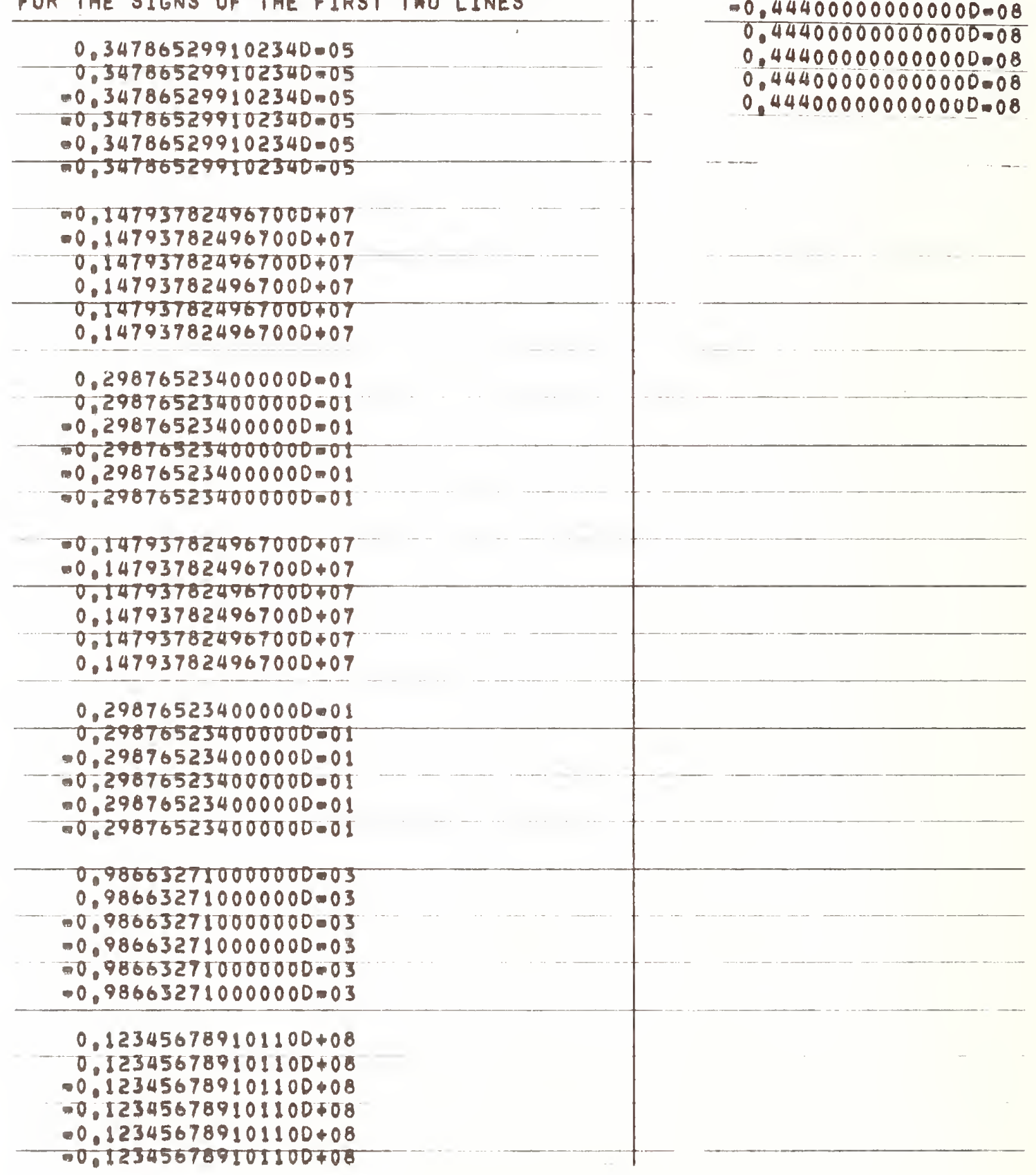


CASGN - (015) COMPLEX ASSIGNMENT STATEMENTS

ASA REFS. -5.1 .1 .47 .1 .1 .1$

RESULTS

LINE 1 OF EACH GROUP IS HOLLERITH INFORMATION

VALUES IN A GROUP SHOULD BE THE SAME

$$
\begin{array}{r}
0.222 E+02 \\
.222 E+02 \\
.222 E+02 \\
.222 E+02 \\
.222 E+02
\end{array}
$$

$$
\begin{array}{r}
0.3956 E+03 \\
.3956 E+03 \\
.3956 E+03 \\
.3956 E+03 \\
.3956 E+03
\end{array}
$$

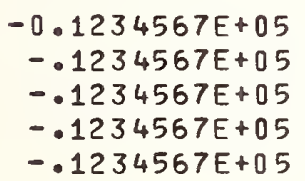

$$
\begin{array}{r}
0.89 E+01 \\
.89 E+01 \\
.89 E+01 \\
.89 E+01 \\
.89 E+01
\end{array}
$$$$
-0.263512 E+04
$$$$
\text { -.263512E+04 }
$$$$
-.263512 E+04
$$$$
-.263512 E+04
$$$$
\text { -.263512E+04 }
$$$$
\begin{array}{r}
0.1 E+02 \\
.1 E+02 \\
.1 E+02
\end{array}
$$$$
0.3 E+03
$$$$
.3 E+03
$$$$
.3 E+03
$$$$
-0.5 E+02
$$$$
-.5 E+02
$$$$
-.5 E+02
$$$$
0.71 E+02
$$$$
.71 E+02
$$$$
.71 E+02
$$

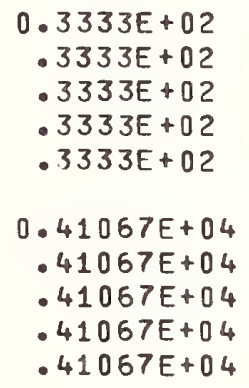

$0.41067 E+04$ $.41067 E+04$ . $41067 E+04$ $.41067 E+04$ . $41067 \mathrm{E}+04$

$-0.1234567 E+04$ $-.1234567 E+04$ -.1234567E+04 -.1234567E+04 -.1234567E+ 04

$-0.91 E+01$

$-.91 E+01$

$-.91 E+01$

-.91E+01

-.91E+01

$0.4621 E+02$

$.4621 E+02$

$.4621 E+02$

$.4621 E+02$

$.4621 E+02$

$$
\begin{array}{r}
0.2 E+02 \\
.2 E+02 \\
.2 E+02 \\
0.4 E+04 \\
.4 E+04 \\
.4 E+04 \\
-0.6 E+03 \\
-.6 E+03 \\
=.6 E+03 \\
-0.92 E+02 \\
-.92 E+02 \\
-.92 E+02
\end{array}
$$

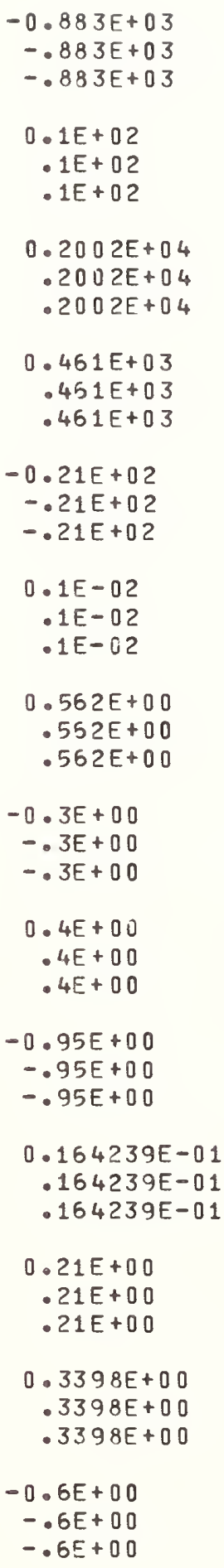

$0.1414 E+04$

$.1414 E+04$

$.1414 E+04$

$0.562 E+03$

. $562 E+03$

$.562 E+03$

$$
\begin{array}{r}
-0.983 E+03 \\
-.983 E+03 \\
-.983 E+03
\end{array}
$$

$$
\begin{array}{r}
-0.165 E+03 \\
-.165 E+03 \\
-.165 E+03 \\
0.122 E+03 \\
.122 E+03 \\
.122 E+03
\end{array}
$$

$$
\begin{array}{r}
0.2 E-02 \\
.2 E-02 \\
.2 E-02 \\
0.562 E+00 \\
.562 E+00 \\
.562 E+00
\end{array}
$$

$-0.3333333 E+00$

-. $3333333 E+00$

-. $3333333 E+00$

$-0.445 E+00$

$-.445 E+00$

$-.445 E+00$

$0.95 E+00$

$.95 E+00$

$.95 E+00$

$0.36 E+00$

- $36 E+00$

$.36 E+00$

$-0.3963 E+00$

-.3963E+00

-. $3963 E+00$

$0.3398 E+00$

$.3398 E+00$

$.3398 E+00$

$0.6 E+00$

- $6 E+00$

- $6 E+00$ 


\begin{tabular}{|c|c|}
\hline $\begin{array}{l}0.0 E+00 \\
0 .\end{array}$ & $\begin{array}{r}0.1 E+01 \\
.1 E+01\end{array}$ \\
\hline $\begin{array}{r}0.4562311 E+07 \\
.4562311 E+07\end{array}$ & $\begin{array}{r}0.789453 E+06 \\
.789453 E+06\end{array}$ \\
\hline $\begin{array}{r}0.449 E+06 \\
.449 E+06\end{array}$ & $\begin{array}{r}0.25 E+04 \\
.25 E+04\end{array}$ \\
\hline $\begin{array}{r}0.22223 E+07 \\
.22223 E+07\end{array}$ & $\begin{array}{r}0.3332 E+05 \\
.3332 E+05\end{array}$ \\
\hline $\begin{array}{r}0.3 E+01 \\
.3 E+01 \\
.3 E+01\end{array}$ & $\begin{array}{r}0.3 E+01 \\
.3 E+01 \\
.3 E+01\end{array}$ \\
\hline $\begin{array}{r}0.9376543 E+05 \\
.9876543 E+05 \\
.9876543 E+05\end{array}$ & $\begin{array}{r}0.8765432 E+04 \\
.8765432 E+04 \\
.8765432 E+04\end{array}$ \\
\hline $\begin{array}{r}0.4444 E+04 \\
.4444 E+04 \\
.4444 E+04\end{array}$ & $\begin{array}{r}0.55555 E-02 \\
.55555 E-02 \\
.55555 E-02\end{array}$ \\
\hline $\begin{array}{r}0.6 E-04 \\
.6 E-04 \\
.6 E-04\end{array}$ & $\begin{array}{r}0.77 E+07 \\
.77 E+07 \\
.77 E+07\end{array}$ \\
\hline $\begin{array}{r}0.142 E+03 \\
.142 E+03 \\
.142 E+03\end{array}$ & $\begin{array}{r}0.2667 E+02 \\
.2667 E+02 \\
.2667 E+02\end{array}$ \\
\hline $\begin{array}{r}-0.36923 E+06 \\
-.36923 E+06 \\
-.36923 E+06\end{array}$ & $\begin{array}{r}-0.234 E+03 \\
-.234 E+03 \\
-.234 E+03\end{array}$ \\
\hline $\begin{array}{r}0.21 E+03 \\
.21 E+03 \\
.21 E+03\end{array}$ & $\begin{array}{r}-0.21 E+03 \\
-.21 E+03 \\
-.21 E+03\end{array}$ \\
\hline $\begin{array}{r}-0.5959 E+03 \\
-.5959 E+03 \\
-.5959 E+03\end{array}$ & $\begin{array}{r}0.4967 E+03 \\
.4967 E+03 \\
.4967 E+03\end{array}$ \\
\hline $\begin{array}{r}0.1 E+01 \\
.1 E+01 \\
.1 E+01\end{array}$ & $\begin{array}{r}0.1 E+01 \\
.1 E+01 \\
.1 E+01\end{array}$ \\
\hline $\begin{array}{r}-0.2 E+01 \\
-.2 E+01 \\
-.2 E+01\end{array}$ & $\begin{array}{r}-0.2 E+01 \\
-.2 E+01 \\
-.2 E+01\end{array}$ \\
\hline $\begin{array}{r}0.492 E+01 \\
.492 E+01 \\
.492 E+01\end{array}$ & $\begin{array}{r}-0.6527 E+04 \\
-.6527 E+04 \\
-.6527 E+04\end{array}$ \\
\hline
\end{tabular}

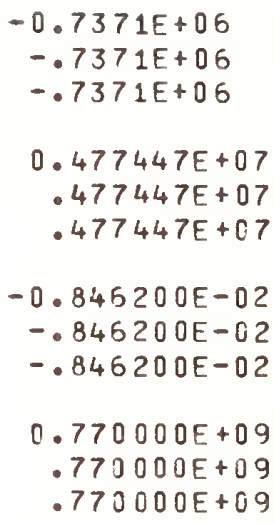

$$
\begin{array}{r}
0.998 E-01 \\
.998 E-01 \\
.998 E-01
\end{array}
$$

$$
\begin{array}{r}
-0.93624 E+00 \\
-.93624 E+00 \\
-.93624 E+00 \\
0.13330 E+03 \\
.13330 E+03 \\
.13330 E+03 \\
0.81625 E+08 \\
.81625 E+08 \\
.81625 E+08
\end{array}
$$

$0.37900 E+06$

. $37900 E+06$

$.37900 E+06$

$0.30000 E+06$ $.30000 E+06$ $.30000 E+06$

$0.299 E+02$ . 299E+ 02 . $299 E+02$

$0.1419 E+02$ $.1419 E+02$ . $1419 E+02$

$0.987 E+03$

. $987 E+03$ $.987 E+03$

$0.4659 E+05$ $.4659 E+05$ $.4659 E+05$

$-0.93296 E+08$

-. $93296 E+08$

$-.93296 E+03$

$-0.6 E+07$

$-.6 E+07$

$-.6 E+07$

$0.16 E+07$

- $16 E+07$

$.16 E+07$

$0.1 E+02$

- $1 E+02$

. $1 E+02$ 


\begin{tabular}{|c|c|}
\hline $\begin{array}{r}-0.2 E-01 \\
-.2 E-01 \\
-.2 E-01\end{array}$ & $\begin{array}{r}-0.2 E-01 \\
-.2 E-01 \\
-.2 E-01\end{array}$ \\
\hline $\begin{array}{r}0.3 E-02 \\
.3 E-02 \\
.3 E-02\end{array}$ & $\begin{array}{r}-0.3 E+04 \\
-.3 E+04 \\
-.3 E+04\end{array}$ \\
\hline $\begin{array}{r}-0.4 E+05 \\
-.4 E+05 \\
-.4 E+05\end{array}$ & $\begin{array}{r}0.4 E-03 \\
.4 E-03 \\
.4 E-03\end{array}$ \\
\hline $\begin{array}{r}0.5 E+06 \\
.5 E+06 \\
.5 E+06\end{array}$ & $\begin{array}{r}-0.5 E-04 \\
-.5 E-04 \\
-.5 E-04\end{array}$ \\
\hline $\begin{array}{r}-0.6 E-05 \\
-.6 E-05 \\
-.6 E-05\end{array}$ & $\begin{array}{r}0.6 E+07 \\
.6 E+07 \\
.6 E+07\end{array}$ \\
\hline $\begin{array}{r}0.39393 E+01 \\
.39393 E+01 \\
.39393 E+01\end{array}$ & $\begin{array}{r}0.62 E+04 \\
.62 E+04 \\
.62 E+04\end{array}$ \\
\hline $\begin{array}{r}0.9 E+00 \\
.9 E+00 \\
.9 E+00\end{array}$ & $\begin{array}{r}0.765765 E+03 \\
.765765 E+03 \\
.755765 E+03\end{array}$ \\
\hline $\begin{array}{r}0.352 E+09 \\
.352 E+09 \\
.352 E+09\end{array}$ & $\begin{array}{r}0.35 E+03 \\
.35 E+03 \\
.35 E+03\end{array}$ \\
\hline $\begin{array}{r}0.147626 E+00 \\
.147626 E+00 \\
.147626 E+00\end{array}$ & $\begin{array}{r}0.891 E-14 \\
.891 E-14 \\
.891 E-14\end{array}$ \\
\hline $\begin{array}{r}\text { 0. } 9 E-07 \\
.9 E-07 \\
.9 E-07\end{array}$ & $\begin{array}{r}0.9999 E+08 \\
.9999 E+08 \\
.9999 E+08\end{array}$ \\
\hline $\begin{array}{r}0.13 E-04 \\
.13 E-04 \\
.13 E-04\end{array}$ & $\begin{array}{r}0.13 E-04 \\
.13 E=04 \\
.13 E=04\end{array}$ \\
\hline $\begin{array}{r}0.77 E+00 \\
.77 E+00 \\
.77 E+00\end{array}$ & $\begin{array}{r}0.77 E+00 \\
.77 E+00 \\
.77 E+00\end{array}$ \\
\hline $\begin{array}{r}0.878 E+01 \\
.878 E+01 \\
.878 E+01\end{array}$ & $\begin{array}{r}-0.878 E+01 \\
-.878 E+01 \\
=.878 E+01\end{array}$ \\
\hline $\begin{array}{r}-0.9797 E+02 \\
-.9797 E+02 \\
-.9797 E+02\end{array}$ & $\begin{array}{r}0.9797 E+02 \\
.9797 E+02 \\
.9797 E+02\end{array}$ \\
\hline
\end{tabular}

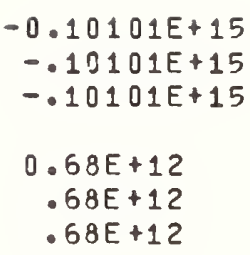

$0.3682 E+01$ - $3682 E+01$ - $3682 E+01$ . $3682 E+01$

$-0.2571 E+09$

$-.2571 E+09$

$-.2571 E+09$

$-.2571 E+09$

$0.1460 E+00$ $.1460 E+00$ . $1460 E+00$ $.1460 E+00$

$$
\begin{array}{r}
-0.10101 E+15 \\
-.10101 E+15 \\
-.10101 E+15 \\
0.357628 E+00 \\
.357628 E+00 \\
.357628 E+00 \\
0.76444 E+00 \\
.76444 E+00 \\
.76444 E+00
\end{array}
$$

$$
\begin{array}{r}
-0.2594 E+05 \\
-.2594 E+05 \\
-.2594 E+05 \\
-0.12 E-04 \\
-.12 E-04 \\
-.12 E-04 \\
-0.6 E+09 \\
-.6 E+09 \\
=.5 E+09 \\
0.9119 E-06 \\
.9119 E-06 \\
.9119 E-06
\end{array}
$$

$$
\begin{array}{r}
-0.39426 E-02 \\
-.39426 E-02 \\
-.39426 E-02 \\
0.45 E+12 \\
.45 E+12 \\
.45 E+12 \\
0.3479 E+06 \\
.3479 E+06 \\
.3479 E+06 \\
.3479 E+06
\end{array}
$$

$0.8236 E+02$ - $8236 E+02$ - $8236 E+02$ - $9236 E+02$

$0.1752 E+09$ . $17525+09$ - $1752 E+09$ $.1752 E+09$

$-0.1064 E+05$

$-.1064 E+05$

$.1064 E+05$

$-.1064 E+05$ 


$\begin{array}{rr}0.1642390 E-01 & 0.3600000 E+00 \\ .1642390 E-01 & .3600000 E+00 \\ .1642390 E-01 & .3600000 E+00 \\ .1642390 E-01 & .3600000 E+00 \\ .1642390 E-01 & .3600000 E+00 \\ 0.1642390 E-01 & .3600000 E+00 \\ .4552311 E+07 & 0.7894530 E+06 \\ .4562311 E+07 & .7894530 E+06 \\ . .4562311 E+07 & .7894530 E+06 \\ .4552311 E+07 & .7894530 E+06 \\ .4552311 E+07 & .7894530 E+06 \\ -0.6000000 E-05 & 0.5000000 E+07 \\ -.6000000 E-05 & .6000000 E+07 \\ -.6000000 E-05 & .6000000 E+07 \\ -.6000000 E-05 & .5000000 E+07 \\ -.5000000 E-05 & .6000000 E+07 \\ -.6000000 E-05 & .6000000 E+07 \\ -0.9119000 E+06 & 0.9119000 E-06 \\ -.9119000 E+06 & .9119000 E-06 \\ -.9119000 E+06 & .9119000 E-06 \\ -.9119000 E+06 & .9119000 E-06 \\ -.9119000 E+06 & .9119000 E-06 \\ -.9119000 E+06 & .9119000 E-06\end{array}$

EACH GROUP SHOULD BE IDENTICAL EXCEPT FOR THE SIGN OF THE FIRST TWO LINES

$$
\begin{array}{r}
0.3000000 E+03 \\
.3000000 E+03 \\
-.3000000 E+03 \\
-.3000000 E+03 \\
-.3000000 E+03 \\
-.3000000 E+03 \\
-0.5000000 E+02 \\
-.5000000 E+02 \\
.5000000 E+02 \\
.5000000 E+02 \\
.5000000 E+02 \\
.5000000 E+02 \\
0.7700000 E+00 \\
.7700000 E+00 \\
-.7700000 E+00 \\
-.7700000 E+00 \\
-.7700000 E+00 \\
-.7700000 E+00
\end{array}
$$

$0.5000000 E+06$ $.5000000 E+06$

$-.5000000 E+06$

-.5000300E+06

$-.5000000 E+06$

$-.5000000 E+06$

$0.4920000 E+01$

$.4920000 E+01$

$-.4920000 E+01$

-.4920000E+01

-.4320000E+01

$-.4920000 E+01$
$-0.6000000 E-05$
-.600000JE-05 $.6000000 E-05$ $.6000000 E-05$ $.6000000 E-05$ $.6000000 E-05$

$0.4444000 E+04$ $.4444000 E+04$ $-.4444000 E+04$ -. $4444000 E+04$ $-.4444000 E+04$ $-.4444000 E+04$

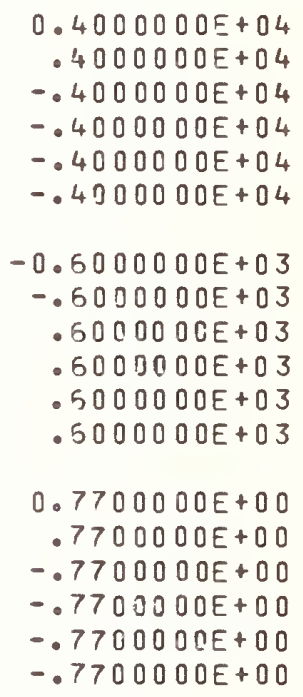

$0.6000000 E+07$ $.6000000 E+07$

-.6000000E+07

-.6000000E+07

$-.5000000 E+07$

-.6000000E+07

$0.5555500 E-02$ . $5555500 E-02$

$-.5555500 E-02$

$-.5555500 E-02$

-. $5555500 E-02$

$-.5555500 E-02$ 
$-0.3692300 E+06$

-. $3692300 E+06$

$.3692300 E+06$

$.3692300 E+06$

$.3692300 E+06$

$.3692300 E+06$
LASGN - (016) ASSIGNMENT OF ASA REFS. -7.1 .1 .2$

RESUL TS

ALL ANSWERS BELOW MUST BE TRUE

$$
\begin{aligned}
& T \\
& T \\
& T \\
& T \\
& T \\
& T \\
& T \\
& T \\
& T \\
& T \\
& T \\
& T \\
& T \\
& T \\
& T \\
& T \\
& T \\
& T \\
& T \\
& T \\
& T
\end{aligned}
$$

ALL ANSWERS BELOW MUST BE FALSE

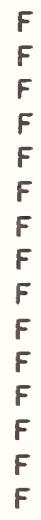


INTRL - (017) ASSIGN INTEGER, REAL, AND DOUBLE PRECISION VALUES

ASA REFS. - 7.1.1.1. 5.1.1.2

RESULTS

ASSIGN INTEGER VARIABLES

1 - TO REAL VARIABLES

$111.0 *$

111.0

$-1111.0 *$

$-1111 \cdot 0$

$-11111.0 *$

$-11111.0$

$$
\begin{aligned}
& 1.0 * \\
& 1.0
\end{aligned}
$$

2 - TO DOUBLE PRECISION VARIABLES

$$
\begin{array}{r}
-0.11111005 \\
-.111110+05 \\
0.1001 \\
.10+01 \\
0.111003 \\
.1110+03 \\
-0.1111004 \ldots \\
-.11110+04
\end{array}
$$

ASSIGN INTEGER CONSTANTS

1 - TO REAL VARIABLES

$$
\begin{array}{r}
-2222.0 \\
-2222.0 \\
222.0 \\
222.0 \\
-22222.0 \\
-22222.0 \\
2.0 \\
2.0
\end{array}
$$

2 - to oouble precision variables

$$
\begin{gathered}
0.2001 * \\
.20+01 \\
-0.22222005^{*} \\
-.222220+05 \\
-0.2222004^{*} \\
-.22220+04 \\
0.222003 . \\
.2220+03
\end{gathered}
$$

ASSIGN BASIC REAL GONSTANTS

1 - to integer variables

$$
\begin{array}{r}
3 \\
3 \\
3 \\
3 \\
-3 \\
-3
\end{array}
$$

2 - to double precision variables

$$
\begin{gathered}
0.33333001 * \\
.333330+01
\end{gathered}
$$

0.3333333001 $.33333330+01$

$$
\begin{gathered}
-0.3333333001 . \\
-.33333330+01 \\
-0.333333001 \\
-.3333330+01
\end{gathered}
$$

ASSIGN REAL VARIABLES

1 - TO INTEGER VARIABLES

$$
\begin{aligned}
& -44 \\
& -44 \\
& -44 \\
& 44 \\
& 44 \\
& 44
\end{aligned}
$$


2 - TO DOUBLE PRECISION VARIABLES

$-0.444002 *$

$-.0440+02$

$-0.4444002 *$

$-.4444 D+02$

0.44444002 *

$.444440+02$

$0.44444002 *$

$.444440+02$

ASSIGN DOUBLE PRECISION VARIABLES

1 - TO INTEGER VARI ABLES

$5555 *$

5555

$$
\begin{aligned}
& 5 \\
& 5 \\
& -5 \\
& -5 \\
& -5
\end{aligned}
$$

$-5$

2 - TO REAL VARIABLES

$-0.5555556 E \quad 01 *$

$-.5555556 E+01$

$-0.5555556 E \quad 01 *$

$-.5555556 E+01$

$0.5555556 E$ O 01 *

$.5555556 E+01$

$0.555555 E \quad 04$ *

$.555555 E+04$
ASSIGN DOUBLE PRECISION CONSTANTS

1 - TO INTEGER VARIABLES

$$
\begin{gathered}
6 * \\
6 \\
-6 * \\
-6 \\
-6 \\
6666 * \\
6666 \\
2-\text { TO REAL VARIABLES } \\
0.6666667 E \text { 14* } \\
.6666667 E+14 \\
0.66666 E \text { 01* } \\
.66666 E+01 \\
-0.5666666 E \text { 01* } \\
-.6666666 E+01 \\
-0.6666667 E 01 * \\
-06666667 E+01
\end{gathered}
$$

ALL TEST OUTPUT SHOULD BE CHECKED AGAINST THE ASTERISKED (*) FIGURE HHICH PRECEDES IT 
UGOTO - (020) UNCONDITIONAL GO TO
STATENENT

ASA REFS. -7.1 .2 .1 .1$

RESULTS

1

2

3

4

5

6

7

8

THIS TEST IS SUCCESSFUL ONLY IF THE NUMBERS LISTED ABOVE ARE SEQUENTIALLY IN ORDER FROM 1 TO 8

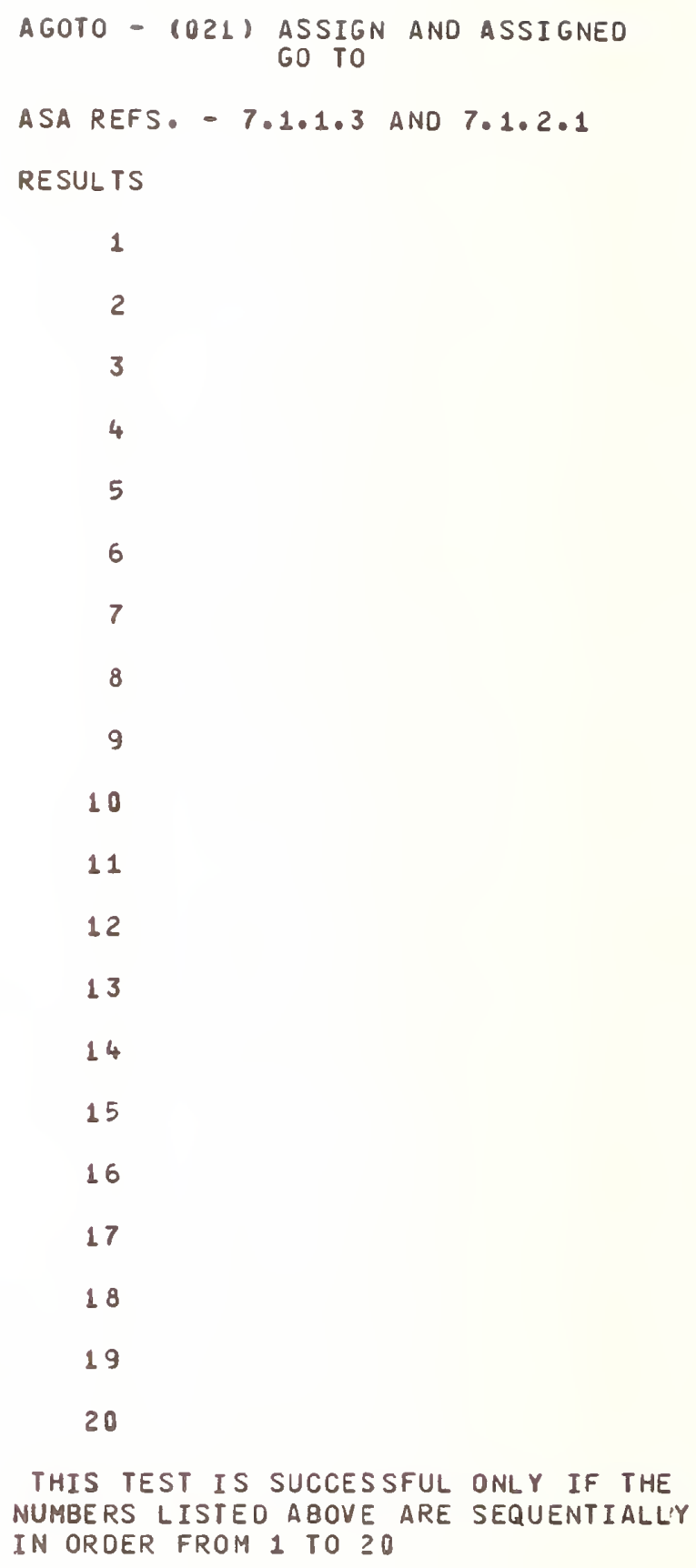


CGOTO - 1022$)$ COMPUTED GO TO

ASA REF. -7.1 .2 .1 .3$

RESULTS

1

2

3

4

5

6

7

8

9

10

11

12

13

14

15

16

17

18

19

20

THIS TEST IS SUCCES.SFUL ONLY IF THE NUMBERS LISTED ABOVE ARE SEQUENTIALLYY IN ORDER FROM 1 TO 20

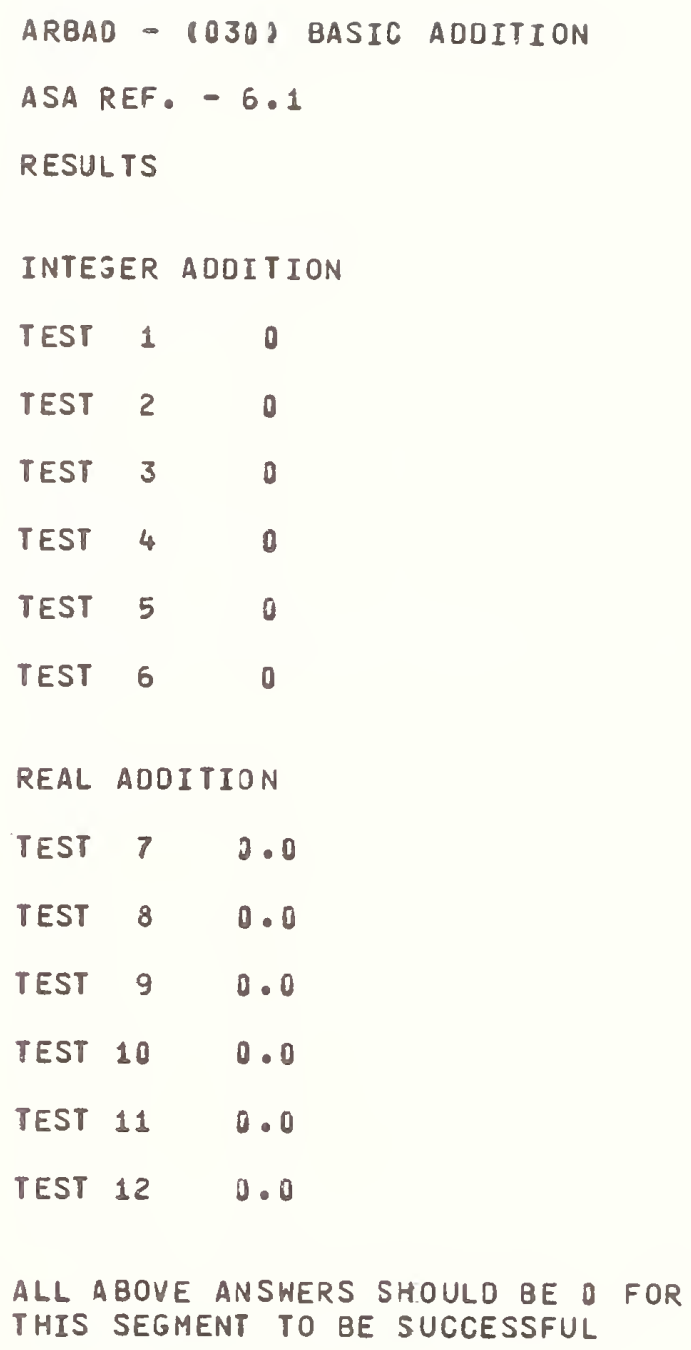


ARFAD - $\$ 031\}$ D.P. ADDITION

ASA REF. - 6.1

RESULTS

0.

0 .

0 .

0.

0 .

THE 5 ANSWERS ABOVE SHOULD BE 0 PLUS OR MINUS AN ERROR FACTOR OF 0.1D-13

ARBSB - (032) BASIC SUBTRACTION

ASA REFS. - 6.1

RESULTS

TEST 1 INTEGER SUBTRACTION

$$
\begin{aligned}
& 0 \\
& 0 \\
& 0 \\
& 0 \\
& 0
\end{aligned}
$$

TEST? REAL SUBTRACTION

$$
\begin{aligned}
& 0.0 \\
& 0.0 \\
& 0.0 \\
& 0.0
\end{aligned}
$$

ALL ABOVE ANSHERS SHOULD BE 0 FOR THIS SEGMENT TO BE SUCCESSFUL

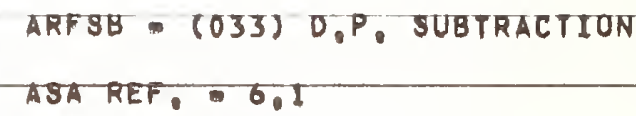

THE ANSWERS ABOVE SHOULD BE 0 PLUS OR MINUS AN ERROR FACTOR OF $0,10=13$

ARBAS - (034) BASIC ADDITION AND SUBTRACTION

ASA REF, 6.4

RESULTS

TESTI INTEGER ADO ANO SUBT

0
0
0
0

TEST2 REAL IDD AND SUBTR$$
0.0
$$$$
0.0
$$$$
0.0
$$

ALL ABOVE ANSWERS SHOULD BE O FOR

THIS SEGMENT TO BE SUCCESSFUL 
F O R T R A N T E S T P R O G R A M S

PREPARED BY NATIONAL BUREAU OF STANDARDS

FOR USE ON LARGE FORTRAN PROCESSORS

IN ACCORDANCE WITH ASA FORTRAN $\times 3.9-1966$

VERSION 3 PART 4

SAMPLE COMPUTER, FORTRAN COMPILER LEVEL

OPERATING SYSTEM VERSION

DATE, INSTALLATION NAME

ARFAS - (035) D.P. ADO AND SUBTR

ASA REF. -6.1

RESULTS

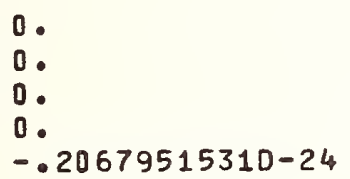

THE ANSWERS ABOVE SHOULD BE O FOR

THIS SEGMENT TO BE SUCCESSFUL.

VALUES WITH EXPONENTS LESS THAN

$10 * *(-14)$ ARE CONSIDEREO ZERO
ARBMI - (036) INTEGER MULTIPLICATION

ASA REF. -6.1

RESULTS

$$
0
$$$$
0
$$$$
0
$$$$
0
$$$$
0
$$

ALL ABOVE ANSWERS SHOULD BE O FOR

THIS SEGMENT TO BE SUCCESSFUL

ARBMR - (037) REAL MULTIPLICATION

ASA REF. - 6.1

RESULTS

$$
\begin{aligned}
& 0.0 \\
& 0.0 \\
& 0.0 \\
& 0.0 \\
& 0.0 \\
& 0.0 \\
& 0.0
\end{aligned}
$$

ALL ABOVE ANSHERS SHOULD BE 0 FOR THIS SEGMENT TO BE SUCCESSFUL

ARFMD $=(038)$ D.P. MULIIPLICATION

ASA REF, 6.1

RESULTS

$$
\begin{aligned}
& 0.00000000000+00 \\
& 0.00000000000+00 \\
& 0.00000000000+00 \\
& 0.00000000000+00 \\
& 0.00000000000+00 \\
& 0.00000000000+00 \\
& 0.00000000000+00 \\
& 0.00000000000 \$ 00
\end{aligned}
$$

THE ANSWERS ABOVE SHOULD BE O FOR

THIS SEGMENT TO BE SUCCESSFUL 


\begin{tabular}{c} 
AREOV - 6039 INTEGER ANO REAL \\
DIVISION \\
\hline ASA REF, 6.1 \\
\hline RESULTS \\
TESTI INTEGER DIVISIUN \\
0 \\
0 \\
0 \\
0 \\
0 \\
TEST2 REAL OIVISION \\
0.0 \\
0.0 \\
0.0 \\
0.0
\end{tabular}

ALL ABOVE ANSWERS SHOULD BE O FOR THIS SEGMENT TO BE SUCCESSFUL

ARFOV - (040) D.P. DIVISION

ASA REF。 -6.1

RESULTS
0.
0 .
0 .
0.
0 .
0 .

THE ANSWERS ABOVE SHOULO BE D FOR THIS SEGMENT TO BE SUCCESSFUL
ARBEX - (041) BASIC EXPONENTIAIION ASA REFS. $=0.1$

RESULIS

INTEGER BY INTEGER

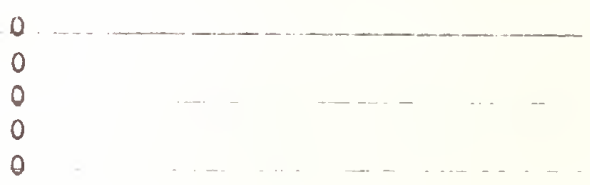

REAL BY INT, REAL BY REAL
0.0
$\theta, \theta$
0.0
0.0
0,0
0.0
0.0

ALL ABUVE ANSWERS SHOULD HE O FOR

THIS SEGMENT TO BE. SUCCESSFUL

ARFEX - 10421 EXPONENTIATION

ASA REF. -6.1

RESUI.TS

$$
\begin{aligned}
& .0000000000 \\
& .0000000000 \\
& .0000000000 \\
& .0000000000 \\
& .0000000000
\end{aligned}
$$

THF ANSWER5 ABOVF SHQULD BF O FOR

THIS SFGMENT TO BE SIJCCESSFUL.

VALUES MTH EXPONENTS LESS THAN

10* $(-14)$ ARE CONSIDERED ZERO 


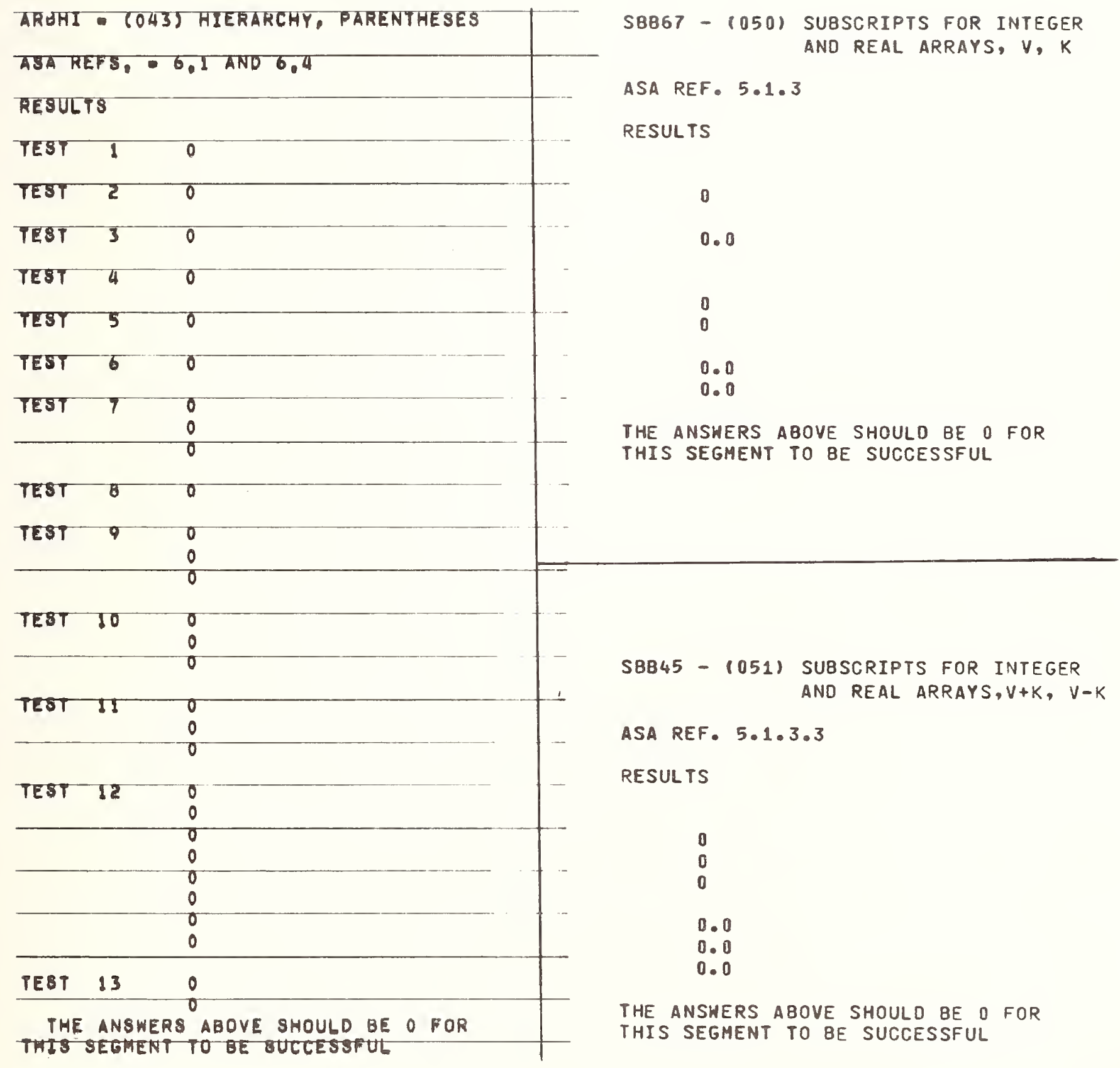


SBB13 - (052) SUBSCRIPTS INTEGER AND

ASA REF. $5 \cdot 1 \cdot 3.3$

RESULTS

$$
\begin{aligned}
& 0 \\
& 0 \\
& 0.0 \\
& 0.0 \\
& \\
& 0 \\
& 0 \\
& 0.0 \\
& 0.0 \\
& 0 \\
& 0.0
\end{aligned}
$$

THE ANSHERS ABOVE SHOULD BE O FOR THIS SEGMENT TO BE SUCCESSFUL REAL, $C * V, C * V-K, C * V+K$

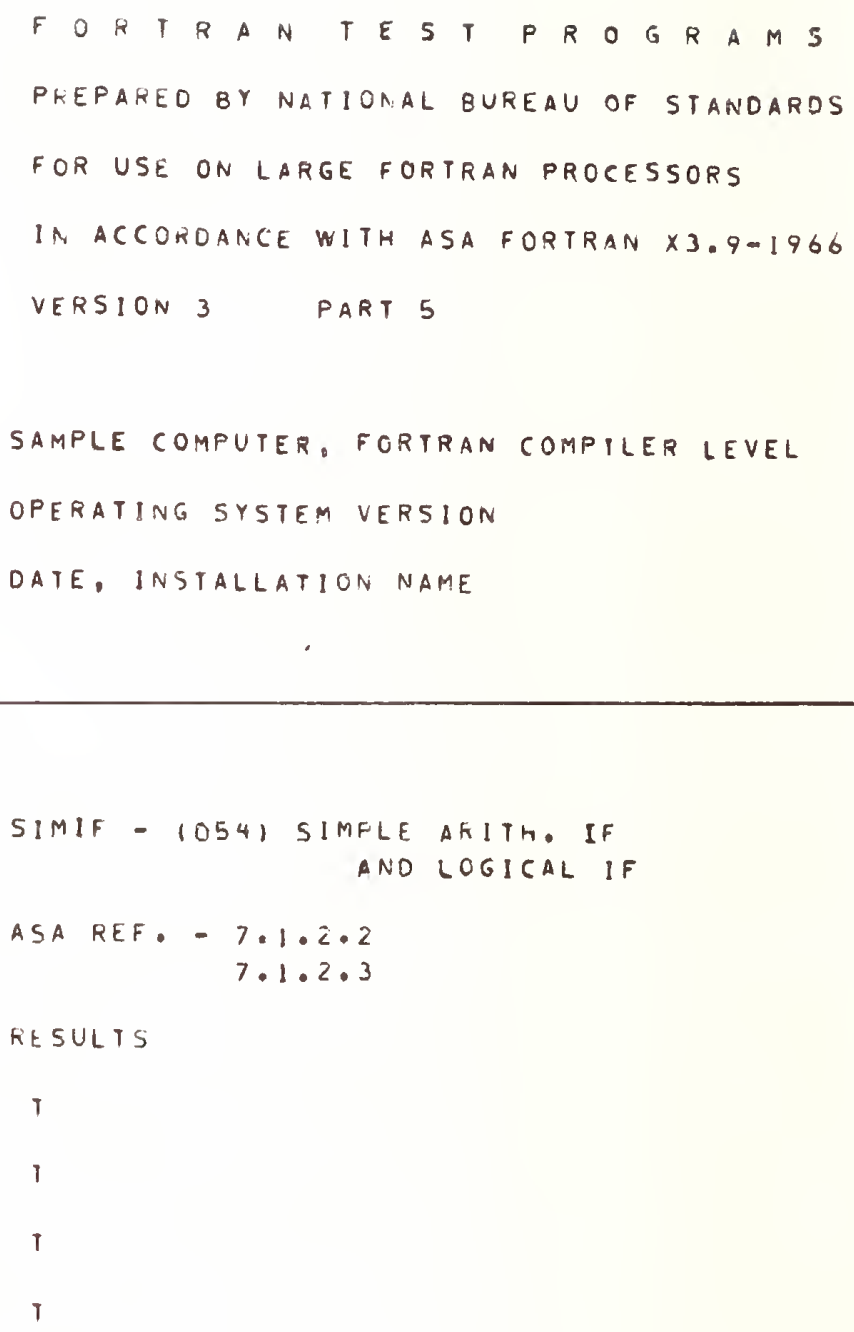

THE ANSWERS ABOVE SHOULD BE O FOR THIS SEGMENT TO-BE SUCCESSFUL. 
IFABS - (055) INTRINSIC FUNCTIONS-ABS, IABS (ABSOLUTE VALUE)

ASA REFS. -8.2

RESULTS

$$
\begin{aligned}
& 0.0 \\
& 0.0 \\
& 0.0 \\
& 0.0 \\
& 0 \\
& 0 \\
& 0
\end{aligned}
$$

THE ABOVE ANSWERS SHIOULD ALL BE O FOR THIS TEST SEGMENT TO BE SUCCESSFUL.

IFFLT - (056) INTRINSIC FUNCTION-FLOAT

ASA REF -8.2

RESULTS

$$
\begin{aligned}
& 0.0 \\
& 0.0 \\
& 0.0
\end{aligned}
$$

THE ABOVE ANSWERS SHOULD ALL BE O FOR THIS TEST SEGMENT TO BE SUCCESSFUL.

IFFIX - (OST) INTRINSIS FUNCTION= If IX

ASA FEF. $=8.2$

RESULTS

$$
\begin{aligned}
& 0 \\
& 0 \\
& 0 \\
& 0 \\
& 0 \\
& 0
\end{aligned}
$$$$
\text { o }
$$

THE ABOVE ANSWERS SHOULD ALL BE O FOR

THIS TEST SEGMENT TO BE SUCCESSFUL.

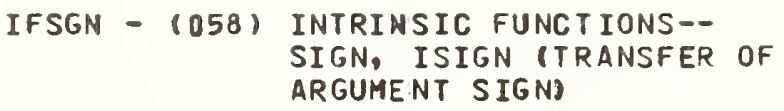

ASA REF. -8.2

RESULTS

$$
\begin{aligned}
& 0.0 \\
& 0.0 \\
& 0.0 \\
& 0.0 \\
& 0.0 \\
& 0 \\
& 0 \\
& 0 \\
& 0 \\
& 0
\end{aligned}
$$$$
0
$$

THE ABOVE ANSHERS SHOULO ALL BE O FOR

THIS TEST SEGMENT TO BE SUCCESSFUL.

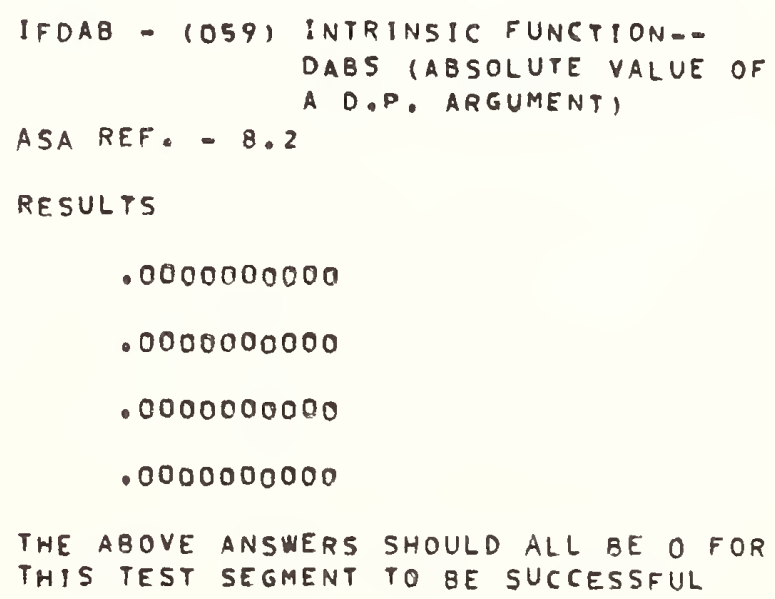




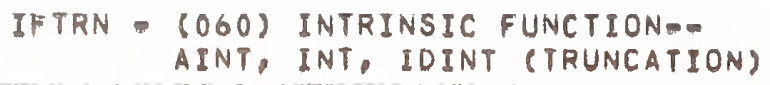

0

0

END OF INT TEST

0

0

0

END OF IDINT TEST

ALL ABOVE ANSWERS SHOULD BE O FOR THIS TEST SEGMENT TO BE SUCCESSFUL

\section{IFMOD - (061) INTRINSIC FUNOTION-- AMOD, MOD (REMAINDERING)}

ASA REF. -8.2

RESULTS

$$
\begin{aligned}
& 0.0 \\
& 0.0 \\
& 0.0 \\
& 0.0
\end{aligned}
$$

END OF AMOD TEST.

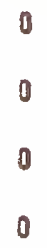

0

0

0

0

END OF MOD TEST.

ALL ABOVE ANSWERS SHIULO BE O FOR THIS TEST SEGMENT TO BE SUCCESSFUL. 

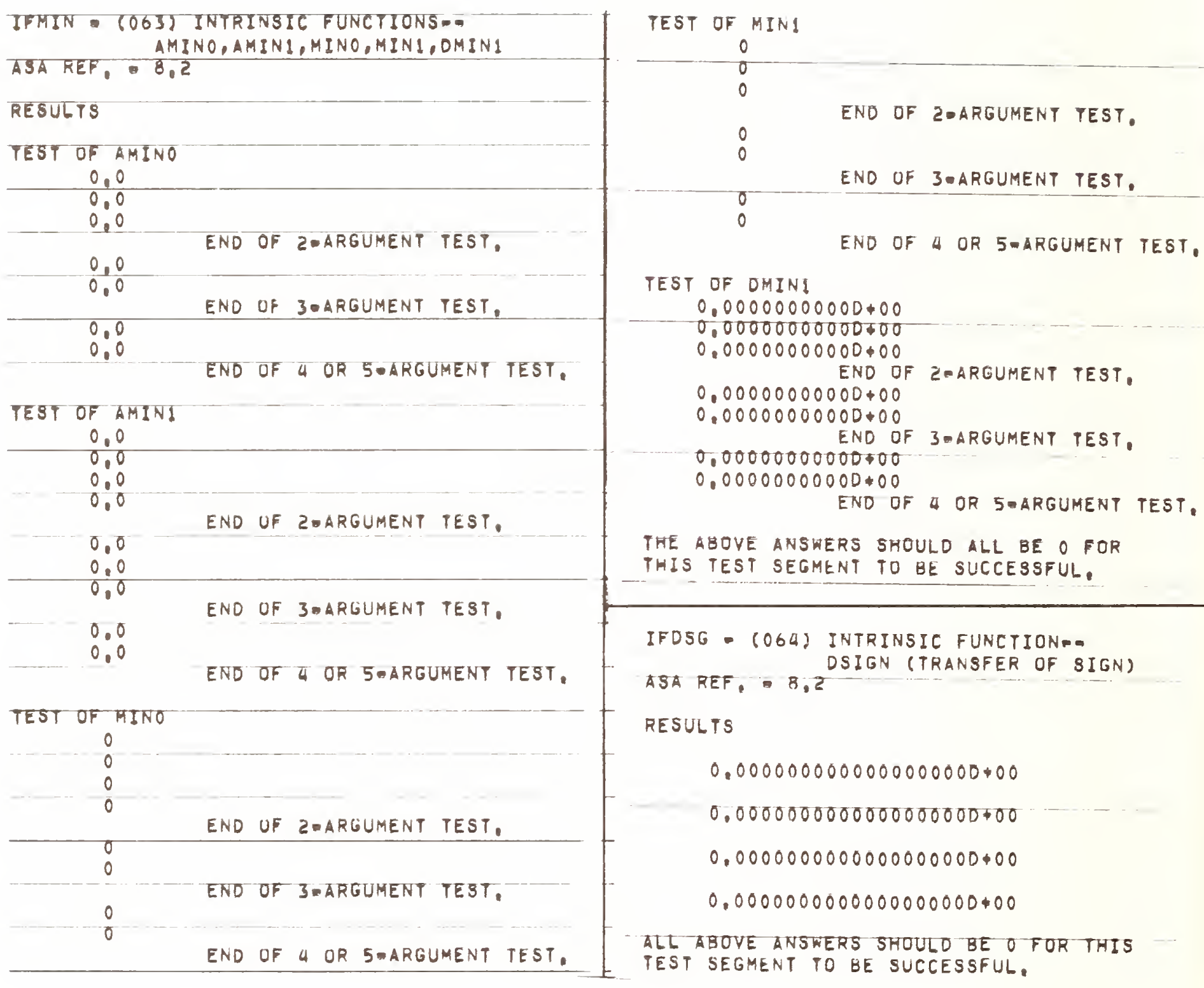
FOR T R A N T E S T P R O G R A M S PREPARED BY NATIONAL BUREAU OF STANDARDS FOR USE ON LARGE FORTRAN PROCESSORS IN ACCORDANCE WITH ASA FORTRAN $\times 3.9-1966$ VERSION 3 PART 6

SAMPLE COMPUTER, FORTRAN COMPILER LEVEL OPERATING SYSTEM VERSION

DATE, INSTALLATION NAME

IFDIM - (065) INTRINSIC FUNCTIONS - DIM ASA REF. $=8.2$ AND IDIM (POSITIVE DIFFERENCE)

RESULTS

$$
\begin{aligned}
& 0.00 \\
& 0.00 \\
& 0.00 \\
& 0.00
\end{aligned}
$$$$
0
$$$$
0
$$$$
0
$$$$
0
$$$$
0
$$

ALL ABOVE ANSWERS SHOULD BE 0 FOR THIS TEST SEGMENT TO BE SUCCESSFUL.

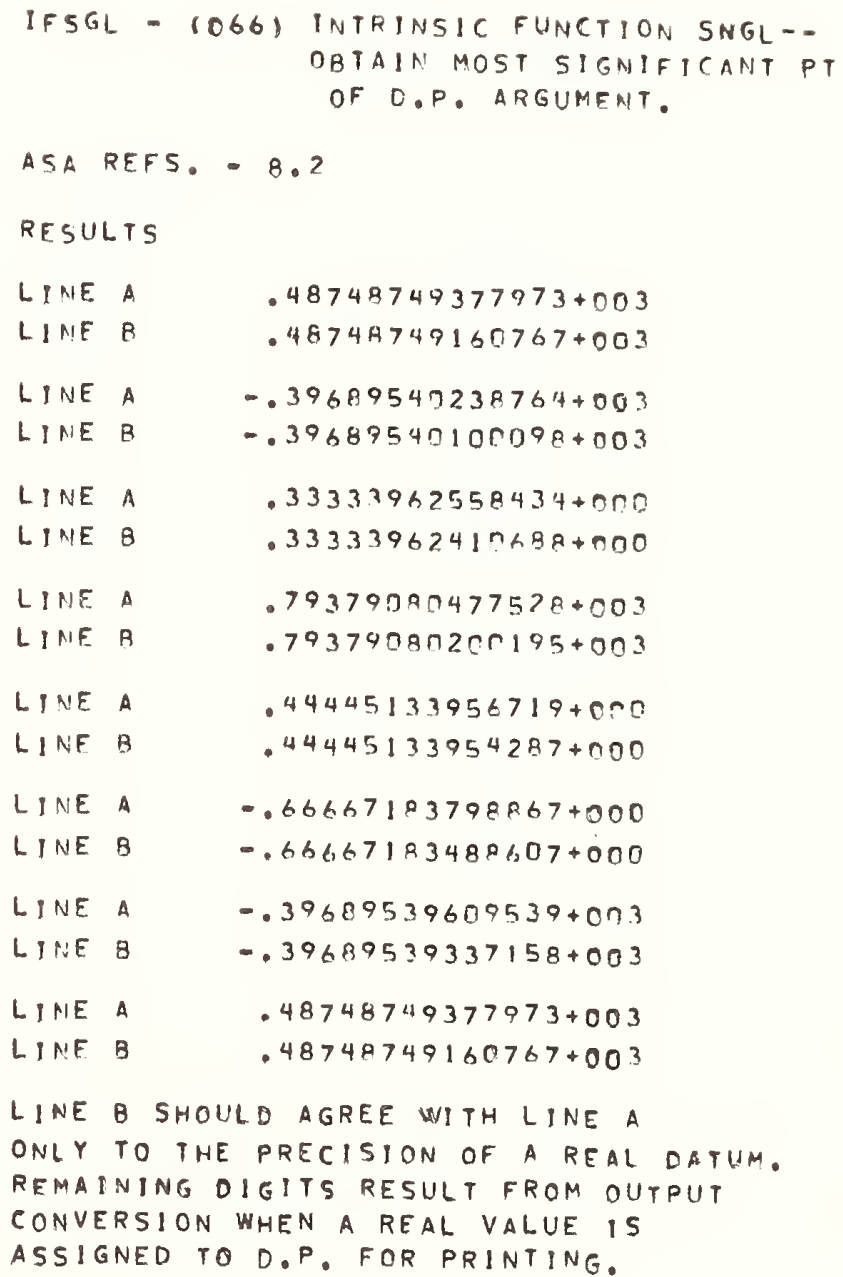


IFREL - (067) INTRINSIC FUNCTION-REAL

ASA REF. -8.2

RESULTS

$$
\begin{aligned}
& 0.0000 \\
& 0.0000 \\
& 0.0000 \\
& 0.0000 \\
& 0.0000 \\
& 0.0000 \\
& 0.0000 \\
& 0.0000 \\
& 0.0000 \\
& 0.0000 \\
& 0.0000 \\
& 0.0000 \\
& \\
& 0.0000 \\
& 0.0000 \\
& 0.0000 \\
& 0.0000 \\
& 0.0000 \\
& 0.0000
\end{aligned}
$$

ALL ABOVE ANSWERS SHOULD BE G FOR THIS TEST SEGMENT TO BE SUCCESSFUL.

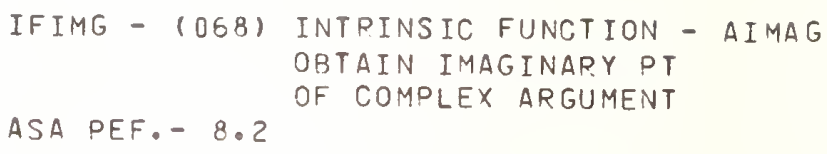

RESULTS

$$
\begin{aligned}
& 0.00000 \\
& 0.00000 \\
& 0.00000 \\
& 0.00000 \\
& 0.00000 \\
& 0.00000 \\
& 0.00000 \\
& 0.00000 \\
& \\
& 0.00000 \\
& 0.00000 \\
& 0.00000 \\
& 0.00000 \\
& 0.01 \\
& 0.00000 \\
& 0.00000 \\
& 0.00000 \\
& 0.00000 \\
& 0.0000000 \\
& 0.00000 \\
& 0.00000 \\
& 0.00000 \\
& 0.00000 \\
& 0.00000 \\
& 0.00000 \\
& 0.00000 \\
& 0.000
\end{aligned}
$$

ALL ABOVE ANSWERS SHOUL? BE O FOR THIS TEST SEGMENT TO BE SUCCESSFUL. 


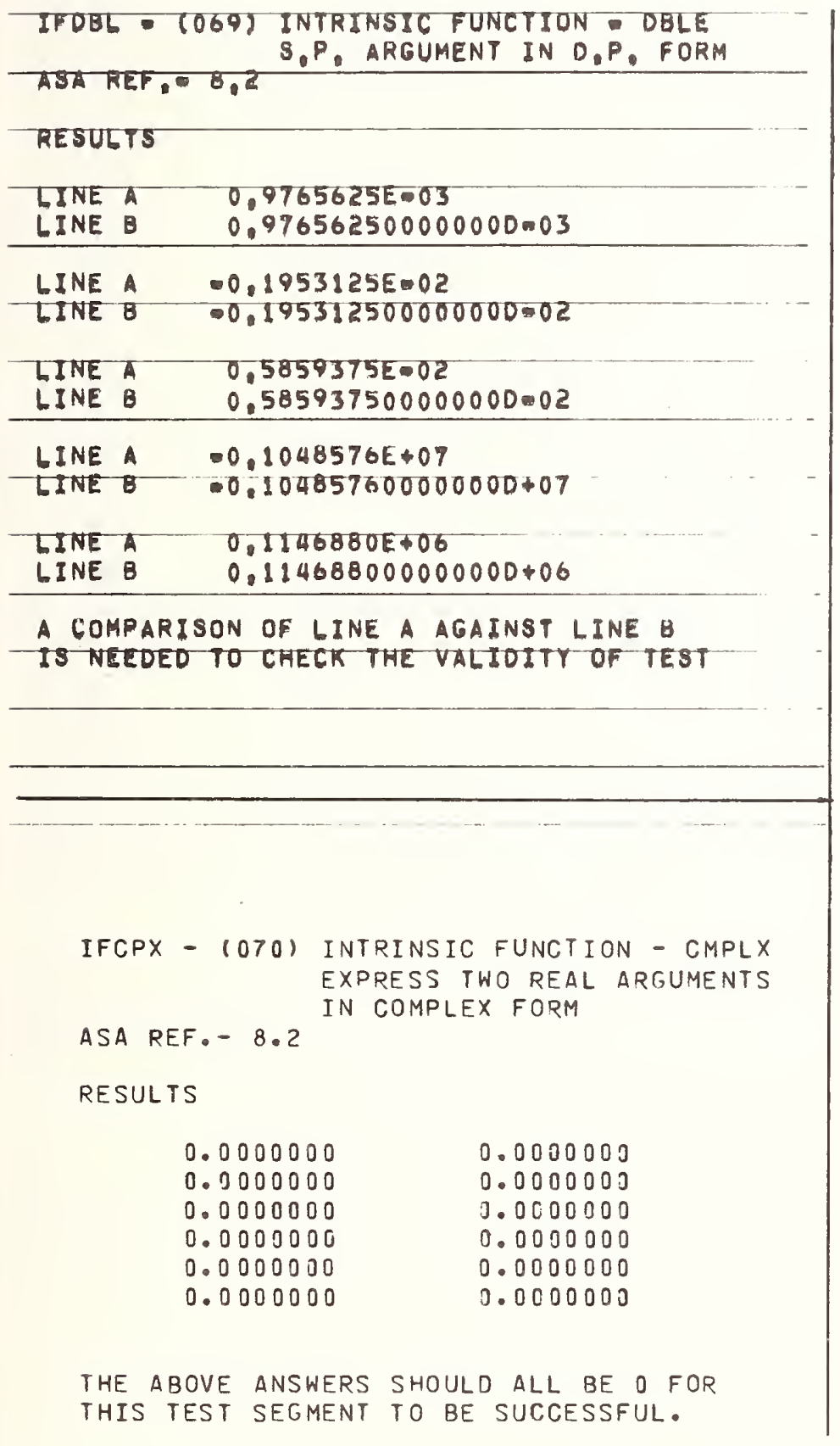

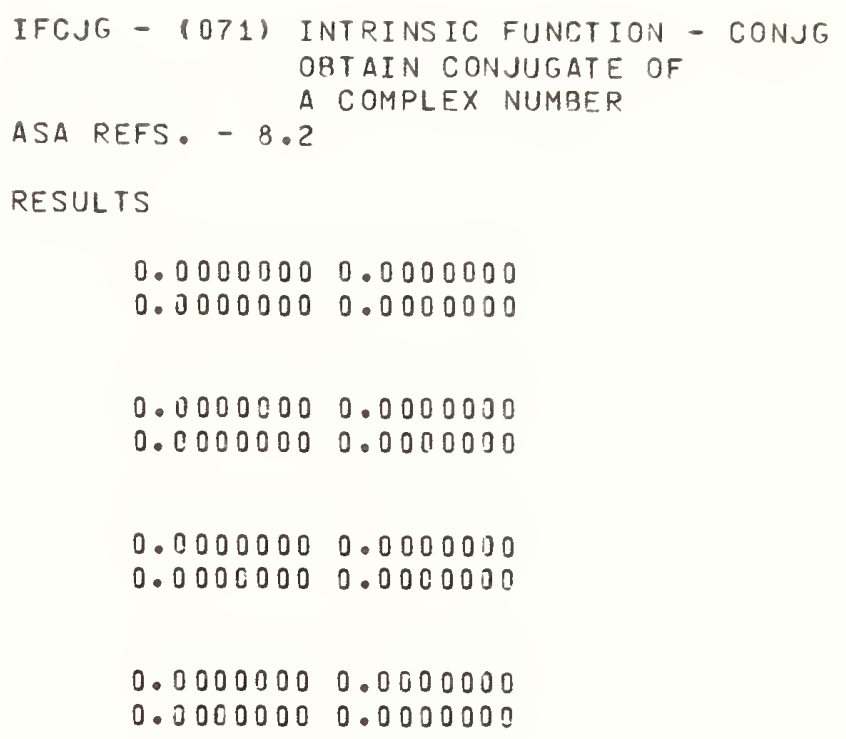

RESULTS

ALL ABOVE ANSWERS MUST BE O FOR THIS TEST SEGMENT TO BE SUCCESSFUL. 


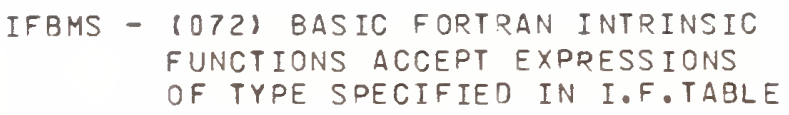

ALL ABOVE ANSWERS SHOULD BE O FOR THIS TEST SEGMENT TO BE SUCCESSFUL.

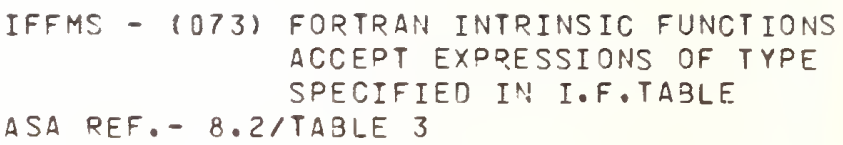

$$
\begin{aligned}
& 0 \\
& 0 \\
& 0 \\
& 0
\end{aligned}
$$

TEST OF AMOD, MOD IN EXDRESSIONS

0.

0 .

0

TEST OF AMAXO,AMAX1, MAXO,MAX1 AND DMAX 0.

0 . 0 0 . 
TEST OF AMINO, AMIN1, MINO, MIN1 AND OMIN

0 . 0
0
0

TEST OF DSIGN AND OBLE IN EXPRESSIONS

$$
\begin{aligned}
& 0 . \\
& 0 . \\
& 0 .
\end{aligned}
$$

TEST OF DIM AND IDIM IN EXPRESSIONS

$$
0 \text {. }
$$

TEST OF SNGL, REAL, AIMAG, CMPLX AND CONJG IN EXPRESSIONS

$$
\begin{aligned}
& 0 . \\
& 0 .
\end{aligned}
$$

TEST OF SOME COMBINATIONS OF ABOVE INTRINSIC FUNCTIONS

$$
0 \text {. }
$$

0.

ALL ABOVE ANSWERS SHOULD BE 0 FOR THIS SEGMENT TO BE SUCCESSFUL.
F O R T R A N TEST PROAR A M S PREPAREO BY NATIONAL GUREAU OF STANDARDS FOR USE ON LARGE FORTRAN PROCESSORS IN ACCORDANCE WITH ASA FORTRAN $\times 3.9-1966$ VERSION 3 PART 7

SAMPLE COMPUTER, FORTRAN COMPILER LEVEL OPERATING SYSTEM VERSION DATE, INSTALLATION NAME

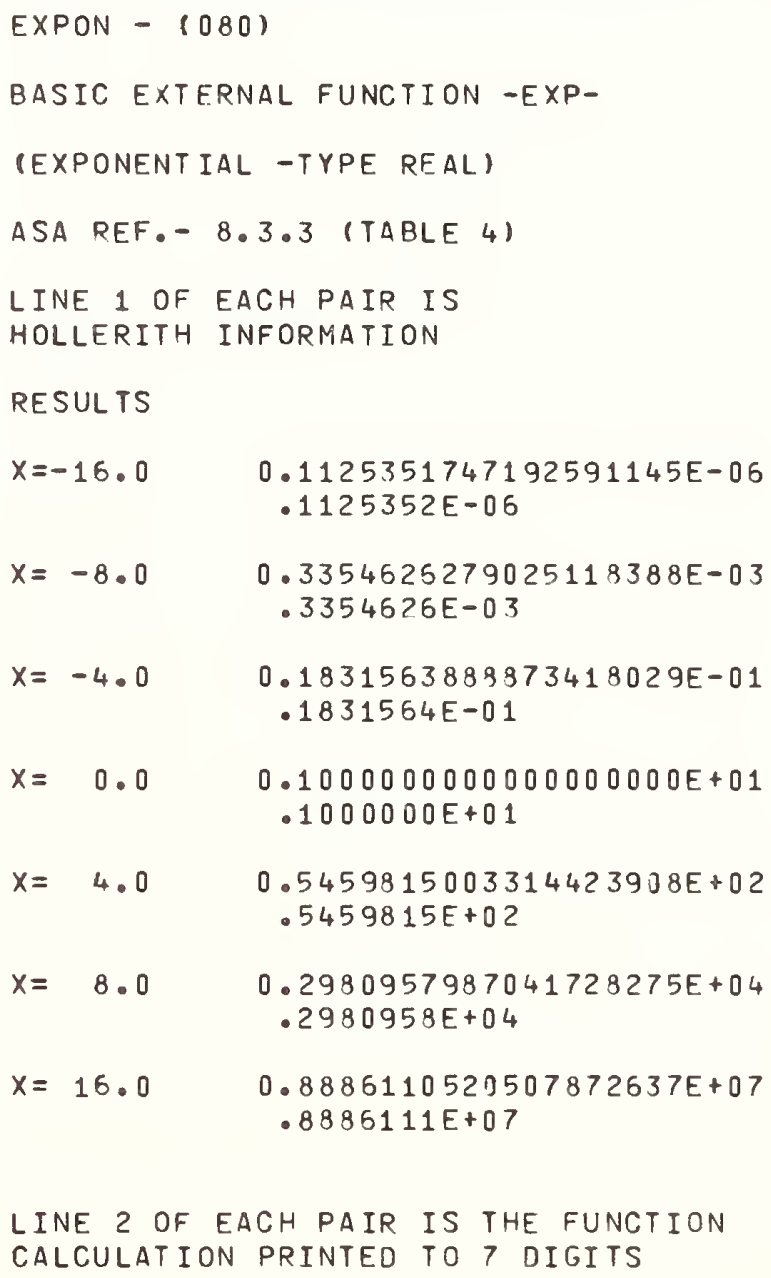


DEXPO - $(081)$

BASIC EXTERNAL FUNCTION - DEXP-

(EXPONENTIAL - TYPE DOUBLE PRECISION) ASA REF.- 8.3 .3 (TABLE 4)

LINE 1 OF EACH PAIR IS

HOLLERITH INFORMATION

RESULTS

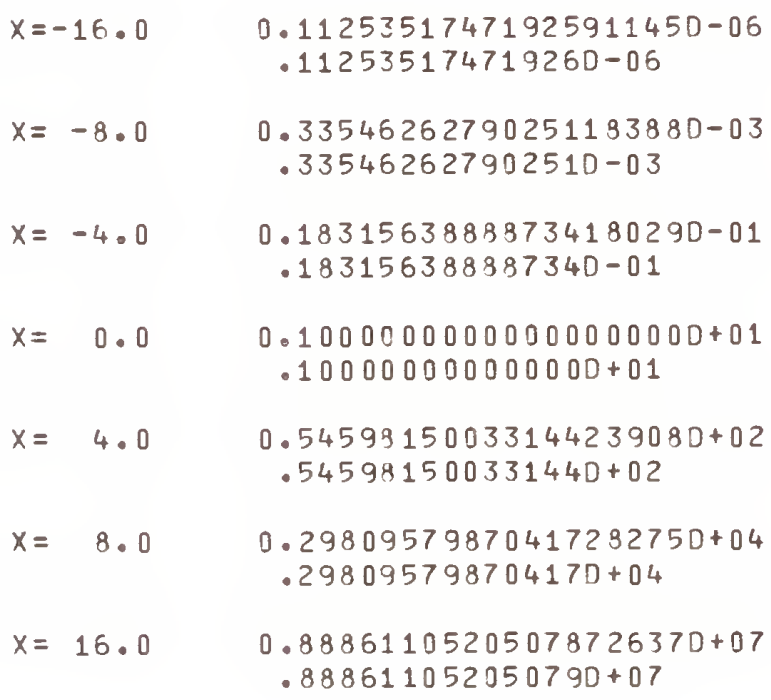

LINE 2 OF EACH PAIR IS THE FUNCTION CALCULATION PRINTED TO 14 DIGITS
CEXPO- $(082)$

BASIC EXTERNAL FUNCTION - CEXF-

(EXPONENTIAL - TYPE COMPLEX)

ASA REF, $8,3,3$ (TABLE 4)

(COMPLEX ARGUMENT)

EXPECTED RESULT

FUNCTION RESULT

$(-0,1611810 E+02,-0.7330383 E+01)$

$0.5000000 E=07 \quad 0.8660254 E=07$

$0.5000000 E-07-0.8660254 E=07$

$(=0,1450866 E+02,-0,7330383 E+01)$

$0.2500000 E-06=0.4330127 E=06$

$0.2500000 E-06 \quad-0.4330127 E-06$

(-0.138I55IE+02, $=0.6283185 E+0 I)$

$0.1000000 E=05 \quad 0.0000000 E+00$

$0.1000000 E=05 \quad 0.0000000 E+00$

$(-0,1220607 E+02,-0,6283185 E+01)$

$0.5000000 E-05 \quad 0.0000000 E+00$

$0.5000000 E-05 \quad 0.0000000 E+00$

$(-0.1151293 E+02,=0.5235988 E+01)$

$0.5000000 E-05 \quad 0.8660254 E=05$

$0.5000000 E=05 \quad 0.8660254 E=05$

$(-0,9903488 E+01, \pi 0.5235988 E+01)$

$0.2500000 E=04 \quad 0.4330127 E=04$

$0.2500000 E=04 \quad 0.4330127 E=04$

$(-0,9210340 E+01,-0.4188790 E+01)$

$-0,5000000 E-04 \quad 0.8660254 E-04$

$-0.5000000 E=04 \quad 0.8660254 E-04$

$(=0,7600902 E+01,-0.4188790 E+01)$

$-0.2500000 E=03 \quad 0.4330127 E=03$

$-0.2500000 E=03 \quad 0.4330127 E-03$

$(-0,6907755 E \$ 01,0.314(593 E+01)$

$-0.1000000 E-02 \quad 0.0000000 E+00$

$=0.1000000 E-02 \quad 0.0000000 E+00$

$(-0.5298317 E+01,=0.3141593 E+01)$

$0.5000000 E-02 \quad 0.0000000 E+00$

$.0 .5000000 E-02$ 0.0000000Eक00 


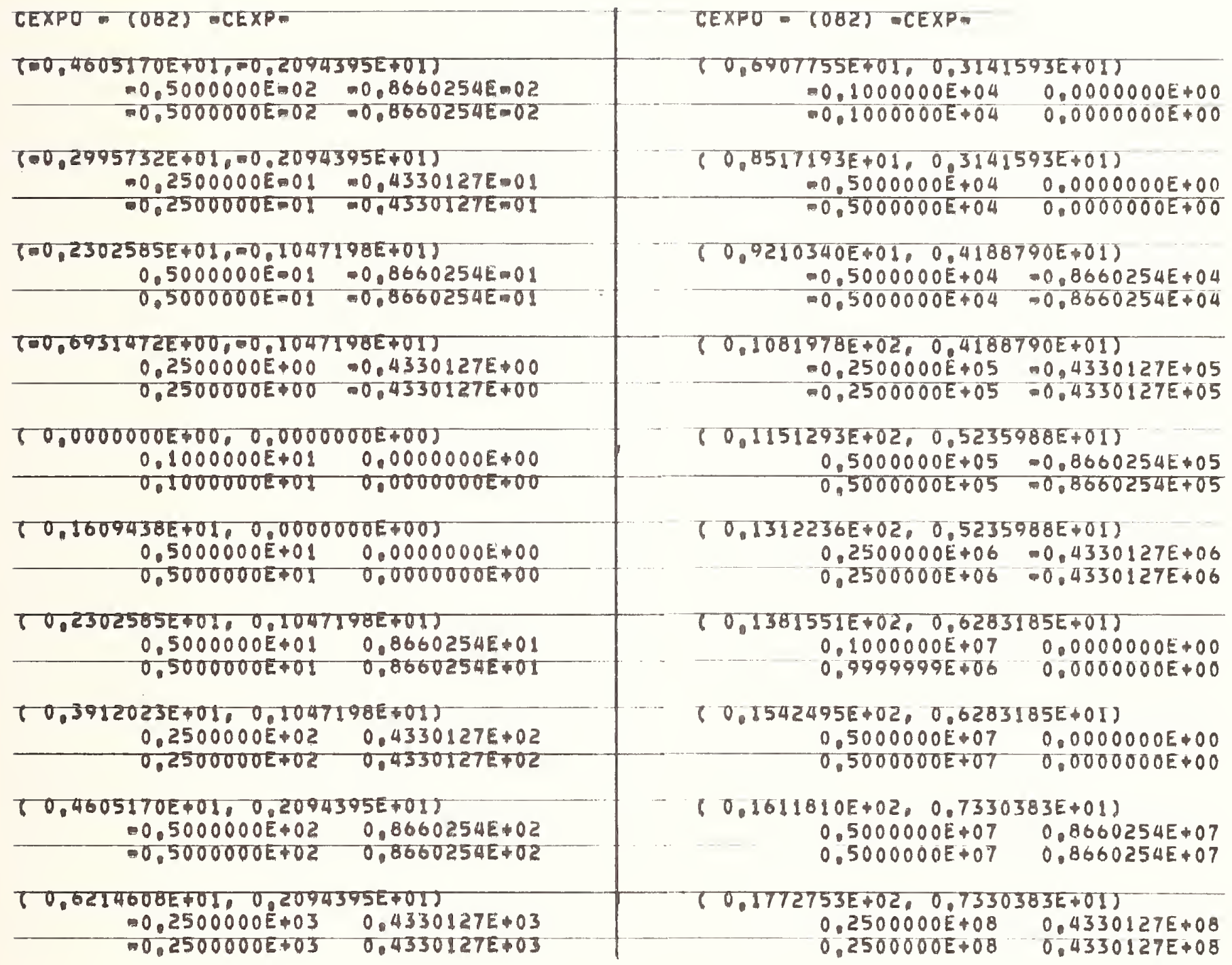


LOGTM - (083)

BASIC EXTERNAL FUNCTION - ALOG-

(NATURAL LOG - TYPE REAL)

ASA REF. - 8.3 .3 (TABLE 4)

LINE 1 OF EACH PAIR IS

HOLLERITH INFORMATION

RESULTS

$x=0.125 \quad-2.0794415416798359$

$-2.079442$

$x=0.25 \quad-1.3862943611198906$

$-1.386 ? 3.4$

$x=0.5 \quad-0.6931471805599453$

$-.6931472$

$x=1.0 \quad 0.0003000000000000$

0.0003000

$x=1.5 \quad 0.4054651081081644$ .4054551

$x=2.0 \quad 0.6931471805599453$ .5931472

LINE 2 OF EACH PAIR IS THE FUNCTION CALCULATION PRINTEO TO 7 DIGITS

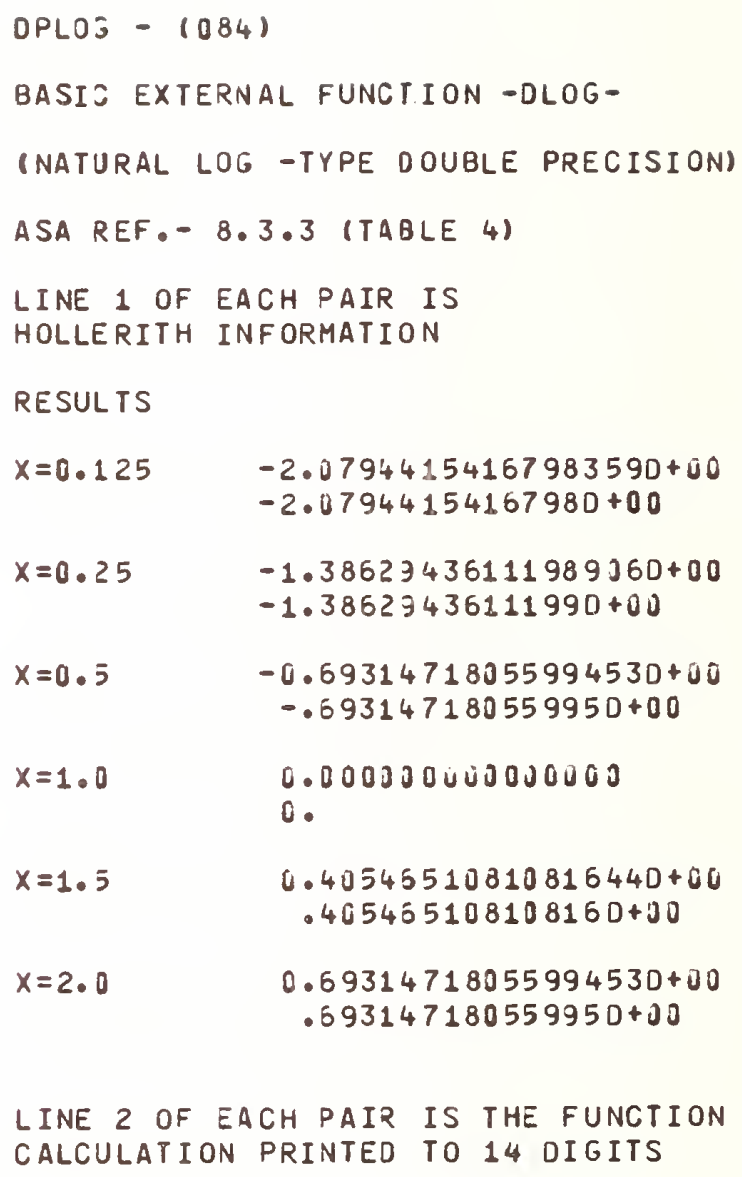

LINE 2 OF EACH PAIR IS THE FUNCTION CALCULATION PRINTED TO 14 DIGITS 
CXLOG-(085)

BASIC EXTERNAL FUNCTION = CLOG-

(NATUKAL LUG - TYPE COMPLEX)

ASA REF.= 8.3.3 (TABLE 4)

(COMPLEX ARGUMENT)

EXPEGTEO AESULT

FUNCTION RESULT

$(0.5000000 E=07.00 .8000254 E=07)$

$=0.1011$ HUE- $12-0.1047198 E-0$

$-0.1611810 E$ O2 $-0.1047198 E \quad 01$

( $0.2500000 E-06 .-0.4330127 E-06)$

$=0.1454$ HOOt $t 2$.

$=0.1450806 E$ U2 $=0.1047198 \mathrm{E}$ U1

$(0.1000000 E-U 5.0 .0000000 E$ OO)

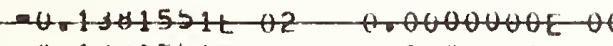

$-0.1381531 E 02$ U. 0000UUOE OO

$(0.5000000 \mathrm{E}=05.0 .0000000 \mathrm{E}, 00)$

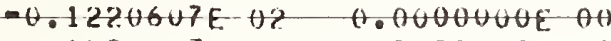

- U.12206U7E U? 0.0000U00E 0O

$(0.5000000 E=05,0.8600754 E-05)$

$=0.11512 \times 3 E$ UL $-0.1647148 E 0$

$-0.11512 y 3 E$ O2 $0.1047198 E$ UI

$(0.2500000 E-04.0 .4330127 E-04)$

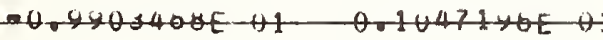

$-0.991) 5408 E$ UI D.1047198E O1

$(=0.5000000 E-04,0.8060254 E-04)$

$=0.9210340 E-01 \quad 0.2094345 E-01$

$-0.9210340 E$ I) 1 0.2094395E 01

$(-0.2500000 E-03.0 .4330127 E-U 3)$

$-0.7000902 E-01-0.2094395 E-01$

$-0.7600902 E$ 01 $0.2094345 E$ OI

$(=0.1000000 E-02,0.0000000 E$ OO)

$-4.5404755 E_{-01}$

- $0.64077 \supset 5 E$ O1 1$) .3141543 E$ O
CXLUG $=(085)-$ CLOG-

$(-0.500000 U E-0<-0), 8 B 60254 E-02)$

-U.HOUS1/0E -11-0. $20943 \times 5 E-21$

$-0.46051101 .01-11.2094395 E$ UI

$(=0.25000(1 \cup E-0) .=0.4330) 1 \angle E=01)$

- U.2945732t fl1 -0.2094395E 41

$\begin{array}{llll}-0.2945732 E & 01 & -0.2094595 F & 1\end{array}$

( U. SUOOOUUE-0I.-1). HAOOPS $4 E=01)$

- U. PUUSUSE D1 - $11.104 / 148 E 01$

$-0.2 S U \angle S O S E$ OI $=0.104 / 1 Y 8 F$ OI

( $0.2500000 \mathrm{E}$ NU. $=0.4330121 \mathrm{E}$ UU)

$=0.04314 / 2 t$ (1) -0.164714 \&E 01

$-0.5 Y 314(2 t$ Un) $\quad 0.10471 y 0 E$ UI

( 0.10UUOUUE 01. 1). (10001100t 00)

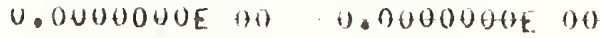
U. OUUDIIUUE UO O.0(10UDUOE UO

( $0.3000000 \mathrm{E} 11.0 .0000$ OOOE OO) $0.100943 b_{k} H 1$ 0.0000000E OO U.100Y43BE U1 O.000UUOUE OU

$(0.5000600 \mathrm{O}$ (91. U.4000254E 1)1) $0.2302505 t$ - 1 - $0.104+148 E-4 t$

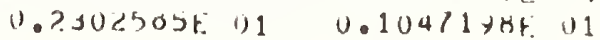

( $0.2500000 E(12,0.43301276$ U2) (1).3\1दU<3E 01 0.10471 × ती औ1 $0.3 \Rightarrow 12023 E$ O 0.10471 प8 01

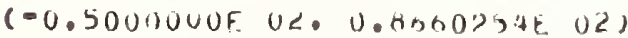
U.46(151/0k ()) $0.2044345 E 01$ U.4 DUS1/UE 1) O. 2 U943पלE UI

$(=0.2500$ UUUE US. $0.433012 / E$ O3) v. $6214603 t$ 1) 1 . $0.2044345 E-(21$ U.6214UUSE O1 $0.2494345 F$ O1 


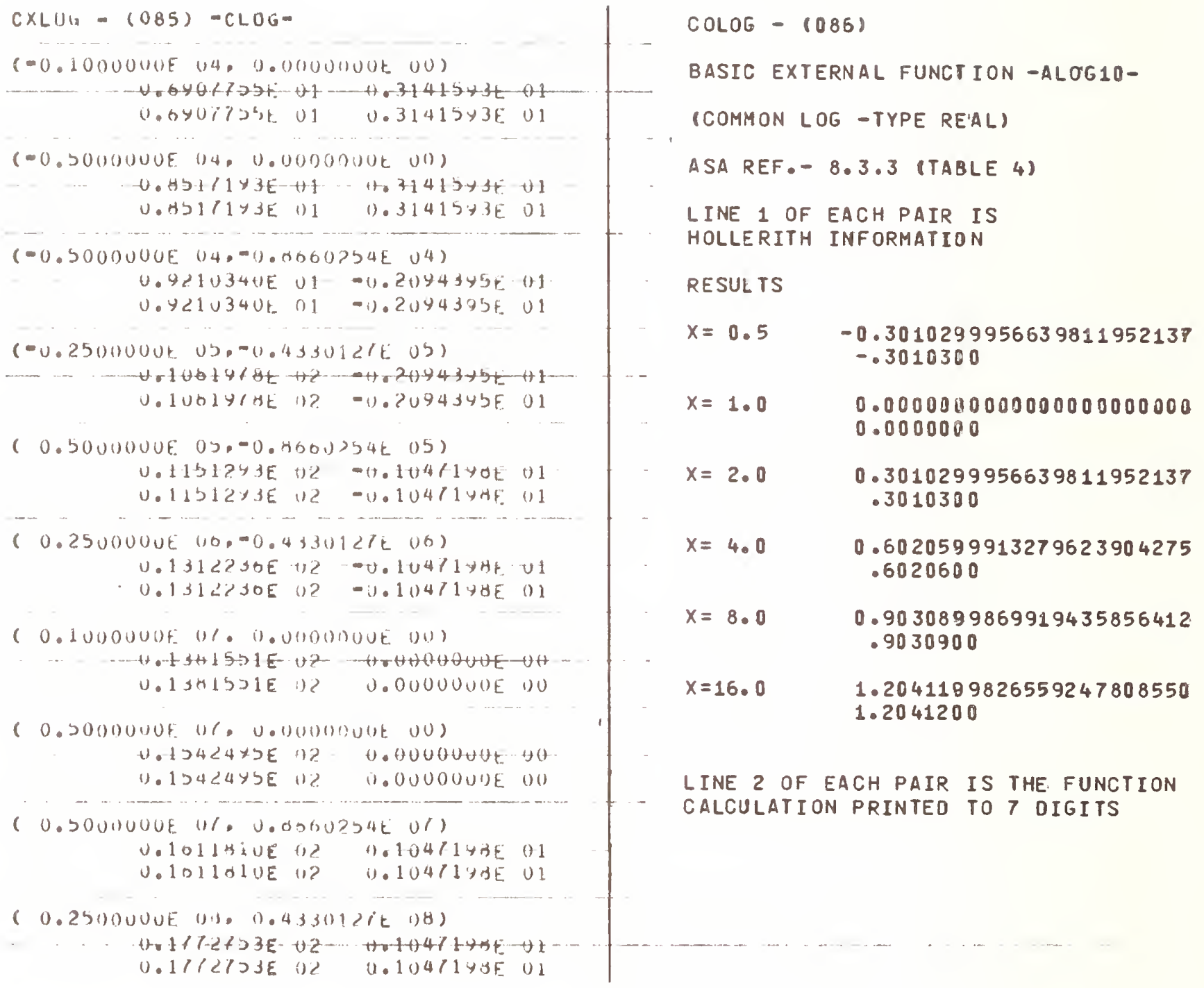




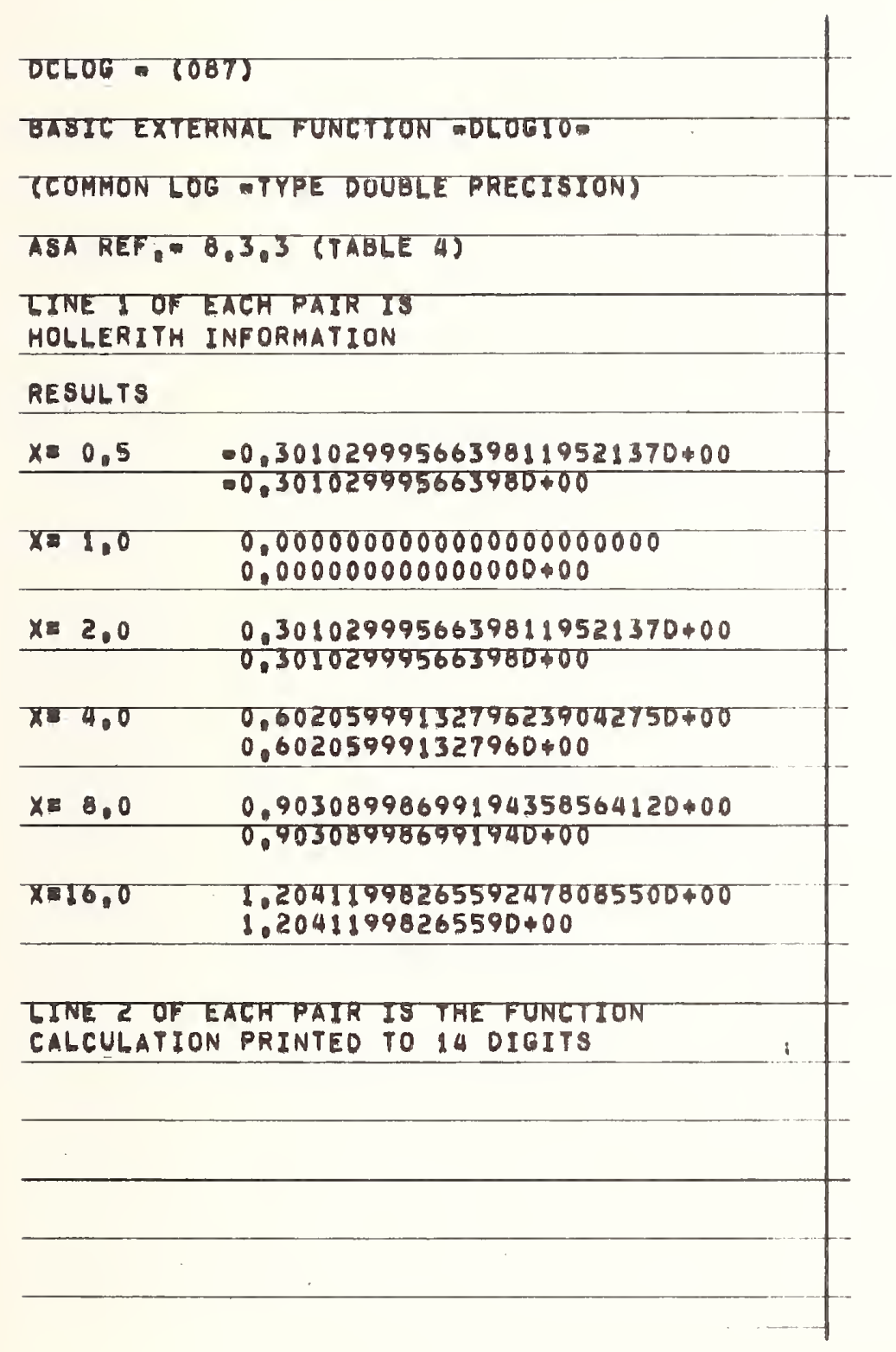

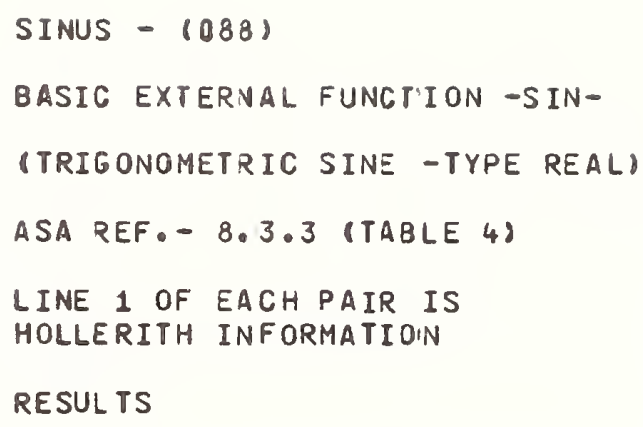

LINE 2 OF EACH PAIR IS THE FUNCTION CALCULATION PRINTED TO 7 DIGITS 


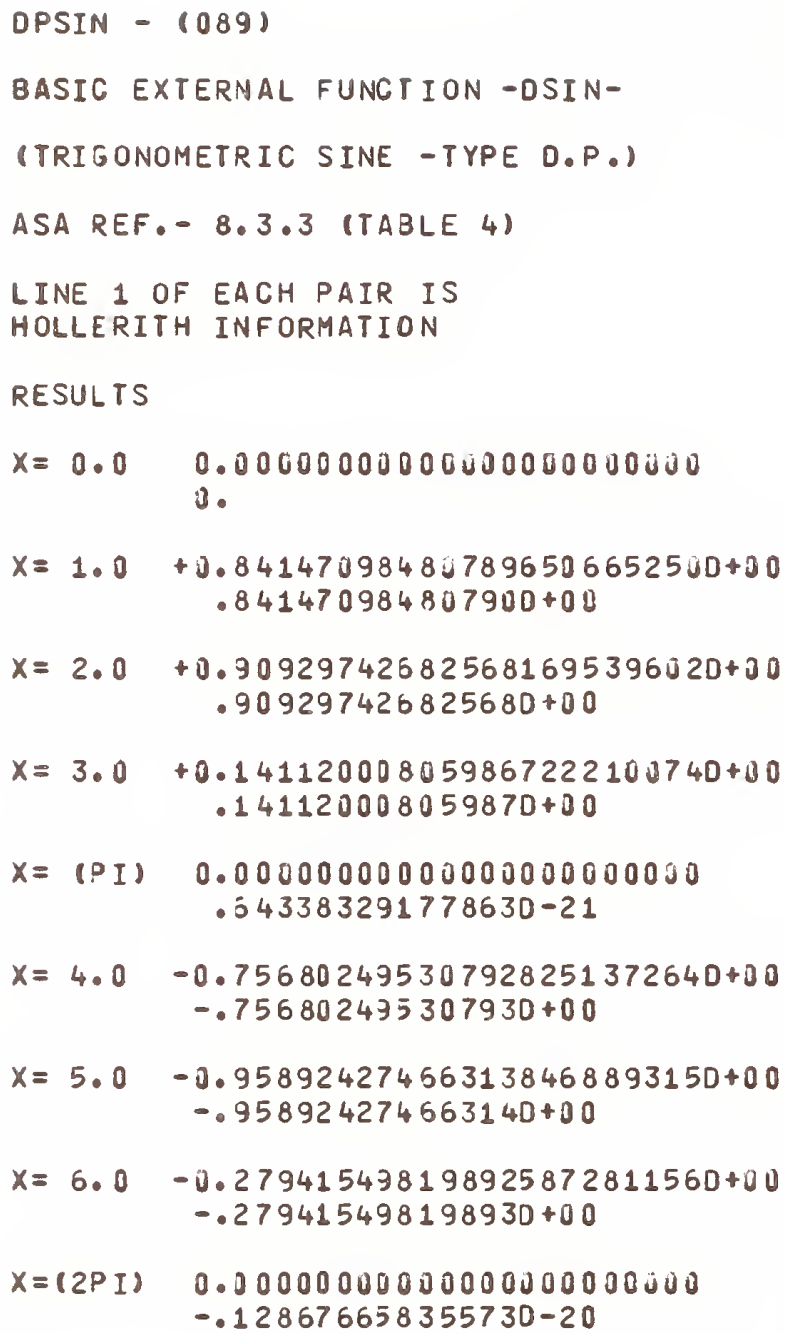

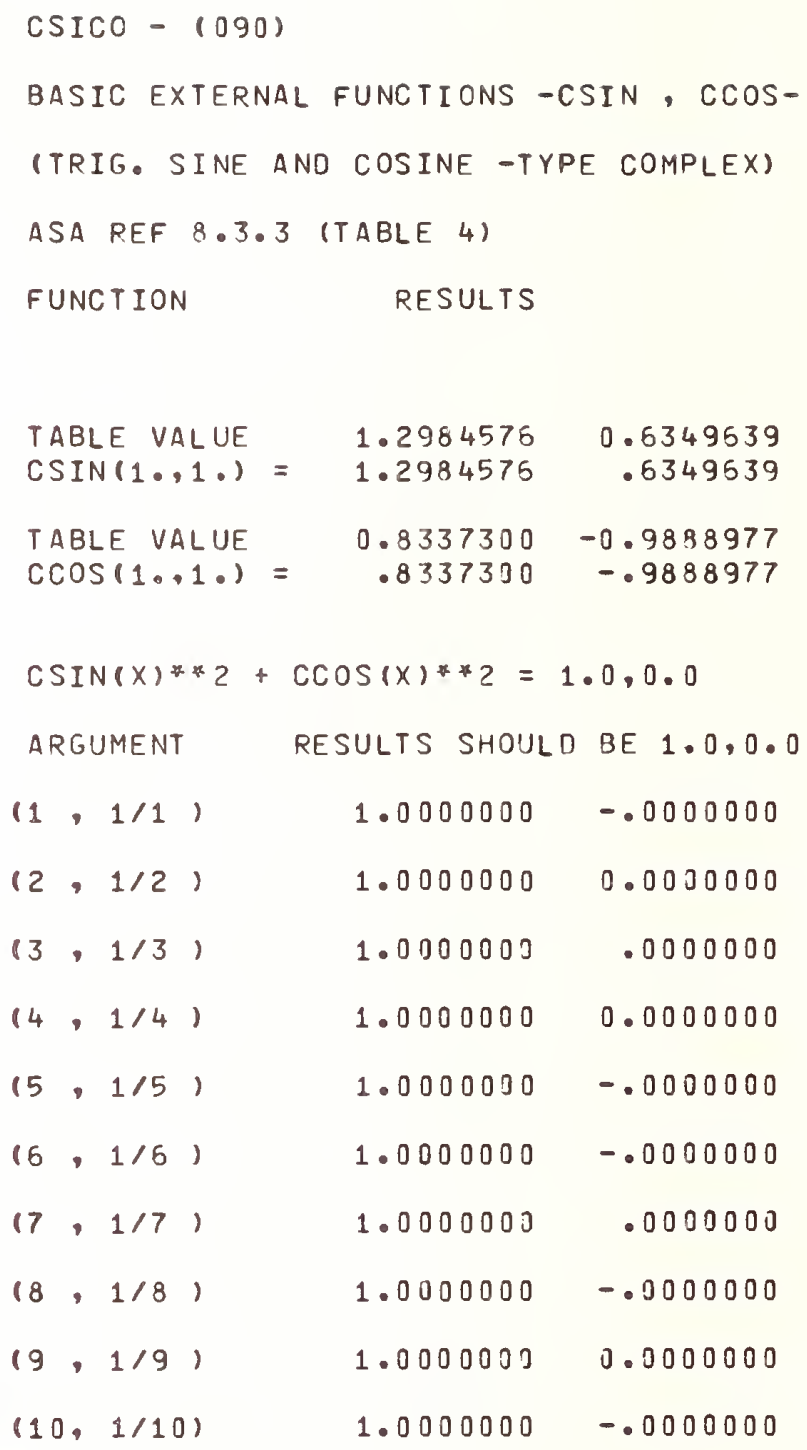




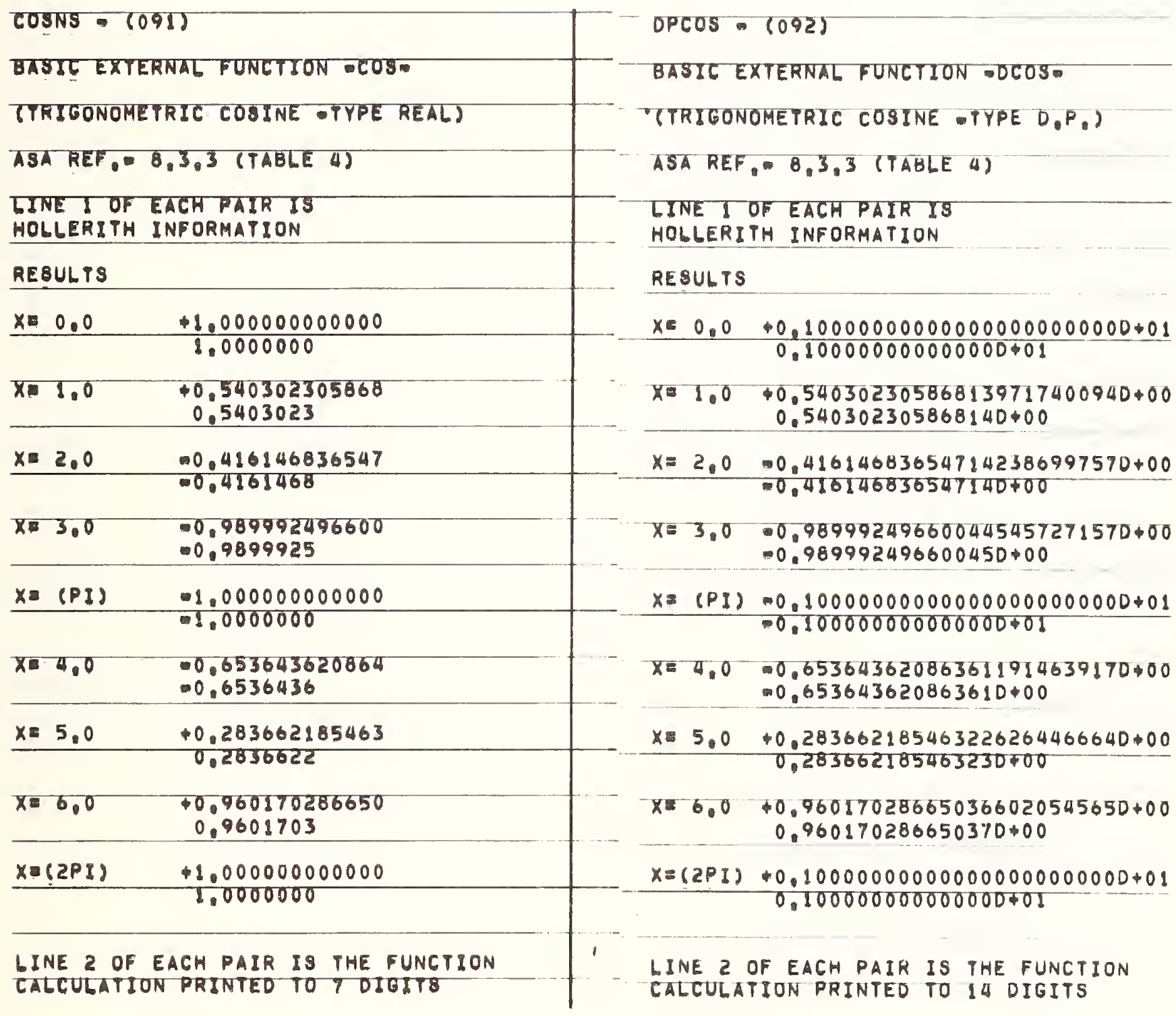


FORTRANTESTTPROGRAM 5 PREPARED BY NATIONAL BUREAU OF STANDARDS FOR USE ON LARGE FORTRAN PRDCESSORS IN ACCORDANCE WITH ASA FORTRAN X3.9-1966 VERSION 3 PART 8

SAMPLE COMPUTER, FORTRAN COMPILER LEVEL OPERATING SYSTEM VERSION DATE, INSTALLATION NAME

TANGH $-(094)$

BASIC EXTERNAL FUNCTION ITANHA (HYPERBOLIC TANGENT -TYPE REAL)

ASA REF $8.3,3$ (TABLE 4)

LINE I OF EACH PAIR IS HOLLERITH INFORMATION

RESULTS

$x=0.0$ $\frac{0.0000000000}{0.0000000}$

$x=2,0$

0.9640275801

0.9640276

$x=2,5$

0.9866142982

0.9866143

$x=4.0$

0.9993292997

0.9993293

$x=6,0$

0.9999877117

0.9999877

$x=8,0$

0.9999997749

0.9999998

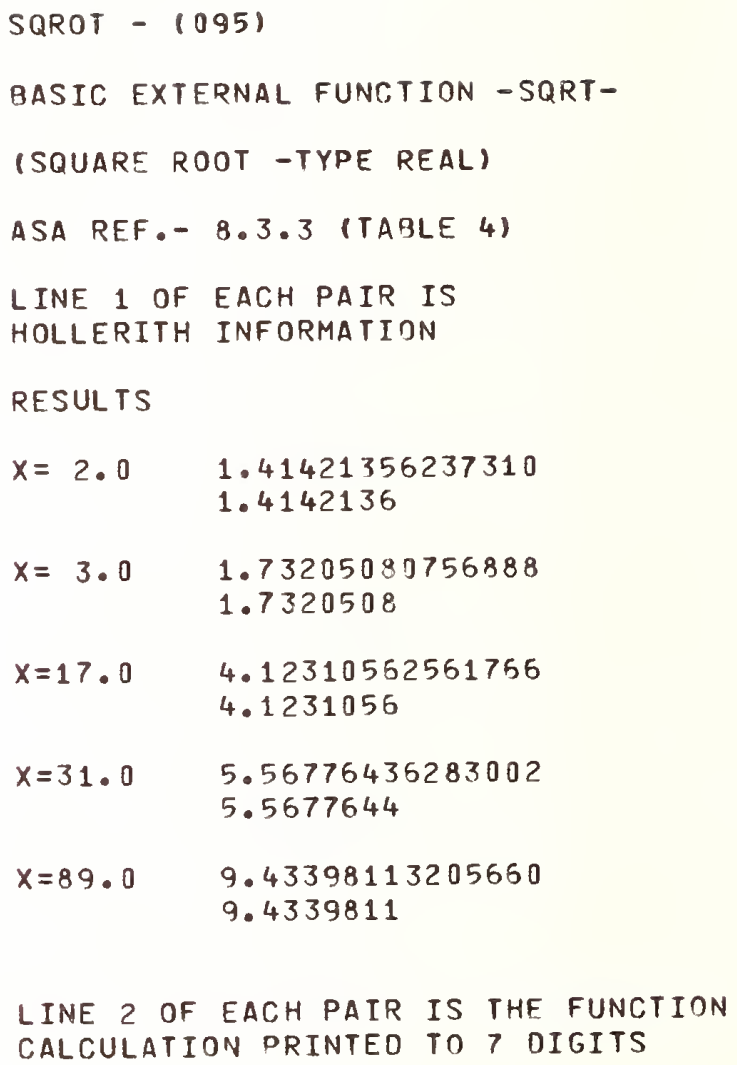




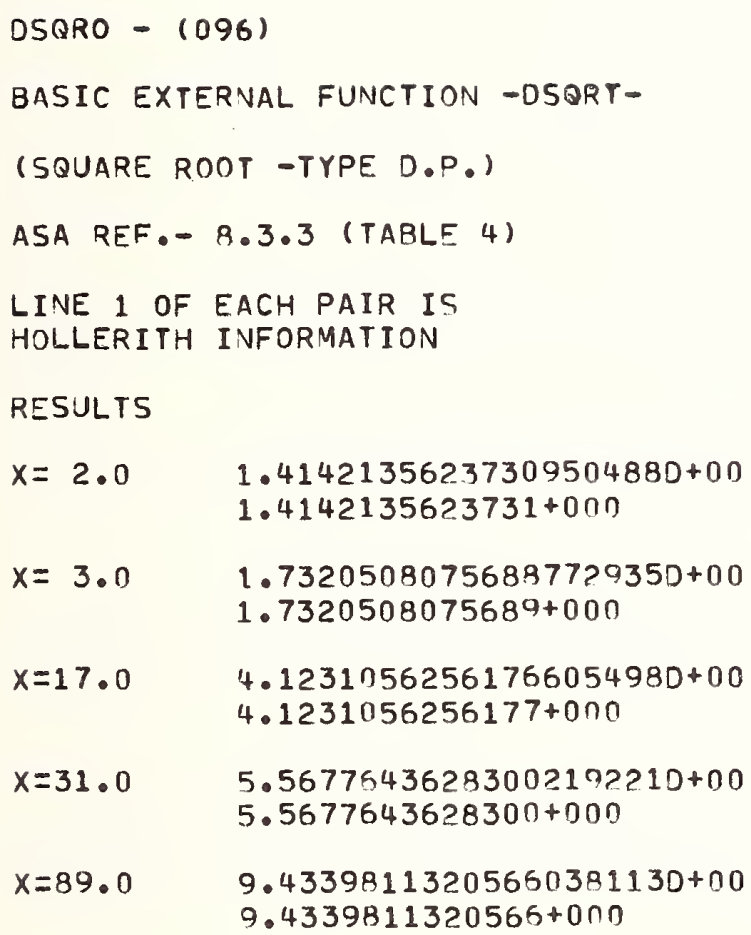

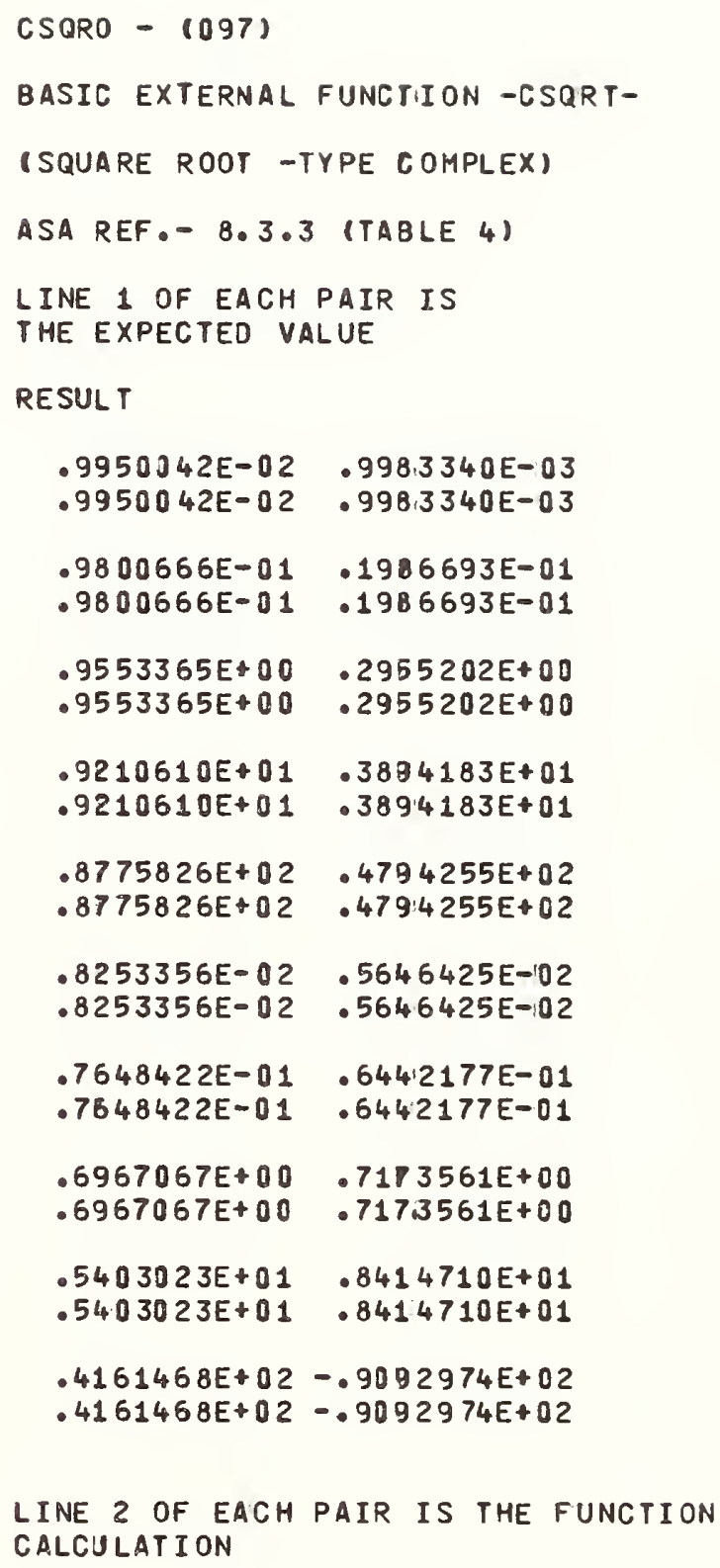

LINE 2 OF EACH PAIR IS THE FUNCTION CALCULATION 


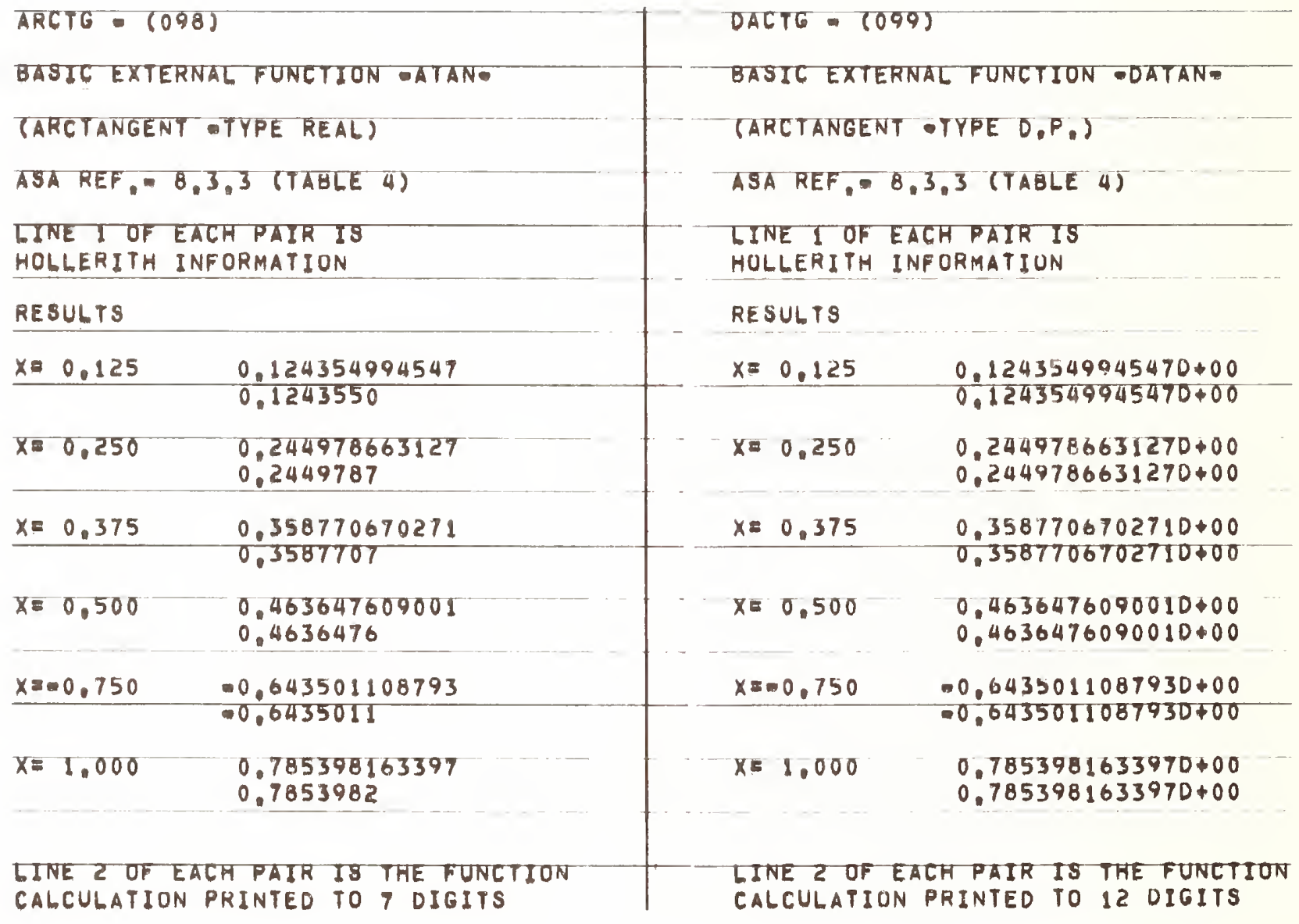




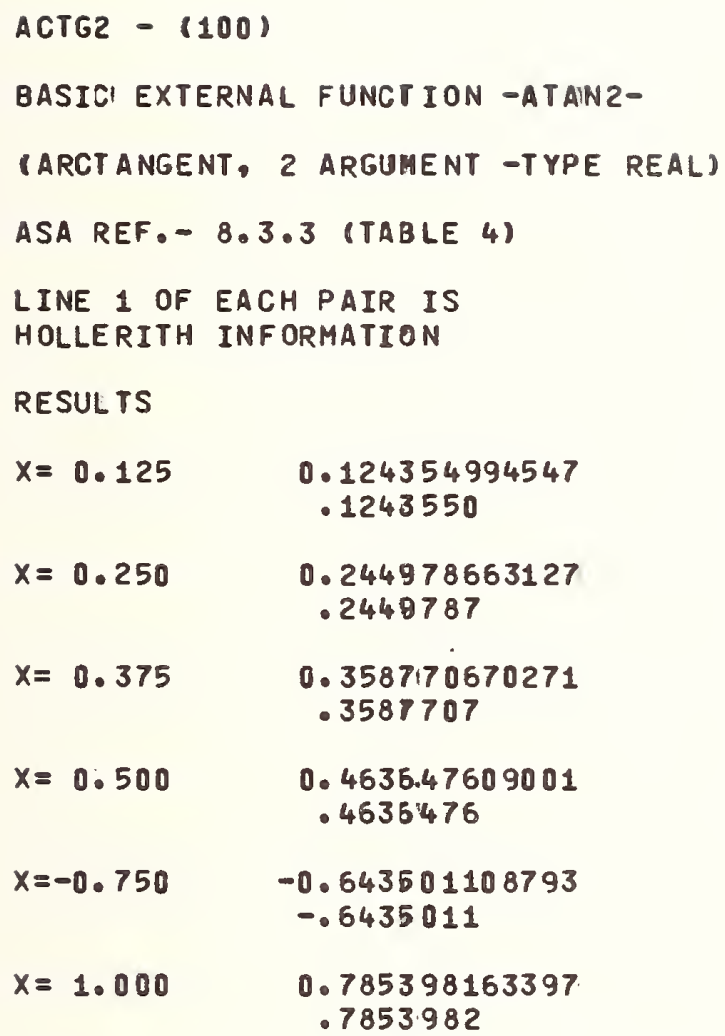

LINE 2 OF EACH PAIR IS THE FUNCTION CALCULATION PRINTED TO 7 DIGITS
DATN2 - (101)

BASIC EXTERNAL FUNCTION -DATANZ(ARCTANGENT, 2 ARGUMENT -TYPE D.P.) ASA REF - 8.3 .3 (TABLE 4)

LINE 1 OF EACH PAIR IS HOLLERITH INFORMATION

RESULTS

$x=0.125$

$0.124354994547 ! n+00$ $.124354994547+000$

$x=0.250$

$0.2449786632270+00$ $.244978663127+000$

$x=0.375 \quad 0.3587706702710+00$ $.358770670271+000$

$x=0.500 \quad 0.4636476090010+00$ $.463647609001+000$

$x=-0.750 \quad-0.6435011087930+00$ $-.643501108793+000$

$x=1.000 \quad 0.7953991633970+00$ $.735398163397+000$

LINE 2. OF EACH PAIR IS THE FUNCTION CALCULATION PRINTEN TO 12 DIGITS 


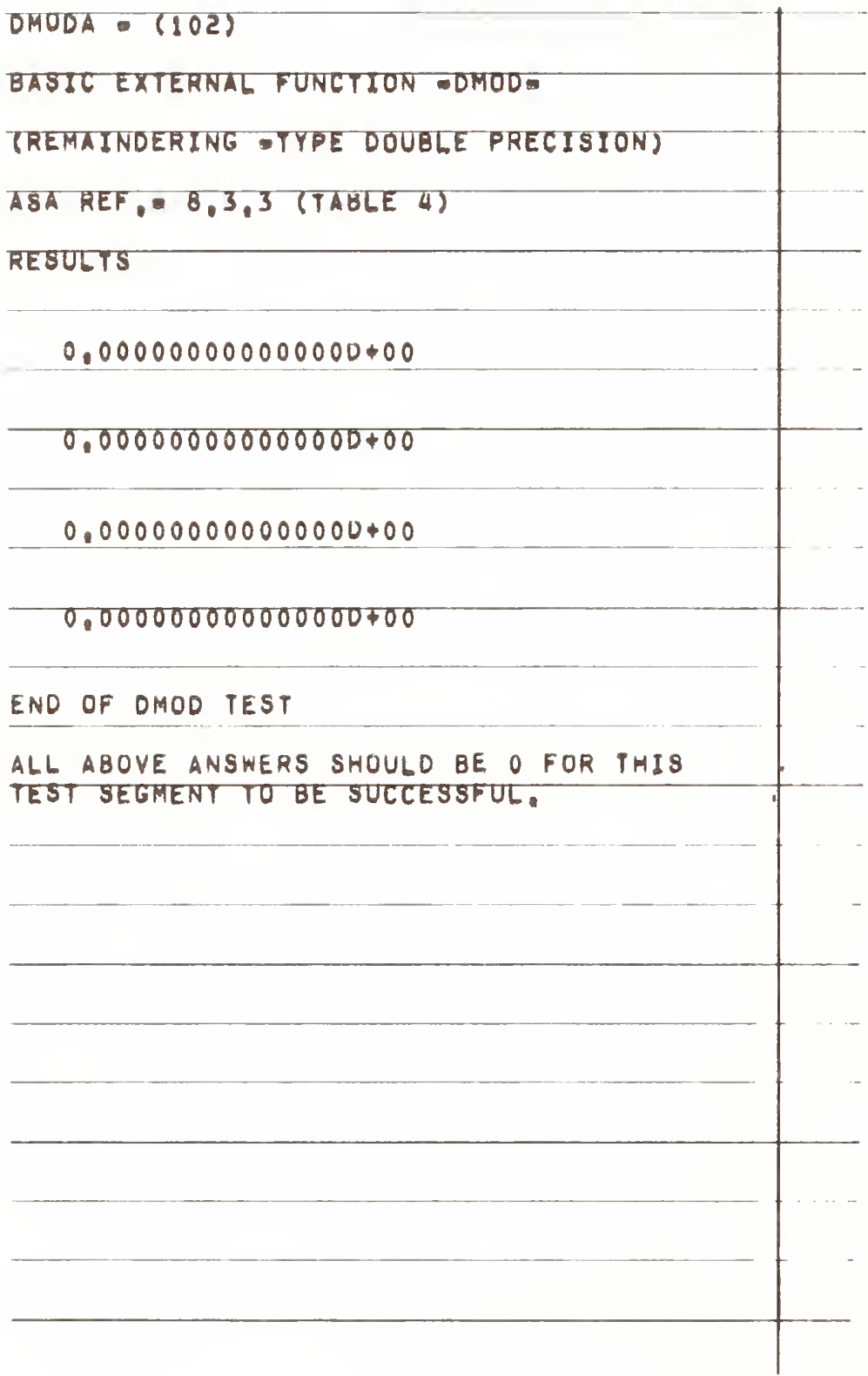

CABSA - (103)

BASIC EXTERNAL FUNGTION - CABS-

(MOOULUS OF A COMPLEX NUMBER)

ASA REF.- 8.3 .3 (TABLE 4)

RESULTS

SET 1

SET 2

$$
\begin{aligned}
& .100000 E-36 \\
& .100000 E-05 \\
& .100000 E-04 \\
& .100000 E-03 \\
& .100000 E-02 \\
& .100000 E-01 \\
& .100000 E+00 \\
& .100000 E+01 \\
& .100000 E+02 \\
& .100000 E+03 \\
& .100000 E+04 \\
& .100000 E+05 \\
& .100000 E+06 \\
& .100000 E+07 \\
& .100000 E+08
\end{aligned}
$$$$
.500000 E-05
$$$$
.500000 E-05
$$$$
.500000 E-04
$$$$
.500000 E-03
$$$$
.500003 E-02
$$$$
.500000 E-01
$$$$
.500000 E+00
$$$$
.500000 E+01
$$$$
.500000 E+02
$$$$
.500000 E+03
$$$$
.500000 E+04
$$$$
.500000 E+05
$$$$
.500000 E+06
$$$$
.503003 E+07
$$$$
.500000 E+08
$$

VALUES IN EACH SET SHOULD BE POSITIVE . 1 FOR SET 1 (.5 FOR SET 2), EXPONENT RANGE FROY - 05 TO +08 IN SERUENCE 


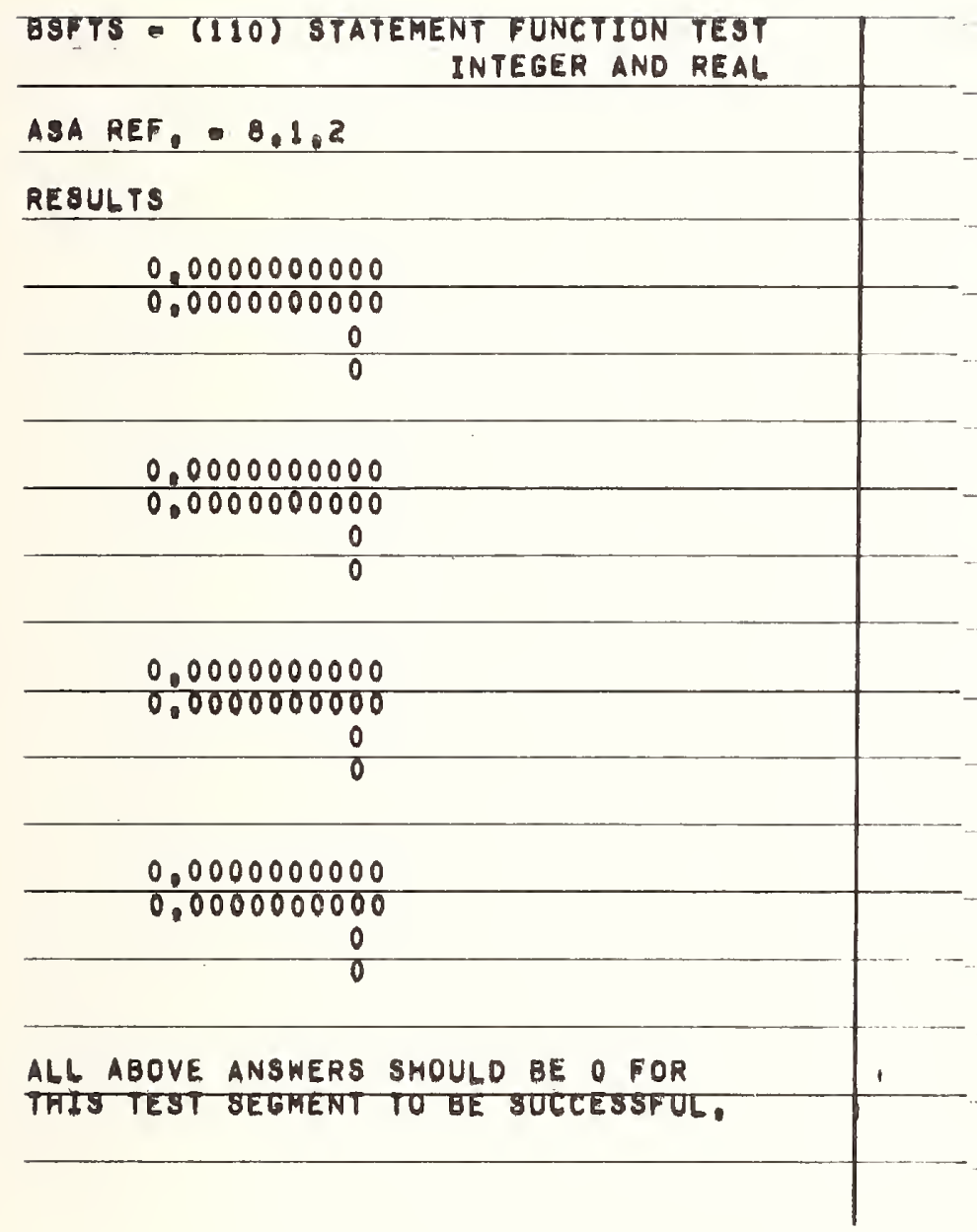

FSFTS - (III) STATEMENT FUNCTION TEST DOUBLE PRECISION, COHPLEX AND LOGICAL ASA REF, $8,1,2$

RESULTS

$0.0000000000000000000+00$

$0,0000000000000000000+00$

$0.0000000000000000000+00$

$0,0000000000000000000+00$

$0.0000000000000000000+00$ $0,0000000000000000000 \$ 00$ $0,0000000000000000000+00$ $0,0000000000000000000+00$

$\begin{array}{ll}0.0000000 & 0.0000000 \\ 0.0000000 & 0.0000000 \\ 0.0000000 & 0.0000000 \\ 0.0000000 & 0.0000000\end{array}$

ALL ABOVE ANSWERS SHOULO BE O FOR THIS TEST SEGMENT TO BE SUCCESSFUL, VALUES

WITH EXPONENTS LESS THAN IO* $(\pi 14)$ ARE CUNSIDERED ZERO

THE FOUR ABOVE ANSWERS SHOULD BE TRUE FOR THIS SEGMENT TO BE SUCCESSFUL 
F O R T R A H T E S T PRA G R A M S

PREPARED BY NATIONAL BUREAU OF SIANDARDS

FOR USE ON LARGE FORTRAN PROCESSORS

IN ACCORDANCE WITH ASA FORTRAN $\times 3.9 .1966$

VERSION 3 PART 9

SAMPLE COMPUTER, FORTRAN COMPILER LEVEL

OPERATING SYSTEM VERSION

DATE, INSTALLATION NAME

CPXAD - (140) COMPLEX ADDITION AND SUBTRACTION

ASA REF. - 6.1

RESULTS

$\begin{array}{ll}.0000 & .0000 \\ .0000 & .0000 \\ .0000 & .0000 \\ .0000 & .0000 \\ .0000 & .0000 \\ .0000 & .0000 \\ .0000 & .0000 \\ .0000 & .0000 \\ .0000 & .0000 \\ .0000 & .0000 \\ .0000 & .0000\end{array}$

TEST IS POSITIVE IF NUMBERS PRINTED ABOVE ARE 0.0 .0 .0
CPXMU - (141) COMPLEX MULTIPLICATION

ASA REF. 6.1

RESULTS

\begin{tabular}{ll}
1.000 & 0.000 \\
\hline 1.000 & 0.000 \\
1.000 & 0.000 \\
1.000 & 0.000 \\
1.000 & 0.000 \\
1.000 & 0.000 \\
1.000 & 0.000 \\
1.000 & 0.000 \\
1.000 & 0.000 \\
1.000 & 0.000 \\
1.000 & 0.000 \\
1.000 & 0.000 \\
1.000 & 0.000 \\
1.000 & 0.000 \\
1.000 & 0.000 \\
1.000 & 0.000 \\
1.000 & 0.000 \\
1.000 & 0.000 \\
1.000 & 0.000 \\
1.000 & 0.000
\end{tabular}

TEST IS POSITIVE IF NUMBERS PRINTED ABOVE ARE $1,0,0,0$

ERROR SHOULD NOT EXCEED + OR -.0OT 
CPXDV - $(142)$ DIVISION OF COMPLEX NUMBERS

ASA REF.- 6.1

RESULTS

$\begin{array}{ll}1.0000 & 1.0000 \\ 1.0000 & 1.0000 \\ 1.0000 & 1.0000 \\ 1.0000 & 1.0000 \\ 1.0000 & 1.0000 \\ 1.0000 & 1.0000 \\ 1.0000 & 1.0000 \\ 1.0000 & 1.0000 \\ 1.0000 & 1.0000 \\ 1.0000 & 1.0000 \\ 1.0000 & 1.0000 \\ 1.0000 & 1.0000 \\ 1.0000 & 1.0000 \\ 1.0000 & 1.0900 \\ 1.0000 & 1.0000 \\ 1.0000 & 1.0000 \\ 1.0000 & 1.0000 \\ 1.0000 & 1.0000 \\ 1.0000 & 1.0000 \\ 1.0000 & 1.0000\end{array}$

TFST IS POSITIVE IF NUMBERS PRINTED ABOVE ARE $1.0,1.0$

ERROR SHOULD NOT EXCEED + OR - .000I

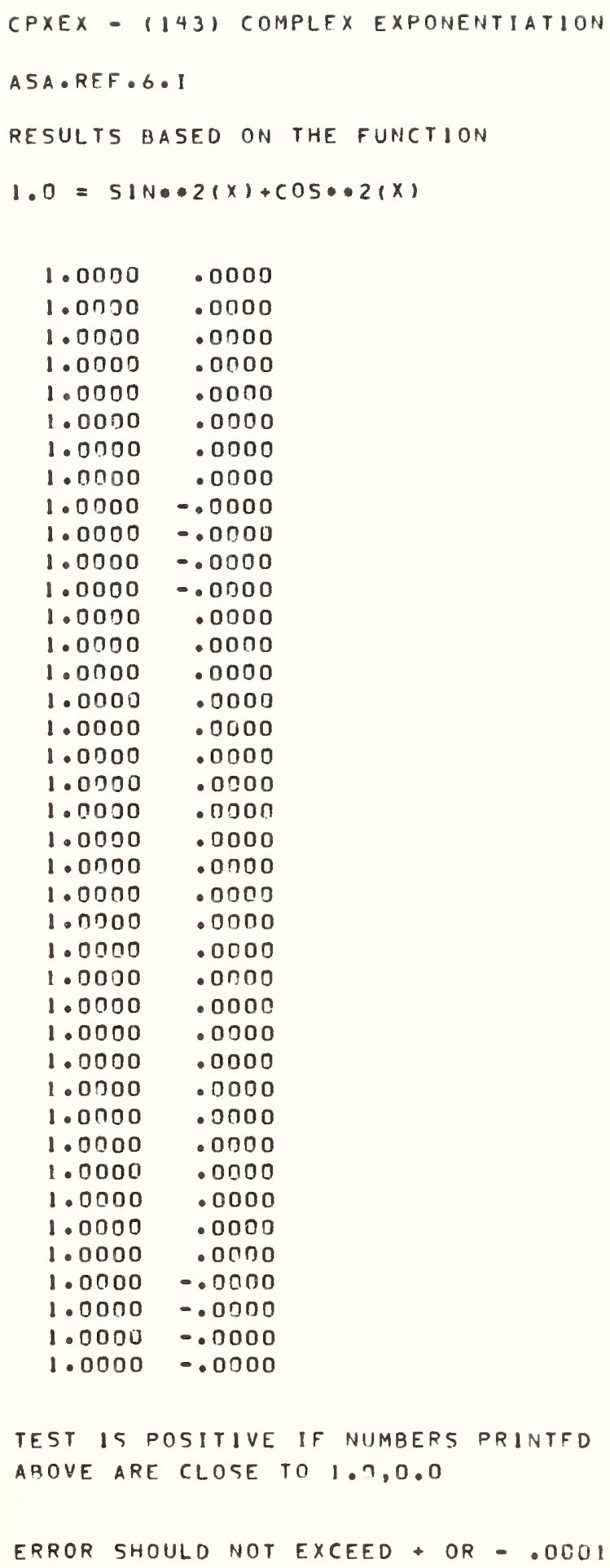




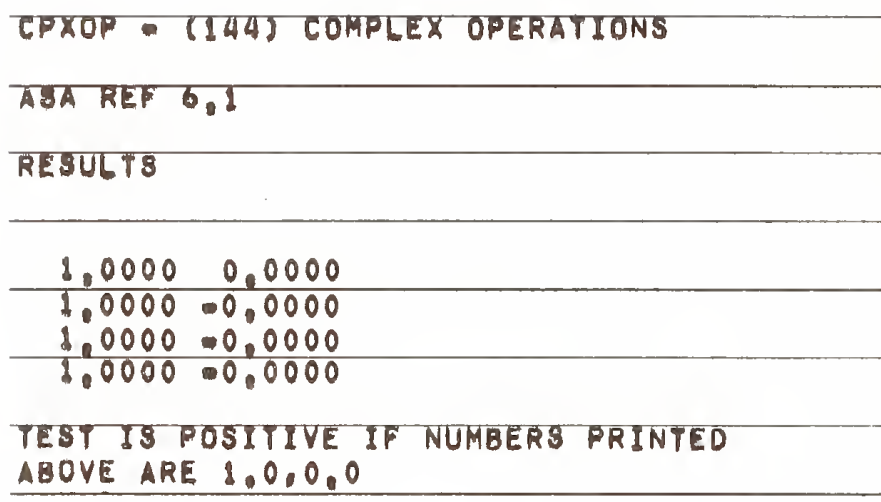

ERROR SHOULO NOT EXCEEO + OR ..00OI

CREAD - (145) MDDITION AND SUBTRACTION OF COMPLEX AND REAL NUMBERS

ASA REF. 6.1

RESULTS

$\begin{array}{ll}.0000 & .0000 \\ .0000 & .0000 \\ .0000 & .0000 \\ .0000 & .0000 \\ .0000 & .0000 \\ .0000 & .0000 \\ .0000 & .0000 \\ .0000 & .0000 \\ .0000 & .0000\end{array}$

TEST IS POSITIVE IF NUMBERS PRINTED

ABOVE ARE $0.0,0.0$
CREMU - (146) MULTIPLICATION OF COMPLEX BY REAL

$A S A \cdot R E F \cdot 6.1$

RESULTS

$$
\begin{array}{ll}
1.0000 & 2.0000 \\
1.0000 & 2.0030 \\
1.0000 & 2.0000 \\
1.0000 & 2.0000
\end{array}
$$

TEST IS POSITIVE IF NUMBERS PRINTED ABOVE ARE $1.0,2.0$

$$
\begin{array}{ll}
1.0000 & 1.0000 \\
1.0000 & 1.0000 \\
1.0000 & 1.0000 \\
1.0000 & 1.0000
\end{array}
$$

TEST IS POSITIVE IF NUMBERS PRINTED ABOVE ARE $1.0,1.0$

ERROR SHOULD NOT EXCEED + OR - .0001
ASA REF 6.1

RESULTS

$\begin{array}{ll}1.0000 & 1.0000 \\ 1.0000 & 1.0000 \\ 1.0000 & 1.0000 \\ 1.0000 & 1.0000 \\ 1.0000 & 1.0000 \\ 1.0000 & 1.0000 \\ 1.0000 & 1.0000 \\ 1.0000 & 1.0000\end{array}$

TEST IS POSITIVE IF NUMBERS PRINTEO ABOVE ARE $1.0,1.0$

ERROR SHOULO NOT EXCEEO + OR - .0001 
CREOP - (148) OPERATIONS ON REAL AND COMPLEX NUMBERS

ASA REF. 6.1

RESULTS

$$
2.0000-1.0000
$$

TEST IS POSITIVE IF NUMBERS PRINTED ABOVE ARE $2.0,-1.0$

$$
1.0000 \quad .0000
$$

TEST IS POSITIVE IF NUMBERS PRINTED ABOVE ARE $1.0,0.0$

ERROR SHOULD NOT EXCEED + OR - .0001

MISC3 - (149) EFFECT OF BLANKS WITHIN STMNT AND CONTINUATION OF STMNT TO 20 LINES

ASA REFS. $3.1 .4 .13 .2 .4 .3 .3 \quad 3.2 .4$ RESULTS

$\mathbf{0}$

.0

TEST IS POSITIVE IF NUMBERS PRINTED ABOVE ARE O

MISC 4 - (150) EFFECT OF 9LANKS WITHIN
STMNT AND CONTINUATION
OF STMNT TO 20 LINES

ASA REFS. $-3.1 .4 .13 .2 .4 .3 .3 \quad 3.2 .4$ RESULTS

$$
\begin{array}{r}
0.0000 \quad 0.0000 \\
.0000 \quad .0000
\end{array}
$$

TEST IS POSITIVE IF NUMBERS PRINTED ABOVE ARE $0.0,0.0$
F O R T R A N T E S T P R O G R A M S

PREPARED BY NATIONAL BUREAU OF SYANDARDS

FOR USE ON LARGE FORTRAN PROCESSORS

IN ACCORDANCE ITH ASA FORTRAN X3.9-1966

VERSION 3 PART IO

SAMPLE COMPUTER, FORTRAN COMPILER LEVEL

OPERATING SYSTEM VERSION

DATE, INSTALLATION NAME

BRFCP - (160) REAL EXTERNAL FUNCTIONS

ASA REF. $=8.3 .1$

RESULTS SHOULD BE POSITIVE

TEST I IS POSITIVE

TEST 2 IS POSITIVE

TEST 3 IS POSITIVE

TEST 4 IS POSITIVE

TEST 5 IS POSITIVE

TEST 6 IS POSITIVE 
BIFCP - (16I) INTEGER EXTERNAL FUNCTIONS WITH INTEGER AND REAL ARGS

ASA REF. - $8.3 \cdot 1$

RESULTS SHOULD BE POSITIVE

TEST I IS POSITIVE

TEST 2 IS POSITIVE

TEST 3 IS POSITIVE

TEST 4 IS POSITIVE

TEST 5 IS POSITIVE

TEST 6 IS POSITIVE

FRFCP - (162) REAL FUNCTIONS WITH LOGICAL, D.P. AND COMPLEX ARGS

ASA REF. 8.3 .1

RESULTS SHOULD BE POSITIVE

TEST I IS POSITIVE.

TEST 2 IS POSITIVE.

TEST 3 IS POSITIVE.

TEST \& IS POSITIVE.

TEST 5 IS POSITIVE.

TEST 6 IS POSITIVE.

TEST 7 IS POSITIVE.
FIFCP - $(163)$ INTEGER FUNCTION IN
FULL FORTRAN

ASA REF. 8.3 .1

RESULTS SHOULD BE POSITIVE

TEST I IS POSITIVE

TEST 2 IS POSITIVE

TEST 3 IS POSITIVE

TEST 4 IS POSITIVE

TEST 5 IS POSITIVE

TEST 6 IS POSITIVE

TEST 7 IS POSITIVE

CFCCP - (164) COMPLEX FUNCTIONS

ASA REFS. $8.3 \cdot 1.8 .3 \cdot 2$

RESULTS

$$
\begin{aligned}
& .0 \text {. - TEST I POSITIVE IF } 0.0 .0 .0 \\
& .0 .0 \text { - TEST } 2 \text { POSITIVE IF } 0.0 .0 .0 \\
& .0 \quad 0 \text { - TEST } 3 \text { POSITIVE IF } 0.0 .0 .0 \\
& .0 \text {.0-- TEST } 4 \text { POSITIVE IF } 0.0 .0 .0 \\
& .0 \text {. } 0 \text { TEST } 5 \text { POSITIVE IF } 0.0 .0 .0 \\
& .0 \text {. } 0 \text { TEST } 6 \text { POSITIVE IF } 0.0 .0 .0 \\
& .0 \quad .0=\text { TEST } 7 \text { POSITIVE IF } 0.0 .0 .0 \\
& .0 \text {.0-DEST } 8 \text { POSITIVE IF } 0.0 .0 .0 \\
& \text { TEST } 9 \text { IS POSITIVE } \\
& \text { TEST IO IS POSITIVE }
\end{aligned}
$$


F O R T R A N T E S T PRO GRA M S PREPARED BY NATIONAL BUREAU OF STANDARDS FOR USE ON LARGE FORTRAN PROCESSORS

IN ACCORDANCE WITH ASA FORTRAN $\times 3.9-1965$ VERSION 3 PART 11

SAMPLE COMPUTER, FORTRAN COMPILER LEVEL OPERATING SYSTEM VERSION DATE, INSTALLATION NAME

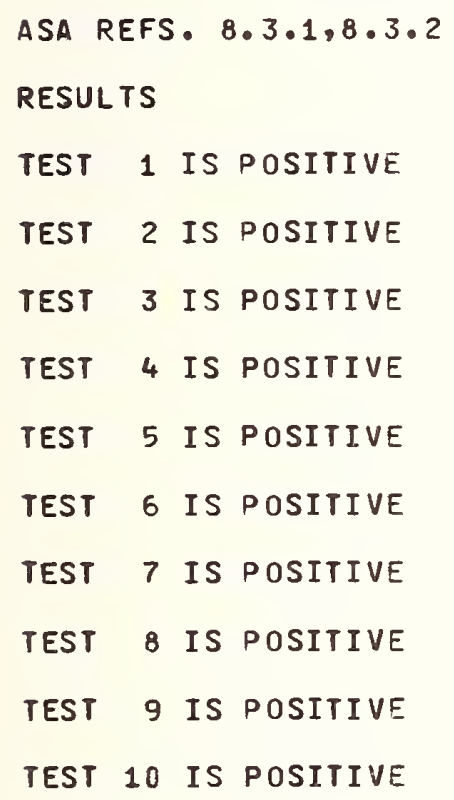

ASA REFS. $8 \cdot 3 \cdot 1,8 \cdot 3 \cdot 2$

RESULTS

TEST 1 IS POSITIVE

TEST 2 IS POSITIVE

TEST 3 IS POSITIVE

TEST 4 IS POSITIVE

TEST 5 IS POSITIVE

TEST 6 IS POSITIVE

TEST 7 IS POSITIVE

TEST 8 IS POSITIVE

TEST 9 IS POSITIVE

TEST 10 IS POSITIVE FUNCTIONS

$0.0 \quad 0.0$

TEST 11 IS POSITIVE IF NUMBERS DRINTED ABOVE ARE $0.0,0.0$

TEST 12 IS POSITIVE
BFCCP - (166) LOGICAL FUNCTIONS

ASA REF 8.3 .1

RESULTS

TEST 1 IS POSITIVE

TEST 2 IS POSITIVE

TEST 3 IS POSITIVE

TEST 4 IS POSITIVE

TEST 5 IS POSITIVE

TEST 6 IS POSITIVE

TEST 7 IS POSITIVE

TEST 8 IS POSITIVE

TEST 9 IS POSITIVE

TEST 10 IS POSITIVE

$0.0000 \quad 0.0000$

TEST 11 IS POSITIVE IF NUMBERS PRINTED ABOVE ARE $0.0,0.0$

END OF (166)

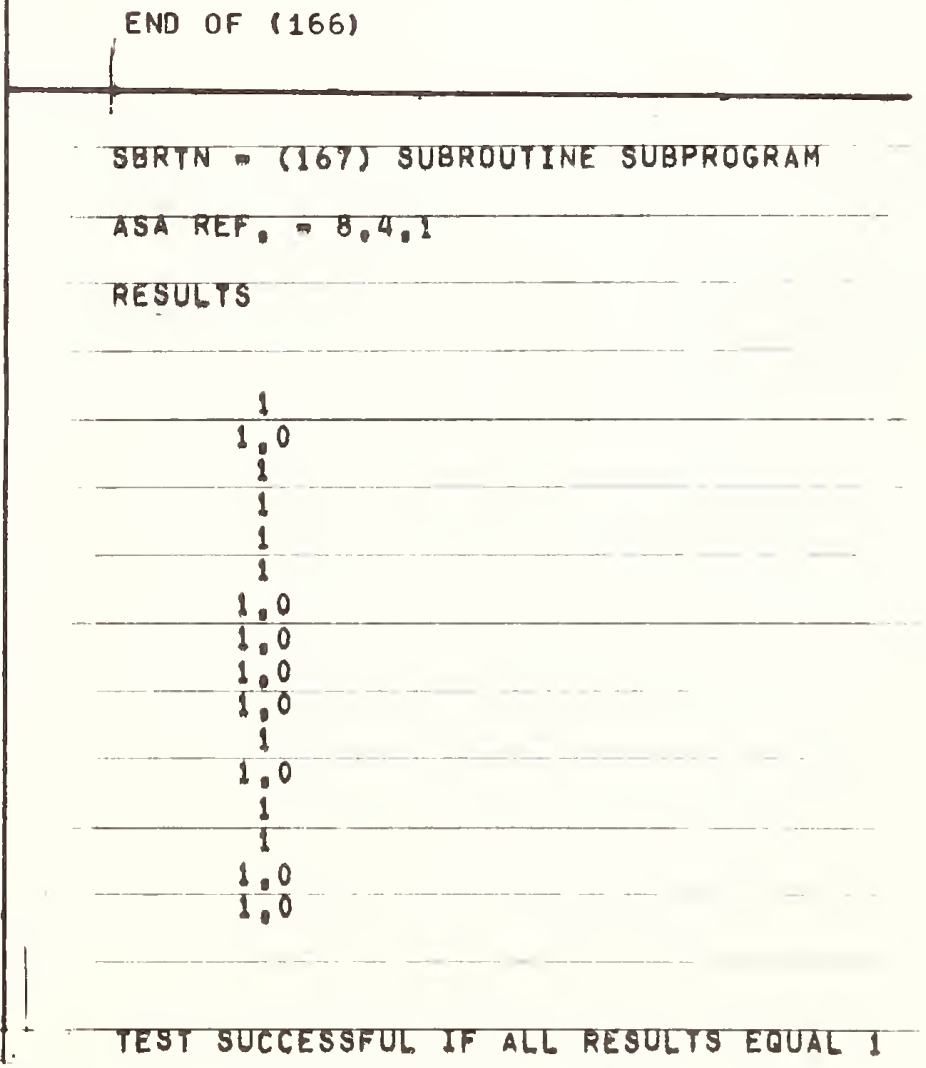

II- C- 57 
FSBRT - $(158)$ SUBROUTINE SUBPROGRAMS

ASA REF. -8.4 .1$

\section{RESULTS}

TEST IS SUCCESSFUL IF EACH GROUP CONTAINS SAME VALUES

1
1
1
1
1
1
1
1
1
1
2.0
2.0
2.0
2.0
2.0
2.0
2.0
2.0
2.0
2.0
$4.00+00$
$4.00+00$
$4.00+00$
$4.00+00$
$4.00+00$
$4.00+00$
$4.00+00$
$4.00+00$

$\begin{array}{ll}6.0 & 6.0 \\ 6.0 & 6.0 \\ 6.0 & 6.0 \\ 6.0 & 6.0 \\ 6.0 & 6.0 \\ 6.0 & 6.0 \\ 6.0 & 6.0 \\ 6.0 & 6.0\end{array}$

\section{BLKDT - (169) BLOCK DATA SUBPROGRAM}

ASA REF. 8.5

RESULTS

TEST IS SUCCESSFUL IF EACH

GROUP CONTAINS SAME VALUES

$$
\begin{aligned}
& 2 \\
& 2 \\
& 2 \\
& 2
\end{aligned}
$$

3.0

3.0

3,0

3,0

$4.00+00$

$4.00+00$

$4.0 D+00$

$4.0 D+00$

$4.0 \quad 5.0$

$4.0 \quad 5.0$

$4.0 \quad 5.0$

$4.0 \quad 5.0$

$T$
$T$
$T$

$A B$

$A B$

$A B$ 
F O R T R A N T E S I P R O G R A M S

PREPARED BY NATIONAL BUREAU OF SIANDARDS

FOR USE ON LARGE FORTRAN PROCESSORS

IN ACCORDANCE WITH ASA FORTRAN $\times 3.9-1966$

VERSION 3 PART 12

SAMPLE COMPUTER, FORTRAN COMPILER LEVEL OPERATING SYSTEM VERSION

DATE, INSTALLATION NAME

\section{BLKDA - (IT9) SEVERAL BLOCK OATA SUBPROGRAMS}

ASA REF. $: 8,5$

\section{RESULTS}

TEST IS SUCCESSFUL IF EACH

\section{GROUP CONTAINS SAME VALUES}

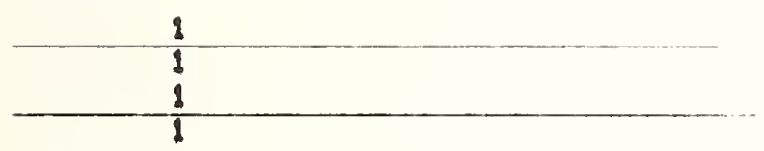

$$
\begin{aligned}
& 2.0 \\
& 2.0 \\
& 2.0 \\
& 2.0
\end{aligned}
$$

$4.00 \$ 00$

4.00400

$4,00+00$

$4.00+00$

$$
\begin{array}{rr}
3.0 & 4.0 \\
3.0 & 4.0 \\
3.0 & 4.0 \\
\hline 3.0 & 4.0
\end{array}
$$

\section{F}

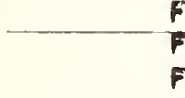

HP

HP

MP
UNFRW - (180) UNFORMATTED READ AND WRITE STATEMENTS

ASA REFS -7.1 .3 .2 .4 AND 7.1 .3 .2 .5 RESULTS

.0000000000 .0000000000

0

.0000000000

.0000000000

.0000000000

.0000000000

.0000000000

.0000000000

.0000000000

.0000000000

.0000000000

.0000000000

.0000000000

.0000000000

.0000000000

.0000000000

0

.0000000000

.0000000000

.0000000000

.0000000000

.0000000000

.0000000000

.0000000000

.0000000000

.0000000000

0

ALL ABOVE ANSWERS SHOULD BE ZERO IF

THE READ AND WRITE RECORDS COMPARE. 


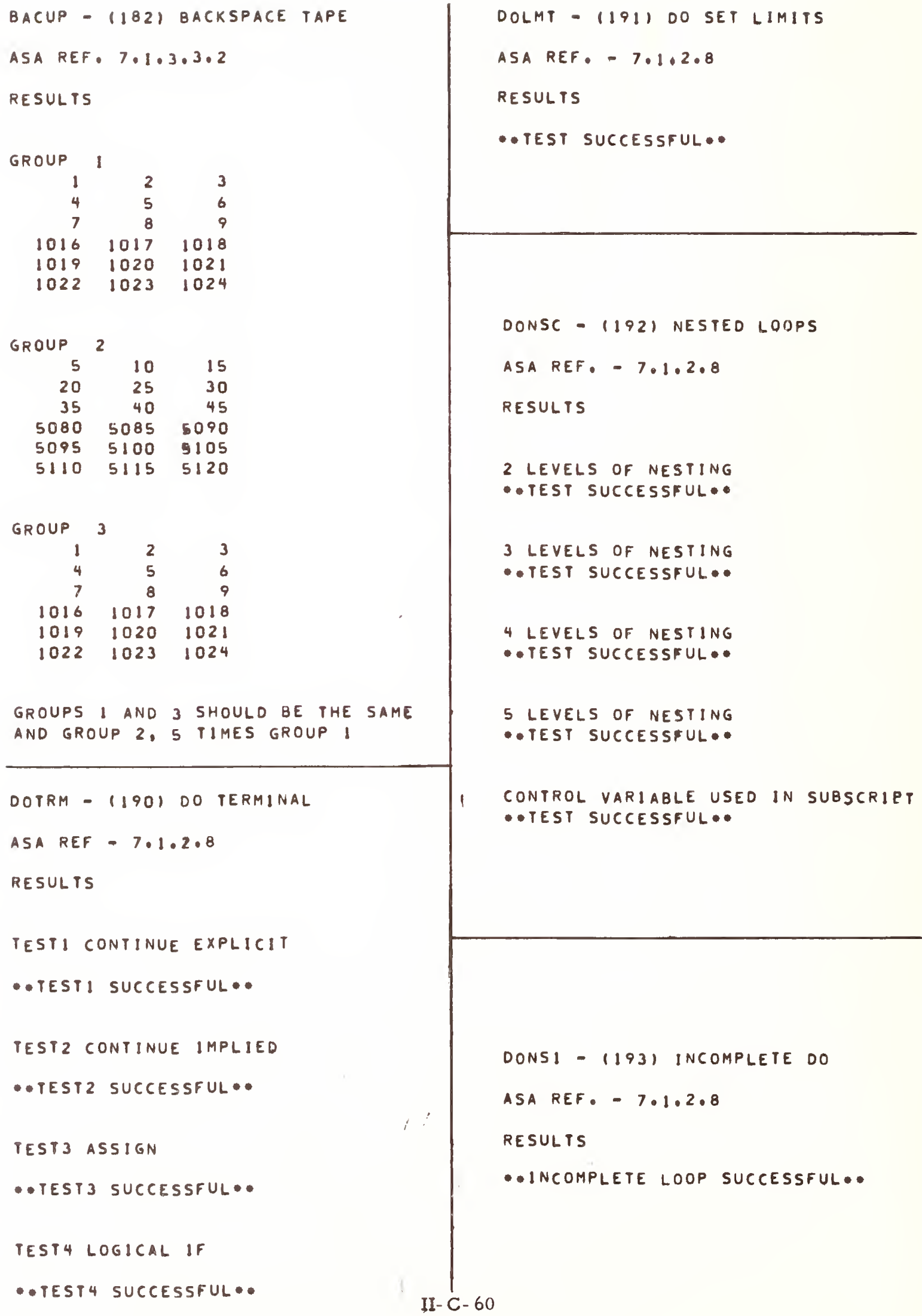

DOLMT - (191) DO SET LIMITS

ASA REF. -7.1 .2 .8$

RESULTS

- test successful *

DONSC - (192) NESTED LODPS

ASA REF. -7.1 .2 .8$

RESULTS

2 LEVELS OF NESTING

- test successFul *

3 LEVELS OF NESTING

- TEST SUCCESSFUL..

4 LEVELS OF NESTING

- TEST SUCCESSFUL..

5 LEVELS OF NESTING

- test successFul.

CONTROL VARIABLE USED IN SUBSCRIET - test successful.

DONSI - $(193)$ INCOMPLETE DO

ASA REF. -7.1 .2 .8$

RESULTS

- incomplete loop successful. 


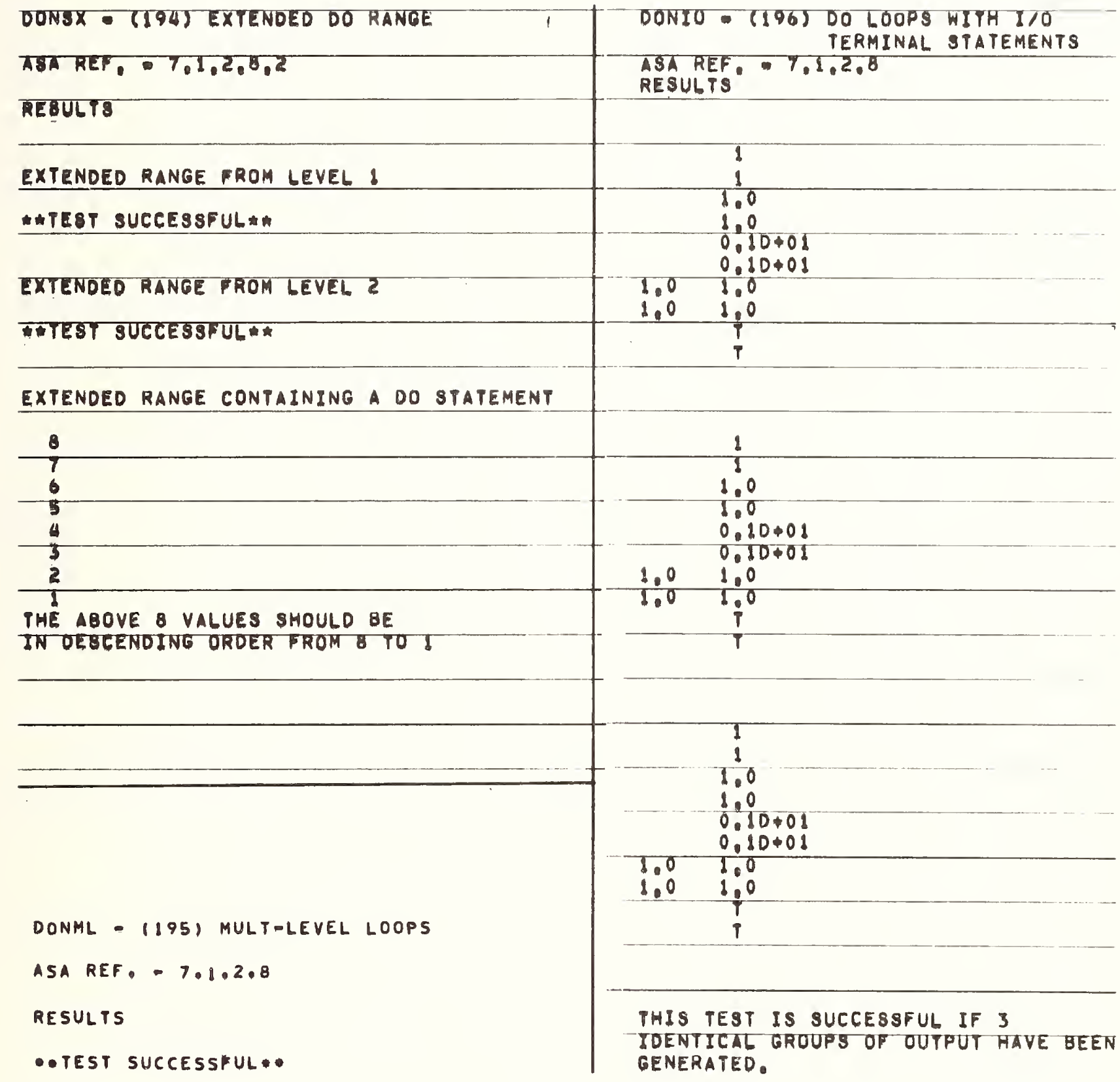


MORDO - (197) A MORE COMPLICATED SEG. OF DO STATEMENTS

ASA REFS -7.1 .2 .8 AND 7.1 .2 .8 .1

RESULTS

THIS SEGMENT SUCCESSFULLY TESTED IF NO ERROR MESSAGES

SUBRI - (200) SUBROUTINE SIIBPROGRAM WITHOUT AN ARGUMENT LIST

ASA REF. - 8.4.1

RESULTS

THIS SEGMENT SUCCESSFULLY TESTED IF NO ERROR MESSAGES.

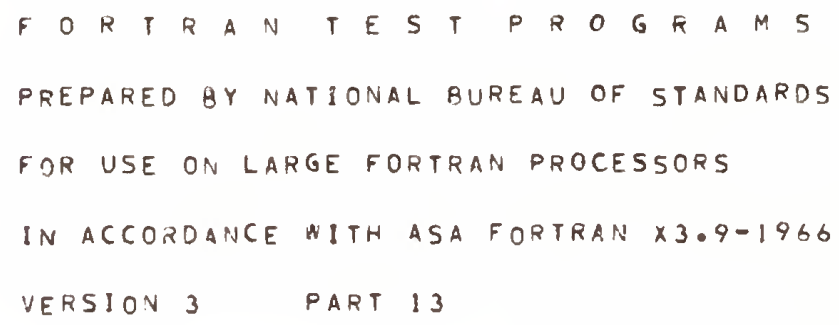

LOGIF - (300) LOGICAL IF STATEMENT

ASA REF. -7.1 .2 .3$

RESULTS

TEST EXPLICITLY WRITTEN-SIGNEO-ZERO

+0 EQUALS - 0

+0.0 EQUALS -0.0

+0.000 EQUALS -0.000

TEST COMPUTATIONAL SIGN OF ZERO

+0 EQUALS - 0

+0.0 EQUaLs - 0.0

+0.0DO EQUALS -0.000

TEST - LOGICAL IF- FOLLOWEO BY

DIFFERENT KINDS OF STATEMENTS

$$
\begin{aligned}
& 0 \\
& 0 \\
& 0 \\
& 0 \\
& 0 \\
& 0 \\
& 0 \\
& 0 \\
& 0
\end{aligned}
$$$$
0
$$

THERE SHOULO $3 E 10$ VALUES A3OVE, IF ONLY 9 , TEST 9 HAS FAILED.

0
0
0
0
0
0
0
0
0

ALL VALUES SHOULD BE ZERO. a VALUE OTHER THAN ZERO HILL BE THE NUMBER OF THE TEST WHICH FAILED. 
BARIF - (301) BASIC FORTRAN ARITHMETIC IF STATEMENT

ASA REF. -7.1 .2 .2$

RESULTS

TEST FOR SIGN OF ZERO - TYPE INTEGER

PATH * FORM OF EXPRESSION *

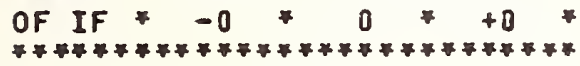

$\begin{array}{cccccccc} & * & & * & & * & & * \\ \text { NEG. } * & 0 & * & 0 & * & 0 & * \\ \text { ZERO } & * & 11 & * & 11 & * & 11 & * \\ \text { POS. } & * & 0 & * & 0 & * & 0 & * \\ * & & * & & * & & *\end{array}$

TEST FOR SIGN OF ZERO - TYPE REAL

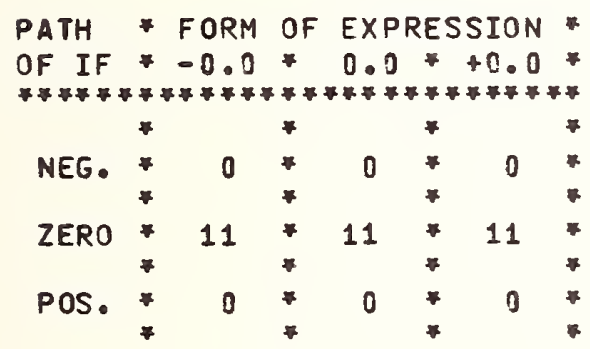

ALL ENTRIES SHOULD BE $O$ EXCEPT THE ZERO PATH, WHICH SHOULO BE 11 IN EACH COLUMN. OTHER TESTS MAY FAIL IF THESE RESULTS DIFFER.

TEST EXPRESSIONS IN IF STATEMENTS

TESTS SUCCESSFUL

\section{FARIF - (302) FULL FORTRAN} ARITHMETIC IF STATEMENTS

ASA REF. -7.1 .2 .2$

RESULTS

SEGMENT 302 TESTED SUCCESSFULLY.
IOFMT " (310) ADDITIONAL FORMATTED I/O

ASA REFS - 7.1.3.2.2 7.1.3.2.3 7.2,3

RESULTS

TEST BLANK INPUT

EACH ANSWER SHOULD BE ZERO

o

0

0

0.0

0.0

0.0

0.0

$0.0 E \$ O O$

$O . O E+O O$

$0.0 E \$ 00$

$0,0 E+00$

$0.00 \$ 00$

$0.00 \$ 00$

$0,00+00$

$0.00 \$ 00$

TEST DEC, PT SPECIFIED BY INPUT

3 LINES IN EACH GROUP SHOULD MATCH

* LINE IS HOLLERITH DATA

1.23456

1.23456

1.23456

$+987654.0$

987654.0

987654.0

म $0.1234 E+01$

$0.1234 E \neq 01$

$0.1234 E+01$

T0.98765पE\$02

$0.987654 E+02$

- $0,987654 E+02$

$-0.2345678910110+06$

$0.2345678910110+06$

$0.2345678910110 \$ 06$

* $-0.1098760-04$

$=0.1098760=04$

.0 .1098760 .04 


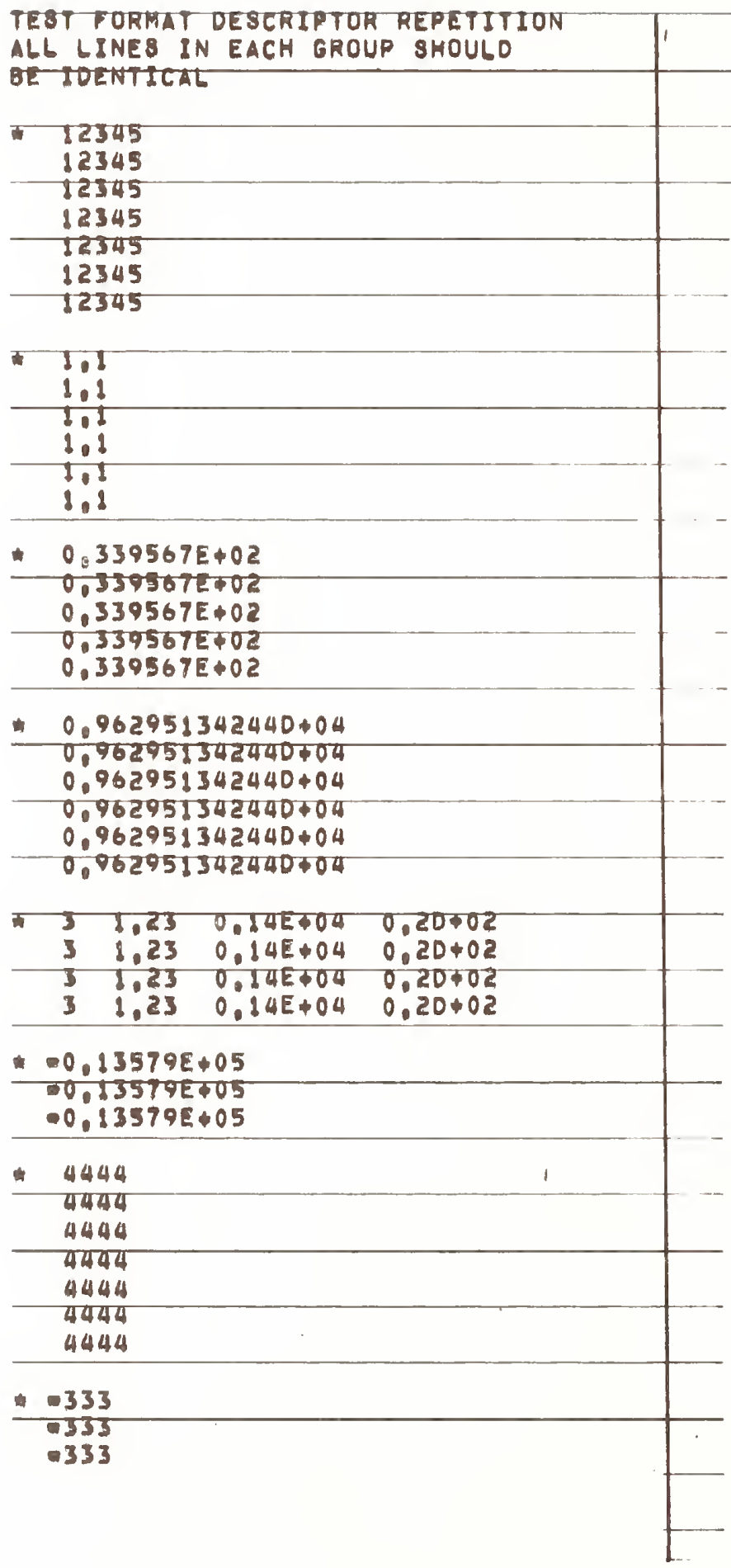

* 5.555

5.555

5.555

$* \quad 0.4545 E-04$ . $4545 E-04$ $.4545 E-34$

* -6.666

$-6.666$

$-6.666$

* $\quad 0.9989 E+12$

- $9989 E+12$

. $9989 E+12$

* $\quad 7.77$

7.77

7.77

$*-0.747 E-02$

$-.747 E-02$

$-.747 E-02$

$* \quad 0.549 E+00$

. $549 E+00$

. $549 E+00$

* 22

22

22

* $0.662 E+00$

. $662 E+00$

$.662 E+00$

$=0.468 E-10$

$.468 E-10$

$.468 E-10$

* 11

11

11

* $0.595420+04$ . $595420+04$ $.595420+04$

* -44.6666

$-44.6666$

$-44.6666$

* $-0.12345678900-03$

-.12345678900-03

$. .12345678900-03$ 


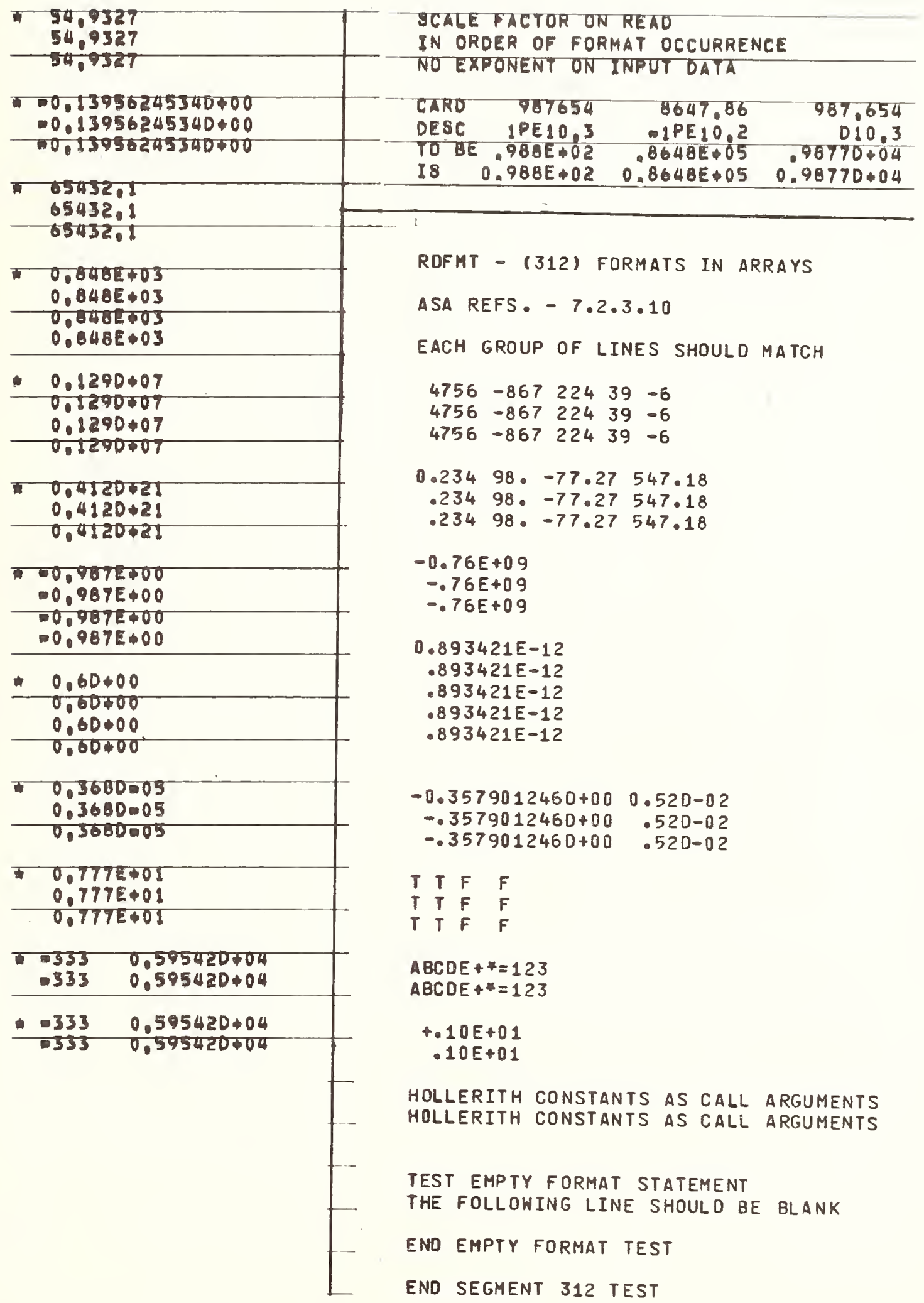

II- C- 65 
F O R T R A N T E S T P R PREPAREO BY NATIONAL BUREAU OF STANDAROS FOR USE ON LARGE FORTRAN PROCESSORS IN ACCORDANCE WITH ASA FORTRAN $\times 3.9-1966$ VERSION 3 PART 14 SAMPLE COMPUTER, FORTRAN COMPILER LEVEL OPERATING SYSTEM VERSION DATE, INSTALLATION NAME

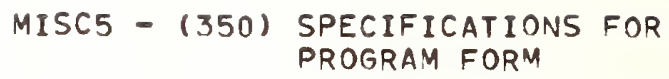

TEST $1.2,3 \cdot 4 \cdot 5 \cdot 6$ CHARACTER VARTABLES AND ARRAY NAMES

**TEST SUCCESSFUL-ALL NAMES ACCEPTED**

TEST PLACEMENT OF STATEMENT LABELS AND LABELS WITH LEADING ZEROS

$$
\begin{aligned}
& 1 \\
& 2 \\
& 3 \\
& 4 \\
& 5 \\
& 6 \\
& 7 \\
& 8 \\
& 9
\end{aligned}
$$

TEST SUCCESSFUL IF 9 NUMBERS IN SEQUENTIAL ORDER FROM 1 TO 9 ARE WRITTEN ABOVE

END OF SEGMENT 350 
FUNMX $-(351)$

THIS SEGMENT FURTHER TESTS

SOME BASIC EXTERNAL FUNCTIONS

BY USING TRIGONOMETRIC FORMULAE

ASA REFS. - 8.3 .3

RESULTS

$$
\begin{array}{r}
0.00000 \\
-.00000 \\
-.00000 \\
.00000 \\
.00000 \\
-.00000 \\
.00000 \\
0.00000 \\
.00000 \\
0.00000 \\
=.00000
\end{array}
$$

ALL ABOVE ANSWERS SHOULD BE O PLUS OR MINUS AN ERROR FACTOR OF NOT MORE THAN $10 * *(-4)$

\section{NAMES - (352)}

TEST OF THE COMPILERS CAPABILITY OF IDENTIFYING DATA NAMES THAT RESEMBLE FORTRAN VERBS AND/OR PREDEFINED

FUNCTION NAMES

ASA REFS. - $10.1 .7 / 4$

\section{RESULTS}
0.00000
0.00000
0.00000
0.00003
0.00000
0.00000
0.00000
0.00000
0.00000
0.00000

$$
0
$$

SPEC2 ( 360$)$ COMMON ANO EGUIVALENCE

ASA REFS - 7.2.1.2 7.2.1.3 7.2.1.4 RESULTS

LINE \& BELOW IS HOLLERITH

$-\frac{2}{2} \frac{2.0}{2.0}$

ANSWERS BELOW SHOULD BE O OR 0.0

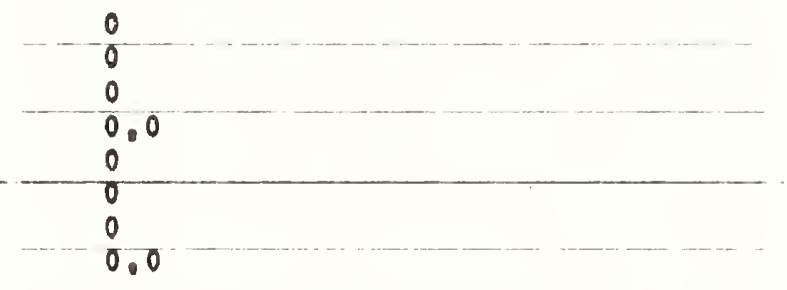

ARITHMETIC IF SUCCESSFUL

ANSWER BELOW SHOULD BE 13,0

$\$ 3.0$

COMPUTED GO TO SUCCESSFUL

TEST EOUIVALENCE EXTENDS COMMON

TEST SUCCESSFUL 



\section{A. GENERAL DESCRIPTION}

This section of the document describes the organization of the NBS FORTRAN Test Programs and data as recorded on magnetic tape for distribution. When the programs have been retrieved and stored in a form more appropriate to utilization, this section of the manual is of no significance.

The distribution tape containing both Version 1 (116 executable test units) and Version 3 (14 executable programs containing the 116 test units) is available in $800 \mathrm{cpi}$ recording density in the following forms:

7 track, even parity, BCD recorded from FORTRAN $H$ set punch card code (See Appendix D X3.9-1966)

9 track, odd parity, EBCDIC recorded from the American National Standard punch card code

9 track, odd parity, ASCII recorded from the American National Standard punch card code

The distribution tape is an unlabeled, fixed block size recorded tape, terminating with two tape mark records.

Version 1 Programs and its data precede Version 3 with its data. Each block contains 720 characters comprised of nine 80-character card image records。 Partial blocks at the end of both Version 1 and Version 3 are filled with blank card images, so that Version 1 begins in Block 1 record 1 and Version 3 begins with Block 1597 record 1.

The differences between the punch card code for the FORTRAN H Set and the American National Standard are reflected in the following four characters:

$\begin{array}{lrr} & \text { H-Set } & \text { Standard } \\ \text { ( left parenthesis } & 0-4-8 & 12-5-8 \\ =\text { right parenthesis } & 12-4-8 & 11-5-8 \\ + \text { equal } & 3-8 & 6-8 \\ \text { plus } & 12 & 12-6-8\end{array}$

The programs and the data are in the same code.

For FORTRAN processors which contain an option on the coded character set for conversion of the FORTRAN programs, but not for the data, or perform a logical conversion only, causing the program listing to print a different character representation for the four characters listed above should perform a character conversion to the test programs and data before performing the tests, because the program listing is considered part of the documentation.

The following tables identify each main program unit, subprogram and data in two different forms:

The Block and Record number identifies the block number and the record within the block of the start of each element of information.

The card image number is the record number for the location of the start of each element of information. 
For Version 3, one table lists the elements in relation to their position on the tape with Version 1 preceding it, and the other table assumes that the tape has been forward spaced over Version 1 (1596 blocks).

Each element of information in the tables is identified by the letter:

$$
\begin{aligned}
& M=\text { main program unit } \\
& F=\text { function subprogram } \\
& S=\text { subroutine subprogram } \\
& B=\text { Block Data subprogram }
\end{aligned}
$$

WARNING - Version 1 and Version 3 each contain the same subprograms. If Version 1 and Version 3 are to be retained as a single file for use, one copy of the subprograms (63 functions and subroutines) must be deleted otherwise duplicate external procedure names will occur.

In Version 1, the Directory (segment 000) recorded as a set of comment lines is included as part of the first test unit, segment 008. This causes this test unit to contain 871 card images. The Directory of 342 card images may be removed and by appending a STOP statement and an END line may be compiled to obtain a program listing. 
Card Image \# B1ock \& Record \# Segment \# Name

Card

Block \& Record \# Segment \# Name

\begin{tabular}{|c|c|c|c|c|c|c|c|c|c|c|c|}
\hline \multirow[t]{2}{*}{1} & 1 & 000 & \multicolumn{2}{|l|}{ * } & \multirow[t]{2}{*}{1} & 652 & \multirow{2}{*}{$\begin{array}{l}9 \\
3\end{array}$} & \multirow{2}{*}{$\begin{array}{l}068 \\
069\end{array}$} & IFIMG & \multirow{2}{*}{$\begin{array}{l}M \\
M\end{array}$} & 5868 \\
\hline & & 008 & FMTRW & $M$ & & 667 & & & IFD BL & & 5997 \\
\hline 97 & 8 & & 46 data cards & & 872 & 673 & 6 & 070 & IFCPX & M & 6054 \\
\hline 102 & 9 & 009 & AFRMT & $\mathrm{M}$ & 918 & 680 & 4 & 071 & IFCJG & M & 6115 \\
\hline 115 & 7 & & 3 data cards & & 1033 & 687 & 7 & 072 & IFBMS & M & 6181 \\
\hline 116 & 1 & 010 & DATA2 & M & 1036 & 702 & 1 & 073 & IFFMS & M & 6310 \\
\hline 133 & 6 & 011 & AASGN & M & 1194 & 722 & 2 & 080 & EXPON & M & 6491 \\
\hline 163 & 4 & 013 & DASGN & $M$ & 1462 & 728 & 8 & 081 & DEXPO & M & 6551 \\
\hline 210 & 1 & 015 & CASGN & M & 1882 & 736 & 4 & 082 & CEXPO & M & 6619 \\
\hline 262 & 2 & 016 & LASGN & M & 2351 & 747 & 3 & 083 & LOGTM & M & 6717 \\
\hline 273 & 9 & 017 & INTRL & M & 2457 & 753 & 6 & 084 & DPLOG & M & 6774 \\
\hline 294 & 5 & 020 & UGOTO & M & 2642 & 761 & 1 & 085 & CXLOG & M & 6841 \\
\hline 302 & 2 & 021 & AGOTO & M & 2711 & 772 & 8 & 086 & COLOG & M & 6947 \\
\hline 318 & 7 & 022 & CGOTO & M & 2860 & 779 & 1 & 087 & DCLOG & M & 7003 \\
\hline 334 & 9 & 030 & ARBAD & M & 3006 & 786 & 4 & 088 & SINUS & M & 7069 \\
\hline 347 & 7 & 031 & ARFAD & M & 3121 & 795 & 4 & 089 & DPSIN & $M$ & 7150 \\
\hline 354 & 1 & 032 & ARBSB & M & 3178 & 804 & 5 & 090 & CSICO & M & 7232 \\
\hline 361 & 5 & 033 & ARFSB & $M$ & 3245 & 811 & 7 & 091 & COSNS & M & 7297 \\
\hline 369 & 5 & 034 & ARBAS & M & 3317 & 820 & 8 & 092 & DPCOS & M & 7379 \\
\hline 378 & 3 & 035 & ARFAS & $M$ & 3396 & 829 & 8 & 094 & TANGH & M & 7460 \\
\hline 384 & 9 & 036 & ARBMI & $M$ & 3456 & 836 & 2 & 095 & SQROT & M & 7517 \\
\hline 392 & 3 & 037 & ARBMR & M & 3522 & 842 & 3 & 096 & DSQRO & M & 7572 \\
\hline 399 & 4 & 038 & ARFMD & M & 3586 & 849 & 3 & 097 & CSQRO & M & 7635 \\
\hline 407 & 3 & 039 & ARBDV & M & 3657 & 857 & 5 & 098 & ARCTG & M & 7709 \\
\hline 415 & 9 & 040 & ARFDV & M & 3735 & 863 & 9 & 099 & DACTG & M & 7767 \\
\hline 423 & 3 & 041 & ARBEX & M & 3801 & 871 & 3 & 100 & $\mathrm{ACTG} 2$ & M & 7833 \\
\hline 433 & 3 & 042 & ARFEX & M & 3891 & 877 & 5 & 101 & DATN2 & M & 7889 \\
\hline 441 & 5 & 043 & ARBHI & M & 3965 & 884 & 8 & 102 & DMODA & M & 7955 \\
\hline 461 & 2 & 050 & SBB67 & M & 4142 & 891 & 8 & 103 & CABSA & M & 8018 \\
\hline 469 & 9 & 051 & SBB45 & M & 4221 & 901 & 2 & 110 & BSFTS & M & 8102 \\
\hline 479 & 6 & 052 & SBB13 & M & 4308 & 913 & 3 & 111 & FSFTS & M & 8211 \\
\hline 492 & 1 & 053 & SBF 17 & M & 4420 & 931 & 7 & 140 & CPXAD & M & 8377 \\
\hline 500 & 8 & 054 & SIMIF & $M$ & 4499 & 940 & 2 & 141 & CPXMU & M & 8453 \\
\hline 509 & 4 & 055 & IFABS & M & 4576 & 955 & 8 & 142 & CPXDV & M & 8594 \\
\hline 516 & 5 & 056 & IFFLT & M & 4640 & 965 & 1 & 143 & CPXEX & M & 8677 \\
\hline 521 & 9 & 057 & IFF IX & M & 4689 & 978 & 9 & 144 & СРXOP & M & 8802 \\
\hline 528 & 5 & 058 & IFSGN & M & 4748 & 985 & 9 & 145 & CREAD & $M$ & 8865 \\
\hline 537 & 6 & 059 & IFDAB & M & 4830 & 993 & 4 & 146 & CREMU & M & 8932 \\
\hline 544 & 8 & 060 & IFTRN & M & 4895 & 1000 & 3 & 147 & CREDV & M & 8994 \\
\hline 556 & 7 & 061 & IFMOD & M & 5002 & 1006 & 7 & 148 & CREOP & M & 9052 \\
\hline 566 & 1 & 062 & IFMAX & M & 5086 & 1014 & 1 & 149 & MISC 3 & $M$ & 9118 \\
\hline 593 & 6 & 063 & IFMIN & $M$ & 5334 & 1024 & 8 & 150 & MISC4 & M & 9215 \\
\hline 618 & 6 & 064 & IFDSG & M & 5559 & 1036 & 5 & 160 & BRFCP & $M$ & 9320 \\
\hline 625 & 1 & 065 & IFDIM & $M$ & 5617 & 1045 & 6 & 400 & AFS & F & 9402 \\
\hline 632 & 7 & 066 & IFSGL & M & 5686 & 1046 & 7 & 420 & BFS & F & 9412 \\
\hline 641 & 6 & 067 & IFREL & M & 5766 & 1047 & 8 & 430 & CFS & F & 9422 \\
\hline & & & & & & 1048 & 9 & 440 & DFS & $\mathrm{F}$ & 9432 \\
\hline *See & $u_{1}$ & & & & & 1050 & 2 & 450 & EFS & $\mathrm{F}$ & 9443 \\
\hline & & & & & & 1051 & 4 & 460 & FFS & $\mathrm{F}$ & 9454 \\
\hline
\end{tabular}

$M=$ Main Program

$F=$ Function Subprogram

$S$ = Subroutine Subprogram

$\mathrm{B}=$ BLOCK DATA Subprogram 
VERSION 1 DISTRIBUTION TAPE ORGANIZATION - continuation

Card Image \# Block \& Record \# Segment \# Name

Card

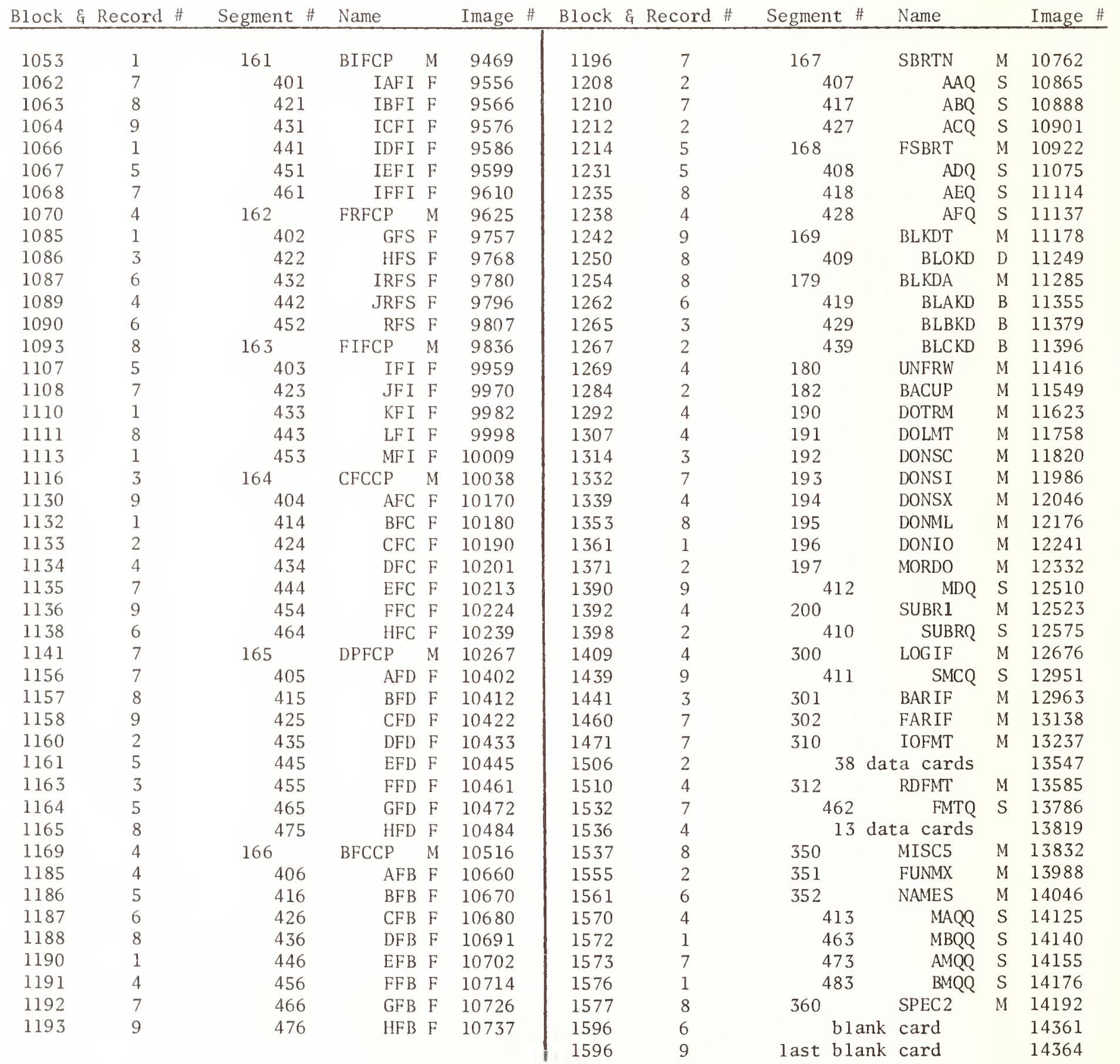


Card

Card Image \# Block \& Record \# Segment \# Name Image \#

\begin{tabular}{|c|c|c|c|c|c|c|c|c|c|c|c|}
\hline Block & \& Record \# & Segment & Name & & Image \# & Block & E Record \# & Segment \# & Name & & Image \\
\hline 1597 & 1 & 008-011 & PART 1 & M & 14365 & 2738 & 1 & $165-169$ & PART 11 & M & 24634 \\
\hline 1716 & 4 & & 19 data cards & & 15439 & 2812 & 7 & 405 & AFD & $\mathrm{F}$ & 25306 \\
\hline 1721 & 8 & 013-015 & PART 2 & M & 15488 & 2813 & 8 & 415 & BFD & $\mathrm{F}$ & 25316 \\
\hline 1824 & 7 & & 6 data cards & & 16414 & 2814 & 9 & 425 & CFD & F & 25326 \\
\hline 1825 & 4 & $016-034$ & PART 3 & M & 16420 & 2816 & 2 & 435 & DFD & F & 25337 \\
\hline 1944 & 3 & & 6 data cards & & 17490 & 2817 & 5 & 445 & EFD & F & 25349 \\
\hline 1944 & 9 & $035-053$ & PART 4 & M & 17496 & 2819 & 3 & 455 & FFD & $\mathrm{F}$ & 25365 \\
\hline 2069 & 1 & & 6 data cards & & 18613 & 2820 & 5 & 465 & GFD & $\mathrm{F}$ & 25376 \\
\hline 2069 & 7 & $054-064$ & PART 5 & M & 18619 & 2821 & 8 & 475 & HFD & $\mathrm{F}$ & 25388 \\
\hline 2197 & 2 & & 6 data cards & & 19766 & 2825 & 4 & 406 & $\mathrm{AFB}$ & F & 25420 \\
\hline 2197 & 8 & $065-073$ & PART 6 & M & 19772 & 2826 & 5 & 416 & BF B & $\mathrm{F}$ & 25430 \\
\hline 2298 & 5 & & 6 data cards & & 20678 & 2827 & 6 & 426 & CFB & F & 25440 \\
\hline 2299 & 2 & $080-092$ & PART 7 & M & 20684 & 2828 & 8 & 436 & DFB & $F$ & 25451 \\
\hline 2409 & 3 & & 6 data cards & & 21675 & 2830 & 1 & 446 & EFB & $\mathrm{F}$ & 25462 \\
\hline 2409 & 9 & $094-111$ & PART 8 & M & 21681 & 2831 & 4 & 456 & FFB & $\mathrm{F}$ & 25474 \\
\hline 2514 & 9 & & 6 data cards & & 22626 & 2832 & 7 & 466 & GF B & F & 25486 \\
\hline 2515 & 6 & $140-150$ & PART 9 & M & 22632 & 2833 & 9 & 476 & HFB & F & 25497 \\
\hline 2622 & 8 & & 6 data cards & & 23597 & 2836 & 7 & 407 & AAQ & S & 25522 \\
\hline 2623 & 5 & $160-164$ & PART 10 & M & 23603 & 2839 & 3 & 417 & $\mathrm{ABQ}$ & S & 25545 \\
\hline 2693 & 9 & 400 & AFS & $\mathrm{F}$ & 24237 & 2840 & 7 & 427 & ACQ & S & 25558 \\
\hline 2695 & 1 & 420 & BFS & $\mathrm{F}$ & 24247 & 2843 & 1 & 408 & $\mathrm{ADQ}$ & S & 25579 \\
\hline 2696 & 2 & 430 & CFS & F & 24257 & 2847 & 4 & 418 & $\mathrm{AEQ}$ & $S$ & 25618 \\
\hline 2697 & 3 & 440 & DFS & $\mathrm{F}$ & 24267 & 2849 & 9 & 428 & AFQ & S & 25641 \\
\hline 2698 & 5 & 450 & EFS & F & 24278 & 2854 & 5 & 409 & BLOKD & B & 25682 \\
\hline 2699 & 7 & 460 & FFS & $\mathrm{F}$ & 24289 & 2858 & 5 & 6 & data cards & & 25718 \\
\hline 2701 & 4 & 401 & IAF I & $\mathrm{F}$ & 24304 & 2859 & 2 & $179-200$ & PART 12 & M & 25724 \\
\hline 2702 & 5 & 421 & IBF I & $\mathrm{F}$ & 24314 & 2998 & 3 & 410 & SUBRQ & S & 26976 \\
\hline 2703 & 6 & 431 & ICF I & $\mathrm{F}$ & 24324 & 3009 & 5 & 412 & MDQ & S & 27077 \\
\hline 2704 & 7 & 441 & IDF I & F & 24334 & 3010 & 9 & 419 & BLAKD & B & 27090 \\
\hline 2706 & 2 & 451 & IEFI & F & 24347 & 3013 & 6 & 429 & BLBKD & B & 27114 \\
\hline 2707 & 4 & 461 & IFF I & F & 24358 & 3015 & 5 & 439 & BLCKD & B & 27131 \\
\hline 2709 & 1 & 402 & GFS & F & 24373 & 3017 & 7 & 6 & data cards & & 27151 \\
\hline 2710 & 3 & 422 & HFS & F & 24384 & 3018 & 4 & $300-312$ & PART 13 & M & 27157 \\
\hline 2711 & 6 & 432 & IRFS & F & 24396 & 3139 & 3 & 411 & MCQ & S & 28245 \\
\hline 2713 & 4 & 442 & JRFS & $\mathrm{F}$ & 24412 & 3140 & 6 & 462 & FMTQ & S & 28257 \\
\hline 2714 & 6 & 452 & RFS & F & 24423 & 3144 & 3 & 57 & data cards & & 28290 \\
\hline 2717 & 8 & 403 & IFI & $\mathrm{F}$ & 24452 & 3150 & 6 & $350-360$ & PART 14 & M & 28347 \\
\hline 2719 & 1 & 423 & JFI & F & 24463 & 3206 & 8 & 413 & MAQQ & S & 28853 \\
\hline 2720 & 4 & 433 & KFI & F & 24475 & 3208 & 5 & 463 & $M B Q Q$ & S & 28868 \\
\hline 2722 & 2 & 443 & LF I & $\mathrm{F}$ & 24491 & 3210 & 2 & 473 & AMQQ & $S$ & 28883 \\
\hline 2723 & 4 & 453 & MF I & F & 24502 & 3212 & 5 & 483 & BMQQ & S & 28904 \\
\hline 2726 & 6 & 404 & AFC & $\mathrm{F}$ & 24531 & 3214 & 3 & & data cards & & 28920 \\
\hline 2727 & 7 & 414 & $\mathrm{BFC}$ & $\mathrm{F}$ & 24541 & 3214 & 8 & 1ast & data card & & 28925 \\
\hline 2728 & 8 & 424 & $\mathrm{CFC}$ & $\mathrm{F}$ & 24551 & 3214 & 9 & (blank fil & 11 er card) & & 28926 \\
\hline 2730 & 1 & 434 & $\mathrm{DFC}$ & $\mathrm{F}$ & 24562 & & & & & & \\
\hline 2731 & 4 & 444 & $\mathrm{EFC}$ & $\mathrm{F}$ & 24574 & $M=N$ & Main Program & & & & \\
\hline 2732 & 6 & 454 & FFC & $\mathrm{F}$ & 24585 & $F=F$ & Function Subl & program & & & \\
\hline 2734 & 3 & 464 & $\mathrm{HFC}$ & $\mathrm{F}$ & 24600 & $S=S$ & Subroutine Si & ubprogram & & & \\
\hline 2737 & 4 & & 6 data cards & & 24628 & $\mathrm{~B}=\mathrm{E}$ & BLOCK DATA S? & ubprogram & & & \\
\hline
\end{tabular}


VERSION 3 DISTRIBUTION TAPE ORGANIZATION

(Listed as if Version 1 (1596 blocks) had been deleted or forward spaced.)

\begin{tabular}{|c|c|c|c|c|c|c|c|c|c|c|c|c|c|}
\hline Block & \& Record \# & Segment & $\#$ & Name & & $\begin{array}{c}\text { Card } \\
\text { Image }\end{array}$ & Block & \& Record \# & Segment & $\#$ & Name & & $\begin{array}{c}\text { Card } \\
\text { Image \# }\end{array}$ \\
\hline 1 & 1 & 008-011 & & PART 1 & M & 1 & 1142 & 1 & $165-169$ & & PART 11 & M & 10270 \\
\hline 120 & 4 & & 19 & data cards & & 1075 & 1216 & 7 & 405 & & $\mathrm{AFD}$ & $\mathrm{F}$ & 10942 \\
\hline 125 & 8 & $013-015$ & & PART 2 & M & 1124 & 1217 & 8 & 415 & & $\mathrm{BFD}$ & $\mathrm{F}$ & 10952 \\
\hline 228 & 7 & & 6 & data cards & & 2050 & 1218 & 9 & 425 & & CFD & $\mathrm{F}$ & 10962 \\
\hline 229 & 4 & $016-034$ & & PART 3 & M & 2056 & 1220 & 2 & 435 & & DFD & $\mathrm{F}$ & 10973 \\
\hline 348 & 3 & & 6 & data cards & & 3126 & 1221 & 5 & 445 & & EFD & $\mathrm{F}$ & 10985 \\
\hline 348 & 9 & $035-053$ & & PART 4 & M & 3132 & 1223 & 3 & 455 & & FFD & $\mathrm{F}$ & 11001 \\
\hline 473 & 1 & & 6 & data cards & & 4249 & 1224 & 5 & 465 & & GFD & $\mathrm{F}$ & 11012 \\
\hline 473 & 7 & $054-064$ & & PART 5 & M & 4255 & 1225 & 8 & 475 & & $\mathrm{HFD}$ & $\mathrm{F}$ & 11024 \\
\hline 601 & 2 & & 6 & data cards & & 5402 & 1229 & 4 & 406 & & $\mathrm{AFB}$ & $\mathrm{F}$ & 11056 \\
\hline 601 & 8 & $065-073$ & & PART 6 & M & 5408 & 1230 & 5 & 416 & & $\mathrm{BFB}$ & $\mathrm{F}$ & 11066 \\
\hline 702 & 5 & & 6 & data cards & & 6314 & 1231 & 6 & 426 & & CF B & $\mathrm{F}$ & 11076 \\
\hline 703 & 2 & $080-092$ & & PART 7 & M & 6320 & 1232 & 8 & 436 & & DFB & $\mathrm{F}$ & 11087 \\
\hline 813 & 3 & & 6 & data cards & & 7311 & 1234 & 1 & 446 & & EFB & $\mathrm{F}$ & 11098 \\
\hline 813 & 9 & $094-111$ & & PART 8 & M & 7317 & 1235 & 4 & 456 & & FF B & $\mathrm{F}$ & 11110 \\
\hline 918 & 9 & & 6 & data cards & & 8262 & 1236 & 7 & 466 & & GF B & $\mathrm{F}$ & 11122 \\
\hline 919 & 6 & $140-150$ & & PART 9 & M & 8268 & 1237 & 9 & 476 & & $\mathrm{HFB}$ & $\mathrm{F}$ & 11133 \\
\hline 1026 & 8 & & 6 & data cards & & 9233 & 1240 & 7 & 407 & & AAQ & S & 11158 \\
\hline 1027 & 5 & $160-164$ & & PART 10 & M & 9239 & 1243 & 3 & 417 & & $A B Q$ & $\mathrm{~S}$ & 11181 \\
\hline 1097 & 9 & 400 & & AFS & $\mathrm{F}$ & 9873 & 1244 & 7 & 427 & & ACQ & S & 11194 \\
\hline 1099 & 1 & 420 & & BFS & $\mathrm{F}$ & 9883 & 1247 & 1 & 408 & & $\mathrm{ADQ}$ & S & 11215 \\
\hline 1100 & 2 & 430 & & CFS & $\mathrm{F}$ & 9893 & 1251 & 4 & 418 & & AEQ & $\mathrm{S}$ & 11254 \\
\hline 1101 & 3 & 440 & & DFS & $\mathrm{F}$ & 9903 & 1253 & 9 & 428 & & $\mathrm{AFQ}$ & S & 11277 \\
\hline 1102 & 5 & 450 & & EFS & $\mathrm{F}$ & 9914 & 1258 & 5 & 409 & & BLOKD & B & 11318 \\
\hline 1103 & 7 & 460 & & FFS & $\mathrm{F}$ & 9925 & 1262 & 5 & & & data cards & & 11354 \\
\hline 1105 & 4 & 401 & & IAF I & $\mathrm{F}$ & 9940 & 1263 & 2 & $179-200$ & & PART 12 & M & 11360 \\
\hline 1106 & 5 & 421 & & IBF I & $\mathrm{F}$ & 9950 & 1402 & 3 & 410 & & SUBRQ & S & 12612 \\
\hline 1107 & 6 & 431 & & ICF I & $\mathrm{F}$ & 9960 & 1413 & 5 & 412 & & MDQ & S & 12713 \\
\hline 1108 & 7 & 441 & & IDF I & $\mathrm{F}$ & 9970 & 1414 & 9 & 419 & & BLAKD & B & 12726 \\
\hline 1110 & 2 & 451 & & IEF I & $\mathrm{F}$ & 9983 & 1417 & 6 & 429 & & BLBKD & B & 12750 \\
\hline 1111 & 4 & 461 & & IFFI & $\mathrm{F}$ & 9994 & 1419 & 5 & 439 & & BLCKD & B & 12767 \\
\hline 1113 & 1 & 402 & & GFS & $\mathrm{F}$ & 10009 & 1421 & 7 & & & data cards & & 12787 \\
\hline 1114 & 3 & 422 & & HFS & $\mathrm{F}$ & 10020 & 1422 & 4 & $300-312$ & & PART 13 & $M$ & 12793 \\
\hline 1115 & 6 & 432 & & IRFS & $\mathrm{F}$ & 10032 & 1543 & 3 & 411 & & SMCQ & $\mathrm{S}$ & 13881 \\
\hline 1117 & 4 & 442 & & JRFS & F & 10048 & 1544 & 6 & 462 & & FMTQ & $\mathrm{S}$ & 13893 \\
\hline 1118 & 6 & 452 & & RFS & $\mathrm{F}$ & 10059 & 1548 & 3 & & 57 & data cards & & 13926 \\
\hline 1121 & 8 & 403 & & IF I & $\mathrm{F}$ & 10088 & 1554 & 6 & $350-360$ & & PART 14 & M & 13983 \\
\hline 1123 & 1 & 423 & & JF I & $\mathrm{F}$ & 10099 & 1610 & 8 & 413 & & MAQQ & S & 14489 \\
\hline 1124 & 4 & 433 & & KFI & $\mathrm{F}$ & 10111 & 1612 & 5 & 463 & & MBQQ & $\mathrm{S}$ & 14504 \\
\hline 1126 & 2 & 443 & & LF I & $\mathrm{F}$ & 10127 & 1614 & 2 & 473 & & AMQQ & $S$ & 14519 \\
\hline 1127 & 4 & 453 & & MFI & $\mathrm{F}$ & 10138 & 1616 & 5 & 483 & & $B M Q Q$ & $\mathrm{~S}$ & 14540 \\
\hline 1130 & 6 & 404 & & $\mathrm{AFC}$ & $\mathrm{F}$ & 10167 & 1618 & 3 & & & data cards & & 14556 \\
\hline 1131 & 7 & 414 & & $\mathrm{BFC}$ & $\mathrm{F}$ & 10177 & 1618 & 8 & & ast & data card & & 14561 \\
\hline 1132 & 8 & 424 & & $\mathrm{CFC}$ & $\mathrm{F}$ & 10187 & 1618 & 9 & (b lank & & 11 er card) & & 14562 \\
\hline 1134 & 1 & 434 & & DFC & $\mathrm{F}$ & 10198 & & & & & & & \\
\hline 1135 & 4 & 444 & & EFC & $\mathrm{F}$ & 10210 & $M=M_{2}$ & ain Program & & & & & \\
\hline 1136 & 6 & 454 & & FFC & $\mathrm{F}$ & 10221 & $\mathrm{~F}=\mathrm{Fr}$ & unction Sub & bprogram & & & & \\
\hline 1138 & 3 & 464 & & $\mathrm{HFC}$ & $\mathrm{F}$ & 10236 & $S=S$ & ubroutine $\mathrm{S}$ & Subprogram & & & & \\
\hline 1141 & 4 & & 6 & data cards & & 10264 & $B=B$ & LOCK DATA S & Subprogram & & & & \\
\hline
\end{tabular}


NBS.114A (REV. 7-73)

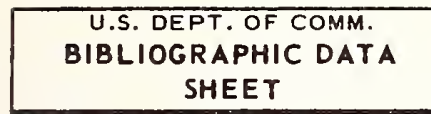

4. TITLE AND SUBTITLE

\begin{tabular}{l}
$\begin{array}{l}\text { 2. Gov'c Accession } \\
\text { No. }\end{array}$ \\
\hline
\end{tabular}

NBS-SP 399 Vol. 1
3. Recipient's Accession No.

5. Publication Date

Octobèr 1974

6. Performing Organization Code

NBS FORTRAN TEST PROGRAMS

7. $\operatorname{AUTHOR}(S)$

Frances E. Holberton Elizabeth G. Parker

9. PERFORMING ORGANIZATION NAME AND ADDRESS

\section{NATIONAL BUREAU OF STANDARDS DEPARTMENT OF COMMERCE WASHINGTON, D.C. 20234}

12. Sponsoring Organization Name and Complete Address (Street, City, State, ZIP)

\section{Same as 9}

Library of ( ongress C atalog C ard Number: 74-12314

15. SUPPLEMENTARY NOTES Volumes 1,2 , and 3 contain the documentation, Version 1 program listing, Version 3 program listing, respectively. The magnetic tape containing the NBS FORTRAN Test Programs is available in 7-track BCD, 9-track ASCII or EBCDIC recording.

16. ABSTRACT (A 200-word or less factual summary of most significant information. If document includes a significant bibliography or literature survey, mention it here.)

The NBS FORTRAN test programs, written in Standard FORTRAN, are designed to test whether a FORTRAN compiler accepts the forms and interpretations of the FORTRAiv language as described in the American National Standard FORTRAN document X3.9-1966. The test programs, comprised of 116 test units, are structured into two versions, each containing approximately 14,500 punch card images. The test units may be used as separate executable FORTRAN programs, or may be linked end to end with other test units, with a minimum of user effort, to improve operating efficiency. Version 1 is structured into 116 executable FORTRAN programs, and Version 3, containing the same 116 test units, is structured into 14 executable FORTRAN programs for use on large FORTRAN processors.

The test program design criteria was to:

- Constrain all test programs to the FORTRAN Standard X3.9-1966.

- Reduce the effect of those areas in which the FORIRAN Standard does not prescribe a method or solution, e.g., range, precision, size of computer, etc.

- Simplify the use of the FORTRAN test programs.

- Test FORTRAN language elements before they are used in support of other tests.

- Maintain an open erided systen so that tests nay be changed or addeu.

The test programs require the use of a card reader, printer and one intermediate tape unit.

17. KEY WORDS (six to twelve entries; alphabetical order; capitalize only the first letter of the first key word unless a proper name; separated by semicolons)

Computer programming language; FORTRAN: FORTRAN validation; language validation; standard FORTRAN; test program design.

18. AVAILABILITY E Unlimited

$\square$ For Official Distribution. Do Not Release to NTIS Xrder From Sup. of Doc., U.S. Government Printing Office
Washington, D.C. 20402 , SD Cat. No.C13.10:399/V.1. Order From National Technical Information Service (NTIS) Springfield, Virginia 22151

\begin{tabular}{|l|c|}
\hline $\begin{array}{l}\text { 19. SECURITY CLASS } \\
\text { (THIS REPORT) }\end{array}$ & 21. NO. OF PAGES \\
UNCL ASSIFIED & 171 \\
\hline $\begin{array}{l}\text { 20. SECURITY CLASS } \\
\text { (THIS PAGE) } \\
\text { UNCLASSIFIED }\end{array}$ & 22. Price \\
$\$ 2.35$ \\
\hline
\end{tabular}





\section{NBS TECHNICAL PUBLICATIONS}

\section{PERIODICALS}

JOURNAL OF RESEARCH reports National Bureau of Standards research and development in physics, mathematics, and chemistry. Comprehensive scientific papers give complete details of the work, including laboratory data, experimental procedures, and theoretical and mathematical analyses. Illustrated with photographs, drawings, and charts. Includes listings of other NBS papers as issued.

Published in two sections, available separately:

- Physics and Chemistry (Section A)

Papers of interest primarily to scientists working in these fields. This section covers a broad range of physical and chemical research, with major emphasis on standards of physical measurement, fundamental constants, and properties of matter. Issued six times a year. Annual subscription: Domestic, $\$ 17.00$; Foreign, $\$ 21.25$.

- Mathematical Sciences (Section B)

Studies and compilations designed mainly for the mathematician and theoretical physicist. Topies in mathematical statistics, theory of experiment design, numerical analysis, theoretical physics and chemistry, logical design and programming of computers and computer systems. Short numerical tables. Issued quarterly. Annual subscription: Domestic, $\$ 9.00$; Foreign, $\$ 11.25$.

DIMENSIONS/NBS (formerly Technical News Bulletin)-This monthly magazine is published to inform scientists, engineers, businessmen, industry, teachers, students, and consumers of the latest advances in science and technology, with primary emphasis on the work at NBS.

DIMENSIONS/NBS highlights and reviews such issues as energy research, fire protection, building technology, metric conversion, pollution abatement, health and safety, and consumer product performance. In addition, DIMENSIONS/NBS reports the results of Bureau programs in measurement standards and techniques, properties of matter and materials, engineering standards and services, instrumentation, and automatic data processing.

Annual subscription: Domestic, $\$ 6.50$; Foreign, $\$ 8.25$.

\section{NONPERIODICALS}

Monographs-Major contributions to the technical literature on various subjects related to the Bureau's scientific and technical activities.

Handbooks-Recommended codes of engineering and industrial practice (including safety codes) developed in cooperation with interested industries, professional organizations, and regulatory bodies.

Special Publications-Include proceedings of high-level national and international conferences sponsored by NBS, precision measurement and calibration volumes, NBS annual reports, and other special publications appropriate to this grouping such as wall charts and bibliographies.

Applied Mathematics Series-Mathematical tables, manuals, and studies of special interest to physicists, engineer's, chemists, biologists, mathematicians, computer programmers, and others engaged in scientific and technical work.
National Standard Reference Data Series-Provides quantitative data on the physical and chemical properties of materials, compiled from the world's literature and critically evaluated. Developed under a world-wide program coordinated by NBS. Program under authority of National Standard Data Act (Public Law 90-396). See also Section 1.2.3.

Building Science Series-Disseminates technical information developed at the Bureau on building materials, components, systems, and whole structures. The series presents research results, test methods, and performance criteria related to the structural and environmental functions and the durability and safety characteristics of building elements and systems.

Technical Notes-Studies or reports which are complete in themselves but restrictive in their treatment of a subject. Analogous to monographs but not so comprehensive in scope or definitive in treatment of the subject area. Often serve as a vehicle for final reports of work performed at NBS under the sponsorship of other government agencies.

Voluntary Product Standards-Developed under procedures published by the Department of Commerce in Part 10, Title 15, of the Code of Federal Regulations. The purpose of the standards is to establish nationally recognized requirements for products, and to provide all concerned interests with a basis for common understanding of the characteristics of the products. The National Bureau of Standards administers the Voluntary Product Standards program as a supplement to the activities of the private sector standardizing organizations.

Federal Information Processing Standards Publications (FIPS PUBS)-Publications in this series collectively constitute the Federal Information Processing Standards Register. The purpose of the Register is to serve as the official source of information in the Federal Government regarding standards issued by NBS pursuant to the Federal Property and Administrative Services Act of 1949 as amended, Public Law 89-306 (79 Stat. 1127), and as implemented by Executive Order 11717 (38 FR 12315, dated May 11, 1973) and Part 6 of Title 15 CFR (Code of Federal Regulations). FIPS PUBS will include approved Federal information processing standards information of general interest, and a complete index of relevant standards publications.

Consumer Information Series-Practical information, based on NBS research and experience, covering areas of interest to the consumer. Easily understandable language and illustrations provide useful background knowledge for shopping in today's technological marketplace.

NBS Interagency Reports-A special series of interim or final reports on work performed by NBS for outside sponsors (both government and non-government). In general, initial distribution is handled by the sponsor; public distribution is by the National Technical Information Service (Springfield, Va. 22151) in paper copy or microfiche form.

Order NBS publications (except Bibliographic Subscription Services) from: Superintendent of Documents, Government Printing Office, Washington, D.C. 20402.

\section{BIBLIOGRAPHIC SUBSCRIPTION SERVICES}

The following current-awareness and literature-survey bibliographies are issued periodically by the Bureau:

Cryogenic Data Center Current Awareness Service (Publications and Reports of Interest in Cryogenics) A literature survey issued weekly. Annual subscription: Domestic, $\$ 20.00$; foreign, $\$ 25.00$.

Liquefied Natural Gas. A literature survey issued quarterly. Annual subscription: $\$ 20.00$.

Superconducting Devices and Materials. A literature survey issued quarterly. Annual subscription: $\$ 20.00$. Send subscription orders and remittances for the pre- ceding bibliographic services to the U.S. Department of Commerce, National Technical Information Service, Springfield, Va. 22151.

Electromagnetic Metrology Current Awareness Service (Abstracts of Selected Articles on Measurement Techniques and Standards of Electromagnetic Quantities from D-C to Millimeter-Wave Frequencies). Issued monthly. Annual subscription: $\$ 100.00$ (Special rates for multi-subscriptions). Send subscription order and remittance to the Electromagnetic Metrology Information Center, Electromagnetics Division, National Bureau of Standards, Boulder, Colo. 80302. 


\section{NBS SPECIAL PUBLICATION 399}

Volume 2

U.S. DEPARTMENT OF COMMERCE / National Bureau of Standards

NBS FORTRAN Test Programs

Volume 2-Listings for Version 1 
The National Bureau of Standards ' was established by an act of Congress March 3, 1901. The Bureau's overall goal is to strengthen and advance the Nation's science and technology and facilitate their effective application for public benefit. To this end, the Bureau conducts research and provides: (1) a basis for the Nation's physical measurement system, (2) scientific and technological services for industry and government, (3) a technical basis for equity in trade, and (4) technical services to promote public safety. The Bureau consists of the Institute for Basic Standards, the Institute for Materials Research, the Institute for Applied Technology, the Institute for Computer Sciences and Technology, and the Office for Information Programs.

THE INSTITUTE FOR BASIC STANDARDS provides the central basis within the United States of a complete and consistent system of physical measurement; coordinates that system with measurement systems of other nations; and furnishes essential services leading to accurate and uniform physical measurements throughout the Nation's scientific community, industry, and commerce. The Institute consists of a Center for Radiation Research, an Office of Measurement Services and the following divisions:

Applied Mathematics - Electricity - Mechanics - Heat - Optical Physics - Nuclear Sciences" - Applied Radiation " - Quantum Electronics ${ }^{3}$ - Electromagnetics ${ }^{3}$ - Time and Frequency " - Laboratory Astrophysics" - Cryogenics".

THE INSTITUTE FOR MATERIALS RESEARCH conducts materials research leading to improved methods of measurement, standards, and data on the properties of well-characterized materials needed by industry, commerce, educational institutions, and Government; provides advisory and research services to other Government agencies; and develops, produces, and distributes standard reference materials. The Institute consists of the Office of Standard Reference Materials and the following divisions:

Analytical Chemistry - Polymers - Metallurgy - Inorganic Materials - Reactor Radiation - Physical Chemistry.

THE INSTITUTE FOR APPLIED TECHNOLOGY provides technical services to promote the use of available technology and to facilitate technological innovation in industry and Government; cooperates with public and private organizations leading to the development of technological standards (including mandatory safety standards), codes and methods of test; and provides technical advice and services to Government agencies upon request. The Institute consists of a Center for Building Technology and the following divisions and offices:

Engineering and Product Standards - Weights and Measures - Invention and Innovation - Product Evaluation Technology - Electronic Technology — Technical Analysis - Measurement Engineering - Structures, Materials, and Life Safety ${ }^{4}$ - Building Environment ${ }^{4}$ - Technical Evaluation and Application " - Fire Technology.

THE INSTITUTE FOR COMPUTER SCIENCES AND TECHNOLOGY conducts research and provides technical services designed to aid Government agencies in improving cost effectiveness in the conduct of their programs through the selection, acquisition, and effective utilization of automatic data processing equipment; and serves as the principal focus within the executive branch for the development of Federal standards for automatic data processing equipment, techniques, and computer languages. The Institute consists of the following divisions:

Computer Services - Systems and Software - Computer Systems Engineering - Information Technology.

THE OFFICE FOR INFORMATION PROGRAMS promotes optimum dissemination and accessibility of scientific information generated within NBS and other agencies of the Federal Government; promotes the development of the National Standard Reference Data System and a system of information analysis centers dealing with the broader aspects of the National Measurement System; provides appropriate services to ensure that the NBS staff has optimum accessibility to the scientific information of the world. The Office consists of the following organizational units:

Office of Standard Reference Data - Office of Information Activities — Office of Technical Publications - Library - Office of International Relations.

1 Headquarters and Laboratories at Gaithersburg, Maryland, unless otherwise noted; mailing address Washington, D.C. 20234.

Part of the Center for Radiation Research.

Y.ocated at Boulder, Colorado 80302 .

- Part of the Center for Buiding Technology. 


\section{NBS FORTRAN Test Programs \\ Volume 2-Listings for Version 1}

Frances E. Holberton and

Elizabeth G. Parker

Institute for Computer Sciences and Technology

National Bureau of Standards

Washington, D.C. 20234

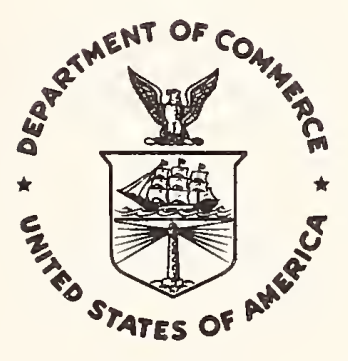

U.S. DEPARTMENT OF COMMERCE, Frederick B. Dent, Secretary NATIONAL BUREAU OF STANDARDS, Richard W. Roberts, Director

Issued October 1974 


\section{Library of Congress Cataloging in Publication Data}

Holberton, Frances E

NBS FORTRAN test programs.

National Bureau of Standards Special Publications 399.

CONTENTS: V. 1. Documentation for versions 1 and 3.-V. 2. Listings for version 1.-V. 3. Listings for version 3.

Supt. of Docs. No.: C 13.10:399.

1. Computer programs-Testing. 2. FORTRAN (Computer program language) 1. Parker, Elizabeth G., joint author. II. United States. National Bureau of Standards. 1II. Title. IV. Series: United States. National Bureau of Standards. Special Publication 399.

QC100.U57 no. 399 [QA76.6]

$389^{\prime} .08$ s $\left[001.6^{\prime} 425\right]$ $74-12314$

\section{National Bureau of Standards Special Publication 399}

Nat. Bur. Stand. (U.S.), Spec. Publ. 399,221 pages (Oct. 1974)

CODEN: XNBSAV

For sale by the Superintendent of Documents, U.S. Government Printing Office, Washington, D.C. 20402 (Order by SD Catalog No. C13.10:399/V.2). Price $\$ 2.85$ 


\section{INTRODUCTION}

This document, Volume 2 of three volumes, contains the program listings and supporting input data for the NBS FORTRAN Test Programs, Version 1, developed by the Institute for Computer Sciences and Technology, National Bureau of Standards. The test programs are written in ASA Standard FORTRAN and test the language elements described in the ASA Standard FORTRAN document X3.9-1966.

The NBS FORTRAN Test Programs, Version 1, contain 116 test units, each structured as an executable FORTRAN program. Test units numbered 008, 009,310 , and 312 contain input data which is listed following the respective test unit.

This listing is in the onder described in Volume I Section III Distribution Tape Organization.

Volume I, Section I describes the system design, the programming techniques and conventions used in the program development and should enable the user to extend, alter or reorganize the test programs.

Volume I, Section II defines the organization and operating procedure for performing the tests and contains a set of representative results obtained from actual running of the test programs on several FORTRAN processors.

Volume, , Section III describes the order and location of each test unit and data as reconded on magnetic tape for distribution.

Volume 2 contains the program listings for the NBS FORTRAN Test Programs, Version 1.

Volume 3 contains the program listings for the NBS FORTRAN Test Programs, Version 3. 
Table of Contents

000

008 - FMTRW

009 - AFRMT

010 - DATA2

003 - DATA1

011 - AASGN

013 - DASGN

015 - CASGN

016 - LASGN

017 - INTRL

020 - UGOTO

021 - AGOTO

022 - CGOTO

030 - ARBAD

031 - ARFAD

032 - ARBSB

033 - ARFSB

034 - ARBAS

035 - ARFAS

036 - ARBMI

037 - ARBMR

038 - ARFMD

039 - ARBDV

040 - ARFDV

041 - ARBEX

042 - ARFEX

043 - ARBHI

050 - SBB67

051 - SBB45

$052-$ SBB13

$053-\mathrm{SBF} 17$

054 - SIMIF

055 - IFABS

056 - IFFLT

057 - IFFIX

058 - IFSGN

059 - IFDAB

060 - IFTRN

061 - IFMOD

062 - IFMAX

063 - IFMIN

064 - IFDSG

065 - IFDIM

066 - IFSGL

067 - IFREL
Directory of Test Programs

Formatted Input/Output

6 Identification Cards and 40 Data Cards

A-Conversion

3 Data Cards

DATA Statement Use

Test Format of Data Statement

Real and Integer Arith Assignmt Stmnts

Simple D.P. Assignment Statements

Simple Complex Assignment Statements

Logical Assignment Statements

Arithmetic Assignment Statements

Unconditional GO TO Statements

GO TO Assignment Statements

Computed GO TO Statements

Basic Addition

Double Precision Addition

Basic Subtraction

Double Precision Subtraction

Basic Addition and Subtraction

Addition and Subtraction of D.P. Values

Multiplication of Integer Values

Multiplication of Real Values

Multiplication of D.P. Values

Division of Integer and Real Values

Division of D.P. Values

Exponentiation of Integer and Real Values

Exponentiation of D.P. Values

Hierarchy of Operators and Parentheses

Subscripts of Integer, Real Arrays v, k

Subscripts of Int., Real Arrays $v+k, v-k$

Subscripts of Int., Real Arrays $c^{*} v, c^{*} v+k$ $c^{*} \mathrm{v}-\mathrm{k}$

Subscripts of D.P. Arrays v, k, $c^{*} v, c^{*} v+k$, $c^{*} \mathrm{v}-\mathrm{k}, \mathrm{v}+\mathrm{k}, \mathrm{v}-\mathrm{k}$

Arith IF, Logical IF followed by GO TO

Intrinsic Functions ABS, IABS

Intrinsic Function FLOAT

Intrinsic Function IFIX

Intrinsic Functions SIGN, ISIGN

Intrinsic Function DABS

Intrinsic Functions AINT, INT, IDINT

Intrinsic Functions AMOD, MOD

Intr Funct AMAXO, AMAXI, MAXO, MAXI, DMAXI

Intr Funct AMINO, AMIN1, MINO, MIN1, DMIN1

Intrinsic Function DSIGN

Intrinsic Functions DIM, IDIM

Intrinsic Function SNGL

Intrinsic Function REAL
Page 
Table of Contents

068 - IFIMG
069 - IFDBL
070 - IFCPX
071 - IFCJG
072 - IFBMS
073 - IFFMS
080 - EXPON
081 - DEXPO
082 - CEXPO
083 - LOGTM
084 - DPLOG
085 - CXLOG
086 - COLOG
087 - DCLOG
088 - SINUS
089 - DPSIN
090 - CSICO
091 - COSNS
092 - DPCOS
094 - TANGH
095 - SQROT
096 - DSQRO
097 - CSQRO
098 - ARCTG
099 - DACTG
100 - ACTG2
101 - DATN2
102 - DMODA
103 - CABSA
110 - BSFTS
005 - BSFDF
111 - FSFTS
006 - FSFDF
140 - CPXAD
141 - CPXMU
142 - CPXDV
143 - CPXEX
144 - CPXOP
145 - CREAD
146 - CREMU
147 - CREDV
148 - CREOP
149 - MISC3
150 - MISC4
160 - BRFCP
400 - AFS
420 - BFS
430 - CFS
440 - DFS
450 - EFS
460 - FFS

Intrinsic Function AIMAG

Intrinsic Function DBLE

Intrinsic Function CMPLX

Intrinsic Function CONJG

Integer and Real Intrinsic Functions 91

Int, Real and D.P. Intrinsic Functions 93

Basic External Function EXP 96

Basic External Function DEXP 97

Basic External Function CEXP 98

Basic External Function ALOG 99

Basic External Function DLOG 100

Basic External Function CLOG 101

Basic External Function ALOG10 103

Basic External Function DLOG10 104

Basic External Function SIN 104

Basic External Function DSIN 106

Basic External Functions CSIN, CCOS 107

Basic External Function COS 108

Basic External Function DCOS 109

Basic External Function TANH 110

Basic External Function SQRT 111

Basic External Function DSQRT 112

Basic External Function CSQRT 113

Basic External Function ATAN 114

Basic External Function DATAN 115

Basic External Function ATAN2 116

Basic External Function DATAN2 117

Basic External Function DMOD 118

Basic External Function CABS 118

Statement Functions - Integer and Real 120

Statement Function Definition 120

Statement Funct - D.P., Complex, Logical 121

Statement Function Definitions 122

Addition and Subtraction of Complex 124

Multiplication of Complex Numbers $\quad 125$

Division of Complex Numbers 127

Exponentiation of Complex Numbers 128

Arithmetic Operations on Complex 130

Add and Subtract Complex and Real Numbers 131

Multiply Complex by Real Numbers 132

Divide Complex by Real and the Reverse 133

Combined Operations on Complex and Real 134

Blanks in, Cont. of Statement to Max Lines 135

Special Characters for Continuations 136

Real External Functions 138

Real Argument 139

Real Arguments $\quad 139$

Integer Argument 139

Integer Arguments 139

Array Name As Argument 139

Different Types of Arguments 140 
Real Argument 141

Real Arguments 141

Integer Argument 141

Integer Arguments 141

Array Name as Argument 142

Different Types of Arguments 142

Real External Functions 142

D.P. Argument 144

Complex Arguments 144

Logical Argument 144

External Procedure 145

Different Types of Arguments 145

Integer External Functions 145

D.P. Argument 147

Complex Arguments $\quad 147$

Logical Argument 147

External Procedure $\quad 148$

Different Types of Arguments 148

Complex External Functions 148

Real Argument 150

Integer Argument 150

Array Name as Argument 150

D.P. Argument 151

Complex Argument 151

Logical Argument 151

Different Types of Arguments 151

Double Precision External Functions 152

Real Argument 153

Integer Argument $\quad 154$

D.P. Arguments 154

Complex Argument $\quad 154$

Logical Argument 154

External Procedure 154

Array Name as Argument 155

Different Types of Arguments 155

Logical External Functions 155

Real Argument 157

Integer Argument 157

D.P. Argument 158

Logical Argument 158

Complex Argument 158

Array Name as Argument 158

External Procedure $\quad 158$

Different Types of Arguments 158

$\begin{array}{ll}\text { Subroutine Subprogram } & 159\end{array}$

Integer, Real Variables, Array Elements 160

Array Elements 161

No Argument List 161

Subroutine Subprogram 161

Different Types of Arguments 163

Array Names and Integer Arguments 164

No Argument List 164 
Table of Contents

169 - BLKDT
409 - BLOKD
179 - BLKDA
419 - BLAKD
429 - BLBKD
439 - BLCKD
180 - UNFRW
182 - BACUP
190 - DOTRM
191 - DOLMT
192 - DONSC
193 - DONSI
194 - DONSX
195 - DONML
196 - DONIO
197 - MORDO
005 - BSFDF
412 - MDQ
200 - SUBRI
410 - SUBRQ
300 - LOGIF
411 - SMCQ
301 - BARIF
302 - FARIF
310 - IOFMT
312 - RDFMT
462 - FMTQ
350 - MISC5
351 - FUNMX
352 - NAMES
413 - MAQQ
463 - MBQQ
473 - AMQQ
483 - BMQQ
360 - SPEC2

BLOCK DATA Test BLOCK DATA Subprogram

BLOCK DATA Test

BLOCK DATA Subprogram

BLOCK DATA Subprogram

BLOCK DATA Subprogram

Unformatted WRITE and READ

BACKSPACE Tape

DO Loops - Terminal Statements

Do Loops - Parameters as Variable Names

DO Loops - Completely Nested Nest

DO Loops - Incomplete DO, Exit by GO TO

Do Loops - Extended Range

DO Loops - Nested Nest

Do Loops - I/O Terminal Statements

DO Loops - I/O, Statmt. Ft., Intr Ft., CALL

Statement Functions

Subroutine Subprogram

Subroutine - Operations Done at Sub Level Subroutine Subprogram - No Arg. List

Logical IF Statements

Subroutine Subprogram

Arithmetic IF Statements - Integer, Real

Arithmetic IF Statements - D.P.

Formatted READ/WRITE - Additional Features

38 Data Cards

Formats in Arrays

Subroutine Subprogram

13 Data Cards

Specifications for Program Form

Basic External Functions - Trig Formulae

Names Resemble FORTRAN Verbs, Functions

Subroutine

(Intrinsic Function Names

Subroutine used as Variable Names in

Subroutine

some Subrts. and as

Subroutine

Functions in others)

COMMON, DIMENSION, EQUIVALENCE

Page

165

166

166

168

168

168

168

170

171

173

174

177

178

180

181

182

182

184

185

185

187

191

191

194

195

200

200

203

204

204

206

207

208

208

209

209

209 



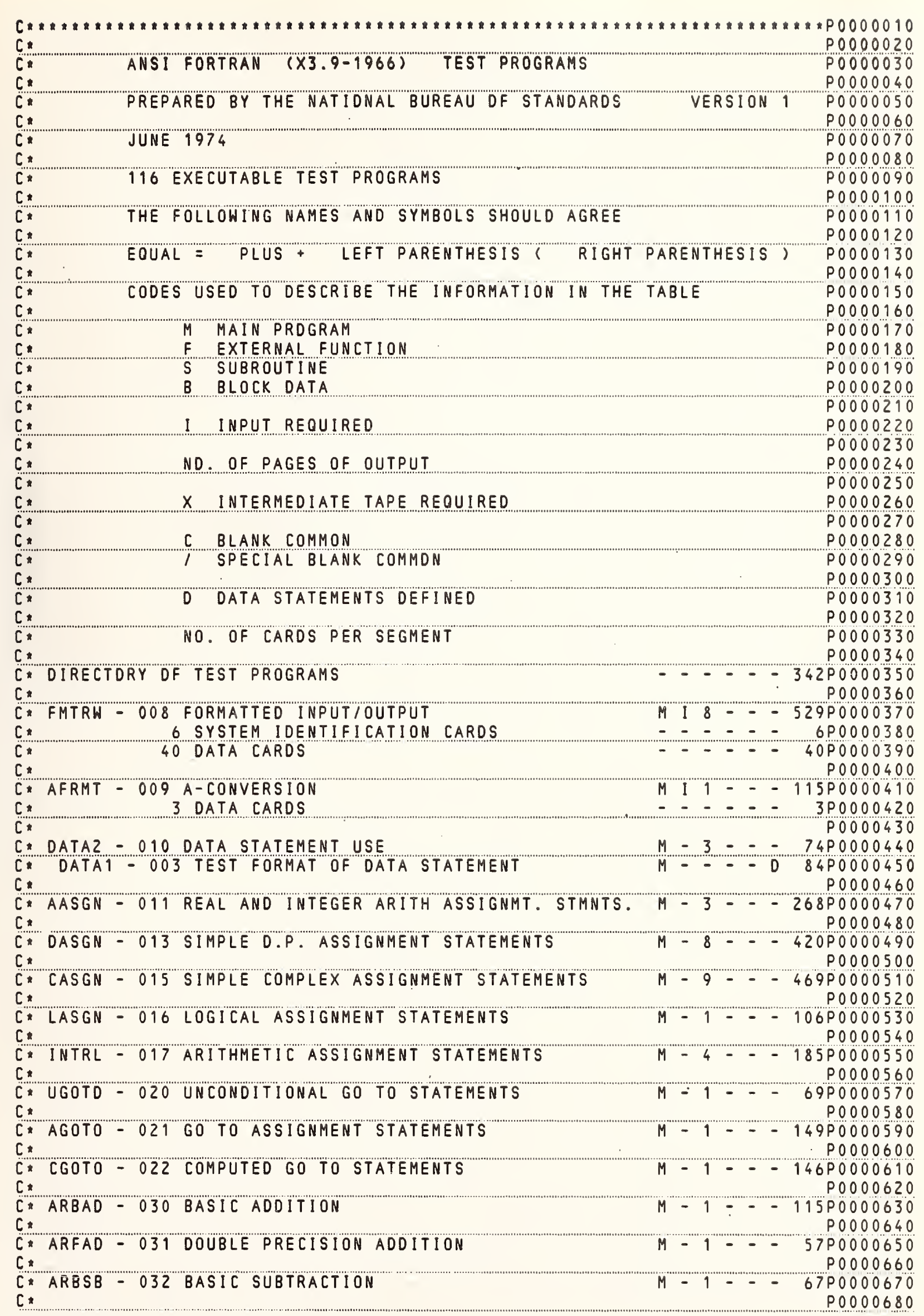


C* ARBMI - O36 MULTIPLICATION OF INTEGER VALUES

$60 P 0000730$

$P 0000740$

C*

C*ARBMR - 037 MULTIPLICATION OF REAL VALUES

66P0000750

$P 0000760$

C* ARFMO - 038 MULTIPLICATION OF D.P. VALUES

$64 P 0000770$

$C *$

ARBDV - 039 OIVISION OF INTEGER AND REAL VALUES

$P 0000780$

$C$.

C*ARFOV - 040 OIVISION OF O.P. VALUES

$M-1-71 P 0000790$
$P 0000800$

$M-1-71 P 0000790$
$P 0000800$

$M-1-78 P 0000810$
$P 0000820$

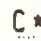

C* ARBEX - 041 EXPONENTIATION OF INTEGER AND REAL VALUES

$C *$

C* ARFEX - 042 EXPONENTIATION OF O.P. VALUES

$C *$

C* ARBHI - 043 HIERARCHY OF OPERATORS ANO PARENTHESES

$M-1-\cdots 660000830$

P0000840

$90 P 0000850$

$P 0000860$

$M-1-7 P 0000870$
$P 0000880$

$7-1-7 P 0000870$
$P 0000880$

$C *$

C* SBB67 - 050 SUBSCRIPTS OF INTEGER, REAL ARRAYS $V, K$

C*

C* SB845-051 SUBSCRIPTS OF INT. .REAL ARRAYS $V+K, V-K$

C.

C* SBB 13 - OS2 SUBSCRIPTS OF INT. REAL ARRAYS C*V.

C*

$C * V+K, \quad C * V-K$

$M-1--177 P 0000890$

P0000900

$79 P 0000910$

P0000920

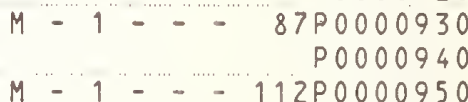

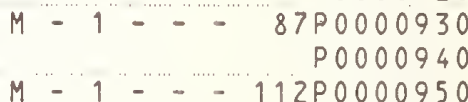

$C$ *

C* SBF 17 - 053 SUBSCRIPTS OF D.P. ARRAYS $V, K, C * V$,

$C *$

$C * V+K, \quad C * V-K, V+K, V-K$

$P 0000960$

P0000970

$M-1-$ - $79 P 0000980$

$P 0000990$

$C$ *

C* SIMIF- 054 ARITH. IF, LOGICAL IF FOLLOWED BY GO TO

P0001000

$C *$

C*IFABS - OS5 INTRINSIC FUNCTIONS ABS. IABS

C* IFFLT - OS6 INTRINSIC FUNCTION FLOAT

C*

C*IFFIX-0S7 INTRINSIC FUNCTION IFIX

$C *$

C* IFSGN - 058 INTRINSIC FUNCTIONS SIGN, ISIGN

$C *$

C* IFDAB- O59 INTRINSIC FUNCTION DABS

$C$

C*IFTRN - 060 INTRINSIC FUNCTIONS AINT, INT, IDINT

$C *$ IFMOO- 061 INTRINSIC FUNCTIONS AMOD, MOD

C*

C*IFMAX - 062 INTR. FUNCT. AMAXO,AMAX1, MAX0, MAX1, DMAX1

$c$ *

C*IFMIN-063 INTR. FUNCT. AMINO,AMINI, MINO,MINI,OMINI

C*IFOSG - 064 INTRINSIC FUNCTION OSIGN

C*IFOIM - 065 INTRINSIC FUNCTIONS DIM, IDIM

C*IFSGL - 066 INTRINSIC FUNCTION SNGL

$M-1-\ldots+77 P 0001010$
$M 0001020$

$M-1-\ldots+77 P 0001010$
$M 0001020$

$P 0001040$

$M-1-\quad-49 P 0001050$

P0001060

$M-1-$ - $59 P 0001070$

P0001080

$M-1$ - - 82P0001090

$P 0001100$

$M=1-\ldots 65 P 0001110$

$P 0001120$

$M-1$ - - 107P0001130 $P 0001140$

$M-1$ - $\quad 84 P 0001150$

$P 0001160$

$M=2-248 P 0001170$

$P 0001180$

$M-2--225 P 0001190$ $P 0001200$

$M-1$ - - 58P0001210 $P 0001220$

M-1 - - $69 P 0001230$

$P 0001240$

$M-1--{ }^{-} 80 P 0001250$ $P 0001260$

C* IFREL - 067 INTRINSIC FUNCTION REAL

C*

C*IFIMG - 068 INTRINSIC FUNCTION AIMAG

C* IFOBL - 069 INTRINSIC FUNCTION OBLE

C* IFCPX - 070 INTRINSIC FUNCTION CMPLX

C* IFCPX - 070 INTRINSIC FUNCTION CMPLX

C IFCJG- 079 INTRINSIC FUNCTION CONJG

$M-1-\ldots P$
$P 0001280$

$M-1=--129 P 0001290$

$P 0001300$

$M-1-$ - $57 P 0001310$

$P 0001320$

$M-1--\quad 61 P 0001330$

$P 0001340$

$M-1-\cdots 66 P 0001350$

$P 0001360$ 
C* IFBMS - 072 INTEGER AND REAL INTRINSIC FUNCTIONS

$M-1--129 P 0001370$

C*IFFMS - 073 INT. REAL AND D.P. INTRINSIC FUNCTIONS M - 2 -

C* $\quad$ P0001400

C*EXPON - O80 BASIC EXTERNAL FUNCTION EXP M - $1--60 P 0001410$

$C *$

C DEXPO- O81 BASIC EXTERNAL FUNCTION DEXP

$P 0001410$
$P 0001420$

C*.......

C* CEXPO - O8Z BASIC EXTERNAL FUNCTION CEXP

$M-1-280001430$
$P 0001440$

C*

C* LOGTM - 083 BASIC EXTERNAL FUNCTION ALOG

C*

C* OPLOG - 084 BASIC EXTERNAL FUNCTION DLOG

$M-1-68 P 0001430$

C.

C*

CXLOG - O85 BASIC EXTERNAL FUNCTION CLOG

CLOG

$M-3-\cdots 9 P 0001450$

$P 0001460$

$M-1--57 P 0001470$
$P 0001480$
$H-1--67 P 0001490$

$M-1--57 P 0001470$
$P 0001480$
$M-1-\cdots \quad 67 P 0001490$

$P 0001500$

$C *$

$C *$

COLOG - 086 BASIC EXTERNAL FUNCTION ALOG10

$M-3--106 P 0001510$

$C *$

$c *$

OCLOG - 087 BASIC EXTERNAL FUNCTION DLOG 10

$P 0001520$

SINUS - O88 BASIC EXTERNAL FUNCTION SIN

IIN

6P0001530

P0001540

$M-1-66 P 0001550$
$P 0001560$

$M-1-66 P 0001550$
$P 0001560$

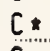

C* OPSIN - O89 BASIC EXTERNAL FUNCTION OSIN

C*

CSICO - 090 BASIC EXTERNAL FUNCTIONS

$\operatorname{csIN}, \cos$

$M-1-81 P 0001570$
$P 0001580$

$C *$

$C:$

COSNS - O91 BASIC EXTERNAL FUNCTION COS

OPCOS - 092 BASIC EXTERNAL FUNCTION OCOS

$M-1--82 P 0001590$

$P 0001600$

$65 P 0001610$

$P S P 001620$
$M-1-1-82 P 001630$
$P 0001640$
$M-1-81 P 001650$
$P 0001660$

$P S P 001620$
$M-1-1-82 P 001630$
$P 0001640$
$M-1-81 P 001650$
$P 0001660$

$P S P 001620$
$M-1-1-82 P 001630$
$P 0001640$
$M-1-81 P 001650$
$P 0001660$

$C *$

C* TANGH - 094 BASIC EXTERNAL FUNCTION TANH

$c$ *

$C *$

$C$.

C*

C*

C:

C.:

$c *$

$C *$ OA

C*

$C *$ ACTGZ - 100 BASIC EXTERNAL FUNCTION ATANZ

Cः DATNZ - 101 BASIC EXTERNAL FUNCTION DATAN

$C *$

C: OMOOA - 102 BASIC "EXTERNAL FUNCTION DMOD

C*

C* CABSA = 103 BASIC EXTERNAL FUNCTION CABS

SOROT = 095 BASIC EXTERNAL FUNCTION SORT

$M=1--37 P 0001670$

$P 0001680$

OSORO - 096 BASIC EXTERNAL FUNCTION DSORT

$M-1-\cdots 55 P 0001690$

$P 0001700$

$M-1-63 P 0001710$
$P 0001720$

$M-1-63 P 0001710$
$P 0001720$

CSORO - 097 BASIC EXTERNAL FUNCTION CSORT

ARCTG = 098 BASIC EXTERNAL FUNCTION ATAN

$4 P 0001740$

$58 P 0001750$

$P 0001760$

$66 P 0001770$

$M-1-60 P 0001770$
$P 0001780$
$M-1-\cdots$

$P 0001800$

$M-1-6 P 0001810$
$P 0001820$

$M-1-6 P 0001810$
$P 0001820$

C*

C* BSFTS - 110 STATEMENT FUNCTIONS - INTEGER ANO REAL

C* BSFOF - OOS STATEMENT FUNCTION DEFINITION

PO

$P 0001840$
$M-1-\cdots$

$P 0001860$

BSEOE O OS STATEMENT EUNCTION OEFINIT ION

$M-1-\cdots 740001870$

$M---35 P 0001880$

C* FSFTS - 111 STATEMENT FUNCT - D.P. COMPLEX, LOGICAL

C FSFOF - 006 STATEMENT FUNCTION DEFINITIONS

$P 0001890$

$c$.

C: CPXAO- 140 ADOITION ANO SUBTRACTION OF COMPLEX

$C *$

$C$ CPXMU - 141 MULTIPLICATION OF COMPLEX NUMBERS

$M-1--108 P 0001900$
$M--\cdots+180001910$

$C *$

$C *$ CPXOV-142 DIVISION OF COMPLEX NUMBERS

$C *$

C CPXEX - 143 EXPONENTIATION OF COMPLEX NUMBERS $P 0001920$

$c *$

C CPXOP - 144 ARITHMETIC OPERATIONS ON COMPLEX

$M-1-\cdots+760001930$

$P 0001940$

$M-1--141 P 0001950$ P0001960

C.

$C:$

C.

CREAO - 145 ADO AND SUBTRACT COMPLEX AND REAL NUMBERS

$M-1---83 P 0001970$

$P 0001980$

$M-1--125 P 0001990$ $P 0002000$

$M-1--63 P 0002010$ $P 0002020$

$67 P 0002030$

$P 0002040$ 
C* CREMU - 146 MULTIPLY COMPLEX BY REAL NUMBERS

C* CREOV - 147 DIVIDE COMPLEX BY REAL AND THE REVERSE

$M-1---58 P 0002070$

C. $P 0002080$

C* CREOP - 148 COMBINED OPERATIONS ON COMPLEX AND REAL

6600002090

$C *$

C.

$C *$

C*

C:

C. BRFCP - 160 REAL EXTERNAL FUNCTIONS

C* AFS -400 REAL ARGUMENT

C* BFS - 420 REAL ARGUMENTS

C* CFS - 430 INTEGER ARGUMENT

C*DFS -440 INTEGER ARGUMENTS

C* EFS - 450 ARRAY NAME AS ARGUMENT

C* FFS - 460 DIFFERENT TYPES OF ARGUMENTS

$P 0002100$

C*

C BIFCP - 161 INTEGER EXTERNAL FUNCTIONS

C. IAFI- 401 REAL ARGUMENT

$C *$ IBFI - 421 REAL ARGUMENTS

C ICFI - I31 INTEGER ARGUMENT

C* IDFI-441 INTEGER ARGUMENTS

$C * \quad I E F I-451 \quad$ ARRAY NAME AS ARGUMENT

$C$ IFFI -46

$P 0002110$

P0002120

C.........

C* FRFCP - 162 REAL EXTERNAL FUNCTIONS

C $\quad G F S-402 \quad$ D.P. ARGUMENT

C*HFS - 422 COMPLEX ARGUMENTS

C* IRFS - 432 LOGICAL ARGUMENT

C* JRFS - 442 EXTERNAL PROCEDURE

C* RFS - 452 DIFFERENT TYPES OF ARGUMENTS

$M-1--105 P 0002130$
$P 0002140$
$M-1-\quad 82 P 0002150$

$M-1--105 P 0002130$
$P 0002140$
$M-1-82 P 0002150$

$F---\quad-010 P 0002160$

$F$ - - - 10P0002170

$F-$ - - 10P0002180

$F-\cdots 11 P 0002190$

$F-F_{-}-11 P 0002200$

$F$ - - - $15 P 0002210$

$P 0002220$

$M-1--\quad 87 P 0002230$

$F----10 P 0002240$

$F-$ - - 10P0002250

$F-=-10 P 0002260$

$F$ - - D $13 P 0002270$

$F----11 P 0002280$

$F-\cdots+\cdots 1590002290$

$P 0002300$

$M-1-C-132 P 0002310$

$F--\quad-\quad 11 P 0002320$

$F-\cdots+12 P 0002330$

$F-$ - - $16 P 0002340$

$F-\cdots+11 P 0002350$

$F$ - - C - 29P0002360

$P 0002370$

C* FIFCP - 163 INTEGER EXTERNAL FUNCTIONS M $\quad 1-C-123 P 0002380$

C* IFI-403 D.P. ARGUMENT F - $--11 P 0002390$

C* JFI-423 COMPLEX ARGUMENTS

C* KFI- 433 LOGICAL ARGUMENT

C* LFI-443 EXTERNAL PROCEDURE

C* MFI -453

DIFFERENT TYPES OF ARGUMENTS

$F-$ - - $12 P 0002400$

$F$ - - - $16 P 0002410$

$F---\ldots 11 P 0002420$

C* $*$ CFCCP -164 COMPLEX EXTERNAL FUNCTION

C* AFC -404 REAL ARGUMENT

C BFC -414 INTEGER ARGUMENT

C* CFC - 424 ARRAY NAME AS ARGUMENT

C* DFC - 434 D.P. ARGUMENT

C* EFC -444 COMPLEX ARGUMENT

$C *$ FFC -454 LOGICAL ARGUMENT

C* HFC - 464 DIFFERENT TYPES OF ARGUMENTS

$F---C-29 P 0002430$

$P 0002440$

C* DPFCP - 165 DOUBLE PRECISION EXTERNAL FUNCTIONS

$C *$ DPFCP -165 DOUBLE PRECISION
$C *$ AFD -405 REAL ARGUMENT

$C * \quad B F D=415 \quad$ INTEGER ARGUMENT

C* CFD - 425 D.P. ARGUMENTS

$C * \quad$ DFD $-435 \quad$ COMPLEX ARGUMENT

C* EFD- -445 LOGICAL ARGUMENT

C* FFD - 455 EXTERNAL PROCEDURE

C* GFD - 465 ARRAY NAME AS ARGUMENT

C* HFD - 475 DIFFERENT TYPES OF ARGUMENTS

$M-1-C-132 P 0002450$

$F$ - _ - 10P0002460

$F=--10 P 0002470$

$F----11 P 0002480$

$F--\cdots 12 P 0002490$

$F----\quad 11 P 0002500$

$F \cdots-\cdots 15 P 0002510$

$F--C-C-28 P 0002520$

P0002530

$M-1-C-135 P 0002540$

$F-\cdots 10 P 0002550$

$F$ - - - $10 P 0002560$

$F$ - - - 11P0002570

$F-$ - - $12 P 0002580$

$F--\cdots 16 P 0002590$

$F$ - - - 11P0002600

$F----12 P 0002610$

$F--C-32 P 0002620$

$P 0002630$

C* BFCCP - 166 LOGICAL EXTERNAL FUNCTIONS M $-1-C-144 P 0002640$

C AFB -406 REAL ARGUMENT F - - - -

C BFB - 416 INTEGER ARGUMENT F - $--\ldots$

C* CFB - $426 \quad$ D.P. ARGUMENT $\quad$ F - - - - $11 P 002670$

C. DFB - $436 \quad$ LOGICAL ARGUMENT

C. EFB - 446 COMPLEX ARGUMENT

C FFB - 456 ARRAY NAME AS ARGUMENT

C GFB - 466 EXTERNAL PROCEDURE

C HFB -476

DIFFERENT TYPES OF ARGUMENTS

$F----11 P 0002680$

$F^{-}-\ldots-12 P 0002690$

$F$ - - - $12 P 0002700$

$F-\cdots-11 P 0002710$

$F--C-25 P 0002720$ 
$C *$ SBRTN - 167 SUBROUTINE SUBPROGRAM

C * AAQ - 407 INTEGER,REAL VARIABLES,ARRAY ELEMENTS

$C * \quad A B Q-417$ ARRAY ELEMENTS

$C * A C O-427$. NO ARGUMENT LIST

$M-1-C-103 P 0002740$

$C *$

C * FSBRT - 168 SUBROUTINE SUBPROGRAM $S--\quad-\quad-13 P 0002760$

$S--C-21 P 0002770$

$P 0002780$

C* $\quad A O Q-408$ OIFFERENT TYPES OF ARGUMENTS

C. $A E O-418$

ARRAY NAMES ANO INTEGER ARGUMENTS

$M-1-C-153 P 0002790$

$A F O-428$ NO ARGUMENT LIST

$S----39 P 0002800$

$C *$

$C * B L K O T-169$ BLOCK OATA TEST

$C * B L O K D-409$ BLOCK DATA SUBPROGRAM

$S$ - - - 23P0002810

$C *$

C*BLKOA - 179 BLOCK OATA TEST.

$C *$ BLAKO - 419 BLOCK OATA SUBPROGRAM

$C * B L B K D=429$ BLOCK OATA SUBPROGRAM

$C * B L C K O-439 \quad$ BLOCK OATA SUBPROGRAM

S - - C - 41P0002820

$P 0002830$

$C *$

C* UNFRW - 180 UNFORMATTEO WRITE ANO REAO

$M-1--$

$71 P 0002840$

B - - - D $36 P 0002850$

$P 0002860$

$M-1---70 P 0002870$
$B--D-0$

$B-\ldots-017 P 0002890$

$B--\quad-0 \quad 20 P 0002900$

P0002910

C*

C* BACUP - 182 BACKSPACE TAPE

$M$ - $1 X$ - $133 P 0002920$ $P 0002930$

c.

C* OOTRM - 190 OO LOOPS - TERMINAL STATEMENTS

$1 \times-1 X-74 P 0002940$
$P 0002950$

C*

OOLMT - 191 00 LOOPS - PARAMETERS AS VARIABLE NAMES

$c$

$C *$

C* DONSC - 192 OO LOOPS - COMPLETELY NESTEO NEST

$M-1$ - - $135 \mathrm{P0002960}$

$M-1-\quad P 0002970$

$C *$ OONSI - 193 DO LOOPS - INCOMPLETE DO, EXIT BY GO TO

P0002990

C* OONSX - 194 DO LOOPS - EXTENDED RANGE

$M-1-166 P 0003000$ P0003010

$60 P 0003020$

$P 0003030$

C* DONML - 195 OO LOOPS - NESTED NEST

P0003050

$c$

C* OONIO - 196 DO LOOPS - $1 / 0$ TERMINAL STATEMENTS M - 1 X - 91P0003080

$5 P 0003060$

$P 0003070$

C*

C* MORDO - 197 O0 LOOPS - I/0,STATMT FT..INTR FT...CALL M $1 X-143 P 0003100$

$C *$ BSFOF - 005 STATEMENT FUNCTIONS

C* MDQ - 412 SUBROUTINE SUBPROGRAM

$M=-\cdots-35 P 0003110$

C* SUBR1 - 200 SUBROUTINE - OPERATIONS DONE AT SUB LEVEL M - 1 X C - 52 P0003140

$C *$ SUBRO - 410 SUBROUTINE SUBPROGRAM - NO ARG. LIST $S=-X c-101 P 0003150$

c.

$C *$ LOGIF $=300$ LOGICAL IF STATEMENTS

C* SMCQ - 411 SUBROUTINE SUBPROGRAM

P 0003160

c

C* BARIF - 301 ARITHMETIC IF STATEMENTS - INTEGER REAL

$C *$

$C *$

FARIF - 302 ARITHMETIC IF STATEMENTS - D.P.

M-1- - $-275 P 0003170$

S - - - 12P0003180 P 0003190

$75 P 0003200$

RIF - 302 ARITHMETIC IF STATEMENTS - D.P.

C* IOFMT - 310 FORMATTEO REAO/WRITE - AOOITIONAL FEATURES M I 5 - - $310 P 0003240$

C* 38 OATA CARDS $\quad-\quad-\quad-38 P 003250$

$C *$ RDFMT -312 FORMATS IN ARRAYS

C* FMTO - 462 SUBROUTINE SUBPROGRAM

C* 13 DATA CAROS

$P 0003260$

$C *$

C*MISC5 - 350 SPECIFICATIONS FOR PROGRAM FORM

MI I - $-0201 \mathrm{P} 0003270$

S - - - 33P0003280

- - - $13 P 0003290$

$P 0003300$

$C *$

C* FUNMX - 351 BASIC EXTERNAL FUNCTIONS - TRIG FORMULAE

$M-1--156 P 0003310$

$P 0003320$

$C *$

C NAMES - 352 NAMES RESEMBLE FORTRAN VERBS, FUNCTIONS

C* MAQQ- 413 SUBROUTINE (INTRINSIC FUNCTION NAMES

$C$ * MBOO-463 SUBROUTINE USED AS VARIABLE NAMES IN

$C * A M O O-473$

SUBROUTINE SOME SUBRTS. ANO AS

$C * B M O O-483$ SUBROUTINE FUNCTIONS IN OTHERS )

$58 \mathrm{P0003330}$

$P 0003340$

$M-1--79 P 0003350$

$S---\quad-15 P 0003360$

S - - - $15 P 0003370$

S - - D $21 P 0003380$

S - - - $16 \mathrm{P} 0003390$

$P 0003400$

NBS FORTRAN Test Programs Version 1 
C* SPECZ - 360 COMMON, DIMENSION, EQUIVALENCE

$M-1-1-169 P 0003410$ $P 0003420$

$C *$ PO 0880020

$C * * * *$

$C * * * *$

FMTRW - $(008)$

$P 0080030$

$C * * * *$

$P 0080040$

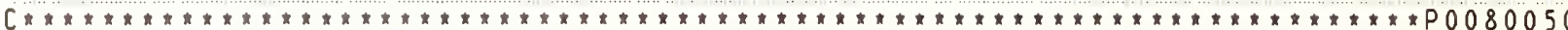

$C * * * *$ GENERAL PURPOSE

C*\#* TO TEST SIMPLE FORMAT AND FORMATTEO I/O STATEMENTS

ASA REFSPO080060

C** PROGRAM SEGMENTS

$C * * * *$ RESTRICTIONS OBSERVEO

$\because *$ * ALL FORMAT STATEMENTS ARE LABELEO

$C * * * *$ H ANO X DESCRIPTORS ARE NEVER REPEATED

$C * *$ FOR W.O OESCRIPTORS, 0 IS ALWAYS SPECIFIED AND

$C * * *$ W IS EQUAL TO OR GREATER THAN 0

$C * * * *$ FIELO WIOTH IS NEVER ZERO

$C * * * * *$ IF THERE IS AN I/O LIST, THE FORMAT STATEMENT

$C * * *$ CONTAINS AT LEAST ONE FIELD OESCRIPTOR (OTHER

$[* * * *$ THAN HOR $X)$

$7.1 .3 .2 .2 P 0080070$

$7.1 .3 .2 .3 P 0080080$

$7.2 .3 \quad P 0080090$

$P 0080100$

$7.2 .3 \quad 157 P 0080110$

$7.2 .3 .3 / 54 P 0080120$

7.2.3.1/31P0080130

$7.2 .3 .1 / 33 P 0080140$

$7.2 .3 / 18 P 0080150$

7.2.3.4/22P0080160

$P 0080170$

P0080180

$[* * * *$ ITEMS IN $1 / 0$ LIST CORRESPOND TO FORMAT OESCRIPTORS $7.2 .3 .4 / 36 P 0080190$

$[* * * *$ NEGATIVE OUTPUT VALUES ARE SIGNEO

$C * * * * *$ FIELO WIOTH NEVER EXCEEOEO BY OUTPUT

$C * * * * *$ FOR I CONVERSION, EXTERNAL INPUT FIELOS ARE

$C * * * *$ INTEGER CONSTANTS

$C * * *$ GENERAL COMMENTS

C***** PLUS SIGNS FOR INPUT FIELOS ARE USUALLY OMITTEO

$7.2 .3 .6 / 56 P 0080200$

7.2 .3 .610190080210

$7.2 .3 \cdot 6 \cdot 1 / 07 P 0080220$ $P 0080230$ P0080240

$C * * * *$

$C * * *$

$C * * * *$

$C * * * *$

$C * * * * *$

$C * * * * *$

STATEMENTS TEST H AND X DESCRIPTORS AND SLASH

$C * * * *$ RE CORD DIVIDERS)

$7.2 .3 .6 / 44 P 0080250$
$P 0080260$ $P 0080270$ $P 0080280$

$7 \cdot 1 \cdot 3 \cdot 2 \cdot 3 / 05 P 0080290$

$7.2 .3 .2144 P 0080300$

$7.2 .3 .8 \quad 109 P 0080310$

7.2.3.9 131P0080320

C INPUT OATA TO THIS SEGMENT CONSISTS OF 40 CARO IMAGES IN COL. 1 - 80 POO80330

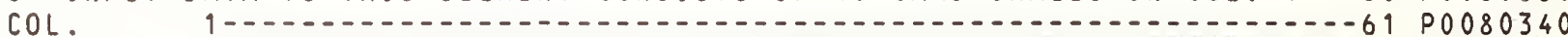

CARD 1 1 999

CARO $2 \quad 555554444$

$\begin{array}{llll}\text { CARD } & 3 & 666 & 777777\end{array}$

CARD 4 333333911112222222255555444444444444

P0080350

P0080360

7.7123456 .7

$\begin{array}{lll}\text { CARD } & 6 & 8.889 .9997 .123456 \\ \text { CARD } & 7 & 5.44446 .5555533 .133 .133 .133 .1444 .1\end{array}$

$\begin{array}{lll}\text { CARD } & 6 & 8.889 .9997 .123456 \\ \text { CARD } & 7 & 5.44446 .5555533 .133 .133 .133 .1444 .1\end{array}$

CARD $8 \quad 5555.15555 .1 \quad 66666.166666 .1 \quad 44.22$

CARD 9 2.12.12.12.12.1666.3334.3334.3334.333

CARO $10-0.1 E+01+0.22 E-010.333 E+02 \quad 0.4444 E+03-0.55555 E-03+0.666666 E+$

COL. $\quad 62-\cdots----\cdots--77$

CARD $10 \quad 00+0.9876543 E+12$

$P 0080370$

$P 0080380$

P0080390

$P 0080400$

P0080410

$P 0080420$

$P 0080430$

COL. $1 \ldots \ldots+\cdots$

CARD $11 \quad 1.05 .522 .066 .633 .123455 .0789$

$P 0080450$

$P 0080460$

$61 P 0080470$

$P 0080480$

CARO $12 \quad 123.00456 .880 .123 E+01+0.987+1-0.2345+02-0.6879 E+2+0.7 E+0$

COL $\quad 62-\cdots-70$

CARD $1230.4 E+03$

$P 0080490$

$P 0080500$

P 0080510

COL.

CARD 13

$0.9876543 E-04+0.1357913 E-04$

$-61 P 0080520$

CARD $14 \quad 19.34+0.2468 E+02+.765+287.643 .960 .5407 E+0243.96+0.5407 E+0$

COL. $\quad 62 \cdots \cdots-\cdots-\cdots-\cdots 78$

$\begin{array}{llll}\text { CARD } 14 & 243.96 & 0.5407+2\end{array}$

$P 0080530$

COL.

CARD 15

$+0.10+06$

CARD 16

$-0.3340-04$

$-.334-4+0.76576540000 .123456789010+10$

CARD $17+0.987654321098760-1+0.987654321098760-01$

COL. $\quad 62-66$

CARD $17-1$

COL.

$-1$

CARO 18

CARD 19

$-.5555555420+03$

$-0.555555542+3$

$P 0080540$

P0080550

P0080560

P0080570

P0080580

P0080590

CARD 20

TABC

FDEFFGHIT*+T१F\$) TF

.98765432109876

P0080600

P0080610

$P 0080620$

P0080630

P0080640

P0080650

P0080660 
CARD 21

CARD 22

CARD 23

CARD 24

COL.

CARD 24

COL.

CARD 25

CARD 26

CARD 27

CARD 28

CARD 29

CARD 30

CARD 31

CARD 32

COL.

CARD 33

COL.

CARD 3345678

COL

CARD 34

COL.

CARD 3

COL.

CARD $35 \quad 9.8765598 .7654 \mathrm{E2}$

COL. 62-......-.-80

CARD $35 \quad 8657.8600 \quad 9876.54$

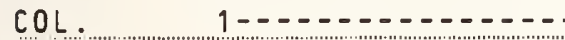

CARD $36 \quad 122333544888611222$

CARD $37 \quad 455666233444966111$

CARD $38 \quad 788999377555899777$

CARD $39 \quad 11112 \quad 334 \quad 559 \quad 880 \quad 11$

CARD $40 \quad 6 \quad 778995 \quad 449 \quad 222 \quad 00$

$C * * * *$

C*\#: S P E C I F I C A T I O N S SEGHENT 008

$C * * * * *$

$C * \pi * * *$

$C * * * * *$

$C * \# * * *$

$C=\quad$ DIMENSION A1S(5),A2S(2,2) , EP1S(33), CMA1S (5), A3S $(3,3,3)$

$C=1, \operatorname{IAC} I I(5), I A C Z I(2,7), A C I S(25), A C Z S(5,6), \operatorname{MCA} 11(5)$

$C=\quad$ INTEGER $12 I(2,2), I 3 I(2,2,2), \operatorname{MCA} 3 I(2,3,3)$

$C=\quad$ LOGICAL MCA $1 B(7), A 1 B(2), A Z B(2,2), A 3 B(2,2,2), A V B, C V B, D V B, A C B V B$

$C=\quad$ DOUBLE PRECISION DPA $10(5), M C A 3 D(1,4,2), 2 Z D V D, A 2 D(2,2), A 3 D(2,2,2)$

$C=1, A C 1 D(10), B C 2 D(7,4), D P A V D, D P B V D$

$C=\quad$ COMPLEX BVC,OAVC, CHAVC, CHBVC, CHCVC, CHOVC

$C=1, L L 1 C(32), \operatorname{LM} C C(8,4), A 1 C(12), A 2 C(2,2), B 3 C(2,2,2), B 1 C(8)$

$[* * * *$

DIMENSION A1S (5), A2S $(2,2)$,EPIS (33), CMA1S(5), A3S $(3,3,3)$

1. IAC II (5), IAC2I $(2,7), A C 1 S(25), A C 2 S(5,6), M C A 1 I(5)$

INTEGER I I $(2,2), 13 I(2,2,2), \operatorname{MCA} 3 I(2,3,3)$

$\angle O G I C A L \operatorname{MCA} 1 B(7), A 1 B(2), A 2 B(2,2), A 3 B(2,2,2), A \vee B, C V B, D V B, M C B V B$

DOUBLE PRECISION DPA1D (5),MCA3D $(1,4,2), Z Z D V D, A Z D(2,2), A 3 D(2,2,2)$

1. $A C 1 D(10), B C 2 D(7,4), D P A V D, D P B \vee D$

COMPLEX BVC, OAVC, CHAVC, CHBVC, CHCVC, CHDVC

$1, L L 1 C(32), L M 2 C(8,4), A 1 C(12), A 2 C(2,2), B 3 C(2,2,2), B 1 C(8)$

$C * \| * * I N P U T-0 U T P U T$ TAPE ASSIGNMENT STATEMENTS

$C * * * *$

C:*:* WHEN EXECUTING ONLY SEGMENT 008 . THE FOLLOWING STATEMENTS

$C * * *$ NUVI $=6$ AND IRVI $=5$ MUST HAVE THE $C=I N$ COLUMNS 1 AND 2 REMOVED.

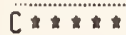

$C=\quad I R V I=5$

$C=\quad$ NUVI $=6$

$I R V I=5$

NUVI $=6$

C*\# IDENTIFY THE SOURCE OF THE TEST PROGRAMS

WRITE (NUVI, 0071)

0071 FORMAT (49H1 F O R T R A N T.E S T P R O GRAMS/I
$P 0080670$

$P 0080680$

$P 0080690$

P0080700

$P 0080710$

$P 0080720$

$P 0080730$

$P 0080740$

P0080750

P0080760

P0080770

P0080780

P0080790

P0080800

P0080810

$-61 P 0080820$

123 P0080830

P0080840

P0 080850

P0080860

P0080870

P0080880

P0080890

P0080900

P0080910

P0080920

P0080930

$1 P 0080940$

P0080950

P0080960

P0080970

P0080980

P0080990

P0081000

P0081010

$P 0010010$

P0010015

P0010020

P0010025

P0010030

P0010035

$P 0010040$

P 0010045

$P 0010050$

P0010055

P0010060

P0010065

P0010070

P008A1

P008A2

P008A3

P008A4

P008A5

P008A6

P008A7

P008A8

P0081020

P0081030

P0070010

$P 0070015$

P 0070020

P0070025

P 0070030

P008B1

P008B2

P0070035

P0070040

P0070045 
$142 H$ PREPARED BY NATIONAL BUREAU OF STANDARDS/I

$523 H$ VERSION 1

C***3 OF 6 INPUT CARDS IDENTIFY THE USERS SYSTEM ANO COMPILER

$P 0070065$

C PREPARED BY USER

$P 0070070$

$P 0070075$

C REAO, NO LIST
C PREPARED BY USER

C REAO, NO LIST

C PREPARED BY USER

C REAO, NO LIST

READ (IRVI,0070)

P0070080

$P 0070085$

$P 0070090$

$P 0070095$

P0070100

$P 0070105$

REAO (IRVI.,0072)

P0070110

REAO(IRV!.0073)

0070 FORMAT $(40 \mathrm{H}$ BASEO ON ASA FORTRAN $\times 3.9-1966$

0072

FORMAT $(40 \mathrm{H}$

TEST PROGRAMS

0073

FORMAT $(40 \mathrm{H}$

FORTRAN COMPILER

/)

1)

i)

P0070115

P0070120

$P 0070125$

P 0070130

P0070135

P0070140

$P 0070145$

$P 0081040$

P0081050

P0081060

$P 0081070$

P0081080

$P 0081090$

P0081100

P0081110

P0081120

P0081130

$C * * *$ FORMAT CONTAINING ALL LETTERS (A-Z) IN"H FIELDS AND

$C * * * * \quad A$ VARIABLE NUMBER OF BLANKS IN H ANO X FIELDS

0082 FORMAT $/ 2 \times, 3$ HAAA, $5 \times, 5 H$. $3 \mathrm{HBBB}, 10 \times, 3 \mathrm{HCCC} / 3 \mathrm{H}$

$19 \mathrm{H} \quad 3 \mathrm{HFFF} / 4 \times, 3 \mathrm{HGGG}, 8 \times, 3 \mathrm{HHHH}, 8 \mathrm{H}$

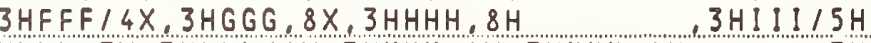

$33 \mathrm{HPPP}, 5 \mathrm{H}$ . $3 H K K K, 7 X, 3 H L L L / 6 X, 3 H M M M, 6 X, 3 H N N N, 6 H$ ,3HOQO, 5X, 3HRRR/ $8 X, 3 H S S S, 4 X, 3 H T T T, 4 H$

$45 \mathrm{H}$ VVV

WRITE (NUVI,0082)

$C * * *$ FORMAT CONTAINING H FIELD WITH ALL" POSSIBLE

$C * * * * \quad$ SPECIAL CHARACTERS

0083 FORMAT $(121 \mathrm{H}=+-, \%$, , \$)

WRITE (NUVI.0083)

C*** FORMAT TO TEST VERTICAL SPACING

$C * * * *$

7154 FORMAT $/ 24 \mathrm{H}$ BEGIN VERTICAL SPACING//3OH FORMAT(14H

W I ) I )

7155 FORMAT $(32 \mathrm{H}$ FORMAT $(15 \mathrm{H}$ SKIP 2 LINES $1 / 1) 1 /)$

WRITE (NUVI, 7155)

7156 FORMAT 33 FORMAT( $16 H$ SKIP 3 LINES $/ 1 /) / 1 /)$

WRITE (NUVI.7156)

0084 FORMATS $32 \mathrm{H}$ IMBEOOED SLASHES - SKIP 1 LINE II

$114 \mathrm{H}$ SKIP 2 LINES/II $14 \mathrm{H}$ SKIP 3 LINES/ $3(\%)$,

$219 \mathrm{H}$ SKIP TO NEXT LINE/ $1 \mathrm{H}, 12 \mathrm{H}$ SKIP 1 LINEI $1 \mathrm{HO}$,

$38 \mathrm{H}$ TEST NO/1H+.9X,14H/1H+,7HAOVANCE/19H SKIP TO NEW PAGEI

$4 \quad 1 H 1$.

WRITE (NUVI,0084)

$/ / / 3 O H$ ENO OF VERTICAL SPACING TEST)

$7.1 .3 .4104 P 0081250$

SKIP 1 LINEPOO 81260 P0081270

P0081280

P0081290

P0081300

P0081310

P 0081320

P0081330

P0081340

P0081350

P0081360

$P 0081370$

P 0081380

C* * FORMATTED REAO AND WRITE STATEMENTS WITH INTEGER $7.1 .3 .2 .1 / 25 P 0081390$

C* VARIABLES AND ARRAY ELEMENTS IN AN I/O LIST. (THE 7.2.3.3/01P0081400

C**** NUMBER OF ITEMS IN THE LIST IS VARIABLE.) SOME

C** FORMAT STATEMENTS CONTAIN REPEATED FIELOS.

$C * * *$ FORMATS CONTAINING I CONVERSION OESCRIPTORS.

C*\#* FIELOS WIOTH IS FROM 1 TO 5 OIGITS. SOME

$C * * *$ FIELOS ARE REPEATED

$P 0081410$

P0081420

$7 \cdot 2 \cdot 3 \cdot 6 \cdot 1 / 03 P 0081430$

$7.2 .3 .3101 P 0081440$

$P 0081450$

0085 FORMAT (//25H BEGIN I CONVERSION TEST/40H EACH PAIR OF LINES SHOPOO81460 IULO BE IOENTICAL/47H LINE I OF EACH GROUP IS HOLLERITH INFORMATIOPOO81470 2N)

WRITE (NUVI,0085)

P0081480

$P 0081490$

$P 0081500$

P0081510

$C * * *$ INPUT CARO
$0086 \quad$ FORMAT $(2 x, 13)$ 
READ (IRVI,0086) JACVI

$C * * * *$ INPUT CARD 2

0087 FORMAT $(1 \times .15 .9 \times .14)$

READ (IRVI,0087) KBCVI, IACII(1)

$C * * * *$ INPUT CARO 3

0088 FORMAT $(2 \times, 13,2 \times, 3(12), 2 \times, 11)$

READ (IRVI, 0088 ) IACZI $(1,2), L C C V I, I A C I I(5)$, IHDVI, MCA3I $(1,2,3)$

$C * * * *$ INPUT CARD 4

0089 FORMAT $(2 \times .2(13) .1(15) .4(12) .5(11) .3(14))$

READ (IRVI, 0089) MDCVI, IAC2I $(2,2), \operatorname{IAC} I I(4), N E C V I, I A C 1 I(3)$,

P0081580 P0081590 $P 0081600$ P0081610

1 IAC2I(2,3); IAC2I(2,1), MRRVI, IGDVI, KGVI, IEDVI, IAC2I(1,1)P0081620 7086 FORMAT IAC1I(2), IAC2I(2,7), MCA3I(2,1,3)

WRITE (NUVI,7086)

WRITE (NUVI, 0086 ) JACVI

7087 FORMAT ( I 11 H 55554444$)$

WRITE (NUVI.7087)

WRITE (NUVI.0087) KBCVI, IACII(1)

7088 FORMAT (1 16H $666 \quad 777777$ 8)

WRITE (NUVI, 7088 )

$P 0021630$

P0081640

POO 81650

P0081660

$P 0081670$

PO081680

$P 0081690$

P0081700

P0081710

WRITE (NUVI,0088) IAC2I (1,2), LCCVI, IACII(5), IHDVI, MCA3I $(1,2,3) P 0081720$

7089 FORMAT ( / $38 \mathrm{H} \quad 333333111112222222255555444444444444)$

WRITE (NUVI.7089)

WRITE (NUVI,0089) MDCVI, IAC2I $(2,2)$, IACII(4), NECVI, IACII(3),

$P 0081730$

$P 0081740$

2 IACII 2 , IAC2I $(2,7), \operatorname{MCA} I(2,1,3)$

P0081770

$C * * * *$ FORMATTED READ AND WRITE STATEMENTS WITH REAL $7.1 .3 .2 .1 / 25 P 0081780$

$C * * * *$ VARIABLES AND ARRAY ELEMENTS IN AN I/O LIST.(THE 7.2.3.6.2/18P0081790

$C * * * *$ NUMBER OF ITEMS IN THE LIST IS VARIABLE.) ONLY $7.2 .3 .3101 P 0081800$

$C * * * * \quad F$ CONVERSION IS USED IN THE FORMAT STATEMENTS.

$C * * * *$ SOME F FIELD DESCRIPTORS ARE REPEATED. FIELD

$C * * * * \quad$ WIDTH ALWAYS CONTAINS 1 POSITION FOR DECIMAL PT.

$C * * * *$ FORMATS CONTAINING F CONVERSION DESCRIPTORS.

$C * * * * \quad$ FIELD WIDTH IS FROM 1 TO 7 DIGITS. PLACEMENT OF

$C * * * *$ DECIMAL POINT IS VARIABLE. SOME F FIELDS ARE

$C * * * *$ REPEATED

P0081810

PO081820

P0081830

7080 FORMAT (I 25H BEGIN F CONVERSION TEST/4OH EACH PAIR OF LINES SHOPOO 1880 IULD BE IDENTICAL)

WRITE (NUVI.7080)

$C * * * *$ INPUT CARD 5

7081 FORMAT $(2 X, F 3.1, F 8.1)$

READ (IRVI,7081) ACVS, CMAVS

C***** INPUT CARD 6

7082 FORMAT $(2 X, F 4.2, F 5,3, F 8.6)$

READ (IRVI.7082) A1S(2), BCVS, CMBVS

$7 \cdot 2 \cdot 3 \cdot 6 \cdot 2118 P 0081840$

P0081860

P0081870

PO081890

$P 0081900$

P0081910

P0081920

P0081930

P0081940

P0081950

P0081960

$C * * *$ INPUT CARD 7

7083 FORMAT $(2 X, F 6.4 . F 7.5 .4(F 4.1), F 5.1)$

READ (IRVI,7083) HHCVS, CMCVS, GGCVS, FFCVS, A1S(1), AC1S(25),

P0081970

P0081980

P0081990

P0082000

P0082010

$C * * * *$ INPUT CARD 8

7084 FORMAT $(2 \times, 2(F 6.1), 2 \times, 2(F 7.1), 2 \times, F 5.2)$

P0082020

READ (IRVI, 7084) AC15(18), AC15(7), AC25(4,4), AC1S(8), AC $15(10) \quad P 0082030$

C***** INPUT CARD 9

7085 FORMAT $(2 X, 5(F 3.1), F 7.3,3(F 5.3))$

READ (IRVI,7085) ACZS(3,3), ACZS(5,1), CCVS, AC1S(12), DCVS,

$1 \quad A C 1 S(13), A C 1 S(5), A 3 S(1,1,2), A C 2 S(3,5)$

7091 FORMAT ( $13 \mathrm{H} 7.7123456 .7)$

WRITE (NUVI.7091)

WRITE (NUVI.7081) ACVS, CMAVS

7092 FORMAT ( / $19 \mathrm{H} 8.889 .9997 .123456)$

WRITE (NUVI.7092)

WRITE (NUVI,7082) A1S(2), BCVS, CMBVS

7093 FORMAT (I $36 \mathrm{H} 5.44446 .5555533 .133 .133 .133 .1444 .1)$

WRITE (NUVI,7093)

WRITE (NUVI.7083) HHCVS, CMCVS, GGCVS, FFCVS, A1S(1), ACIS( 25 )

$1 \quad A C 2 S(4,1)$

7094 FORMAT ( $137 \mathrm{H} 5555.15555 .166666 .166666 .1 \% 44.22)$

WRITE (NUVI,7094)

$P 0082040$

P0082050

P0082060

P0082070

P0082080

P0082090

P0082100

P0082110

PU082120

P0082130

POO 22140

P008 2150

P0082160

P0082:70

P0082180

P0082190

NBS FORTRAN TEst Programs Version 1 
WRITE (NUVI.7084) AC1S(18), AC1S(7), AC2S(4.4), AC1S(8), AC1S(10)PO082200

7095 FORMAT $(139 \mathrm{H} 2.12 .12 .12 .12 .1666 .3334 .3334 .3334 .333)$

$P 0082210$

WRITE (NUVI,7095)

WRITE (NUVI,7085) ACZS(3,3), AC2S(5,1), CCVS, AC1S(12), DCVS,

P0082220 $A C 1 S(13), A C 1 S(5), A 3 S(1,1,2), A C 2 S(3,5)$

\begin{tabular}{|c|c|}
\hline & \\
\hline$C * * * * *$ & FORMATTED READ AND WRITE STATEMENTS WITH REAL \\
\hline$C * * * * *$ & VARIABLES AND ARRAY ELEMENTS IN AN I/O LIST. \\
\hline$C * * * *$ & E CONVERSION IS USED IN THE FORMAT STATEMENTS \\
\hline$C * * *$ & SOME E FIELD DESCRIPTORS ARE REPEATED \\
\hline$C * * * *$ & (FIELD WIDTH ALWAYS INCLUDES 6 EXTRA POSITIONS \\
\hline$C * \| *$ & TO PROVIDE FOR SIGN, DECIMAL \\
\hline$C * * * *$ & PROVISION IS ALWAYS MADE FOR \\
\hline 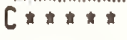 & DECIMAL POINT) \\
\hline$C * * * *$ & THE NUMBER OF DECIMAL PLACES \\
\hline$C * \cdots \cdots$ & TO 7 DIGITS. \\
\hline
\end{tabular}

P0 082240

$7.1 .3 .2 .1 / \quad P 0082250$

$7.2 .3 .6 .21 \quad P 0082260$

$7.2 .3 .3 / P 0082270$

$P 0082280$

$7.2 .3 \cdot 6.2 .1 / 47 P 0082290$

$7.2 .3 .6 / 01 P 0082300$

$7 \cdot 2 \cdot 3 \cdot 6 \cdot 2 \cdot 1 / 04 P 0082310$

$P 0082320$

$P 0082330$

$P 0082340$

7110 FORMAT (//25H BEGIN E CONVERSION TEST/4OH EACH PAIR OF LINES SHOPOO82350

IULD BE IDENTICAL)

WRITE (NUVI,7110)

$P 0082360$

$P 0082370$

C*** INPUT CARD 10

7111 FORMAT (E8.1,E9.2,E10.3,E11.4,E12.5,E13.6,E14.7)

READ (IRVI,7111) AVS, BVS, EP1S(5), ACZS $(1,5), C V S, A C 2 S(5,4)$,

$1 \quad A 3 S(2,1,2)$

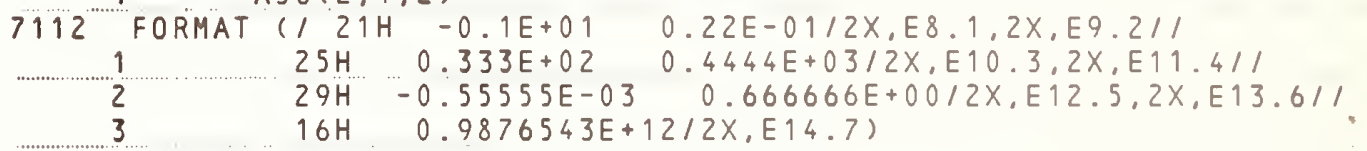

VI.7112) AVS, BVS, EP1S(5), AC2S(1,5), CVS, AC2S(5,4), P0082460

$1 \quad$ A3S $(2,1,2)$....

C****VARIABLES AND ARRAY ELEMAENTS IN AN I/O LIST.

C*\#* E AND F CONVERSION ARE USED IN THE FORMAT

C*** STATEMENTS. SOME FORMAT DESCRIPTORS ARE REPEATED $7.2 .3 .3 \quad 101$ PO0 82510

7118 FORMAT ( 31 H BEGIN COMPLEX CONVERSION TEST/32H EACH GROUP SHOULPOO 82520 10 BE IDENTICAL)

WRITE (NUVI,7118)

$C * * * *$ INPUT CARD 11

7119 FORMAT ( 2(F3.1), 2(F4.1), 2(F7.4))

READ (IRVI,7119) CHAVC, CHBVC, A1C(2)

C**** INPUT CARDS 12,13

7120 FORMAT (2(F6.2), 2(E10.3), 2(E11.4), 2(E8.1)/2(E14.7))

READ (IRVI,7120) AZC (1,2), B3C (2,2,1), CHCVC, A1C (1), CHDVC

C**** INPUT CARD 14

7122 FORMAT (F5.2, E11.4,E10.3,F4.1, 3(F5.2,E11.4))

READ (IRVI,7122) AZC (2,1), BVC, OAVC, LMZC (1,2), LL1C (2)

7123 FORMAT ( / 10H 1.0 5.5/2X,F3.1.2X,F3.1 /

$112 \mathrm{H} 22.0 \quad 66.612 \mathrm{X}, \mathrm{F} 4.1,2 \mathrm{X}, \mathrm{F} 4.1 / 1$

$218 \mathrm{H} 33.123455 .0789 / 2 X, F 7.4,2 X, F 7.4$ )

WRITE (NUVI,7123) CHAVC, CHBVC, A1C(2)

7124 FORMAT (I $16 \mathrm{H} 123.00456 .88 / 2 \mathrm{X}, \mathrm{F} 6.2,2 \mathrm{X}, \mathrm{F} 6.21 /$

$124 H \quad 0.123 E+01 \quad 0.987 E+01 / 2 X, E 10.3,2 X, E 10.3 / 1$

$226 H-0.2345 E+02-0.6879 E+0212 X, E 11.4,2 X, E 11.411$

$320 \mathrm{H} \quad 0.7 E+03 \quad 0.4 E+03 / 2 X, E 8.1,2 x, E 8.1 / 1$

$\begin{array}{lll}432 H & 0.9876543 E-04 & 0.1357913 E-04 / 2 X, E 14.7,2 X, E 14.7)\end{array}$

WRITE (NUVI,7124) AZC (1,2), B3C(2,2,1), CHCVC, A1C (1), CHDVC

7126 FORMAT ( / $20 \mathrm{H} 19.34 \quad 0.2468 \mathrm{E}+02 / 2 X, F 5.2,2 X, E 11.4 / 1$

$118 \mathrm{H} \quad 0.765 E+0287.6 / 2 X, E 10.3,2 X, F 4.1 / 1$

$218 \mathrm{H} \quad 43.960 .5407 E+021 \quad 3(F 7.2, E 11.41))$

WRITF (NUVI,7126) AZC (2,1), BVC, OAVC, LMZC (1,2), LL1C(2)

$P 0082530$

$P 0082540$

$P 0082550$

$P 0082560$

$P 0082570$

$P 0082580$

$P 0082590$

$P 0082600$

P0082610

$P 0082620$

$P 0082630$

$P 0082640$

$P 0082650$

$P 0082660$

$P 0082670$

$P 0082680$

$P 0082690$

P0082700

P0082710

P0082720

P 0082730

$P 0082740$

P0082750

P0082760

$P 0082770$

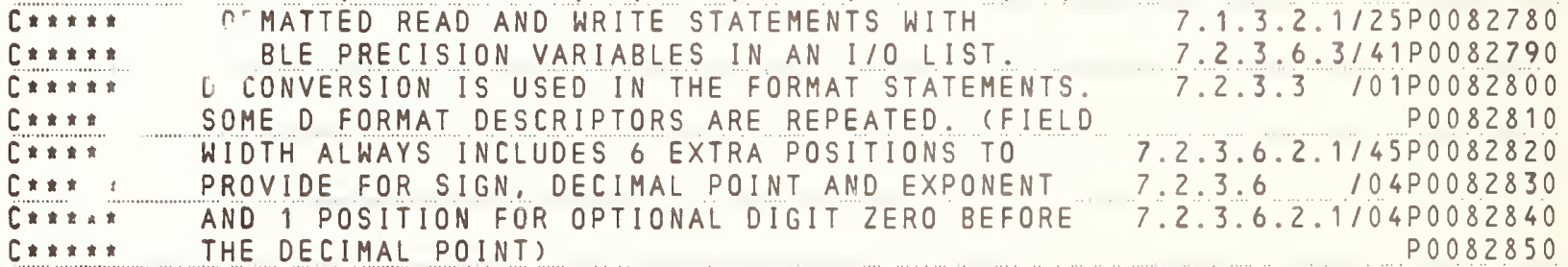

7127 FORMAT $125 \mathrm{H}$ BEGIN D CONVERSION TEST/32H EACH GROUP SHOULD BE IPOO 2860 IDENTICAL)

P0082870 
7131 FDRMAT (1 $12 \mathrm{H}-0.3340-0412 \mathrm{x}, 010.3 / 2 \mathrm{x}, 010.311$

P0082970

"P0082980

$116 \mathrm{H} \quad 0.76576540+00 / 2 x_{0} 014.7 / 1$

P0082990

$220 \mathrm{H} 10.123456789010+10 \% 2 \mathrm{X}, 018.11 / 1$

P0083000

$323 \mathrm{H} \quad 0.987654321098760-01 / 2 x_{0} .021 .1412 x_{0} .021 .14 / 2 x_{0} 021.14 / 190083020$

$418 H \quad-0.5555555420+0312 X, 016.91,2 X, 016.9)$

WRITE (NUVI,7131) MCA3D(1,2,2), AC1D(2), BC2D(3,1), AC1D(1)

P0083030

1 ZZDVD, AC10(3), DPBVD, MCA3D(1,2,1), BC2D (1,2)
FORMATTEO READ AND WRITE STATEMENTS WITH LOGICAL

C***** VARIABLES AND ARRAY ELEMENTS IN AN IID LIST

C***** SOME L DESCRIPTORS ARE REPEATED.

7132 FORMAT / / /25H BEGIN L CONVERSIDN TEST/33H LINES BELOW SHOULO BE DDENTI CAL )

C***** L CDNVERSION IS USEO IN THE FORMAT STATEMENTS

WRITE (NUVI.7132)

C*****INPUT CARO 19

7133 FORMAT (L4)

READ (IRVI.7133) A2B(2,1)

C***** INPUT CARD 20

7134 FDRMAT $(2(L 4), L 3, L 2, L 3,2(L 1))$

READ (IRVI, 7134) MCA1B(1), MCBVB, $A 2 B(1,1), A 3 B(1,1,1), C V B$,

7135 FORMAT ( $1 / 24 H \quad$ T F F T T FTF/ $2 X, 3(L 4), L 3, L 2, L 3$,

$12(L 1))$

WRITE (NUVI,7135) AZB (2,1), MCA1B(1), MCBVB, AZB $(1,1), A 3 B(1,1,1)$,

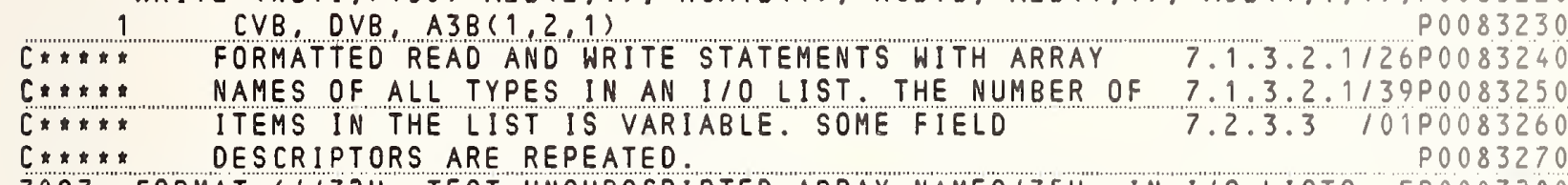

7097 FORMAT (//32H TEST UNSUBSCRIPTED ARRAY NAMES/35H IN 1/O LISTS. EP0083280 IACH GROUP OF LINES/2ZH SHOULO BE IDENTICAL.)

WRITE (NUVI,7097)

$C * * * *$ INPUT CAROS 21,22

7098 FORMAT $(2 X .8(F 3.1), 8 \mathrm{~F} 3.1 / 8(2(\mathrm{~F} 3.1)))$

READ (IRVI.7098) B1C,B3C

$C * * * *$ INPUT CAROS $23,24,25$

7099 FORMAT $(2 \times .4(F 4.1) / 4(09.2), 409.215(12))$

READ (IRVI,7099) AZS, A30, MCA1I

C***** INPUT CAROS $26,27,28$

7100 FDRMAT $(2 X, 4(09.2) / 27$ (F2.1)/5(L1),5L1)

READ (IRVI,7100) A2D, A3S, A1B, A3B

C***** INPUT CARDS 29,30

7101 FDRMAT $(2 X, 4(12), 5(D 9.2) / 4(2(F 3.1)), 8(12), 4(L 1), 5(F 3.1))$

READ (IRVI.7101) 121, DPA10,A2C, 131, AZB, CMA1S

7102 FDRMAT ( $26 \mathrm{H} 9.91 .19 .92 .29 .93 .39 .94 .4 / 2 \times .8(F 3.1) / 2 \times .8(F 3.1))$ WRITE (NUVI,7102) BIC

7103 FORMAT $(/ 18 \mathrm{H}-9.9-9.9-9.9-9.9 / 2 \times, 4(\mathrm{~F} 4.1) / /$

$138 \mathrm{H}-0.990+01-0.990+01-0.990+01-0.990+01 / 2 x_{0} 4(09.2) / 2 X_{0} 4(09.2) / / \quad P 0083460$

POO 083290

P0 083300

P0083310

P0 083320

P0083330

P 0083340

P0083350

P0083360

P0083370

P0083380

P0083390

P 0083400

P0083410

P 0083420

P0083430

P0083440

P0083450 $\begin{array}{lllllll}212 H \quad 9999999999 / 2 X & 5(12) \quad / 138 H \quad 0.990+01 \quad 0.990+01 \quad 0.990+010.9 P 0083470\end{array}$ $390+01 / 2 X, 4(D 9.2) / 1 \quad 37 \mathrm{H} \quad 0.90 .90 .90 .90 .90 .90 .90 .90 .9 / 1 X, P 0083480$ $49(F 4.1) / 1 \times, 9(F 4.1) ! 1 \times, 9(F 4.1) / 4 H 1 T F / 2 X, 2(L 1))$

WRITE (NUV1,7103) AZS, A30, MCA11, A2O,A3S, A1B

P0083490

P0083500

7104 FORMAT ( / 10H TFTFTFTF/ 2x, $8(\mathrm{~L} 1) / 110 \mathrm{H} 99999999 / 2 x_{0} 4(12) / / P 0083510$ $111 \mathrm{H} \quad 0.990+01 / 5(011.2 \mathrm{I}) \quad 126 \mathrm{H} 9.95 .59 .96 .69 .97 .79 .98 .8 / 2 X, \quad \mathrm{P} 0083520$ $28(F 3.1) / 2 \times .8(F 3.1) / 2 \times .8(F 3.1) / 118 H \quad 9999999999999999 / 2 \times .8(12) / /$

$36 H^{2}$ TFFT/ $\left.2 X, 4(L 1) / 117 H \quad 9.99 .99 .99 .99 .9 / 2 X, 5(F 3.1)\right)$

WRITE (NUVI,7104) A3B, 121, DPA1D, A2C, B3C, 131, A2B, CMA1S

P0083530

P 0083540

P0083550 
7090 FORMAT ( $130 \mathrm{H}$ LEADING BLANK INSERTION TEST/40H EACH PAIR OF LINEP0083600 IS SHOULD BE IDENTI (AL)

WRITE (NUVI,7090)

$P 0083610$

$\begin{array}{cccccc}7096 \text { FORMAT (1 3H } 8 / 13 / 14 \mathrm{H} & 22 / 14 / 15 \mathrm{H} & 22 / 15 / 16 \mathrm{H} & 22 / 16 / 1 \\ 17 \mathrm{H} & 22 / 17 / 1 / 5 \mathrm{H} & 7.7 / F 5.1 / 17 \mathrm{H} & 8.88 / F 7.21 & 9 \mathrm{H} 9 & 9.999 /\end{array}$

$\begin{array}{rrrrrr}2 & F 9.3 / 1 & 11 \mathrm{H} & 5.4444 / \mathrm{F} 11.41 / & 13 \mathrm{H} & 6.55555 / \mathrm{F} 13.51 / \\ 3 & 15 \mathrm{H} & 7.123456 / \mathrm{F} 15.6 / 1 & 10 \mathrm{H} & 0.21 \mathrm{E}+01 / \mathrm{E} 10.21 /\end{array}$

$P 0083620$

$412 \mathrm{H} \quad 0.331 \mathrm{E}+02 / \mathrm{E} 12.3 / / 14 \mathrm{H} \quad 0.4441 \mathrm{E}+03 / \mathrm{E} 14.4 / 1$

$516 \mathrm{H} \quad 0.55551 \mathrm{E}+04 / \mathrm{E} 16.5 / / 18 \mathrm{H} \quad 0.666661 \mathrm{E}+05 / \mathrm{E} 98.6 / 1$

$620 \mathrm{H} \quad 0.1234567 E+06 / E 20.7)$

WRITE (NUVI,7096) MCA3I $(1,2,3)$, IACII(3), NECVI, IACII(3),

1 IACZI $(2,3)$, ACVS, A $1 S(2), B C V S$, HHCVS, CMCVS, CMBVS,

2 DCVS, AC1S(25), ACZS $(4,1), A C 1 S(7), A C 1 S(8)$, CMAVS

7105 FORMAT (/ 9H $0.10+00 / 09.1 / / 90 \mathrm{OH} 0.10+00 / 010.1 / 1$

$119 \mathrm{H} \quad 0.10+00 / 019.1 / / 12 \mathrm{H} \quad 0.1 \mathrm{D}+00 / 012.1 / 1$

$210 \mathrm{H} \quad 1.0 \quad 5.5 / 2(\mathrm{~F} 5.1) / / 12 \mathrm{H} \quad 9.9 \quad 5.5 / 2(\mathrm{~F} 6.1) 1 /$

$\begin{array}{llllllll}3 & 14 \mathrm{H} & 9.9 & 5.5 / 2(\mathrm{~F} 7.1) / 1 & 16 \mathrm{H} & 1.0 & 5.5 / 2(\mathrm{~F} 8.1))\end{array}$

WRITE (NUVI,7105) AC1D(3), ZZDVD, ZZDVD。

1 ZZDVD, CHAVC, B3C $(1,1,1), B 3 C(1,1,1), C H A V C$ $P 0083630$

P 0083640

P0083650

$P 0083660$

$P 0083670$

$P 0083680$

$P 0083690$

$P 0083700$

P0 083790

$P 0083720$

P0 083730

$P 0083740$

$P 0083750$

$P 0083760$

P0 083770

P0 0083780

C*****.. FORMATTED READ AND WRITE STATEMENT TO TEST THAT $7.2 .3 .7 / 03 P 0083790$

C***: OPTIONAL BLANKS MAY PRECEDE A LOGICAL INPUT FIELD 7.2.3.7/06P0083800

7138 FORMAT ( 33 H TEST LOGICAL FIELDS WITH BLANKS/33H LINES BELOW SHP0083810

IOULD BE IDENTICAL)

WRITE (NUVI,7138)

C**** INPUT CARD 31

7939 FORMAT ( L6, L4, L10, L5)

READ (IRVI,7139) AVB, $M C A 1 B(2), A 2 B(1,2), A 3 B(2,1,2)$

7140 FORMAT (1/27H TH F F T $2 X, L 6, L 4, L 10, L 5)$

$P 0083820$

P0 0083830

P 0083840

$P 0083850$

P 0083860

WRITE (NUV1,7940) AVB, $\operatorname{MCA} 1 B(2), A 2 B(1,2), A 3 B(2,1,2$ )

$C * * *$ FORMATTED READ AND WRITE TO TEST F DESCRIPTORS

$C * * *$ WHERE $D$ IS EQUAL TO ZERO AND WHERE W EQUALS D

P 0083870

$P 0083880$

C***** (2ND TEST APPLIES ONLY TO READ STMNTS.)

7108 FORMAT (//36H TEST $D=0, W=D+1$ (PAIRS OF LINES/ $28 \mathrm{H}$

ILD BE IDENTICAL))

WRITE (NUVI,7908)

C***** INPUT CARD 32

7149 FORMAT $(2 X, F 5.0, F 5.5)$

READ (IRVI,7141) ACVS, BVS

7109 FORMAT (//7H 4444.I2X,F5.0//9H .55555/3X.F6.5)

WRITE (NUVI, 7109 ) ACVS, BVS
C***** FORMATS WITH G CONVERSIONS

$7.2 .3 .1 / 3190083890$

$7.2 .3 .4 / 40 P 0083900$

$P 0083910$

C***** INPUT CARD 33

7142 FORMAT $3(611.4), 3611.4)$

READ (IRVI,7142) AC1S(14), AC1S(15), AC1S(16), AC1S(17)

1 ACIS(21), ACIS(22)

7143 FORMAT ( / $2 X, 23$ HBEGIN G CONVERSION

$12 \times, 38 H E A C H$ PAIR OF LINES SHP0084050

$P 0083930$

$P 0083940$

$P 0083950$

$P 0083960$

$P 0083970$

$P 0083980$

$P 0083990$

P 0084000

$P 0084010$

$P 0084020$

$P 0084030$

$P 0084040$

$10 U L D$ BE IDENTICAL//36H $.1235 E+05$

$2614.4,4 X, 2611.4 / 1 / 3 X, 33 \mathrm{H} \quad 12.35$

$3 \quad 614.4,4 X, 2611.4)$

WRITE(NUVI,7943) AC1S(14), AC1S(15), AC 1S(16), AC1S(17),

1 AC1S(21), ACIS(22)

C**** SCALE FACTOR APPLIED TO F,E,D,G DESCRIPTORS

$C * * * *$ ON READ, BUT NOT ON WRITE

C**** INPUT CARD 34

7144 FORMAT(2PF8.3,-2PE9.4,F9.4,0PG9.4,09.4,-2PE9.4,F9.4,09.4,2PG9.4)

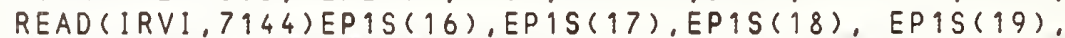

$1 \quad B C 2 D(1,4), E P 1 S(20), E P 1 S(22), B C 2 D(2,1), E P 1 S(23)$

1235 . $123.5 \% \quad P 0084060$

P 0084070

P 0084080

$P 0084090$

$P 0084100$

P 0084110

P 0084120

$P 0084130$

P0084140

$P 0084150$

P 0084160

7145 FORMAT $22 \mathrm{H} 1$ SCALE FACTOR ON READ/31H IN ORDER OF FORMAT OCCURRENCP0084170

TE//4OH CARD $9876.5498 .7654 E 2 \quad 9876.54 \%$

$240 \mathrm{H}$ DESC 2PF8.3 2 -2PE9.4

3 4 $40 \mathrm{H}$ TO BE $98.7654 \quad .9877 \mathrm{E}+04 \quad 987654.001$

$44 \mathrm{H}$ IS, F12.4, E12.4, F12.211

$540 \mathrm{H}$ CARD $987.654 \quad 8647860-4$

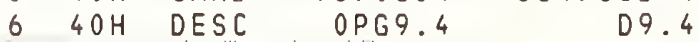

$$
\begin{array}{r}
86.4786 E 21 \\
-2 \text { PE } 9.41
\end{array}
$$

P 0084180

P0084190

$P 0084200$

$P 0084210$

P0 0084220

$P 0084230$ 
$7.40 H$ TD BE $987.654 \quad .8648 D-02 \quad .8648 E+041$

$84 \mathrm{H}$ IS. F12.3.012.4, E 12.411

$9 \quad 40 \mathrm{H}$ CARO $86.4786 \quad 8657.8700 \quad 9876.541$

$\begin{array}{llll}\text { A } 40 H \quad O E S C & \text { F9.4 } & 09.4 & 2 P G 9.41\end{array}$

98.771

$P 0084250$

P0084260

$P 0084270$

P0 084280

(4H IS,F12.3, $012.4,616.4)$

WRITE (NUVI,7145) EP1S(16), EP1S(17), EPIS(18),EP1S(19),

$1 \quad B C 2 D(1,4), E P 1 S(20), E P 1 S(22), B C 2 D(2,1), E P 1 S(23)$

P0084290

P 0084300

$P 0084310$

$P 0084320$

SCALE FACTDR APPLIED TD F, E, D, G DESCRIPTORS

$C * * * * * \quad$ ON WRITE, BUT, NOT DN READ

$P 0084330$

C**\# INPUT CARD 35

7152 FORMAT(F8.2,E9.4,F9.2,G9.3,D9.0,E9.4,F9.4,D9.2,G9.4)

$P 0084340$

$P 0084350$

REAO(IRVI, 7152) ACIS(1),ACIS(2),ACIS(3), ACIS(4),

$P 0084360$

$1 A C 1 D(4), A C 1 S(20), A C 1 S(23), A C 10(5), A C 15(24)$

$P 0084370$

7153 FDRMAT $/ 23 \mathrm{H}$ SCALE FACTOR ON WRITE/31H IN OROER DF FDRMAT DCCURREPOO84380 INCE//40H CARO $9.87655 \quad 98.7654 E 2 \quad 9876.541$

$240 \mathrm{H}$ DESC 2PF12.2 -2 PE $12.4 \quad$ F12.41

$340 \mathrm{H}$ TO BE $987.65 \quad .0099 E+06 \quad 98.76541$

$4 \quad 4 \mathrm{H}$ IS, 2PF12.2, - 2PE $12.4, \mathrm{~F} 12.41 \%$

$540 \mathrm{H}$ CARO $987.654 \quad 8647860-3 \quad 86.4786 E 21$

$640 \mathrm{H}$ DESC IPG12.2 $\quad$ D $12.4 \quad-2 P E 12.41$

$740 \mathrm{H}$ TD BE $9.88 \mathrm{E}+02 \quad 8.64790+02 \quad .0086 \mathrm{E}+061$

$8 \quad 4 \mathrm{H}$ IS, IPG $12.2,012.4,-2 \mathrm{PE} 12.411$

$9 \quad 40 H \quad$ CARO $86.4786 \quad 8657.8600 \quad 9876.541$

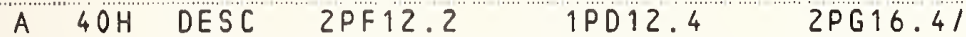

B $40 \mathrm{H}$ TO BE $8647.86 \quad 8.65790+03 \quad 9877.1$

C $4 \mathrm{H}$ IS, 2PF12.2, 1PD12.4, 2PG16.411

H28H THE LAST TWO LINES OF EACH/24H SET SHDULD BE THE SAME)

WRITE(NUVI, 7153) ACIS(1), AC1S(2), AC 1S(3), AC1S(4),

$1 A C 1 D(4), A C 1 S(20), A C 1 S(23), A C 1 D(5), A C 1 S(24)$

$C * * *$ 1/D FDRMAT RESCAN

$C * * * *$ INPUT CARDS $36,37,38$

7146 FDRMAT( $11,12,13)$

READ (IRVI,7146) I2I, IAC II

7147 FORMAT / 37 FDRMAT RESCAN - THE SECONO GROUP OF/38H EACH SET SHPOO 84580 IDULD AGREE WITH THE FIRST //15H $1222 \quad 333 / 15 H \quad 4 \quad 55 \quad 666 / P 0084590$

$115 \mathrm{H} 7 \mathrm{7} 89991 \mathrm{H})$

WRITE (NUVI,7147)

7148 FORMAT $(14,15,16)$

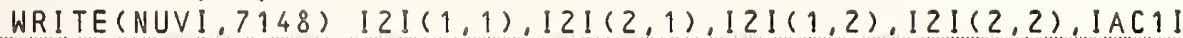

C***** INPUT CAROS 39,40

7149 FORMAT $(14,2(11,1 \times, 12))$

READ ( IRVI,7149) I2I, IACII

7150 FDRMAT $(121 \mathrm{H} 2 * 4 \$ \$ \quad 6 \quad(17 \mathrm{H} \quad 8 \$ 11 \mathrm{H})$

WRITE( NUVI,7950)

7151 FORMAT $(14,3 \mathrm{H} *, 1(14,3 \mathrm{H} \$ \$,(14,3 \mathrm{H}(()))$

WRITE( NUVI,7151) I2!(2,1), I2!(2,2), IACII(2), IACII(4)

$C * * *$ END OF TEST SEGMENT 008
$C * * *$ WHEN EXECUTING DNLY SEGMENT 008 . THE STDP AND END CARDS

$C * * *$ WHICH APPEAR AS COMMENT CARDS MUST HAVE THE $C=$ IN CDLUMNS

C*****1 AND 2 REMOVEO

$C=S T O P$

$C=\quad$ ENO

STOP

ENO

PO084600

$P 0084610$

$P 0084620$

$P 0084630$

P0084640

$P 0084650$

P0084660

$P 0084670$

P 0084680

P0084690

P0 084700

$P 0084710$

P0084720

P0084730

$P 0084740$

$P 0084750$

P0084760

P008C1

POO $8 \mathrm{C2}$

PREPARED BY USER

DD NDT READ OR WRITE RECORO 2 . ODUBLE SPACE ON OUTPUT."IO"

PREPAREO BY USER

OO NOT READ OR WRITE RECDRO 4 . OOUBLE SPACE DN DUTPUT I0 4

PREPAREO BY USER

OO NOT REAO OR WRITE RECORD 6 OOUBLE SPACE ON OUTPUT IO 6

999

555554444

$\begin{array}{lll}666 & 777777 \quad 8\end{array}$

333333111112222222255555444444444444

7.7123456 .7

8.889 .9997 .123456

5.44446 .5555533 .133 .133 .133 .1444 .1 
$5555.15555 .166666 .166666 .1 \quad 44.22$

$2.12 \cdot 12 \cdot 12 \cdot 12 \cdot 1666.3334 .3334 .3334 .333$

$-0.1 E+01+0.22 E-010.333 E+020.4444 E+03-0.55555 E-03+0.666666 E+00+0.9876543 E+12$

1.05 .522 .066 .633 .123455 .0789

$123.00456 .880 .123 E+01+0.987+1-0.2345+02-0.6879 E+2+0.7 E+030.4 E+03$

$0.9876543 E-04+0.1357913 E-04$

$19.34+0.2468 E+02+.765+287.643 .960 .5407 E+0243.96+0.5407 E+0243.96 \quad 0.5407+2$ $+0.10+06$

$-0.3340-04-.334-4+0.7657654000 \quad 0.123456789010+10$

$+0.987654321098760-1+0.987654321098760-01 \quad .98765432109876-1$

-. $5555555420+03-0.555555542+3$

TABC

FDEFFGHIT*+T1F\$) TF

9.91 .19 .92 .29 .93 .39 .94 .49 .91 .19 .92 .29 .93 .39 .94 .4

9.95 .59 .96 .69 .97 .79 .98 .89 .95 .59 .96 .69 .97 .79 .98 .8

$-9.9-9.9-9.9-9.9$

$-0.990+01-0.990+01-0.990+01-0.990+01-0.990+01-.990+01-.99+01-.99+1$

9999999999

$+0.990+010.990+01+.99001+.9901$

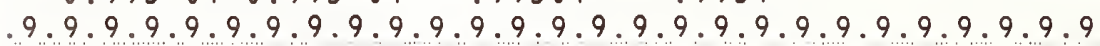

TFTFTFTFTF

$99999999+0.990+010.990+010.990+01+0.990+01 \quad .9901$

$9.95 .59 .96 .69 .97 .79 .98 .89999999999999999 T F F T 9.99 .99 .99 .99 .9$

$$
T . F
$$

4444.55555

$123.45678 \mathrm{E2} \quad 1234.5678 \quad 123.45678 \quad 12.345678 \quad 1.2345678 \quad .12345678$

$9876.5498 .7654 E 29876.54 \quad 987.6548647860-486.4786 E 286.4786 \quad 8657.8600 \quad 9876.54$

$9.8765598 .7654 E 2 \quad 9876.54 \quad 987.6548647860-386.4786 E 286.4786 \quad 8657.8600 \quad 9876.54$

122333544888611222

455666233444966111

788999377555899777

$11112 \quad 334 \quad 559 \quad 880 \quad 11$

677899544922200

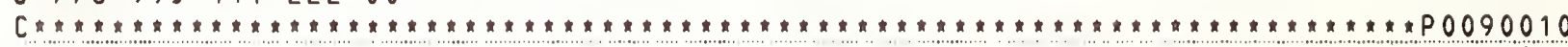

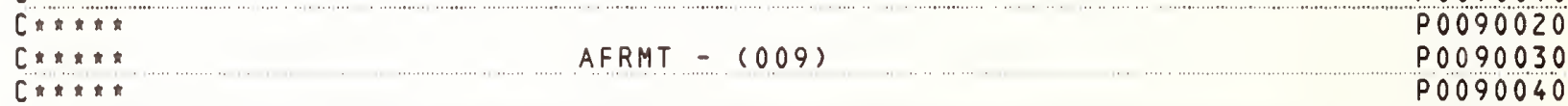

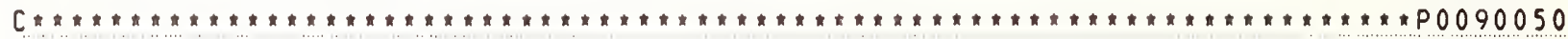

C* GENERAL PURPDSE ASA REFSPOO90060

C* TD TEST SIMPLE FDRMAT AND FDRMATTED I/D STATEMENTS $7.1 .3 .2 .2 P 0090070$

C*\# WHICH USE A-CDNVERSIDN SD THAT THIS FEATURE MAY $\quad 7.1 .3 .2 .2 P 0090080$

C*** BE USED IN DTHER SEGMENTS $\quad 7.1 .3 .2 .3 P 0090090$

$\begin{array}{rr}C * * * & 7.2 .3 \\ C * * * 2.30090100\end{array}$

$C * * * \ldots \ldots . .28090110$

C**:RESTRICTIONS DBSERVED

$\because * *$ ALL FDRMAT STATEMENTS ARE LABELED

$C * * *$ H AND X DESCRIPTDRS" ARE NEVER REPEATED

$C * * *$ FIELD WIDTH IS NEVER 2ERD

C\#\# IF THERE IS AN I/D LIST, THE FDRMAT STATEMENT

$C * * *$ CDNTAINS AT LEAST ONE FIELD DESCRIPTDR (DTHER

$7.2 .3 .8 \quad P 0090110$
$P 0090120$

$C * *$ THAN HOR $X$ )

$7.2 .3 \quad 157 P 0090130$

$7.2 .3 .3 / 54 P 0090140$

$7.2 .3 / 18 P 0090150$

$7.2 .3 .4 / 22 P 0090160$ $P 0090170$ $P 0090180$

$C * *$ ITEMS IN I/O LIST CORRESPDND TD FORMAT DESCRIPTDRS $7.2 .3 .4 / 36 P 0090190$

C**** FIELD WIDTH NEVER EXCEEDED BY DUTPUT

$C * * * * \quad P 0090210$

C*\# READ AND WRITE STATEMENTS FDR ENTIRE SEGMENT FOLLDW POOQOZZO

C**** P* P0090Z30

$C * *$ FDRMATTED READ AND WRITE STATEMENTS WITH ALL $\quad 7.1 .3 .2 .1 / 25 P 0090240$

C* TYPES DF FIELDS. DNLY A (HDLLERITH) CDNVERSIDN $7.2 .3 .8116 P 0090250$

C* IS USED IN THE FDRMAT STATEMENTS. SOME A FDRMAT $7.2 .3 .3101 P 0090260$

$C * *$ DESCRIPTORS ARE REPEATED PO090270

C INPUT DATA TD THIS SEG. CDNSISTS DF 3 DATA CARD IMAGES IN CDLS. 1 - 55POO90Z8O

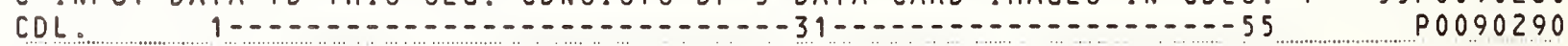

CARD $1 \quad B=E F-J K L /() 012 T U V W+\ldots \$ X$ YZACDGHIPORSMND678(C)B2\$9+A345 POO90300

CARD $2 \quad 0 Z 1 * A \quad P O 090310$

CARD 3 ABCDEFGHIJKLMNDPORSTUVWXYZ

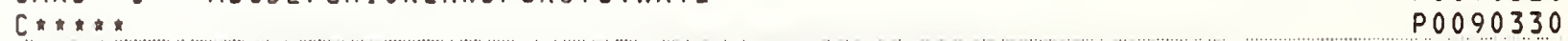

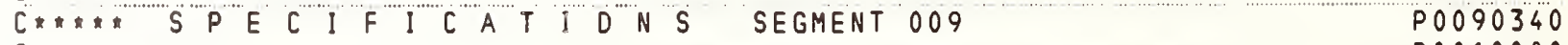

$C * * * * \quad P 0010080$ 
C***** WHEN EXECUTING ONLY SEGMENT 009 . THE SPECIFICATION STATEMENTS $C * * * *$ WHICH APPEAR AS COMMENT CAROS MUST HAVE THE C

$C * * * *$ IN COLUMNS 1 AND 2 REMOVEO.

$C * * * * *$

$C=$ DIMENSION A1S(5),A3S(3,3,3),EP1S(33), IACZI(2,7),AC2S(5,6)

$C=\quad 1, M C A 1 I(5), C M A 1 S(5)$

$C=\quad$ INTEGER BVI, MAVI, LAVI, MCA3I $(2,3,3)$

$C=\quad$ REAL MVS, CVS, BCVS

$C=\quad \operatorname{LGICAL} M C A 1 B(7), A 1 B(2), A Z B(2,2), A 3 B(2,2,2), A \vee B, E V B$

DIMENSION A1S(5), A3S $(3,3,3), \operatorname{EP} 1 S(33), \operatorname{AC} 2 I(2,7), A C 2 S(5,6)$

1. MCA1 I (5), CMA1S(5)

INTEGER BVI, MAVI, LAVI, MCA3I $(2,3,3)$

REAL MVS, CVS, BCVS

$L O G I C A L M C A 1 B(7), A 1 B(2), A 2 B(2,2), A 3 B(2,2,2), A \vee B, E V B$

$C * * * * *$

$C_{* * * *}$ I $N$ P U T $-0 \cup T P U T$ T A P E ASSIGNMENT STATEMENTS

$C * \# * * *$

$C * * * * *$

$C * * * * *$

$C * * * * *$

$C=$

$C=$

WRITE (NUVI,0090)

READ (IRVI,0091) MVS, IACZI(2,2), MAVI,ACZS(4.2), MCAII(1),LAVI,

$1 A Z B(1,2), A 1 B(2), B C V S, M C A 1 B(2), B V I, C V S, E V B, A 1 S(2), E P 1 S(9)$,

$2 A 3 S(1,1,1), A 3 B(2,2,1), \operatorname{MCA} 3 I(1,2,3), \operatorname{MCA} 3 !(2,1,2), \operatorname{MCA} 3\lfloor(1,1,3)$

WRITE (NUVI,0092) BVI, MVS, CVS, MAVI, EVB, MCA1I(1), EP1S(9),

1

2

3

$C * * * *$

$C * * * * *$

$[* * * * *$

\section{WRITE (NUVI,0093)}

READ (IRVI,0094) CMA1S(2), CMA1S(1), LCCVI, AVB, BVI

WRITE (NUVI,0095) BVI, AVB, CMA1S(2), LCCVI, CMA1S(1)

$C * * *$ FORMATTED READ AND WRITE TO TEST HOLLERITH FIELDS

C*** WHERE FIELD WIDTH IS GREATER THAN THE WORO LENGTH

$C * * * * * \quad$ CAPACITY OF THE MACHINE

WRITE (NUVI,0096)

READ (IRVI,0097) MRRV!

WRITE (NUVI,0098) MRRVI

$C * * * *$

$C * \cdots * *$

$C * * * * *$

$C * * * * *$

$C * * * *$

$C * * * * *$

FORMAT STATEMENTS FOR THE ENTRIRE SEGMENT FOLLOW FORMATS TO TEST A CONVERSION. FIELO WIOTH IS

FROM 1 TO 4 CHARACTERS. SOME A DESCRIPTORS ARE REPEATED.

0090 FORMAT ( 1 H $1,1 \mathrm{X}, 26$ HAFRMT - $(009)$ A-CONVERS $10 N / 12 \mathrm{X}$.

117 HASA REF - 7.2.3.8/140H EACH PAIR OF LINES SHOULD BE IDENTICAL/POO90630 $28 \times, 26 H F O R$ COMPUTERS STORING FOUR/8X,27HOR MORE CHARACTERS PER WORDPOO90640 3)

0091 FORMAT $(2(A 1), 2(A 2), 3(A 3), 3(A 4), A 1, A 2, A 3, A 4,6(A 3))$

0092 FORMAT ( $1 / 29 H$ ABCOEFGHIJKLMNOPORSTUVWX YZI $2 X, 2(A 1), 2(A 2)$,

$$
3(A 3), 3(A 4) / / 12 H=-* /()+, \$ 12 X, A 1, A 2, A 3, A 411
$$$$
20 H \quad 0123456789+A B 2 \$(C) / 2 \times, 6 A 3 \text { ) }
$$

$C * * * *$ FORMATS TO TEST A CONVERSION WHERE FIELO WIOTH
$C * * * *$ IS LESS THAN THE WORO LENGTH CAPACITY OF MACHINE

0093 FORMAT ( $/ 135 \mathrm{H}$ TEST A CONVERSION - ADDING BLANKS/4OH

1 LINES SHOULD BE IOENTICAL)

0094 FORMAT $(5(\mathrm{~A} 1))$

0095 FORMAT (//4H A / $3 \times, A 3 / / 4 H$ A $3 \times, A 3 / / 4 H$ A $3 \times, A 3 / 1$

$14 \mathrm{H} \quad 1 / 3 \times, A 3 / / 4 \mathrm{H} \quad 2 / 3 X, A 3)$

C*** FORMATS TO TEST A CONVERSION WHERE FIELO WIDTH

$C * * * *$ IS GREATER THAN HORD LENGTH CAPACITY OF MACHINE

P0090580
P0010085

P0010090

P0010095

P0010100

P0010105

P0010110

P0O10115

P0010120

P0010125

P009A1

P0O9AZ

P0O9A3

P009A4

P009A5

PO010130

P0090350

P0070150

P0070155

P0 070160

P0070165

P0070170

P0070175

P009B1

P $009 B 2$

$P 0,070180$

P0090360

P0090370

P0090380

P 0090390

P 0090400

P 0090410

P0090420

P0090430

$3.8 / 22 P 0090440$

$7.2 .3 .8128 P 0090450$

$P 0090460$

P0 090470

P0090480

$P 0090490$

$7.2 .3 .8120 P 0090500$

$7.2 .3 .8 / 25 P 0090510$

P0090520

$P 0090530$

$P 0090540$

P0090550

$P 0090560$

P0090570

$7.2 .3 .8116 P 0090590$

$7.2 .3 .3101 P 0090600$

P 0090610

$P 0090620$

$P 0090650$

$P 0090660$ P0090670 $P 0090680$ P0090690

$7.2 .3 .8122 P 0090700$

$7.2 .3 .8 / 28 P 0090710$ EACH PAIR OFP0090720 $P 0090730$ $P 0090740$ P 0090750 P0090760 7.2 .3 .812000090770 $7.2 .3 .8 / 25 P 0090780$ 
0096 FORMAT(/25H TEST A FIELD TRUNCATION/37H 2ND LINE SHOULD PARTIALLP0090790 1Y MATCH 1ST)

0097 FDRMAT ( A26)

0098 FORMAT ( 11 28H ABCOEFGHIJKLMNOPORSTUVWXYZI $2 X$, A26)
$C * \# \#$ END DF TEST SEGMENT OO9

P0090800

$P 0090810$

P0090820

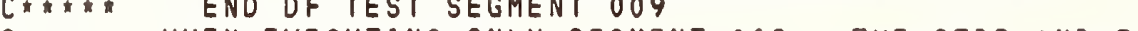

C***** WHEN EXECUTING ONLY SEGMENT 009, THE STDP AND END CARDS P PO090840

C***\# WHICH APPEAR AS COMMENT CARDS MUST HAVE THE C = IN COLUMNS P0090850

$C * * * * 1$ AND 2 REMOVED

$C=$ STOP

$C=E N D$

STOP

P0090860

P 0090870

P0 090880

END

$B=E F-* J K L /() 012$ TUVW..$+ \$ X$ YZACOGHIPORSMN0678(C)B259+A345

P009C1

POO9C2

$Q Z 1 * A$

ABCOEFGHIJKLMNOPORSTUVWXYZ

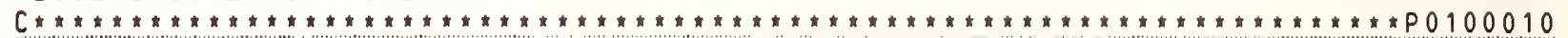

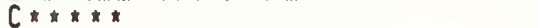

$C * \pi * *$
$C * \pi * *$

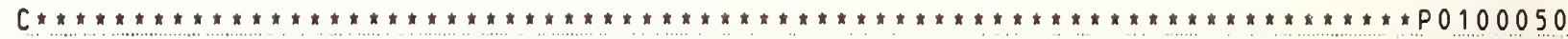

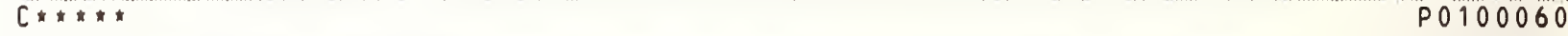

C**** GENERAL PURPDSE POP 100070

C**** TO TEST CONTENTS OF VARIABLES THAT WERE FORMEO BY PO PO 100080

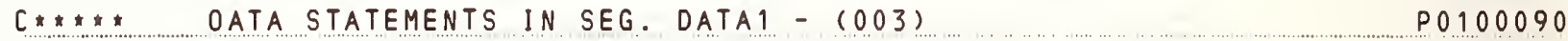

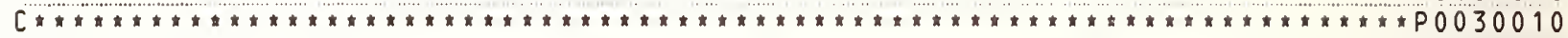

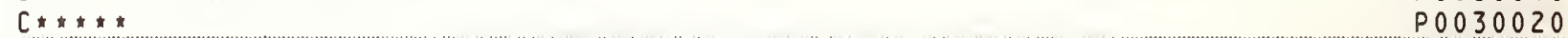

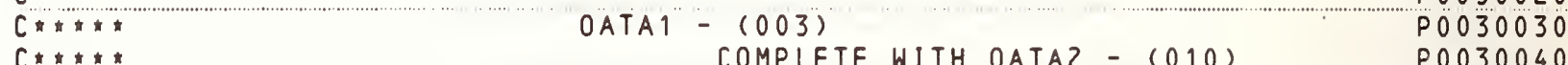

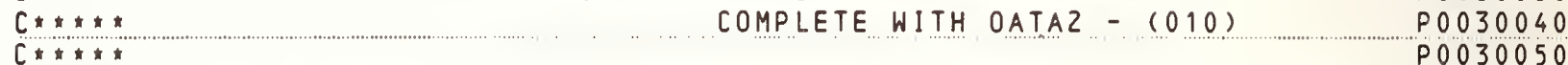

C

$C * \#$ GENERAL PURPOSE ASA REFSP0030070

C*\#* TO TEST FORMAT DF OATA STATEMENT $\quad 7.2 .2 \quad$ POO

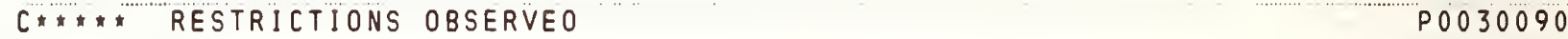

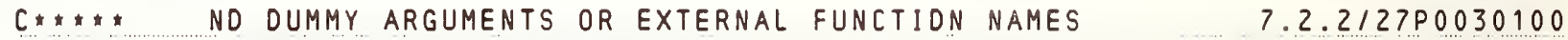

C*\#* APPEAR IN DATA STATEMENTS 8.4.1.1/40P0030110

C*\#** $\quad 10.1 .2108 P 0030120$

$C \# \#$ NO INITIALY OEFINED ITEMS APPEAR IN BLANK COMMON $\quad 7.2 .2139 P 0030130$

C\#\#\#\# $\quad 10.2 .4147 P 0030140$

$C * \pi * \cdots$ STORAGE UNITS INITIALIZEO ONLY ONCE

C**** SUBSCRIPTS ARE INTEGER CONSTANTS $\quad 7.2 .2128 P 0030160$

$C * \pi * *$ EXPLICIT VARIABLES P0030170

C*\#**AVI IS INTEGER $\quad$ PO030180

C*\#\#* JVS IS REAL P0030190

C\#\#\#* P0030200

C*\#\# S P E C I F I C A T I O N S SEGMENTS 003 ANO $010 \quad$ P0030210

$C * * * * *$ S

$C * \#$ WHEN EXECUTING ONLY SEGMENTS 003 ANO 010, THE SPECIFICATIDN PON 10140

$C * * * *$ STATEMENTS WHICH APPEAR AS COMMENT CAROS MUST HAVE THE C $=\quad$ PO0 10145

$C * \# \#$ IN COLUMNS 1 ANO 2 REMOVEO. PO010150

C*\#* $\quad$ P0010155

$C=$ DIMENSIONIACZI $(2,7), \operatorname{EP} 1 S(33), \operatorname{ACZS}(5,6) \quad P 0010160$

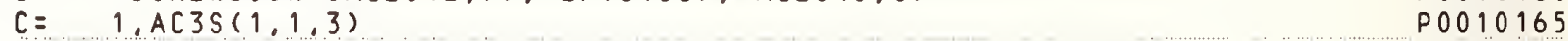

$C=$ INTEGER AVI,MCA3I(2,3,3), I1I(5) P0010170

$C=$ REAL JVS $\quad P 0010175$

$C=\quad$ LOGICAL MAVB, MBVB,MCVB, MCA1B(7),GHZB(1,2),GI3B(1,1,2),GG1B(2) P0010180

$C=\quad$ DOUBLE PRECISION AVO,BVO,CVO,OVO

$C=1,0 P A 2 O(2,2), M C A 30(1,4,2), A 1 D(4)$

$C=\quad$ CDMPLEX ADSVC,BCVC,CHEVC,OCVC,LL1C(32),LMZC (8,4),LN3C(9,2,2)

$C * * * *$

DIMENSION IAC2I $(2,7), \operatorname{EP} 1 S(33), \operatorname{AC} 2 S(5,6)$

$1, A C 3 S(1,1,3)$

INTEGER AVI, MCA $I(2,3,3), I 11(5)$

REAL JVS

LOGICAL MAVB, MBVB,MCVB, $M C A 1 B(7), G H 2 B(1,2), G I 3 B(1,1,2), G G 1 B(2)$

DOUBLE PRECISIDN AVD, BVO, CVO, OVO

1. DPA2O(2,2),MCA30 (1,4,2),A10(4)

CDMPLEX ADSVC, BCVC, CHEVC,OCVC,LL1C $(32), L M Z C(8,4), L N 3 C(9,2,2)$

P0010185

PO010190

P0010195

$P 0010200$

P003A1

P003AZ

$P 003 A 3$

POOZA4

P003A5

POO3A6

P003A7

P003A8

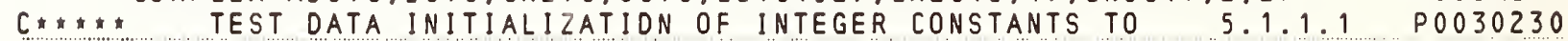


DATA III(1), MCA3I(1,2,1), MCA3I(2,2,2), IAC2I(2,5), IAC2I(2,6), P0030250

AMCA3I $(2,1,1) / 0,2 * 10,3 * 2461$

$C * * * * \quad$ TEST OATA INITIALIZATION OF REAL CONSTANTS TO

$P 0030260$

$C * * * *$ REAL VARIABLES

DATA EP1S(8),EP1S(10),EP1S(12),AC2S(5,5),EP1S(11),AC2S(5,3),

$P 0030270$

$A A C 2 S(5,2) / 2 * 0.2 *-750.05, .24615 E 3,2.4615 E 2.3 .54674 E+31$

$C * * * *$ TEST DATA INITIALIZATION OF DP CONTANTS TO

$C * * * *$ DP VARIABLES

$5 \cdot 1 \cdot 1 \cdot 3$

$P 0030290$

DATA BVD DPAZD $(2,1)$ CVD DPA2D $(1,2)$ DVD DPA2D $(2,2) 1+34567890,1$

$A 3.345 .6789010+2.112233 .50-08.19 .223350-4.3 .4012 .0 .340131 \quad P 0030340$

$C * * *$ TEST DATA INITIALIZATION OF COMPLEX CONSTANTS TO 5.1 .1 .4

$C * * * *$ COMPLEX VARIABLES

DATA ADSVC, LN $3 C(9,1,2), L L 1 C(30), L N 3 C(8,2,2), L M 2 C(8,3), L N 3 C(9,1,1), P 0030370$

$A L L 1 C(32), L N 3 C(8,1.2) / 2 *(11.1,22.22),(-3.45 E 1 .-67.8 E-1), \quad P 0030380$

$B(-34.5 E 0,-6.78 E 0),(10 . E 0,-20 . E 0),(1.0 E 1,-2.0 E 1),(-20.0 E 1,+4 . E 3), P 0030390$

$C(-200 . E 0 .+4000 . E 0) l$

C***** TEST DATA INITIALIZATION OF LOGICAL CONSTANTS TO 5.1 .1 .5

$C * * * *$ LOGICAL VARIABLES

DATA MAVB, MCA1B(6), MBVB/2*.TRUE, .FALSE./

C*** TEST DATA INITIALIZATION OF HOLLERITH CONSTANTS 5.1 .1 .6

DATA GI3B $(1,1,2), G G 1 B(1), E P 1 S(15) / 2 H N 0,2 * 2 H A D /$

C**** TEST DATA INITIALIZATION OF A MIXTURE OF ALL TYPES OF

$C * * * * \quad$ CONSTANTS AND VARIABLES IN ONE DATA STATEMENT

DATA I1I(2), IACZI(1,5), IACZI $(1,3)$, I1I(5), IAC2I(2,4),

$A M C A 3 I(1,1,2), A V I, E P 1 S(13), A C Z S(2,6), A C 2 S(1,6), A C 3 S(1,1,1)$,

$\operatorname{BAC} 2 S(3,6), A C 3 S(1,1,2), A C Z S(4,6), A V D, A 1 D(1), D P A 2 D(1,1)$.

$C M C A 3 D(1,1,1), A 1 D(2), M C A 3 D(1,1,2), L L 1 C(29), L N 3 C(8,2,1), B C V C$,

P 0030400

$P 0030410$

$P 0030420$

$P 0030430$

DLMZC $(8,4), G H Z B(1,1), G I 3 B(1,1,1), M C V B / 3 * 0,4 *-750,2 * 0 \ldots, 2 * 246.15$.

$E 354674 . E-2.354 .674 E+1,35467.4 E-01,3 *-29505,-29.5 D+3$,

$F 3456.78901 D+01,0.345678901 D+5,2 *(1.11 E 1,+222.2 E-1),(-34.5,-6.78)$.

G(-.345E2,-678.E-2), 2*.TRUE...FALSE./, I1 I(3), I II(4),

$P 0030440$

$P 0030450$

$P 0030460$

00030470

$P 0030480$

$P 0030490$

P0030500

$P 0030510$

P 0030520

$P 0030530$

$P 0030540$

POO30550

HMCA3I $(1,2,2), A C Z S(5,6)$, JVS ,EP1S(14),AC3S(1,1,3),IACZI(1,4).

ICHEVC,LL1C (31), DCVC, LMZC (8,2),A1D(3), MCA3D(1,3,1),A1D(4),

P0030560

JMCA3D $(1,4,1), \quad M C A 1 B(7), G H 2 B(1,2)$

$K-.75005 E 03,-7.5005 E+02,2 H B C, 2 H *=, 2 H P, 2 *(10 \ldots,-20$.$) ,$

$12 * 10+246, P 0030580$

$M 34.0011,0.034014,2 *$ FALSE./

$C * *$ END OF SEGMENT 003

$C * * * * * * 0$
$C * *$ U T U U T T P E ASSIGNMENT STATEMENT. NO INPUT TAPE

$P 0030600$

$P 0030610$

P 0030620

$P 0100100$

$C * * * * *$

C*** WHEN EXECUTING ONLY SEGMENTS 003 AND 010 THE FOLLOWING STATEMENTP

$C * * * *$ NUVI $=6$ MUST HAVE THE $C=$ IN COLUMNS 1 AND 2 REMOVED.

$C=N \cup V !=6$

NUVI $=6$

$C * * * * *$

WRITE (NUVI, 100)

100 FORMAT $(1 \mathrm{H} 1,1 \mathrm{X}$.

A $12 X .17$ HASA REFS. $-7.2 .21 / 2 X, 7$ HRESULTS)

32 HDATAZ - $(010)$ DATA STATEMENT USEI

Po 100110

P0100120

WRITE (NUVI, 101)

101 FORMAT(/35H LINE 1 OF EACH GROUP IS HOLLERITH/36H INFORMATION. TPO 100170 AEST IS SUCCESSFUL IF/37H EACH GROUP CONTAINS THE SAME VALUES)

WRITE (NUVI,102) I1I(1), III(2), IAC2I(1,5), IAC2I(1,3),

A MCA3I $(1,2,1), \operatorname{MCA} 3(2,2,2)$, I1I(3), III (4),

B IAC2I $(2,5), \operatorname{IAC} 2 I(2,6), \operatorname{MCA} I(2,1,1)$.

$\operatorname{MCA} I(1,2,2)$, I II(5), IACZI $(2,4), \operatorname{MCA} 3[(1,1,2)$.

C

102 FORMAT (125X.1HO/4(126/)/I

$\begin{array}{ll}A & 24 \times, 2 \mathrm{H} 1014(126 /) 11 \\ B & 23 \times, 3 H 24614(1261) 11\end{array}$

C $22 \times, 4 H-750 / 4(126 /))$

POP 0070190

$P 0070195$

P 01081

P0070205

P0100130

$P 0100140$

$P 0100150$

$P 0100160$

PO $100180^{\circ}$

P0100190

P0100200

P 0100210

P0100220

P0100230

Po 100240

P0100250

P0100260

P0100270

WRITE (NUVI, 103) EP1S (8), EP1S $(10), \operatorname{EP1S}(13), A C 2 S(2,6)$,

$P 0100280$

A $\quad A C 2 S(1,6), A C 3 S(1,1,1), E P 1 S(11), A C 2 S(5,3)$

P0100290

$B \quad A C 2 S(3,6), A C 2 S(5,2), A C 3 S(1,1,2), A C 2 S(4,6)$,

EP1S(12), $A C 2 S(5,5), A C 2 S(5,6)$, JVS

PO 100300

$P 0100310$

P0100320

103 FORMAT ( $122 \times, 4 \mathrm{HO} 0.0014(\mathrm{~F} 26.21) 11$

A $\quad 20 \times .6 \mathrm{H} 246.15 / 4(\mathrm{~F} 26.21) 11$

P 0100330 


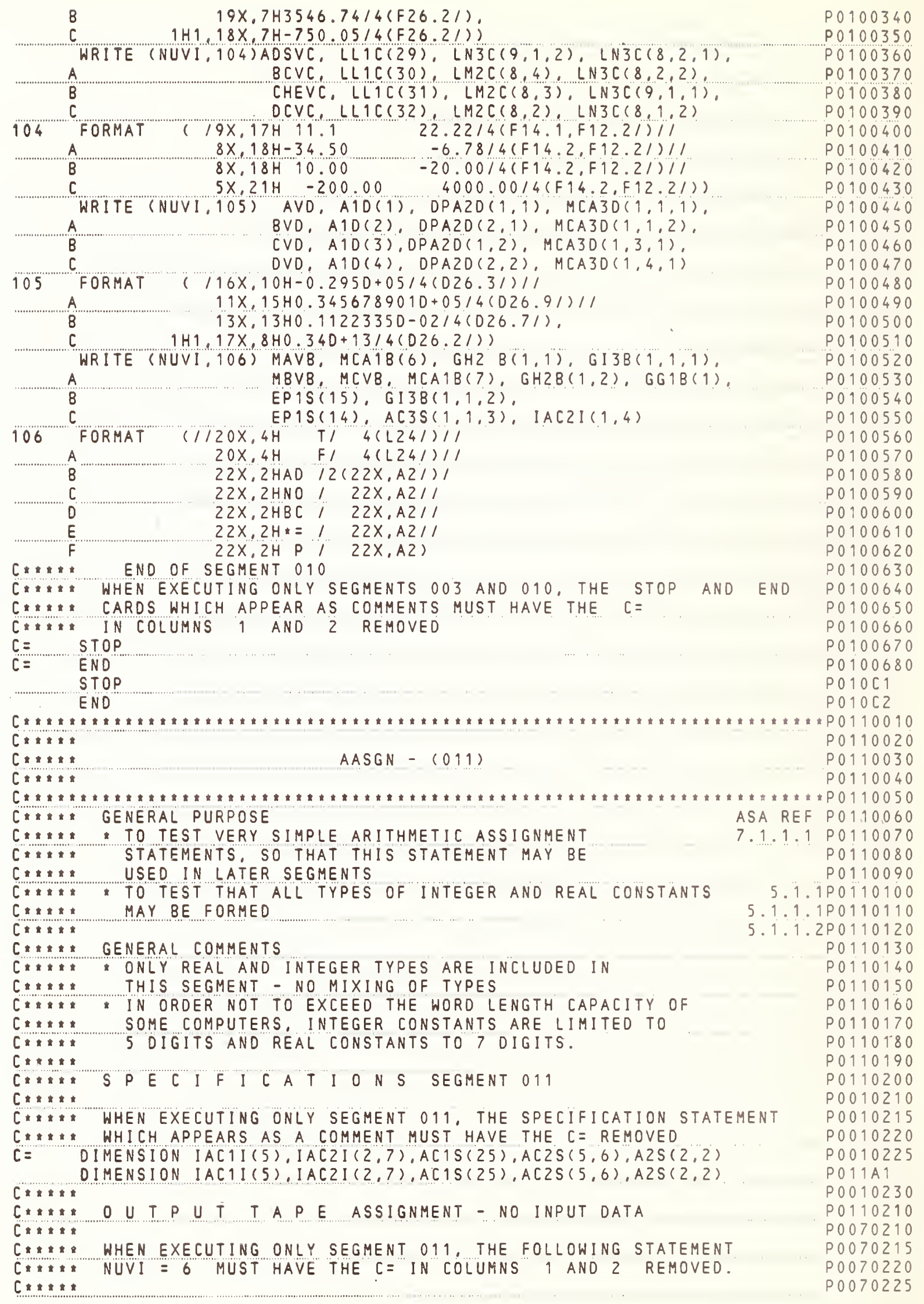


110 FORMAT (1H1,1X,37HAASGN- 611$)$ SIMPLE REAL AND INTEGER/10X,32HARPO110230

1 I THMETIC ASSIGNMENT STATEMENTS/2X,16HASA REF. - $7.1 .1 / 134 \mathrm{H}$ LINE 1 P0190240

2 OF EACH PAIR IS HOLLERITH/13H INFORMATION/117H INTEGER RESULTSIP0110250

C****. HEADER FOR SEGMENT 011 WRITTEN

$C * * *$ TEST ASSIGNMENT OF UNSIGNED INTEGER CONSTANTS

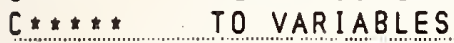

MRRVI $=1$

JACVI $=12345$

$K B C V I=000$

C***** TEST ASSIGNMENT OF SIGNED INTEGER CONSTANTS TO

\section{$C * * * *$ VARIABLES}

$M C A V I=+2$

LCCVI $=-3$

MDCVI $=-8765$

NECVI $=+6912$

C**** TEST ASSIGNMENT OF UNSIGNED INTEGER CONSTANTS $C * * * *$ TO ARRAYS

$I A C 1 I(1)=0$

IACZI $(2,1)=02468$

IACZI $(2,2)=00$

IACII (3) $=4444$

C**** TEST ASSIGNMENT OF SIGNED INTEGER CONSTANTS

$C * * * *$ TO ARRAYS

$I A C 2 I(1,1)=+45$

IACII $(4)=+4321$

$I A C 11(2)=-23$

$I A C Z I(1,2)=-3123$

C*\# TEST ASSIGNMENT OF UNSIGNED REAL CONSTANTS

$C * * * *$ TO VARIABLES (BASIC REAL CONSTANTS)

$A C V S=1.0$

$B C V S=358.6724$

C***** TEST ASSIGNMENT OF SIGNED REAL CONSTANTS

$C * \# *$ TO VARIABLES (BASIC REAL CONSTANTS)

$C * * * * *$

CCVS $=-2.0$

DCVS $=+3.0$

ECVS $=-2714.250$

FCVS $=+29.30542$

C*** TEST ASSIGNMENT OF UNSIGNED REAL CONSTANTS

C*** TO ARRAYS (BASIC REAL CONSTANTS)

$C * * * *$

$A C 1 S(2)=86.27$

$A C 2 S(1.2)=1034.2$

$A C 1 S(1)=0.0$

$A C Z S(1.1)=0.00000$

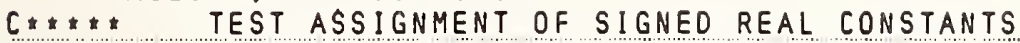

C***** TO ARRAYS (BASIC REAL CONSTANTS)

$C * * * *$

$\operatorname{AC2S}(2,2)=+345.678$

$A C 1 S(3)=-2.5$

$A C 2 S(2,1)=-5.66$

$A C 1 S(4)=+1.111111$

$C * * * *$ TEST ASSIGNMENT OF UNSIGNED AND SIGNED REAL

C**** CONSTANTS WITH NO OECIMAL DIGITS TO BOTH

C**** VARIABLES AND ARRAYS

GCVS $=1$.

HCVS $=-2$.

AADVS $=+3$.

$A C Z S(3,1)=4$.

$A C 2 S(1,3)=+5$.

$A C 1 S(5)=-6$.

C**** TEST ASSIGNMENT OF UNSIGNED AND SIGNED REAL

$c * * *$ CONSTANTS WITH NO INTEGER PART TO BOTH

$C * * *$ VARIABLES AND ARRAYS
$P 0110260$

$7.1 .1 .1140 P 0110270$

$5 \cdot 1.1 .1 / 15 P 0110280$ P0110290

P0110300

PO 110310

$7.1 .1 .1 / 40 P 0110320$

$5.1 .1 / 1190110330$

P0110340

P 0110350

$P 0110360$

PO110370

$7.1 .1 .1 / 40 P 0110380$

$5.1 .1 .1 / 15 P 0110390$ P0110400

PO 110410

PO 110420

$P 0110430$

7.1.1.1/40P0110440

$5.1 .1 / 111 \mathrm{PO} 110450$ P0110460 P0110470 P0 110480

Po110490

$7.1 .1 .1 / 40 P 0110500$

5.1 .1 .211890110510 P0110520 $P 0110530$

$7.1 .1 .1 / 40 P 0110540$

$5.1 .1 .2 / 18 P 0110550$

$5.1 .1 / 11 P 0110560$ P0110570 P0110580 PO 110590

P0110600

$7.1 .1 .1 / 40 P 0110610$

$5.1 .1 .2 / 18 P 0190620$

$5.1 .1 / 11 P 0110630$

PO 110640

P0110650

P0110660

PO 110670

$7.1 .1 .1 / 40 P 0110680$

5.1.1.2/18P0110690

5.1.1/11P0110700

PO 110710

P0110720

PO 110730

P0110740

$5.1 .1 .2 / 22 P 0110750$

$P 0110760$

PO 110770

P0110780

PO $110790^{\circ}$

P0110800

P0110810

P0110820

PO 110830

$5.1 .1 .2122 P 0110840$

PO 110850

P0110860 
BBDVS $=.0$

CCDVS $=+.23$

PO 110870

DDDVS $=-.716$

$A C 1 S(6)=-.7$

$\operatorname{AC2S}(4,1)=.81$

$A C 1 S(7)=+.9$

C**** TEST ASSIGNMENT OF UNSIGNED AND SIGNEO REAL

$C *$ CDNSTANTS WITH UNSIGNED AND SIGNED DECIMAL

C**** EXPDNENTS TD BDTH VARIABLES AND ARRAYS

EEDVS $=1.05 E 02$

FFDVS $=-7.6 E 1$

GGDVS $=+332.4 E 0$

HHOVS $=51.32 \mathrm{E}-1$

DDDVS $=+5.34 E-3$

PPDVS $=-14.19 \mathrm{E}-2$

OODVS $=-9.9 E+2$

RRDVS $=+10.5210 E+3$

SSDVS $=4.56 E+1$

$A C 2 S(1,4)=665.2 E O$

$A C 1 S(11)=-52.9 E 01$

$A C 1 S(9)=+78.564 E 2$

$A C 2 S(5,1)=-3.4567 E+3$

$A C 2 S(1,5)=61.62 E+2$

$A C 1 S(10)=+0.023 E+1$

$A C 1 S(8)=94.333 E-1$

$A C 1 S(12)=+0.3524 E-2$

$A C 2 S(3.2)=-743.2 E-3$

C\#\#\# TEST ASSIGNMENT DF UNSIGNED AND SIGNED REAL

$C * * *$ CONSTANTS (NO DECIMAL PART) WITH DECIMAL

$C * * * *$ EXPDNENTS TD BDTH VARIABLES AND ARRAYS

TTDVS $=1 . E 0$

UUDVS $=+123 \cdot E 2$

VVDVS $=-11 . E 3$

WWDVS $=144 . E-1$

XXDVS $=-12 \cdot E-2$

YYDVS $=+3645 \cdot E-3$

$22 D V S=1 . E+4$

CMAVS $=-200 \cdot E+1$

CMBVS $=+99 . E+2$

$A C 1 S(13)=+0 . E 00$

$A C 2 S(2,5)=-1512 . E 2$

$\operatorname{AC2S}(4,3)=214 . E 3$

$A C 1 S(15)=34 \cdot E-1$

$A C 1 S(14)=-4 \cdot E-2$

$A C 2 S(3,4)=+53214 \cdot E-4$

$\operatorname{AC2S}(4,4)=+6 \cdot E+3$

$\operatorname{AC2S}(2,3)=72 \cdot E+4$

$A C 1 S(16)=-813 \cdot E+1$

C*** TEST ASSIGNMENT DF UNSIGNED AND SIGNED REAL

C**** CDNSTANTS (ND INTEGER PART) WITH DECIMAL

C**** EXPONENTS TD BOTH VARIABLES AND ARRAYS

CMCVS $=.234 E 0$

CMDVS $=-.3 E 2$

CMEVS $=+.44 E 1$

CMFVS $=.36 E-3$

CMGVS $=+.9 E-4$

CMHVS $=-.10 \mathrm{E}-2$

CMOVS $=.777 \mathrm{E}+1$

CMPVS $=-.29 E+3$

CMOVS $=+.04 E+2$

$A C 1 S(17)=.90 E 1$

$A C 2 S(4,2)=+.810 E 0$

$A C 1 S(19)=-.7 E 3$

$A C 2 S(3,3)=.62 E+3$

$A C 1 S(21)=+.5310 E+1$

$\operatorname{A2S}(1,2)=-.442 E+2$

$A C 1 S(18)=.3 E-4$

$\operatorname{AC2S}(2,4)=+.25 E-03$

P0110880

P0110890

PO 110900

P0110910

P0110920

$5.1 .1 .2 / 25 P 0110930$

$5.1 .1 .2132 P 0110940$

P0110950

P0110960

P0110970

P0110980

P0110990

PO 111000

P0111010

P0111020

PO111030

PO 111040

P0111050

P0111060

P0 111070

P0111080

$P 0111090$

P0111100

P0111110

P0111120

PO 111130

$5.1 .1 .2 / 22 P 0111140$

5.1.1.2/26P0111150

$P 0111160$

$P 0111170$

P0111180

$P 0111190$

PO 111200

PO 111210

PO111220

PO 111230

P0111240

PO 111250

$P 0111260$

$P 0111270$

$P 0111280$

$P 0111290$

$P 0111300$

PO111310

PO 0111320

PO 111330

$P 0111340$

$5.1 .1 .2 / 22 P 0111350$

$5.1 .1 .2126 P 0111360$

PO 111370

P0111380

PO 111390

P0111400

P0111410

P0111420

PO 111430

P0111440

PO111450

$P 0111460$

P0111470

PO 111480

P0111490

P0111500

P0111510

P0111520

P0111530

P0111540 
$A 2 S(2,1)=-.163 E-2$

$\begin{array}{ll}C * * * * & \text { TEST ASSIGNMENT OF UNSIGNED AND SIGNED REAL } \\ C * * * * & \text { CONSTANTS (FORMED BY PLACING DECIMAL EXPONEN }\end{array}$

P0111550

$C * * * *$

$C * * * * *$ AFTER INTEGER CONSTANT) TO BOTH VARIABLES AND

$5.1 .1 .2134 P 0111560$ ARRAYS

$A V S=709 E 3$

BVS $=+81842 E 0$

CVS $=-9 E 5$

DVS $=627 E+2$

EVS $=+53 E+3$

$F V S=-4 E+04$

GVS $=1463 E-2$

$H V S=+2 E-3$

PVS $=-355 E-1$

$A C 1 S(24)=29 E 5$

$A C 1 S(20)=+4072 E 3$

$A C 2 S(5,4)=-61835 E 2$

$A C 2 S(3,5)=829 E+1$

$A C 1 S(22)=+03 E+2$

$A C 1 S(25)=-1 E+3$

$A C 2 S(4,5)=3404 E-4$

$A 2 S(2,2)=+55 E-5$

$A C 1 S(23)=-761 E-1$

$C * * * * \quad$ VERIFY CORRECTNESS OF ASSIGNMENT BY WRITING

$C * * * *$ THE INFORMATION

WRITE (NUVI, 111) MRRVI, JACVI, KBCVI, MCAVI, LCCVI, MOCVI, NECVI,

$1 \quad(I A C I I(I V I), I V I=1,4),((I A C Z I(I V I, J V I), I V I=1,2), J V I=1,2)$

WRITE (NUVI,112)

WRITE (NUVI, 113) ACVS, BCVS, CCVS, DCVS, ECVS, FCVS, AC1S(2),

$A C 2 S(1,2), A C 1 S(1), A C 2 S(1,1), A C 2 S(2,2)$

$A C 1 S(3), A C 2 S(2,1), A C 1 S(4)$, GCVS, HCVS,

AADVS, ACZS $(3,1)$

$P 0111570$

P0111580

$P 0111590$

P0111600

Po111610

$P 0111620$

$P 0111630$

$P 0111640$

$P 0111650$

$P 0111660$

Po111670

P0111680

Po111690

P0111700

$P 0111710$

P0111720

Po111730

P0111740

P0111750

P0111760

Po111770

P0111780

P0111790

P0111800

Po111810

P0111820

$P 0111830$

P 0111840

$P 0111850$

P0111860

WRITE (NUVI, 114) ACZS (1,3), AC1S(5), BBDVS, CCDVS, DDDVS, AC1S(6),P0111870

1

$A C 2 S(4,1), A C 1 S(7)$, EEDVS, FFDVS, GGDVS, HHDVS,

OODVS, PPDVS, OODVS, RRDVS, SSDVS

WRITE (NUVI, 115) AC2S $(1,4), A C 1 S(11), A C 1 S(9), A C 2 S(5,1)$,

$A C 2 S(1,5), A C 1 S(10), A C 1 S(8), A C 1 S(12)$,

AC2S $(3,2)$, TTDVS, UUDVS, VVDVS, WWDVS, XXDVS YYDVS

WRITE (NUVI, 116) CMAVS, CMBVS, AC1S(13), AC2S(2,5), AC2S $(4,3)$,

1

$A C 1 S(15), A C 1 S(14), A C 2 S(3,4), A C 2 S(4,4)$

$A C 2 S(2,3)$ AC1S(16), CMCVS, CMDVS, CMEVS, ZZDVS

WRITE (NUVI, 197)

CMFVS, CMGVS, CMHVS, CMOVS, CMPVS, CMOVS,

$A C 1 S(17), A C 2 S(4,2), A C 1 S(19), A C 2 S(3,3)$,

$A C 1 S(21), A Z S(1,2), A C 1 S(18), A C 2 S(2,4), A Z S(2,1)$

$P 0111880$

P 0111890

P0111900

PO111910

$P 0111920$

Po111930

PO 111940

$P 0111950$

$P 0111960$

$P 0111970$

$P 0111980$

1

AVS, BVS, CVS, DVS, EVS, FVS, GVS, HVS, PVS,

$P 0111990$

WRITE (NUVI, 118)

$A C 1 S(24), A C 1 S(20), A C 2 S(5,4), A C 2 S(3,5)$,

$A C 1 S(22), A C 1 S(25), A C 2 S(4,5), A Z S(2,2)$

$P 0112000$

P 0112010 $A C 1 S(23)$

$P 0112020$

$P 0112030$

P0112040

111 FORMAT $(17 x, 1 H 1,7 x, 5 H 12345,13 x, 1 H 0 / 1 X, 17,5 x, 17,7 x, 1711$

$17 x, 1 H 2,10 X, 2 H-3,8 x, 6 H-8765 / 1 x, 17,5 x, 17,7 x, 17 / 1$

$23 x, 5 H$ 6912, 11X, 1HO, 11X, 3H-23/ 1X, 17, 5x, 17, 7X, 17/1

$34 X, 4 H 4444,7 X, 5 H 4321,12 X, 2 H 45 / 1 X, 17,5 X, 17,7 X, 1711$

Po 112050

P0112060

P0112070

$44 X, 4 H 2468,6 X, 6 H-3123,13 X, 1 H 011 X, 17,5 X, 17,7 X, 17)$

P0112080

112 FORMAT (114H REAL RESULTS)

P0112090

113 FORMAT $(/ 3 X, 3 H 1.0,10 X, 8 H 358.6724,6 X, 4 H-2.0 / 1 X, F 5,1,6 X, F 12,4,2 X, P 0112100$

$1 \mathrm{~F} 8.1 / 13 \mathrm{X}, 3 \mathrm{H3}, 0,8 \mathrm{X}, 9 \mathrm{H}-2714.250,7 \mathrm{X}, 8 \mathrm{H} 29.30542 / 1 \mathrm{X}, \mathrm{F} 5.1,6 \mathrm{X}, \mathrm{F} 11.3,3 \mathrm{X}, \mathrm{PO} 112110$

$2 \mathrm{~F} 12.5 / 12 \mathrm{X}, 5 \mathrm{H86}, 27,8 \mathrm{X}, 6 \mathrm{H} 1034.2,10 \mathrm{X}, 3 \mathrm{HO}, 0 / 1 \mathrm{X}, \mathrm{F} 6.2,5 \mathrm{X}, \mathrm{Fg}, 1,5 \mathrm{X}, \mathrm{F} 8.1 / 1 \mathrm{PO} 112120$

$33 X, 3 H 0.0,10 X, 7 H 345.678,7 X, 4 H-2.5 / 1 X, F 5.1,6 X, F 11.3,3 X, F 8.1119 P 0112130$

$42 X, 5 H-5.66,11 X, 8 H 1.111111,5 X, 3 H 1,0 / 1 X, F 6,2,5 X, F 14,6, F 8.1 / 1$

$52 X, 4 H-2.0,12 X, 3 H 3.0,10 X, 3 H 4.0 / 1 X, F 5.1,6 X, F 9.1,5 X, F 8.1)$

114 FORMAT $(13 \mathrm{X}, 3 \mathrm{H} 5.0,11 \mathrm{X}, 4 \mathrm{H}-6.0,10 \mathrm{X}, 3 \mathrm{H} 0.0 / 1 \mathrm{X}, \mathrm{F5}, \mathrm{1}, 6 \mathrm{X}, \mathrm{FO}, 1,5 \mathrm{X}, \mathrm{F} 8.1 / 1$

$13 X, 4 H 0.23,10 X, 6 H-0.716,7 X, 4 H-0.7 / 1 X, F 6.2,5 X, F 11.3,3 X, F 8.1 / 1$

$23 X, 4 H 0.81,11 X, 3 H 0.9 / 1 X, F 6.2,5 X, F 9.1 / 1 H 1,2 X, 9 H 0.105 E+03,3 X$,

$39 H-0.76 E+02,5 X, 10 H 0.3324 E+03 / E 12.3, E 12.2, E 15.4 \%$

$43 X, 10 H 0.5132 E+01,3 X, 9 H 0.534 E-02,3 X, 11 H-0.1419 E+00 / E 13,4, E 12.3$,

$5 E 14.4 / / 2 X .9 H-0.99 E+03,5 X, 12 H 0.105210 E+05,10 H \quad 0.456 E+02 / E 11.2$,

6 E $17,6, E 10.3)$

$P 0112140$

$P 0112150$

$P 0112160$

$P 0112170$

P0112180

P0112190

P0112200

P0112210

P0112220 
FORMAT $(/ 3 X, 10 H 0.6652 E+03,2 X, 10 H-0.529 E+03,4 X, 11 H 0.78564 E+04 / E 13.4, P 0112230$ E12.3, E15.5//2X,12H-0.34567E+04,2X,10H0.6162E+04,3X,8H0.23E+00!

$E 14.5, E 12.4, E 11.2 / / 3 X, 11 H 0.94333 E+01,2 X, 10 H 0.3524 E-02,2 X$,

$11 H-0.7432 E+00 / E 14.5, E 12.4, E 13.4 / 13 X, 7 H 0.1 E+01,6 X, 9 H 0.123 E+05$. $3 X, 9 H-0.11 E+05 / E 10.1, E 15.3, E 12.2 / 13 X, 9 H 0.144 E+02,3 X, 9 H-0.12 E+00$ $5 X, 10 H 0.3645 E+01 / E 12.3, E 12.2, E 15.4)$

116 FORMAT $(1 / 2 \mathrm{H}-0.200 \mathrm{E}+04,4 \mathrm{X}, 8 \mathrm{HO} .99 \mathrm{E}+04,5 \mathrm{X}, 7 \mathrm{HO} .0 \mathrm{E}+00 / \mathrm{E} 12.3, \mathrm{E} 12.2$. $E 12.1 / / 2 X, 11 H-0.1512 E+06,3 X, 9 H 0.214 E+06,4 X, 8 H 0.34 E+01 / E 13.4$, $E 12.3, E 12.2 / / 2 X, 8 H-0.4 E-01,6 X, 11 H 0.53214 E+01,2 X, 7 H 0.6 E+04 / E 10.1$ 。 $3 E 17.5, E 9.1 / / 3 X, 8 H 0.72 E+06,4 X, 10 H-0.813 E+04,4 X, 9 H 0.234 E+00 / E 11.2$, $E 14.3, E 13.3 / / 2 X, 8 H-0.3 E+02,6 X, 8 H 0.44 E+01,5 X, 7 H 0.1 E+05 / E 10.1$, E14.2, E12.1)

117 FORMAT $(/ 3 X, 8 H 0.36 E-03,5 X, 7 H 0.9 E-04,5 X, 9 H-0.10 E-02 / E 11.2, E 12.1$, $\mathrm{E} 14.2 / / 3 \mathrm{X}, 9 \mathrm{HO} .777 \mathrm{E}+01,3 \mathrm{X}, 9 \mathrm{H}-0.29 \mathrm{E}+03,5 \mathrm{X}, 7 \mathrm{H} 0.4 \mathrm{E}+01 / \mathrm{E} 12.3, \mathrm{E} 12.2$, $E 12.1 / / 3 X .8 H 0.90 E+01,5 X, 9 H 0.810 E+00,3 X, 8 H-0.7 E+03 / E 11.2, E 14.3$, $\mathrm{E} 11.1 / / 3 \mathrm{X}, 8 \mathrm{H0} .62 \mathrm{E}+03,5 \mathrm{X}, 10 \mathrm{H} 0.5310 \mathrm{E}+01,2 \mathrm{X}, 10 \mathrm{H}-0.442 \mathrm{E}+02 / \mathrm{E} 11.2$. $4 E 15.4, E 12.3 / / 3 X, 7 H 0.3 E-04,6 X, 8 H 0.25 E-03,4 X, 10 H-0.163 E-02 / E 10.1$, E14.2, E14.3/1H1)

$E 9.1 / / 3 X, 9 H 0.627 E+05,4 X, 8 H 0.53 E+05,4 X, 8 H-0.4 E+05 / E 12.3, E 12.2$,

$E 12.1 / / 3 X, 10 H 0.1463 E+02,3 X, 7 H 0.2 E-02,5 X, 10 H-0.355 E+02 / E 13.4$,

$E 10.1, E 15.3 / / 3 X, 8 H 0.29 E+07,5 X, 10 H 0.4072 E+07,2 X, 12 H-0.61835 E+07 /$

$E 11.2, E 15,4, E 14.5 / 13 X, 9 H 0.829 E+04,4 X, 7 H 0.3 E+03,5 X, 8 H-0.1 E+04 /$

E12.3, E11.1, E13.1//3X,10H0.3404E+00,3X,8H0.55E-03,4X,10H-0.761E+0P $62 / E 13.4, E 11.2, E 14.3$ )

C***** END OF TEST SEGMENT 011

C**\#\# WHEN EXECUTING ONLY SEGMENT 011, THE STOP AND END CARDS

$C * * *$ HHICH APPEAR AS COMMENT CARDS MUST HAVE THE $C=$ C*\#**IN COLUMNS 1 AND 2 REMOVED

$C=\operatorname{STOP}$

$C=\quad$ END

STOP

END

P0112240

PO 112250

$P 0112260$

$P 0112270$

P0112280

P0112290

P0112300

P0112310

$P 0112320$

P0112330

$P 0112340$

P0112350

$P 0112360$

$P 0112370$

$P 0112380$

$P 0112390$

$P 0112400$

$P 0112420$

P0112430

P0112440

P0112450

OP 0112460

$P 0112470$

P0112480

P0112490

$P 0112500$

P0112510

$P 0112520$

$P 0112530$

P011C1

Po11C2

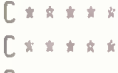

$C * * * *$

$C * * *$

P0130010

P0130020

P0 130030

P0130040

$C * * *$

GENERAL PURPOSE

$C * * * * * * *$

* to test all possible methods of forming double PRECISION CONSTANTS

$C * * * t$

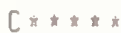
TO TEST THAT D.P. VARIABLES AND ARRAY

$C * * * *$

$C * * * *$

C**** ELEMENTS MAY BE REFERENCED

DASGN - $(013)$

$P 0130050$

$C * * \pm \leqslant$

C * * * क

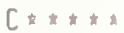

C $* * *$

C * $* * *$

$C * * * * *$

C****

$C=$

DOUBLE PRECISION MCAVD, MCBVD, MCCVD, MCDVD, MCEVD, MCFVD, MCGVD. TO TEST VERY SIMPLE ARITHMETIC ASSIGNMENT STATEMENTS, SO THAT THIS FEATURE CAN BE USED FOR INITIALIZATION IN LATER SEGMENTS

ASA REF P0130060 5.1.1 THRUPO 130070 P.1. 15 P0130080 $5.1 .2 / 5 \quad P 0130090$ $5.1 .3 .1 / 16 P 0130100$ 7.1.1.1 P0130110 TABLE 1 PO 130120 $S$ P E C I F I C A T I O N S SEGMENT 013

P0130130 P0 130140

WHEN EXECUTING ONLY SEGMENT 013. THE SPECIFICATION STATEMENTS $P 0010240$ P0010245 WHICH APPEAR AS COMMENT CARDS MUST HAVE THE $C=$ P0010250 P0010255 P0010260 P0010265 $P 0010270$ 1 MCHVD, MCIVD, EEDVD, ACVD, BCVD, CCVD,DCVD, DDDVD, CCDVD, FFDVD, GGDVD 2 HHDVD,EP1D(43),AC1D(10),BC2D(7,4),CC3D(7,2,2),FC2D(5,5)

DOUBLE PRECISION DPAVD,DPBVD, DPCVD,DPDVD,DPEVD,DPFVD,DPGVD,DPHVD,

$C=1$ DPIVD,DPJVD,DPKVD,DPLVD,DPMVD,DPNVD,DPOVD,DPPVD,

$C=2$ AADVD,BBDVD,PPDVD,RRDVD, SSDVD, TTDVD, UUDVD, VVDVD, WWDVD, XXDVD,

$C=3$ YYDVD, 2ZDVD,ECVD,FCVD,GCVD,HCVD,RC3D(3,3,3),MCJVD,MCKVD

DOUBLE PRECISION MCAVD, MCBVD, MCCVD, MCDVD, MCEVD, MCFVD, MCGVD,

$1 M C H V D, M C I V D, E E D V D, A C V D, B C V D, C C V D, D C V D, D D D V D, C C D V D, F F D V D, G G D V D$,

2 HHDVD,EP1D(43),AC1D(10),BC2D(7,4),CC3D(7,2,2), FC2D(5,5) $P 0010275$ $P 0010280$ PO010285 POO 10290 P0010295 P0010300 P013A1 P013A2 $P 013 A 3$ DOUBLE PRECISION DPAVD,DPBVD,DPCVD,DPDVD,DPEVD,DPFVD,DPGVD, DPHVD, DPIVD, DP JVD, DPKVD, DPLVD,DPMVD, DPNVD, DPOVD, DPPVD, P013A4 $P 013 A 5$ P013A6 AADVD, BBDVD, PPDVD, RRDVD, SSDVD, TTDVD, UUDVD, VVDVD, WWDVD, XXDVD, YYDVD,22DVD,ECVD,FCVD,GCVD,HCVD,RC3D $(3,3,3), M C J V D, M C K V D$ $P 013 A 7$ C*\#* OUTPUT T A P E ASSIGNMENT STATEMENT. NO INPUT TAPE. P0 130150 
2 2X.7HRESULTS)

131 FORMAT $(/ 2 \times, 23 \mathrm{HLINE} 1$ OF EACH GROUP ISI

$P 0130190$

$P 0130200$

$P 0130210$

A $2 X, 21$ HHOLLERITH INFORMATION)

$C * * * *$ HEADER FOR THIS SEGMENT WRITTEN

$C * * * * *$ TEST ASSIGNMENT OF UNSIGNED DP CONSTANTS WITH

$P 0130220$

$C * * * * *$ UNSIGNED EXPONENTS TO VARIABLES AND ARRAY ELEMENTS

$C * * * * *$

$C * * * * *$

$M C A V D=3.401$

$P 0130230$

$P 0130240$

$7.1 .1 .1 / 41 P 0130250$

$5.1 .1 .3 / 40 P 0130260$

5.1.1/114P0130270

$5.1 .1 .3 / 36 P 0130280$

5.1.1.2126P0130290

MCBVD $=123456.7891011002$

$P 0130300$

P0130310

$A C 1 D(1)=3.401$

$A C 1 D(2)=123456.7891011002$

$P 0130320$

$B C 2 D(1,1)=3.401$

$P 0130330$

$B C 2 D(2,1)=123456.7891011002$

$P 0130340$

$C C 3 D(1,1,1)=3.401$

$P 0130350$

$C C 3 D(2,1,1)=123456.789101102$

00130360

$P 0130370$

$C * * * * *$ ASSIGNMENT OF UNSIGNED DP CONSTANTS WITH

$C * * * * *$ SIGNED EXPONENTS TO VARIABLES AND ARRAY ELEMENTS 5.1.1.2126P0130390

MCCVD $=29.8765234 D-3$

$M C D V D=345.100005550+4$

$P 0130400$

$A C 10(3)=29.8765234 D-3$

$A C 1 D(4)=345.100005550+4$

$B C 2 D(3.1)=29.8765234 D-3$

$B C 2 D(4,1)=345.100005550+4$

$C C 3 D(3,1,1)=29.87652340-3$

CC $30(4,1,1)=345.100005550+4$

$C * * * * *$ ASSIGNMENT OF UNSIGNED DP CONSTANTS (NO DECIMAL

$C * * * * *$ PART) WITH UNSIGNED EXPONENTS TO VARIABLES

$C * * * *$ AND ARRAY ELEMENTS

MCEVD $=22232425.000$

$A C 1 D(5)=22232425.000$

$B C 2 D(5,1)=22232425.000$

$C C 3 D(5,1,1)=22232425 . D 00$

$C * * * * *$ ASSIGNMENT OF UNSIGNED DP CONSTANTS (NO

$[* * * *$ INTEGER PART) WITH UNSIGNED EXPONENTS TO VARIABLES AND ARRAY ELEMENTS

$P 0130410$

$P 0130420$

$P 0130430$

$P 0130440$

$P 0130450$

$P 0130460$

$P 0130470$

$C * * * * *$

MCFVD $=.28142005$

$A C 10(6)=.28142005$

$B C 2 D(6,1)=.28142005$

$C C 30(6,1,1)=.28142005$

$C * * * *$ ASSIGNMENT OF UNSIGNED DP CONSTANTS (NO DECIMAL

$[* * * * *$ PART) WITH SIGNED EXPONENTS TO VARIABLES AND

$C * * * *$ ARRAY ELEMENTS

MCGVD $=4455667788 . D+6$

MCHVD $=35692483569248.0-02$

$A C 1 D(7)=4455667788.0+6$

$A C 1 D(8)=35692483569248 . D-02$

$B C 2 D(7,1)=4455667788 . D+6$

$B C 2 D(1,2)=35692483569248 . D-02$

$C C \cdot 3 D(7,1,1)=4455667788 . D+6$

$C C 3 D(1,2,1)=35692483569248 . D-2$

5.1.1.2/22P0130480

$P 0130490$

$P 0130500$

P0130510

$P 0130520$

$P 0130530$

$P 0130540$

$5.1 .1 .2122 P 0130550$

$P 0130560$

$P 0130570$

$P 0130580$

$P 0130590$

$P 0130600$

P0130610

$P 0130620$

P0130630

$P 0130640$

P0130650

$P 0130660$

P0130670

$P 0130680$

P0130690

$P 0130700$

P0130710

P0130720

$C * * * * *$ ASSIGNMENT OF UNSIGNED DP CONSTANTS (NO

$P 0130730$

C***** INTEGER PART) WITH SIGNED EXPONENTS TO

$P 0130740$

$C * * * * *$ VARIABLES AND ARRAY ELEMENTS

$A C V D=.6549876 D-3$

$B C V D=.780+10$

P0130750

$P 0130760$

P 0130770

$A C 1 D(9)=.65498760-3$

P0130780 
$A C 1 D(10)=.780+10$

$B C 2 D(2,2)=.65498760-3$

P0130790

$B C 2 D(3.2)=.780+10$

$P 0130800$

$\operatorname{CC} 3 D(2.2 .1)=.65498760-3$

$P 0130810$

$C C 3 D(3,2,1)=.780+10$

P0130820

ASSIGNMENT OF SIGNED DP CONSTANTS WITH

UNSIGNED EXPONENTS TO VARIABLES AND ARRAY

P0130830

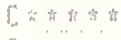
ELEMENTS

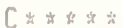

CCVD $=+0.000$

DCVD $=-17263544.503$

EP1O(1) $=+0.000$

EP1D(2) $=-17263544.503$

$8 C 20(4,2)=+0.0000$

$8 C 20(5,2)=-17263544.503$

$C C 30(4,2,1)=+0.000$

$C\{3 D(5,2,1)=-17263544.503$

$C * * * * *$ ASSIGNMENT DF SIGNED DP CONSTANTS WITH

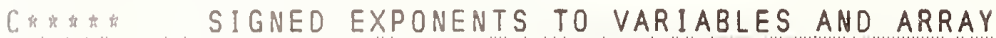

C 4 क

$E C V D=+1987.620+1$

$F C V D=-2.543966210+2$

GCVD $=+34.7865299102340-7$

HCVD $=-44.40-10$

EP1D(3) $=+1987.620+1$

EP1D(4) $=-2.543966210+2$

EP1D (5) $=+34.7865299102340-7$

$E P 1 D(6)=-44.4 D-10$

$B C 2 D(6,2)=+1987.620+1$

$B C 2 D(7,2)=-2.543966210+2$

$B C 2 D(1,3)=+34.7865299102340-7$

$B C 20(2,3)=-44.40-10$

$C C 30(6,2,1)=+1987.620+1$

$C[30(7,2,1)=-2.543966210+2$

$C C 3 D(1,1,2)=+34.7865299102340-07$

$\operatorname{CC3D}(2,1,2)=-44.40-10$

$5.1 .1 \quad 112 P 0130840$

$P 0130850$

$P 0130860$

P0130870

$P 0130880$

$P 0130890$

P0130900

$P 0130910$

$P 0130920$

$P 0130930$

$P 0130940$

$P 0130950$

$P 0130960$

$P 0130970$

$P 0130980$

$P 0130990$

$P 0131000$

P0131010

$P 0131020$

$P 0131030$

$P 0131040$

$P 0131050$

$P 0131060$

P 0131070

P0131080

$P 0131090$

P0131100

P0131110

P0131120

P 0131130

$P 0131140$

C****** ASSIGNMENT OF SIGNED DP CONSTANTS (NO DECIMAL

$P 0131150$

$C * * * *$ PART) WITH SIGNED EXPONENT TO VARIABLES AND

$P 0131160$ ARRAY ELEMENTS

$A A D V D=+0 . D+1$

$B B D V D=-123.0+17$

CCDVD $=+3692468.0-8$

DODVD $=-147937824967.0-5$

$P 0131170$

$P 0131180$

$P 0131190$

$P 0131200$

EP1D (7) $=+0.0+1$

$E P 1 D(8)=-123 \cdot D+17$

EP1D(9) $=+3692468.0-8$

$P 0131210$

$P 0131220$

EP1D(10) $=-147937824967.0-5$

$P 0131230$

$P 0131240$

$B C 2 D(3,3)=+0 . D+1$

$B C 2 D(4,3)=-123 \cdot D+17$

$B C 2 D(5,3)=+3692468.0-8$

$P 0131250$

$P 0131260$

$P 0131270$

$B C 2 D(6,3)=-147937824967.0-5$

$P 0131280$

$C C 3 D(3,1,2)=+0 . D+1$

P0 131290

$C[30(4,1,2)=-123.0+17$

$P 0131300$

$C C 3 D(5,1,2)=+3692468.0-8$

$C C 30(6,1,2)=-147937824967.0-5$

$P 0131310$

$P 0131320$

PO 131330

$P 0131340$

ASSIGNMENT OF SIGNED DP CONSTANTS (NO INTEGER

$P 0131350$ ARRAY ELEMENTS

$C * * * *$

EEDVD $=+.9277861749850+2$

P0131360

P 0131370

FFDVD $=-.593549142236190+0$

GGOVD $=+.986632710-03$

HHDVD $=-.10-15$

EP1D(11) $=+.9277861749850+2$

$P 0131380$

$P 0131390$

$P 0131400$

$P 0131410$

EP1D(12) $=-.593549142236190+0$

EP1D(13) $=+.986632710-03$

$E P 1 D(14)=-.10-15$

$B C 2 D(7,3)=+.9277861749850+2$

$B C 2 D(1,4)=-.593549142236190+0$

$P 0131420$

$P 0131430$

P0131440

P0 131450

$B C 2 D(2,4)=+.986632710-03$

P0131460 
$B C 2 D(3,4)=-.1 D-15$

$P 0131470$

$C C 30(7,1.2)=+.9277861749850+2$

$P 0131480$

$C C 30(1.2,2)=-.593549142236190+0$

$P 0131490$

$\operatorname{cc} 30(2,2,2)=+.986632710-3$

$C C 3 D(3,2,2)=-.10-15$

$P 0131500$

$P 0131510$

$C * * * \quad$ ASSIGNMENT OF SIGNED DP CONSTANTS (NO DECIMAL

*** PART) WITH UNSIGNED EXPONENTS TO VARIABLES

$C * * *$ ANO ARRAY ELEMENTS

PPDVD $=+3261294675.012$

RRDVD $=-969492909.04$

$E P 1 D(15)=+3261294675.012$

$E P 1 D(16)=-969492909.04$

$B C 2 D(4.4)=+3261294675.012$

$B C 2 D(5,4)=-969492909.04$

$C C 3 D(4,2,2)=+3261294675.012$

$C C 3 D(5,2,2)=-969492909.04$

C*** ASSIGNMENT OF SIGNED DP CONSTANTS (NO INTEGER

$C * * * *$ PART) WITH UNSIGNED EXPONENTS TO VARIABLES

$c * * *$ AND ARRAY ELEMENTS

SSOVD $=+.00124608503$

TTDVD $=-.5902$

$E P 1 D(17) \quad=+.00124608503$

$E P 1 D(18)=-.5902$

$B C 2 D(6,4)=+.00124608503$

$B C 2 D(7,4)=-.5902$

$C C 30(6,2,2)=+.00124608503$

$C C 3 D(7,2,2)=-.5902$

$C \because * \because \quad$ ASSIGNMENT OF DP CONSTANTS FORMED BY ADDING

$C * * *$ UNSIGNED EXPONENTS TO UNSIGNED INTEGERS

UUDVD $=79828139225300$

$E P 1 D(19)=79828139225300$

$F C 20(1,1)=79828139225300$

$R C 30(1,1,1)=79828139225300$

$C * * * *$ ASSIGNMENT OF DP CONSTANTS FORMED BY ADDING

$C * * * *$ SIGNED EXPONENTS TO UNSIGNED INTEGERS

VVDVD $=429210+6$

WWDVD $=7936854430-4$

$E P 1 D(20)=429210+6$

$E P 10(21)=7936854430-4$

$F C 2 D(2,1)=429210+6$

$F(20(3,1)=7936854430-4$

$R C 30(2,1,1)=429210+6$

$R C 30(3,1,1)=7936854430-4$

$C * * *$ ASSIGNMENT OF OP CONSTANTS FORMED BY ADDING

$C * * *$ UNSIGNED EXPONENTS TO SIGNED INTEGERS

XXDVD $=+3334445556602$

YYDVD $=-22233344401$

$E P 10(22)=+3334445556602$

$E P 10(23)=-22233344401$

$F C 2 D(4,1)=+3334445556602$

$F C 2 D(5,1)=-22233344401$

$R C 30(1,2,1)=+3334445556602$

$R C 30(2,2,1)=-22233344401$

$C * * * *$ ASSIGNMENT OF DP CONSTANTS FORMED BY AODING

$C * * * *$ SIGNED EXPÖNENTS TO SIGNED INTEGERS

ZZOVD $=+10+1$

$M C I V D=-2 D+2$

$M C J V D=+333333333333330-3$

$M C K V D=-4444444440-4$

$E P 1 D(24)=+10+1$

EPID (25) $=-2 D+2$

$E P 1 D(26)=+333333333333330-3$

EP1D(27) $=-4444444440-4$

$F C 2 D(1,2)=+1 D+1$

$F C 2 D(2,2)=-2 D+2$

$F C 2 D(3,2)=+33333333333333 D-3$

$F C 2 D(4,2)=-444444444 D-4$

$R C 3 D(3,2,1)=+1 D+1$

$5.1 .1 .3 / 42 P 0131740$

$P 0131750$

$P 0131760$

$P 0131770$

$P 0131780$

P0131790

P0131800

P0131810

P0131820

PO131830

P0131840

P0131850

P0131860

$P 0131870$

P0131880

$P 0131890$

$P 0131900$

P0131910

$P 0131920$

P0131930

P0131940

P0131950

$P 0131960$

P0131970

P0131980

P0131990

$P 0132000$

$P 0132010$

P0132020

P0132030

$P 0132040$

P0132050

$P 0132060$

$P 0132070$

P0132080

P0132090

$P 0132100$

P0132110

P0132120

P0132130

PO 132140 
$R C 30(1,3,1)=-20+2$

$R C 30(2,3,1)=+333333333333330-3$

P0132150

$R C 30(3,3,1)=-4444444440-4$

$P 0132160$

$P 0132170$

$C * * * *$ ASSIGNMENT OF UNSIGNED DP VARIABLES AND ARRAY

$C * * * *$ ELEMENTS TO OP VARIABLES AND ARRAY ELEMENTS

$C * *$ (BOTH PLUS AND MINUS VALUES ARE ASSIGNED IN THIS

7.1.1.1/41P0132180

$C * * * * *$ WAY

DPAVD = MCAVD

DPBVD = DCVD

DPCVD $=E P 1 D(1)$

DPOVD $=E P 1 D(2)$

$D P E V D=B C 2 D(2,2)$

$D P F V D=B C 2 D(4,2)$

DPGVD $=\operatorname{CC} 30(3,1,1)$

DPHVD $=C C 3 D(7,2,1)$

$P 0132190$

$P 0132200$

$P 0132210$

$P 0132220$

P 0132230

P0132240

$P 0132250$

$P 0132260$

$P 0132270$

$P 0132280$

$E P 1 D(28)=D P A V D$

$P 0132290$

$E P 1 D(29)=D P B V D$

$E P 1 D(30)=E P 1 D(1)$

$E P 10(31)=E P 10(2)$

$P 0132300$

$P 0132310$

$E P 1 D(32)=B C 2 D(2,2)$

$P 0132320$

$P 0132330$

$E P 1 D(33)=B C 2 D(4,2)$

P0132340

$E P 1 D(34)=C[3 D(3,1,1)$

$P 0132350$

$E P 1 D(35)=C C 3 D(7,2,1)$

$P 0132360$

$F C 2 D(5,2)=D P A V D$

$P 0132370$

$F C 2 D(1,3)=D P B \vee D$

$F C 2 D(2,3)=E P 1 D(1)$

$F C 2 D(3,3)=E P 1 D(2)$

$F C 2 D(4,3)=B C 2 D(2,2)$

$F C 2 D(5,3)=B C 2 D(4,2)$

$F C 2 D(1,4)=C C 3 D(3,1,1)$

$F C 2 D(2,4)=C C 3 D(7,2,1)$

$P 0132380$

$P 0132390$

$P 0132400$

P0132410

$P 0132420$

$P 0132430$

$P 0132440$

$\operatorname{RC} 30(1,1,2)=\operatorname{MCAVD}$

$P 0132450$

$P 0132460$

$\operatorname{RC3D}(2,1,2)=D C V D$

$R C 3 D(3,1,2)=E P 1 D(1)$

$P 0132470$

$R C 3 D(1,2,2)=E P 1 D(2)$

$P 0132480$

$P 0132490$

$R C 3 D(2.2 .2)=B C 2 D(2.2)$

$P 0132500$

$R C 3 D(3,2,2)=B C 2 D(4,2)$

$P 0132510$

$R C 3 D(1,3,2)=C C 3 D(3,1,1)$

$P 0132520$

$R C 3 D(2,3,2)=C C 3 D(7,2,1)$

$C * * * * *$ ASSIGNMENT OF SIGNED DP VARIABLES AND ARRAY

$C * * * *$ ELEMENTS TO DP VARIABLES AND ARRAY ELEMENTS

$P 0132530$

$P 0132540$

$C * * * *$ (UNARY MINUS USED TO REVERSE BOTH PLUS AND

$(* * \cdots \cdots$ MINUS VALUES)

$P 0132550$

OPIVD = -GCVD

DPJVD $=-$ DDDVD

DPKVD $=-A C 1 D(3)$

$D P L V D=-E P 10(10)$

DPMVD $=-B C 2 D(3,1)$

OPNVD $=-B C 2 D(2,4)$

DPOVD $=-C C 30(2,1,1)$

DPPVD $=-C C 3 D(2,1,2)$

EP1D(36) = -GCVD

EP1D(37) $=-$ DDOVD

$E P 1 D(38)=-A C 10(3)$

$E P 1 D(39)=-E P 1 D(10)$

$E P 10(40)=-B C 2 D(3,1)$

$E P 1 D(41)=-B C 20(2,4)$

$E P 1 D(42)=-C C 3 D(2,1,1)$

$E P 10(43)=-C C 30(2,1,2)$

$F C 2 D(3,4)=-G C V D$

$F(20(4,4)=-D O D V D$

$F C 2 D(5,4)=-A C 10(3)$

$F C 2 D(1.5)=-E P 1 D(10)$

$F C 2 D(2,5)=-B C 2 D(3,1)$

$F C 2 D(3,5)=-B C 2 D(2,4)$

$F C 2 D(4,5)=-C C 3 D(2,1,1)$

$F C 2 D(5,5)=-C C 3 D(2,1,2)$

$R C 30(3,3,2)=-G C V D$

$P 0132570$

$P 0132580$

$P 0132590$

P0132600

$P 0132610$

$P 0132620$

$P 0132630$

$P 0132640$

$P 0132650$

$P 0132660$

$P 0 \uparrow 32670$

$P 0132680$

$P 0132690$

$P 0132700$

$P 0132710$

$P 0132720$

P 0132730

$P 0132740$

P 0132750

P 0132760

$P 0132770$

P 0132780

P 0132790

$P 0132800$

P 0132810

$P 0132820$ 
$\operatorname{RC} 30(1,1,3)=-D D D V D$

$R C 3 D(2,1,3)=-A C 10(3)$

$P 0132830$

$\operatorname{RC} 30(3,1,3)=-E P 10(10)$

$P 0132840$

$R C 30(1,2,3)=-B C 2 D(3,1)$

PO 132850

$R C 30(2,2,3)=-B C 20(2,4)$

$R C 30(3,2,3)=-C C 3 D(2,1,1)$

$P 0132860$

P0132870

$R C 30(1,3,3)=-C C 30(2,1,2)$

$P 0132880$

P0132890

WRITE RESULTS FOR THIS SEGMENT

WRITE (NUVI,132) MCAVD, AC1D(1), BC2D(1,1), CC3D(1,1,1), MCBVD,

$A \quad A C 1 D(2), B C 2 D(2,1), C C 30(2,1,1), M C C V D, A C 1 D(3), B C 2 D(3,1)$,

B $\quad C C 3 D(3,1,1), M C D V D, A C 10(4), B C 2 D(4,1), C C 3 D(4,1,1), M C E V D$,

$C \quad A C 1 D(5), B C 2 D(5,1), C C 3 D(5,1,1), \operatorname{MCFVD,} A C 1 D(6), B C 20(6,1)$,

D CC3D(6,1,1), MCGVD, AC1D(7), BC2D(7,1), CC3D(7,1,1), MCHVD,

$E \quad A C 10(8), B C 2 D(1,2), C C 3 D(1,2,1), A C V D, A C 10(9), B C 2 D(2,2)$,

F $\quad C C 30(2,2,1), B C V D, A C 1 D(10), B C 20(3,2), C C 30(3,2,1), C C V D$,

G $\quad E P 10(1), B C 2 D(4,2), C C 3 D(4,2,1), \quad D C V D, E P 10(2), B C 2 D(5,2)$,

H CC3D(5,2,1), ECVD, EP1D(3), BC20(6,2), CC30(6,2,1), FCVD.

$1 \quad E P 1 D(4), B C 2 D(7,2), C C 30(7,2,1), G C V D, E P 10(5), B C 20(1,3)$,

$J \quad C C 30(1,1,2), H C V D, E P 10(6), B C 20(2,3), C C 30(2,1,2)$, AADVD,

$K \quad E P 1 D(7) \quad B C 2 D(3,3) \quad C C 3 D(3,1,2), B B D V D, E P 1 D(8) \quad B C 2 D(4,3)$,

$L \quad C C 30(4,1,2), C C O V D, \operatorname{EP} 10(9), B C 20(5,3), C C 30(5,1,2)$, DDOVD,

$M \quad E P 1 D(10), B C 2 D(6,3), C C 3 D(6,1,2)$

WRITE (NUVI, 133) EEDVD, EP1D(11), BC2D(7,3), CC3O(7,1,2), FFOVD,

$1 \quad E P 1 D(12), B C 2 D(1,4), C C 3 D(1,2,2), G G D V D, E P 1 D(13), B C 2 D(2,4)$,

$2 \quad C C 3 D(2,2,2), H H D V D, E P 10(14), B C 2 D(3,4), C C 30(3,2,2), P P D V D$,

3 $E P 10(15), \quad B C 2 D(4,4), C C 3 D(4,2,2), \quad R R D V D, E P 10(16), B C 2 D(5,4)$ CC3D $(5,2,2)$, SSDVD, EP1D(17), BC2D(6,4), CC3D(6,2,2), TTDVD, $E P 1 D(18), B C 2 D(7,4), \quad C C 30(7,2,2)$

WRITE (NUVI,134) UUDVD, EP1D(19), FC2D(1,1), RC30(1,1,1), VVDVD,

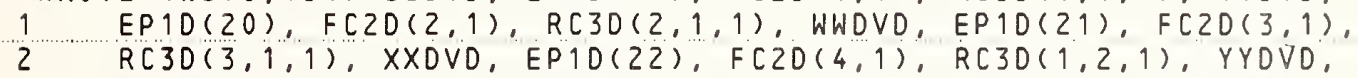
$E P 10(23), F C 2 D(5,1), R C 3 D(2,2,1), Z Z D V D, E P 1 D(24), F C 2 D(1,2)$, $R C 30(3,2,1), M C I V D, E P 10(25), F C 2 D(2,2), \operatorname{RC} 30(1,3,1), M C J V D$, $E P 10(26), F C 2 D(3,2), R C 30(2,3,1), M C K V D, E P 10(27), F C 20(4,2)$, $R C 30(3,3,1)$

WRITE (NUVI, 135) MCAVD, OPAVD, EP1D(28), $F C 20(5,2), \quad R C 3 D(1,1,2)$, A DCVD, DPBVD, EP1D(29), $F C 20(1,3), R C 3 D(2,1,2), E P 1 D(1)$,

B DPCVD, EP1D(30), FC2D(2,3), RC3O(3,1,2), EP1O(2), DPDVD, EP1O(31), FC2D(3,3), RC3O(1,2,2), BC2D(2,2), OPEVD, EP1D(32), PO 133210

$D \quad F C 2 D(4,3), R C 30(2,2,2), B C 2 D(4,2), \quad O P F V D, E P 1 D(33), F C 2 D(5,3), P($

E RC3O(3,2,2), CC3O(3,1,1), OPGVO, EP1D(34), FC2D(1,4),

$F \quad R C 3 D(1,3,2), C C 3 D(7,2,1), \quad D P H V D, E P 1 D(35), F C 2 D(2,4)$,

G RC30(2,3,2), GCVD, DPIVD, EP1D(36), FC2D(3,4), RC3D(3,3,2).

H DDDVD, DPJVD, EP1D(37), FC2D(4,4), RC3D(1,1,3), AC $10(3)$,

I DPKVD, EP1O(38), FC2D(5,4), RC3O(2,1,3), EP1D(10), DPLVD,

$J \quad E P 1 D(39), F C 2 D(1,5), R C 3 D(3,1,3), B C 2 D(3,1), \quad D P M V D, E P 10(40)$,

$K \quad F C 2 D(2,5), \quad R C 3 D(1,2,3), B C 2 D(2,4), \quad D P N V D, E P 1 D(41), F C 2 D(3,5)$

$L \quad R C 30(2,2,3), C C 30(2,1,1), \quad O P O V D, \operatorname{EP} 10(42), \operatorname{FC} 20(4,5)$,

$M \quad R C 3 D(3,2,3), C C 3 D(2,1,2), \quad D P P V D, \operatorname{EP} 10(43), F C 2 D(5,5)$,

$N \quad R C 3 D(1,3,3)$

132 FORMAT (/ $6 \times .8 H 0.340+02 / 4(014.21) /$

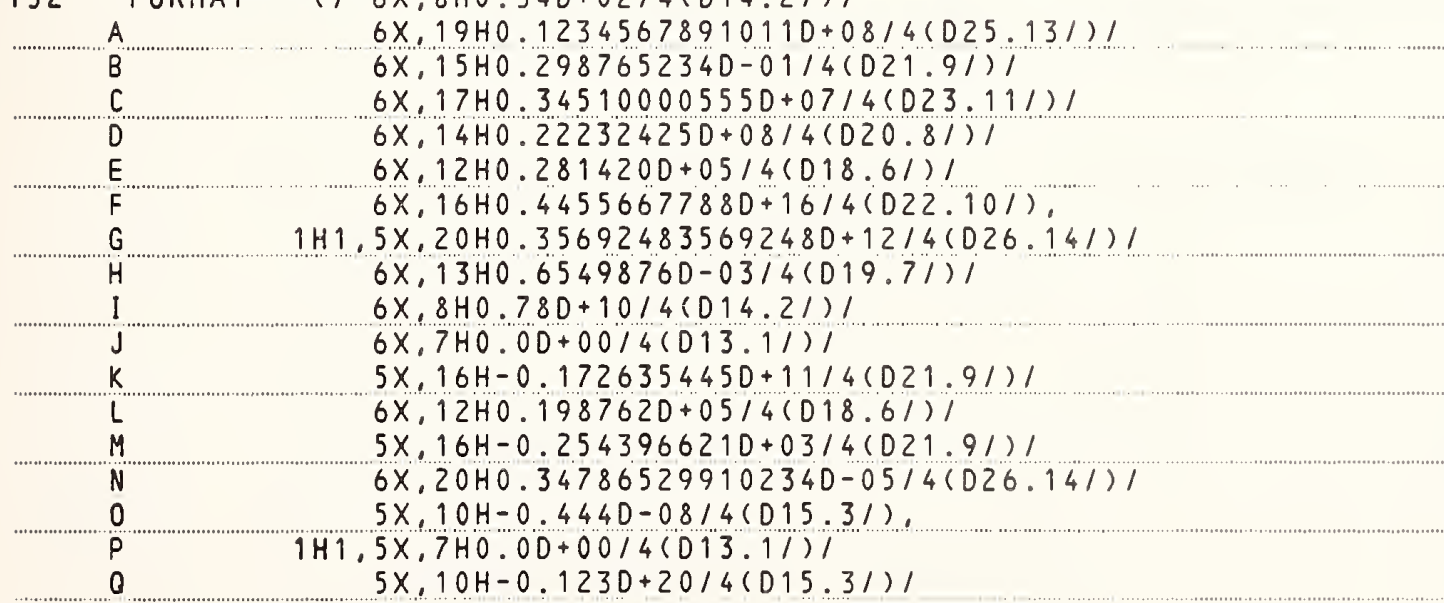

P0132900

$P 0132910$

$P 0132920$

$P 0132930$

$P 0132940$

$P 0132950$

P0132960

P0132970

P0132980

$P 0132990$

P0133000

P0133010

$P 0133020$

P0133030

$P 0133040$

P0133050

$P 0133060$

$P 0133070$

$P 0133080$

$P 0133090$

P0133100

P0133110

P0133120

PO133130

$P 0133140$

P0133150

P0133160

P0133170

P0133180

P0133190

P0133210

P0133220

P0133230

$P 0133240$

P0133250

P0133260

P 0133270

$P 0133280$

P0133290

P0133300

P 0133310

P 0133320

P0133330

$P 0133340$

$P 0133350$

P0133360

P0133370

$P 0133380$

P 0133390

P0133400

P0133410

$P 0133420$

PO133430

P0133440

P0133450

P0133460

P 0133470

P0133480

00133490

P0133500 


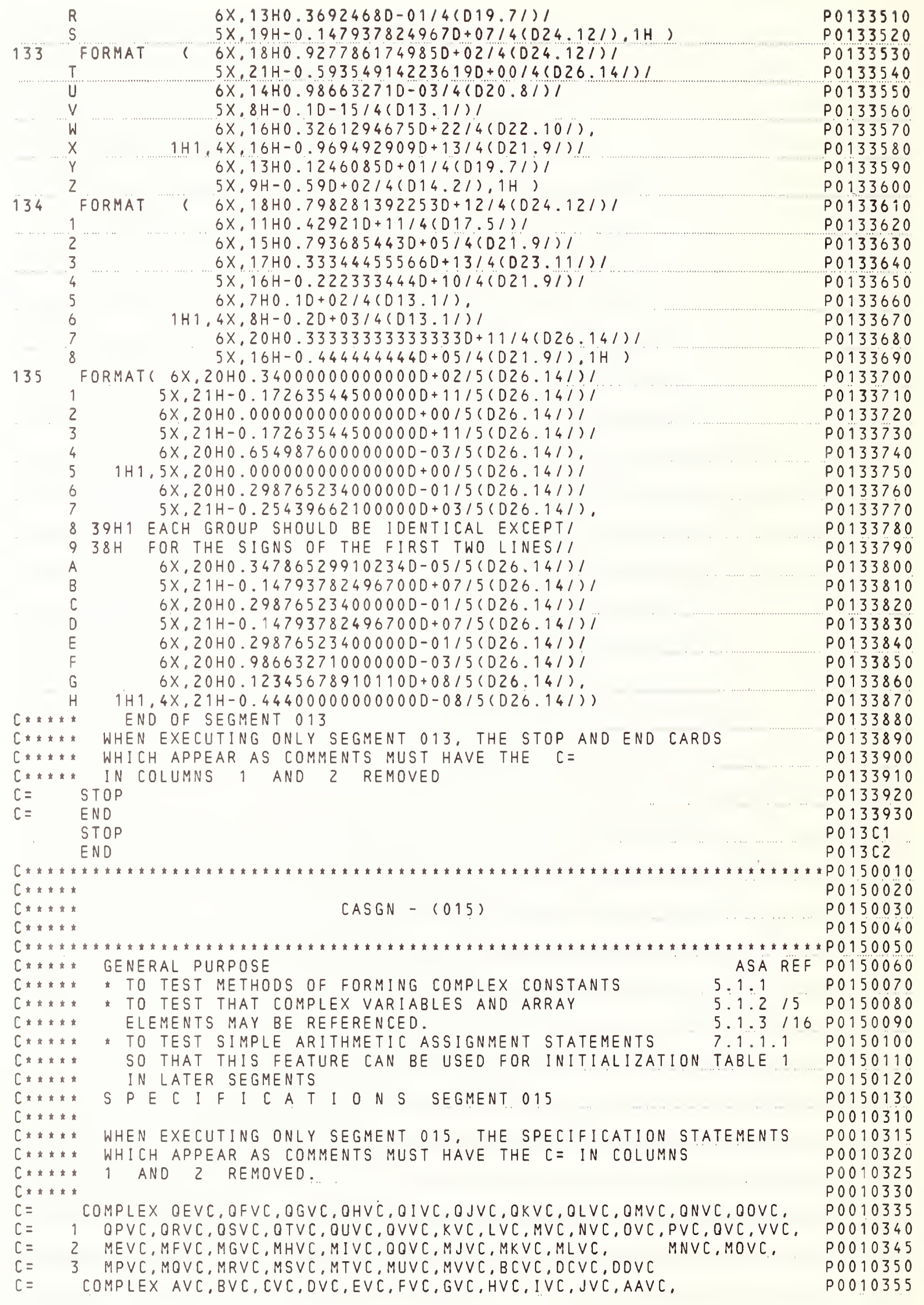


$C=1 \quad A B V C, B A V C, B B V C, C C V C, C D V C, C A V C, D A V C, A S V C, B S V C, C S V C$,

$C=2$

$D S V C, A A A V C, A B A V C, A C A \vee C, A D A V C, C H C V C$

$C=\quad \operatorname{COM}$

COMPLEX NUMVC.

OAVC, OBVC, OCVC, ODVC,RVC, SVC, TVC, UVC

$P 0010360$

$P 0010365$

P0010370

$C=\quad$ COMPLEX LL1C(32) LMZC(8.4) LN C $(9,2,2), A 1 C(12), A 2 C(2,2)$

$P 0010375$

\section{$[* * * * * \cdots$}

COMPLEX OEVC, OFVC,OGVC,OHVC, OIVC,OJVC, OKVC,OLVC, OMVC, ONVC,OOVC,

$1 \quad O P V C, O R V C, O S V C, O T V C, O U V C, O V V C, K V C, L V C, M V C, N V C, O V C, P V C, O V C, V V C$,

2 MEVC,MFVC,MGVC,MHVC, MIVC, OQVC, MJVC,MKVC,MLVC,

$M N \vee C, M O V C$.

$3 \quad M P V C, M O V C, M R V C, M S V C, M T V C, M U V C, M V V C, B C V C, D C V C, D D V C$

COMPLEX AVC, BVC, CVC, DVC, EVC, FVC, GVC, HVC, IVC, JVC, AAVC,

$1 \quad A B V C, B A V C, B B V C, C C V C, C D V C, C A V C, D A V C, A S \vee C, B S V C, C S V C$,

2 DSVC, AAAVC, ABAVC,ACAVC, ADAVC, CHCVC

COMPLEX NUMVC, OAVC,OBVC,OCVC,ODVC, RVC, SVC, TVC,UVC

1 MAVC,MBVC,MCVC,MDVC,B1C $(8), B 2 C(4,2), B 3 C(2,2,2)$

$P 0010385$

COMPLEX LL1C $(32), L M 2 C(8,4), L N 3 C(9,2,2), A 1 C(12), A 2 C(2,2), A 3 C(2,2,1)$

$C * * * * 0$ U T $P$ U T T A P E ASSIGNMENT STATEMENT. NO INPUT TAPE.

$C * * * * *$

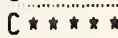

WHEN EXECUTING ONLY SEGMENT 015, THE FOLLOWING STATEMENT

$C * * * *$ NUVI $=6$ MUST HAVE THE $C=$ IN COLUMNS 1 AND 2 REMOVED.

$C * * * * *$

$C=\quad$ NUVI $=6$

$C * * * * *$

NUVI $=6$

WRITE (NUVI.150)

WRITE (NUVI, 151)

150 FORMAT(1H1,1X.32HCASGN-(015) COMPLEX ASSIGNMENT/

$116 X, 10 H S T A T E M E N T S / 2 X .28$ HASA REFS $-5.1 .1 .47 .1 .1 .1 / 1$

$22 X .7 H R E S U L T S / / 2 X, 23 H L I N E$ I OF EACH GROUP IS/

$32 X, 21 H H O L L E R I T H$ (NFORMATION/)

151 FORMAT ( $2 X, 36 H V A L U E S$ IN A GROUP SHOULD BE THE SAME)

$C * * * *$ HEADER FOR SEGMENT 015 WRITTEN

$C * * *$ BEGINNING OF TEST OF COMPLEX CONSTANT ASSIGNMENTS. IN

$C * * * *$ THE FOLLOWING 22 BLOCKS, BOTH PARTS OF THE CONSTANT

$C * * *$ HAVE THE SAME METHOD OF FORMATION

$C * * * *$ TEST ASSIGNMENT OF COMPLEX CONSTANTS FORMEO FROM

$C * * * *$ UNSIGNED BASIC REAL CONSTANTS

$O A V C=(22.2,33.33)$

$L L 1 C(1)=(22.2 .33 .33)$

$L M 2 C(1.1)=(22.2 .33 .33)$

$\operatorname{LN} 3 C(1.1 .1)=(22.2 .33 .33)$

$C * * * *$ TEST ASSIGNMENT OF COMPLEX CONSTANTS FORMED FROM

$C * * * *$ SIGNED BASIC REAL CONSTANTS

$O B \vee C=(+395.6,+4106.7)$

$O C V C=(-12345.67 .-1234.567)$

ODVC $=(+8.9,-9.1)$

QEVC $=(-2635.12,+46.21)$

$L L 1 C(2)=(+395.6,+4106.7)$

$L L 1 C(3)=(-12345.67,-1234.567)$

$L L 1 C(4)=(+8.9,-9.1)$

$L L 1 C(5)=(-2635.12 .+46.21)$

$\operatorname{LM} 2 C(2,1)=(+395.6,+4106.7)$

$\operatorname{LMCC}(3,1)=(-12345.67,-1234.567)$

$\operatorname{LM} 2 C(4,1)=(+8.9,-9.1)$

$\operatorname{LMZC}(5,1)=(-2635.12 .+46.21)$

$\operatorname{LN} 3 C(2,1,1)=(+395.6,+4106.7)$

$\operatorname{LN} 3 C(3,1,1)=(-12345.67,-1234.567)$

$\operatorname{LN} 3 C(4,1,1)=(+8.9,-9.1)$

$\operatorname{LN} 3 C(5,1.1)=(-2635.12 .+46.21)$

$C * * * *$ TEST ASSIGNMENT OF COMPLEX CONSTANTS FORMED FROM

C**** UNSIGNED AND SIGNED REAL CONSTANTS (INTEGER PART

$[* * * * \quad O N(Y)$

$O F \vee C=(10 \ldots 20$.

OGVC $=(+300 \ldots+4000 \ldots)$

OHVC $=(-50 . .-600$.

OIVC $=(+71 \ldots-92$.

OJVC $=(-883 \ldots+1414$.

OKVC $=(10 \ldots+562$.

P015A1

$P 015 A 2$

PO15A3

PO15A4

P015A5

P015A6

P015A7

P015A8

PO15A9

$P 015 A A$

$P 0150140$

$P 0070260$

$P 0070265$

$P 0070270$

P 0070275

$P 0070280$

P0070285

P015B1

P0150150

$P 0150160$

P0150170

$P 0150180$

P0150190

P0150200

P0 150210

$P 0150220$

P0150230

$P 0150240$

P 0150250

$P 0150260$

P0150270

$P 0150280$

P0150290

$P 0150300$

P0150310

P0150320

$P 0150330$

P0150340

P0150350

$P 0150360$

P0150370

P0150380

P0150390

$P 0150400$

P0150410

$P 0150420$

P0150430

$P 0150440$

P0150450

$P 0150460$

P0150470

$P 0150480$

$P 0150490$

$P 0150500$

P0150510

$P 0150520$

P0150530

$P 0150540$

P0150550

$P 0150560$

P0150570

P0150580 
QLVC $=(2002 \ldots-983$.

$P 0150590$

QMVC $=(+461 \ldots-165$.

$P 0150600$

QNVC $=(-21 \ldots+122$.

$P 0150610$

$L L 1 C(6)=(10 \ldots 20$.

$\operatorname{LMZC}(6,1)=(+300 \ldots+4000$.

$\operatorname{LN} 3 C(6,1,1)=(-50,-600$.

$P 0150620$

$L L 1 C(7)=(+71 \ldots-92$.

$L M Z C(7,1)=(-883 \ldots+1414$,

$P 0150630$

$P 0150640$

$P 0150650$

$\operatorname{LN} 3 C(7,1,1)=(10 \ldots+562$.

$L L 1 C(8)=(2002 \ldots-983$.

$P 0150660$

$P 0150670$

$\operatorname{LMZC}(8,1)=(+461 \ldots-165$.

$\operatorname{LN} 3 C(8,1,1)=(-21 \ldots+122$.

$P 0150680$

$P 0150690$

$P 0150700$

C*** TEST ASSIGNMENT OF COMPLEX CONSTANTS FORMED FROM

$P 0150710$

C**** UNSIGNED AND SIGNED REAL CONSTANTS (DECIMAL PART

$C * * * * \quad O N L Y$

QOVC $=(.001, .00200)$

QPVC $=(+.562,+.562)$

QQVC $=(-.3,-.3333333)$

QRVC $=(+.4,-.445)$

QSVC $=(-.95,+.95)$

QTVC $=(.0164239 .+.36)$

QUVC $=(.21,-.3963)$

QVVC $=(+.3398 . .3398)$

NUMVC $=(-.6, .6)$

$L L 1 C(9)=(.001, .00200)$

$L M Z C(1,2)=(+.562,+.562)$

$\operatorname{LN} 3 C(1,2,1)=(-.3,-.3333333)$

$L L 1 C(10)=(+.4,-.445)$

$\operatorname{LMZC}(2,2)=(-.95,+.95)$

$\operatorname{LN} 3 C(2,2,1)=(.0164239,+.36)$

$L L 1 C(11)=(.21,-.3963)$

$\operatorname{LMZC}(3,2)=(+.3398, .3398)$

$\operatorname{LN} 3 C(3,2,1)=(-.6, .6)$

C***** TEST ASSIGNMENT OF COMPLEX CONSTANTS FORMED FROM

$P 0150720$

$P 0150730$

$P 0150740$

$P 0150750$

$P 0150760$

$P 0150770$

$P 0150780$

$P 0150790$

P0150800

$P 0150810$

P0150820

P0150830

$P 0150840$

$P 0150850$

$P 0150860$

$P 0150870$

$P 0150880$

$P 0150890$

P0150900

$P 0150910$

$P 0150920$

$P 0150930$ $A V C=(0.0 E 0,1.0 E 0)$

$L L 1 C(12)=(456231.1 E 1,789.453 E 3)$

$\operatorname{LMZC}(4,2)=(44.9 E 4,2.5 E 3)$

$\operatorname{LN} 3(4,2,1)=(2222.3 E 3,333.2 E 2)$

C**** TEST ASSIGNMENT OF COMPLEX CONSTANTS FORMED FROM

$P 0150940$

$P 0150950$

$P 0150960$

$P 0150970$

$P 0150980$

$P 0150990$

P0151000

$B V C=(3 \cdot O E+0,3 \cdot O E+0)$

$C V C=(987654.3 E-1,876543.2 E-2)$

$D V C=(4.444 E+3,55.555 E-4)$

$E V C=(6.0 E-5,7.7 E+6)$

$L L 1 C(13)=(3.0 E+0,3.0 E+0)$

$\operatorname{LMZC}(5.2)=(987654.3 E-1.876543 .2 E-2)$

$\operatorname{LN} 3 C(5,2,1)=(4.444 E+3,55.555 E-4)$

$L L 1 C(14)=(6.0 E-5,7.7 E+6)$

C**** TEST ASSIGNMENT OF COMPLEX CONSTANTS FORMED FROM

$C * *$ SIGNED REAL CONSTANTS WITH UNSIGNED EXPONENTS

$F V C=(+14.2 E 1,+26.67 E 0)$

$G \vee C=(-36.923 E 4,-0.234 E 03)$

HVC $=(+2.1 E 2,-2.1 E 2)$

IVC $=(-595.9 E 00,+4.967 E 2)$

$\operatorname{LMZC}(6,2)=(+14.2 E 1,+26.67 E 0)$

$\operatorname{LN} 3 C(6,2,1)=(-36.923 E 4,-0.234 E 03)$

$L L 1 C(15) \quad=(+2.1 E 2,-2.1 E 2)$

$\operatorname{LMZC}(7,2)=(-595.9 E 00,+4.967 E 2)$

$C * * *$ TEST ASSIGNMENT OF COMPLEX CONSTANTS FORMED FROM

C*** SIGNED REAL CONSTANTS WITH SIGNED EXPONENTS

$J V C=(+1.0 E+0,+1.0 E+0)$

$K V C=(-2.0 E-0,-2.0 E-0)$

$L V C=(+49.2 E-1,-65.27 E+2)$

$M V C=(-737.1 E+3,+99.8 E-3)$

$N V C=(+4774.47 E+03,-9362.4 E-4)$

OVC $=(-846.2 E-5 .+13.33 E+1)$

$P 0151010$

$P 0151020$

$P 0151030$

$P 0151040$

$P 0151050$

$P 0151060$

$P 0151070$

P0151080

P0151090

P0151100

P0151110

$P 0151120$

P0151130

P0151140

$P 0151150$

P0151160

P0151170

P0151180

P0151190

P0151200

P0151210

P0151220

P0151230

$P 0151240$

P0151250

$\operatorname{LN} 3 C(7,2,1)=(+1.0 E+0,+1.0 E+0)$

P0151260 
$L L 1 C(16)=(-2.0 E-0,-2.0 E-0)$

$\operatorname{LM} 2 C(1,3)=(+49.2 E-1,-65.27 E+2)$

$P 0151270$

$\operatorname{LN} 3 C(1,1,2)=(-737.1 E+3,+99.8 E-3)$

P0151280

$L L 1 C(17)=(+4774.47 E+03,-9362.4 E-4)$

$L M Z C(2,3)=(-846.2 E-5,+13.33 E+1)$

$P 0151290$

P0151300

P0151310

$P 0151320$

$P 0151330$

$P 0151340$

$P 0151350$

$P 0151360$

$L L 1 C(18)=(77 . E 7,816248 . E 2)$

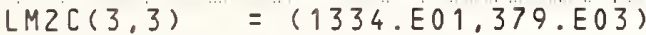

$\operatorname{LN} 3[(2,1,2)=(1334 . E 01,379 . E 03)$

$C * * * *$ TEST ASSIGNMENT OF COMPLEX CONSTANTS FORMED FROM

C***** UNSIGNED REAL CONSTANTS (NO DECIMAL PART) WITH

C***** SIGNED EXPONENTS

$Q V C=(3 . E+5,3 \cdot E+05)$

$R V C=(299 . E-4,299 . E-1)$

$S V C=(1419 \cdot E+2,1419 \cdot E-2)$

$T V C=(76 . E-3.987 . E+0)$

$L L 1 C(19)=(3 \cdot E+05,3 \cdot E+5)$

$\operatorname{LMZC}(4,3)=(299 . E-4,299 . E-1)$

$\operatorname{LN} 3 C(3,1,2)=(1419 . E+2,1419 . E-2)$

P 0151370

P0151380

$P 0151390$

P0151400

PO 151410

P0151420

$P 0151430$

$P 0151440$

$P 0151450$

$P 0151460$

$P 0151470$

P0151480

$L L 1 C(20)=(76 \cdot E-3,987 . E+0)$

$P 0151490$

$C * * * *$ TEST ASSIGNMENT OF COMPLEX CONSTANTS FORMED FROM

C*****) SIGNED REAL CONSTANTS (NO DECIMAL PART) WITH

C***** UNSIGNED EXPONENTS

$U V C=(+31 . E 0,+4659 . E 1)$

$V V C=(-728 . E 2,-93296 . E 3)$

$M A V C=(+6 . E 6,-6 . E 6)$

$M B V C=(-7914 \cdot E 3,+16 \cdot E 5)$

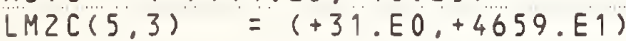

$\operatorname{LN} 3 C(4,1,2)=(-728 . E 2,-93296 . E 3)$

$L L 1 C(21)=(+6 . E 6,-6 . E 6)$

$\operatorname{LMZC}(6,3)=(-7914 . E 3,+16 . E 5)$

$C * * * *$ TEST ASSIGNMENT OF COMPLEX CONSTANTS FORMED FROM

$C * * * * *$ SIGNED REAL CONSTANTS (NO DECIMAL PART) WITH

$C * * * * *$ SIGNED EXPONENTS

$M C V C=(+1 \cdot E+1,+1 \cdot E+1)$

MDVC $=(-2 . E-2,-2 \cdot E-2)$

$M E V C=(+3 \cdot E-3,-3 \cdot E+3)$

$M F V C=(-4 \cdot E+4,+4 \cdot E-4)$

$M G V C=(+5 . E+5,-5 \cdot E-5)$

P0151500

PO 151510

P0151520

PO151530

$P 0151540$

P0151550

P0151560

P0151570

P0151580

P0151590

P0151600

PO151610

PO151620

P0151630

P0151640

$P 0151650$

$P 0151660$

P0151670

P0151680

MHVC $=(-6 \cdot E-6,+6 \cdot E+6)$

$P 0151690$

$\operatorname{LN} 3 C(5,1,2)=(+1 . E+1,+1 . E+1)$

$L L 1 C(22)=(-2 \cdot E-2,-2 \cdot E-2)$

P0151700

$\operatorname{LMZC}(7,3)=(+3 \cdot E-3,-3 \cdot E+3)$

$P 0151710$

P0151720

$\operatorname{LN} 3 C(6,1,2)=(-4 . E+4,+4 \cdot E-4)$

PO 151730

$L L 1 C(23)=(+5 \cdot E+5,-5 \cdot E-5)$

$P 0151740$

$\operatorname{LM} C(1,4)=(-6 . E-6,+6 . E+6)$

PO 151750

$C * * * *$ TEST ASSIGNMENT OF COMPLEX CONSTANTS FORMED FROM

$C * * * *$ UNSIGNED REAL CONSTANTS (NO INTEGER PART) WITH

C***** UNSIGNED EXPONENTS

$M I V C=(.39393 E 01, .62 E 04)$

$L L 1 C(24)=(.39393 E 01 . .62 E 04)$

$\operatorname{LM} 2 C(2,4)=(.009 E 2, .765765 E 3)$

$\operatorname{LN} 3 C(7,1,2)=(.009 E 2, .765765 E 3)$

$C * * * *$ TEST ASSIGNMENT OF COMPLEX CONSTANTS FORMED FROM

$C * * * *$ UNSIGNED REAL CONSTANTS (NO INTEGER PART) WITH

$C * * * *$ SIGNED EXPONENTS

$M J V C=(.352 E+09, .352 E+3)$

$M K V C=(.147626 E+0, .891 E-14)$

$M L V C=(.9 E-7, .9999 E+8)$

$M N V C=(.13 E-04.13 E-04)$

$L L 1 C(25)=(.352 E+09, .352 E+3)$

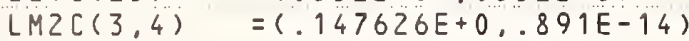

$L N 3 C(1.2,2)=(.9 E-7, .9999 E+8)$

P0151760

PO 151770

P0151780

P0151790

P0151800

P0151810

P0151820

P0151830

PO 151840

P0151850

P0151860

PO 151870

$P 0151880$

P0151890

$P 0151900$

P 0151910

P0151920

$L N 3 C(2,2,2)=(.13 E-4.13 E-4)$

P0151930

$C * * *$ TEST ASSIGNMENT OF COMPLEX CONSTANTS FORMED FROM

P0151940 


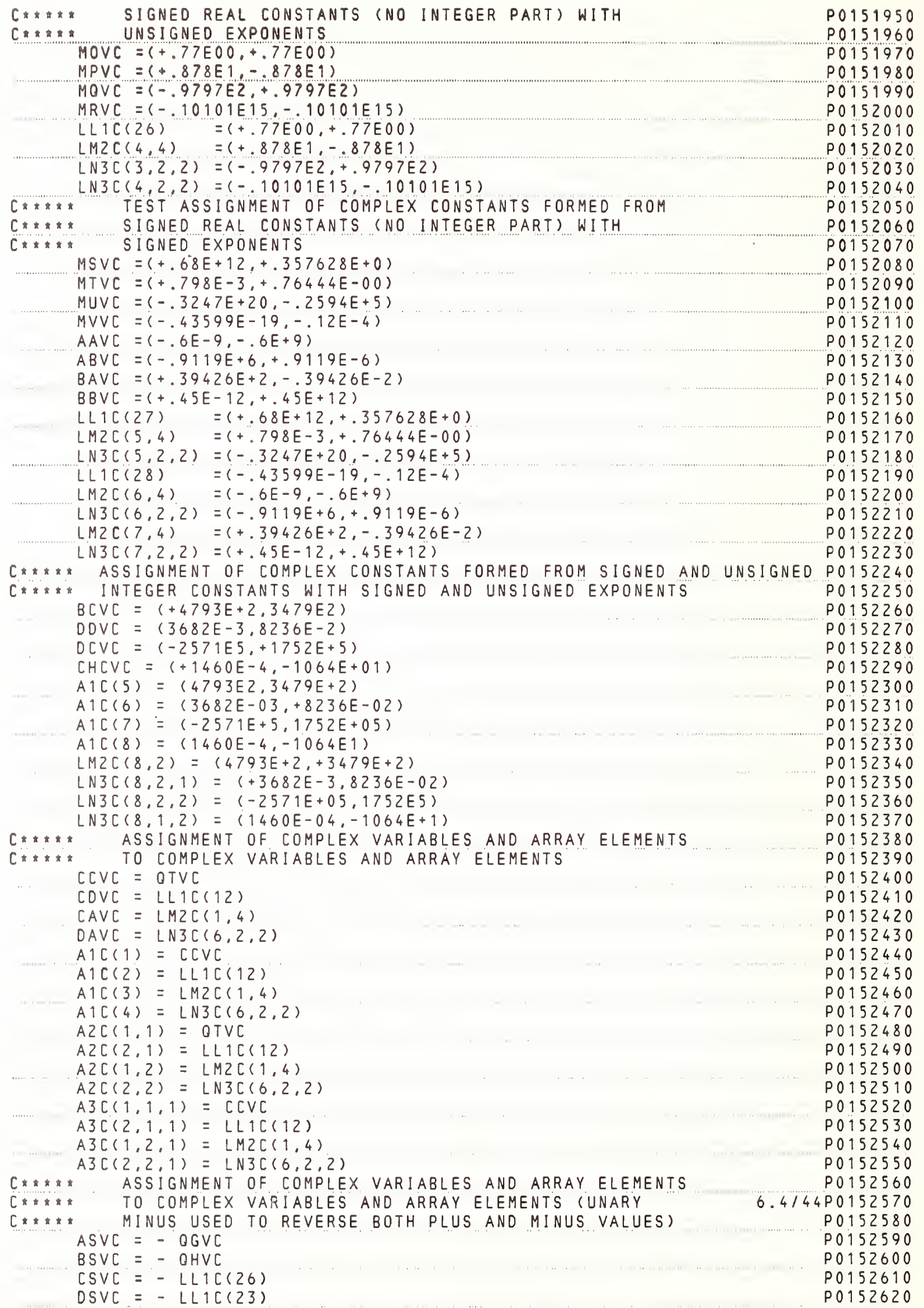


AAAVC $=-L M 2 C(1,3)$

$A A A V C=-\operatorname{LM} C C(1,3)$

$A B A V C=-L M 2 C(1,4)$

P0152650

$A C A V C=-\operatorname{LN} 3 C(5,2,1)$

P0152660

$A D A V C=-\operatorname{LN} 3 C(6,2,1)$

Po152670

$B 1 C(1)=-Q G V C$

$B 1 C(2)=-O H \vee C$

$B 1 C(3)=-L L 1 C(26)$

$B 1 C(4)=-L L 1 C(23)$

$B 1 C(5)=-\operatorname{LMZC}(1,3)$

$B 1 C(6)=-\operatorname{LM} C(1,4)$

$B 1 C(7)=-\operatorname{LN} 3 C(5,2,1)$

$B 1 C(8)=-\operatorname{LN} 3 C(6,2,1)$

$B 2 C(1,1)=-O G V C$

$B Z C(2,1)=-O H V C$

$B 2 C(3,1)=-L L 1 C(26)$

$B 2 C(4.1)=-\operatorname{LL} 1 C(23)$

$B 2 C(1,2)=-\operatorname{LM} C(1,3)$

$B Z C(2,2)=-\operatorname{LMZC}(1,4)$

$B 2 C(3,2)=-\operatorname{LN} 3 C(5,2,1)$

$B 2 C(4,2)=-\operatorname{LN} 3 C(6,2,1)$

$B 3 C(1,1,1)=-O G V C$

$B 3 C(2,1,1)=-O H V C$

$B 3 C(1,2,1)=-\operatorname{LL} 1 C(26)$

$B 3 C(2,2,1)=-L L 1 C(23)$

$B 3 C(1,1,2)=-\operatorname{LM} 2 C(1,3)$

$B 3 C(2,1,2)=-\operatorname{LM} C(1,4)$

$B 3 C(1,2,2)=-\operatorname{LN} 3 C(5,2,1)$

$B 3 C(2,2,2)=-\operatorname{LN} 3 C(6,2,1)$

$C * * * *$ WRITE RESULTS FOR THIS TEST SEGMENT

WRITE (NUVI, 152) OAVC, LL1C(1), LMZC (1,1), LN3C $(1,1,1), \quad O B V C$,

$1 \quad \operatorname{LL} 1 C(2), \operatorname{LMZC}(2,1), \operatorname{LN} 3 C(2,1,1), \operatorname{OCV} C, \operatorname{LL} 1 C(3), \operatorname{LM} Z C(3,1)$,

$2 \quad \operatorname{LN} 3 C(3,1,1)$, ODVC, LLIC (4), LMZC $(4,1), \operatorname{LN} 3 C(4,1,1)$, OEVC,

$3 \quad L L 1 C(5), \operatorname{LMZC}(5,1), \operatorname{LN} 3 C(5,1,1), O F V C, L L 1 C(6), 0 G V C$,

$4 \quad L M Z C(6,1), O H V C, \operatorname{LN} 3 C(6,1,1), O I V C, L L 1 C(7), 0 J V C, L M Z C(7,1)$,

$5 \quad$ OKVC, $L N 3 C(7,1,1), \quad O L V C, L L 1 C(8), \quad O M V C, L M Z C(8,1), \quad O N V C$,

$6 \quad \operatorname{LN} 3 C(8,1,1), \quad 0 O V C, \quad L L 1 C(9), \quad O P V C, L M Z C(1,2), \quad 0 O V C$,

$7 \quad L N 3 C(1,2,1), \quad O R V C, L L 1 C(10), \quad Q S V C, L M 2 C(2,2), O T V C$, LN $3 C(2,2,1)$

WRITE (NUVI, 153) OUVC, LL1C(11), OVVC, LMZC $(3,2)$, NUMVC,

P 0152680

P0152690

P0152700

P 0152710

P0152720

P0152730

P0152740

P0152750

P0152760

P0152770

P0152780

P0152790

P0152800

P0152810

P0152820

P0152830

P0152840

P0152850

P0152860

P 0152870

P0 0152880

P0152890

P0152900

P0152910

P0152920

P0152930

P0152940

P0152950

P0152960

PO152970

P0152980

P0152990

P0153000

P0153010

P0153020

$1 \quad L N 3 C(3,2,1), A V C, L L 1 C(12), L M Z C(4,2), L N 3 C(4,2,1), B V C$.

$2 \quad L L 1 C(13), C V C, L M 2 C(5,2), D V C, L N 3 C(5,2,1), E V C, L L 1 C(14)$,

$3 \quad F V C, \operatorname{LM} C C(6,2), G \vee C, \operatorname{LN} 3 C(6,2,1), H V C, L L 1 C(15)$, IVC,

$P 0153030$

P0153040

PO 153050

P0153060

$P 0153070$

5 MVC, LN3C $(1,1,2)$, NVC, LL $1 C(17)$

WRITE(NUV1,8873) OVC, $L M Z C(2,3), P V C$,

$1 \quad$ LL $1 C(18), \operatorname{LMZC}(3,3), \operatorname{LN} 3 C(2,1,2)$, OVC, LLIC (19)

WRITE (NUVI, 154) RVC, $\operatorname{LMZC}(4,3), S V C, \operatorname{LN} 3 C(3,1,2), T V C$,

1 LLIC(20), UVC, LMZC (5,3), VVC, LN3C(4,1,2), MAVC, LLIC (21),

$2 \quad M B V C, \operatorname{LMZC}(6,3), M C V C, L N 3 C(5,1,2), M D V C, L L 1 C(22), M E V C$,

$3 \quad \operatorname{LMZC}(7,3), M F V C, \operatorname{LN} 3 C(6,1,2), M G V C, L L 1 C(23), M H V C$,

$4 \quad \operatorname{LMZC}(1,4), M I V C, L L 1 C(24), \operatorname{LMZC}(2,4), \operatorname{LN} 3 C(7,1,2)$

WRITE (NUVI, 8870) MJVC, LL $1 C(25)$, MKVC, LMZC $(3,4)$, MLVC,

- $\quad \operatorname{LN} 3 C(1,2,2)$, MNVC, $\operatorname{LN} 3 C(2,2,2)$, MOVC, LL1C(26).

$+\quad$ MPVC, LMZC $(4,4)$, MOVC, LN3C(3,2,2), MRVC,

LN3C $(4,2,2), M S V C, \operatorname{LL} C(27), M T V C, \operatorname{LMZC}(5,4)$, MUVC, LN3C $(5,2,2)$, MVVC, LLIC $(28), A A V C$.

$L M Z C(6,4), A B \vee C, L N 3 C(6,2,2), B A V C, L M Z C(7,4)$, $B B \vee C, L N 3 C(7,2,2)$

WRITE(NUVI, 8872) BCVC,A1C(5), LMZC ( 8, 2), DDVC, $A 1 C(6), \operatorname{LN} 3 C(8,2,1)$,

P0153080

P0153090

P0153100

P0153110

P0153120

P0153130

P0153140

P0153150

P0153160

P0153170

P0153180

P0153190

P0 153200

P0153210

P0153220

P0153230

$1 \quad D C V C, A 1 C(7), L N 3 C(8,2,2), C H C V C, A 1 C(8), L N 3 C(8,1,2)$

OWRITE (NUVI, 8871) OTVC, $C C V C, A 1 C(1), A Z C(1,1), A 3 C(1,1,1)$,

$P 0153240$

P0153250

LL1C(12), CDVC, $A 1 C(2), A 2 C(2,1), A 3 C(2,1,1), \operatorname{LM} C C(1,4)$,

$P 0153260$

$2 \quad C A V C, A 1 C(3), A 2 C(1,2), A 3 C(1,2,1), \quad L N 3 C(6,2,2), D A V C$,

$P 0153270$

$A 1 C(4), A 2 C(2,2), A 3 C(2,2,1), \quad 0 G V C, A S V C, B 1 C(1), B 2 C(1,1)$,

P0153280

LL1C $(26), \quad C S \vee C, B 1 C(3), B 2 C(3,1), B 3 C(1,2,1), L L 1 C(23)$,

P0153290

$6 \quad D S V C, B 1 C(4), B Z C(4,1), B 3 C(2,2,1), \operatorname{LMZC}(1,3), A A A V C, B 1 C(5), P 0153300$ 
$7 \quad B 2 C(1,2), B 3 C(1,1,2), L M Z C(1,4), A B A V C, B 1 C(6), B 2 C(2,2)$,

$8 \quad B 3 C(2,1,2), \quad L N 3 C(5,2,1), \quad A C A V C, B 1 C(7), B 2 C(3,2), B 3 C(1,2,2)$,

$9 \quad L N 3 C(6,2,1), A D A V C, B 1 C(8), B 2 C(4,2), B 3 C(2,2,2)$

C** FORMAT STATEMENTS FOR THIS SEGMENT

152 FORMAT ( 6 X.9HO.222E+02.9X, $10 H 0.3333 E+02 / 4(E 15.3, E 19.4 /) /$

A $\quad 6 \times, 10 H 0.3956 E+03,8 X, 11 H 0.41067 E+04 / 4(E 16.4 . E 19.51) 1$

B $\quad 5 X, 14 H-0.1234567 E+05.4 X, 14 H-0.1234567 E+04 / 4(E 19.7, E 18.7 /) /$

C $\quad 6 X, 8 H 0.89 E+01,9 X, 9 H-0.91 E+01 / 4(E 14.2, E 18.21) 1$

D $\quad 5 X, 13 H-0.263512 E+04.6 X, 10 H 0.4621 E+02 / 4(E 18.6, E 16.4 /) /$

E $\quad 6 X, 7 H 0.1 E+02,11 X, 7 H 0.2 E+02 / 2(E 13.1, E 18.1 /) /$

F $\quad 6 X, 7 H 0.3 E+03,11 X, 7 H O .4 E+04 / 2(E 13.1, E 18.1 /) /$

G $\quad 5 X, 8 H-0.5 E+02,10 X, 8 H-0.6 E+03 / 2(E 13.1, E 18.1 /) 1$

$H \quad 6 X, 8 H O .71 E+02,9 X, 9 H-0.92 E+02 / 2(E 14.2, E 18.2 /) /$

$11 H 1,4 X, 10 H-0.883 E+03,9 X, 10 H 0.1414 E+04 / 2(E 15.3, E 19.4 /) /$

J $6 X, 7 H O .1 E+02,11 X, 9 H 0.562 E+03 / 2(E 13.1, E 20.3 /) /$

X $\quad 6 X, 10 H 0.2002 E+04,7 X, 10 H-0.983 E+03 / 2(E 16.4, E 17.3 /) 1$

L $\quad 6 X, 9 H 0.461 E+03,8 X, 10 H-0.165 E+03 / 2(E 15.3, E 18.3 /) /$

$M \quad 5 X, 9 H-0.21 E+02,10 X, 9 H 0.122 E+03 / 2(E 14.2, E 19.3 /)$ !

N $6 X, 7 H 0.1 E-02,11 X, 7 H 0.2 E-02 / 2(E 13,1, E 18.1 /) /$

$0 \quad 6 X, 9 H 0.562 E+00,9 X, 9 H 0.562 E+00 / 2(E 15.3, E 18.3 /) /$

P $\quad 5 X, 8 H-0.3 E+00,10 X, 14 H-0.3333333 E+00 / 2(E 13.1, E 24.7 /) /$

$06 X, 7 H 0.4 E+00,10 X, 10 H-0.445 E+00 / 2(E 13.1, E 20.3 /) /$

R $5 X, 9 H-0.95 E+00,10 X, 8 H 0.95 E+00 / 2(E 14.2, E 18.2 /) /$

S $\quad 6 X, 12 H 0.164239 E-01,6 X, 8 H 0.36 E+00 / 2(E 18.6, E 14.21), 1 H)$

153 FORMAT ( $6 X .8 H 0.21 E+00.9 X, 11 H-0.3963 E+00 / 2(E 14.2 . E 20.4 /) /$

A $\quad 6 X, 10 H 0.3398 E+00,8 X, 10 H 0.3398 E+00 / 2(E 16.4, E 18.4 /) /$

B $5 X, 8 H-0.6 E+00,11 X, 7 H 0.6 E+00 / 2(E 13.1, E 18.1 /) /$

C $1 \mathrm{H} 1,5 \mathrm{X}, 7 \mathrm{HO} .0 \mathrm{E}+00,11 \mathrm{X}, 7 \mathrm{HO} .1 \mathrm{E}+01 / \mathrm{E} 13.1, \mathrm{E} 18.1 / 1$

D $6 X, 13 H 0.4562311 E+07,5 X, 12 H 0.789453 E+06 / E 19.7, E 17.6 / 1$

$6 \mathrm{X}, 9 \mathrm{HO} .449 \mathrm{E}+06,9 \mathrm{X}, 8 \mathrm{HO} .25 \mathrm{E}+04 / \mathrm{E} 15.3, \mathrm{E} 17.2 / 1$

$6 X, 11 H 0.22223 E+07,7 X, 10 H 0.3332 E+05 / E 17.5, E 17.41 /$

$6 X, 7 H 0.3 E+01,11 X, 7 H 0.3 E+01 / 2(E 13.1, E 18.1 /) /$

$6 X, 13 H 0.9876543 E+05,5 X, 13 H 0.8765432 E+04 / 2(E 19.7 . E 18.7 /) /$

$6 X, 10 H 0.4444 E+04,8 X, 11 H 0.55555 E-02 / 2(E 16.4, E 19.5 /) /$

$6 X, 7 H 0.6 E-04,11 X, 8 H 0.77 E+07 / 2(E 13.1, E 19.21) 1$

$6 X, 9 H 0.142 E+03,9 X, 10 H 0.2667 E+02 / 2(E 15.3 . E 19.41) !$

$5 X .12 H-0.36923 E+06.6 X .10 H-0.234 E+03 / 2(E 17.5 . E 16.3 /) /$

$6 X, 8 H 0.21 E+03,9 X, 9 H-0.21 E+03 / 2(E 14.2, E 18.2 /) /$

$5 X, 11 H-0.5959 E+03,8 X, 10 H 0.4967 E+03 / 2(E 16.4, E 18.4 /) /$

$6 X, 7 H 0.1 E+01,11 X, 7 H 0.1 E+01 / 2(E 13.1, E 18.1 /) /$

$5 X, 8 H-0.2 E+01,10 X, 8 H-0.2 E+01 / 2(E 13.1, E 18.1 /) /$

$6 X, 9 H 0.492 E+01,8 X, 11 H-0.6527 E+04 / 2(E 15.3, E 19.4 /)$,

$\mathrm{R} 1 \mathrm{H} 1,4 \mathrm{X}, 11 \mathrm{H}-0.7371 \mathrm{E}+06,8 \mathrm{X}, 9 \mathrm{HO} .998 \mathrm{E}-01 / 2(\mathrm{E} 16.4, \mathrm{E} 17.3 /) /$

S $\quad 6 X, 12 H 0.477447 E+07,5 X, 12 H-0.93624 E+00 / 2(E 18.6, E 17.5 /), 1 H)$

8873 FORMAT $(5 X, 13 H-0.846200 E-02,6 X, 11 H 0.13330 E+03 / 2(E 18.6, E 17.5 /) /$

U $\quad 6 X, 12 H 0.770000 E+09,6 X, 11 H 0.81625 E+08 / 2(E 18.6, E 17.5 /) !$

$V \quad 6 X, 12 H 0.133400 E+05.6 X, 11 H 0.37900 E+06 / 2(E 18.6, E 17.5 /) /$

H $\quad 6 X, 12 H 0.300000 E+06,6 X, 11 H 0.30000 E+06 / 2(E 18.6, E 17.5 /), 1 H)$

154 FORMAT ( $6 X, 9 H 0.299 E-01,9 X, 9 H 0.299 E+02 / 2(E 15.3, E 18.3 /) /$

A $\quad 6 X, 10 H 0.1419 E+06,8 X, 10 H 0.1419 E+02 / 2(E 16,4, E 18.4 /) /$

B $\quad 6 X, 8 H 0.76 E-01,10 X, 9 H 0.987 E+03 / 2(E 14.2, E 19.3 /) /$

C $\quad 6 X, 8 H 0.31 E+02,10 X, 10 H 0.4659 E+05 / 2(E 14.2, E 20.41) 1$

D $\quad 5 X, 10 H-0.728 E+05,8 X, 12 H-0.93296 E+08 / 2(E 15.3, E 20.5 /) /$

E $\quad 6 \times, 7 H 0.6 E+07,10 X, 8 H-0.6 E+07 / 2(E 13.1, E 18.1 /) 1$

$F \quad 5 X, 11 H-0.7914 E+07,8 X, 8 H 0.16 E+07 / 2(E 16,4, E 16.21) /$

G $6 X, 7 H 0.1 E+02,11 X, 7 H 0.1 E+02 / 2(E 13.1, E 18.1 /)$,

H1H1, 4X,8H-0.2E-01,10X,8H-0.2E-01/2(E13.1,E18.1/)/

$1 \quad 6 X, 7 H 0.3 E-02,10 X, 8 H-0.3 E+04 / 2(E 13,1, E 18.1 /) /$

J $5 X, 8 H-0.4 E+05,11 X, 7 H 0.4 E-03 / 2(E 13.1, E 18.1 /) /$

K $\quad 6 X, 7 H 0.5 E+06,10 X, 8 H-0.5 E-04 / 2(E 13.1, E 18.1 /) /$

L $\quad 5 X .8 H-0.6 E-05.11 X, 7 H 0.6 E+07 / 2(E 13.1, E 18.1 /) /$

M $\quad 6 X, 11 H 0.39393 E+01,7 X, 8 H 0.62 E+04 / 2(E 17.5, E 15.21) /$

N $\quad 6 X, 7 H 0.9 E+00,11 X, 12 H 0.765765 E+03 / 2(E 13.1, E 23.6 /), 1 H)$

8870 FORMAT ( $6 X, 9 H 0.352 E+09,9 X, 8 H 0.35 E+03 / 2(E 15.3, E 17.21) /$ $6 X, 12 H 0.147626 E+00,6 X, 9 H 0.891 E-14 / 2(E 18.6, E 15.3 /) 1$ $6 X, 7 H 0.9 E-07.11 X, 10 H 0.9999 E+08 / 2(E 13.1 ; E 21.41) 1$ $6 \mathrm{X}, 8 \mathrm{HO} 0.13 \mathrm{E}-04,10 \mathrm{X}, 8 \mathrm{HO} 0.13 \mathrm{E}-04 / 2(\mathrm{E} 14.2, \mathrm{E} 18.21) /$ $6 X, 8 H 0.77 E+00,10 X, 8 H 0.77 E+00 / 2(E 14.2, E 18.21) 1$

P0153490

P0153500

P0153510

$P 0153520$

P0153530

P0153540

P0153550

P0153560

P0153570

$P 0153580$

P0153590

P0153600

P0153610

P0153620

$P 0153630$

PO 153640

$P 0153650$

P0153660

P0153670

P0153680

$P 0153690$

P0153700

PO 153710

P0153720

PO 153730

P0153740

P0153750

P0153760

P0153770

P0153780

P0153790

P0153800

P0153810

P0153820

PO 153830

P0153840

P 0153850

P0153860

P0153870

P0153880

PO 153890

P0153900

P0153910

P0153920

P 0153930

P0153940

P0153950

PO 153960

P0153970

P0153980 


$\begin{array}{ll}\text { A } & \\ \text { A } & 1 \mathrm{H1} \\ & \\ - & \\ + & \\ 1 & \\ 2 & \\ 3 & \\ 4 & \\ 5 & \end{array}$

8872 FORMAT 7 8 9

8871 FORMAT $(1 H 1,5 X, 13 H 0.1642390 E-01,5 X, 13 H 0.3600000 E+00 / 5(E 19.7 . E 18.7 /)$ $1 \quad / 6 X, 13 H 0.4562311 E+07.5 X, 13 H 0.7894530 E+06 / 5(E 19.7, E 18.7 /) /$ $2 \quad 5 X, 14 H-0.6000000 E-05,5 X, 13 H 0.6000000 E+07 / 5(E 19.7, E 18.7 /) /$ $39 \mathrm{H1}$ $5 X, 14 H-0.9119000 E+06,5 X, 13 H 0.9119000 E-06 / 5(E 19.7, E 18.7 /)$

EACH GROUP SHOULD BE IDENTICAL EXCEPT/ 7 8

9

A $\ldots \ldots \ldots \ldots \ldots \ldots . . . . . . . . .16$

B

C

0

$[* * * * *$

$C * * * * *$

$[* * * * *$

$[* * * * *$

$C=$

$C=$

$6 X, 13 H 0.3000000 E+03,5 X, 13 H 0.4000000 E+04 / 5(E 19.7 . E 18.7 /) /$

$5 X, 14 H-0.5000000 E+02,4 X, 14 H-0.6000000 E+03 / 5(E 19.7, E 18.7 /) /$ $6 X, 13 H 0.7700000 E+00,5 X, 13 H 0.7700000 E+00 / 5(E 19.7, E 18.7 /) /$ $6 X, 13 H 0.5000000 E+06,4 X, 14 H-0.5000000 E-04 / 5(E 19.7, E 18.7 /) /$ $6 X, 13 H 0.4920000 E+01,4 X, 14 H-0.6527000 E+04 / 5(E 19.7, E 18.7 /) /$ $5 X, 14 H-0.6000000 E-05,5 X, 13 H 0.6000000 E+07 / 5(E 19.7, E 18.7 /) /$ $6 \mathrm{X}, 13 \mathrm{HO} .4444000 \mathrm{E}+04,5 \mathrm{X}, 13 \mathrm{HO} 0.5555500 \mathrm{E}-02 / 5(\mathrm{E} 19.7, \mathrm{E} 18.7 /) 1$

$1 H 1,4 X, 14 H-0.3692300 E+06,4 X, 14 H-0.2340000 E+03 / 5(E 19.7, E 18.7 /))$ ENO OF TEST SEGMENT O 15

WHEN EXECUTING ONLY SEGMENT 015, THE STOP AND END CARDS

WHICH APPEAR AS COMMENTS MUST HAVE THE C = IN COLUMNS

\section{AND 2 REMOVED.}

STOP

END

STOP

END

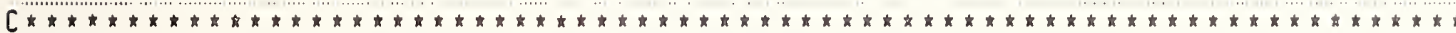

$C * * * *$

$[* * * * *$

$C \star \star \star \star \star * t$

$[* * * * * *$

$[* * * * *$

$[* * * * *$

$[* * * * *$

$C * * * * *$

$C * * * * *$

$C * * * * *$

$[* * * * *$

$C * * * * *$

$[* * * * *$

$C=$ DIMENSION IACII(5)

$C=\quad$ LOGICAL MCAVB, MCBVB, MCCVB, MCOVB, MCEVB, MCFVB, MCGVB, MCHVB, MCIVB,

$C=1 \quad M C J \vee B, M C K \vee B, M C L \vee B, M C M \vee B, M C N \vee B, M C A 1 B(7)$

$C=\quad \operatorname{LOGICALA} A B(2), A 2 B(2,2), A 3 B(2,2,2), A \vee B, B \vee B, C \vee B$

$C * * * * *$

DIMENSION IAC 1 I ( 5$)$

LOGICAL MCAVB, MCBVB, MCCVB, MCOVB, MCEVB, MCFVB, MCGVB, MCHVB, MCIVB, $M C J \vee B, M C K \vee B, M C L \vee B, M C M \vee B, M C N \vee B, M C A 1 B(7)$

LOGICAL $A 1 B(2), A 2 B(2,2), A 3 B(2,2,2), A \vee B, B \vee B, C V B$

$[* * * * \quad 0$ U T P U T T A P E ASSIGNMENT STATEMENT. NO INPUT TAPE.

$C * * * *$ WHEN EXECUTING ONLY SEGMENT 016 , THE FOLLOWING STATEMENT

$C * * * *$ NUVI $=6$ MUST HAVE THE $C=$ IN COLUMNS 1 AND 2 REMOVEO.

$[* * * * *$

$C=$

NUVI $=6$

$[* * * * *$
P0 153990

$P 0154000$

P0154010

$P 0154020$

P0 154030

P0154040

P0154050

$P 0154060$

P0154070

$P 0154080$

P0154090

P0154100

P0154110

P0154120

P0154130

P0154140

P0154150

P0154160

$P 0154170$

P0154180

P0154190

$P 0154200$

P0154210

$P 0154220$

P0154230

$P 0154240$

P0154250

P0 154260

P0154270

$P 0154280$

P0 154290

$P 0154300$

P0154310

P0 154320

P 0154330

$P 0154340$

P015C1

$P 015 C 2$

P0160010

$P 0160020$

P0 160030

$P 0160040$ ASA REFP 0160060 7.1.1.2P0160070

$P 0160080$

$P 0160090$

$P 0160100$

P 0010390

$P 0010395$

$P 0010400$

P0010405

P0010410

P 0010415

$P 0010420$

$P 0010425$

$P 0010430$

P016A1

P016A2

$P 016 A 3$

P016A4

$P 0160110$

$P 0070290$

P0070295

$P 0070300$

$P 0070305$

P016B1

$P 0160120$ 
$I A C 1 I(1)=25$

$P 0160130$

$I A C 1 \llbracket(2)=10$

$P 0160140$

$I A C 1 \square(3)=15$

P0160150

$I A C I I(4)=25$

P0160160

WRITE HEADER FOR THIS SEGMENT

Po 160170

WRITE (NUVI, 160)

P0160180

160 FORMAT (1H1.28H LASGN - (016) ASSIGNMENT OF/ 16X,17HLOGIGAL VARIABPO 160190

ALES/21H ASA REFS. - 7.1.1.2//9H RESULTS)

C**** TEST THE ASSIGNMENT OF RELATIONAL EXPRESSIONS $\quad 6.2 \quad$ P0160210

C***** TO LOGICAL VARIABLES ANO ARRAYS

$M C A V B=I A C 1 I(2) \ldots L T, I A C 1 I(3)$

$M C B V B=I A C 1 I(3) \cdot L T \cdot I A C I I(2)$

$M C C V B=I A C 1 I(1)=E O . \quad I A C I I(4)$

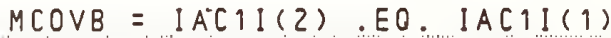

$M C E V B=$ IACII(1) $\cdot$ LE. IACII(4)

$M C F V B=I A C 1 I(2) \cdot L E \cdot I A C 1 I(1)$

$M C G V B=I A C 1 I(1) \cdot L E \cdot I A C 1 !(2)$

$M C H V B=I A C 1 I(1) \cdot E O \cdot 25$

$M C I V B=I A C 1 I(2), E O, \quad I A C 1 I(4)$

$P 0160220$

P0160230

P 0160240

P0160250

$P 0160260$

$P 0160270$

$P 0160280$

$P 0160290$

$P 0160300$

$M C A 1 B(1)=I A C 1 I(2) \cdot N E \cdot I A C 1 I(3)$

$P 0160310$

$M C A 1 B(2)=\lfloor A C 1 \llbracket(1) \cdot N E \cdot \square A C 1 \llbracket(4)$

$P 0160320$

$M C A 1 B(3)=I A C 11(1) \cdot G T \cdot I A C 11(2)$

P0160330

$M C A 1 B(4)=\square A C 1 I(2), G T \cdot \square A C 1 !(1)$

P0160340

$M C A 1 B(5)=\square A C 1 !(1) \cdot G E \cdot \square A C 1 !(2)$

$P 0160350$

$A 1 B(1)=\square A C 1 \square(1) \cdot G E \cdot I A C 1 \llbracket(4)$

$A 1 B(2)=I A C 1 I(2) \cdot G E \cdot I A C 1 \llbracket(1)$

P0160360

P 0160370

P0160380

$C * * * *$ TEST THE ASSIGNMENT OF A MIXTURE OF RELATIONAL AND

P0160390

C**** LOGICAL EXPRESSIONS TO LOGICAL VARIABLES AND ARRAYS

$A Z B(1,1)=$.TRUE.

$A 2 B(1,2)=$.FALSE.

$A V B=A 2 B(1,2)$. AND. NOT. $A 2 B(1,1)$

P0160400

P0160410

$P 0160420$

PO 160430

$B V B=A 2 B(1,2) \cdot O R \cdot$. NOT. $A 2 B(1,1)$

PO 160440

$C V B=I A C I I(2) \cdot L T \cdot I A C 1 I(3) \cdot A N D \cdot(A Z B(1,1) \cdot O R \ldots N O T \cdot A Z B(1,2)) \cdot O R \cdot A Z B(P 0160450$

$A 1,1)$. ANO..NOT.AZB $(1,2)$. AND. IACII (1).GT.IACII(4)

P0160460

$A 2 B(2,1)=$.NOT. (CVB.AND.MCIVB).AND. IAC1I(2), NE. IAC1I(3), AND.PO160470

$1 \quad$ IACI!(2) . LT. IACII(3) .AND. IACII(1) .EO. IAC1I(4) P0160480

$A 2 B(2,2)=A 2 B(1,2) \cdot A N D$. IACII (1).$E O$. IAC1I(4)

$A 3 B(1,1,1)=I A C 1 I(2) \cdot L T \cdot I A C 1 I(3) \cdot A N D \cdot A 2 B(1,2)$

P0 160490

P0160500

$A 3 B(1,1,2)=I A C 1 I(2) \cdot G T \cdot I A C 1 I(3) \cdot A N D \cdot A 2 B(1,1) \quad P 0160510$

$A 3 B(1,2,1)=$.NOT. MCA $1 B(5)$. AND.

$1 \quad A 2 B(1,1) . O R$. IACII(1) .EO. IAC1 I(4)

$A 3 B(1,2,2)=$.NOT. $(A Z B(1,2)$. ANO. IACI!(1) .EO. IAC1I(4)) .OR.

$1 \quad A 2 B(1,1) . O R . A 2 B(1,2)$

$A 3 B(2,1,1)=A 2 B(1,2), O R$. IAC1I(1) .EO. IAC II (4)

P0160520

$P 0160530$

P0160540

$P 0160550$

P0 160560

$A 3 B(2,2,1)=$.NOT.MCCVB.ANO.MCHVB.OR. IAC II(1), NE. IAC1I(4), OR.PO160570

$1 \quad$ IACII (1) .LT. IACII $(4), O R \cdot A 2 B(1,2)$

$P 0160580$

$A 3 B(2,1,2)=$.NOT. $A 3 B(1,1,2)$. ANO.

$1 \quad(A 2 B(1,1)$. ANO. . NOT. $A 2 B(1,2))$

P 0160590

P0160600

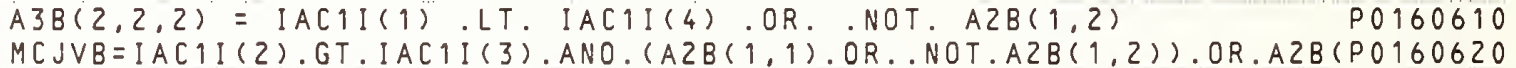

$A 1,2)$. ANO..NOT.AZB $(1,2)$. ANO.IACII ( 1$)$.GT. IAC II $(4)$

$M C K V B=I A C 1 I(2) \cdot L T \cdot I A C 1 I(3) \cdot A N D \cdot A Z B(1,1) \cdot O R \cdot A Z B(1,2)$

$M C L V B=(I A C I I(2) \cdot L T \cdot \operatorname{IAC} 1 I(3) \cdot A N O \cdot A 2 B(1,2)) \cdot O R \cdot A 2 B(1,1)$

$M C M V B=A 2 B(1,2) \cdot O R \cdot I A C 1 I(2) \cdot L T \cdot I A C I I(3) \cdot A N O \cdot A 2 B(1,1)$

$M C N \vee B=A Z B(1,2) \cdot O R \cdot(I A C 1 I(2) \cdot L T \cdot \operatorname{IAC} 1 I(3) \cdot A N O \cdot A Z B(1,1))$

C*** WRITE VARIABLES THAT ARE TRUE

WRITE (NUVI, 161) MCAVB, MCCVB, MCEVB, MCFVB, MCHVB, MCA 1B(1),

$P 0160630$

P0 160640

P0160650

$P 0160660$

P0160670

P0160680

P0160690

P0160700

$A \quad \operatorname{MCA} B(3), \operatorname{MCA} B(5), A \backslash B(1), A 2 B(1,1), A 2 B(2,1)$,

$B \quad A 3 B(1,2,1), A 3 B(1,2,2), A 3 B(2,1,1), A 3 B(2,1,2)$,

C $A 3 B(2,2,2), C \vee B, M C K V B, M C L V B, M C M V B, M C N V B$

161 FORMAT (//32H ALL ANSWERS BELOW MUST BE TRUE//21(L16/)//)

C*\#"* WRITE VARIABLES THAT ARE FALSE

$P 0160710$

$P 0160720$

$P 0160730$

P0160740

WRITE (NUVI, 162) MCBVB, MCOVB, MCGVB, MCIVB, MCA1B(2), MCA1B(4), P0160750

$A \quad A 1 B(2), A 2 B(1,2), A 2 B(2,2), A 3 B(1,1,1), A 3 B(1,1,2)$,

$B \quad A 3 B(2,2,1), A \vee B, B \vee B, M C J V B$

162 FORMAT (33H ALL ANSWERS BELOW MUST BE FALSE//15(L16/))

C*:** ENO OF SEGMENT 016

$C \div \div \div$ 
$C * * * *$ WHEN EXECUTING ONLY SEGMENT 016. THE STOP AND END

$C * * * *$ GENERAL PURPOSE

C**** TO TEST ARITHMETIC ASSIGNMENT STATEMENTS WHERE

$C * * * *$ REAL CONSTANTS AND VARIABLES, INTEGER VARIABLES

$C * * * *$ AND ARRAY ELEMENTS, AND DOUBLE PRECISION CON-

$C * * * *$ STANTS AND VARIABLES ARE ASSIGNED TO EACH OTHER

$C * * * * *$

$C * * * * *$

$C * * * *$

$C * * * * *$

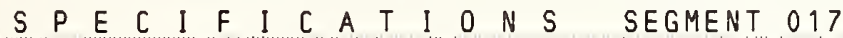

C*****

$C * * * *$

$C=\quad$ INTEGER MCA3I $(2,3,3)$

$C=$ DOUBLE PRECISION ACID(10),BC2D(7,4),CC3D(7,2,2), DPAVD $C * * * *$

DIMENSION A1S(5), AZS $(2,2), A 3 S(3,3,3)$, IAC1 I (5), IACZI $(2,7)$

INTEGER MCAZI $(2,3,3)$

DOUBLE PRECISION AC1D(10),BC2D(7,4),CC3D(7,2,2), DPAVD

$[* * * * \quad 0 U T P U T$ T A P E ASSIGNMENT STATEMENT. NO INPUT TAPE:

$C * * * * *$

$C * * * *$ WHEN EXECUTING ONLY SEGMENT 017 . THE STATEMENT NUVI = 6

$C * * *$ MUST HAVE THE $C=$ IN COL 1 AND 2 REMOVED.

$C * * * * *$

$C=$ NUVI $=6$

$C * * * * *$

NUVI $=6$

WRITE (NUVI, 170)

170 FORMAT (1H1,1X,39HINTRL - (017) ASSIGN INTEGER, REAL, ANDI

$116 X, 23 H D O U B L E$ PRECISION VALUES/2X.29HASA REFS. - 7.1 .1 .1$. $22 / 2 X, 7$ HRESULTS $/$ )

$C * * * *$ TEST ASSIGNMENT OF INTEGER VARIABLES

$\operatorname{IAC} 21(2,3)=-1111$

171

172
JACVI $=1$

$I A C 1 I(3)=+111$

$\operatorname{MCA} 3(2,1,2)=-11111$

$A C V S=I A C 1 I(3)$

$A 1 S(2)=I A C Z I(2,3)$

$\operatorname{AZS}(2,1)=\operatorname{MCA} 31(2,1,2)$

$A 3 S(2,1,2)=J A C V I$

DPAVD $=\operatorname{MCA} I(2,1,2)$

$A C 10(7)=J A C V I$

$B C 20(7,4)=\mid A C 1](3)$

$C C 3 D(5,1,2)=I A C 2 I(2,3)$

WRITE (NUVI.171)

FORMAT ( / $2 X, 24$ HASSIGN INTEGER VARIABLES/ $/ 3 X$.

IREAL VARIABLES)

$1 \% 7$

FORMAT $(/ 8 X .8 \mathrm{H} 111.0 * / F 14.1 / 1$

$1 \quad 7 X .9 H-1111.0 * / F 14.11 \%$

$2 \quad 4 X .12 \mathrm{H}-11111.0 * / F 14.1 / 1$

$11 \times 5 \mathrm{H}_{1.0} 0 / \mathrm{F} 14.1 / 13 \mathrm{X}, 33 \mathrm{~Hz}$

$1 / 4 \mathrm{X} .16 \mathrm{H}-0.11911005 * / 018.5 / 1$

$11 \times .9 \mathrm{HO}^{\circ} 1 \mathrm{0} 01 * / 018.1 / 1$

$9 \times .11 H 0.111003 * / 018.3 / 1$

TABLE 1/LN 5,9P0170180

$P 0170190$

$P 0170200$

$\mathrm{P} 0170210$

$P 0170220$

P0170230

$P 0170240$

$P 0170250$

$P 0170260$

$P 0170270$

P0170280

$P 0170290$

P0170300

$P 0170310$

$21 H 1-$ TOP0170320

$P 0170330$

$P 0170350$

$P 0170360$

$P 0170370$

$P 0170380$

5

6 
$A 1 S(2)=+222$

P0170460

$\operatorname{A} 2 S(2,1)=-22222$

$P 0170470$

$A 3 S(2,1,2)=2$

PO 170480

DPAVD $=2$

$A C 1 D(7)=-22222$

$P 0170490$

$B C 2 D(7,4)=-2222$

$P 0170500$

$C C 3 D(5,1,2)=+222$

$P 0170510$

WRITE (NUVI, 173)

173 FORMAT (/2X,24HASSIGN INTEGER CONSTANTS//3X,

P0170520

$P 0170530$

1EAL VARIABLES)

$21 H 1$ - TO RP0170540

$P 0170550$

WRITE (NUVI, 174) ACVS,A1S(2), AZS (2,1), A3S(2,1,2), DPAVD,AC1D(7), BC2DP0170560

$1(7,4), C[30(5,1,2)$

174 FORMAT $(16 \mathrm{X}, 9 \mathrm{H}-2222.0 * / \mathrm{F} 13.1 / 1$

$P 0170570$

$1 \quad 8 X, 7 \mathrm{H} 222.0 * / \mathrm{F} 13.1 / 1$

$P 0170580$

$3 \times .12 \mathrm{H}-22222.0 * / F 13.1 / 1$

$P 0170590$

$3 \quad 10 X, 5 H 2,0 * 1 F 13.1135 \mathrm{H} 12$

$112 \times .9 H 0.2001 * / 019.1 / 1$

$5 \times, 16 H-0.222220 \quad 05 * 1019.511$

$8 \mathrm{X}, 13 \mathrm{H}-0.2222 \mathrm{O} 04 * 1019.411$

$10 X .11 H 0.222003 * / 019.311$

C*\#*\# TEST ASSIGNMENT OF BASIC REAL CONSTANTS $J A C V I=3.3$

$I A C 1 I(3)=+333 \cdot 3 E-2$

$1 A C 2 !(2,3)=.3333 E+1$

$M C A 3 !(2,1,2)=-.0033333 E 3$

OPAVD $=+3.3333$

$A C 1 D(7)=.3333333 E 1$

$B C 2 D(7,4)=-333.3333 E-2$

$C C 3 D(5,1,2)=-.0333333 E+2$

WRITE (NUVI,7173)

7173 FORMAT ( $/ 2 X, 27$ HASSIGN BASIC REAL CONSTANTS//3X,

1 TO INTEGER VARIABLES)

P 0170610

$P 0170620$

$P 0170630$

$P 0170640$

$P 0170650$

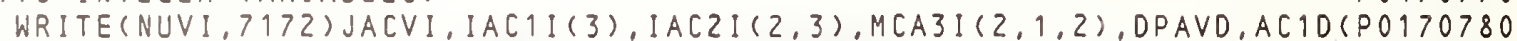
$17), B C 2 D(7,4), C C 30(5,1,2)$

TABLE $1 / L N \quad 2,10 P 0170660$

$P 0170670$

$P 0170680$

$P 0170690$

$P 0170700$

P0170710

$P 0170720$

$P 0170730$

$P 0170740$

$P 0170750$

7172 FORMAT $/ 9 X, 3 H 3 * / 3(110 /) / 8 X, 4 H-3 * / 110 / / 3 X, 33 H 2$ - TO DOUBLE PRECISPO170800 IION VARIABLES//

$2 \quad 8 X, 13 H 0.33333001 * 1019.511$

$6 x, 15 H 0.3333333001 * 1019.711$

P0170820

$5 X, 16 H-0.3333333001 * / 019.7 / 1$

$P 0170830$

$6 \mathrm{X}, 15 \mathrm{H}-0.333333001 * / 019.61)$

$P 0170840$

$P 0170850$

$P 0170860$

P0 0170870

P 0170880

P0 170890

P0170900

$A 3 S(2,1,2)=4.4444 E+1$

$P 0170910$

$J A C V I=A 2 S(2,1)$

$\triangle A C 1 I(3)=A 1 S(2)$

$P 0170920$

$P 0170930$

$\operatorname{IAC2} I(2,3)=\operatorname{ABS}(2,1,2)$

$P 0170940$

$\operatorname{MCA} I(2,1,2)=A C V S$

$P 0170950$

P 0170960

$A C 1 D(7)=A 1 S(2)$

$B C 2 D(7,4)=A 3 S(2,1,2)$

$C C 3 D(5,1,2)=A C V S$

WRITE (NUVI,175)

175 FORMAT (/23H ASSIGN REAL VARIABLES//

1ER VARIABLES)

$P 0170970$

P 0170980

$P 0170990$

WRITE (NUVI,176) JACVI, IAC1I(3), IAC2!(2,3), MCA3!(2,1,2), DPAVD, AC1D(P0171020

$17), B C 20(7,4), C C 30(5,1,2)$

$P 0171030$

176 FORMAT (17X,5H-44*/2(110/)/8X,4H44*/2(I10/), 35H12 - TO DOUBLPO171040 IE PRECISION VARIABLESII

$26 \mathrm{X}, 12 \mathrm{H}-0.444 \mathrm{D} 02 * 1016.311$

$5 \mathrm{X} .13 \mathrm{H}-0.4444 \mathrm{O} 02 * 1016.411$

$5 X .13 H 0.44444002 * 1016.5 / 1$

$5 X, 13 H 0.44444002 * 1016.51)$

$C *$ TEST ASSIGNMENT DF D.P. VARIABLES

$P 0171050$

$P 0171060$

$P 0171070$

$P 0171080$

$P 0171090$

TABLE 1/LN 3,6P0171100 
DPAVO $=5555.55$

$A C 10(7)=+55555555555555.0-13$

$P 0171110$

$B C 20(7.4)=-.0000055555555506$

$P 0179120$

$C C 3 D(5,1,2)=-.055555555555550+2$

JACVI $=$ DPAVD

$I A C 1 I(3)=A C 1 D(7)$

IAC2I $(2,3)=B C 2 D(7,4)$

$\operatorname{MCA} 3(2,1,2)=C C 30(5,1,2)$

$A C V S=C C 3 D(5,1,2)$

$A 1 S(2)=B C 2 D(7,4)$

$A 2 S(2,1)=A C 10(7)$

$A 3 S(2,1,2)=$ DPAVD

WRITE (NUVI,177)

177 FORMAT ( $/ 2 X, 33$ HASSIGN DOUBLE PRECISION VARIABLES I

$1 / 3 \times, 24 H 1-$ TO INTEGER VARIABLES)

$P 0171130$

P0171140

$P 0171150$

$P 0171160$

$P 0171170$

$P 0171180$

$P 0171190$

P 0171200

P0 0171210

P0171220

P 0171230

WRITE (NUVI, 178) JACVI, IACII(3), IACZI (2,3),MCA3I(2,1,2),ACVS, A1S (2)P0171260

$1, A 2 S(2,1), A 3 S(2,1,2)$

178 FORMAT(/3X,9H $5555 * / 110 / 19 X, 3 H 5 * / 110 / / 8 X, 4 H-5 * / 2(110 /) / 3 X, 21 H P 0171280$ 12 - TO REAL VARIABLES/I

$2 \quad 3 X .16 \mathrm{H}-0.5555556 \mathrm{E} \quad 01 * 1 \mathrm{E} 17.711$

$3 \quad 3 X, 16 \mathrm{H}-0.5555556 \mathrm{E} 01 . / \mathrm{E} 17.711$

$3 \times .16 \mathrm{H} \quad 0.5555556 \mathrm{E} \quad 01 * / \mathrm{E} 17.711$

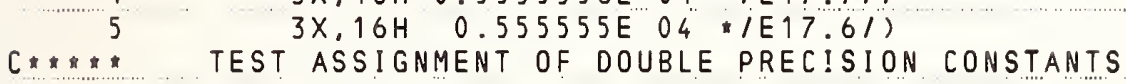

JACVI $=66666.0-4$

$\operatorname{IACII(3)=-.0000006666666607}$

IAC2I $(2.3)=-.0666666666666660+2$

$\operatorname{MCA3I}(2,1,2)=66666.66666666600-1$

ACVS $=666666666666666.00$

$A 1 S(2)=+66666.0-4$

$\operatorname{AZS}(2,1)=-.0000000666666608$

$A 3 S(2,1,2)=-.0666666666666660+2$

WRITE (NUVI,179)

179 FORMAT ( 35 HI ASSIGN DOUBLE PRECISION CONSTANTSI

$1 / 3 X, 24 H 1 .-10$
WRITEGER VARIABLES)

$P$
$P 0171280$

$1, A Z S(2,1), A 3 S(2,1,2)$

7170 FORMATC I 9X,3H6*/I10/18X,4H-6*/2(110/)/3X,9H 6666*/110/I

$13 X .21 H 2$ - TO REAL VARIABLES /I

$2 \quad 3 \times, 16 H \quad 0.6666667 \mathrm{E} \quad 14: 1 \mathrm{E} 17.7 ! 1$

$3 \quad 3 \times .16 \mathrm{H} \quad 0.66666 \mathrm{E} 01 * / \mathrm{E} 17.511$

$4 \quad 3 X .16 \mathrm{H}-0.6666666 \mathrm{E} 01 * / \mathrm{E} 17.7 ! 1$

$5 \quad 3 X, 16 H-0.6666667 \mathrm{E} 01 *(E 17.7 i)$

WRITE (NUVI,7171)

7171 FORMAT(//34H ALL̈ TEST OUTPUT SHOULD BE CHECKED/

$\begin{array}{cc}1 & 34 H \text { AGAINST THE ASTERISKED (*) FIGUREI } \\ 2 & 18 \text { WHICH PRECEDES IT) } \\ \text { C\#\#* END OF TEST SEGMENT } 017\end{array}$

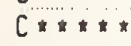

C*\#\# WHEN EXECUTING ONLY SEGMENT 017. THE STOP AND END

$C * * * *$ CAROS WHICH APPEAR AS COMMENTS MUST HAVE THE C $C=$

C*****IN COL 1 AND 2 REMOVED.

$C * \| * *$

$C=S T O P$

$C=\quad E N D$

STOP

END

$P 0171300$

Po 171310

$P 0171320$

$P 0171330$

$P 0171340$

P0171350

P0 171360

Po171370

P0 171380

P0 171390

P0 171400

P0171410

P0171420

Po 171430

P0171440

$P 0171450$

P0 171470

P0 171480

P0171490

P0 171500

Po171510

P0171520

$P 0171530$

P0171540

P0171550

P0 171560

P0171570

P0 171580

P0171590

P0171600

P0171610

P0 171620

P0171630

P0171640

P0171650

P017C1

P017C2

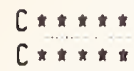

$C * * * *$

C*⿻**

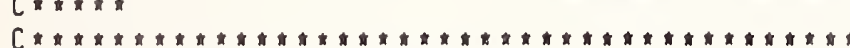

UGOTO- $(020)$

P0200010

P0200020

P0200030

P 0200040

C***** GENERAL PURPOSE

C**\# TO TEST UNCONDITIONAL GO TO STATEMENTS

C*\#** RESTRICTION OBSERVED

C** GO TO STATEMENTS CAUSE BRANCHES ONLY TO

C*\#** EXECUTABLE STATEMENTS

EXECUTABLE STATE

$\because P 0200050$

$C * * *$ GENERAL COMMENTS

ASA REF P0200060

7.1.2.1.1.1P0200070

P0200080

$7.1 .2 / 54 P 0200090$

PO200100

P0200110 
C* WHEN EXECUTING ONLY SEGMENT 020 . THE STATEMENT NUVI = 6 $P 0070340$

$C * * * *$ MUST HAVE THE $C=$ IN COL 1 AND 2 REMOVED.

$P 0070345$

$C * * * *$

$C=$ NUVI $=6$

$P 0070350$

C*****

NUVI $=6$

$P 0070360$

WRITE (NUVI,200)

200 FORMAT ( 1 H1, $1 \times, 33$ HUGOTO - (020) UNCONOITIONAL GO TO/16X, 19 HSTATEMENT//2X.

2 21HASA REFS - $7.1 .2 .1 .1 / 12 \times, 7$ HRESULTS )

C**** HEADER FOR SEGMENT 020 WRITTEN

$C * * * *$ TEST BRANCH FORWARD GO TO 201

203 MRRVI $=3$

WRITE (NUVI,7200) MRRVI

7200 FORMAT $(14 \mathrm{X}, 11)$

$P 0070365$

PO20B1

$P 0200150$

P0200160

$P 0200170$

P0200180

P 0200190

$P 0200200$

P0200210

PO 200220

P0 0200230

GO TO 204

207 MRRVI $=7$

"WRITE (NUVI,7200)" MRRVI

GO TO 208

202 MRRVI $=2$

WRITE (NUVI,7200) MRRVI

$C *$ TEST BRANCH BACKWARD

GOTO 203

201 MRRVI $=1$

WRITE (NUVI,7200) MRRVI

GO TO 202

208 MRRVI $=8$

WRITE (NUVI.7200) MRRVI

GO TO 209

206 MRRVI $=6$

WRITE (NUVI,7200) MRRVI

GO TO 207

204 MRRVI = 4

WRITE (NUVI,7200) MRRVI

P0200240

P0200250

$P 0200260$

P0200270

P0200280

P0200290

P0200300

P0200310

P0200320

P0200330

P0200340

P0200350

PC2 200360

$P 0200370$

P0200380

P0200390

P0200400

P 0200410

P0200420

P0200430

C***** TEST BRANCH TO STATEMENT IMMEOIATELY AFTER

C***** UNCONDITIONAL GO TO

gO TO 205

205 MRRVI $=5$

WRITE (NUVI, 7200) MRRVI

GO TO 206

209 WRITE (NUVI,7201)

7201 FORMAT (//2X,35HTHIS TEST IS SUCCESSFUL ONLY IF THE/

$12 X, 37$ HNUMBERS LISTED ABOVE ARE SEQUENTIALLYI

$22 X, 2 O H I N$ ORDER FROM 1 TO 8 )

C***** END OF TEST SEGMENT O 20

$[* * * * *$

$C * * * *$ WHEN EXECUTING ONLY SEGMENT 020 , THE STOP AND END

$C * * *$ CARDS, WHICH APPEAR AS COMMENTS, MUST HAVE THE C=

$[* * *$ IN COL 1 AND 2 REMOVED.

$C=$ STOP

$C=\ldots N D$

STOP

P0200440

$P 0200450$

$P 0200460$

$P 0200470$

P 0200480

P0200490

$P 0200500$

P0200510

$P 0200520$

P0200530

$P 0200540$

P0200550

P0200560

P0200570

$P 0200580$

P 0200590

P0200600

END

P $020 C 1$

P $020 C 2$

C*****

$C * * * * *$

C $* * * *$

$P 0210010$

P0210020

P0210030

P0210040

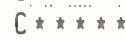
AGOTO - (021)

P0210050

$C * * *$ GENERAL PURPOSE

$C * * * *$ TO TEST GO TO.ASSIGNMENT STATEMENTS

$C * * * *$ AND ASSIGNED GO TO STATEMENTS

$[* * * *$ RESTRICTIONS OBSERVEO

$C * * * *$ INTEGER VARIABLE USED IN ASSIGN STATEMENTS
ASA REF PO210060

$7.1 .1 .3 \quad P 0210070$

7.1.2.1.2.2P0210080

$P 0210090$

$7.1 .1 .3 \quad 106 P 0210100$ 
$C * * * *$ IS NEVER REFERENCEO ELSEWHERE IN THIS SEGMENT

$C * * * *$ ASSIGNED GO TO STATEMENTS CAUSE BRANCHES ONLY

$7 \cdot 1 \cdot 1 \cdot 3$

$103 P 0210120$

$C * * * * *$ TO EXECUTABLE STATEMENTS

7.1 .2

$154 \mathrm{PO} 210130$

$C * * * *$ INTEGER VARIABLE ALWAYS CONTAINS STATEMENT

$7 \cdot 1 \cdot 2 \cdot 1.2 / 20 P 0210140$

$C * * * * *$ LABEL FROM THE ASSIGNED GO TO LIST

$P 0210950$

$C * * * *$ GENERAL COMMENTS

$C * * * *$ IGVI ANO KGVI ARE IMPLICITLY OEFINEO

5.3

$P 0210160$

$C * * * * * \quad$ GTVI IS EXPLICITLY OEFINED

$C * * * *$ ASSIGN ANO ASSIGNEO GO TO ALSO TESTEO IN

$7 \cdot 2 \cdot 1 \cdot 6$

$107 P 0210170$ $C * * * * \quad$ SEGMENT 190

$C * * * * *$

$C * * * * *$

$C * * * * *$

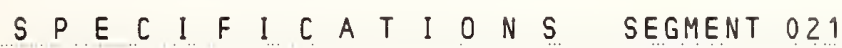

$C * * * * *$ WHEN EXECUTING ONLY SEGMENT 021 . THE SPECIFICATION STATEMENTS

$C * * * * *$ WHICH APPEAR AS COMMENTS, MUST HAVE THE $C=$ IN COL

$C * * * * 1$ ANO 2 REMOVEO

$C=$ INTEGER GTVI

$C * * * * *$

INTEGER GTVI

$C * * * * 0$ U T P U T T A P E ASSIGNMENT STATEMENT. NO INPUT TAPE.

$C * * * * *$

$C * * * * *$ WHEN EXECUTING ONLY SEGMENT 021, THE STATEMENT NUVI = 6

$C * * * *$ MUST HAVE THE C= IN COL 1 AND 2 REMOVED.

$C * * * * *$

$C=$ NUVI $=6$

$C * * * * *$

NUVI $=6$

WRITE (NUVI, 210)

210 FORMAT ( 1 H1, 1 X, 33HAGOTO - $(021)$ ASSIGN AND ASSIGNEO/16X, 15 HGO TO//2X,

231HASA REFS. -7.1 .1 .3 AND $7.1 .2 .1 / 12 \times .7$ HRESULTS)

$C * * * * *$ HEAOER FOR SEGMENT O21. WRITTEN

$C * * * *$ TEST FORWARO BRANCHING GO TO WITH ONLY ONE

C***** LABEL IN THE BRANCH LIST

ASSIGN 211 TO IGVI

GO TO IGVI, (211)

C* TEST FORWARO BRANCHING GO TO WHICH BRANCHES

$C * * * * *$ TO IMMEOIATELY FOLLOWING STATEMENT

212 MRRVI $=2$

WRITE (NUVI,8212) MRRVI

ASSIGN 213 TO GTVI

GO TO GTVI, (213)

$C * * * * *$ TEST FORWARO BRANCHING GO TO WHERE ALL BRANCHES

$C * * * *$ ARE IDENTICAL

213 MRRVI $=3$

WRITE (NUVI.8212) MRRVI

ASSIGN 214 TO GTVI

GO TO GTVI, $(214,214,214)$

$C * *$ TEST FORWARD BRANCHING GO TO WITH SEVERAL UNIQUE

$C * * * *$ BRANCHES IN THE LIST

215 MRRVI $=5$

WRITE (NUVI,8212) MRRVI

ASSIGN 217 TO KGVI

ASSIGN 216 TO IGVI

GO TO IGVI, (217,218,216,219)

$C * * * *$ TEST BACKWARO BRANCHING GO TO WHERE BRANCHES

$C * * * *$ ARE IDENTICAL

$214 \quad$ MRRVI $=4$

WRITE (NUVI,8212) MRRVI

ASSIGN 215 TO IGVI

GO TO IGVI, (215,215)

$C * * * *$ TEST BACKWARD BRANCHING GO TO WITH ONLY ONE LABEL

$C * * * *$ IN THE BRANCH LIST

211 MRRVI $=1$

WRITE (NUVI.8212) MRRVI

ASSIGN 212 TO GTVI

GO TO GTVI, ( 212 )

$C * * * *$ IN THE FIRST PART OF THIS TEST, ALL GO TO STATEMENTS

$\mathrm{P} 0210190$

P0210200

PO210210

$P 0210220$

$P 0010480$

$P 0010485$

P0010490

$P 0010495$

$P 0010500$

$P 0010505$

P021A1

P0210230

$P 0070370$

$P 0070375$

$P 0070380$

$P 0070385$

$\mathrm{P} 0070390$

P0070395

P021B1

P0210240

PO210250

P0210260

P0210270

PO210280

P0210290

P0210300

P0210310

$P 0210320$

$P 0210330$

$P 0210340$

$P 0210350$

$P 0210360$

P0210370

$P 0210380$

$P 0210390$

$P 0210400$

P0210410

$P 0210420$

$P 0210430$

$P 0210440$

$P 0210450$

P0210460

$P 0210470$

P0210480

$P 0210490$

P0210500

P0210510

PO210520

$P 0210530$

$P 0210540$

PO210550

$P 0210560$

P0210570

P0210580

$P 0210590$

P0210600

$P 0210610$

$P 0210620$

$P 0210630$

P0210640

NBS FORTRAN Test Programs Version 1 


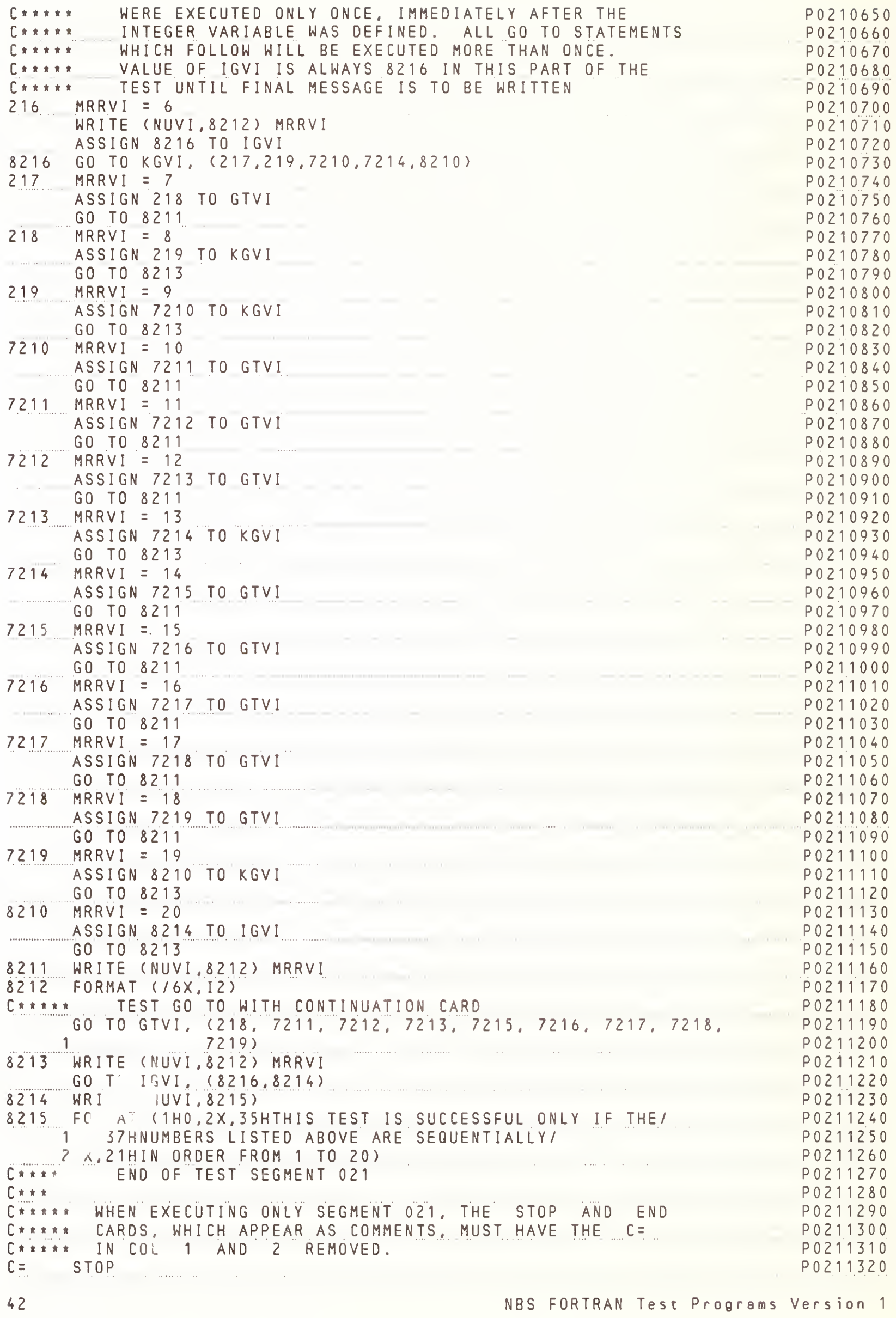




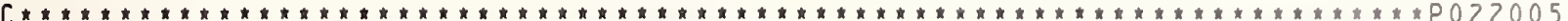

$C * * * *$ GENERAL PURPOSE

$C * * * *$ TO TEST COMPUTEO GO TO STATEMENTS

$C * * * *$ RESTRICTIONS OBSERVEO

C***** VALUE OF INTEGER VARIABLE IS NEVER LESS THAN 1

$C * * * *$ ANO NEVER LARGER THAN THE NUMBER OF BRANCHES

C**** INTEGER VARIABLES USEO IN COMPUTEO GO TO STMNTS.

$C * * * *$ ARE NOT EQUATED TO AVOID SECONO LEVEL

$[* * * *$ OEFINITION PROBLEMS

$C * * * *$ GENERAL COMMENTS

C***** IGVI ANO KGVI ARE IMPLICITLY DEFINEO

$C * * * * *$ GTVI IS EXPLICITLY OEFINED

$C * * * * *$

$C * * * * *$

$C * * * * *$

$C * * * * *$

$C * t \star \star * t$

$C * * * *$ COMPUTEO GO TO ALSO TESTEO

\section{$S P E C I F I C A T I O N$ S SEGMENT 022}

$C * * * * *$

C*

$C=\quad$ INTEGER GTVI

$C * * * * *$

INTEGER GTVI

$C * * * * 0$ U T $P$ U T $T$ A P E ASSIGNMENT STATEMENT. NO INPUT TAPE.

$C * * * * *$

$C * * * *$ WHEN EXECUTING ONLY SEGMENT 022, THE STATEMENT NUVI $=6$

$C * * *$ MUST HAVE THE $C=$ IN COL 1 AND 2 REMOVED.

$C * * * *$

$C=$

NUVI $=6$

$C * * * * *$

NUVI $=6$

WRITE (NUVI.220)

220 FORMAT ( 1 H $1,12,28 H C G O T O$ - (022) COMPUTED GO TO $/ 12 \mathrm{X}$.

120 HASA REF. - $7.1 .2 .1 .3 / 12 \times$.7HRESULTS)

$C * * * *$ HEAOER FOR SEGMENT 022 WRITTEN

$C * * * *$ TEST FORWARD BRANCHING GO TO WITH ONLY ONE

$C * * * * \quad$ LABEL IN BRANCH LIST

$I G V I=1$

GO TO (221) IGVI

C***\# TEST FORWARO BRANCHING GO TO WHICH BRANCHES

$C * * * *$ TO IMMEOIATELY FOLLOWING STATEMENT

222 MRRVI $=2$

WRITE (NUVI.8222) MRRVI

$C * * * *$

GOTO (223), GTVI

$C * * * * *$ TEST FORWARO BRANCHING GO TO WHERE SOME BRANCHES

C***** ARE IOENTICAL

223 MRRVI $=3$

WRITE (NUVI.8222) MRRVI

GTVI $=2$

GO TO $(225,224,225)$, GTVI

C**** TEST FORWARO BRANCHING GO TO WITH SEVERAL UNIOUE

$C * * * *$ BRANCHES IN LIST

$225 \quad$ MRRVI $=5$

WRITE (NUVI,8222) MRRVI

KGVI $=1$

$I G V I=3$

GO TO $(227,228,226,229)$. IGVI

C**\# TEST BACKWARO BRANCHING GO TO WHERE SOME

$C * * * *$ BRANCHES ARE IDENTICAL

224 MRRVI $=4$
ASA REF 90220060

7.1.2.1.3 P0220070

$P 0220080$

$7.1 .2 .1 .3 / 33 P 0220090$

$P 0220100$

$10.2 .8 \quad 109 P 0220110$

$10.3 \quad / 13 P 0220120$

$P 0220130$

P0220140

$5.3 \quad 107 P 0220150$

$7.2 .1 .6 / 55 P 0220160$

P0220170

P0220180

P0220190

P0010510

$P 0010515$

$P 0010520$

P0010525

$P 0010530$

P0010535

P0010540

P022A1

P0220200

P0 070400

P0 070405

P0070410

$P 0070415$

PO0 070420

P0070425

P022B

P0220210

P0220220

P0220230

P0220240

PO 220250

P0220260

PO 220270

P0220280

P0220290

P0220300

P0220310

P0220320

PO 220330

P0220340

P0220350

P0220360

P0220370

P0220380

P0220390

P0220400

P0220410

P0220420

P0220430

P0220440

P0220450

P0220460

P0220470

P0220480

P0220490

P0220500 
WRITE (NUVI.8222) MRRVI

$P 0220510$

IGVI $=4$

GO TO (226.226,226.225)..IGVI

PO 220520

$P 0220530$

$\begin{array}{ll}C * & \text { TEST BACKWARO BRANCHING GO TO WITH ONLY ONE } \\ C * \because \text { LABEL IN BRANCH LIST } & \end{array}$

$P 0220540$

$221 \quad$ MRRVI $=1$

WRITE (NUVI, 8222 ) MRRVI

P0220550

$P 0220560$

GTV! $=1$

GO TO $(222)$, GTVI

C: $*$ IN THE FIRST PART OF THIS TEST, ALL GO TO STATEMENTS

$P 0220570$

P0220580

P0220590

$P 0220600$

$C * * *$ WERE EXECUTED ONLY ONCE, IMMEDIATELY AFTER THE

P0220610

$C *$ INTEGER VARIABLE WAS DEFINEO... ALL GO TO STATEMENTS

$P 0220620$

C***WHICH FOLLOW WILL BE EXECUTEO MORE THAN ONCE.

$C * * *$ VALUE OF IGVI IS ALWAYS 1 IN THIS PART OF THE TEST

C**** UNTIL THE FINAL MESSAGE IS TO BE WRITTEN

226 MRRVI $=6$

$I G V I=1$

WRITE (NUVI,8222) MRRVI

8226 GO TO $(227,229,7220,7224,8220)$, KGVI

227 MRRVI $=7$

GTVI $=1$

GO TO 8221

$228^{-M R R V I}=8$

KGVI $=2$

GO TO 8223

P0220630

$P 0220640$

$P 0220650$

$P 0220660$

$P 0220670$

P0220680

$P 0220690$

$P 0220700$

P0220710

$P 0220720$

$P 0220730$

P0220740

$P 0220750$

$P 0220760$

MRRVI $=9$

$P 0220770$

KGVI $=3$

7220 MRRVI $=10$

P0220780

P0220790

GTVI $=$ ?

GO TO 8221

7221 MRRVI $=11$

P0220800

$P 0220810$

$P 0220820$

GTVI $=5$

GO TO 8221

7222 MRRVI $=12$

P02220830

$P 0220840$

GTVI $=4$

P0220850

P0220860

GO TO 8221

7223 MRRVI $=13$

P0220870

P 0220880

KGVI $=4$

GO TO 8223

P0220890

P0220900

P0220910

P0220920

GTVI $=6$

GO TO 8221

7225 MRRVI $=15$

P0220930

P0220940

P0220950

GTVI $=7$

GO TO 8221

P0220960

P0220970

P0220980

P0220990

$P 0221000$

P0221010

P0221020

P0221030

P0221040

P0221050

P0221060

P0221070

P0221080

P0221090

$P 0221100$

P0221110

$P 0221120$

P0221130

PO221140

P0221150

P0221160

PO221170

P0221180

8223 WRITE (NUVI.8222) MRRVI

GO TO $(8226.8224)$, IGVI 
8224 WRITE (NUVI.8225)

8225 FORMAT ( 1 HO, 2X,35HTHIS TEST IS SUCCESSFUL ONLY IF THEI

$C * * * * *$

C**** WHEN EXECUTING ONLY SEGMENT 022, THE STOP AND END

PO221240

$C * * * *$ CAROS, WHICH APPEAR AS COMMENTS, MUST HAVE THE $C=$

$C * * * *$ IN COL 1 ANO 2 REMOVEO.

$C=S T O P$

$C=E N D$

STOP

$P 0221250$

$P 0221260$

P 0221270

$P 0221280$

P0221290

END

PO22C 1

$\mathrm{PO} 22 \mathrm{C2}$

$C * * * * *$
$C * * * *$

$C * * * * *$

$A R B A O-(030)$

$P 0300020$

$P 0300030$

$P 0300040$

$\cot * * * x$

$C * * * * * * * * * * * * * * * * * * * * * *$
$C * * * *$ GENERAL PURPOSE

$C * * * *$

TEST THAT EXPRE

ARBAO

P 0300050

$C * * * *$ OF INTEGER OR REAL VALUES MAY BE FORMED

ASA REF P0 300060 GENERAL COMMENTS

$C * * * * *$ TYPES ARE NEVER MIXEO.
$C * * * * *$ VARIABLES, ARRAY ELEMENTS ANO CONSTANTS ARE USED

IN A VARIETY OF COMBINATIONS.

$C * * * * *$

$C * * * * *$

$C * * * *$ S P E C I F I C A T I O N S SEGMENT O 30

$6.1 \quad P 0300070$

P0300080

P0300090

P0300100

$P 0300110$

$P 0300120$

$P 0300130$

$P 0300140$

$C * * * *$

C*****

WHEN EXECUTING ONLY SEGMENT 030, THE SPECIFICATION STATEMENTS

$P 0010550$

$C * * *$ WHICH APPEAR AS COMMENTS, MUST HAVE THE $C=$ IN COL

$C * * * * i$ AND 2 REMOVED

$C * * * * *$

$C=\quad$ OIMENSION A1S(5), A2S $(2,2), \operatorname{IAC} 1 \mathrm{I}(5), \operatorname{IAC} 2 I(2,7)$

$C * * * * *$

DIMENSION A1S(5), A2S $(2,2)$, IAC1I $(5), I A C 2 I(2,7)$

$C * * * * 0 U T P U T$ T A P E ASSIGNMENT STATEMENT. NO INPUT TAPE.

P0010555

P0010560

$P 0010565$

$P 0010570$

$P 0010575$

$P 0010580$

P030A1

$C * * * * *$

WHEN EXECUTING ONLY SEGMENT 030, THE STATEMENT NUVI = 6

$C * * * *$ WHEN EXECUTING ONLY SEGMENT 030 , THE STATE
$C * * * *$ MUST HAVE THE C=IN COL 1 ANO 2 REMOVEO.

$[* * * * *$

$C=\quad$ NUVI $=6$

$[* * * * *$

NUVI $=6$

WRITE (NUVI, 301 )

301 FORMAT ( 1 H1, 1X,28HARBAO - (030) BASIC AODITION/IZX,

-14HASA REF. - $6.1 / 12 X, 7$ HRESULTS)

C***** HEADER FOR SEGMENT 030 WRITTEN

WRITE (NUVI, 302)

302 FORMAT ( / / X, 16HINTEGER AODITION)

C***** TEST 1 - AOO 2 INTEGER VARIABLES (ONE CONTAINS MINUS VALUE) MRRVI $=1$

JACVI $=2$

$K B C V I=-2$

$I H O V I=J A C V I+K B C V I$

WRITE (NUVI, 303 ) MRRVI, IHOVI

303 FORMAT $(16 \mathrm{H}$ TEST, I3, I6)

$C * * *$ TEST 2 - REVERSE VARIABLES IN TEST 1

MRRVI $=2$

I GOVI =KBCVI+JACVI

WRITE (NUVI,303) MRRVI, IGDVI

C***** TEST 3 - AOO 2 CONSTANTS

MRRVI $=3$

IAC II $(1)=2+(-2)$

WRITE (NUVI,303) MRRVI, IAC I I 1 )

C**** TEST 4 - AOO 2 ARRAY ELEMENTS (ONE CONTAINS MINUS VALUE)

MRRVI $=4$

$I A C 1 I(3)=3$

$\operatorname{IAC} I(1,3)=-3$

0300150

$P 0070430$

$P 0070435$

$P 0070440$

$P 0070445$

$P 0070450$

$P 0070455$

PO30B 1

$P 0300160$

P0300170

$P 0300180$

$P 0300190$

$P 0300200$

P0300210

$P 0300220$

P0300230

$P 0300240$

$P 0300250$

$P 0300260$

$P 0300270$

$P 0300280$

$P 0300290$

$P 0300300$

$P 0300310$

$P 0300320$

$P 0300330$

$P 0300340$

P0300350

$P 0300360$

P0300370

P0300380

P0300390

$P 0300400$

NBS FORTRAN Test Programs Version 1 
$\operatorname{IAC2} I(2,2)=\mid A C 1 I(3)+\operatorname{IAC} 2 I(1,3)$

WRITE (NUVI,303) MRRVI, I AC2I $(2,2)$

$P 0300410$

$P 0300420$

$C * \#$ TEST 5 - ADD 8 INTEGER VARIABLES

$P 0300430$

MRRVI $=5$

LCCVI $=-6$

$P 0300440$

MDCVI $=-2$

NECVI $=+18$

$I F D V I=J A C V I+K B C V I+L C C V I+M D C V I+M D C V I+L C C V I+K B C V I+N E C V I$

$P 0300450$

$P 0300460$

$P 0300470$

WRITE (NUVI,303) MRRVI, IFOVI

C* TEST 6 - ADD COMBINATION OF VARIABLES, ARRAY ELEMENTS

$C * * *$ AND CONSTANTS

MRRVI $=6$

$\operatorname{IAC} 2 I(2,2)=-2$

IFDVI $=I A C 1 I(3)+I A C 2 I(1,3)+I A C 2 I(2,2)+J A C V I+K B C V I+L C C V I+7+1$

WRITE (NUVI,303) MRRVI, IFDVI

C**** TEST 7 - ADO 2 REAL VARIABLES

WRITE (NUVI,304)

304 FORMAT (//15H REAL ADDITION)

MRRVI $=7$

$A C V S=-2.0$

$B C V S=2.0 E 0$

HHCVS = ACVS+BCVS

WRITE (NUVI, 305) MRRVI, HHCVS

305 FORMAT (16H TEST, I3,F7.1)

C**** TEST 8 - REVERSE ORDER OF VARIABLES IN TEST 7

MRRVI $=8$

GGCVS $=$ BCVS + ACVS

WRITE (NUVI,305) MRRVI, GGCVS

C***TEST 9 - ADD 4 REAL VARIABLES

MRRVI $=9$

$F F C V S=A C V S+B C V S+A C V S+B C V S$

WR!TE (NUVI,305) MRRVI, FFCVS

C\# TEST 10 - ADO 2 REAL CONSTANTS MRRVI $=10$

$A Z S(1,2)=3.5+(-3.5)$

WRITE (NUVI,305) MRRVI, A2S(1,2)

C TEST 11 - ADD REAL ARRAY ELEMENTS

MRRVI $=11$

$A 1 S(1)=-25 . E-1$

ACVS $=2.5$

$A Z S(1,1)=-7.0$

FFCVS $=A 1 S(1)+A 2 S(1,1)+9.5$

$P 0300480$

P0300490

$P 0300500$

P0300510

P0300520

$P 0300530$

P0300540

$P 0300550$

P0300560

P0300570

P0300580

P0300590

$P 0300600$

P0300610

$P 0300620$

$P 0300630$

$P 0300640$

$P 0300650$

P0300660

P0300670

P0300680

P0300690

$P 0300700$

P0300710

P0300720

P0300730

P0300740

P0300750

P0300760

$P 0300770$

P0300780

P0300790

P0300800

P0 300810

P0300820

WRITE ( NUVI,305) MRRVI, FFCVS

$P 0300830$

$C * * *$ TEST 12 - ADD COMBINATION OF VARIABLES, ARRAY ELEMENTS

P0 0300840

C*AND CONSTANTS

MRRVI $=12$

FFCVS $=A 1 S(1)+A C V S+7.0+A 2 S(1,1)$

WRITE (NUVI, 305 ) MRRVI, FFCVS

WRITE (NUVI, 306)

306 FORMAT (//35H ALL ABOVE ANSWERS SHOULD BE O FOR/

$131 \mathrm{H}$ THIS SEGMENT TO BE SUCCESSFUL)

C**** END OF TEST SEGMENT 030

$C * \# *$ WHEN EXECUTING ONLY SEGMENT 030 , THE STOP AND END

C*** CARDS, WHICH APPEAR AS COMMENTS, MUST HAVE THE C

$C * * * *$ IN COL 1 AND 2 REMOVED.

$C=$ STOP

$C=E N D$

STOP

$P 0300850$

$P 0300860$

$P 0300870$

P0 300880

$P 0300890$

P0300900

$P 0300910$

$P 0300920$

$P 0300930$

P0300940

P0300950

$P 0300960$

$P 0300970$

PO3 00980

PO30C 1

END

P030C2

$C \cdots \cdots *$
$C * \cdots \cdots$
$C * \cdots * *$

$P 0310010$

PO310020

PO310030

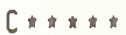

ARFAD - $(031)$

P0310040

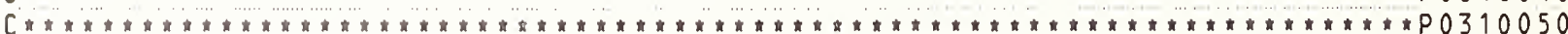

C**** GENERAL PURPOSE

ASA REF P0310060

C***** TEST THAT EXPRESSIONS INVOLVING THE ADDITION OF

$C * * *$ DOUBLE PRECISION VALUES MAY BE FORMED

6.1 P0310070

P0310080 
$C * * * *$ GENERAL COMMENTS

C**** VARIABLES, ARRAY ELEMENTS ANO CONSTANTS ARE USED IN A

$P 0310090$

C**** VARIETY OF COMBINATIONS

$P 0310100$

$C * * * * *$

$C * * * * *$

S P E C I F I C A T I O N S SEGMENT 031

P0310110

$P 0310120$

P0310130

$[* * * * *$

$C * * * * *$

WHEN EXECUTING ONLY SEGMENT 031, THE SPECIFICATION STATEMENTS

$C * * * *$ WH!CH APPEAR AS COMMENTS, MUST HAVE THE $C=$ IN COL

$C * * * * 1$ AND 2 REMOVED

$C * * * *$

$C=$ DOUBLE PRECISION ACVD,BCVD,FFCVD,GGCVD, HHCVD

$C=1, E P 1 D(43), B C 2 D(7,4), C C 3 D(7,2,2)$

$C * * * * *$

DOUBLE PRECISION ACVD, BCVD, FFCVD, GGCVD, HHCVD

1, EP 10(43), BC2D(7,4),CC3D(7,2,2)

$C * * * * \quad 0$ U T P U T T A P E ASSIGNMENT STATEMENT. NO INPUT TAPE.

$C * * * * *$

$C * * * *$ WHEN EXECUTING ONLY SEGMENT 031 . THE STATEMENT NUVI = 6

$C * * * *$ MUST HAVE THE $C=$ IN COL 1 AND 2 REMOVED.

$C * * * * *$

$C=\quad$ NUVI $=6$

$[* * * * *$

NUVI $=6$

WRITE (NUVI, 310)

310 FORMAT (1H1,1X,27HARFAD - (031) D.P. ADDITION/I

$-16 \mathrm{H}$ ASA REF. - $6.1 / 19 \mathrm{H}$ RESULTS)

$[* * * *$ HEADER FOR SEGMENT 031 WRITTEN

$A C V D=-.0141421356237309502$

$P 0010590$

$P 0010595$

P0010600

$P 0010605$

$P 0010610$

$P 0010615$

$P 0010620$

$P 0010625$

$P 031 \mathrm{~A} 1$

$P 031 \mathrm{A2}$

$P 0310140$

$P 0070460$

$P 0070465$

$P 0070470$

$P 0070475$

$P 0070480$

$P 0070485$

$P 031 B 1$

$P 0310150$

$P 0310160$

$P 0310170$

$P 0310180$

$P 0310190$

$B C V D=14.142135623730950-1$

$E P 1 D(20)=-4.1231056256176600$

$P 0310200$

$B C 2 D(6,3)=.20615528128088301$

$P 0310210$

HHCVD $=A C V D+B C V D$

$P 0310220$

$G G C V D=B C V D+A C V D$

$P 0310230$

$P 0310240$

$E P 1 D(34)=.00303+(-300.00-2)$

$P 0310250$

$F F C V D=B C V D+A C V D+A C V D+B C V D$

$P 0310260$

$C C 3 D(7,1,1)=E P 1 D(20)+B C 20(6,3)+206.1552812808830-2+41.23105625617 P 0310270$

$1660-1+E P 10(20)$

WRITE (NUVI,312) HHCVD, GGCVD, FFCVD, EP1D(34), CC 3D $(7,1,1)$

P 0310280

P0310290

312 FORMAT ( $/ / 5(022.10 / /) / / 38$ H THE 5 ANSWERS ABOVE SHOULD BE O PLUS/ PO310300

$137 \mathrm{H}$ OR MINUS AN ERROR FACTOR OF $0.10-13$ )

P0310310

$P 0310320$

$C * * * *$ END OF TEST SEGMENT 031

C*****

C*** WHEN EXECUTING ONL̈Y SEGMENT 031, THE STOP AND END

$C * * * *$ CAROS, WHICH APPEAR AS COMMENTS, MUST HAVE THE $C=$

C***** IN COL 1 AND 2 REMOVED.

$C=E N D$

$C=\quad$ STOP

STOP

$P 0310330$

P0310340

P0310350

P0310360

P0310370

P0310380

P031C1

END

P031C2

$C * * * * *$
$C * * * *$

P0320010

$C * * * * *$

$A R B S B-(032)$

$P 0320020$

P0320030

$C * * * *$

$P 0320040$

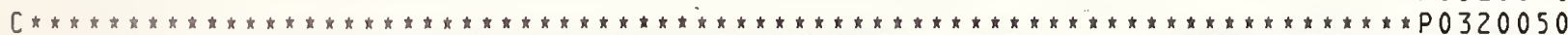

C****) GENERAL PURPOSE ASA REF P0320060

$C * * * *$ TEST THAT EXPRESSIONS INVOLVING THE SUBTRACTION OF

C***** INTEGER OR REAL VALUES MAY BE FORMED

$P 0320090$

GENERAL COMMENTS

TYPES ARE NEVER MIXED

$[* * * * *$

$[* * * *$ VARIABLES, ARRAY ELEMENTS AND CONSTANTS ARE USED IN A

$P 0320100$

PO 0320110

$[* * * *$ VARIETY OF COMBINATIONS.

$C * * * *$ S P E C I F I C A T I O N S SEGMENF 032

$P 0320120$

$P 0320130$

$P 0010630$

$P 0010635$

C***** WHEN EXECUTING ONLY SEGMENT 032. THE SPECIFICATION STATEMENTS

$C * * * *$ WHICH APPEAR AS COMMENTS, MUST HAVE THE $C=$ IN COLUMNS

$C * * * * 1$ AND 2 REMOVED

$C * * * * *$

$C=\quad$ DIMENSION A1S(5), A2S $(2,2), \operatorname{IAC} 1 \mathrm{I}(5), \operatorname{IAC} 2 I(2,7)$

$P 0010640$

P0010645

$P 0010650$

P0010655

NBS FORTRAN Test Programs Version 1 
DIMENSION AIS(5), AZS $(2,2)$, IACII $(5)$, IACZI $(2,7)$
OUT P UT T A P E ASSIGNMENT STATEMENT.

P032A1

$C * * * * 0 U T P U T$ T A P E ASSIGNMENT STATEMENT. NO INPUT TAPE.

P0320140

$C * * * * * \ldots+$ HHEN EXECUTING ONLY SEGMENT OJZ THE

$P 0070490$

C**** WHEN EXECUTING ONLY SEGMENT 032. THE STATEMENT NUVI $=6$

$P 0070495$

$C * * *$ MUST HAVE THE $C=$ IN COL 1 ANO 2 REMOVED.

$C * * * *$

$C=\ldots$ NUVI $=6$

P0070500

P0070505

P0070510

$C * * * *$

NUVI $=6$

P0070515

P03281

WRITE (NUVI, 320)

320 FORMAT ( $1 H 1,1 X, 31$ HARBSB - (O32) BASIC SUBTRACTION/I

$P 0320150$

$117 \mathrm{H}$ ASA REFS. - 6.1//2X,7HRESULTS)

$P 0320160$

$P 0320170$

P0320180

MRRVI $=1$

P0320190

WRITE (NUVI, 321)MRRVI

321 FORMAT (//2X,4HTEST, 11,1X,19HINTEGER SUBTRACTION)

$P 0320200$

P0320210

JACVI $=3$

IAC II $(1)=3$

$I H D V I=J A C V I-I A C 1 \mid(1)$

$P 0320220$

$P 0320230$

$P 0320240$

$I G O V I=I A C I I(1)-J A C V I$

IFOVI = JACVI-IAC II(1)-IAC II(1)+JACVI

$P 0320250$

$\operatorname{IAC} 2 I(2,3)=3-2-1$

$I A C 2 I(1,1)=6-J A C V I-I A C 1 I(1)$

$P 0320260$

$P 0320270$

$P 0320280$

WRITE (NUVI,323) IHOVI, IGOVI, IFOVI, IAC2I 2,3$),$ IAC2I(1,1) PO320290

323 FORMAT $(15(111 /))$

$P 0320300$

MRRVI $=2$

328 WRITE (NUVI,329)MRRVI

329 FORMAT (//2X,4HTEST, I1, 1X, 16HREAL SUBTRACT!ON)

$P 0320310$

$P 0320320$

$A C V S=5.1 E 1$

P0320330

$P 0320340$

$B C V S=.51 E 2$

$P 0320350$

HHCVS = ACVS - BCVS

$G G C V S=B C V S-A C V S$

$F F C V S=A C V S-B C V S+B C V S-A C V S$

P0320360

$P 0320370$

$P 0320380$

$A 2 S(1,2)=2.1 E 1$

$A 1 S(1)=A C V S-A 2 S(1.2)-30.0$

WRITE (NUVI,324) HHCVS, GGCVS, FFCVS, A1S(1)

P0320390

$P 0320400$

$P 0320410$

324 FORMAT (/4(F11.1/)/34H ALL ABOVE ANSWERS SHOULO BE 0 FOR/

$131 \mathrm{H}$ THIS SEGMENT TO BE SUCCESSFUL)

$[* * * * *$ ENO OF TEST SEGMENT 032

C***** W*** WHEN EXECUTING ONLY SEGMENT 032. THE STOP AND END

$C * * * *$ CAROS, WHICH APPEAR AS COMMENTS, MUST HAVE THE $C=$

$C * * * *$ IN COL 1 AND 2 REMOVED.

$C=S T O P$

$C=\quad$ END

STOP

P0320420

$P 0320430$

$P 0320440$

$P 0320450$

$P 0320460$

$P 0320470$

$P 0320480$

$P 0320490$

$P 0320500$

$P 032 C 1$

ENO

$P 032 C 2$

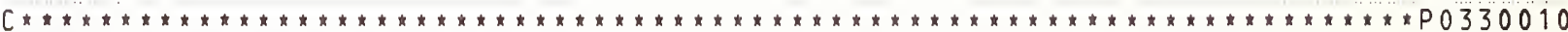

$[* * * * *$

$P 0330020$

$C * * * * *$

ARFSB - $(033)$

$P 0330030$

$P 0330040$

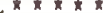

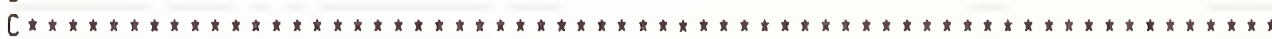

$C * * * *$ GENERAL PURPOSE
$C * * * *$ TEST THAT EXPRESSIONS INVOLVING THE SUBTRACTION OF

$\begin{array}{ll}C * * * * * & \text { TEST THAT EXPRESSIONS INVOLVING THE SUE } \\ C * * * * * & \text { DOUBLE PRECISION VALUES MAY BE FORMEO }\end{array}$

$[* * * *$ GENERAL COMMENTS

$[* * * *$ VARIABLES, ARRAY ELEMENTS AND CONSTANTS ARE USEO IN A

$C * * * * \quad$ VARIETY OF COMBINATIONS

$C * * * * *$
$C * * * * *$

C**** WHEN EXECUTING ONLY SEGMENT 033. THE SPECIFICATION STATEMENTS

$C * * * *$ WHICH APPEAR AS COMMENTS, MUST HAVE THE C $=$ IN COLUMNS

$C * * * \cdots 1$ AND 2 REMOVED

$C * * * * *$ 辛

$C=\quad$ OOUUBLE PRECISION ACVO, BCVO, CCVO,OCVO, GGCVO, HHCVD,DPCVO, FFCVO

ASA REF PO330060

6.1 P0330070

$P 0330080$

$P 0330090$

$P 0330100$

$P 0330110$

$P 0330120$

$P 0330130$

$P 0010670$

$P 0010675$

$P 0010680$

$P 0010685$

$P 0010690$

P0010695

$P 0010700$ 
DOUBLE PRECISIDN ACVD,BCVD,CCVD,OCVD,GGCVD,HHCVD,OPCVD,FFCVD

1.AC $10(10), A 2 D(2,2), A 3 D(2,2,2)$

$C * D \cup T P U T$ T A P E ASSIGNMENT STATEMENT. NO INPUT TAPE.

$C * \# * \#$ WHEN EXECUTING DNLY SEGMENT 033 , THE STATEMENT NUVI = 6

$P 0330140$

C**** MUST HAVE THE C $=$ IN CDL 1 AND 2 REMDVED.

$P 0070520$

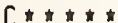

$C=$ NUVI $=6$

$C * * * * *$

NUVI $=6$

WRITE (NUVI, 330)

330 FORMAT ( 1 H $1,1 X, 30$ HARFSB- $-(033)$ D.P. SUBTRACTION/I

$P 0070525$

$P 0070530$

$P 0070535$

$P 0070540$

$P 0070545$

$\mathrm{PO} 33 \mathrm{~B} 1$

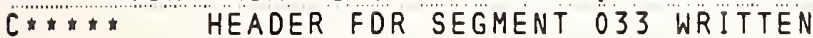

$P 0330150$

P0330160

$A C V D=1.002$

$B C V D=.301$

$C C V D=15.00$

$A C 10(1)=60.0-1$

$A 20(1,1)=-.0202$

$A 30(1,2,1)=4000.0-3$

$C * * * *$ TWD TERM SUBTRACTION

HHCVD $=A C V D-B C V D$

$H H C V D=H H C V D-97.0 D 0$

$G G C V D=1.000-A C 10(1)$

$G G C V D=G G C V D+5.000$

$D C V D=4.000-A 3 D(1,2,1)$

WRITE (NUVI, 331) HHCVD, GGCVD, DCVD

$C * * * *$ THREE TERM SUUBTRACT IDN

HHCVO $=A C V D-B C V D-97.000$

GGCVD $=16.000-C C V D-1.000$

$D C V D=A 3 D(1,2,1)-A 2 D(1,1)-6.000$

WRITE (NUVI.331) HHCVD, GGCVD, DCVD

$C * * * * *$ FOUR TERM SUBTRACTIDN

DPCVD $=6.8556546004010400$

FFCVD $=(+.34278273002005201)$

GGCVD = DPCVD - FFCVD - 42.7827300200520-2-300D-2

$P 0330170$

$P 0330180$

$P 0330190$

$P 0330200$

$P 0330210$

P0 330220

$P 0330230$

$P 0330240$

$P 0330250$

$P 0330260$

$P 0330270$

$P 0330280$

$P 0330290$

$P 0330300$

$P 0330310$

P 0330320

$P 0330330$

$P 0330340$

$P 0330350$

$P 0330360$

$P 0330370$

P0330380

$P 0330390$

HHCVD $=A C V D-A C 10(1)-A C 1 D(1)-8.801$

$D C V D=C C V D-A 2 D(1.1)-110.0-1-A C 1 D(1)$

$P 0330400$

$P 0330410$

WRITE (NUVI,332) HHCVD, DCVD, GGCVD

P 0330420

$P 0330430$

331 FDRMAT $(1 / 3(122.10 /))$

332 FDRMAT ( $/ 13(022.10 /) / 136 H$ THE ANSWERS ABOVE SHOULD BE O PLUS/

P0330440

$137 \mathrm{H}$ OR MINUS AN ERRDR FACTDR DF $0.10-13$ )

$C * * * *$ END DF TEST SEGMENT 033

$C * * * * *$

$[* * *$ WHEN EXECUTING ONLY SEGMENT 033 , THE STOP AND END

$P 0330460$

$P 0330470$

$P 0330480$

$C * * * *$ CARDS, WHICH APPEAR AS COMMENTS, MUST HAVE THE $C=$

$C * * * *$ IN CDL 1 AND 2 REMDVED.

$C=$ STDP

$C=E N D$

STOP

$P 0330490$

P0330500

$P 0330510$

P 0330520

P0330530

P033C1

END

$P 033 C 2$

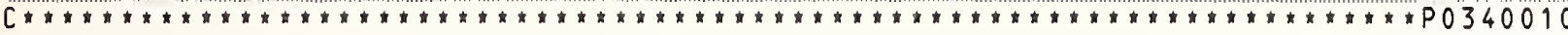

$C \star \star \star \star *$

$C * * * *$

$A R B A-(034)$

$P 0340020$

P0340030

P0340040

$C * * * *$

ARBAS - $(034)$

P0340050

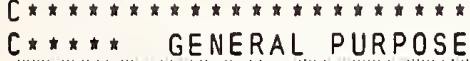

C** TEST THAT EXPRESSIDNS INVDLVING THE ADOITION AND

$C * * * *$ SUBTRACTIDN (CDMBINED) DF INTEGER DR REAL VALUES MAY BE

$C * * * *$ FORMEO

$C * \star * *$ GENERAL CDMMENTS

$C * * * *$ TYPES ARE NEVER MIXED.

$C * * * *$ VARIABLES, ARRAY ELEMENTS AND CDNSTANTS ARE USED IN

$C * * *$ A VARIETY OF COMBINATIONS.

$C * * * *$ S P E C I F I C A T I O N S SEGMENT 034

ASA REF PO340060

6.1 PO340070

$P 0340080$

P0340090

P0340100

P0340110

P0340120

P0340130

$P 0340140$

$C * * * *$

$P 0340150$

P0010710 
$C * * * *$ WHEN EXECUTING ONLY SEGMENT 034, THE SPECIFICATION STATEMENTS $C * * * *$ WHICH APPEAR AS COMMENTS, MUST HAVE THE $C=I N$ COLUMNS

$C * * * * * 1$ AND 2 REMOVED

$C * * * * *$

$C=$ DIMENSION AZS $(2,2), A 3 S(3,3,3)$

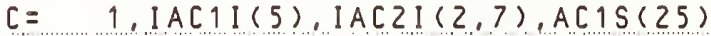

$C=\quad$ INTEGER MCA3I $(2,3,3)$

$C * * * * *$

DIMENSION AZS $(2,2), A 3 S(3,3,3)$

$1, I A C 1 I(5), I A C 2 I(2,7), A C 1 S(25)$

INTEGER MCA I $(2,3,3)$

$C * * * * \quad 0 \cup T P U T$ T A P E ASSIGNMENT STATEMENT. NO INPUT TAPE.

$C * * * * *$ WHEN EXECUTING ONLY SEGMENT 034 , THE STATEMENT NUVI = 6

$C * * * *$ MUST HAVE THE $C=I N$ COL" 1 AND 2 REMOVED.

$C * * * * *$

$C=N U V I=6$

$C * * * * *$

NUVI $=6$

WRITE (NUVI, 340)

340 FORMAT ( 1 H $1,1 X, 32$ HARBAS - (034) BASIC ADDITION AND/ $44 X$, $113 \mathrm{H}$. SUBTRACTION//16H ASA REF. - $6.4 / 1$

$22 X, 7$ HRESULTS )

$C * * * *$ HEADER FOR SEGMENT 034 WRITTEN

WRITE (NUVI.3"31)

341 FORMAT (//2X,26HTEST1 INTEGER ADD AND SUBT)

JACVI $=5$

$K B C V I=1$

LCCVI $=10$

$M D C V I=-2$

$I A C 1 I(2)=3$

$I A C 2 \perp(2,2)=-3$

$\llbracket H D V I=J A C V I+K B C V I-L C C V I+M D C V I-I A C 1 I(2)+9$

$\llbracket$ GDVI $=(J A C V I+K B C V I)-(M D C V I-I A C I I(2))-11$

IFDVI $=(6+(K B C V I-(L C C V I+M D C V I)))+1$

$\operatorname{MCA} 3 !(1,1,1)=I A C 2 I(2,2)-J A C V I-M D C V I-K B C V I+7+0$

WRITE (NUVI,342) IHOVI,IGOVI, IFDVI, MCA3I $(1,1,1)$

342 FORMAT (/4(111/))

$C * * * *$ HEADER FOR TEST 2

WRITE (NUVI,344)

344 FORMAT ( $2 X, 24 H T E S T 2$ REAL ADD AND SUBTR)

$A C V S=5.0$

$B C V S=1.0$

CCVS $=10.0$

$D C V S=-.2 E+1$

$A C 1 S(1)=30 . E-1$

$A 2 S(2,1)=6.0$

HHDVS $=$ ACVS + BCVS - CCVS + DCVS + 9.0-AC1S(1)

GGDVS $=$ (ACVS +1.0)-11.0-( DCVS-AC1S(1))

FFDVS $=(6.0+(B C V S-(C(V S+D C V S)))+1.0$

$A 3 S(1,1,2)=A 2 S(2,1)-$ CCVS $+8.0-4.0$

WR!TE (NUVI,343) HHDVS, GGDVS, FFDVS, A3S $(1,1,2)$

343 FORMAT ( $1 / 4(\mathrm{~F} 11.1 /) / 35 \mathrm{H}$ ALL ABOVE ANSWERS SHOULD BE 0 FOR/

$131 \mathrm{H}$ THIS SEGMENT TO BE SUCCESSFUL)

$C * * * *$ END OF TEST SEGMENT 034

$C * * * * *$ WHEN EXECUTING ONLY SEGMENT 034 , THE STOP AND END

$C * * * *$ CARDS WHICH APPEAR AS COMMENT CARDS MUST HAVE THE $C=$

$C * * * *$ IN COLUMNS 1 AND 2 REMOVED.

$C=\quad S T O P$

$C=\quad E N D$

STOP

END

$C * * *$

$C * * * * *$

$C * * * * *$

$C * * * * *$

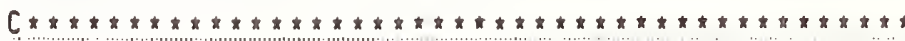

P 0010715

$P 0010720$

$P 0010725$

$P 0010730$

$P 0010735$

$P 0010740$

$P 0010745$

$P 0010750$

P034A1

P034A2

P034A3

P0340160

P0070550

P0070555

$P 0070560$

$P 0070565$

P0070570

$P 0070575$

$P 034 B 1$

$P 0340170$

P0340180

P0340190

P0340200

P0340210

P0340220

P0 340230

P0340240

P0 340250

P0340260

P0340270

P0340280

$P 0340290$

P0340300

$P 0340310$

P0340320

P 0340330

P 0340340

$P 0340350$

P0340360

$P 0340370$

P0340380

$P 0340390$

P0 340400

P0340410

P0340420

$P 0340430$

P0340440

$P 0340450$

P 0340460

P0340470

$P 0340480$

P0340490

$P 0340500$

$P 0340510$

P0340520

P0340530

P0340540

$P 0340550$

P0340560

P0340570

P0340580

$P 034 C 1$

$P 034 C 2$

0350010

$P 0350020$

P0 350030

$P 0350040$

*P0350050 
$C * * * * \quad$ VARIABLES, ARRAY ELEMENTS AND CONSTANTS ARE USED IN A

PO350100 VARIETY DF CDMBINATIDNS

PO350110

$C * * * *$

$C * \# \#$ S P E C I F I C A T I D N S SEGMENT 035

$P 0350120$

P0350130

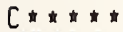

$C * \hbar * *$

WHEN EXECUTING ONLY SEGMENT 035, THE SPECIFICATIDN STATEMENTS

$C * * * *$ WHICH APPEAR AS COMMENT CARDS MUST HAVE THE C

$C * \#$ IN COLUMNS 1 AND 2 REMOVED.

$[* * * * *$

$C=$ DOUBLE PRECISION ACVO,BCVD,CCVO,OCVD,FFOVD, GGOVD, HHOVD

$C=1, A C\{D(10), B C 2 D(7,4), C[3 D(7,2,2)$

DOUBLE PRECISIDN ACVD,BCVD,CCVD,DCVD,FFDVD, GGDVD, HHOVD

$1, A C 1 D(10), B C 2 D(7,4), C C 3 D(7,2,2)$

$C * * * * *$

$C * * * * 0 \cup T P U T$ T A P E ASSIGNMENT STATEMENT. ND INPUT TAPE.

$P 0350140$

$P 0010760$

$P 0010765$

$P 0010770$

$P 0010775$

$P 0010780$

$P 0010785$

$P 0010790$

$P 035 \mathrm{~A} 1$

$P 035 A 2$

P0010795

C*** WHEN EXECUTING DNLY SEGMENT 035 . THE FOLLDWING STATEMENT

$C * * * *$ NUVI $=6$ MUST HAVE THE $C=$ IN CDLUMNS 1 AND 2 REMDVED.

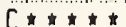

$c=$

NUVI $=6$

NUVI $=6$

WRITE (NUVI,350)

350 FDRMAT ( 1 H $1.1 X .32$ HARFAS - $(035)$ D.P. ADD AND SUBTR//2X,

$P 0350150$

$P 0070580$

$P 0070585$

$P 0070590$

$P 0070595$

PO35B 1

$P 0350160$

-14HASA REF. - 6.1//2X.7HRESULTS)

$C * * * *$ HEADER FDR SEGMENT 035 WRITTEN

$A C V D=5.0 D O$

$B C V D=10.00-1$

CCVD $=10.000$

DCVD $=-0.201$

$A C 10(1)=300.00-2$

$B C 2 D(6,3)=400 . D-2$

$A C 1 D(2)=.2481632642481605$

$B C 2 D(5,3)=-.1240816321240805$

HHDVD $=A C V D+B C V D-C C V D+D C V D+9.0 D O-A C 1 D(1)$

PO350170

PO350180

PO 350190

PO 350200

$P 0350210$

PO3 30220

PO350230

PO 350240

PO350250

P0350260

PO 350270

PO350280

GGDVD $=(A C V D+1.0 E O)-11.0 E O-(D C V D-A C 1 D(1))$

FFOVD $=(6.0 D 0+(B C V D-(C C V D+D C V D)))+10.00-1$

$C C 3 D(6,1,1)=C C V D-D C V D+B C 2 D(6,3)-A C V D-11.000$

$C C 3 D(5,1,2)=A C 1 D(2)+B C 2 D(5,3)-12408.16321240800$

PO350290

P0350300

P0350310

P0350320

WRITE (NUVI, 351) HHOVD, GGOVD, FFDVD, $C C 30(6,1,1), C C 3 D(5,1,2)$

351 FDRMAT (//5(D22.10/)//35H THE ANSWERS ABDVE SHDULD BE O FDR/

PO350330

$132 \mathrm{H}$ THIS SEGMENT TO BE SUCCESSFUL.136H VALUES WITH EXPDNENTS LEP0350350

2SS THAN $131 \mathrm{H} \quad 10 *(-14)$ ARE CONSIDERED ZERD)

$C * * * *$ END DF TEST SEGMENT 035

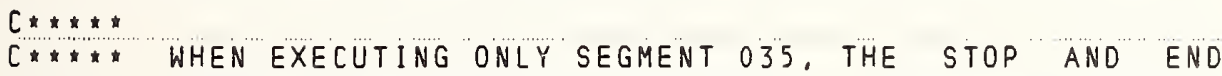

$C * * * *$ CARDS, WHICH APPEAR AS CDMMENTS, MUST HAVE THE $c=$

$C * * * *$ IN COL 1 AND 2 REMOVED.

$C=\quad S T D P$

$C=\ldots$ END

STOP

PO350360

P 0350370

P0350380

P0350390

$P 0350400$

P0350410

P. 0350420

P0 350430

P035C1

END

P035C2

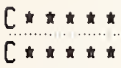

$C * * * * *$

$C * * * * *$

$C * * * * * * *$

$C * * * * *$

$[* * * * *$

$C * * * * *$

$C * * * *$

$C * * * * *$

$C * * * *$

$C * * * *$

$C * * * *$

GENERAL PURPDSE

TEST THAT EXPRESSIONS INVDLVING MULTIPLICATIDN DF INTEGER VALUES MAY BE FORMED.

GENERAL CDMMENTS

INTEGER SUBTRACTION ASSUMED WORKING

* VARIABLES, aRRAy Elements and CDNSTANTS aRe used IN A VARIETY DF COMBINATIDNS.

ARBMI - $(036)$

$P 0360010$

P0360020

P0360030

P0360040

ASA REF P0360060

$6.1 \quad P 0360070$

P0360080

P0360090

$P 0360100$

P0360110

P0360120

P0360130 
$C * * * *$ WHEN EXECUTING ONLY SEGMENT 036, THE SPECIFICATION STATEMENTS $C * * *$ WHICH APPEAR AS COMMENTS, MUST HAVE THE C $\quad$ IN COLUMNS

$C * \ldots$ AND 2 REMOVED.

$C * * * * *$

$C=$ DIMENSION IAC1 I (5), IAC2I $(2,7)$

DIMENSION IACII(5), IACZI $(2,7)$

$P 0010810$

P 0010815

P0010820

$P 0010825$

$C * * \# * *$

P036A1

$C * * * * 0 \cup T P U T$ T A P E ASSIGNMENT STATEMENT. NO INPUT TAPE.

$P 0010830$

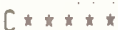

$C * * * * *$

WHEN EXECUTING ONLY SEGMENT 036, THE FOLLOWING STATEMENT

$P 0360150$

$C * \pm \cdots$

NUVI $=6$

TING ONLY SEGMENT
MUST HAVE THE

$C=$

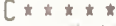

$C=$ NUVI $=6$

NUVI $=6$

$P 0070600$

$P 0070605$

$P 0070610$

$P 0070615$

$C * * * *$

WRITE (NUVI,360)

360 FORMAT ( 1 H1, 1X,36HARBMI - (036) INTEGER MULTIPLICATION//

$116 \mathrm{H}$ ASA REF - $6.1 / / 2 X, 7 \mathrm{HRESULTS})$

C*\#*\# HEADER FOR SEGMENT 036 WRITTEN

$J A C V I=1$

$K B C V I=2$

$L C C V I=0$

MDCVI $=-5$

$I A C 1 I(Z)=-10$

$\operatorname{IACZI(1,2)=3}$

$I H D V I=J A C V I * K B C V I$

$I G D V I=K B C V I * M D C V I * L C C V I$

$I F D V I=M D C V I * J A C V I * I A C I I(2) * 3$

$I E D V I=-3 * J A C V I *(-M D C V I) * J A C V I * K B C V I$

$I D D V I=K B C V I * K B C V I * K B C V I * K B C V I * K B C V I * J A C V I$

$I C D V I=(-I A C 1 I(2)) * J A C V I * K B C V I * J A C V I * K B C V I * J A C V I * 1$

$I A C 2 I(1,1)=I A C 2 I(1,2) * M D C V I * I A C 1 I(2) * 2$

IHDVI = IHDVI - 2

IFDVI = IFDVI - 150

IEDVI = IEDVI + 30

IDDVI = IDDVI - 32

ICDVI = ICDVI - 40

$\operatorname{IAC} 2 I(1,1)=I A C 2 I(1,1)-300$

WRITE (NUVI,361) IHDVI, IGDVI, IFDVI, IEDVI, IDDVI, ICDVI,

$P 0070620$

P036B1

$P 0070625$

$P 0360160$

$P 0360170$

P0360180

$P 0360190$

P0360200

P0360210

$P 0360220$

$P 0360230$

$P 0360240$

P0360250

$P 0360260$

$P 0360270$

$P 0360280$

P0360290

$P 0360300$

P0360310

$P 0360320$

P0360330

P0360340

$P 0360350$

$P 0360360$

P0360370

$P 0360380$ 1 I $A C 2 I(1,1)$

$P 0360390$

$P 0360400$

P0360410

$P 0360420$

P0360430

P0360440

C*\#***

$31 \mathrm{H}$ THIS SEGMENT TO BE SUCCESSFUL)

C****WHEN EXECUTING ONLY SEGMENT 036, THE STOP AND END

$C * * * *$ CARDS, WHICH APPEAR AS COMMENTS, MUST HAVE THE C

$C * * * *$ IN COL 1 AND 2 REMOVED.

$C=S T O P$

$C=\quad E N D$

STOP

$P 0360450$

P0360460

$P 0360470$

$P 0360480$

$P 0360490$

$P 036 C 1$

END

$P 036 C 2$

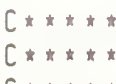

$P 0370010$

P0370020

C*\#\#***

ARBMR - (037)

P0370030

$P 0370040$

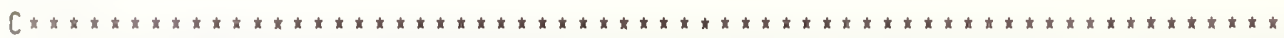

C*****) GENERAL PURPOSE

$C * * * *$ TEST THAT EXPRESSIONS INVOLVING MULTIPLICATION OF

REAL VALUES MAY BE FORMED

C**** GENERAL COMMENTS

C*\#** REAL SUBTRACTION ASSUMED WORKING

$C * * * *$ *VARIABLES, ARRAY ELEMENTS AND CONSTANTS ARE USED IN A

C**** VARIETY OF COMBINATIONS.

$C * * * * *$

$[* * * * *$

$S P E C$ I F I C A T I O N S SEGMENT 037

$P 0370050$

ASA REF PO370060

6.1 P0370070

$P 0370080$

$P 0370090$

$P 0370100$

$P 0370110$

$P 0370120$

$P 0370130$

$P 0370140$

$P 0010840$ 
C $* * * *$ WHEN EXECUTING ONLY SEgMENT 037. THE SPECIFICATION STATEMENTS

$C * * *$ WHICH APPEAR AS COMMENTS, MUST HAVE THE C= IN COLUMNS

$C * * * * * 1$ ANO 2 REMOVEO.

$C * * * * *$

$C=$ DIMENSION AZS $(2,2), A C 1 S(25)$

DIMENSION A2S(2,2),AC1S(25)

P0010845

P0010850

P0010855

P0010860

P0010865

P037A1

$[* * * * *$

C*****0 0 U T P U T T A P E ASSIGNMENT STATEMENT. NO INPUT TAPE.

P0010870

$C * * * * *$

C*** WHEN EXECUTING ONLY SEGMENT 037. THE FOLLOWING STATEMENT

$C * * * *$ NUVI $=6$ MUST HAVE THE $c=$ IN COLUMNS 1 AND 2 REMOVED.

$C * * * * *$

$C=$ NUVI $=6$

NUVI $=6$

$P 0370150$

P0070630

P0070635

P0070640

P0070645

P0070650

P03781

$C * * * * *$

WRITE (NUVI, 370)

370 FORMAT ( $1 H 1,1 X, 33$ HARBMR - $(037)$ REAL MULTIPLICATION $/ Z Z$,

114 HASA REF. - $6.1 / / 2 X, 7$ HRESULTS )

$C * * * *$ HEADER FOR SEGMENT O37 WRITTEN

ACVS $=1.0$

P0070655

P0370160

P0370170

P0370180

BCVS $=0.2 \mathrm{EL}$

CCVS $=-1.0$

DCVS $=0.0$

$A C 1 S(1)=.5 E+1$

$P 0370190$

P0370200

P0370210

P0370220

PO370230

P0 370240

HHOVS $=A C V S * B C V S$

$G G D V S=B C V S * B C V S * 1.0$

$F F D V S=2.0 * A C 1 S(1) * A C V S * A C V S$

$P 0370250$

$P 0370260$

$P 0370270$

$E E D V S=A C V S * B C V S * C C V S * D C V S * A C 1 S(1)$

DDDVS $=A C 1 S(1) * A C V S * B C V S * 1.0 E 1 * A C V S * A C V S$

$C C D V S=C C V S * C C V S * C C V S * 3 . E O * A C V S * A C V S * A C V S$

$P 0370280$

P0370290

P0370300

$A 2 S(1,1)=A C V S * C C V S * 2$.

HHDVS = HHDVS -20.0

PO370310

$P 0370320$

GGDVS = GGOVS -400.0

$P 0370330$

FFDVS = FFDVS -10.0

P0370340

DODVS $=$ DDOVS -1000.0

CCDVS $=$ CCDVS +3.0

$P 0370350$

$A 2 S(1,1)=A 2 S(1,1)+2$.

P0370360

PO370370

WRITE (NUVI, 371) HHDVS, GGDVS, FFOVS, EEDVS, DODVS, CCOVS,

P0370380

1 A2S $(1,1)$

371 FORMAT (//7(F11.1/)//35H ALL ABOVE ANSWERS SHOULO BE O FOR/

$P 0370390$

1 3IH THIS SEGMENT TO BE SULCESSFUL)

C***** END OF TEST SEGMENT O37

$C * * * *$ WHEN EXECUTING ONLY SEGMENT 037. THE STOP AND END CARDS

$C * * * *$ WHICH APPEAR AS COMMENT CARDS MUST HAVE THE C= IN COLUMNS

$C * * * * * 1$ AND 2 REMOVED.

$C=\quad S T O P$

$C=\quad E N D$

STOP

END

$P 0370400$

PO 370410

P0370420

$P 0370430$

P0370440

$P 0370450$

P0370460

P 0370470

$P 037 C 1$

$P 037 C 2$

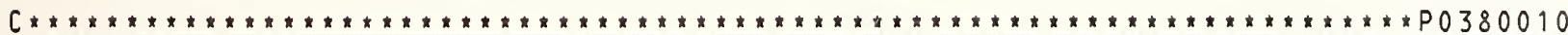

$C * * * * *$

$[* * * * *$

ARFMD - $(038)$

P0380020

$C * * * * *$

P 0380030

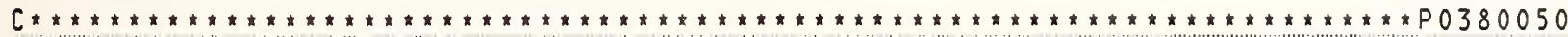

$C * * * *$ GENERAL PURPOSE

C***** TEST THAT EXPRESSIONS INVOLVING THE MULTIPLICATION 6.1 P0380070

$C * * * *$ OF DOUBLE PRECISION VALUES MAY BE FORMED

OF DOUBLE PRECIS

$C * * * * *$ GENERAL COMMENTS
$C * * * * *$ OP ADOITION AND SUBTRACTION ASSUMED WORKING.

C****** VARIABLES, ARRAY ELEMENTS ANO CONSTANTS ARE USEO IN A

C***** VARIETY OF COMBINATIONS.

$C * * * * *$

$C * * *$ S P E C I F I C A T I O N S SEGMENT 038

$C * * * * *$

$C * * * *$ WHEN EXECUTING ONLY SEGMENT 038 , THE SPECIFICATION STATEMENTS

$C * * * *$ WHICH APPEAR AS COMMENTS, MUST HAVE THE C = IN COLUMNS

$[* * * * 1$ AND 2 REMOVED.

$C * * * * *$

P0380080

P0380090

P0380100

P0380110

P0380120

P0380130

P0380140

P0010880

P 0010885

P0010890

P0010895

P0010900 
$C=$ DOUBLE PRECISION ACVD,BCVD,CCVD,DCVD,EEDVD, DDDVD,CCDVD

$C=1, F F D V D, G G D V D, H H D V D, A C 1 D(10), B C 2 D(7,4), C C 3 D(7,2,2)$

DOUBLE PRECISION ACVD, BCVD, CCVD,DCVD, EEDVD, DDDVD, CCDVD

1.FFDVD, GGDVD, HHDVD,AC $1 D(10), B C 2 D(7,4), C C 3 D(7,2,2)$

P0010905

$P 0010910$

P038A1

$C * * \# *$

$C * * * * \quad 0, U T P U T$ T A P E ASSIGNMENT STATEMENT... NO INPUT TAPE.

P038A2

$C * * * * *$

C**** WHEN EXECUTING ONLY SEGMENT 038. THE FOLLOWING STATEMENT

P0010915

$C *$ NUVI $=6$ MUST HAVE THE $C=$ IN COLUMNS 1 AND 2 REMOVED.

$C * * * * *$

$C=N$ NUVI $=6$

NUVI $=6$

$P 0380150$

$P 0070660$

$P 0070665$

P 0070670

$P 0070675$

$P 0070680$

$C * * * *$

WRITE (NUVI,380)

380 FORMAT ( 1 H $1,1 \mathrm{X}, 33$ HARFMD - $(038)$ D.P. MULTIPLICATION/ $2 X$,

$P 038 B 1$

$P 0070685$

$P 0380160$

- $15 H$ ASA REF. - 6.1//2X,7HRESULTS)

P0380170

C***** HEADER FOR SEGMENT 038 WRITTEN

$P 0380180$

$A C V D=1.000$

$P 0380190$

$P 0380200$

$B C V D=2.0$

$C C V D=-30.00-1$

$D C V D=1.0 D 1$

$A C 1 D(1)=1.101$

$C C 3 D(3,1,2)=.26214406$

$\operatorname{CC} 3 D(6,1,2)=-2000.0-3$

$P 0380210$

$P 0380220$

P0380230

$P 0380240$

$P 0380250$

$C C 3 D(3,2,2)=409.601$

$P 0380260$

$P 0380270$

$H H D V D=A C V D * B C V D$

$G G D V D=A C V D * 0.0 D O * C C V D$

$F F D V D=A C 1 D(1) * A C V D * A C V D * A C V D$

$P 0380280$

P0380290

$P 0380300$

$E E D V D=C C V D * C C V D * A C V D * 1.0 D O * B C V D$

$P 0380310$

$D D D V D=A C V D * 2.0 D 1 * A C V D * D C V D * 1.0 E 0 * C C V D$

$C C D V D=A C V D * B C V D * C C V D * C C V D * C C V D * B C V D * A C V D$

$P 0380320$

$B C 2 D(3,4)=D C V D *(400 . D-2) * C C 3 D(6,1,2)$

$P 0380330$

$P 0380340$

$B C 2 D(2,3)=C C 3 D(3,1,2) * C C 3 D(3,2,2)$

HHDVD = HHDVD -2.000

FFDVD = FFDVD - 11.000

EEDVD = EEDVD - 18.000

DDDVD = DDDVD + 600.000

CCDVD $=$ CCDVD +108.000

$B C 2 D(3,4)=B C 2 D(3.4)-(-80.00)$

$B C 2 D(2,3)=B C 2 D(2,3)-1.07374182409$

WRITE (NUVI,381) HHDVD, GGDVD, FFDVD, EEDVD, DDOVD, CCDVD.

\section{$1 \quad B C 2 D(3,4), B C 2 D(2,3)$}

381 FORMAT (/18(D22.10/)//35H THE ANSWERS ABOVE SHOULD BE 0 FOR/

131 THIS SEGMENT TO BE SUC(ESSFUL)

C $*$ END OF TEST SEGMENT 038

C.* WHEN EXECUTING ONLY SEGMENT 038 , THE STOP AND END CARDS

C* WHICH APPEAR AS COMMENT CARDS MUST HAVE THE C $C=$ IN COLUMNS

C*\#** 1 AND 2 REMOVED.

$C=$ STOP

$C=\quad E N D$

STOP

P0380350

$P 0380360$

$P 0380370$

$P 0380380$

$P 0380390$

$P 0380400$

$P 0380410$

$P 0380420$

$P 0380430$

$P 0380440$

P0 0380450

$P 0380460$

P0380470

P0380480

P0380490

$P 0380500$

P0380510

P0 380520

PO38C1

END

PO38C2

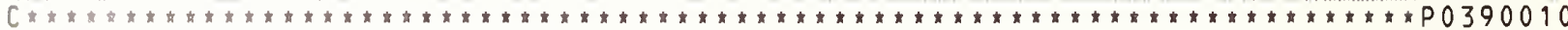

$C * * * *$

$C * \cdots *$

ARBDV - $(039)$

$P 0390020$

$P 0390030$

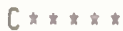

$P 0390040$

$C * * * * * *+2)$

C $* * * * *$

$C * * * * *$

$C \neq * * * *$

C $* * * *$

$C * * * * *$

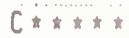

$C * * * *$

$C * \cdots *$

$C * * * * *$

$C * * * *$

$C *\|*\|$

GENERAL PURPOSE

TEST BASIC DIVISION.

INTEGER AND REAL (SP) TYPES ONLY

ASA REF PO390070

$S P E C I F$ I C A T I O N S SEGMENT 039

6.1 P0390080

$P 0390090$

P0390100

P0 390110

P0010920

WHEN EXECUTING ONLY SEGMENT 039. THE SPECIFICATION STATEMENTS

$P 0010925$

WH!CH APPEAR AS COMMENTS, MUST HAVE THE $C=1 N$ COLUMNS

1 AND 2 REMOVED.

P0010930

P0010935

P0010940 
390 FORMAT ( 1 H1, 1X,30HARBDV - (039) INTEGER AND REAL/15X,

P $039 B 1$

$P 0070715$

$P 0390130$

- 9 H DIVISION//2X, 14 HASA REF. - $6.1 / / 2 X, 7$ HRESULTS)

C** HEADER FOR SEGMENT 039 WRITTEN

$P 0390140$

$P 0390150$ WRITE (NUVI, 391)

391 FORMAT (//2X,22HTESTI INTEGER DIVISION)

P0390160

$P 0390170$

$P 0390180$

$J A C V I=1$

KBCVI $=2$

$L C C V I=0$

$M D C V I=10$

$I A C 1 I(2)=1$

$\operatorname{IAC} 2 I(1,4)=-8$

I HDVI $=K B C V I / J A C V I$

I GDVI = MDCVI/KBCVI/ JACVI

I FDVI =LCCVI/JACVI/JACVI/1

IEDVI = MDCVI/KBCVI/IAC I I (2)/IAC1I(2)/JACVI

$P 0390190$

P0390200

$P 0390210$

$P 0390220$

$P 0390230$

$P 0390240$

$P 0390250$

$P 0390260$

$P 0390270$

$P 0390280$

$I A C 2 I(1,2)=I A C 2 I(1,4) / 4 / K B C V I$

IHDVI $=$ IHDVI -2

IGDVI = IGDVI - 5

$P 0390290$

$P 0390300$

P0390310

IEDVI = IEDVI - 5

$I A C 2 I(1,2)=I A C 2 I(1.2)+1$

$P 0390320$

$P 0390330$

WRITE (NUVI.392) IHDVI, IGDVI, IFDVI,IEDVI, IACZI $(1,2)$

$P 0390340$

392 FORMAT $(/ / 5(110 /))$

$P 0390350$

WRITE (NUVI, 393 )

393 FORMAT ( $/ 2$ X, 19 HTEST 2 REAL DIVISION)

$A C V S=1.0$

$P 0390360$

$P 0390370$

$P 0390380$

$B C V S=0,0$

CCVS $=1.0 \mathrm{E} 1$

$P 0390390$

$D C V S=20.0 E-1$

$P 0390400$

$P 0390410$

$A C 1 S(1)=100.0 E-2$

$P 0390420$

$A 2 S(1,1)=-200 \cdot E-2$

HHDVS = ACVS/ACVS

GGDVS $=[C V S / A C V S /(-A C V S)$

FFDVS $=$ BCVS/CCVS/DCVS/ACVS

EEDVS $=$ CCVS/AC1S(1)/DCVS/ $(-1.0) / A C V S$

$A 2 S(1,2)=A 2 S(1,1) / A C 1 S(1) / A C V S /(-2.0 E 0)$

HHDVS $=$ HHDVS -1.0

GGDVS $=$ GGDVS +10.0

EEDVS = EEDVS + 5.0

$P 0390430$

P0390440

$P 0390450$

P0390460

$P 0390470$

P0390480

$P 0390490$

P0390500

$P 0390510$

$A Z S(1,2)=A 2 S(1,2)-1$.

WRITE (NUV!.394) HHDVS GGOVS, FFOVS, EEOVS, $A 2 S(1,2)$

394 FORMAT (//5(F11.1/)//35H ALL ABOVE ANSWERS SHOULO BE 0 FOR/

P0390520

$P 0390530$

$12 X, 29 H T H I S$ SEGMENT TO BE SUCCESSFUL)

$C * * *$ END OF TEST SEGMENT 039

$C * * * *$ WHEN EXECUTING ONLY SEGMENT 039 , THE STOP ANO END CAROS

C* WHICH APPEAR AS COMMENT CARDS MUST HAVE THE C $=$ IN COLUMNS

$[* * * * 1$ AND 2 REMOVED.

$C=\operatorname{STOP}$

$C=\quad$ END

STOP

P0390540

$P 0390550$

P0390560

$P 0390570$

P0390580

$P 0390590$

P0390600

$P 0390610$

P039C1

END

P039C2

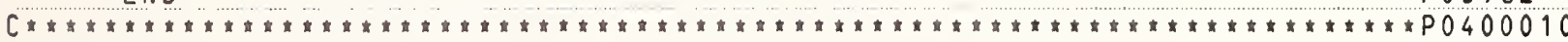

$C * t * *$

$C * * *+1$

ARFDV $-(040)$

P0 400020

P0 400030

C $* * * *$

P 0400040

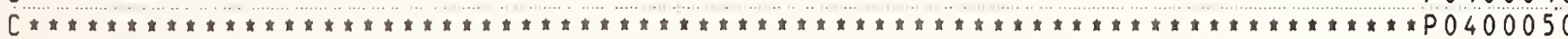

$C * * * *$ GENERAL PURPOSE ASA REFPO 400060 


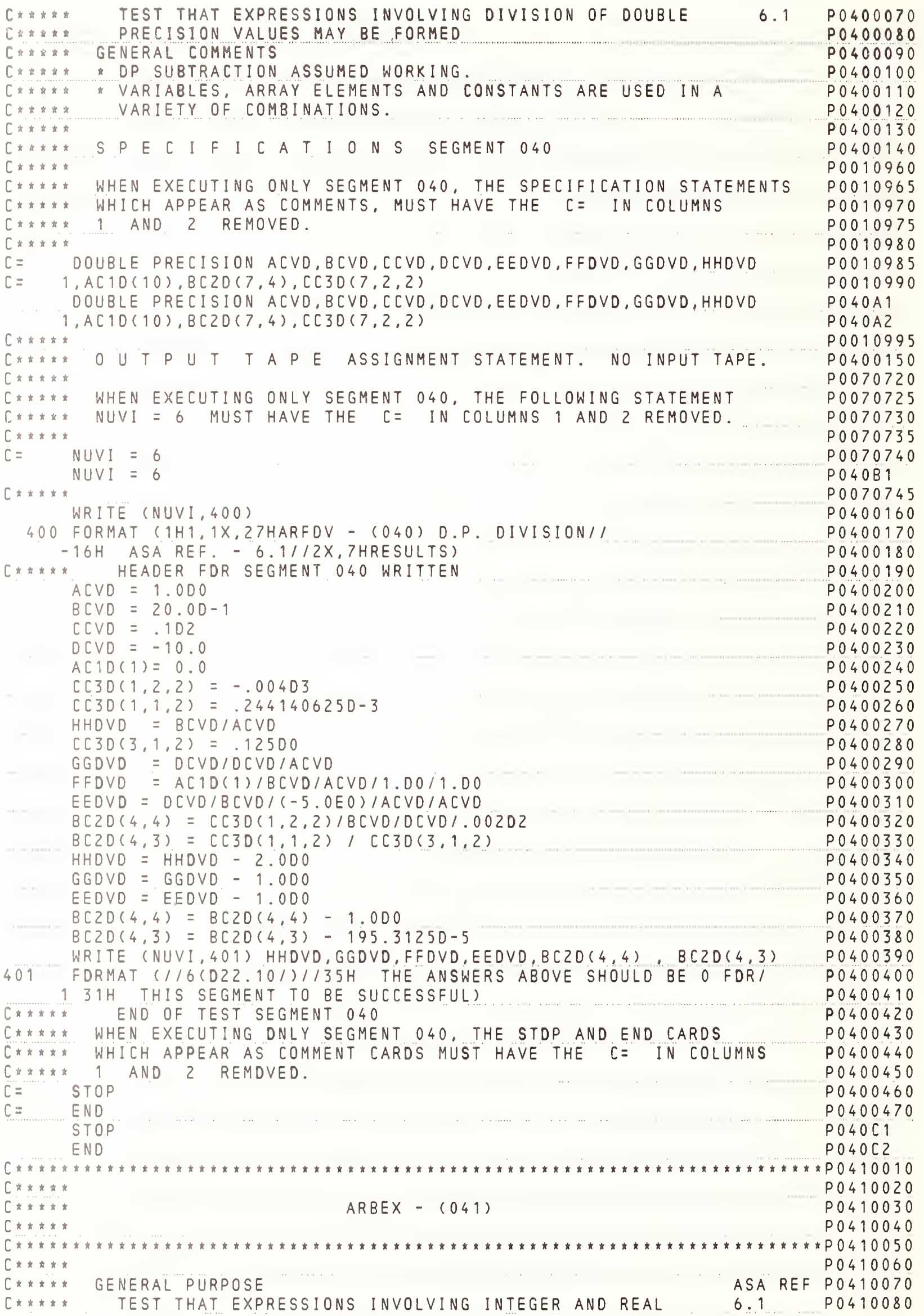


$C * * * *$ EXPDNENTIATIDN MAY BE FDRMED

6.1

REAL (SP) BY INTEGER GIVING REAL (SP)

$C * * * *$ REAL (SP) BY REAL (SP) GIVING REAL (SP)

$C * * * *$ RESTRICTIONS OBSERVEO

$C * * * * *$

$C * * * * *$ SPECIFICATTION S SEGMENT 041

$C * * * * *$

C***** WHEN EXECUTING ONLY SEGMENT 041, THE SPECIFICATION STATEMENTS

$C * * * * *$ WHICH APPEAR AS COMMENTS, MUST HAVE THE $C=$ IN COLUMNS

$C * * * * 1$ AND 2 REMOVED.

$C * * * * *$

$C=$ DIMENSION AZS $(2,2)$, IAC1I(5), IACZI(2,7), AC1S(25)

D!MENSION AZS $(2,2)$, IACII $(5)$, IACZI $(2,7), A C 1 S(25)$

$C * * * * *$

$C * * * *$ D U T P U T. T A P. E ASSIGNMENT STATEMENT. ND INPUT TAPE.

$C * * * * *$

$C * * * * *$

WHEN EXECUTING DNLY SEGMENT 041, THE FOLLDWING STATEMENT

$C * * * *$ NUVI $=6$ MUST HAVE THE $C=$ IN COLUMNS 1 ANO 2 REMOVEO.

$C * * * * *$

$C=\quad$ NUVI $=6$

$C * * * *$

NUVI $=6$

WRITE (NUVI, 410)

410 FDRMAT (1H1, 1 X,34HARBEX - $(041)$ BAS!C EXPONENTIATIDN/I

- 2X.15HASA REFS. - 6.1/12X, 7HRESULTS)

$C * * *$ HEAOER FOR SEGMENT 041 WRITTEN

WRITE (NUVI, 411)

411 FDRMAT (//2X,18HINTEGER BY INTEGER)

JACVI $=1$

$K B C V I=0$

$L C C V I=2$

MDCVI $=-1$

$I A C 1 I(2)=3$

I $A C 2$ I $(1,4)=3$

IHDVI $=$ LCCVI* IACII(2)

$I G D V I=K B C V I * J A C V I$

IFOVI $=J A C V I * * K B C V I$

IEOVI = MOCVI* $I A C 1 \rrbracket(2)$

IDOVI $=(L C C V I *$ LCCVI $) * *(J A C V I * * M D C V I)$

$I A C 2 I(1,2)=(\operatorname{LCCVI} *$ IAC2I $(1,4)) *$ JACVI

IHDVI = IHDVI -8

IFOVI = IFDVI -1

IEDVI = IEDVI + 1

IDDVI = IDOVI - 4

$I A C 2 I(1,2)=I A C 2 I(1,2)-8$

412 WRITE (NUVI, 412)

FDRMAT ( $/ / 6(1110 /)$
WRITE (NUVI, 413$)$

413 FORMAT ( / / $2 x, 25 H R E A L$ BY INT, REAL BY REAL)

$A C V S=1.0$

$B C V S=0.0$

$C C V S=0.5 E 0$

$D C V S=20.0 E-1$

$A C 1 S(1)=1.21 E 0$

$A Z S(1,1)=300 \cdot E-2$

HHOVS = ACVS**JACV!

GGDVS=BCVS**JACVI

FFDVS = DCVS *IAC II (2)

EEDVS=ACVS**ACVS

DDDVS $=A C 1 S(1) *$ CCVS

CCDVS $=(D C V S * * 1) * *(2.0 * A C V S)$

$A 2 S(2,1)=(A 2 S(1,1) * * D C V S) * B C V S$

HHDVS $=$ HHDVS -1.0

FFOVS $=$ FFOVS -8.0

EEDVS = EEDVS -1.0
$P 0410120$

PO410130

P0 410140

P0 410150

P0410160

P0410170

P0011000

PO011005

P0011010

P 0011015

P0011020

P0011025

P041A1

P0011030

$P 0410180$

$P 0070750$

P 0070755

$P 0070760$

$P 0070765$

$P 0070770$

P041B1

$P 0070775$

P0 410190

P0 410200

P0410210

$P 0410220$

$P 0410230$

P0 410240

P0410250

P0410260

$P 0410270$

P0 0410280

P0 0410290

P0410300

P0410310

P0410320

$P 0410330$

P0 0410340

P0410350

P0 0410360

$P 0410370$

P0 0410380

P0410390

P0 010400

P0 010410

P0 0410420

$P 0410430$

$P 0410440$

PO410450

P0 0410460

$P 0410470$

$P 0410480$

P0 410490

P0410500

P0410510

P0410520

$P 0410530$

P0 410540

P0 410550

P0 410560

P0410570

P0410580

$P 0410590$

P0 410600

P0410610 
WRITE (NUVI, 414) HHDVS, GGDVS, FFDVS, EEDVS, DDOVS, CCDVS, A2S(2,1)P0410650

414 FORMAT (//7(F $11.1 / 1) / / 35 \mathrm{H}$ ALL ABOVE ANSWERS SHOULD BE O FOR'

PO410660

12X, 29HTHIS SEGMENT TO BE SUCCESSFUL)

C**\#* END OF TEST SEGMENT 041

C***** WHEN EXECUTING ONLY SEGMENT 041, THE STOP AND END CARDS

C*\#\#* WHICH APPEAR AS COMMENT CARDS MUST HAVE THE C= IN COLUMNS

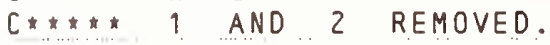

$C=S T O P$

$C=\quad E N D$

PO 410670

PO 410680

P0 0410690

P0 410700

P0 410710

$P 0410720$

P0 410730

STOP

P041C1

END

P041C2

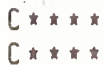

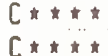

ARFEX - $(042)$

P0420010

C*****

P0420020

P0 420030

P0 420040

C

$[* * * *$ GENERAL PURPOSE

C** TEST EXPONENTIATION OF DOUBLE PRECISION ITEMS

$[* * * *$ THE FOLLOWING TYPES OF DP EXPONENTIATION ARE TESTED -

ASA REFP0420060

$C * * * *$ DP BY REAL GIVING OP

$6.1 \quad P 0420070$

$\begin{array}{ll}C * * * * * & \text { REAL BY DP GIVING DP } \\ C * * * * * & \text { DP BY DP GIVING DP }\end{array}$

$C * * * * *$ GENERAL COMMENTS

C**** \# DP ADDITION AND SUBTRACTION ASSUMED WORKING.

$[* * * *$ VARIABLES, ARRAY ELEMENTS AND CONSTANTS ARE USED IN A

$C * * * * *$ VARIETY OF COMBINATIONS.

$C * * * *$ RESTRICTION OBSERVED

C**** NEGATIVE VALUED ITEMS ARE NEVER RAISED TO A REAL OR

$[* * * *$ DP EXPONENT

C $* \pi * *$

$C * * * *$ S P E C I F I C A T I O N S SEGMENT 042

$P 0420080$

P0 420090

P0420100

$P 0420110$

P0 420120

$P 0420130$

PO420140

$P 0420150$

P0 420160

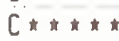

$[* * * * *$

S P E C I F I C A T I O N S SEGMENT 042

$C * *$ WHICH APPEAR AS COMMENTS, MUST HAVE THE $C=$ IN COLUMNS

$C * * * * 1$ AND 2 REMOVED.

$C * * * * *$

$C=\quad$ DOUBLE PRECISION ACVD,BCVD, CCVD,EEDVD,FFDVD, GGDVD,HHDVD

$C=\quad$ DOUBLE PRECISION ACID(10),BC2D(7,4),CC3D(7,2,2)

DOUBLE PRECISION ACVD,BCVD, CCVD, EEDVD, FFDVD, GGDVD, HHDVD

DOUBLE PRECISION ACID $(10), B C 2 D(7,4), C C 3 D(7,2,2)$

$[* * * * *$

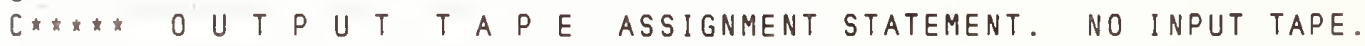

$[* * * * *$

C*\#* WHEN EXECUTING ONLY SEGMENT 042. THE FOLLOWING STATEMENT

$C * * * *$ NUVI $=6$ MUST HAVE THE $C=$ IN COLUMNS 1 AND 2 REMOVED.

$[* * * *$

$C=\quad$ NUVI $=6$

$[* * * * *$

$$
\text { NUVI }=6
$$

WRITE (NUVI, 420)

420 FORMAT ( 1 H $1,1 \times .28$ HARFEX - (042) EXPONENTIATION $/ 1$

- 16 H ASA REF. - 6.1/12X.7HRESULTS)

C***** HEADER FOR SEGMENT 042 WRITTEN

$C * * * *$ DEFINE VARIABLES AND ARRAY ELEMENTS

ACVS $=1.0$

$B C V S=0.0$

CCVS $=0.5$

$O C V S=20.0 E-1$

$A C V D=1.000$

$B C V D=80.0 D-1$

$C C V D=0.0$

$A C 10(1)=1.0$

$B C 2 D(2,4)=3000 \cdot 0-3$

HHOVD $=A C V D * B C V S$

$G G D V D=A C V S * A C V D$

$6.4 / 12 P 0420170$

$P 0420180$

PO420190

$P 0420200$

$P 0011040$

$P 0011045$

$P 0011050$

$P 0011055$

P0011060

$P 0019065$

P0011070

P042A1

P042A2

P 0011075

P0420210

$P 0070780$

P 0070785

$P 0070790$

P 0070795

P0070800

P $042 B 1$

P0070805

P0420220

P0 020230

P0 420240

P0420250

$P 0420260$

P0 420270

P0420280

$P 0420290$

P0420300

P0420310

P 0420320

P 0420330

$P 0420340$

P0420350

P0420360

P0420370 
$132 \mathrm{H}$ THIS SEGMENT TO BE SUCCESSFUL. $136 \mathrm{H}$ VALUES WITH EXPONENTS LEPO420480 ZSS THAN $131 \mathrm{H} 10 *(-14)$ ARE CONSIDERED ZERO)

\section{$C * * * * *$ END OF TEST SEGMENT 042}

$C * * * * *$ WHEN EXECUTING ONLY SEGMENT 042, THE STOP AND END CARDS

$C * * * *$ WHICH APPEAR AS COMMENT CAROS MUST HAVE THE C $=$ IN COLUMNS

$C * * * * * 1$ AND 2 REMOVED.

$C=$ STOP

$C=E N D$

$P 0420490$

P0420500

$P 0420510$

$P 0420520$

P0 420530

P0420540

STOP

$P 0420550$

END

$P 042 C 1$

$P 042 C 2$

$C * * * *$
$C * * * *$

$C * * * *$

$C * * * * *$

P0 030020

$C * * * * * *$

C***** GENERAL PURPOSE

C***** TESTS THAT HIERARCHY OF OPERATORS AND PARENTHESES

$C * * * *$ ARE HANDLED CORRECTLY. OPERATORS SHOULD FOLLOW

C***** THIS ORDER - ** (EXPONENTIATION)

$C * * * * * \quad *$ AND! (MULTIPLICATION,DIVISION)

$P 0430030$

$P 0430040$

+ AND - (ADDITION, SUBTRACTION)

$C * * * *$

$C * * * *$ GENERAL COMMENTS

$C * * * *$ ONLY INTEGER EXPRESSIONS ARE USED SINCE THIS TEST IS

$C * * * *$ CONCENTRATING ON OPERATORS AND PARENTHESES

$C * * * *$ * ADDITION, SUBTRACTION, MULTIPIICATION, DIVISION,

$C * * * *$ EXPONENTIATION ASSUMED TO FOLLOW LAWS OF

$C * * * *$ ASSOCIATION AND COMMUTATION UNLESS PARENTHESES

$C * * * * *$ REGROUP EXPRESSIONS

C****** INTEGER DIVISION MUST BE EVALUATED FROM LEFT TO

$C * * * * * \quad$ RIGHT

$C * * * * *$ RESTRICTIONS OBSERVED

$C * * * * *$ aLL ELEMENTS EVALUATED ARE MATHEMATICALLY DEFINED

$C * * * * *$ NO NEGATIVE VALUES ARE RAISED TO A REAL

$C * * * *$ EXPONENT

$C * * * * *$ NO ZERO VALUEO PRIMARY"IS RAISED TO A ZERO

$C * * * * *$ VALUED EXPONENT

$C * * * * *$

$C * * * * *$

$C * * * * *$

$C * * * * *$

$C * * * *$

$C * * * * *$

$C * * * * *$

$C=\quad$ DIMENSION IAC $1(5), I A C 2 I(2,7)$

DIMENSION IACII $(5)$, I $A C Z$ I $(2,7)$

\section{C*****}

$C * * * * *$

$C * * * * *$

$C * * * * *$

$C * * * *$

$C * * * * *$

$C=$ NUVI $=6$

$C * * * * *$

$$
\text { NUVI }=6
$$

WRITE (NUVI, 430)

430 FORMAT ( 1 H $1,1 X, 36$ HARBHI - (043) HIERARCHY, PARENTHESES//2X,

1 '23HASA REFS. - 6.1 AND 6.411

$22 X, 7 H R E S U L T S$ )
ASA REF PO430060

$6.1 / 07 P 0430070$

$P 0430080$

$6.4 / 4190430090$

$P 0430100$

$P 0430110$

P0430120

$P 0430130$

$P 0430140$

$6.4 / 49 P 0430150$

$P 0430160$

P0430170

$P 0430180$

$6.4 / 56 P 0430190$

$P 0430200$

P0 030210

$6.4 / 16 P 0430220$

$6.4 / 12 P 0430230$

$P 0430240$

$6.4 / 14 P 0430250$

$P 0430260$

$P 0430270$

$P 0430280$

P0011080

P0011085

$P 0011090$

P0011095

P0011100

P0011105

PO43A1

P0011110

$P 0430290$

P0070810

P0070815

$P 0070820$

$P 0070825$

$P 0070830$

P $043 B 1$

$P 0070835$

P 0430300

P0 430310

P0 430320

P 0430330 
JACVI $=1$

$K B C V I=2$

LCCVI $=-5$

MDCVI $=0$

NECVI $=36$

$I A C 1 I(Z)=10$

**** TEST THAT ADDITION IS COMMUTATIVE (TEST 1)

$P 0430340$

$P 0430350$

$P 0430360$

P0 430370

$P 0430380$

$P 0430390$

MRRVI $=1$

IHDVI = JACVI + KBCVI

$I G D V I=K B C V I+J A C V I$

IFDVI = IHDVI - IGDVI

P 0430400

P 0430410

$P 0430420$

$P 0430430$

WRITE (NUVI, 431) MRRVI, IFDVI

C*\# TEST IHAT MULTIPLICATION IS COMMUTATIVE (TEST 2)

MRRVI $=2$

$I H D V I=J A C V I * K B C V I$

$I G D V I=K B C V I * J A C V I$

IFDVI = IHDVI - IGDVI

P 0430440

P 0430450

P 0430460

P0 0430470

P0 0330480

P0430490

WRITE (NUVI, 431) MRRVI, IFDVI

C*\#\#\# TEST THAT SUBTRACTION IS COMMUTATIVE (TEST 3 )

MRRVI $=3$

$I H D V I=K B C V I-J A C V I$

$I G D V I=-J A C V I+K B C V I$

IFDVI = IHDVI - IGDVI

WRITE (NUVI, 431) MRRVI, IFDVI

C***** TEST THAT ADDITION IS ASSOCIATIVE (TEST 4)

MRRVI $=4$

IHDVI $=(I A C 1 I(2)+J A C V I)+K B C V I$

$I G D V I=I A C 1 I(2)+(J A C V I+K B C V I)$

IFDVI = IHDVI - IGDVI

WRITE (NUVI, 431) MRRVI, IFDVI

C***** TEST THAT MULTIPLICATION IS ASSOCIATIVE (TEST 5)

MRRVI $=5$

IHDVI $=($ IACII (2) * LCCVI) *KBCVI

$I G D V I=I A C I I(2) *(L C C V I * K B C V I)$

$P 0430500$

P0 430510

P0 030520

P0 030530

P0 430540

$P 0430550$

P0430560

$P 0430570$

P0 430580

$P 0430590$

P0 430600

P0 430610

$P 0430620$

$P 0430630$

$P 0430640$

P0430650

P 0430660

$P 0430670$

IFDVI = IHDVI - IGDVI

WRITE (NUVI, 431) MRRVI, IFDVI

C*\#\# TEST THAT MULTIPLICATION IS DONE BEFORE ADDITION

C*\#** OR SUBTRACTION (TEST 6). ANSWER SHOULD BE ZERO

MRRVI $=6$

$I H D V I=J A C V I+K B C V I * L C C V I-1+I A C 1 I(2)$

WRITE (NUVI, 431 ) MRRVI, IHDVI

C**** REGROUP TEST 6 EXPRESSION (SLIGHTLY CHANGED) WITH

C* PARENTHESES. ANSWERS SHOULD BE NON-ZERO (TEST7).

P 0430680

P 0430690

P0 0430700

P 0430710

P 0430720

P 0430730

$P 0430740$

P0 430750

P0 0430760

MRRVI $=7$

$I G O V I=(J A C V I+K B C V I) * L C C V I+9$

IFDVI = JACVI + KBCVI*(LCCVI + 9$)$

$I E D V I=(J A C V I+K B C V I) *(L C C V I+9)$

$I A C 1 I(1)=I G D V I+6$

$I A C I I(3)=I F O V I-9$

IACII $(4)=$ IEDVI - 12

WRITE (NUVI, 432) MRRVI, IACII(1), IACII(3), IAC1 I(4)

P0 430770

P0 430780

P0 430790

P0 430800

P0 430810

PO 430820

P0 430830

P0 430840

P0 430850

P0 430860

P0 430870

P0430880

P0 430890

P0 430900

P0430910

P0 430920

P0 430930

P0 430940

P0 430950

P 0430960

P 0430970

P0 430980

P0 430990

P0 431000

P0431010

C*\#\#TEST THAT EXPONENTIATION IS DONE BEFORE 
$C * * * *$ ANY OTHER OPERATION (TEST 10 ). ANSWERS SHOULO

$P 0431020$

$C * * * *$ BE ZERO.

MRRVI $=10$

IHOVI $=K B C V I+3 * * 2-11$

IGOVI $=$ IACII(2)*KBCVI*3=96

IFOVI = NECVI / LCCVI * KBCVI - 1

WRITE (NUVI.432) MRRVI, IHOVI, IGDVI, IFOVI

C***** REGROUP TEST 10 EXPRESSIONS WITH PARENTHESES (TEST 11)

$C * * * *$ ANSWERS SHOULO BE NON-ZERO

$P 0431030$

P0 431040

P0431050

$P 0439060$

P0 0431070

$P 0431080$

MRRVI $=11$

IHOVI $=(K B C V I+3) * 2-11$

IGDVI $=(I A C 1 I(2) * K B C V I) * 3-80$

IFDVI = (NECVI , LCCVI) * KBCVI - 1

$I A C I I(1)=I$ HOVI -14

IACII $(3)=$ IGDVI -13744

IACI I (4) = IFDVI - 35

WRITE (NUVI,432) MRRVI, IACII(1), IACII(3), IACII(4)

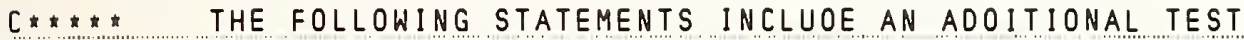

C**** OF OPERATOR HIERARCHY. A VARIETY OF OPERATORS IS USEO

C***** BOTH VARIABLES AND ARRAY ELEMENTS ARE USEO. ALL

C***** ANSWERS SHOULO BE ZERO (TEST 12).

MRRVI $=12$

$L C C V I=-5$

$I A C 1 I(2)=10$

IEOVI $=J A C V I+K B C V I * L C C V I-I A C 1 I(2) / 2-I A C 1 I(2) / 2 / 5+15$

IOOVI $=$ KBCVI**3*4+162/(3*(KBCVI*2))+MDCVI-34

IHDVI $=K B C V I *(J A C V I+K B C V I *(I A C I I(2)-K B C V I))-34$

$I G D V I=I A C 1 I(2) / K B C V I+70 /(L C C V I *(K B C V I * 2+3))-3$

IFDVI $=K B C V I *(K B C V I+I A C I I(2) *(K B C V I+3 *(J A C V I+K B C V I)))-224$

P0 431090

P0 431100

P0 431110

Po 431120

P0 431130

P0 431140

P0 431150

P0 431160

P0 431170

P0 431180

P0 431190

P0 431200

P0 431210

P0 431220

P0 431230

P0 431240

P0 431250

P0 431260

P0 431270

P0 431280

P0 431290

$I A C 1 I(1)=K B C V I *(K B C V I+K B C V I *(K B C V I+K B C V I *(K B C V I+K B C V I *$

$-(K B(V I+K B(V I))))-92$

$I A C 2 I(1,4)=I A C 1 I(2)+L C C V I+J A C V I+K B C V I+K B C V I-J A C V I-9$

$I A C 2 I(1,2)=I A C I I(2) /(L C C V I+J A C V I+K B C V I) *(K B C V I * *$

$1(K B C V I-J A C V I))+10$

WRITE (NUVI, 433) MRRVI, IEOVI, IODVI, IHDVI, IGDVI, IFDVI,

$P 0431300$

$P 0431310$

$P 0431320$

$P 0431330$

$P 0431340$

$P 0431350$

$P 0431360$

$P 0431370$

C**** EVALUATION MAY PROCEEO ACCOROING TO ANY VALIO FORMATION SEQUENCEP0431380

C***** EVALUATION OF INTEGER TERM CONTAINING OIVISION

MRRVI $=13$

NECVI $=7$

$P 0431390$

$P 0431400$

P0 0431410

$K B C V I=2$

P0 431420

$\angle C C V I=4$

IGOVI = NECVI/KBCVI* LCCVI

$I F D V I=L C C V I * N E C V I / K B C V I$

$P 0431430$

$P 0431440$

P0 0431450

IACII $(1)=$ IGDVI -12

P0 0331460

IACII $(2)=I F D V I-14$

WRITE (NUVI,434) MRRVI, IACII(1), IAC1I(2)

P0 431470

P0 431480

P0 431490

$P 0431500$

P0 0431510

FORMAT $(12 X, 4$ HTEST, 14, 16)

432 FORMAT $(12 X, 4$ HTEST, 14, 16/ $116 / 116)$

$P 0431520$

433 FORMAT(/2X, 4HTEST,14,16/6(116/),116)

FORMAT(/2X,4HTEST, $14,16 / 196 / 2 X, 35 \mathrm{H}$ THE ANSWERS ABOVE SHOULD BE 0 PO 0439530
IFOR/31H THIS SEGMENT TO BE SUCCESSFUL) P

C***** ENO OF TEST SEGMENT 043

C $* * * *$ WHEN EXECUTING ONLY SEGMENT 043. THE STOP ANO ENO CARDS

$C * * * * *$ WHICH APPEAR AS COMMENT CAROS MUST HAVE THE C = IN COLUMNS

$C * * * * * \quad 1$ AND 2 REMOVED.

$C=\quad S T O P$

$C=E N O$

STOP

$P 0431550$

$P 0431560$

P0 431570

$P 0431580$

P0 431590

P0 431600

P $043 C 1$

END

P0 $43 C 2$

$C * * * * * * * * *$

P0500010

C********

$P 0500020$

$C * * * * * *$

$S B B 67-(050)$

P0 500030

P0 500040

$C * * * * * *$

PO500050

C****** GENERAL PURPOSE

$C * * * * * *$ TEST FORMATION OF SUBSCRIPTS FOR INTEGER

ASA REFPO500060

NBS FORTRAN TEst Programs Version 1 
C***** WHEN EXECUTING ONLY SEGMENT 050., THE SPECIFICATION STATEMENTS

$C * * *$ WHICH APPEAR AS COMMENTS, MUST HAVE THE C $=$ IN COLUMNS

C*****1 AND 2 REMOVED.

$C * * * * *$

$C=$ DIMENSION AZS $(3,3,3)$

$C=\quad$ DIMENSION IACII $(5), \operatorname{IAC} I(2,7), \operatorname{AC} 1 S(25), \operatorname{ACZS}(5,6)$

$C=\quad$ INTEGER MCA3! $(2,3,3)$

DIMENSION A3S $(3,3,3)$

DIMENSION IAC1I (5), IACZI $(2,7), A C 1 S(25), A C 2 S(5,6)$

$P 0500100$

$P 0011120$

$P 0011125$

$P 0011130$

$P 0011135$

$P 0011140$

POO11145

$P 0011150$

P0011155

P050A1

INTEGER MCA3! $(2,3,3)$

C*******

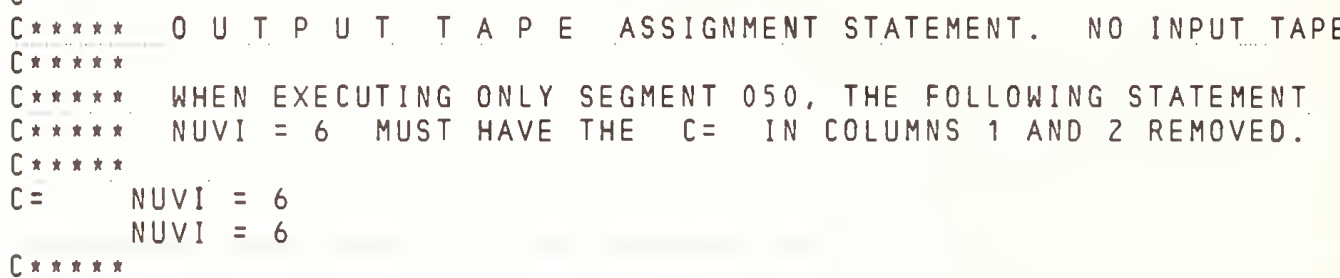

PO5OAZ

P050A3

P0011160

$P 0500110$

$P 0070840$

$P 0070845$

$P 0070850$

$P 0070855$

$P 0070860$

$P 05081$

$P 0070865$

WRITE (NUVI,501)

501 FORMAT ( 1 H $1,1 X, 36 H S B B 67$ - (050) SUBSCRIPTS FOR INTEGER/

- 16X, 21 HAND REAL ARRAYS, V, K//2X,14HASA REF. 5.1.3//2X,

- 7HRESUL.TS)

$I A C 1 \mid(5)=3$

I ACZ I $(1,3)=4$

$\operatorname{MCA} 3](2,2,1)=-7$

$A C 1 S(20)=1.0$

$A C 2 S(4,1)=-2 \cdot 1 E 1$

$\operatorname{A3S}(1,2,2)=-22.0$

$J A C V !=I A C 1 I(5)+I A C 2 I(1,3)+\operatorname{MCA} I(2,2,1)$

HHCVS $=A C 1 S(20)-A C 2 S(4,1)+A 3 S(1,2,2)$

WRITE (NUVI, 502) JACVI, HHCVS

502 FORMAT $(/ / 19 / / F 11.1)$

$504 \quad$ JACVI $=1$

$A C V S=1.0$

$I A C 1 I(J A C V I)=10$

$I A C 2 !(J A C V ! 3)=12$

IACZI(2,JACVI) $=-6$

MCA3I (JACVI, JACVI, 3) $=-1$

$M C A 3 I(2, J A C V I, J A C V I)=-1$

$M C A 3 I(J A C V I, 3, J A C V I)=-2$

$A C 1 S(J A C V I)=A C V S$

$A C Z S(J A C V I, 2)=3.0$

$A C 2 S(5, J A C V I)=60.0 E-1$

$A 3 S(J A C V I, J A C V I, 3)=+1.0$

$A 3 S(2, J A C V I, J A C V I)=+1.0$

$A 3 S($ JACVI, 3, JACVI) $=+0.0$

P0500120

P0 500130

$P 0500140$

P0500150

P0500160

P0500170

P0500180

P0500190

P 0500200

P0 500210

P0 050220

P0500230

$P 0500240$

P0500250

P0 500260

P0 0500270

P0500280

P 0500290

P0 500300

P0500310

P0 500320

P0 0500330

$P 0500340$

P0 0500350

P 0500360

$P 0500370$

P0500380

P 0500390

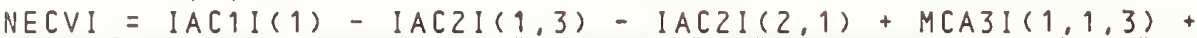

$P 0500400$

$1 \quad \operatorname{MCA} I(2,1,1)+\operatorname{MCA} 3 I(1,3,1)$

MOCVI $=$ IACII(JACVI) - IACZI(JACVI, 3)-IACZI(2,JACVI)+

1 MCA3I(JACVI,JACVI,3) + MCA3I(2, JACVI, JACVI) +

$2 \quad M C A 3 I(J A C V I, 3, J A C V I)$

P0500410

P0 500420

$P 0500430$

P0500440

HHCVS $=A C 1 S(1)+A C 2 S(1,2)-A C 2 S(5,1)+A 3 S(1,1,3)+A 3 S(2,1,1) P 0500450$

$1+\operatorname{ABS}(1,3,1)$

GGDVS $=A C 1 S(J A C V I)+A C Z S(J A C V I, 2)-A C Z S(5, J A C V I)+$

P0 500460

P0 500470

P0500480

P0 500490

P0 0500500

WRITE (NUVI,508) NECVI, MDCVI, HHCVS, GGDVS

508 FORMAT ( / $2(19 /) / 2(F 11.1 /) / 35 H$ THE ANSWERS ABOVE SHOULO BE OPO500510

FOR/31H THIS SEGMENT TO BE SUCCESSFUL)

$C * * * *$ END OF TEST SEGMENT 050

$C * * * *$ WHEN EXECUTING ONLY SEGMENT 050, THE STOP AND END CARDS

$C * * *$ WHICH APPEAR AS COMMENT CARDS MUST HAVE THE C IN COLUMNS

$P 0500530$

$P 0500540$

$P 0500550$

$P 0500560$ 
$C * * * * *$ GENERAL PURPOSE

$C * * * * *$ TEST FORMATION OF SUBSCRIPTS FOR INTEGER

$C * * * * *$

$C * * * \pi *$

$C * * * * *$

$C * * * * *$

$C * * * * *$

$C * * * * *$

$C * * * * *$

$C * * * * *$

$c=$

$S$ P E C I F I C A T I O N S SEGMENT 051

1 AND 2 REMOVED.

INTEGER MCA3I $(2,3,3)$
AND SINGLE PRECISION ARRAYS IN FORM $V+K$ AND $V-K$

WHEN EXECUTING ONLY SEGMENT O51. THE SPECIFICATION STATEMENTS WHICH APPEAR AS COMMENTS, MUST HAVE THE C = IN COLUMNS

OIMENSION IACII (5), IAC2 I $(2,7), \operatorname{AC} 1 S(25), \operatorname{AC} 2 S(5,6), A 3 S(3,3,3)$

OIMENSION IACII (5), IAC2I $(2,7), A C 1 S(25), A C 2 S(5,6), A 3 S(3,3,3)$

INTEGER MCA3I $(2,3,3)$

$C * * * * *$

$C * * * * *$

$C * * * * *$

$C * * * * *$

$C * * * *$

$C * * * *$

$C=$ NUVI $=6$

$C * * * * *$

NUVI $=6$

WRITE (NUVI , 511)

511 FORMAT ( 1 H1, 1X,36HSBB45 - (051) SUBSCRIPTS FOR INTEGER/

$-16 X, 24 H A N D$ REAL ARRAYS, $V+K, V-K / / 2 X, 16 H A S A$ REF, $5.1 .3 .3 / / 2 X$,

- 7 HRESULTS)

JACVI $=4$

IAC 1I (JACVI+1) =1

$I A C 1 !(J A C V I-1)=2$

$\triangle A C 2 !(J A C V I-2,1)=3$

$I A C 2 I(J A C V I-2,2)=4$

$\operatorname{IAC} 2 I(2, J A C V I+3)=5$

IAC2I $(1, J A C V I-0)=-3$

$A C 1 S(J A C V I+1)=1.0$

$A C 1 S(J A C V I-1)=2.0$

$A C 2 S(J A C V I+0,1)=3.0$

$A C 2 S(J A C V I-2,2)=4.0$

$A C 2 S(2, J A C V I+2)=5.0$

$A C 2 S(1, J A C V I-0)=-3.0 E 0$

NECVI $=I A C 1 I(5)+I A C 1 I(3)+I A C 2 I(2,1)+I A C 2 I(2,2)$

$-+\operatorname{IAC} 2 I(2,7)+\operatorname{IAC} 2 I(1,4)-12$

$K B C V I=I A C 1 I(J A C V I+1)+I A C 1 I(J A C V I-1)+I A C 2 I(J A C V I-2,1)+$

ASA REFPO5 10060

5.1.3.3P0510070

P0510080

$P 0510090$

P0510100

$P 0011170$

$P 0011175$

PO011180

P0011185

POO11190

P0011195

P0011200

P051A1

POS1AZ

P0011205

P0510110

P0070870

P 0070875

$P 0070880$

$P 0070885$

$P 0070890$

PO51B1

$P 0070895$

P0510120

P0510130

00510140

P0510150

00510160

P0510170

P0510180

P0 510190

P0510200

P0510210

P0510220

P0510230

P0510240

P0510250

$P 0510260$

$P 0510270$

P0510280

$P 0510290$

P0510300

$P 0510310$

1

HHCVS $=A C 1 S(5)+A C 1 S(3)+A C 2 S(4,1)+A C 2 S(2,2)+A C 2 S(2,6)$

$1 \quad A C Z S(1,4)-12.0$

GGOVS $=A C 1 S(J A C V I+1)+A C 1 S(J A C V I-1)+A C 2 S(J A C V I+0,1)+$

$1 \quad A C Z S(J A C V I-2,2)+A C 2 S(2, J A C V I+2)+A C 2 S(1, J A C V I-0)-12.0$

JACVI $=2$

MCAZI (JACVI, JACVI+1,1) $=12$

$M C A 3 !(1, J A C V I+1,3)=-4$

$M C A 3 I(1,2, J A C V I+0)=+2$

$M C A 3 I(J A C V I-1,1, J A C V I-1)=-6$

$M C A 3 I(J A C V I, J A C V I-0,2)=15$

$M C A 3 I(2, J A C V I-1, J A C V I-1)=-11$

$M C A 3 I(J A C V I-0, J A C V I+1, J A C V I+0)=-8$

$M C A 3 I(J A C V I, J A C V I+1, J A C V I+1)=\operatorname{MCA} I(J A C V I, J A C V I+1,1)+$

1

$\operatorname{MCA} 3 I(1, J A C V I+1,3)+M C A 3 I(1,2, J A C V I+0)+$

2

MCA3I (JACVI-1,1, JACVI-1) + MCA3I JACVI, JACVI-0,2)+
$P 0510320$

$P 0510330$

$P 0510340$

$P 0510350$

PO510360

$P 0510370$

$P 0510380$

$P 0510390$

P0 0510400

P0 510410

PO5 10420

$P 0510430$

$P 0510440$

P0510450

P0 510460

P0510470 
515 FORMAT (//3(19/)/3(F11.1/)/35H THE ANSWERS ABOVE SHOULD BE O FOR/PO510610

$131 \mathrm{H}$ THIS SEGMENT TO BE SUC(ESSFUL)

$C * * \pi *$ END OF TEST SEGMENT OS1

C***\# WHEN EXECUTING ONLY SEGMENT 051, THE STOP AND END CARDS

$C * * *$ WHICH APPEAR AS COMMENT CARDS MUST HAVE THE C= IN COLUMNS

$C * * * * 1$ AND 2 REMOVED.

$C=S T O P$

$C=\quad E N D$

STOP

$P 0510620$

$P 0510630$

P0510640

$P 0510650$

$P 0510660$

P0510670 END

P0510680

P051C1

$P 051 C 2$

$C * * * * *$
$C * * * * *$
$C * * * * *$

P0520010

SBB13-(052)

$P 0520020$

$P 0520030$

$C * * * * *$

P0520040

$C * * * *$

$C * * * *$ GENERAL PURPOSE

$C * * * *$ TEST FORMATION OF SUBSCRIPTS FOR INTEGER

$C * * * *$ AND SINGLE PRECISION ARRAYS

$C * * *$ FORM $C * V, C * V-K, C * V+K$

$C * * * * *$

$C * * * * *$

S P E C I F I C A T I O N S SEGMENT 052

$C * * \star * *$

$C * * * * *$

WHEN EXECUTING ONLY SEGMENT 052, THE SPECIFICATION STATEMENTS

$C * * * *$ WHICH APPEAR AS COMMENTS, MUST HAVE THE C $=$ IN COLUMNS

$C * * * * ;$ AND 2 REMOVED.

$C * * * * *$

$C=\quad$ DIMENSION IAC1 I (5), IAC2I $(2,7), A C 1 S(25), A 3 S(3,3,3), A C 2 S(5,6)$

$C=\quad$ INTEGER MCA $3 !(2,3,3)$

DIMENSION IACII(5), IACZI(2,7),AC1S(25),A3S(3,3,3),ACZS(5,6)

ASA REFSPO520060

5.1.3.3P0520070

$P 0520080$

P0520090

P0520100

P0520110

$P 0011210$

P0011215

$P 0011220$

$P 0011225$

$P 0011230$

$P 0011235$

$P 0011240$

P052A1

INTEGER MCA3I $(2,3,3)$

$C * * * * *$

$C * * * * 0$ Ü T P U T T A P E ASSIGNMENT STATEMENT. NO INPUT TAPE.

PO5ZAZ

P0011245

P0520120

$P 0070900$

$C * * * * \ldots$ WHEN EXECUTING ONLY SEGMENT OS2. THE FOLLLOWIING STATEMENT

$C * * *$ NUVI $=6$ MUST HAVE THE C $=$ IN COLUMNS 1 AND 2 REMOVED.

$C * * * * *$

$C=\quad$ NUVI $=6$

$C * * * * *$

NUVI $=6$

$P 0070905$

$P 0070910$

$P 0070915$

$P 0070920$

P052B1

$P 0070925$

WRITE (NUVI, 520)

520 FORMAT ( 1 H1.1X,36HSBB13-(052) SUBSCRIPTS INTEGER AND/

$-16 X, 23 H R E A L, C * V, C * V-K, C * V+K / / 2 X, 16 H A S A R E F, 5,1.3 .3 / 12 X$,

- 7 HRESULTS)

JACV! $=2$

KACVI $=1$

LCCVI $=-2$

$I A C 1 !(2 * J A C V I)=1$

IAC2 I $(1 * J A C V I, 1)=2$

$\triangle A C Z I(1,3 * K A C V I)=3$

$P 0520130$

P0520140

P0520150

P0520160

P0520170

P0520180

P0520190

PO520200

$P 0520210$

P0520220

$A C 1 S(2 * J A C V !)=1.0$

$A C Z S(1 * J A C V !, 1)=2.0$

P0520230

P0520240

$P 0520250$

$A C 2 S(3,3 * K A C V I)=30 . E-1$

P0 520260

MDCVI $=I A C 1 !(2 \times J A C V I)+I A C Z !(1 * J A C V !, 1)+\lfloor A C Z I(1,3 * K A C V I)-6$

$N E C V I=I A C 1 !(4)+I A C Z I(2,1)+I A C Z I(1,3)-6$

P0 520270

GGDVS $=A C 1 S(2 * J A C V I)+A C Z S(1 * J A C V I, 1)+A C Z S(3,3 * K A C V I)-6.0$

POS 20280 
HHCVS $=A C 1 S(4)+A C 2 S(2,1)+A C 2 S(3,3)-6.0$

$I A C 1 I(2 * J A C V I+1)=-6$

IAC 1I $(1, J A C V]-1)=-4$

PO520320

IAC2 I $(1 * J A C V I-1,2)=3$

P0520330

IAC2I $(2 * J A C V I-3,1)=4$

PO520340

$I A C 2 I(2,1 * J A C V I+4)=2$

PO520350

IAC2 I $(1,3 * J A C V I-2)=1$

P0 0520360

$A C 1 S(2 * L C C V I+9)=-6.0$

PO5 520370

$A C 1 S(1 * L C C V I+3)=-4.0$

PO5 520380

$A C 2 S(1 * L C C V I+3.2)=3.0$

PO520390

$A C 2 S(2 * J A C V I+0,3)=4.0$

P0520400

PO 520410

$A C 2 S(3,1 * J A C V I+3)=2,0$

PO520420

$A C 2 S(3,3 * J A C V I-2)=1.0$

PO 520430

MDCVI $=I A C 1 I(2 * J A C V I+1)+I A C 1 I(1 * J A C V I-1)+I A C 2 I(1 * J A C V I-1,2)+P 0520440$

1

2

$I A C 2 I(1,3 * J A C V I-2)$

$I A C 2 I(1 * K A C V I+0,1)+I A C 2 I(2,2 * J A C V I+2)+$

NECVI = IAC1I(5)+IAC1I(1)+ IAC2I $(1,2)$

$-+I A C 21(1,1)+I A C 2 I(2,6)+I A C 2 I(1,4)$

GGDVS $=A C 1 S(2 * J A C V I+1)+A C 1 S(1 * J A C V I-1)+A C 2 S(1 * J A C V I-1,2)+$

1 $A C 2 S(2 * J A C V I+0,3)+A C 2 S(3,1 * J A C V I+3)+A C 2 S(3,3 * J A C V I-2)$

HHCVS $=A C 1 S(5)+A C 1 S(1)+A C 2 S(1,2)$

$-+A C 2 S(4,3)+A C 2 S(3,5)+A C 2 S(3,4)$

WRITE (NUVI, 524) MDCVI, NECVI, GGDVS, HHCVS

$M C A 3 I(2 * K A C V I, 1,1)=-1$

$\operatorname{MCA} 3 I(2,2 * K A C V I, 2)=-2$

$\operatorname{MCA} I I(1,1,1 * K A C V I)=-3$

$M C A 3 I(1 * K A C V I+1,2,3)=1$

$M C A 3 I(2,1 * K A C V I+2,2)=2$

$M C A 3 I(1,2,3 * K A C V I+0)=3$

$M C A 3 I(4: K A C V I-2,1,3)=40$

MCA3I $(1,6 \times K A C V I-3,2)=5$

$M C A 3 I(2,3,10 * K A C V I-9)=-40$

MCA3I $(2 * K A C V 1,5 * K A C V I-4,2 * K A C V I+0)=-5$

$M C A 3 I(1 * K A C V I-0,3,2 * K A C V I+1)=M C A 3 I(2 * K A C V I, 1,1)+$

$M C A 3 I(2,2 * K A C V 1,2)+M C A 3 I(1,1,1 * K A C V I)+M C A$

$2+M C A 3 I(2,1 * K A C V I+2,2)+M C A 3 I(1,2,3 * K A C V I+0)$
$3+M C A 3 I(4 * K A C V I-2,1,3)+M C A 3 I(1,6 * K A C V I-3,2)$

$4+M C A 3 I(2,3,10 * K A C V I-9)+M C A 3 I(2 * K A C V I, 5 * K A C V I-4,2 * K A C V I+0)$

$A 3 S(3 * K A C V I, 1,1)=-1.0$

$A 3 S(2,2 * K A C V 1,2)=-2.0$

$A 3 S(1,1,1 \pm K A C V I)=-3.0$

$A 3 S(2 * K A C V I+1,2,3)=1.0$

$A 3 S(3,1 * K A C V I+2,2)=2.0$

$A 3 S(1,2,3 * K A C V I+0)=3.0$

A SS $(4 \times K A C V I-2,1,3)=40.0$

$A 3 S(1,6 * K A C V I-3,2)=5.0$

$A 3 S(2,3,10 * K A C V I-8)=-40.0$

$A 3 S(3 * K A C V 1,5 * K A C V I-4,2 * K A C V I+0)=-5.0$

$A 3 S(1 * K A C V I-0,3,2 * K A C V I+1)=A 3 S(3 * K A C V I, 1,1)+A 3 S(2,2 * K A C V I, 2)$ $A 3 S(1,1,1 * K A C V I)+A 3 S(2 * K A C V I+1,2,3)+A 3 S(3,1 * K A C V I+2,2)+$ $A 3 S(1,2,3 * K A C V I+0)+A 3 S(4 * K A C V I-2,1,3)+A 3 S(1,6 * K A C V I-3,2)$

\section{3}

$A 3 S(2,3,10 * K A C V 1-8)+A 3 S(3 * K A C V I, 5 * K A C V I-4,2 * K A C V I+0)$

525

WRITE (NUVI, 525) MCA3I $(1,3,3), \operatorname{ABS}(1,3,3)$

527 FORMAT ( $1 / 35 \mathrm{H}$ THE ANSWERS ABOVE SHOULD BE 0 FOR/

FORMAT (//19//F11.1)

WRITE (NUVI, 527)

$131 \mathrm{H}$ THIS SEGMENT TO BE SUCCESSFUL)

C***** END OF TEST SEGMENT 052

$C * * * *$ WHEN EXECUTING ONLY SEGMENT 052 . THE STOP AND END CARDS

$C * * *$ WHICH APPEAR AS COMMENT CARDS MUST HAVE THE C $C=$ IN COLUMNS

$C * * * * 1$ AND 2 REMOVED.

$C=S T O P$

$C=\quad$ END

STOP

END

$C * x * * * * * x$

NBS FORTRAN TESt Programs Version 1
P0520450

P0520460

PO520470

P0520480

P0520490

P0 520500

P0520510

P0520520

P0520530

P0520540

P0520550

P0520560

P0520570

P0520580

P0520590

P0520600

P0520610

P0520620

P0520630

P0520640

P0520650

P0520660

P0520670

P0520680

P0 520690

P0520700

P0520710

P0520720

P0520730

P0520740

PO520750

P0520760

P0 520770

P0520780

+P0520790

P0520800

+P0520810

P0520820

P0520830

P0520840

P0520850

P0 520860

P0520870

P0 520880

P0 520890

P0520900

P0520910

P0520920

P0520930

P052C1

P052C2

*P0530010 

TEST FORMATION OF SUBSCRIPTS FOR DOUBLE PRECISION

PO530060 $P 0530060$

$C * * *$ ARRAYS

$C * * * *$ FORMS V, K, C V ,

$C * V-K, \quad c * V+K, V+K, V-K$

ASA REF PO530070

$C *\|*\|$

$C * * *$

S P E C I F I C A T I O N S SEGMENT 053

$C * * * *$

C****WHEN EXECUTING ONLY SEGMENT 053, THE SPECIFICATION STATEMENTS

$C * * *$ WHICH APPEAR AS COMMENTS, MUST HAVE THE C = IN COLUMNS

$C * * * * * 1$ AND 2 REMOVED.

C*****

$C=\quad$ DOUBLE PRECISION AC1D(10),BC2D(7,4),CC3D(7,2,2),EP1D(43),

$C=1$ VTAVD, WTAVD, AADVD

DOUBLE PRECISION AC1D(10),BC2D(7,4),CC3D(7,2,2), EP 1D(43),

1 VTAVD, WTAVD, AADVD

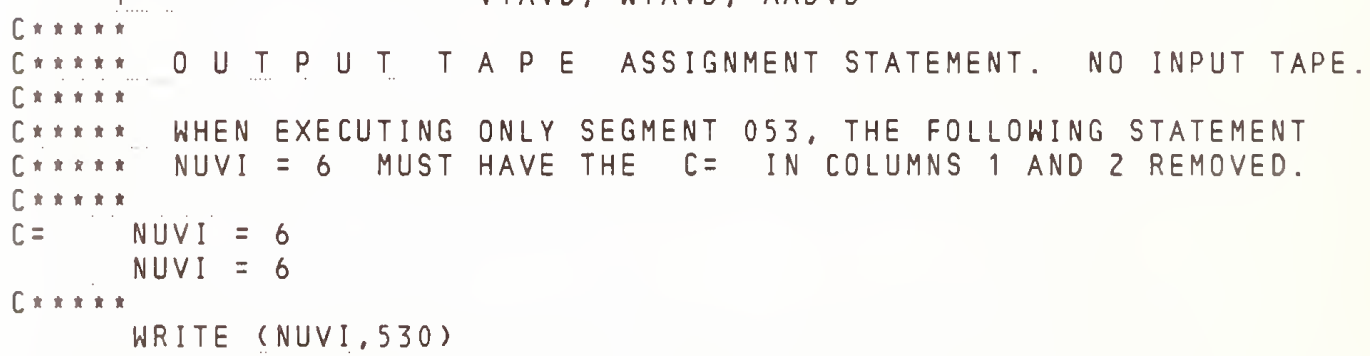

530 FORMAT ( 1 H $1,1 X, 33$ HSBF 17 - (053) SUBSCRIPTS FOR D.P. I

$-16 X, 17$ HARRAYS, ALL FORMS//2X,18HASA REF. $-5.1 .3 .3 / / 2 X, 7$ HRESULTS) NACVI $=10$

JACVI $=1$

KACVI $=+2$

LCCVI $=-1$

$E P 1 D(10)=1.00$

$B C 2 D(6,3)=4.000$

$C C 3 D(4,1,1)=-60.0 D-1$

$A C 1 D(J A C V I)=30.00-1$

$B C 2 D(J A C V I, 3)=1.000$

CC $30(J A C V I, 1,1)=2.000$

$B C 2 D(3, J A C V I)=5.000$

$C C 3 D(2, J A C V I, 1)=-2.000$

$C C 3 D(3,2, J A C V I)=.401$

VTAVD $=E P 1 D(10)+B C 2 D(6,3)+C C 3 D(4,1,1)+A C 1 D(1)$

$=+B C 2 D(1,3)+C C 3 D(1,1,1)+B C 2 D(3,1)+C C 3 D(2,1,1)$

$-+C C 3 D(3,2,1)-12.000$

$A A D V D=E P 1 D(10)+A C 1 D(J A C V I)+B C 2 D(J A C V I, 3)+B C 2 D(6,3)+$ 1

2 $C C 3 D(4,1,1)+C C 3 D(J A C V I, 1,1)+B C 2 D(3, J A C V I)+$

$C C 3 D(2, J A C V I, 1)+C[3 D(3,2, J A C V I)-12.000$

$A C 1 D(3 *$ JACVI $)=-0.60+1$

$A C 1 D(3 * J A C V I-2)=70.0 D-1$

$A C 10(5 * J A C V I+3)=1.000$

$A C 10(\mathrm{JACVI}+3)=1.000$

$A C 10(N A C V I-3)=-1.000$

$B C 2 D(6 * J A C V I, 2 * K A C V I-1)=2.000$

$B C 2 D(8 * J A C V I-2,1 * L C C V I+5)=10.000$

$C C 30(3 * J A C V I, 2,4 * K A C V I-6)=-8.000$

$C C 3 D(10 * J A C V I-3,1,1 * L C C V I+3)=-6.000$

WTAVD $=A C 1 D(3)+A C 1 D(1)+A C 1 D(8)+B C 2 D(6,3)+$

$-B C 2 D(6,4)+C C 3 D(3,2,2)+C C 3 D(7,1,2)+A C 1 D(4)+A C 1 D(7)$

$C C 30(2 * K A C V I+1, N A C V I-8,2 * J A C V I)=A C 1 D(3 * J A C V I)+$ $A C 10(3 * J A C V I-2)+A C 10(5 * J A C V I+3)+A C 10(J A C V I+3)+$ $A C 10(N A C V I-3)+B C 2 D(6 * J A C V I, 2 * K A C V I-1)+$ $B C 2 D(8 * J A C V I-2,1 * J A C V I+3)+C C 3 D(3 * J A C V I, 2,4 * K A C V I-6)+$

$4 \quad C C 3 D(10 * J A C V I-3,1,1 * J A C V I+1)$

WRITE (NUVI,531) VTAVD, WTAVD, AADVD, CC3D(5,2,2)

P 0530090

P 0530100

$P 0530110$

P 0530120

$P 0011250$

$P 0011255$

$P 0011260$

$P 0011265$

$P 0011270$

$P 0011275$

$P 0011280$

P053A1

$P 053 A 2$

$P 0011285$

P0 530130

P0070930

P0070935

$P 0070940$

$P 0070945$

P0070950

P053B1

P0070955

$P 0530140$

$P 0530150$

P0530160

P0530170

$P 0530180$

$P 0530190$

P0530200

$P 0530210$

$P 0530220$

P0 030230

P0530240

P0 530250

P0530260

P0530270

P0530280

P0 530290

P0530300

P0 530310

P0 530320

P0530330

P0530340

P0 030350

P 0530360

P0530370

P 0530380

P0530390

P0530400

P0530410

P0530420

P0530430

P0 530440

P0 530450

P0 530460

P0 530470

P0 030480

P0 0530490

P0 530500

P0530510

P0530520 
531 FORMAT (//4(018.5/)/35H THE ANSWERS ABOVE SHOULO BE 0 FOR/

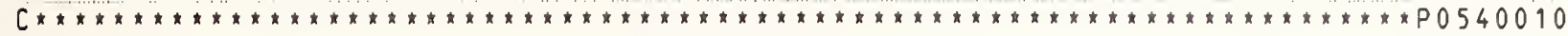

$C * * * * *$

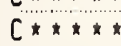

$C * * * * *$

$C * * * * * * * * * *$

$C * * * * *$ GENERAL PURPOSE

C**** TO TEST ARITHMETIC IF STATEMENT

$C * * * * *$ AND LOGICAL IF FOLLOWED BY GO TO

$C * * * *$ SO THAT THESE STATEMENTS MAY BE USED

$C * * * *$ IN SUBSEQUENT TEST SEGMENTS.

$C * * * *$

$C * * * * *$ ARITHMETIC EXPRESSIONS ARE -

$C * * * *$ INTEGER VARIABLE

$C * * * *$ INTEGER VARIABLE + OR - A CONSTANT

C**** LOGICAL EXPRESSIONS ARE -

$[* * * *$ LOGICAL VARIABLE

$C * * * * *$.NOT. LOGICAL VARIABLE

$C * * * * *$

$C * * * * *$

$C * * * * *$

$C * * * * *$

$C * * * * *$

$[* * * *$

$C * * * * *$

$C=$

$C * * * * *$

LOGICAL LVB, L1B(10), LNVB

LOGICAL LVB, L1B(10), LNVB

$C * * * * \quad 0 \quad U T P U T$ T A P E ASSIGNMENT STATEMENT. NO INPUT TAPE.

$C * * *$ WHEN EXECUTING ONLY SEGMENT 054 , THE FOLLOWING STATEMENT

$[* * * *$ NUVI $=6$ MUST HAVE THE $C=$ IN COLUMNS 1 AND 2 REMOVED.

$[* * * * *$

$C=\quad$ NUVI $=6$

NUVI $=6$

WRITE (NUVI, 7540)

IVI $=-8$

JVI $=0$

$K V I=2$

$M V I=-4$

LVB $=$ TRUE.

LNVB $=$.FALSE.

$C * * *$ LOGICAL ARRAY LIB SHOULD CONTAIN ALL. TRUE. IF TEST IS CORRECT. NVI $=1$

IF (IVI) 541, 542,542

544 IF (JVI) $542,541,542$

545 IF (KVI) $542,542,541$

$C * * * *$ ZERO IS NEITHER POSITIVE NOR NEGATIVE

$546 \mathrm{NAVI}=$ IVI* JVI

If (NAVI) 542,541, 542

547 NAVI $=$ JVI * MVI

IF (NAVI) 542,541, 542

548 NAVI $=$ JVI $/$ MVI

If (NAVI) $542,541,542$

549 IF (MVI + 4) $542,541,542$

7543 IF (KVI - 2) 542,541,542

C***** LOGICAL IF FOLLOWED BY GO TO

7544 IF (LVB) GO TO 541

GO TO 542

7545 IF (.NOT.LNVB) GO TO 541
$P 0540020$

$P 0540030$

$P 0540040$

$7.1 .2 .2 P 0540070$

7.1 .2 .3 P0540080

$4.2 \quad P 0540090$

$P 0540100$

$P 0540110$

P0540120

P0 540130

P0 540140

P0 540150

P0 540160

P0 540170

P0 540180

P 0540190

P0011290

P0011295

$P 0011300$

P0011305

$P 0011310$

P0011315

P054A1

P 0011320

$P 0540200$

$P 0070960$

$P 0070965$

$P 0070970$

$P 0070975$

P $054 \mathrm{~B} 1$

$P 0540210$

P0 0540220

P0 0540230

P0 0540240

P0 0540250

P0 540260

P0540270

P0540280

P0540290

P0540300

P0540310

P0540320

$P 0540330$

P 0540340

P0540350

P0 0540360

$P 0540370$

$P 0540380$

P0540390

P0 040400

P0540410

P0540420

P0540430

P0540440

$P 0540450$ 
$542 L 18(N V I)=$.FALSE.

P0540460

GO TO 543

P0540470

$541 L 18$ (NVI) =.TRUE.

P0 540480

$543 N V I=N V I+1$

GO TO $(544,544,545,546,547,548,549,7543,7544.7545,7546)$. NVI

PO540490

P 0540500

7546 WRITE (NUVI,7541) L1B

WRITE (NUVI,7542)

P0540510

PO5 40520

7540 FORMAT (2HI .30HSIMIF- 1054$)$ SIMPLE ARITH. IF/19X.14HAND LOGICAL PO540530 -IF//20H ASA REF. $-7.1 .2 .2 / 13 \mathrm{X}, 7 \mathrm{H} 7.1 .2 .3 / 19 \mathrm{H}$ RESULTS) PO540540

7541 FORMAT (/L4)

7542 "FORMAT ( $136 \mathrm{H}$ THE TEN ANSWERS ABOVE MUST BE TRUE)

C**** END OF TEST SEGMENT 054

C** WHEN EXECUTING ONLY SEGMENT 054, THE STOP AND END CARDS

$C * * * *$ WHICH APPEAR AS COMMENT CAROS MUST HAVE THE $C=$

$C * * * *$ IN COLUMNS 1 AND 2 REMOVED.

$C=S T O P$

$C=\quad E N D$

PO5 PO5 050550

PO540560

P0540570

PO540580

P0540590

P0540600

P0540610

PO540620

STOP

PO54C?

END

PO5 4 C2

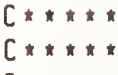

C $* * * * *$

C $* * * * *$

IFABS - (055)

*P0550010

C $* * * *$

IFABS - $(055)$

PO550020

PO550030

P0550040

$C * * * *$ GENERAL PURPOSE

$C * * * *$

$[* * * *$

O U T P U T T A P E ASSIGNMENT STATEMENT. NO INPUT TAPE.

$[* * * * *$

$C * * * *$

WHEN EXECUTING ONLY SEGMENT 055, THE FOLLOWING STATEMENT.

$[* * *$ NUVI $=6$ MUST HAVE THE C=IN COLUMNS 1 AND 2 REMOVED.

C*****

$C=$ NUVI $=6$

$C * * * *$

NUVI $=6$

WRITE (NUVI, O550)

0550 FORMAT(37H1 IFABS - $(055)$ INTRINSIC FUNCTIONS--/10X,26HABS, IABS 1ABSOLUTE VALUE)//17H ASA REFS. - $8.2 / 19 \mathrm{H}$ RESULTS)

$C * * * *$ HEADER FOR SEGMENT OS5 WRITTEN

C**** SINGLE PRECISION REAL ARGUMENT

MCGVI $=1$

CMAVS $=1.000789$

CMBVS $=-0.2 E 2$

CMCVS $=-2.0$

CMDVS $=2.0$

CMFVS $=-4.0$

CMEVS $=A B S$ (CMAVS)

CMEVS $=$ CMEVS - 1.000789

WRITE (NUVI,0557) CMEV̈S

CMBVS $=A B S(C M B \vee S)$

CMEVS $=$ CMBVS $-0.2 E 2$

WRITE (NUVI.0557) CMEVS

CMEVS $=2.0 *$ CMCVS+ABS $(2.0 *$ CMFVS+ABS (CMCVS*CMDVS*MCGVI) $)$

WRITE (NUVI,0557) CMEVS

CMEVS = CMFVS+CMDVS+ABS (CMCVS+ABS (CMFVS)-ABS (CMDVS-CMCVS)

WRITE (NUVI,0557) CMEVS

0557 FORMAT $(12 \times, F 15.1)$

0558 FORMAT (/2X,37HTHE ABOVE ANSWERS SHOULD ALL BE 0 FOR/2X,

13 SHTHIS TEST SEGMENT TO BE SUCCESSFUL.)

$C * * * *$ INTEGER ARGUMENT

$M C A V I=25$

MCBVI $=4$

MCCVI $=-129$

MCDVI $=-2$

MCEVI $=2$

MCFVI = IABS (MCAVI)

MCFVI $=M C F V I-25$

WRITE (NUVI,0551) MCFVI

ASA REF POS50060

8.2 P0550070

$P 0550080$

P0550090

P0070980

P0070985

$P 0070990$

$P 0070995$

P0071000

P05581

P0071005

P0550100

( $P 0550110$

P0550120

P0550130

P0550140

PO550150

P0550160

P0550170

P0550180

P0550190

P0550200

PO5 50210

P0 550220

PO550230

P0550240

P0550250

P0550260

P0550270

P0550280

P0550290

P0550300

PO5 50310

PO5 50320

P0550330

PO5 50340

PO5 50350

P0550360

PO550370

P0 550380

P0550390

PO550400

P0 550410

P0550420 
MCFVI $=I A B S(M C O V I+I A B S(M C B V I / M C O V I)-I A B S(M C E V I * 2))-M C B V I$

$C=$ STOP

$C=\quad$ END

STOP

$P 0550470$

$P 0550480$

P0 550490

P0550500

$P 0550510$

P0550520

PO5 050530

$P 0550540$

PO550550

END

P055C1

PO55C2

C*********t.

$C * * * * *$

$I F F T-(056)$

PO560020

$C * * * * *$

$P 0560030$

$P 0560040$

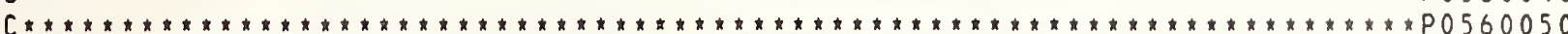

C**** GENERAL PURPOSE

$C * * * *$ TEST INTRINSIC FUNCTION FLOAT (CONVERSION FROM

$[* * * *$ INTEGER TO REAL)

$C * * * * *$

$C * * * * *$

$[* * * * *$

$C * * * *$

$C * * * * *$

$C * * * * *$

$C=\quad$ NUVI $=6$

$C * * * * *$

$$
\text { NUVI }=6
$$

WRITE (NUVI.0560)

0560 FORMAT ( 1 H $1,1 \mathrm{X}, 34 \mathrm{HIFFLT}$ - (056) INTRINSIC FUNCTION-- $/ 16 \mathrm{X}$,

15HFLOAT/ $2 X, 14$ HASA REF. - 8.2 /2X,7HRESULTS)

$C * * * * *$ HEADER FOR SEGMENT 056

$C * * * *$ ARGUMENT IS INTEGER, FUNCTION IS REAL

$M C A V I=64$

MCBVI $=-512$

$M C C V I=2$

MCOV! $=4$

MCEVI $=8$

CMAVS $=$ FLOAT (MCAVI)

CMBVS $=$ CMAVS -64.0

WRITE (NUVI.0561) CMBVS

CMAVS $=$ FLOAT (MCBVI)

CMBVS $=$ CMAVS + 512.0

WRITE (NUVI,0561) CMBVS

CMBVS $=$ FLOAT $(-2$ *MCEVI) +FLOAT(MCCVI*MCDVI)*FLOAT (MCEVI/MCOVI) -

- FLOAT(MCDVI*MCCVI) + 16.0

WRITE (NUVI,0561) CMBVS

WRITE (NUVI.0562)

WRITE (NUVI,0563)

0561 FORMAT $(/ 2 X, F 15.1)$

0562 FORMAT ( $/ 2 X, 37 H T H E$ ABOVE ANSWERS SHOULD ALL BE O FOR)

0563 FORMAT (2X,35HTHIS TEST SEGMENT TO BE SUCCESSFUL.)

$C * * *$ END OF TEST SEGMENT 056

$C * * * * *$ WHEN EXECUTING ONLY SEGMENT 056, THE STOP AND END CARDS

$C * * * * *$ WHICH APPEAR AS COMMENT CAROS MUST HAVE THE $C=$

$C * * * *$ IN COLUMNS 1 ANO 2 REMOVED.

$C=S T O P$

$C=E N D$

STOP

END

ASA REF P0560060

$8.2 P 0560070$

(TABLE 3)PO560080

P0560090

P0560100

P0071010

P0071015

P0071020

P0071025

$P 0071030$

P05681

$P 0071035$

P0560110

P0560120

P0560130

P0560140

P0560150

P0560160

P0560170

P0560180

P0560190

P0560200

P0560210

P0560220

P0560230

P0560240

P0 560250

P0560260

P0560270

P0 560280

P0560290

P0560300

P0560310

P0560320

P0560330

P0560340

P0560350

$P 0560360$

P0560370

P0560380

P0 560390

P0560400

P056C1

$P 056 C 2$

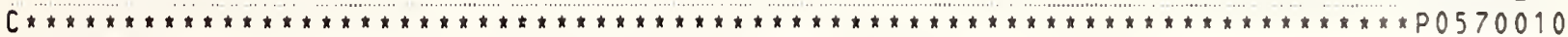

$C * * * * *$

$C * * * * \cdots+1057)$

P0 570020

P0 570030

P 0570040 


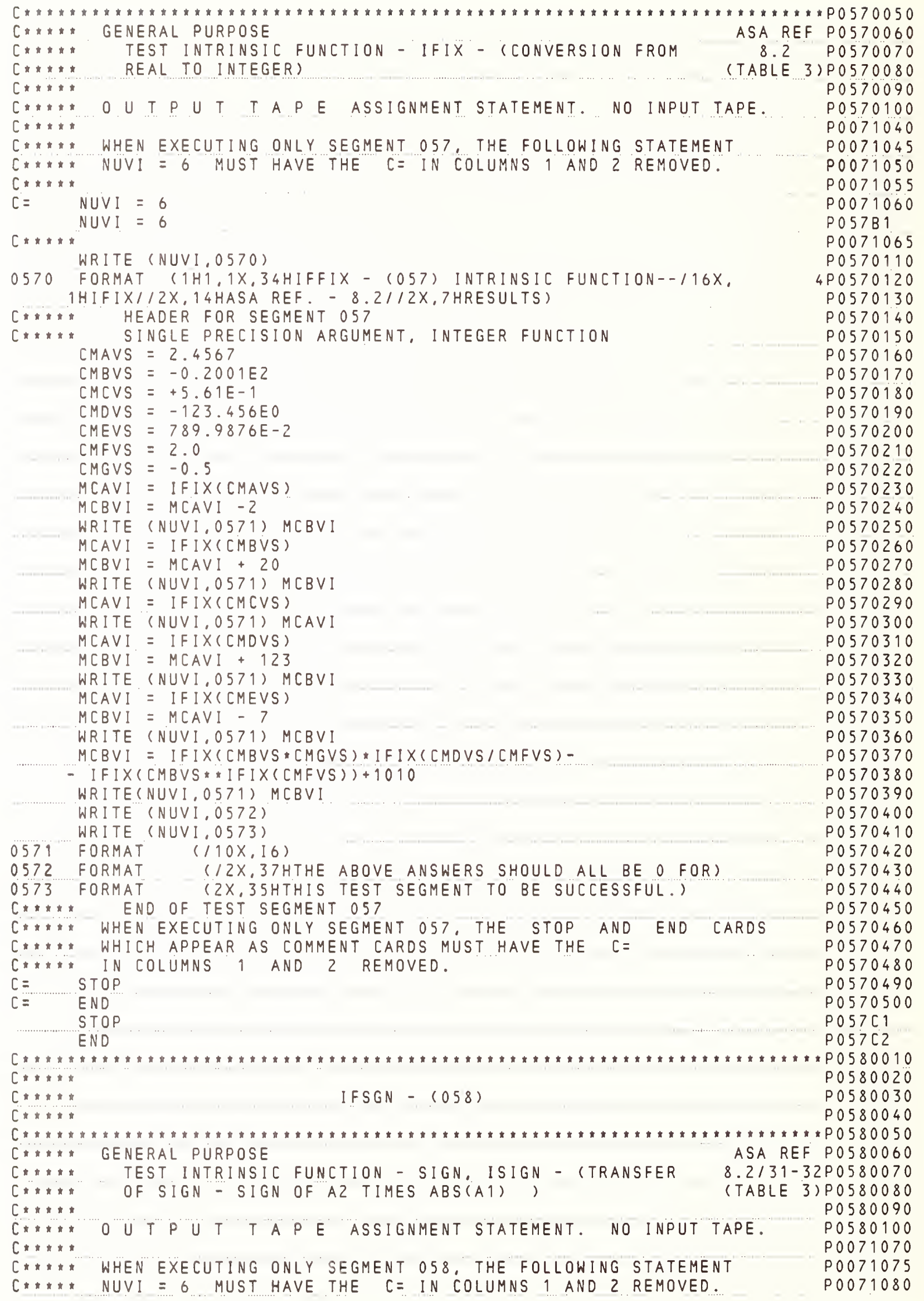


0580 FORMAT (1HI 1X,35HIFSGN - (058) INTRINSIC FUNCTIONS- $116 X, 24$

P0580110

1 HSIGN, ISIGN (TRANSFER OF/16X,14HARGUMENT SIGN)//2X,14HASA REF. PO580130

$2-8.2 / / 2 X, 7$ HRESULTS)

$[* * * *$ HEADER FOR SEGMENT 058

$[* * * *$ ARGUMENTS AND FUNCTION ARE ALL REAL-TYPE (SIGN)

P0580140

$P 0580150$

CMAVS $=1.078$

CMBVS $=-23.0 E 1$

CMCVS $=-5.4567$

CMDVS $=7.567 E-1$

CMGVS $=+2.0$

CMHVS $=-4.0$

CMIVS $=+8.0$

CMEVS $=$ SIGN(CMAVS, CMBVS)

CMFVS $=$ CMEVS +1.078

WRITE (NUVI,0581) CMFVS

CMEVS $=$ SIGN(CMAVS, CMDVS)

CMFVS $=$ CMEVS -1.078

WRITE (NUVI,0581) CMFVS

CMEVS $=$ SIGN(CMBVS, CMCVS)

CMFVS $=$ CMEVS + $23.0 E 1$

WRITE (NUVI,0581) CMFVS

P0580160

P0580170

P0580180

P0580190

P0580200

P0580210

$P 0580220$

P0 0580230

$P 0580240$

$P 0580250$

$P 0580260$

$P 0580270$

$P 0580280$

P0 580290

$P 0580300$

$P 0580310$

CMEVS $=$ SIGN (CMBVS, CMOVS)

$P 0580320$

P 0580330

CMFVS = CMEVS - $23.0 E 1$

WRITE (NUVI,0581) CMFVS

$P 0580340$

CMFVS = SIGN(CMGVS, CMHVS)*SIGN(CMHVS, CMIVS) +

P0580350

- SIGN(SIGN (CMIVS, CMBVS), SIGN(CMHVS, CMGVS))

$P 0580360$

WRITE (NUVI.0581) CMFVS

C**** ARGUMENTS AND FÜCTION ARE ALL INTEGER-TYPE (ISIGN)

$P 0580370$

$P 0580380$

MCAVI $=24$

$M C B V I=+167$

$M C C V I=-5980$

MCOVI $=-12345$

$M C G V I=2$

MCHVI $=-4$

MCIVI $=8$

MCEVI = ISIGN(MCAVI, MCBVI)

$P 0580390$

$P 0580400$

$P 0580410$

$P 0580420$

P 0580430

$P 0580440$

P0 0580450

P0 0580460

$P 0580470$

MCFVI = MCEVI - 24

P 0580480

WRITE (NUVI,0582) MCFVI

$P 0580490$

MCEVI = ISIGN(MCBVI, MCCVI)

$P 0580500$

MCFVI $=$ MCEVI +167

WRITE (NUVI.0582) MCFVI

P0580510

MCEVI = ISIGN(MCCVI,MCDVI)

P0580520

MCFVI = MCEVI + 5980

P0580530

P0580540

WRITE (NUVI.0582) MCFVI

MCEVI $=$ ISIGN(MCOVI, MCAVI)

MCFVI = MCEVI - 12345

WRITE (NUVI.0582) MCFVI

P0 0580550

$P 0580560$

$P 0580570$

MCFVI $=$ ISIGN(ISIGN(MCGVI*MCHVI+(2*MCIVI), MCIVIIMCGVI+MCCVI)+

$P 0580580$

1 ISIGN $(+8, M C H V I / M C G V I+M C C V I), M C I V I)-M C H V I * 2$

WRITE (NUVI.0582) MCFVI

P0580590

$P 0580600$

$P 0580610$

WRITE (NUVI,0583)

WRITE (NUVII,0584)

$P 0580620$

P0580630

$P 0580640$

0581 FORMAT $\quad(12 \times, F 15.1)$

0582 FORMAT (/10X.15)

0583 FORMAT (I2X,37HTHE ABOVE ANSWERS SHOULO ALL BE $O$ FOR)

P0 0580650

$P 0580660$

( $2 X, 3$ SHTHIS TEST SEGMENT TO BE SUCCESSFUL.)

P0 0580670

$[* * * *$ END OF TEST SEGMENT 058

$C * \#$ WHEN EXECUTING ONLY SEGMENT 0 58, THE STOP AND END CARDS

$P 0580680$

$C * * * *$ WHICH APPEAR AS COMMENT CARDS MUST HAVE THE $C=$

$C * * *$ IN COLUMNS 1 AND 2 REMOVED.

$C=\quad S T O P$

$C=\quad$ END

STOP

$P 0580690$ $P 0580700$

$P 0580710$

$P 0580720$

P0 0580730

P0 58 C 1 
C** WHEN EXECUTING ONLY SEGMENT 059" THE" SPECIFICATION STATEMENTS

$C * * * *$ WHICH APPEAR AS COMMENT CARDS, MUST HAVE THE $C=$

(TABLE 3) PO590080

C*****) IN COLUMNS 1 AND 2 REMOVED.

$C * * * * *$

$C=\quad$ DOUBLE PRECISION OPAVD,OPBVD,OPCVD,OPDVD,OPEVD,OPFVD,DPGVD

$C * * * * *$

DOUBLE PRECISION OPAVD,OPBVD,OPCVD,OPDVD,OPEVD,OPFVD,OPGVD

P0590090

$P 0590100$

P0011330

$P 0011335$

$P 0011340$

P0011345

P0011350

POO11355

P059A1

C***** O U T P U T T A P E ASSIGNMENT STATEMENT. NO INPUT TAPE.

$P 0011360$

$C * * * * *$

$C * * * * *$

WHEN EXECUTING ONLY SEGMENT O59. THE FOLLOWING STATEMENT

P0590110

$C * * * * *$

NUVI $=6$ MUST HAVE THE C $=I N$ COLUMNS 1 AND 2 REMOVED.

$C * * * * *$

$C=\quad$ NUVI $=6$

$C * * * * *$

NUVI $=6$

WRITE (NUVI,0590)

0590 FORMAT ( 1 H1, 1X,34HIFOAB - (059) INTRINSIC FUNCTION--i16X, 123 HDABS (ABSOLUTE VALUE OF/16X,16HA D.P. ARGUMENT)/ $2 X$,

214 HASA REF. -8.211

$32 \times, 7$ HRESULTS)

$C * * * *$ HEADER FOR SEGMENT O59 WRITTEN

C*****ARGUMENT AND FUNCTION ARE DOUBLE PRECISION

OPAVD $=1.234567890123400$

DPBVD $=-2.000$

$D P C V D=-39.4680246813570-1$

OPDVO $=2.000$

OPGVD $=-4.000$

DPEVD $=1.000$

DPEVD = DABS (DPAVD)

OPFVD = DPEVD - 1.234567890123400

$P 0071100$

$P 0071105$

P0071110

P0071115

$P 0071120$

P059B1

P0071125

PO590120

P0590130

P0590140

P0590150

P0590160

PO590170

P0590180

P0590190

P0590200

P0590210

P0590220

P0590230

P0590240

P0590250

P0590260

WRITE (NUVI, 0591) DPFVD

OPEVD $=2.0 D O * D P B V D+D A B S(D P O V D * D P G V D+D A B S(D P G V D /(2.0 D O * D P D V D)$

P0590270

$P 0590280$

- *DPDVD**2))

WRITE (NUVI.0591) DPEVD

OPEVO $=3.000$

DPEVO = DABS(DPCVD)

DPFVD = DPEVD - 39.4680246813570-1

WRITE (NUVI,0591) DPFVO

OPEVO $=4.000$

$D P E V D=D P G V D+D P D V D+D A B S(D P B V D+D A B S(D P G V D)-D A B S(D P D V D-D P B V D))$

WRITE (NUVI.0591) DPEVD

WRITE (NUVI,0592)

WRITE (NUVI,0593)

0591 FORMAT (I D22.10)

0592 FORMAT ( / 39 H THE ABOVE ANSWERS SHOULD ALL BE 0 FOR)

0593 FORMAT ( $36 \mathrm{H}$ THIS TEST SEGMENT TO BE SUCCESSFUL)

P0590290

P0590300

P0590310

P0590320

P0590330

P0590340

P0590350

P0590360

P0590370

P0590380

P0590390

P0590400

P0590410

$P 0590420$

C***** END OF TEST SEGMENT O59

$C * * * *$ WHEN EXECUTING ONLY SEGMENT 059. THE STOP AND END CARDS

PO590430

$C *$ WHICH APPEAR AS COMMENT CARDS MUST HAVE THE C $=$

$C * * * *$ IN COLUMNS 1 AND 2 REMOVED.

$C=$ STOP

$C=\ldots \ldots N D$

STOP

END

$P 0590440$

P0590450

P0590460

P0590470

P0 590480

PO59C1

PO59C2

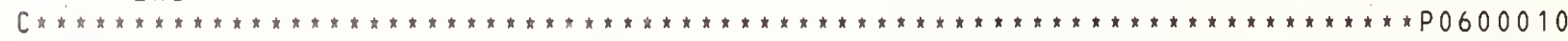

$[* * * * *$

$P 0600020$ 
$[* * * * * * * * * * * * * * * * * * * * * * *$
$C * * * *$ GENERAL PURPOSE $\begin{array}{ll}C * * * * & \text { TEST INTRINSIC FUNCTIONS AINT. INT AND IOINT - } \\ C * * * * * & \text { TRUNCATION (SIGN OF A * LARGEST INTEGER LE ABS(A) }\end{array}$

$C * * * * *$ TRUNCATION (SI

$C * * * * *$

$C * * * * *$ $S P E C I F I C A T$ I O N S SEGMENT 060

$C * * * * *$

$C * * * *$ WHEN EXECUTING ONLY SEGMENT 060, THE SPECIFICATION STATEMENTS

$C * * * *$ WHICH APPEAR AS COMMENT CARDS, MUST HAVE THE $C=$

$C * * * * *$ IN COLUMNS 1 AND 2 REMOVED.

$C * * * * *$

$C=\quad$ DOUBLE PRECISION OPAVD,OPBVD,DPCVD,DPDVD

DOUBLE PRECISION DPAVD,DPBVD,DPCVD,DPDVD

$\begin{array}{lll}C * * * * & 0 \\ C * * * * & 0 & T\end{array}$ U U T T A P E ASSIGNMENT STATEMENT. NO INPUT TAPE.

$[* * * * *$

$C * * *$ WHEN EXECUTING ONLY SEGMENT 060. THE FOLLOWING STATEMENT

$C * * * *$ NUVI $=6$ MUST HAVE THE $C=$ IN COLUMNS 1 AND 2 REMOVED.

$C * * * * *$

$C=$

NUVI $=6$

$C * * * * *$

NUVI $=6$

ASA REF

8.2

(TABLE

P0600050

$P 0600060$

P0600070

PP0600080

P0600090

P0600100

P0011370

P0011375

P0011380

P0011385

P0011390

P0011395

P060A1

PO0 11400

P0600110

P0071130

P0071135

P 0071140

P0071145

$P 0071150$

P060B1

P0071155

WRITE (NUVI.0600)

$P 0600120$

0600 FORMAT (1H1, 1X,34HIFTRN - (060) INTRINSIC FUNCTION- $110 X, 29 H A 1 N T, P 0600130$

1 INT, IOINT (TRUNCATION)1116H ASA REF. - $8.21 / 2 X, 7 H R E S U L T S$ )

$C * * * *$ HEADER FOR SEGMENT O6O WRITTEN

C*** TEST OF AINT - REAL ARGUMENT AND "REAL FUNCTION

CMAVS $=1.999$

CMBVS $=999.001$

CMCVS $=-0.45678$

CMOVS $=-9876.0$

CMEVS $=1.0$

CMEVS $=$ AINT (CMAVS)

CMFVS $=$ CMEVS -1.0

WRITE (NUVI.0601) CMFVS

CMEVS $=2.0$

CMEVS $=$ AINT (CMBVS)

CMFVS $=$ CMEVS -999.0

WRITE (NUVI, 0601 ) CMFVS

CMEVS $=3.0$

CMEVS $=$ AINT(CMCVS)

CMFVS = CMEVS

WRITE (NUVI,0601) CMFVS

CMEVS $=4.0$

CMEVS $=$ AINT (CMDVS)

CMFVS $=$ CMEVS +9876.0

WRITE (NUVI.0601) CMFVS

WRITE (NUVI,0603)

$C * * * *$ TEST OF INT - REAL ARGUMENT BUT INTEGER FUNCTION

MCAVI $=5$

MCAVI = INT (CMAVS)

MCBVI = MCAVI - 1

WRITE (NUVI, 0604) MCBVI

MCAVI $=6$

MCAVI $=$ INT (CMBVS)

MCBVI = MCAVI - 999

WRITE (NUVI.0604) MCBVI

MCAVI $=7$

MCAVI $=$ INT (CMCVS)

WRITE (NUVI,0604) MCAVI

MCAVI $=8$

MCAVI $=I N T(C M D V S)$

MCBVI $=$ MCAVI +9876

WRITE (NUVI,0604) MCBVI

WRITE (NUVI,0605)

$C * * * * *$ TEST OF IDINT - DOUBLE PRECISION ARGUMENT AND FUNCTION

P0600140

P0600150

$P 0600160$

P0600170

P0600180

P0600190

P0600200

P0600210

$P 0600220$

$P 0600230$

P0600240

P0600250

P0600260

$P 0600270$

P0600280

P0600290

P0600300

P0600310

P0600320

$P 0600330$

P0600340

P0600350

P0600360

P0600370

P0600380

P0600390

$P 0600400$

P0600410

P0600420

P0600430

P0600440

P0600450

P0600460

$P 0600470$

P0600480

P0600490

P0600500

P0600510

P0600520

P0600530

P0600540

P0600550

NBS FORTRAN Test Programs Version 1 
DPAVD $=1.999999999999901$

$P 0600560$

DPBVD $=+99.00050018912300$

$P 0600570$

OPCVO $=-0.987654321019800$

$P 0600580$

DPDVD $=-456.7890987651401$

$P 0600590$

MCAVI $=9$

MCAVI = IDINT(DPAVD)

MCBVI = MCAVI - 19

WRITE (NUVI,0606) MCBVI

$P 0600600$

$P 0600610$

$P 0600620$

$P 0600630$

MCAVI $=10$

MCAVI = IDINT(DPBVD)

MCBVI = MCAVI - 99

$P 0600640$

$P 0600650$

WRITE (NUVI,0606) MCBVI

$P 0600660$

$P 0600670$

MCAVI $=11$

MCAVI = IDINT(DPCVD)

WRITE (NUVI,0606) MCAVI

$P 0600680$

$P 0600690$

$P 0600700$

MCAVI $=12$

MCAVI $=$ IDINT(DPDVD)

MCBVI = MCAVI + 4567

$P 0600710$

$P 0600720$

$P 0600730$

WRITE (NUVI,0606) MCBVI

$P 0600740$

WRITE (NUVI,0607)

WRITE (NUVI,0608)

$P 0600750$

$P 0600760$

0601 FORMAT (/F11.1)

0603 FORMAT ( $2 X .16$ HEND OF AINT TEST)

0604 FORMAT (/I10)

0605 FORMAT ( $2 X, 15$ HENO OF INT TEST)

0606 FORMAT (/I10)

0607 FORMAT ( $2 X, 17$ HEND OF IOINT TEST)

0608 FORMAT ( $40 \mathrm{H}$ ALL ABOVE ANSWERS SHOULO BE O FOR THIS/

$131 \mathrm{H}$ TEST SEGMENT TO BE SUCCESSFUL)

$P 0600770$

$P 0600780$

$P 0600790$

$P 0600800$

$P 0600810$

$P 0600820$

$P 0600830$

$P 0600840$

$P 0600850$

C**** END OF TEST SEGMENT 060

C*** WHEN EXECUTING ONLY SEGMENT 060, THE STOP ANO END CARDS

$C * * *$ WHICH APPEAR AS COMMENT CAROS MUST HAVE THE C

C**** IN COLUMNS 1 AND 2 REMOVED.

$C=S T O P$

$C=\quad$ ENO

STOP

$P 0600860$

$P 0600870$

$P 0600880$

$P 0600890$

$P 0600900$

$P 060 C 1$

END

$P 060 C 2$

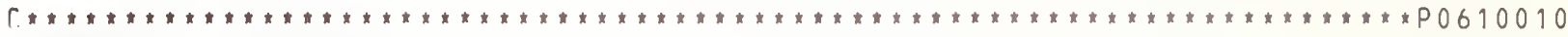

し*****

C $* * * *$

IFMOO - (061)

$P 0610020$

P0610030

$P 0610040$

$C * * * *$

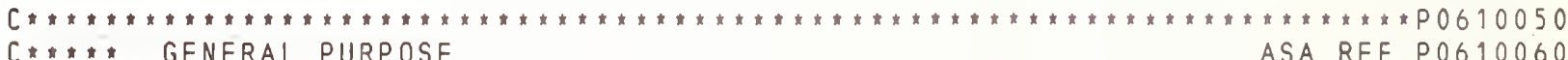

C**** TEST INTRINSIC FUNCTION AMOD ANO MOD - REMAINOERING,

C**** WHICH IS DEFINED AS A1-(A1/A2)AZ WHERE (X) IS AN

8.2 P0610070

$[* * *$ INTEGER WHOSE MAGNITUDE IS LE ABS $(X)$ AND WHOSE SIGN

(TABLE 3)P0610080

$P 0610090$

$C * *$ IS THE SAME AS $X$.

$P 0610100$

$C * * * *$

$C * * * *$

O Ü T P U T T A P ASSIGNMENT STATEMENT. NO INPUT TAPE.

$C * * * * *$

C**** WHEN EXECUTING ONLY SEGMENT 061, THE FOLLOWING STATEMENT

$C * * * *$ NUVI $=6$ MUST HAVE THE $C=$ IN COLUMNS 1 AND 2 . REMOVEO.

C*****

$c=$

NUVI $=6$

NUVI $=6$

C*****

WRITE (NUVI.0610)

P0610110

$P 0610120$

$P 0071160$

$P 0071165$

$P 0071170$

$P 0071175$

$P 0071180$

P061B1

$P 0071185$

$P 0610130$

0610 FORMA T (1H1, 1X,34HIFMOD - (061) INTRINSIC FUNCTION- 116 X,24HAMO0,P0610140 $1 \mathrm{MO}^{\circ}$ 'M EMAINDERING)//16H ASA REF. $-8.2 / 12 \mathrm{X}, \quad \mathrm{P} 0610150$

$27 !$ LTS)

C**** HLADER FOR SEGMENT 061 WRITTEN

$[* * * *$ TEST OF AMOD - REAL ARGUMENTS AND REAL FUNCTION

¿MAVS $=16.0625$

$P 0610160$

$P 0610170$

$P 0610180$

P 0610190

$P 0610200$

CMBVS $=-4.0$

CMCVS $=-8.125$

CMOVS $=2.5$

$P 0610210$

$P 0610220$

P0610230

CMEVS $=-1.0$

CMFVS $=1.0$

P0610240 
CMFVS $=A M O D$ (CMAVS, CMBVS)

$P 0610250$

CMGVS $=$ CMFVS -0.0625

P0610260

WRITE (NUVI.0611) CMGVS

P0610270

CMFVS $=2.0$

CMFVS = AMOD (CMCVS,CMDVS)

P0610280

P0610290

CMGVS $=$ CMFVS + 0.625

WRITE (NUVI.0611) CMGVS

CMFVS $=3.0$

CMFVS $=$ AMOD (CMBVS, CMEVS)

CMGVS $=$ CMFVS +0.0

WRITE (NUVI.0611) CMGVS

CMFVS $=4.0$

CMFVS $=$ AMOO (CMBVS, CMAVS)

CMGVS $=$ CMFVS + 4.0

WRITE (NUVI,0611) CMGVS

WRITE (NUVI,0612)

$C * * * *$ TEST OF MOD - INTEGER ARGUMENTS ANO INTEGER FUNCTION

$M C A V I=35$

$M C B V I=-5$

$M C C V I=-998$

$M C D V I=9$

MCEVI $=10$

$M C F V I=1$

$M C F V I=M O D(M C A V I, M C B V I)$

$M C G V I=M C F V I+0$

WRITE (NUVI,0613) MCGVI

$M C F V I=2$

$M C F V I=M O D(M C C V I, M C D V I)$

$M C G V I=M C F V I+8$

WRITE (NUVI,0613) MCGVI

$M C F V I=3$

MCFVI $=$ MOD (MCAVI, MCDVI)

MCGVI = MCFVI - 8

WRITE (NUVI,0613) MCGVI

$M C F V I=4$

$M C F V I=M O D(M C B V I, M C E V I)$

MCGVI $=$ MCFVI +5

WRITE (NUVI,0613) MCGVI

WRITE (NUVI,0614)

0611 FORMAT (/F11.1)

0612 FORMAT ( $/ / 2 X, 17$ HEND OF AMOD TEST.)

0613 FORMAT (/110)

0614 FORMAT (//2X,16HEND OF MOD TEST.//2X,

$138 \mathrm{HALL}$ ABOVE ANSWERS SHOULO BE O FOR THIS/2X.

23OHTEST SEGMENT TO BE SUCCESSFUL..)

$P 0610300$

P0610310

$P 0610320$

$P 0610330$

P0 0610340

$P 0610350$

P0610360

P0 0610370

P06 10380

$P 0610390$

P0610400

$P 0610410$

$P 0610420$

P0610430

P0610440

P0610450

P0610460

P 0610470

P0610480

P0610490

P0610500

P0610510

P0610520

P0610530

P0610540

P0610550

P0610560

PD 610570

P0610580

P0610590

P0610600

P0610610

P0610620

P0610630

$\mathrm{P} 0610640$

P0610650

P0610660

P0610670

P0610680

P0610690

$C * * * * *$ END OF TEST SEGMENT 061

C***** WHEN EXECUTING ONLY SEGMENT 061. THE STOP AND END CARDS

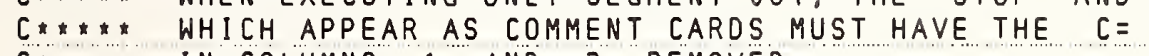

C***** IN COLUMNS 1 AND 2 REMOVED.

$C=S T O P$

$C=E N D$

STOP

$P 0610700$

$P 0610710$

P0610720

P0610730

P0610740

P0610750

P061C1

END

$P 061 C 2$

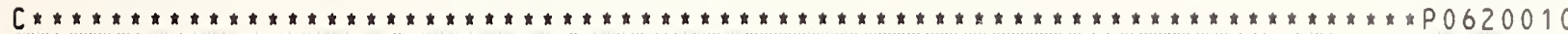

$C * * * *$

$P 0620020$

$C * * * * *$

IFMAX - $(062)$

P0620030

$[* * * * *$

$P 0620040$

C

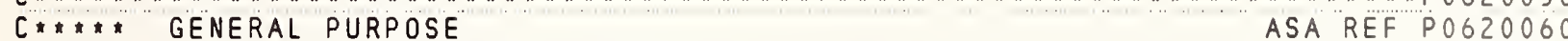

$C * * * * *$ TEST OF INTRINSIC FUNCTION AMAX0, AMAX1, MAXO,MAX1 AND . $8.2 \quad$ PO 620070

C**** DMAX1 - CHOOSING LARGEST VALUE

(TABLE 3)POO20080

$P 0620090$

$P 0620100$

C*\#\# S P E C I F I C A T I O N S SEGMENT O6Z

$P 0011410$

$[* * * * *$

WHEN EXECUTING ONLY SEGMENT 062, THE SPECIFICATION STATEMENTS

$P 0011415$

$C * * * *$ WHICH APPEAR AS COMMENT CARDS, MUST HAVE THE $C=$

$P 0011420$

$P 0011425$

$C * * * *$ IN COLUMNS 1 AND 2 REMOVEO.

P0011430 
$[* *$ WHEN EXECUTING ONLY SEGMENT 062, THE FOLLOWING STATEMENT

P0620110

P0071190

$C * * *$ NUVI $=6$ MUST HAVE THE $C=I N$ COLUMNS 1 AND 2 REMOVED.

$P 0071195$

$[* * * * *$

$C=\quad$ NUVI $=6$

NUVI $=6$

P0071200

$P 0071205$

$P 0071210$

C****

P062B1

WRITE (NUVI,0620)

P0071215

$P 0620120$

0620 FORMAT ( $1 \mathrm{H} 1,1 \mathrm{X}, 35 \mathrm{HIFMAX}$ - (062) INTRINSIC FUNCTIONS- $113 \times, 28 \mathrm{HAMAXPO620130}$

10, AMAX1, MAX0, MAX1,DMAX1 / 2X,14HASA REF, - 8.2//2X,7HRESULTS) P0620140

C**** TEST OF AMAXO - INTEGER ARGUMENTS, REAL FUNCTION $8.2119 P 0620150$

$[* *$ TWO ARGUMENTS FOR AMAXO

$P 0620160$

WRITE (NUVI,0625)

$P 0620170$

MCAVI $=128$

$P 0620180$

MCBVI $=64$

$M C C V I=-8$

MCDVI $=-4096$

$P 0620190$

$P 0620200$

CMEVS $=1.0$

$P 0620210$

CMEVS = AMAXO (MCAVI, MCBVI)

CMFVS $=$ CMEVS -128.0

$P 0620220$

P0620230

WRITE (NUVI,0621) CMFVS

$P 0620240$

$P 0620250$

CMEVS $=2.0$

CMEVS = AMAXO (MCCV!, MCCVI)

CMFVS $=$ CMEVS +8.0

P0620260

$P 0620270$

$P 0620280$

WRITE (NUVI,0621) CMFVS

CMEVS $=3.0$

CMEVS = AMAXO (MCAVI, MCCVI)

$P 0620290$

CMFVS $=$ CMEVS -128.0

WRITE (NUVI,0621) CMFVS

CMEVS $=4.0$

CMEVS $=$ AMAXO(MCCVI, MCOVI)

CMFVS $=$ CMEVS +8.0

WRITE (NUVI,0621) CMFVS

CMEVS $=5.0$

$P 0620300$

$P 0620310$

$P 0620320$

$P 0620330$

$P 0620340$

$P 0620350$

$P 0620360$

$P 0620370$

$P 0620380$

CMEVS = AMAXO(MCDVI, MCBVI)

CMFVS $=$ CMEVS -64.0

WRITE (NUVI,0621) CMFVS

$M C G V I=2$

WRITE (NUVI,0622) MCGVI

C**** THREE ARGUMENTS FOR AMAXO

CMEVS $=6.0$

CMEVS $=$ AMAXO (MCCVI, MCBVI, MCAV!)

CMFVS $=$ CMEVS -128.0

WRITE (NUVI.0621) CMFVS

CMEVS $=7.0$

CMEVS = AMAXO (MCDVI, MCBVI, MCCVI)

CMFVS $=$ CMEVS -64.0

WRITE (NUVI,0621) CMFVS

$P 0620390$

$P 0620400$

P0620410

$P 0620420$

$P 0620430$

$P 0620440$

$P 0620450$

$P 0620460$

$P 0620470$

$P 0620480$

$P 0620490$

P0620500

P0620510

P0620520

CMEVS $=8.0$

P0620530

CMEVS = AMAXO (MCDVI, MCCVI, MCCVI)

$P 0620540$

CMFVS $=$ CMEVS +8.0

WRITE (NUVI,0621) CMFVS

$M C G V I=3$

WRITE (NUVI,0622) MCGVI

$C * *$ FOUR OR FIVE ARGUMENTS FOR AMAXO

CMEVS $=9.0$

CMEVS = AMAXO (MCAVI, MCBVI, MCCVI, MCDVI)

CMFVS $=$ CMEVS -128.0

WRITE (NUVI,0621) CMFVS

CMEVS $=10.0$

CMEVS = AMAXO (MCAVI, MCBVI, MCCVI, MCOVI, MCAVI)

CMFVS $=$ CMEVS -128.0

$P 0620550$

$P 0620560$

$P 0620570$

$P 0620580$

$P 0620590$

$P 0620600$

$P 0620610$

$P 0620620$

$P 0620630$

$P 0620640$

$P 0620650$

$P 0620660$

$P 0620670$

WRITE (NUVI.0621) CMFVS

WRITE (NUVI,0623)

$P 0620680$ 
C***** TEST OF AMAXI - REAL ARGUMENTS AND FUNCTION

$C * * * *$ THO ARGUMENTS FOR AMAX 1

8.212000620690 WRITE (NUVI.0624)

$P 0620700$

CMAVS $=102.0 E 0$

P0620710

CMBVS $=+76.12$

$P 0620720$

CMCVS $=-85.43 E 1$

P0620730

CMDVS $=-0.986$

CMEVS $=A M A X 1$ (CMAVS, CMBVS)

CMFVS = CMEVS - $102.0 \mathrm{EO}$

WRITE (NUVI,0621) CMFVS

CMEVS = AMAX1 (CMBVS, CMCVS)

CMFVS $=$ CMEVS -76.12

WRITE (NUVI,0621) CMFVS

P0620740

P0620750

$P 0620760$

P0620770

$P 0620780$

$P 0620790$

CMEVS = AMAX1 (CMDVS, CMCVS)

CMFVS $=$ CMEVS +0.986

WRITE (NUVI,0621) CMFVS

P0620800

P0620810

$P 0620820$

P0620830

P0620840

$M C G V I=2$

WRITE (NUVI,0622) MCGVI

$C * * * *$ THREE ARGUMENTS FOR AMAX 1

$P 0620850$

P0620860

$P 0620870$

CMEVS = AMAX1 (CMCVS, CMBVS, CMAVS)

$P 0620880$

CMFVS = CMEVS - 102.0EO

WRITE (NUVI.0621) CMFVS

$P 0620890$

CMEVS = AMAX1 (CMDVS, CMBVS, CMCVS)

$P 0620900$

CMFVS = CMEVS - 76.12

WRITE (NUVI.0621) CMFVS

P0620910

$P 0620920$

CMEVS = AMAX1 (CMCVS, CMCVS, CMCVS)

P0620930

$P 0620940$

$P 0620950$

CMFVS = CMEVS - CMCVS

WRITE (NUVI,0621) CMFVS

MCGVI $=3$

WRITE (NUVI,0622) MCGVI

$C * * * * *$ FOUR OR FIVE ARGUMENTS FOR AMAXI

$P 0620960$

P0620970

P0620980

$P 0620990$

P0621000

CMEVS = AMAX1 (CMAVS, CMBVS, CMCVS, CMDVS)

$P 0621010$

CMFVS $=$ CMEVS - $102.0 E 0$

WRITE (NUVI,0621) CMFVS

CMEVS = AMAX1 (CMAVS, CMCVS, CMDVS, CMBVS, CMAVS)

$P 0621020$

CMFVS = CMEVS - $102.0 E 0$

P0621030

$P 0621040$

WRITE (NUVI,0621) CMFVS

$P 0621050$

WRITE (NUVI,0623)

$C * * * *$ TEST OF MAXO - INTEGER ARGUMENTS AND FUNCTION

C***** TWO ARGUMENTS FOR MAXO

P0621060

WRITE (NUVI,0628)

MCEVI = MAXO(MCAVI, MCBVI)

MCFVI = MCEVI - 128

WRITE (NUVI,0626) MCFVI

MCEVI = MAXO(MCCVI, MCDVI)

$P 0621070$

P0621080

P0621090

P0621100

$P 0621110$

$P 0621120$

$P 0621130$

$P 0621140$

MCFVI = MCEVI + 8

$P 0621150$

WRITE (NUVI,0626) MCFVI

MCEVI $=M A X O(M C B V I, M C C V I)$

P0621160

MCFVI = MCEVI - 64

WRITE (NUVI,0626) MCFVI

$P 0621170$

P0621180

MCEVI = MAXO(MCCVI, MCCVI)

$P 0621190$

MCFVI = MCEVI - MCCVI

WRITE (NUVI,0626) MCFVI

P0621200

P0621210

$M C G V I=2$

WRITE (NUVI,0622) MCGVI

$C * * * *$ THREE ARGUMENTS FOR MAXO

$P 0621220$

P0621230

$P 0621240$

MCEVI = MAXO(MCCVI, MCBVI, MCAVI)

P0621250

P0621260

MCFVI = MCEVI - 128

WRITE (NUVI,0626) MCFVI

MCEVI $=$ MAXO(MCDVI,MCDVI,MCCVI)

P0621270

$P 0621280$

$P 0621290$

WRITE (NUVI,0626) MCFVI

MCGVI $=3$

WRITE (NUVI,0622) MCGVI

$C * * * *$ FOUR OR FIVE ARGUMENTS FOR MAXO

$P 0621300$

P0621310

$P 0621320$

P0621330

MCEVI = MAXO(MCDVI, MCCVI, MCBVI, MCAVI)

P 0621340

MCFVI = MCEVI - 128

P0621350

WRITE (NUVI,0626) MCFVI

P0621360 
MCEVI $=M A X O(M C A V I, M C C V I, M C B V I, M C D V I, M C B V I)$

$P 0621370$

MCFVI $=$ MCEVI - 128

$P 0621380$

WRITE (NUVI,0626) MCFVI

$P 0621390$

WRITE (NUVI, 0623)

C*** TEST DF MAX1 - REAL ARGUMENTS AND INTEGER FUNCTION

$P 0621400$

$C * * * *$ TWD ARGUMENTS FOR MAX 1

WRITE (NUVI,0629)

MCEVI $=$ MAXI (CMAVS, CMBVS)

MCFVI = MCEVI - 102

WRITE (NUVI,0626) MCFVI

MCEVI $=$ MAX1(CMBVS, CMCVS)

MCFVI $=$ MCEVI -76

WRITE (NUVI,0626) MCFVI

MCEVI $=$ MAX1 (CMOVS, CMCVS)

$M C F V I=M C E V I+0$

WRITE (NUVI,0626) MCFVI

MCGVI $=2$

WRITE (NUVI,0622) MCGVI

$C * * * *$ THREE ARGUMENTS FOR MAX1

MCEVI $=\operatorname{MAX} 1$ (CMCVS, CMBVS, CMAVS)

MCFVI $=$ MCEVI -102

WRITE (NUVI,0626) MCFVI

MCEVI $=$ MAX1 (CMOVS,CMCVS,CMBVS)

MCFVI $=$ MCEVI - 76

WRITE (NUVI,0626) MCFVI

$M C G V I=3$

WRITE (NUVI,0622) MCGVI

C**** FDUR DR FIVE ARGUMENTS FOR MAX 1

MCEVI $=$ MAX 1 (CMAVS, CMBVS, CMCVS,CMDVS)

MCFVI = MCEVI - 102

WRITE (NUVI,0626) MCFVI

MCEVI $=$ MAX1(CMAVS, CMCVS, CMBVS, CMAVS, CMDVS)

MCFVI = MCEVI - 102

WR!TE (NUVI,0626) MCFVI

WRITE (NUVI,0623)

C**** TEST OF DMAX! - DOUBLE PRECISIDN ARGUMENTS AND FUNCTIDN $8.2 / 23 P$

$C * * * *$ TWD ARGUMENTS FOR DMAX1

WRITE (NUVI, 9999)

$M C A V D=23.00-1$

$M C B V D=111.78978900$

MCCVD $=-99.660-1$

$M C D V D=-456.12300$

$M C E \vee D=D M A X 1$ (MCAVD, MCBVD)

MCFVD = MCEVD - 111.78978900

8. 2/22P0621410

$P 0621420$

$P 0621430$

$P 0621440$

$P 0621450$

$P 0621460$

$P 0621470$

$P 0621480$

$P 0621490$

$P 0621500$

$P 0621510$

$P 0621520$

$P 0621530$

$P 0621540$

$P 0621550$

$P 0621560$

$P 0621570$

$P 0621580$

P0621590

P0621600

$P 0621610$

$P 0621620$

$P 0621630$

$P 0621640$

$P 0621650$

$P 0621660$

$P 0621670$

$P 0621680$

$P 0621690$

$P 0621700$

$P 0621710$

WRITE (NUVI.0627) MCFVD

$M C E V D=D M A X 1$ ( MCAVD, MCCVD)

$M C F V D=M C E V D-23.00-1$

WRITE (NUVI,0627) MCFVD

MCEVD = DMAX1 (MCDVD, MCCVD)

$M C F V D=M C E V D+99.660-1$

WRITE (NUVI.0627) MCFVD

$M C E V D=D M A X 1$ (MCOVD, MCDVD)

MCFVD = MCEVD - MCDVD

WRITE (NUVI,0627) MCFVD

MCGVI $=2$

WRITE (NUVI,0622) MCGVI

$C * * * *$ THREE ARGUMENTS FOR DMAX 1

MCEVD = DMAX1 (MCAVD, MCCVD, MCBVD)

MCFVD = MCEVD - 111.78978900

WRITE (NUVI,0627) MCFVD

$M C E V D=D M A X 1$ (MCCVD, MCDVD, MCAVD)

MCFVD = MCEVD - 23.0D-1

WRITE (NUVI,0627) MCFVD

MCEVO = OMAX1 (MCCVD, MCCVD, MCOVD)

MCFVD $=$ MCEVD + 99.66D-1

WRITE (NUVI,0627) MCFVD

MCGVI $=3$

WRITE (NUVI,0622) MCGVI

$P 0621730$

$P 0621740$

$P 0621750$

$P 0621760$

P0621770

$P 0621780$

P0621790

$P 0621800$

$P 0621810$

$P 0621820$

$P 0621830$

$P 0621840$

$P 0621850$

$P 0621860$

$P 0621870$

$P 0621880$

$P 0621890$

$P 0621900$

$P 0621910$

$P 0621920$

$P 0621930$

P0621940

$P 0621950$

$P 0621960$

$P 0621970$

$P 0621980$

$P 0621990$

P0622000

$P 0622010$

$P 0622020$

$P 0622030$

$P 0622040$ 
0621 FORMAT (F11.1)

0622 FORMAT ( $15 \mathrm{X}, 9 \mathrm{H}$ END OF, 12,15H-ARGUMENT TEST.)

0623 FORMAT ( $15 \times, 31 \mathrm{H}$ END OF $4-$ OR 5 -ARGUMENT TEST.)
0624 FORMAT ( $12 \times 15$ HTEST OF AMAX1--)

P0622140

$P 0622150$

0624 FORMAT ( $12 \times, 15$ HTEST OF AMAX1--)

0625 FORMAT ( $12 \times, 15$ HTEST OF AMAXO--)

0626 FORMAT (110)

0627 FORMAT (D22.10)

0628 FORMAT (2H1, 14 HTEST OF MAXO--)

0629 FORMAT ( $12 \times, 14$ HTEST OF MAX1--)

9998 FORMAT ( $39 H$ THE ABOVE ANSWERS SHOULDALL BE O FOR/2X.

$P 0622160$

P0622170

$P 0622180$

$P 0622190$

$P 0622200$

P0622210

$P 0622220$

135 HTHIS TEST SEGMENT TO BE SUCCESSFUL.)

$P 0622230$

$P 0622240$

9999 FORMAT ( /2X, 15 HTEST OF DMAX1--)

\section{C**** END OF TEST SEGMENT 062}

C*** WHEN EXECUTING ONLY SEGMENT 062, THE STOP AND END CAROS

$P 0622250$

$C * * * *$ WHICH APPEAR AS COMMENT CARDS MUST HAVE THE $C=$

$C * * * * *$ IN COLUMNS 1 AND 2 REMOVED.

$C=S T O P$

$C=E N D$

STOP

END

$P 0622260$

$P 0622270$

$P 0622280$

P 0622290

$P 0622300$

P0622310

P062C1

$P 062 C 2$

C

$[* * * * * *$

$[* * * * *$

IFMIN - (063)

P0630020

$[* * * * *$

P0 0630030

$P 0630040$

$C * * * * * *$

$C * * * *$

GENERAL PURPOSE

$C * * * *$

TEST INTRINSIC FUNCTIONS AMINO, AMINI, MINO,MINI AND

$[* * * * *$ DMINI - CHOOSING SMALLEST VALUE.

ASA REF PO630060

8.2 P0630070

$C * * * *$ S P E C I F I C A T I O N S SEGMENT 063

( TABLE 3)P0630080

$P 0630090$

$P 0630100$

$C * * * * *$

$[* * * * *$

WHEN EXECUTING ONLY SEGMENT 063 , THE SPECIFICATION STATEMENTS

$P 0011450$

$[* * * * *$ WHICH APPEAR AS COMMENT CARDS, MUST HAVE THE $C=$

POO11455

C*****IN COLUMNS 1 AND" 2 REMOVED.

P0011460

P0011465

$C * * * * *$

$C=\quad$ DOUBLE PRECISION MCAVD, MCBVD,MCCVD, MCDVD, MCEVD, MCFVD

$P 0011470$

P 0011475

POO 011480

DOUBLE PRECISION MCAVD, MCBVD, MCCVD, MCOVD, MCEVD, MCFVD

$C * * * *$ OUTPU T T A P E ASSIGNMENT STATEMENT. NO INPUT TAPE.

P063A1

$C * * * *$

$C * * * * *$

WHEN EXECUTING ONLY SEGMENT 063. THE FOLLOWING STATEMENT

$C * * * *$ NUVI $=6$ MUST HAVE THE $C=$ IN COLUMNS 1 AND 2 REMOVED.

P0630110

$P 0071220$

$P 0071225$

$[* * * * *$

$C=$ NUVI $=6$

$C * * * * \ldots$

NUVI $=6$

P0071230

$P 0071235$

$P 0071240$

P063B1

P0071245

WRITE (NUVI,0630)

$P 0630120$

0630 FORMAT ( 1 H1, 1X,35HIFMIN - (063) INTRINSIC FUNCTIONS--113X, 27HAMINOPO630130 1. AMIN1, MINO,MIN1,OMIN1/2X,14HASA REF. - 8.2/12X,7HRESULTS)

C*\#** TEST OF AMINO - INTEGER ARGUMENTS, REAL FUNCTION

$C * * * *$ TWO ARGUMENTS FOR AMINO

$P 0630140$

WRITE (NUVI,0635)

8.2124P0630150

$P 0630160$

$P 0630170$

$P 0630180$

MCAVI $=128$

$P 0630190$

MCBVI $=64$

$M C C V I=-8$

MCDVI $=-4096$

CMEVS $=A M I N O$ (MCAVI, MCBVI)

$P 0630200$

P0630210

$P 0630220$

CMFVS $=$ CMEVS -64.0

P0630230

WRITE (NUVI,0631) CMFVS

$P 0630240$ 
CMEVS = AMINO(MCDVI, MCCVI)

$P 0630250$

CMFVS $=$ CMEVS + 4096.0

$P 0630260$

WRITE (NUVI.0631) CMFVS

$P 0630270$

CMEVS $=$ AMINO(MCBVI, MCCVI)

$P 0630280$

CMFVS $=$ CMEVS +8.0

$P 0630290$

WRITE (NUVI,0639) CMFVS

$P 0630300$

$M C G V I=2$

WRITE (NUVI,0632) MCGVI

$P 0630310$

$P 0630320$

THREE-ARGUMENT TEST FOR AMINO

$P 0630330$

CMEVS = AMINO (MCAVI, MCCVI, MCBVI)

CMFVS $=$ CMEVS +8.0

WRITE (NUVI.0631) CMFVS

CMEVS = AMINO(MCBVI, MCBVI, MCDVI)

CMFVS = CMEVS + 4096.0

WRITE (NUVI.0631) CMFVS

P0630340

$P 0630350$

$P 0630360$

$P 0630370$

$P 0630380$

$P 0630390$

$P 0630400$

MCGVI = 3

WRITE (NUVI, 0632) MCGVI

$C * * * *$ FOUR OR FIVE ARGUMENTS FOR AMINO

P 0630410

$P 0630420$

CMEVS = AMINO(MCAVI, MCCVI, MCDVI, MCBVI)

$P 0630430$

CMFVS = CMEVS + 4096.0

WRITE (NUVI,0631) CMFVS

$P 0630440$

$P 0630450$

CMEVS = AMINOC (MCCVI, MCBVI, MCCVI, MCAVI, MCDVI)

$P 0630460$

CMFVS $=$ CMEVS + 4096.0

WRITE (NUVI.0631) CMFVS

$P 0630470$

WRITE (NUVI,0633)

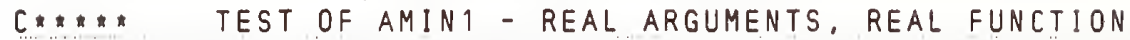

$P 0630480$

$P 0630490$

TWO ARGUMENTS TEST FOR AMIN 1

$8.2125 P 0630500$

$P 0630510$

WRITE (NUVI.0634)

$P 0630520$

CMAVS $=26.5$

CMBVS $=9.6666$

CMCVS $=-1.65$

CMDVS $=-10.001$

CMEVS $=$ AMIN 1 (CMBVS, CMDVS)

CMFVS $=$ CMEVS +10.001

WRITE (NUVI.0631) CMFVS

CMEVS = AMIN1 (CMAVS, CMBVS)

CMFVS $=$ CMEVS -9.6666

WRITE (NUVI.0631) CMFVS

CMEVS = AMIN1 (CMCVS, CMDVS)

CMFVS $=$ CMEVS +10.001

WRITE (NUVI,0631) CMFVS

CMEVS = AMIN1 (CMCVS, CMCVS)

CMFVS = CMEVS + 1.65

WRITE (NUVI.0631) CMFVS

$P 0630530$

P0630540

$P 0630550$

$P 0630560$

$P 0630570$

$P 0630580$

$P 0630590$

P0630600

P0630610

P0630620

$P 0630630$

P0630640

$P 0630650$

$P 0630660$

P0630670

$P 0630680$

MCGVI $=2$

WRITE (NUVI.0632) MCGVI

$C * * * *$ THREE-ARGUMENT TEST FOR AMIN 1

$P 0630690$

$P 0630700$

$P 0630710$

$P 0630720$

CMEVS $=$ AMIN1(CMBVS, CMCVS, CMDVS)

$P 0630730$

P0630740

WRITE (NUVI,0631) CMFVS

$P 0630750$

CMEVS = AMIN1 (CMBVS, CMBVS, CMBVS)

CMFVS = CMEVS - 9.6666

WRITE (NUVI,0631) CMFVS

$P 0630760$

$P 0630770$

CMEVS = AMIN 1 (CMAVS, CMBVS, CMCVS)

$P 0630780$

CMFVS = CMEVS + 1.65

WRITE (NUVI.0631) CMFVS

$P 0630790$

P0630800

MCGVI $=3$

WRITE (NUVI.0632) MCGVI

C*** FOUR OR FIVE-ARGUMENT TEST FOR AMIN 1

$P 0630810$

P 0630820

$P 0630830$

$P 0630840$

CMEVS = AMIN 1 (CMAVS, CMBVS, CMCVS, CMDVS)

CMFVS = CMEVS + 10.001

WRITE (NUVI.0631) CMFVS

$P 0630850$

$P 0630860$

$P 0630870$

CMEVS = AMIN 1 (CMAVS, CMCVS, CMBVS, CMCVS, CMOVVS)

CMFVS = CMEVS + 10.001

WRITE (NUVI,0631) CMFVS

$P 0630880$

$P 0630890$

WRITE (NUVI.0633)

$P 0630900$

C**** TEST OF MINO - INTEGER ARGUMENTS, INTEGER FUNCTION

$C * * * *$ TWO-ARGUMENT TEST FOR MINO

$8.2126 P 0630910$

$P 0630920$ 
WRITE (NUVI.0636)

$P 0630930$

MCEVI = MINO(MCBVI, MCAVI)

$P 0630940$

MCFVI = MCEVI - 64

$P 0630950$

WRITE (NUVI,0639) MCFVI

P0630960

MCEVI $=M I N O$ (MCBVI, MCCVI)

$P 0630970$

MCFVI = MCEVI + 8

WRITE (NUVI,0639) MCFVI

P0630980

MCEVI $=$ MINO(MCCVI, MCDVI)

P0630990

$M C F V I=M C E V I+4096$

$P 0631000$

WRITE (NUVI.0639) MCFVI

$P 0631010$

MCEVI $=$ MINO (MCAVI, 0$)$

WRITE (NUVI,0639) MCEVI

P0631020

MCGVI $=2$

WRITE (NUVI.0632) MCGVI

$C * * * * *$ THREE-ARGUMENT TEST FOR MINO

P0631030

P0631040

P0631050

$P 0631060$

P0631070

P0631080

MCEVI = MINO (MCAVI, MCCVI, MCBVI)

P0631090

MCFVI = MCEVI + 8

WRITE (NUVI.0639) MCFVI

MCEVI = MINO(MCCVI, MCAVI, MCDVI)

P0631100

P0631110

MCFVI = MCEVI + 4096

WRITE (NUVI, 0639) MCFVI

MCGVI $=3$

$P 0631920$

WRITE (NUVI,0632) MCGVI

$C * * * *$ FOUR OR FIVE-ARGUMENT TEST FOR MINO

MCEVI = MINO(MCBVI, MCAVI, MCCVI, MCDVI)

P0631130

$P 0631140$

$P 0631150$

P0631160

P0631970

P0631180

MCFVI = MCEVI + 4096

WRITE (NUVI,0639) MCFVI

MCEVI = MINO(MCAVI, MCBVI, MCAVI, MCCVI, MCOVI)

$P 0631190$

MCFVI $=$ MCEVI + 4096

WRITE (NUVI,0639) MCFVI

$P 0631200$

$P 0631210$

P0631220

WRITE (NUVI,0633)

C***** TEST OF MIN 1 - REAL ARGUMENTS, INTEGER FUNCTION

$P 0631230$

$C * * * *$ TWO-ARGUMENT TEST FOR MINI

WRITE (NUVI,0637)

MCEVI = MINI(CMAVS, CMBVS)

MCFVI = MCEVI - 9

WRITE (NUVI,0639) MCFVI

MCEVI = MINI(CMCVS, CMDVS)

$M C F V I=M C E V I+10$

WRITE (NUVI,0639) MCFVI

MCEVI = MIN1 (CMAVS, CMCVS)

$P 0631240$

$P 0631250$

$P 0631260$

P0631270

$P 0631280$

$P 0631290$

P0631300

P0631310

$P 0631320$

P0631330

MCFVI = MCEVI + 1

WRITE (NUVI,0639) MCFVI

$M C G V I=2$

WRITE (NUVI, 0632) MCGVI

$C * * *$ THREE-ARGUMENT TEST FOR MIN 1

$P 0631340$

$P 0631350$

$P 0631360$

$P 0631370$

P0631380

$P 0631390$

MCEVI = MIN 1 (CMAVS, CMCVS, CMBVS)

P0631400

WRITE (NUVI,0639) MCFVI

P0631410

MCEVI = MIN1 (CMAVS, CMCVS, CMOVS)

$P 0631420$

MCFVI = MCEVI + 10

WRITE (NUVI,0639) MCFVI

MCGVI $=3$

WRITE (NUVI,0632) MCGVI

$C * * * *$ FOUR OR FIVE-ARGUMENT TEST FOR MINI

$P 0631430$

P0631440

$P 0631450$

$P 0631460$

P0631470

P0631480

MCEVI = MIN1(CMAVS, CMBVS, CMDVS, CMCVS)

P 0631490

MCFVI = MCEVI +10

WRITE (NUVI,0639) MCFVI

MCEVI = MIN1 (CMAVS, CMBVS, CMCVS, CMCVS, CMDVS)

P0631500

$M C F V I=M C E V I+10$

WRITE (NUVI,0639) MCFVI

WRITE (NUVI,0633)

C***** TEST OF DMIN 1 - DOUBLE PRECISION ARGUMENTS, FUNCTION

C***** TWO-ARGUMENT TEST FOR DMIN 1

WRITE (NUVI,0638)

MCAVD $=61.123400$

PO631510

P0631520

$P 0631530$

$P 0639540$

$8.2128 P 0631550$

$P 0631560$

P0631570

P0631580

$P 0631590$

$M C B V D=2.001$

$M C C V D=-999.0090-1$

P0631600 
$M C O V D=-1.900$

$P 0631610$

$M C E V D=D M I N 1$ (MCAVD, MCBVD)

P0 631620

MCFVD = MCEVD -2.001

P0 031630

WRITE (NUVI, 9996) MCFVD

$P 0631640$

MCEVD = DMINI (MCCVD, MCDVD)

P0 631650

MCFVD $=$ MCEVD + $999.009 D-1$

P0 031660

WRITE (NUVI, 9996) MCFVD

MCEVD = DMIN 1 (MCAVD, MCDVD)

$P 0631670$

MCFVD = MCEVD + 1.900

WRITE (NUVI,9996) MCFVD

P0631680

P0631690

MCGVI $=2$

WRITE (NUVI,0632) MCGVI

$C * * * *$ THREE-ARGUMENT TEST FOR DMIN 1

MCEVD = DMIN 1 (MCAVD, MCBVD, MCDVD)

$M C F V D=M C E V D+1.900$

WRITE (NUVI, 9996) MCFVD

MCEVD = DMIN 1 ( MCAVD, MCCVD, MCBVD)

MCFVD = MCEVD + $999.009 D-1$

WRITE (NUVI, 9996) MCFVD

MCGVI $=3$

WRITE (NUVI,0632) MCGVI

C*\#\# FOUR OR FIVE-ARGUMENT TEST FOR DMIN 1

MCEVD = DMIN1 (MCAVD, MCCVD, MCBVD, MCDVD)

MCFVD $=$ MCEVD + $999.009 D-1$

WRITE (NUVI, 9996) MCFVD

MCEVD = DMIN1 (MCBVD, MCAVD, MCBVD, MCDVD, MCCVD)

MCFVD $=$ MCEVD + $999.009 D-1$

WRITE (NUVI,9996) MCFVD

WRITE (NUVI,0633)

WRITE (NUVI,9997)

0631 FORMAT ( F11.1)

0632 FORMAT( $15 \mathrm{X}, 8 \mathrm{H}$ END OF, 12,15H-ARGUMENT TEST.)

0633 FORMAT ( $15 X, 30 \mathrm{H}$ END OF 4 OR 5-ARGUMENT TEST.)

0634 FORMAT ( $116 \mathrm{H}$ TEST OF AMIN 1)

0635 FORMAT ( $/ 16 \mathrm{H}$ TEST OF AMINO)

0636 FORMAT ( $116 \mathrm{H}$ TEST OF MINO)

0637 FORMAT ( $16 \mathrm{H} 1$ TEST OF MIN1)

0638 FORMAT ( $/ 16 \mathrm{H}$ TEST OF DMIN1)

0639 FORMAT ( I10)

9996 FORMAT ( D22.10)

9997 FORMAT ( $/ 39 H$ THE ABOVE ANSWERS SHOULD ALL BE O FOR/1X,

P0631700

P0631710

P0631720

P0631730

P0631740

P0631750

P0631760

P0631770

P0631780

P0631790

P0631800

P0631810

P0631820

P0631830

P0631840

P0631850

P0631860

P0631870

P0631880

P0631890

P0631900

P 0631910

P0631920

P0631930

P0631940

P0 631950

P0631960

P0631970

P0631980

P0631990

P0632000

P0632010

$136 \mathrm{H}$ THIS TEST SEGMENT TO BE SUCCESSFUL.)

C*\#* END OF TEST SEGMENT 063

C***** WHEN EXECUTING ONLY SEGMENT 063. THE STOP AND END CARDS

$C * *$ WHICH APPEAR AS COMMENT CARDS MUST HAVE THE $c=$

$C * \because *$ IN COLUMNS 1 AND 2 REMOVED.

$C=S T O P$

$C=E N D$

STOP

$P 0632020$

P0 0632030

$P 0632040$

$P 0632050$

$P 0632060$

$P 0632070$

P0632080

P063C1

END

$P 063 C 2$

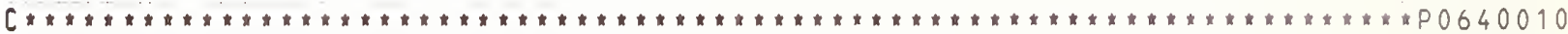

C*******?

$C * * *$

IFDSG - (064)

00640020

$P 0640030$

$P 0640040$

$C * * * *$

P0

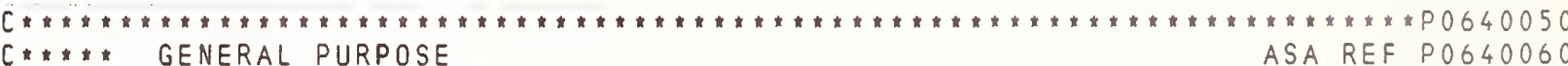

C**** TEST INTRINSIC FUNCTION DSIGN (TRANSFER OF SIGN WITH

$[* * *$ DOUBLE PRECISION ARGUMENTS AND FUNCTION)

$8.2 / 33 P 0640070$

$C * * *$

$[* * * *$ S P E C I F I C A T I O N S SEGMENT 064

(TABLE 3)P0640080

$P 0640090$

$P 0640100$

P0011490

$C * * *$ WHEN EXECUTING ONLY SEGMENT 064, THE SPECIFICATION STATEMENTS

$C * * *$ WHICH APPEAR AS COMMENT CARDS, MUST HAVE THE $C=$

$C * *$ IN COLUMNS 1 AND 2 REMOVED.

$C * * * * *$

$C=$ DOUBLE PRECISION

$M C A V D, M C B V D, M C C V D, M C D V D, M C E V D, M C F V D$

$P 0011495$

$P 0011500$

P0011505

P 0011510

$P 0011515$

P064A1

$C * * * *$

DOUBLE PRECISION

$M C A \vee D, M C B \vee D, M C C V D, M C D V D, M C E V D, M C F \vee D$

P0011520 
$C * * * *$ O U T P U T T A P E ASSIGNMENT STATEMENT. NO INPUT TAPE. PO640110

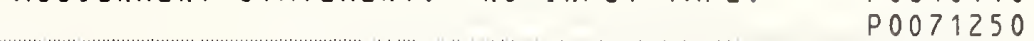

$C * * *$ WHEN EXECUTING ONLY SEGMENT 064 . THE. FOLLOWING STATEMENT

$C * * * * * \quad N U V I=6$ MUST HAVE THE $C=I N$ COLUMNS 1 AND 2 REMOVED.

$C * * * * *$

$C=$ NUVI $=6$
NUVI $=6$

$C * * * * *$

WRITE (NUVI.0640)

$P 0071255$

$P 0071260$

P0071265

$P 0071270$

P06481

P0071275

$P 0640120$

0640 FORMAT (1H1.1X,34HIFDSG- (064) INTRINSIC FUNCTION--116X,24HDSIGN P0640130 1(TRANSFER OF SIGN)/ 2X,14HASA REF. - 8.2/12X,7HRESULTS) P0640140

$C * * * *$ HEADER FOR SEGMENT 064 WRITTEN

$M C A V D=+9.500$

$M C B \vee D=123.456701$

$M C C V D=-5.66501$

MCDVD $=-75.570-0$

$M C E V D=D S I G N(M C A \vee D, M C B \vee D)$

MCFVD = MCEVD - 9.500

WRITE (NUVI.0641) MCFVD

$M C E V D=D S I G N(M C B \vee D, M C C V D)$

$M C F V D=M C E V D+123.456701$

WRITE (NUVI,0641) MCFVD

$M C E V D=D S I G N(M C C V D, M C D V D)$

$M C F \vee D=M C E V D+5.66501$

WRITE (NUVI,0641) MCFVD

$M C E V D=D S I G N(M C D V D, M C D V D)$

$M C F V D=M C E V D+75.5700$

WRITE (NUVI.0641) MCFVD

WRITE (NUVI.0642)

0641 FORMAT (1HO.030.18)

0642 FORMAT ( 1 HO, 1 X, 38 HALL ABOVE ANSWERS SHOULD BE $O$ FOR THIS/ $12 X, 30 H T E S T$ SEGMENT TO BE SUCCESSFUL.)

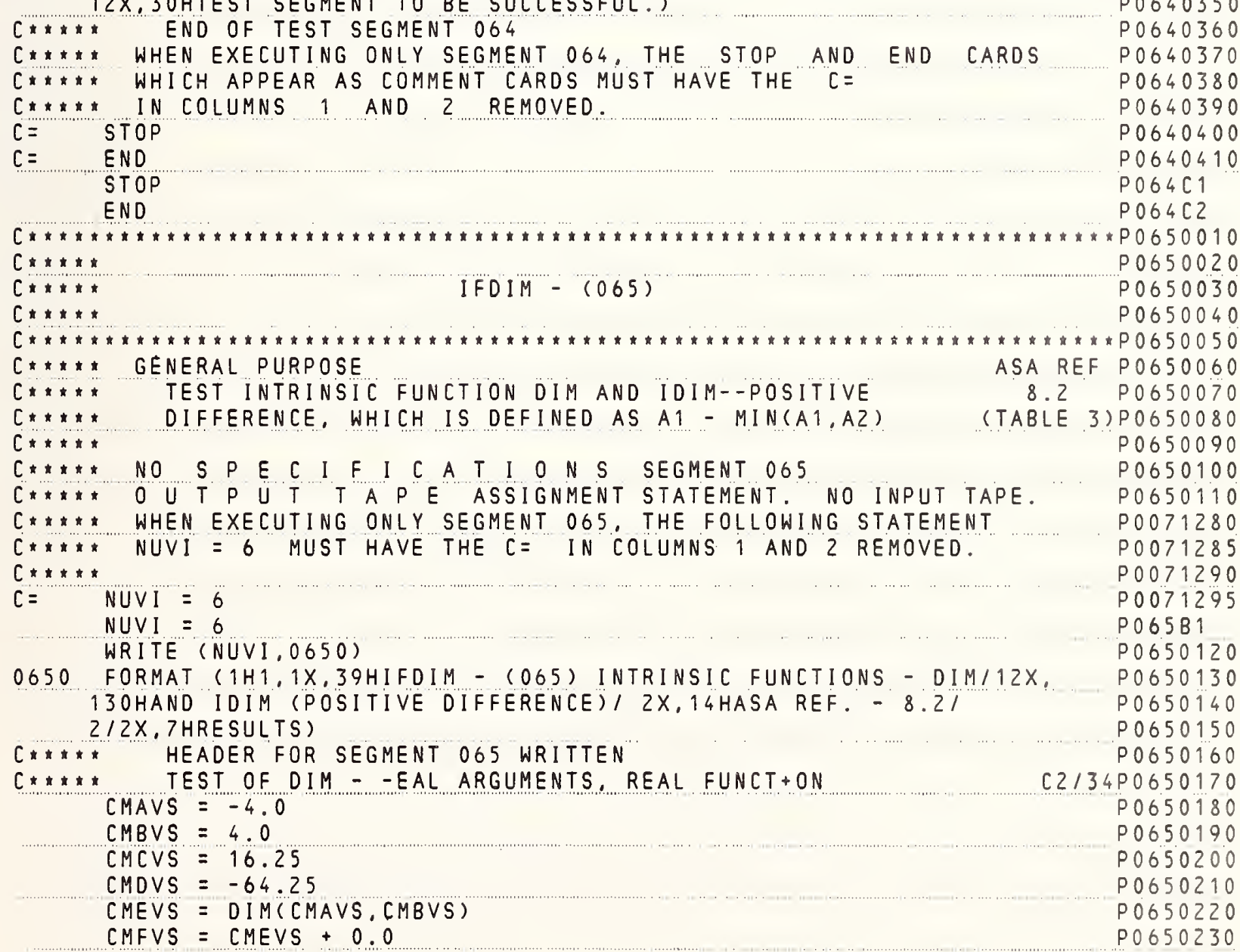


WRITE (NUVI.0651) CMFVS

$P 0650240$

CMEVS $=$ DIM(CMCVS, CMDVS)

$P 0650250$

CMFVS = CMEVS -80.5

P0650260

WRITE (NUVI.0651) CMFVS

P0650270

CMEVS = DIM(CMCVS, CMBVS)

P0650280

CMFVS = CMEVS - 12.25

$P 0650290$

WRITE (NUVI.0659) CMFVS

P0650300

CMEVS = DIM(CMOVS, CMAVS)

$P 0650310$

CMFVS $=$ CMEVS -0.0

$P 0650320$

WRITE (NUVI.0651) CMFVS

$P 0650330$

C*\#** TEST OF IDIM - INTEGER ARGUMENTS, INTEGER FUNCTION

MCAVI $=02468$

$M C B V I=+36$

$M C C V I=-3$

MCDVI $=-23$

MCEVI = IDIM(MCAVI, MCBVI)

MCFVI = MCEVI - 2432

WRITE (NUVI,0652) MCFVI

MCEVI = IDIM(MCBVI, MCCVI)

MCFVI = MCEVI - 39

WRITE (NUVI,0652) MCFVI

MCEVI = IDIM(MCDVI,MCCVI)

MCFVI = MCEVI + 0

WRITE (NUVI,0652) MCFVI

MCEVI = IDIM(MCCVI, MCCVI)

WRITE (NUVI,0652) MCEVI

MCEVI = IDIM(MCCVI, MCBVI)

WRITE (NUVI.0652) MCEVI

WRITE (NUVI,0653)

0651 FORMAT (1HO,F17.2)

0652 FORMAT (1HO, 10X, I 5)

0653 FORMAT ( 1 HO, $1 X, 34 \mathrm{H}$ ALL ABOVE ANSWERS SHOULD BE 0 FOR/2X,

135 HTHIS TEST SEGMENT TO BE SUCCESSFUL.)

C***** END OF TEST SEGMENT 065

$C * * * *$ WHEN EXECUTING ONLY SEGMENT 065 THE STOP AND END CARDS

$C * * * *$ WHICH APPEAR AS COMMENT CARDS MUST HAVE THE C=

$C * * * *$ IN COLUMNS 1 AND 2 REMOVED.

$C=S T O P$

$C=\quad E N D$

STOP

$P 0650340$

$P 0650350$

P0650360

P0650370

P0650380

PO 0650390

P0650400

P0650410

P0650420

P0650430

P0650440

P0650450

P0650460

P0 650470

P0650480

P0650490

P0650500

P0650510

P0650520

P0650530

P0650540

P0650550

P0650560

P0650570

P0650580

P0650590

P0650600

P0650610

P0650620

P065C1

END

P065C2

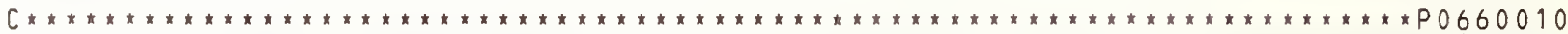

$C * * * * *$

$C * * * * *$

IFSGL - (066)

$P 0660020$

$P 0660030$

$P 0660040$

$C * * * * *$

$[* * * *$ GENERAL PURPOSE

ASA REF P0660060

C***** TEST INTRINSIC FUNCTION SNGL - OBTAIN MOST SIGNIFICANT $8.2 / 36 P 0660070$

$C * * * *$ PART OF DOUBLE PRECISION ARGUMENT.

$C * * * *$ GENERAL COMMENTS

C***** ASSIGNED GO TO STATEMENT ASSUMED WORKING.

(TABLE 3)P0660080

$P 0660090$

$P 0660100$

$P 0660110$

$P 0660120$

$C * * * *$ S P E C I F I C A T I O N S SEGMENT 066

$P 0011530$

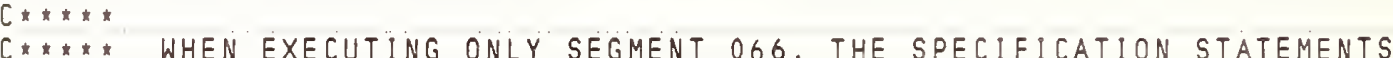

$C * * * *$ WHICH APPEAR AS COMMENT CARDS MUST HAVE THE C

$P 0011535$

$P 0011540$

$P 0011545$

P0011550

C*:* IN COLUMNS I AND 2 REMOVED.

$C * * * *$ DOUBLE PRECISION MCAVD, MCBVD, MCCVD, MCDVD, MCEVD, MCFVD,

$C=1$ CMAVD, CMBVD, CMCVD

DOUBLE PRECISION MCAVD, MCBVD, MCCVD, MCDVD, MCEVD, MCFVD,

$P 0011555$

$P 0011560$

P066A1

$P 066 A 2$

P0011565

P0660130

$P 0079300$

$P 0071305$

$P 0071310$

P0071315

$C * * * *$ NUVI $=6$ MUST HAVE THE $C=$ IN COLUMNS 1 AND 2 REMOVED.

$C=\quad$ NUVI $=6$ 

126 HOBTAIN MOST SIGNIFICANT PT/16X.

$218 \mathrm{H}$ OF D.P. ARGUMENT I/2X,15HASA REFS - $8.21 / 2 \mathrm{X}, 7$ HRESULTS C***** HEADER FOR SEGMENT 066 WRITTEN

MCAVD $=.4874874874874803+.570-5+.56046450-6+.317865095470-7$

MCBVD $=-39.68953960953901-.57 D-5-.5604645 D-6-.31786509547 D-7$

$M C C V D=.3333333333333300+.570-5+.56046450-6+.317865095470-7$

MCDVD $=-.6666666666666600-.570-5+.56046450-6-.317865095470-7$

MCEVD $=.4874874874874803+.570-5+.56046450-6+.317865095470-7$

MCFVD $=-39.6895396095390+1$

AVS $=0.0$

BVS $=0.0$

CVS $=0.0$

IVI $=2$

$P 0660150$

$P 0660160$

$P 0660170$

P0 0600180

$P 0660190$

P0 060200

$P 0660210$

$P 0660220$

$P 0660230$

P0660240

$P 0660250$

P0 060260

P0660270

P0 0660280

$[* * * *$ EXPRESSION RESULTS ASSIGNED TO D.P. RESULT FOR VISUAL COMPARISONP0660290

C****ARGUMENTS OF SNGL - VARIABLE, SIMPLE EXPRESSION

CMAVD = AVS + SNGL(MCAVD) - BVS

WRITE (NUVI,661) MCAVD,CMAVD

CMAVD = CVS + SNGL (MCBVD) + AVS

WRITE (NUVI,661) MCBVD, CMAVD

CMAVD = SNGL (MCCVD)

WRITE (NUVI,661) MCCVO,CMAV̈D

$C M B \vee D=-M C B \vee D$

$C M A \vee D=-S N G L(M C B \vee D-C M B \vee D)$

$C M C V D=-(M C B V D+M C B \vee D)$

WRITE (NUVI, 661) CMCVO, CMAVD

CMCVD = MCDVD*MCDVD

CMAVD = BVS + SNGL(MCDVD*IVI) + CVS

WRITE (NUVI,661) CMCVD, CMAVD

$P 0660300$

$P 0660310$

$P 0660320$

$P 0660330$

P0 0660340

$P 0660350$

P0660360

$P 0660370$

$P 0660380$

$P 0660390$

$P 0660400$

$P 0660410$

$P 0660420$

$P 0660430$

$C * * * *$ ARGUMENT OF SNGL - INTRINSIC FUNCTION WITH DIFFERENT NO. OF ARG CMAVD $=-(C V S+S N G L(D A B S(M C O V D))+B V S)$

WRITE (NUVI,661) MCDVD, CMAVD

CMAVD = AVS - BVS + SNGL(DMINI(MCEVD,MCFVD))

WRITE (NUVI,661) MCFVD, CMAVD

CMAVD = CVS + BVS + SNGL(DMAX1(MCCVD,MCEVD,MCFVD))

WRITE (NUVI,661) MCEVD, CMAVD

WRITE (NUVI, 662)

661 FORMAT (1H0,1X,6HLINE A,025.14/2X,6HLINE B,025.14)

$P 0660440$

$P 0660450$

$P 0660460$

$P 0660470$

$P 0660480$

$P 0660490$

P0660500

$P 0660510$

$P 0660520$

662 FORMAT 33 HO LINE B SHOULD AGREE WITH LINE A $140 \mathrm{OH}$ ONLY TO THE PRECPO660530

AISION OF A REAL DATUM. I37H REMAINING OIGITS RESULT FROM OUTPUT IPO66054O

B $33 \mathrm{H}$ CONVERSION WHEN A REAL VALUE IS I $32 \mathrm{H}$ ASSIGNED TO D.P. FOR P0660550

CPRINTING.)

$C * * * * *$ END OF SEGMENT 066

$C * * *$ WHEN EXECUTING ONLY SEGMENT 066. THE STOP AND ENO CAROS

$C * * * * *$ WHICH APPEAR AS COMMENT CARDS MUST HAVE THE $C=$ IN COLUMNS

$C * * * 2$ AND 2 REMOVED.

$C=\operatorname{STOP}$

$C=\quad E N D$

STOP

ENO

$P 0660560$

$P 0660570$

P0660580

$P 0660590$

P0 0660600

$P 0660610$

P0660620

P066C1

$P 066 C 2$

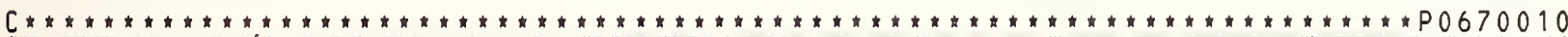

$C * * * *$

$C \star \star \star \star \star *$

IFREL - (067)

$P 0670020$

P0 0670030

$[* * * *$

$P 0670040$

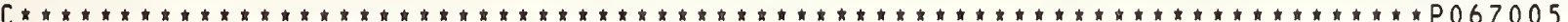

$[* * *$ GENERAL PURPOSE

$C * * * *$ TEST INTRINSIC FUNCTION REAL (OBTAIN REAL PART OF

$C * * * *$ COMPLEX ARGUMENT).

ASA REF P0670060

$8.2139 P 0670070$

$C * * * * *$

$C * * * * *$

$C * * * * *$

$S$ S E C I F I C A T I O N S SEGMENT 067

(TABLE 3) P0670080

$P 0670090$

$P 0670100$

$P 0011570$

WHEN EXECUTING ONLY SEGMENT 067, THE SPECIFICATION STATEMENTS

P0011575

$C * \hbar \star \star \star *$

WHICH APPEAR AS COMMENT CAROS MUST HAVE THE $C=$

$P 0011580$

$C * * * *$ IN COLUMNS 1 AND 2 REMOVEO.

$P 0011585$

P0011590 
$12 X .14$ HASA REF. -8.211

2 2X,7HRESULTS

C**** HEADER FOR SEGMENT 067 WRITTEN

$P 0670130$

CHAVC $=(3.2,1.86)$

CHBVC $=(2.1,0.0)$

CHCVC $=(3.7,-1.2)$

CH.DVC $=(+45.1,+2.2)$

CHEVC $=(-16.0,0.0)$

CHFVC $=(-32.0,-1.1)$

CMAVS = REAL (CHAVC)

CMBVS $=$ CMAVS -3.2

CMAVS $=$ REAL (CHBVC)

CMCVS $=$ CMAVS -2.1

CMAVS = REAL (CHCVC)

$P 0670140$

$P 0670150$

$P 0670160$

$P 0670170$

$P 0670180$

$P 0670190$

$P 0670200$

$P 0670210$

CMDVS $=$ CMAVS -3.7

$P 0670220$

$P 0670230$

$P 0670240$

$P 0670250$

$P 0670260$

$P 0670270$

CMAVS = REAL (CHDVC)

$P 0670280$

CMEVS = CMAVS -45.1

$P 0670290$

CMAVS $=A B S(R E A L(C H E V C)+R E A L(C H F V C))$

$P 0670300$

CMFVS $=$ CMAVS -48.0

$P 0670310$

CMAVS = AMAX1(REAL (CHAVC), REAL (CHBVC), REAL (CHEVC-CHFVC))

$P 0670320$

CMGVS $=$ CMAVS -16.0

WRITE (NUVI,0671) CMBVS, CMCVS, CMDVS,CMEVS, CMFVS, CMGVS

C*** REAL CONSTANTS HAVING ONLY FRACTIONAL PARTS(NO EXPONENT)

$P 0670330$

$P 0670340$

$P 0670350$

CHAVC $=(.789, .12)$

CHBVC $=(.13,1.2)$

CHCVC $=(.507,-2.2)$

CHOVC $=(+.5401,+.5)$

CHEVC $=(-.5,0.25)$

CHFVC $=(-.0625,1.1)$

CMAVS $=$ REAL (CHAVC)

$P 0670360$

$P 0670370$

$P 0670380$

$P 0670390$

$P 0670400$

$P 0670410$

CMBVS $=$ CMAVS -.789

$P 0670420$

$P 0670430$

CMAVS = REAL (CHBVC)

P0670440

CMCVS $=$ CMAVS -0.13

$P 0670450$

CMAVS $=$ REAL (CHCVC)

$P 0670460$

CMDVS $=$ CMAVS -0.507

$P 0670470$

CMAVS = REAL (CHOVC)

$P 0670480$

CMEVS $=$ CMAVS -0.5401

$P 0670490$

CMAVS = REAL (CHEVC+CHFVC)

$P 0670500$

CMFVS $=$ CMAVS +0.5625

$P 0670510$

CMAVS $=$ REAL (CHEVC) - REAL (CHFVC)

$P 0670520$

$P 0670530$

CMGVS $=$ CMAVS +0.4375

WRITE (NUVI,0671) CMBVS, CMCVS, CMDVS, CMEVS, CMFVS, CMGVS

$P 0670540$

$P 0670550$

$C * * *$ REAL CONSTANTS HAVING ONLY INTEGRAL PARTS (NO EXPONENT)

$P 0670560$

CHAVC $=(23,0.1)$

$5 \cdot 1 \cdot 1.2 / 22 P 0670570$

$P 0670580$

CHBVC $=(12 \ldots+1.2)$

$P 0670590$

$C H C V C=(1, .-2.3)$

$P 0670600$

CHCVC $=(+45 \ldots+.6)$

$P 0670610$

CHEVC $=(19.0,1.0)$

$P 0670620$

CHFVC $=(-32.0,2.0)$

$P 0670630$

CMAVS = REAL (CHAVC)

$P 0670640$

CMBVS $=$ CMAVS -23.0

$P 0670650$

CMAVS $=$ REAL (CHBVC)

$P 0670660$

$C M C V S=$ CMAVS -12.0

CMAVS = REAL (CHCVC)

$P 0670670$

CMOVS $=$ CMAVS -1.0

$P 0670680$

$P 0670690$ 
CMAVS = REAL (CHOVC)

CMEVS $=$ CMAVS -45.0

$P 0670700$

CMAVS = SIGN(DIM(REAL(CHEVC), REAL(CHFVC)), REAL(CHFVC))

P0670716

CMFVS $=$ CMAVS +51.0

CMAVS $=$ REAL $((16.0 .1 .0)+$ CHEVC + CHFVC $)$

$P 067072$ ?

P0670730

$P 0670740$

CMGVS = CMAVS -3.0

WRITE (NUVI,0671) CMBVS, CMCVS, CMOVS, CMEVS, CMFVS, CMGVS

P0670750

WRITE (NUVI,0672)

0671 FORMAT $(16(\mathrm{~F} 20.4 /))$

0672 FORMAT ( $140 \mathrm{H}$ ALL ABOVE ANSWERS SHOULD BE O FOR THIS I

P0670760

$P 0670770$

$P 0670780$

$P 0670790$

$132 \mathrm{H}$ TEST SEGMENT TO BE SUCCESSFUL.)

$P 0670800$

$[* * * *$ END OF TEST SEGMENT 067

C**** WHEN EXECUTING ONLY SEGMENT 067\%, THE STOP AND END CARDS

$P 0670810$

$[* * *$ WHICH APPEAR AS COMMENT CARDS MUST HAVE THE $C=$

C****IN COLUMNS 1 AND 2 REMOVED.

$C=\operatorname{STOP}$

$C=\quad$ END

STOP

$P 0670820$

$P 0670830$

$P 0670840$

$P 0670850$

P0670860

P067C 1

END

$P 067 C 2$

$[* * * * * *$
$C * * * * *$

$[* * * *$

$[* * * * *$

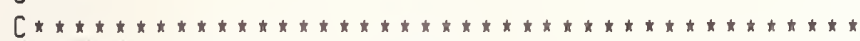

IFIMG - $(068)$

$P 0680010$

$P 0680020$

P0680030

$P 0680040$

$[* * * *$ GENERAL PURPOSE

C***** TEST INTRINSIC FUNCTION AIMAG COBTAIN IMAGINARY PART

$C * * * *$ OF COMPLEX ARGUMENT)

*P0680050

$C * * * * *$

$C * * * *$ S P E C I F I C A T I O N S SEGMENT 068

C******* WHEN EXECUTING ONLY SEGMENT 068, THE SPECIFICATION STATEMENTS

$C * * * *$ WHICH APPEAR AS COMMENT CARDS MUST HAVE THE $C=$

C**** IN COLUMNS 1 AND 2 REMOVED.

$C * * * * *$

$C=\quad$ COMPLEX CHAVC, CHBVC, CHCVC, CHDVC, CHEVC, CHFVC, CHGVC, CHHVC, CHIVC,

$C=\quad 1 C H J V C, C H K V C, C H L V C$

COMPLEX CHAVC, CHBVC, CHCVC, CHDVC, CHEVC, CHFVC, CHGVC, CHHVC, CHIVC,

1 CHJVC, CHKVC, CHLVC

$C * * * * *$

$C * * * * \quad 0 U T P U T$ T A P E ASSIGNMENT STATEMENT. NO INPUT TAPE.

$C * * * * *$

C***** WHEN EXECUTING ONLY SEGMENT 068. THE FOLLOWING STATEMENT

$C * * * *$ NUVI $=6$ MUST HAVE THE $C=I N$ COLUMNS 1 AND 2 REMOVED.

$C=\quad$ NUVI $=6$

$C * * * *$

NUVI $=6$

WRITE (NUVI.0680)

0680 . FORMAT (1H1,1X,40HIFIMG - (068) INTRINSIC FUNCTION - AIMAG/16X, 119 HOBTAIN IMAGINARY PT/16X,19HOF COMPLEX ARGUMENT/ $2 X$,

213 HASA REF. - 8.2 (12X,7HRESULTS)

$[* * * *$ HEADER FOR SEGMENT 068 WRITTEN

C**** IMAGINARY PARTS OF COMPLEX NUMBERS HAVING BOTH INTEGRAL

$[* * * *$ AND FRACTIONAL PARTS. (NO EXPONENT)

CHAVC $=(3.2,1.86)$

CHBVC $=(2.1,0.0)$

CHCVC $=(37.0,-1.2)$

CHDVC $=(+45.1,+2.2)$

CMAVS $=$ AIMAG (CHAVC)

CMBVS $=$ CMAVS -1.86

CMAVS $=$ AIMAG (CHBVC)

CMCVS $=$ CMAVS -0.0

CMAVS $=$ AIMAG (CHCVC)

CMOVS $=$ CMAVS +1.2

CMAVS $=$ AIMAG (CHDVC)

ASA REF P0680060

8.2/41P0680070

(TABLE 3)P0680080

$P 0680090$

P0680100

$P 0011610$

P0011615

P0011620

P0011625

$P 0011630$

P0011635

$P 0011640$

P068A1

P068A2

POO 011645

P0680110

$P 0071360$

P0071365

P0071370

$P 0071375$

P068B1

$P 0071380$

P0680120

P0680130

P0680140

P0680150

P0680160

P0680170

P0680180

P0680190

P0680200

P0680210

P0680220

P0680230

P0680240

P0680250

P0680260

P0680270

P0680280

P0680290

CMEVS = CMAVS -2.2

P0680300

WRITE (NUVI,0681) CMBVS, CMCVS, CMOVS, CMEVS

C*** IMAGINARY PARTS OF COMPLEX NUMBERS HAVING ONLY FRACTIONAL

P0680310

P0680320

$[* * * *$ PARTS (NO EXPONENT)

P0680330

NBS FORTRAN Test Programs Version 1 
CHCVC $=(+4.56,-.456)$

CHDVC $=(-12.3,+.001)$

P0680360

CMAVS $=A I M A G(C H A V C)$

$P 0680370$

CMBVS $=$ CMAVS -0.0

P0 0680380

CMAVS $=A \amalg M A G(C H B \vee C)$

$P 0680390$

CMCVS $=$ CMAVS -.789

$P 0680400$

CMAVS $=$ AIMAG (CHCVC)

$P 0680410$

CMOVS $=$ CMAVS +.456

$P 0680420$

CMAVS $=$ AIMAG (CHOVC)

$P 0680430$

CMEVS $=$ CMAVS -0.001

P 0680440

$P 0680450$

WRITE (NUVI,0681) CMBVS,CMCVS,CMOVS,CMEVS

CH* IMAGINARY PARTS OF COMPLEX NUMBERS HAVING ONLY INTEGRAL

$P 0680460$

C*** PARTS (NO EXPONENT)

$P 0680470$

$P 0680480$

CHAVC $=(-12,12$,

CHBVC $=(+1.23,0$.

$C H C V C=(0.0,-16.0)$

CHDVC $=(-1.1,-32.0)$

$P 0680490$

$P 0680500$

$P 0680510$

CMAVS $=$ AIMAG (CHAVC)

$P 0680520$

CMBVS $=$ CMAVS -12.0

$P 0680530$

CMAVS $=$ AIMAG $($ CHBVC)

P0 0680540

CMCVS $=$ CMAVS +0.0

$P 0680550$

CMAVS $=A B S(A I M A G(C H C V C)+A ! M A G(C H D V C))$

$P 0680560$

CMDVS $=$ CMAVS -48.0

$P 0680570$

CMAVS $=A M A X 1(A ! M A G(C H A V C), A I M A G(C H B \vee C), A \perp M A G(C H C V C-C H O V C))$

P0680580

CMEVS $=$ CMAVS -16.0

$P 0680590$

WRITE (NUVI,0681) CMBVS, CMCVS, CMOVS, CMEVS

$P 0680600$

$P 0680610$

$C * * * * *$ IMAGINARY PARTS OF COMPLEX NUMBERS HAVING A DECIMAL EXPONENT. CHAVC $=(2.3 E 0,1.2 E 0)$

CHBVC $=(1.2, .56 E 2)$

CHCVC $=(.24,1 . E 1)$

CHDVC $=(1 \ldots+7.8 E+1)$

CHEVC $=(1.5,16.0)$

CHFVC $=(1.0,-32.0)$

CHGVC $=(1 . E 0,-7.99 E-1)$

CHHVC $=(27.00, .55 E-1)$

$C H I V C=(1 . E 0,2 \cdot E-0)$

$C H J V C=(1.2,1 . E+1)$

$C H K V C=(1 . E-1,+7 . E 0)$

CHLVC $=(1.7,-99 . E-1)$

CMAVS $=A \rrbracket M A G(C H A V C)$

CMBVS $=$ CMAVS $-1.2 E 0$

CMAVS $=A I M A G(C H B V C)$

CMCVS $=$ CMAVS $-.56 E 2$

CMAVS $=$ AIMAG $(C H C V C)$

CMDVS $=$ CMAVS $-1 . E 1$

CMAVS $=$ AIMAG (CHDVC)

CMEVS $=$ CMAVS $-7.8 E+1$

WRITE (NUVI,0681) CMBVS, CMCVS, CMOVS, CMEVS

CMAVS $=S I G N(D I M(A I M A G(C H E V C), A I M A G(C H F V C)), A I M A G(C H F V C))$

CMBVS $=$ CMAVS +48.0

CMAVS $=$ AIMAG $((1.0,16.0)+$ CHEVC + CHFVC $)$

CMCVS $=$ CMAVS +0.0

CMAVS $=$ AIMAG (CHGVC)

CMDVS $=$ CMAVS $+7.99 E-1$

CMAVS $=$ AIMAG (CHHVC)

CMEVS $=$ CMAVS - .55E-1

WRITE (NUVI,0681) CMBVS, CMCVS, CMOVS, CMEVS

CMAVS $=$ AIMAG (CHIVC)

CMBVS $=$ CMAVS $-2 \cdot E-0$

CMAVS $=$ AIMAG $(C H J V C)$

CMCVS $=$ CMAVS $-1 \cdot E+1$

CMAVS $=$ AIMAG (CHKVC)

CMDVS $=$ CMAVS $-7 . E O$

CMAVS $=$ AIMAG (CHLVC)

CMEVS $=$ CMAVS $+99 . E-1$

WRITE (NUVI, 0681 ) CMBVS, CMCVS, CMDVS, CMEVS

$P 0680620$

$P 0680630$

P0680640

P0 0680650

$P 0680660$

$P 0680670$

P0680680

$P 0680690$

P0680700

P0 0680710

$P 0680720$

P0680730

P0 0680740

P0680750

P0680760

P0 0680770

P0 0680780

P0680790

P0680800

P0680810

P0680820

P0 080830

P0680840

P0680850

P0 0680860

P0 680870

P0 080880

P0680890

P0680900

P0680910

P0680920

$P 0680930$

P0680940

P0680950

P0 0680960

P0 680970

P0680980

P0680990

P0681000

P0681010 
WRITE (NUV!,0682)

0681 FORMAT $(14(F 20.51))$

0682 FORMAT ( $140 \mathrm{H}$ ALL ABOVE ANSWERS SHOULD BE O FOR THIS,

$132 \mathrm{H}$ TEST SEGMENT TO BE SUCCESSFUL.)

$P 0681040$

$C * * * * *$ END OF TEST SEGMENT 068

$C * * * *$ WHEN EXECUTING ONLY SEGMENT 068, THE STOP AND END CARDS

$P 0681050$

$C * * * *$ WHICH APPEAR AS COMMENT CARDS MUST HAVE THE $C=$

$C * * * *$ IN COLUMNS 1 AND 2 REMOVED.

$C=\operatorname{STOP}$

$C=\quad E N D$

STOP

END

$P 0681070$

$P 0681080$

$P 0681090$

$P 0681100$

$P 0681110$

$P 068 C 1$

P068C2

C

$C * \star \star \star \star *$

$I F D B L-(069)$

$C * * * *$

$P 0690020$

$C * * * * *$

$P 0690030$

$C * * * *$ GENERAL PURPOSE

$C * * * *$ TEST INTRINSIC FUNCTION DBLE (EXPRESS S.P. ARGUMENT

$C * * * * *$ IN DOUBLE PRECISION FORM )

C***** INTRINSIC FUNCTIONS DABS,DSIGN,DMIN1, DMAXI, AMAXI

$P 0690040$

$C * * * * *$ ASSUMED WORKING.

$C * * * * *$

$C * * * * *$ S P E C I F I C A T I O N S SEGMENT 069

$C * * *$

$C * * * *$ WHEN EXECUTING ONLY SEGMENT 069, THE SPECIFICATION STATEMENTS

$C * * * *$ WHICH APPEAR AS COMMENT CARDS MUST HAVE THE $C=$

$C * * * *$ IN COLUMNS 1 AND 2 REMOVED.

$C * * * * *$

$C=\quad$ DOUBLE PRECISION MCAVD, MCBVD,MCCVD,MCDVD, MCEVD, MCFVD, MCGVD

$C * * * * *$

DOUBLE PRECISION MCAVD, MCBVD, MCCVD, MCDVD, MCEVD, MCFVD, MCGVD

ASA REF PO690060

8.2143P0690070

(TABLE 3)P0690080

$P 0690090$

$P 0690100$

P0690110

$P 0690120$

P0011650

$P 0011655$

$P 0011660$

$P 0011665$

$P 0011670$

P0011675

P069A1

P 0011680

$[* * * * \quad 0$ U T P U T T A P E ASSIGNMENT STATEMENT. NO INPUT TAPE.

$C * * * * *$

$C * * * *$ WHEN EXECUTING ONLY SEGMENT 06 , THE FOLLOW+NG STATEMENT

$C * * * *$ NUVI $=6$ MUST HAVE THE $C=$ IN COLUMNS 1 AND 2 REMOVEO.

$C=\quad$ NUVI $=6$

$C * * * * *$ NUVI $=6$

WRITE (NUVI,0690)

0690 FORMAT ( $1 \mathrm{H} 1,1 \mathrm{X}, 39 \mathrm{HIFOBL}$ - (069) INTRINSIC FUNCTION - DBLE/16X,

P0690130

$P 0071390$

$P 0071395$

$P 0071400$

$P 0071405$

$P 069 B 1$

$P 0071410$

$P 0690140$

$P 0690150$

126HS.P. ARGUMENT IN D.P. FORM / 2X,13HASA REF. - 8.2/12X,7HRESULTSIP0690160

$C * * * *$ HEADER FOR SEGMENT 069 WRITTEN

CMAVS $=0.9765625 E-3$

CMBVS $=-.1953125 \mathrm{E}-2$

CMCVS $=.5859375 \mathrm{E}-2$

CMOVS $=-.1048576 E+7$

CMEVS $=+114688.0$

MCAVD $=0.000$

$M C B \vee D=M C A \vee D * D B L E(C M A \vee S)$

$M C C V D=D M I N 1(D B L E(C M A \vee S), D B L E(C M E V S))$

$M C D V D=M C A V D * M C B \vee D-D A B S(D B L E(C M B \vee S))$

$M C E V D=M C A V D-D S I G N(D B L E$ (CMCVS), DBLE(CMBVS))

$M C F V D=-D A B S(D B L E(C M D V S))+M C A V D$

$M C G V D=D M A X 1(D B L E(A M A X 1(C M D V S, C M E V S)), M C B V D)$

WRITE(NUVI,691) CMAVS, MCCVD, CMBVS, MCDVD,

1

CMCVS, MCEVD, CMDVS, MCFVD, CMEVS, MCGVD

691 FORMAT(1HO,1X,6HLINE A, E18.718H LINE B, 025.14)

WRITE (NUVI, 692)

692 FORMAT(1HO,38H A COMPARISON OF LINE A AGAINST LINE B/1X,

$140 \mathrm{H}$ IS NEEDED TO CHECK THE VALIDITY OF TEST)

$C * * * *$ END OF TEST SEGMENT 069

$C * * * *$ WHEN EXECUTING ONLY SEGMENT 069 , THE STOP AND END CARDS

$C * * * *$ WHICH APPEAR AS COMMENT CAROS MÚST HAVE THE $C=$

$C * * * * *$ IN COLUMNS 1 AND 2 REMOVED.

$C=\operatorname{STOP}$

$C=\quad$ END

STOP

$P 0690170$

P0690180

$P 0690190$

$P 0690200$

P0690210

$P 0690220$

$P 0690230$

$P 0690240$

$P 0690250$

$P 0690260$

$P 0690270$

P 0690280

$P 0690290$

$P 0690300$

$P 0690310$

$P 0690320$

$P 0690330$

$P 0690340$

$P 0690350$

$P 0690360$

$P 0690370$

$P 0690380$

$P 0690390$

P0690400

$P 0690410$

P069C 1 
$[* * * * *$

$C * * * * *$

$C * * * * *$

$C * * * * *$

$C * * * * *$

$[* * * * *$

$C * * * *$

C ******

$C * * * * *$

$[* * * * *$

$[* * * * *$

$C * * * *$

c

C

$C * * * * *$

C**** OU U T U T T A P E ASSIGNMENT STATEMENT. NO INPUT TAPE.

$C * * * *$ WHEN EXECUTING ONLY SEGMENT 070, THE FOLLOWING STATEMENT

$C * * * *$ NUVI $=6$ MUST HAVE THE $C=$ IN COLUMNS 1 ANO 2 REMOVEO.

$C=N U V I=6$

NUVI $=6$

WRITE (NUVI,0700)

0700 FORMAT (1H1, 1X,40HIFCPX - (070) INTRINSIC FUNCTION - CMPLX/16X,

126 HEXPRESS TWO REAL ARGUMENTS/16X,15HIN COMPLEX FORM/15H ASA REF.

$2-8.2 / 12 X, 7$ HRESULTS)

C** HEAOER FOR SEGMENT O 0 WRITTEN

CMAVS $=23.123$

CMBVS $=-.78$

CMCVS $=+17$.

CMOVS $=157 . E-1$

CMEVS $=-.985 E 1$

CMFVS $=+88 . E+0$

CHAVC = CMPLX (CMAVS, CMBVS)

CHBVC $=$ CHAVC $-(23.123,-.78)$

CHAVC $=$ CMPLX (CMBVS, 15.0)

CHCVC $=$ CHAVC $-(-.78,15.0)$

CHAVC $=$ CMPLX (CMOVS, CMFVS)

CHOVC $=$ CHAVC $-(157 . E-1,+88 . E+0)$

CHAVC $=$ CMPLX $(0.0,0 . E O)$

CHEVC = CHAVC

CHAVC $=$ CMPLX (CMEVS, CMFVS)

CHFVC $=$ CHAVC $-(-.985 E 1,+88 . E+0)$

CHAVC $=$ CMPLX(CMCVS, $-0.0 E-1)$

CHGVC $=$ CHAVC $-(+17.0,0.0)$

WRITE (NUVI, 0702) CHBVC, CHCVC, CHOVC, CHEVC, CHFVC, CHGVC

WRITE (NUVI,0701)

0701 FORMAT (//2X,37HTHE ABOVE ANSWERS SHOULO ALL BE 0 FOR/1X,

$136 \mathrm{H}$ THIS TEST SEGMENT TO BE SUCCESSFUL.)

0702 FORMAT $(6(/ F 17.7, F 17.7))$

$[* \#$ ENO OF TEST SEGMENT 070

C* WHEN EXECUTING ONLY SEGMENT 070, THE STOP ANO ENO CARDS

$C * * *$ WHICH APPEAR AS COMMENT CAROS MUST HAVE THE C $=$

$C * * * *$ IN COLUMNS 1 AND 2 REMOVED.

$C=\quad$ STOP

$C=\ldots N O$

STOP

END

$C * * * * *$
$C * * \cdots * *$
$C * * * * *$

$C * * * *$

$C * * * * *$

$I F C J G-(071)$

$P 0700030$

P0700040

*Po700050

$8.2145 P 0700070$

(TABLE 3)P0700080

P0700090

$P 0700100$

$P 0700110$

$P 0700120$

$P 0011690$

P0011695

$P 0011700$

$P 0011705$

P0011710

POO11715

PO70A1

P0011720

$P 0700130$

$P 0071420$

$P 0071425$

P 0071430

P070B 1

$P 0700140$

P0700150

$P 0700160$

$P 0700170$

$P 0700180$

P0700190

$P 0700200$

$P 0700210$

$P 0700220$

P0700230

$P 0700240$

$P 0700250$

P0700260

$P 0700270$

$P 0700280$

P0 0700290

$P 0700300$

P0700310

$P 0700320$

$P 0700330$

P0700340

P 0700350

P0 0700360

$P 0700370$

$P 0700380$

$P 0700390$

P0700400

P0 0700410

$P 0700420$

$P 0700430$

$P 0700440$

$P 0700450$

$P 0700460$

$P 0700470$

$P 070 C 1$

POTOC2

P 0710010

P0710020

P0710030

P0710040

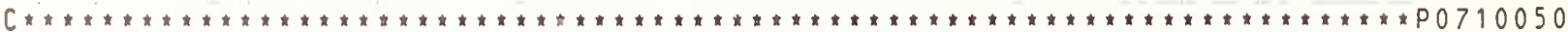

$C * * * *$ GENERAL PURPOSE

ASA REF P0710060 
C***** TEST INTRINSIC FUNCTION CONJG COBTAIN CONJUGATE OF A

$C * * * *$

\section{S P $C$ I F I C A T I O N S SEGMENT 071}

$P 0710110$

$C * * * * *$ WHEN EXECUTING ONLY SEGMENT 071, THE SPECIFICATION STATEMENTS

$C * * * * *$ IN COLUMNS 1 AND 2 REMOVED.

$C * * * * *$

$C=\quad$ COMPLEX CHAVC, CHBVC, CHCVC, CHDVC, CHEVC

$C * * * * *$

COMPLEX CHAVC, CHBVC, CHCVC, CHDVC, CHEVC

\section{$C * * * *$ OU T P U T T A P E ASSIGNMENT STATEMENT. NO INPUT TAPE.}

$C * * * * *$

$C * * * *$ WHEN EXECUTING ONLY SEGMENT 071 , THE FOLLOWING STATEMENT

$C * * * *$ NUVI $=6$ MUST HAVE THE $C=$ IN COLUMNS 1 AND 2 REMOVED.

$C=$ NUVI $=6$

$[* * * * *$

NUVI $=\dot{6}$

PO 0710120

$P 0011730$

$P 0011735$

$P 0011740$

$P 0011745$

$P 0011750$

PO71A1

$P 0011755$

$P 0710130$

$P 0071440$

$P 0071445$

$P 0071450$

$P 0071455$

$P 071 \mathrm{~B} 1$

WRITE (NUVI,0710)

0710 FORMAT (1H1, 1X,40HIFCJG - (071) INTRINSIC FUNCTION - CONJG/16X, 119 HOBTAIN CONJUGATE OF/16X,16HA COMPLEX NUMBER/

$217 \mathrm{H}$ ASA REFS. - $8.21 / 2 \times, 7$ HRESULTS

$C * * * * *$ HEADER FOR SEGMENT 071 WRITTEN

CHAVC $=(1.1,+2.1)$

CHBVC $=$ CONJG(CHAVC)

CHCVC $=$ CHBVC $-(1.1,-2.1)$

CHEVC $=(-2 . E 0,-3 . E-1)$

CHBVC $=$ CONJG (CHEVC)

CHDVC $=$ CHBVC $-(-2 . E 0,3 . E-1)$

WRITE (NUVI,0711) CHCVC, CHDVC

CHAVC $=(-.2,+.3)$

CHBVC $=$ CONJG(CHAVC)

CHCVC $=$ CHBVC $-(-.2,-.3)$

CHAVC $=(23.1 E-1,1 . E-2)$

CHBVC $=$ CONJG(CHAVC)

CHDVC $=$ CHBVC $-(23.1 E-1,-1 . E-2)$

WRITE (NUVI,0711) CHCVC,CHDVC

CHBVC $=$ CONJG $((1.2,2.2))$

CHCVC $=$ CHBVC $-(1.2,-2.2)$

CHBVC $=\operatorname{CONJG}((-1.0,2.0 E-1))$

CHDVC $=$ CHBVC $-(-1.0,-2.0 E-1)$

WRITE (NUVI,0711) CHCVC, CHDVC

CHBVC $=$ CONJG( $(.1, .2 E 0))$

CHCVC $=$ CHBVC $-(.1,-.2 E 0)$

CHOVC $=$ CONJG $((.0,-0 . E 0))$

WRITE (NUVI,0711) CHCVC, CHDVC

WRITE (NUVI,0712)

0711 FORMAT (4(1,F17.7, F10.7))

0712 FORMAT (//38H ALL ABOVE ANSWERS MUST BE $O$ FOR THIS/IX,

131 H TEST SEGMENT TO BE SUCCESSFUL.)

\section{$C * * * * *$ END OF TEST SEGMENT 071}

$C * * * * *$ WHEN EXECUTING ONLY SEGMENT 071 , THE STOP AND END CARDS

$C * * * *$ WHICH APPEAR AS COMMENT CARDS MUST HAVE THE $C=$

$C * * * *$ IN COLUMNS 1 AND 2 REMOVED.

$C=\operatorname{STOP}$

$C=\quad$ END

\section{STOP}

END

$P 0071460$

$P 0710140$

$P 0710150$

$P 0710160$

$P 0710170$

$P 0710180$

$P 0710190$

$P 0710200$

$P 0710210$

P0710220

$P 0710230$

P0710240

$P 0710250$

P0710260

$P 0710270$

P 0710280

P0710290

$P 0710300$

$P 0710310$

P0710320

$P 0710330$

$P 0710340$

$P 0710350$

$P 0710360$

$P 0710370$

$P 0710380$

P0710390

P0710400

$P 0710410$

$P 0710420$

$P 0710430$

$P 0710440$

$P 0710450$

$P 0710460$

$P 0710470$

$P 0710480$

$P 0710490$

P0710500

P0710510

$P 071 C 1$

$P 071 C 2$

\section{$C * * * * *$
$C * * * * *$}

$C * * * * *$

$C * * * * *$

IFBMS - $(072)$

$P 0720020$

$P 0720030$

$P 0720040$

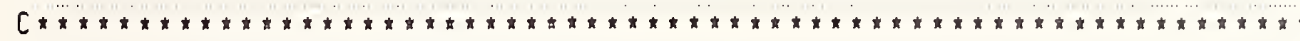

$C * * * *$ GENERAL PURPOSE

$C * * \pi$ TEST THAT ALL INTRINSIC FUNCTIONS WOULD ACCEPT

$C * * * *$ ANY EXPRESSION OF THE TYPE SPECIFIED IN THE
ASA REF P

8. 213200720070

(TABLE 3)PO 20080 
C*** WHEN EXECUTING ONLY SEGMENT 072, THE FOLLOWING STATEMENT
$[* * *$ NUVI $=6$ MUST HAVE THE $C=$ IN COLUMNS 1 AND 2 REMOVED.

$C=$ NUVI $=6$

$[* * * * *$

NUVI $=6$

$P 0720130$

$P 0720140$

$P 0071470$

P0071475

$P 0071480$

$P 07281$

WRITE (NUVI, 0720)

$P 0071485$

P0720150

0720 FORMAT (1H1,1X,37HIFBMS - (072) BASIC FORTRAN INTRINSIC/10X,

$P 0720160$

128 HFUNCTIONS ACCEPT EXPRESSIONS/10X,30HOF TYPE SPECIFIED IN I.F.TAPO7Z0170

2BLE//15H ASA REF.- $8.2 / / 2 X, 7 H R E S U L T S$ )

$C * * * *$ HEADER FOR SEGMENT 072 WRITTEN

C**** TEST ABS - ABSOLUTE VALUE OF REAL ARGUMENT

CMAVS $=0.5$

CMBVS $=-.25$

CMCVS $=16.0$

CMDVS $=-4.0$

CMEVS $=$ ABS (CMAVS + CMBVS $)+1.0$

CMFVS $=$ CMEVS $-(0.5-.25)-1.0$

CMEVS $=A B S(0.0-A B S(C M A V S-C M C V S+C M D V S))$

CMGVS $=$ CMEVS + $(0.5-16.0-4.0)$

CMEVS $=$ ABS(CMAVS + $1.0-($ CMCVS + CMDVS $)+0.5 * 8.0)$

CMHVS $=$ CMEVS $+(0.5+1.0-(16.0-4.0)+4.0)$

CMEVS $=\operatorname{ABS}(1.0 E 0+(1.0 * 1.0,1.0) * 2)$

CMIVS $=$ CMEVS -2.0

WRITE (NUVI,0721) CMFVS, CMGVS , CMHVS, CMIVS

C*** TEST OF IABS - ABSOLUTE VALUE OF INTÉGER ARGUMENT

$M C A V I=2$

MCBVI $=10$

MCCVI $=$ IABS (MCAVI+MCBVI)

$M C D V I=M C C V I-12$

$M C C V I=I A B S(M C A V I * 2+M C B V I / 2)+1$

P0720180

$P 0720190$

MCEVI $=$ MCCVI -10

MCCVI $=\operatorname{IABS}(-M C B V I /(-2)-\operatorname{MCBVI} * 1+(1 * 2 * 3 / 2-3)-10$

$1+10+$ MCBVI / MCAVI - 5)

$M C F V I=M C C V I-5$

$M C C V I=\operatorname{IABS}(0-\operatorname{IABS}(-5 * 1 / 5-5 * \operatorname{IABS}(-1)))$

$M C G V I=M C C V I-6$

WRITE (NUVI, 0722 ) MCDVI, MCEVI, MCFVI, MCGVI

C**** TEST OF' FLOAT - CONVERSION FROM INTEGER TO REAL

CMEVS = FLOAT (MCAVI+ MCBVI)

CMFVS $=$ CMEVS - 12.0

CMEVS = FLOAT(MCAVI $* 2 / 4+$ MCBVI**1)

CMGVS $=$ CMEVS -11.0

CMEVS $=$ FLOAT $(23+46) / 69+10-$ MCBVI $) * 2.0+1.5$

CMHVS $=$ CMEVS -3.5

CMEVS $=(76.5 * 1.0-$ FLOAT $(76 * 1)) * 4.0$

CMIVS $=$ CMEVS -2.0

WRITE (NUVI,0723) CMFVS, CMGVS, CMHVS, CMIVS

$C * * * * *$ TEST OF IFIX - CONVERSION FROM REAL TO INTEGER

$M C C V I=I F I X(C M A V S-C M B V S)$

MCDVI $=$ MCCVI

$M C C V I=I F I X(C M A V S * 1.0+C M B V S / C M B V S-(C M C V S-$ CMDVS $)$ )

MCEVI $=$ MCCVI +18

$M C C V I=1+\operatorname{IFIX}(2.5 * 2.0)-\operatorname{IFIX}(10.0 / 2.0)$

$M C F V I=M C C V I-1$

$M C C V I=2+\operatorname{IFIX}(2.5 * * 1.0+(10.65+3.45))$

$M C G V I=M C C V I-18$

WRITE (NUVI,0724) MCDVI, MCEVI, MCFVI, MCGVI

$C * * * *$ TEST OF SIGN - TRANSFER OF'SIGN WITH REAL ARGUMENTS

CMEVS = SIGN(CMAVS+CMDVS, CMDVS-CMBVS)

CMFVS $=$ CMEVS - (CMAVS + CMDVS)

CMEVS $=\operatorname{SIGN}(25.0+0.0 * 4.0 .-24.4 / 6.1 * 1.0)$

CMGVS $=$ CMEVS +25.0

$8.2111 P 0720200$

$P 0720210$

$P 0720220$

$P 0720230$

$P 0720240$

$P 0720250$

$P 0720260$

P 0720270

P 0720280

$P 0720290$

P0720300

$P 0720310$

$P 0720320$

$P 0720330$

8. $2 / 12 P 0720340$

$P 0720350$

$P 0720360$

$P 0720370$

$P 0720380$

$P 0720390$

$P 0720400$

P0720410

$P 0720420$

$P 0720430$

$P 0720440$

P0720450

P0720460

8. $2129 P 0720470$

$P 0720480$

$P 0720490$

$P 0720500$

$P 0720510$

$P 0720520$

$P 0720530$

P0720540

$P 0720550$

$P 0720560$

8. $2130 P 0720570$

$P 0720580$

P0720590

$P 0720600$

$P 0720610$

$P 0720620$

$P 0720630$

$P 0720640$

$P 0720650$

$P 0720660$

8. 2/31P0720670

$P 0720680$

$P 0720690$

$P 0720700$

P 0720710 
CMEVS $=\operatorname{SIGN}(10.5, \operatorname{SIGN}(2,0,-4.5))$

CMHVS $=$ CMEVS +10.5

$P 0720730$

CMEVS $=\operatorname{SIGN}(1.0, \operatorname{SIGN}(-2.0, \operatorname{SIGN}(2,0,-1,0)))$

PO720740

CMIVS $=$ CMEVS + $1 \cdot 0$

WRITE (NUVI,0725) CMFVS, CMGVS, CMHVS, CMIVS

P0720750

$P 0720760$

$C * * * *$ TEST OF ISIGN - TRANSFER OF SIGN WITH INTEGER ARGUMENT

MCCVI = ISIGN(MCAVI, MCAVI + MCBVI - 13)

MCDVI $=$ MCCVI + 2

MCCVI $=\operatorname{ISIGN}(10,-5-10 / 2+1 * 2)$

MCEVI $=$ MCCVI +10

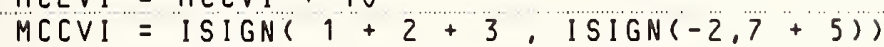

MCFVI $=$ MCCVI - 6

MCCVI $=\operatorname{ISIGN}(1, \operatorname{ISIGN}(-1, \operatorname{ISIGN}(+1,-1)))$

MCGVI $=$ MCCVI + 1

WRITE (NUVI,0726) MCDVI, MCEVI, MCFVI, MCGVI

C***** TEST OF COMBINATION OF ABS, IABS, FLOAT, IFIX, SIGN, ISIGN

CMEVS $=$ FLOAT(IABS(IFIX(ABS $(-5.0+\operatorname{SIGN}(-1.0 .2 .0))))$

CMFVS = CMEVS - 4.0

MCCVI $=$ IFIX(FLOAT(ISIGN(1+2,IABS(1+ISIGN(1,-1)))))

MCDVI = MCCVI - 3

CMEVS $=$ SIGN(ABS $(1.0+F L O A T(-20))$, FLOAT $(I F I X(1.0)))$

CMGVS = CMEVS - 19.0

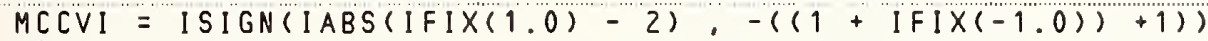

$M C E V I=M C C V I+1$

WRITE (NUVI,0727) CMFVS, CMGVS, MCDVI, MCEVI

CMEVS $=A B S(S I G N(1.0+2.0 . F L O A T(I A B S(-2))))$

CMFVS $=$ CMEVS -3.0

$M C C V I=I A B S(I F I X(S I G N(-2.0,2.0)))$

$M C D V I=M C C V I-2$

CMEVS $=1.2+$ FLOAT $(1+5-1$ SIGN $(-1,6))$

CMGVS $=$ CMEVS -6.2

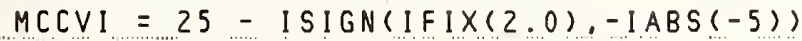

MCEVI = MCCVI - 27

WRITE (NUVI,0728) CMFVS, CMGVS, MCDVI, MCEVI

C***** END OF TEST STATEMENTS

0721 FORMAT ( I 30H TEST OF ABS IN EXPRESSIONS $-14(F 17.1 / 1)$ )

0722 FORMAT ( $31 \mathrm{H}$ TEST OF IABS IN EXPRESSIONS $-14(1151)$ )

0723 FORMAT ( $32 \mathrm{H}$ TEST OF FLOAT IN EXPRESSIONS $-14(\mathrm{~F} 17.1 / 1)$ )

0724 FORMAT ( $31 \mathrm{H}$ TEST OF IFIX IN EXPRESSIONS $-14(115 /)$ )

0725 FORMAT ( $31 \mathrm{H}$ TEST OF SIGN IN EXPRESSIONS $-14(\mathrm{~F} 17.1 / 1)$ )

0726 FORMAT ( $32 \mathrm{H}$ TEST OF ISIGN IN EXPRESSIONS $-14(1151)$ )

0727 FORMAT ( $40 \mathrm{H}$ COMBINATION OF ALL INTRINSIC FUNCTIONS,

$12(/ F 17.1), 2(1 / 195))$

0728 FORMAT ( $2(F 17.1 /) .2(115 /) / 35 \mathrm{H}$ ALL ABOVE ANSWERS SHOULD BE O FOPO721150

$1 R / 2 X, 35 H T H I S$ TEST SEGMENT TO BE SUCCESSFUL.)

C***** END OF TEST SEGMENT 072

$C * * *$ WHEN EXECUTING ONLY SEGMENT 072, THE STOP AND END CARDS

C**** WHICH APPEAR AS COMMENT CARDS MUST HAVE THE C $=$

C**** IN COLUMNS 1 AND 2 REMOVED.

$C=\quad S T O P$

$C=E N D$

STOP

END

$8.2132 P 0720770$

P0720780

P0720790

P0720800

P0720810

P0720820

P0720830

P0720840

P0720850

P0720860

P0720870

P0720880

P0720890

P0720900

P0720910

P0720920

P0720930

P0720940

P0720950

P0720960

P0720970

P0720980

P0720990

P0721000

P0721010

$P 0721020$

P0721030

P0721040

P0721050

P0721060

P0721070

P0721080

P0721090

P0721100

P0721110

P0721120

P0721130

P0721140

$P 0721160$

$P 0721170$

P0721180

$P 0721190$

P0721200

P0721210

$P 0721220$

$P 072 C 1$

$P 072 C 2$

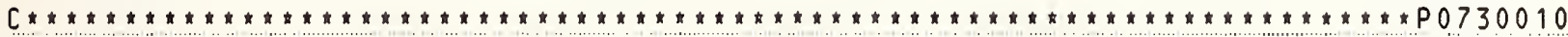

$C * * * * t$

$C * * * * *$

IFFMS - $(073)$

PO 730020

P0730030

C $* * \pi * *$

P0730040

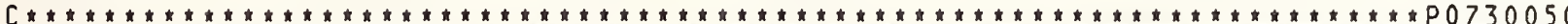

$[* * * *$ GENERAL PURPOSE

ASA REF P0730060

$8.2107 P 0730070$

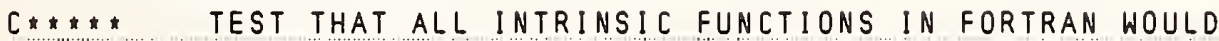

$C * * * * *$ ACCEPT ANY EXPRESSION OF THE TYPE SPECIFIED IN THE

C***** INTRINSIC FUNCTION TABLE - ASA REFS - 8.2/TABLE 3

(PG 24)P0730080

$P 0730090$

C**** SEGMENTS 055 - 071 ASSUMED WORKING.

$C * * * * *$

C*\#* S P E C I F I C A T I O N S SEGMENT 073

$C * * * * *$

$[* * * *$ WHEN EXECUTING ONLY SEGMENT 073. THE SPECIFICATION STATEMENTS

$P 0730100$

$P 0730110$

$P 0730120$

$P 0011760$

P0011765

$C * * * *$ WHICH APPEAR AS COMMENT CARDS MUST HAVE THE $C=$

P0011770

NBS FORTRAN Test Programs Version 1 
C*\# IN CDLUMNS 1 AND 2 REMOVED.

P0011775

$C * * * * *$

$C=\quad$ DIMENSION MCAII $(5), A C 2 S(5,6)$

$P 0011780$

$C=\quad$ INTEGER MCA3I $(2,3,3)$

$C=\quad$ DOUBLE PRECISION DPAV̈D, DPBVD, DPCVD,OPOVD, DPEVD,OPFVO,OPGVD,

$C=\quad$ IDPA1D(5), FC2D(5,5)

DIMENSION MCA I (5), ACZS $(5,6)$

INTEGER MCA3I $(2,3,3)$

DOUBLE PRECISION DPAVO,DPBVD, DPCVD, DPOVD, DPEVD, OPFVO, DPGVD. IDPA1D(5), FC2D(5,5)

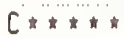

$[* * * * \quad 0 \quad U T P U T$ T A P E ASSIGNMENT STATEMENT.. NO INPUT TAPE.

C***** WHEN EXECUTING ONLY SEGMENT 073, THE FDLLOWING STATEMENT

C***NAUI=6 MUST HAVE THE C= IN COLUMNS 1 AND 2 REMOVED.

$C=\quad$ NUVI $=6$

NUVI $=6$

$C * * * * *$

WRITE (NUVI,0730)

0730 FORMAT ( 1 H1, 1X,41HIFFMS - (073) FORTRAN INTRINSIC FUNCTIONS/16X,

126 HACCEPT EXPRESSIONS OF TYPE/16X,22HSPECIFIED IN I.F.TABLE/

$223 \mathrm{H}$ ASA REF.- 8.2/TABLE 3//2X,7HRESULTS)

C*\#\# HEADER FOR SEGMENT 073 WRITTEN

C**** TEST OF DABS IN EXPRESSIONS

DPAVD $=1.2500$

DPBVD $=-10.000$

$D P C V D=D A B S(D P A V D+D P B V D)$

DPDVD = DPCVD $-10.000+1.2500$

$D P C V D=D A B S(1.000+2 . D O-3.0 D 0 * 50.0-1)$

$D P E V D=D P C V D-12.00$

DPCVD = DABS( DPAVD*1.DO-1.25D0+DPBVD/2.DO)+1.D0

$D P F V D=D P C V D-6.0 D O$

DPGVD $=1.000+D A B S(2.500-1.500 * 1.000)-2.00$

WRITE (NUVI, 0731 ) DPDVD, DPEVD, DPFVD, DPGVD

$C * * * * *$ TEST DF AINT IN EXPRESSIDNS

CMAVS $=1.23$

CMBVS $=27.998$

CMCVS $=-9.007 E 0$

CMDVS = AINT(CMAVS + CMBVS - CMCVS)

CMEVS $=$ CMDVS -38.0

CMDVS $=\operatorname{AINT}(1.0+2.0 / 1.0-3.0 * 2 . E 0)$

CMFVS $=$ CMDVS +3.0

CMDVS $=\operatorname{AINT}(4 .+\operatorname{AINT}(2 . E O+$ CMCVS $))$

CMGVS $=$ CMDVS +3.0

CMOVS $=\operatorname{AINT}(\operatorname{AINT}(\operatorname{AINT}(1.4-2.7)))$

CMHVS $=$ CMDVS +1.0

WRITE (NUVI, 0732) CMEVS, CMFVS, CMGVS, CMHVS

$C * * *$ TEST OF INT IN EXPRESSIDNS

$M C A V I=I N T(1.0+2.1+3.2-8.4 / 2.5 * 2.6)$

$M C B V I=M C A V I+2$

MCAVI $=I N T(100.0 / 6.0-(2.0 * 4.0)+((2.0-3.0)+4.0) * 2.0))$

MCCVI $=$ MCAVI -6

MCAVI $=I N T((100.2 / 6.1 / 5.0+4.10) / 2.0)$

MCDVI $=$ MCAVI -3

MCAVI $=I N T(9.0 / 2.0)+I N T(5.1 / 4.0)$

MCEVI = MCAVI - 5

WRITE (NUVI,0733) MCBVI, MCCVI, MCOVI, MCEVI

$C * \#$ TEST OF IDINT IN EXPRESSIONS

OPA1D(1) $=2.501$

$M C A V I=10 I N T(D P B V D / 2.000+1.500)$

MCBVI = MCAVI + 3

MCAVI $=$ IDINT $(1.001+5.00 * 2.01 / 49.01)+1$

MCCVI = MCAVI - 11

MCAVI = IOINT(DPA1O(1))

$M C O V I=M C A V I-25$

MCAVI $=$ IDINT $(D P A 10(1)+$ OPA1D(1)/4.000)

MCEVI = MCAVI- 31

WRITE (NUVI,0734) MCBVI, MCCVI, MCDVI, MCEVI
P0011785

$P 0011790$

$P 0011795$

$P 0011800$

P073A1

$P 073 A 2$

$P 073 A 3$

P073A4

P0011805

$P 0730130$

$P 0071490$

P0071495

$P 0071500$

P0071505

P 07381

$P 0071510$

$P 0730140$

$P 0730150$

$P 0730160$

$P 0730170$

$P 0730180$

8. $2 / 13 P 0730190$

$P 0730200$

$P 0730210$

P0730220

$P 0730230$

P0730240

$P 0730250$

P 0730260

$P 0730270$

$P 0730280$

$P 0730290$

$8.2 / 14 P 0730300$

$P 0730310$

$P 0730320$

$P 0730330$

$P 0730340$

$P 0730350$

$P 0730360$

$P 0730370$

$P 0730380$

$P 0730390$

$P 0730400$

$P 0730410$

$P 0730420$

$8.2 / 15 P 0730430$

$P 0730440$

$P 0730450$

$P 0730460$

$P 0730470$

$P 0730480$

$P 0730490$

$P 0730500$

$P 0730510$

$P 0730520$

$8.2 / 16 P 0730530$

$P 0730540$

$P 0730550$

$P 0730560$

$P 0730570$

$P 0730580$

$P 0730590$

$P 0730600$

$P 0730610$

$P 0730620$

P0730630 
$C * * *$ TEST OF AMDD. MOD IN EXPRESSIONS

$8.2 / 17-18 P 0730640$ $A C 2 S(1,1)=27.0$

$P 0730650$

CMDVS $=A M D D(25.0+A C Z S(1.1), 1.0 * 5.0)$

$P 0730660$

CMEVS $=$ CMDVS -2.0

CMDVS $=\operatorname{AMOD}(99.0 . \operatorname{AMOD}(25.0+27.0,5.0))$

$P 0730670$

CMFVS $=$ CMDVS -1.0

$P 0730680$

$M C A 3 I(1,2,3)=5$

MCAVI $=\operatorname{MOD}(98+1, \operatorname{MOD}(25+27,5))$

$P 0730690$

MCBVI = MCAVI - 1

MCAVI $=$ MDO (MCA3I $(1,2,3), 2)$

MCCVI = MCAVI - 1

WRITE (NUVI,0735) CMEVS, CMFVS, MCBVI, MCCVI

WRITE (NUVI,0735) CMEVS, CMFVS, MCBVI, MCCVI
$C * * *$ TEST DF AMAXO, AMAX1, MAXO。 MAX1 AND DMAX1 IN EXPRESSIONS
$C * * * 2119-23 P 0730770$

$F C 2 D(1,1)=27.000$

8.2/19-23P0730770

$P 0730780$

CMDVS $=\operatorname{AMAXO}(5+9, \operatorname{MAXO}(14 * 2, \operatorname{MAX} 1(2.0 / 1.0,1.0)))$

$P 0730790$

CMEVS $=$ CMDVS -28.0

CMDVS $=$ AMAX1((AMAXO $((\operatorname{MAXO}(29,-100)), 5+10)), 2.0 * 2.0)$

$P 0730800$

CMFVS $=$ CMDVS -29.0

MCAVI $=\operatorname{MAX} 1((\operatorname{MMAXO}(25,-(1 * 5))), 100.0)$

MCBVI = MCAVI - 100

$D P C V D=D M A X 1(F C 2 D(1,1), D M A X 1(1.0 D 0,0 . D 0 * F C 2 D(1,1)))$

DPDVD = DPCVD - 27.000

WRITE (NUVI,0736) CMEVS, CMFVS, MCBVI, DPDVD

$P 0730810$

$P 0730820$

P0730830

P0730840

P0730850

P0730860

P0730870

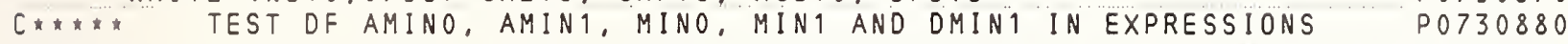

$[* * * * *$

CMDVS $=\operatorname{AMIN} 1(2.5+\operatorname{ACZS}(1,1), \operatorname{AMINO}(-5, \operatorname{MINO}(0,1)))$

$8.2124-27 P 0730890$

CMEVS $=$ CMDVS +5.0

MCAVI $=\operatorname{MINO}((\operatorname{MIN} 1(-99.100 .0-1.0 * 99)), 2$.

MCBVI = MCAVI + 99

MCAVI $=\operatorname{MIN1}(2.0, \operatorname{AMIN1}(5.3 .0,-9.0 /(-9.0)))$

$M C C V I=M C A V I-1$

DPCVD $=$ DMIN $1(F C 2 D(1,1), D M I N 1(2.00-1,0.000))$

DPDVD = DPCVD - $0.0 D 0$

WRITE (NUVI.0737) CMEVS, MCBVI, MCCVI, DPDVD

$C * * * *$ TEST DF DSIGN, AND DBLE IN EXPRESSIONS

$D P C V D=D S I G N(F C 2 D(1.1), 1.001,-1.000)$

$P 0730900$

$P 0730910$

$P 0730920$

$P 0730930$

$P 0730940$

$P 0730950$

$P 0730960$

$P 0730970$

$P 0730980$

DPDVD = DPCVD+27.001

DPCVD $=$ DSIGN ( $D S I G N(2.000,-1.000)+0.000), .000)$

DPEVD = DPCVD - 2.0DO

$D P C V D=D B L E(2.0 * 4.0+A C 2 S(1,1))$

DPFVD = DPCVD - 35.000

$D P C V D=D B L E(-32.00 / 8.0) * D B L E(-2.0)$

$D P G V D=D P C V D-8.0 D 0$

WRITE (NUVI, 0738 ) DPDVD, DPEVD, DPFVD, DPGVD

$C * * *$ TEST DF DIM AND IDIM IN EXPRESSIDNS

CMDVS $=\operatorname{DIM}(2.0 * 3.5 / 7.0, \operatorname{ACZS}(1,1))$

$8.2 / 33,8.2143 P 0730990$

$P 0731000$

$P 0731010$

$P 0731020$

$P 0731030$

$P 0731040$

$P 0731050$

$P 0731060$

$P 0731070$

$P 0731080$

CMEVS $=$ CMDVS -0.0

CMDVS $=\operatorname{DIM}(D I M(9.0,-5.5), \operatorname{DIM}(6.0,0.0))$

CMFVS $=$ CMDVS -8.5

$M C A 1 I(1)=8$

MCCVI $=\operatorname{IDIM}(M C A 1 I(1) * 1,-(\operatorname{IDIM}(0,-3)))$

MCDVI $=$ MCCVI -11

$M C C V I=\operatorname{IDIM}(((4+2+3) / 3),-2)$

MCEVI $=$ MCCVI - 5

WRITE (NUVI,9995) CMEVS, CMFVS, MCDVI, MCEVI

$8.2134-35 P 0731090$

P0731100

P0731110

P0731120

$P 0731130$

P0731140

P0731150

P0731160

$P 0731170$

P0731180

P0731190

$\begin{array}{rl}C * * * * & \text { TEST DF SNGL, REAL, AIMAG, CMPLX AND CDNJG IN EXPRESSIONS } \\ C * * * * * & 8.2136-47 P 0731210\end{array}$

CMEVS $=$ SNGL $(1.000 * 2.01+A C 2 S(1.1))$

$P 0731220$

$P 0731230$

CMFVS $=$ CMEVS -47.0

CMEVS $=\operatorname{REAL}(\operatorname{CDNJG}((1.0,-2.0)))+\operatorname{AIMAG}((99.0,-7.0))$

CMGVS $=$ CMEVS +6.0

CMEVS $=\operatorname{AIMAG}(C M P L X(\operatorname{REAL}((2.0,1.0)), \operatorname{SNGL}(1.000)))$

CMHVS $=$ CMEVS -1.000

WRITE (NUVI,0739) CMFVS, CMGVS, CMHVS

$C * * *$ SOME CDMBINATIONS OF ABOVE INTRINSIC FUNCTIONS

CMEVS $=\operatorname{AMIN1}((\operatorname{FLOAT}(10 I M(1+2,0))),(\operatorname{AIMAG}(C M P L X(1.0,2.0))))$

CMFVS $=$ CMEVS -2.0

$P 0731240$

$P 0731250$

$P 0731260$

$P 0731270$

$P 0731280$

$P 0731290$

$P 0731300$

P0731310 
CMEVS $=\operatorname{REAL(CMPLX(SNGL(DABS(-DSIGN(DBLE(2.0),1.0D0))),~CMAVS))~}$

$P 0731320$

CMGVS = CMEVS - 2.0

WRITE (NUVI, 9994) CMFVS, CMGVS

C**** END OF TEST STATEMENTS FOR SEGMENT 073

0731 FORMAT ( $/ 30 \mathrm{H}$ TEST OF DABS IN EXPRESSIONS / $14(023.81)$ )

0732 FORMAT ( $30 \mathrm{H}$ TEST OF AINT IN EXPRESSIONS //4(E19.6/))

0733 FORMAT ( $30 \mathrm{H}$ TEST OF INT IN EXPRESSIONS $/ 14(110 /))$

0734 FORMAT ( $30 \mathrm{H}$ TEST OF IDINT IN EXPRESSIONS//4(I10/))

0735 FORMAT ( $35 \mathrm{H}$ TEST OF AMOD, MOD IN EXPRESSIONS /I

$12(E 19.6 /), 2(110 /))$

0736 FORMAT ( $40 \mathrm{H}$ TEST OF AMAXO, AMAX1, MAXO, MAX1 AND DMAXII

$P 0731330$

PO 0731340

$P 0731350$

$P 0731360$

P0731370

$P 0731380$

$P 0731390$

$P 0731400$

P 0731410

PO 0731420

$12(E 19.6 /), 110 / 023.8)$

0737 FORMAT ( $40 H 1$ TEST OF AMINO, AMIN1, MINO,MINI AND DMINII

P 0731430

$P 0731440$

$1 E 19.6 / 2(110 /), 023.8)$

0738 "FORMAT ( $/ 39 \mathrm{H}$ "TEST OF" DSIGN AND DBLE IN EXPRESSIONS//4(D23.8/))

0739 FORMAT ( $35 \mathrm{H}$ TEST OF SNGL,REAL, AIMAG, CMPLX AND I

$123 \mathrm{H}$ CONJG IN EXPRESSIONS //3(E19.6/))

9994 FORMAT ( $36 \mathrm{H}$ TEST OF SOME COMBINATIONS OF ABOVEI

$P 0731450$

$P 0731460$

P0731470

P0731480

P0731490

$122 \mathrm{H}$ INTRINSIC FUNCTIONS $/ / 2(E 19.6 /) / 40 \mathrm{H}$ ALL ABOVE ANSWERS SHOULPO731500

2D BE O FOR TH!S/27H SEGMENT TO BE SUCCESSFUL.)

9995 FORMAT ( $137 \mathrm{H}$ TEST OF DIM AND IDIM IN EXPRESSIONSIZ(E19.6/),

$P 0731510$ $12(110 /))$

\section{C***** END OF TEST SEGMENT 073}

C***** WHEN EXECUTING ONLY SEgMENT 073. THE STOP AND END CARDS

$C * * *$ WHICH APPEAR AS COMMENT CARDS MUST HAVE THE C=

C*****IN COLUMNS 1 AND 2 REMOVED.

$C=S T O P$

$C=\quad E N D$

STOP

END

$P 0731520$

$P 0731530$

$P 0731540$

$P 0731550$

$P 0731560$

P0731570

P0731580

$P 0731590$

P073C1

P073C2

$C * * * * *$

P0800010

C*****

EXPON - 080

P0800020

$C * * * * *$

P0800030

P0800040

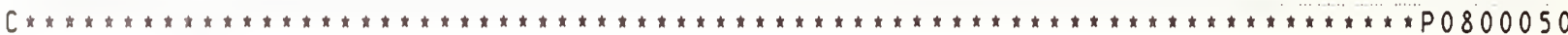

C****: GENERAL PURPOSE

$C * * * *$.TO TEST BASIC EXTERNAL FUNCTION - EXP - EXPONENTIAL

C***** .USED IN SIMPLE ARITHMETIC EXPRESSIONS

C*****.INTRINSIC FUNCTIONS ABS AND SIGN ASSUMED WORKING

$C * * * *$ ARGUMENTS ARE POWERS OF 2

ASA REFP0800060

$8.3 .3 \quad P 0800070$

TABLE 4 P0800080

$P 0800090$

P0800100

$[* * * * *$

C\#\#\# NOSPECIIFI C A T I O N S SEGMENT OSO

P0800110

P0800120

$C * * * * *$

$C * * * *$

o u t p u t t a p e assignment statement. nO InPUT tape.

P0800130

$C * *$ WHEN EXECUTING ONLY SEgMENT 080, THE FOLLOWING STATEMENT

$C * * * *$ NUVI $=6$ MUST HAVE THE $C=$ IN COLUMNS 1 AND 2 REMOVED.

$C * * * * *$

$C=$ NUVI $=6$

NUVI $=6$

WRITE (NUVI, 800)

800 FORMAT(15H9 EXPON - $(080) / / 39$ H BASIC EXTERNAL FÜNCTION -EXP-

P0800140

$P 0071520$

P0071525

$P 0071530$

$P 0071535$

P080B1

$P 0800150$

$1 / / 26 \mathrm{H}$ (EXPONENTIAL - TYPE REAL)

3 HOLLERITH INFORMATION//9H RESULTS)

P 0800170

LINE 1 OF EACH PAIR IS/ $23 \mathrm{H}$ PO800180

PO 0800190

C**** HEADER FOR SEGMENT 080 WRITTEN

C*** ARGUMENT RANGE FROM - 16.0 TO +16.0

P0800210

AVS $=-16.0$

CVS $=4.0$

$B V S=\operatorname{EXP}(A \vee S)$

WRITE (NUVI, 801) BVS

$B V S=\operatorname{EXP}(2 . * C V S+A V S)$

WRITE (NUVI, 802) BVS

$B V S=\operatorname{EXP}(A V S+(3 . *$ CVS $))$

WRITE (NUVI, 803 ) BVS

$B V S=\operatorname{EXP}(A B S(A \vee S)+A V S)$

WRITE (NUVI, 804) BVS

P0800220

P0800230

P0800240

$P 0800250$

P0800260

P0800270

P0800280

P0800290

P0800300

P0800310

$B V S=\operatorname{EXP}(-A V S /$ (VS)

P0800320

WRITE (NUVI, 805) BVS

P0800330 
$B V S=\operatorname{EXP}(S I G N(A V S+C V S * 2.0, C V S))$

$P 0800340$

WRITE (NUVI, 806) BVS

$P 0800350$

$B V S=\operatorname{EXP}(C V S+A B S(A V S)-4.0)$

$P 0800360$

WRITE (NUVI, 807) BVS

WRITE (NUVI, 808 )

801

802

803

804

805

806

807

808

FORMAT ( $9 H O \quad X=-16.0,5 X, 25 H 0.1125351747192591145 E-06 / E 27.7)$

FORMAT( $9 H O \quad X=-8.0,5 X, 25 H 0.3354626279025118388 E-03 / E 27.7)$

FORMAT( $9 H 0 \quad X=-4.0,5 X, 25 H 0.1831563888873418029 E-01 / E 27.7)$

FORMAT ( $9 H O \quad X=0.0,5 X .25 H 0.1000000000000000000 E+01 / E 27.7$ )

FORMAT( $9 H 0 X=4.0 .5 X, 25 H 0.5459815003314423908 E+02 / E 27.7)$

FORMAT( $9 H O X=8.0,5 X .25 H 0.2980957987041728275 E+04 / E 27.7)$

FORMAT ( $\left.9 H 0 \quad X=16^{\circ} .0,5 X, 25 H 0.8886110520507872637 E+07 / E 27.7\right)$

$P 0800370$

$P 0800380$

$P 0800390$

P0800400

PO 800410

$P 0800420$

$P 0800430$

P 0800440

$P 0800450$

FORMAT $/ / 37 \mathrm{H}$ LINE 2 OF EACH PAIR IS THE FUNCTION/25H CALCULATIONPO800460

1 PRINTED TO, 8 H DIGITS)

C**** END OF TEST SEGMENT 080

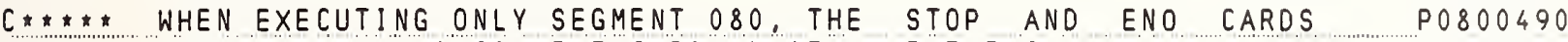

$C * * * *$ WHICH APPEAR AS COMMENT CARDS MUST HAVE THE $C=$

$C * * * *$ IN COLUMNS 1 ANO 2 REMOVED.

$C=$ STOP

$C=\quad E N O$

STOP

$P 0800470$

$P 0800480$
$P 0800490$

P0 0800500

P0800510

$P 0800520$

P0800530

ENO

P080C 1

$P 080 C 2$

$C * * * *$

$C * * * * *$

$C * * * *$

$C * * * * *$

P0810010

$P 0810020$

P0810030

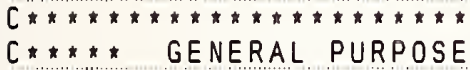

$P 0810040$

$C * * * * *$ TO TEST BASIC EXTERNAL FUNCTION - DEXP - EXPONENTIAL

$C * * * * *$ USED IN SIMPLE ARITHMETIC EXPRESSIONS - SAME AS

$C * * * *$ SEGMENT 080 EXCEPT OOUBLE PRECISION

$C * * * * *$ INTRINSIC FUNCTIONS OABS ANO OSIGN ASSUMEO WORKING

P08 10060 $C * * * *$ ARGUMENTS RANGE FROM -16.000 T0 +16.000, POWERS OF 2

ASA REFP0 810070

8.3.3 P0810080

TABLE $4 P 0810090$

P0810100

$C * * * * *$

P0810110

$P 0810120$

$P 0810130$

P0011810

$[* * * * *$ WHEN EXECUTING ONLY SEGMENT 081 , THE SPECIFICATION STATEMENTS

$C * * * * *$ WHICH APPEAR AS COMMENT CAROS MUST HAVE THE $C=$

$C * * * *$ IN COLUMNS 1 ANO 2 REMOVEO.

$C * * * * *$

$C=\quad$ DOUBLE PRECISION AVO, BVO, CVD

DOUBLE PRECISION AVO, BVD, CVD

$C * * * * *$

$C * * * * 0$ U T P U T T A P E ASSIGNMENT STATEMENT... NO INPUT TAPE.

$C * * * * *$

WHEN EXECUTING ONLY SEGMENT 081. THE FOLLOWING STATEMENT

$C * * * *$ NUVI $=6$ MUST HAVE THE C= IN COLUMNS 1 ANO 2 REMOVEO.

$C=\ldots$ NUVI $=6$

NUVI $=6$

$C * * * * *$

810 FORMAT $(15 H 1$ DEXPO- $(081) / 132 \mathrm{H}$ BASIC EXTERNAL FUNCTION-OEXP-

$1 / 138 \mathrm{H}$ (EXPONENTIAL - TYPE OOUBLE PRECISION)

$2 / 127 \mathrm{H}$ ASA REF.- 8.3 .3 (TABLE 4)//24H LINE $1 "$ OF EACH PAIR IS/23H

3 HOLLERITH INFORMATION//9H RESULTS)

WRITE (NUVI, 810)

$C * * * *$ HEAOER FOR SEGMENT 081 WRITTEN

$A \cup D=-16.000$

$C V D=4.000$

$B \vee O=O E \times P(A \vee O)$

WRITE (NUVI, 811) BVD

$B V D=O E X P(2 . * C V O+A V O)$

WRITE (NUVI, 812) BVD

$B \vee D=0$ EXP(AVD + (3.* (VD))

WRITE (NUVI, 813) BVO

$B \vee O=O E X P(O A B S(A \vee O)+A \vee O)$

WRITE( NUVI, 814) BVO

BVO $=$ OEXP(-AVO / CVO)

P0011815

$P 0011820$

$P 0011825$

$P 0011830$

$P 0011835$

P081A1

P 0011840

$P 0810140$

$P 0071540$

$P 0071545$

$P 0071550$

$P 0071555$

P081B 1

$P 0071560$

P0810150

P0810160

WRITE (NUVI, 815) BVD

$P 0810170$

$P 0810180$

P0810190

$P 0810200$

$P 0810210$

P0810220

$P 0810230$

$P 0810240$

$P 0810250$

$P 0810260$

P0810270

P0810280

P0810290

$P 0810300$

$P 0810310$

P0810320 
BVD $=\operatorname{DEXP(DSIGN(AVD+CVD*2.0DO,CVD)})$

P08 10330

WRITE (NUVI, 816) BVD

$P 0810340$

$B V D=D E X P(C V D+D A B S(A V D)-4.0)$

WRITE (NUVI, 817) BVD

P0810350

WRITE (NUVI, 818)

811 FORMAT $(9 H O X=-16.0 .5 X, 25 H 0.11253517471925911450-06 / 034.14)$

812 FORMAT( $9 H O X=-8.0,5 X, 25 H O .33546262790251183880-03 / 034.14)$

813 FORMAT( $9 H O X=-4.0,5 X, 25 H 0.18315638888734180290-01 / 034.14)$

814 FORMAT( $9 H 0 X=0.0,5 X .25 H 0.10000000000000000000+01 / 034.14)$

815 FORMAT( $9 H O X=4.0,5 X, 25 H 0.54598150033144239080+02 / 034.14)$

816 FORMAT( 9 HO $X=8.0,5 X, 25 H 0.29809579870417282750+04 / 034.14)$

$P 0810360$

$P 0810370$

$P 0810380$

P0810390

$P 0810400$

$P 0810410$

$P 0810420$

$P 0810430$

FORMAT( $9 H O X=16.0,5 X, 25 H 0.88861105205078726370+07 / 034.14)$

$P 0810440$

817

FORMAT ///37H LINE 2 OF EACH PAIR IS THE FUNCTION/25H CALC

A PRINTED TO, 9 H 14 DIGITS)

$C * *$ END OF TEST SEGMENT 081

$C * * * *$ WHEN EXECUTING ONLY SEGMENT 081 , THE STOP AND END CARDS

$C * * * *$ WHICH APPEAR AS COMMENT CARDS MUST HAVE THE $C=$

$C * * *$ IN COLUMNS 1 AND 2 REMOVED.

$C=\quad$ STOP

$C=\quad$ END

STOP

$P 0810450$

P0810460

P0810470

$P 0810480$

P0810490

P0810500

P0810510

P0810520

END

P081C1

$P 081 C 2$

$C * * * * *$
$C * * * * *$

$C * * * *$

CEXPO - $(082)$

P0820020

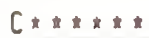

P0820030$$
\begin{aligned}
& C * * * * * * * * * * * * * * * * * * * * * * t \\
& C * * * * * \text { GENERAL PURPOSE }
\end{aligned}
$$

$C * * * *$ TENO TEST THE BASIC EXTERNAL FUNCTION- CEXP

$C * * *$.TESTING RANGE EXTENDS FROM 0 TO 16 FOR MODULUS

C*** AND ARGUMENT, VARIES BY STEPS OF PI/3 MAGNITUDE

$C * * * * *$.INTRINSIC FUNCTIONS CMPLX, SNGL, MOD ASSUMED WORKING

$C * * * *$

$C * * *$ S P E C I F I C A T I O N S SEGMENT 082

$C * * * * *$

$C * * *$

$C * * * * *$

$[* * * * *$

$C * * * * *$

$C=\quad C O M P L E X E P 1 C(30), A \vee C, B \vee C$

$C=\quad$ DOUBLE PRECISION AVD, BVD

COMPLEX EP1C $(30), A \vee C, B \vee C$

DOUBLE PRECISION AVD, BVD

$[* * * * *$

C*:**OU T PU T T A P E ASSIGNMENT STATEMENT. NO INPUT TAPE.

$C * * * * *$

C** WHEN EXECUTING ONLY SEGMENT 082, THE FOLLOWING STATEMENT

$C * * * *$ NUVI $=6$ MUST HAVE THE $C=I N$ COLUMNS 1 AND 2 REMOVED.

$C=\quad$ NUVI $=6$

$C * * * * *$

NUVI $=6$

WRITE(NUVI, 820)

820 FORMAT $(15 H 1$ CEXPO - $(082) / / 32 \mathrm{H}$ BASIC EXTERNAL FUNCTION - CEXP-

$1 / / 29 H$ (EXPONENTIAL - TYPE COMPLEX)//27H ASA REF.- 8.3.3 (TABLE 4P0820160

2) $/ 120 \mathrm{H}$ (COMPLEX ARGUMENT)/8X, 15 HEXPECTED RESULT /8X,15HFUNCTION RPO 820170

3ESULT)

$C: \angle O Q G$ OF 10

$B V D=2.302585092994000$

C* SINE OF 60 DEGREES

AVD $=.8660254037844400$

$C$ INITIALIZE EPIC (EXPECTED VALUES)

$E P 1 C(1)=C M P L X(0.5 E-7$, SNGL $(-A V D * 1 . D-7))$

$E P 1 C(2)=C M P L X(2.5 E-7, S N G L(-A V D * 5.0-7))$

$E P 1 C(3)=(1 . E-6,0.0)$

$E P 1 C(4)=(5 . E-6,0.0)$

$E P 1 C(5)=C M P L X(0.5 E-5, S N G L(A V D * 1.0-5))$

$E P 1 C(6)=C M P L X(2.5 E-5, S N G L(A \vee D * 5 . D-5))$

$E P 1 C(7)=C M P L X(-.5 E-4, S N G L(A V D * 1 . D-4))$
ASA REF.PO820060

8.3.3 PO820070

(TABLE 4)P0820080

P0820090

P0820100

P0820110

P0820120

P0011850

$P 0011855$

$P 0011860$

$P 0011865$

POO 011870

$P 0011875$

P0011880

P08ZA 1

PO82A2

P0011885

$P 0820130$

$P 0071570$

$P 0071575$

$P 0071580$

$P 0071585$

P082B1

P 0071590

$P 0820140$

$P 0820150$

$R P 0820170$
$P 0820180$

P0 820190

$P 0820200$

P0820210

P0820220

P0 820230

P0 820240

P0 820250

P0 820260

P0820270

P0820280

P0820290

P0 820300 
$E P 1 C(8)=C M P L X(-2.5 E-4, S N G L(A V D * 5 . D-4))$

P0820310

$E P 1 C(9)=(-1, E-3,0.0)$

$P 0820320$

$E P 1 C(10)=(-5 . E-3,0.0)$

$E P 1 C(11)=C M P L X(-0.5 E-2, S N G L(-A V D * 1 . D-2))$

P0 0820330

$E P 1 C(12)=C M P L X(-2.5 E-2.5 N G L(-A V D * 5 . D-2))$

$P 0820340$

$E P 1 C(13)=C M P L X(0,5 E-1, S N G L(-A V D * 1 . D-1))$

$P 0820350$

$E P 1 C(14)=C M P L X(2.5 E-1 . S N G L(-A \vee D * 5 . D-1))$

$P 0820360$

$E P 1 C(15)=(1.0,0.0)$

$E P 1 C(16)=(5.0,0.0)$

$E P 1 C(17)=C M P L X(0.5 E 1, S N G L(A V D * 1 . D 1))$

$E P 1 C(18)=C M P L X(2.5 E 1, S N G L(A V D * 5 . D 1))$

$E P 1 C(19)=C M P L X(-0.5 E 2, S N G L(A \vee D * 1 . D 2))$

$P 0820370$

P0 0820380

$P 0820390$

$P 0820400$

$E P 1 C(20)=C M P L X(-2.5 E 2 . S N G L(A \vee D * 5 . D 2))$

P0820410

$P 0820420$

$E P 1 C(21)=(-1 . E 3,0.0)$

$E P 1 C(22)=(-5 . E 3,0.0)$

EP1C(23) $=$ CMPLX(-0.5E4.SNGL (-AVD*1.D4))

$E P 1 C(24)=$ CMPLX(-2.5E4, SNGL(-AVD*5.D4))

$E P 1 C(25)=C M P L X(0.5 E 5, S N G L(-A \vee D * 1.05))$

$E P 1 C(26)=C M P L X(2.5 E 5 . S N G L(-A V D * 5 . D 5))$

$E P 1 C(27)=(1 . E 6,0.0)$

$E P 1 C(28)=(5 . E 6,0.0)$

$E P 1 C(29)=$ CMPLX(0.5E7,SNGL(AVD*1.07))

$P 0820430$

$P 0820440$

$P 0820450$

P0820460

P0 820470

$P 0820480$

$P 0820490$

$P 0820500$

$P 0820510$

$P 0820520$

EP1C(30) $=C M P L X(2.5 E 7 . S N G L(A \vee D * 5 . D 7))$

P0 0820530

IVI $=0$

821 IVI $=I V I+1$

IF (MOD(IVI, 2).EO.0) GO TO 822

$X I V S=((I V I+1) / 2)-8$

AVS $=$ BVD*XIVS

GO TO 823

$C * * * * * 1.609$ IS LOG OF 5

822 XIVS $=($ IVI $/ 2)-8$

$A V S=B V D * X I V S+1.609437912434100$

$C * * * * 1.047$ IS PI/3

$823 \quad A V C=$ CMPLX(AVS,SNGL(1.047197551196600*XIVS))

$B \vee C=\operatorname{CEXP(A\vee C)}$

WRITE(NUVI, 824) AVC, EP TC(IVI), BVC

IF (IVI - 10$) 825,827,825$

825 IF (IVI - 20) 826,827,826

826 IF (IVI - 30$) 821,828,828$

827 WRITE(NUVI, 829)

GO TO 821

828 CONTINUE

829 FORMAT (22H1 CEXPO- $(082)-C E X P-)$

824 FORMAT $(3 H O(, E 14.7,1 H, . E 14.7,1 H), 2(18 X, 2 E 16.7))$

C***** END OF TEST SEGMENT 082

$C * * * *$ WHEN EXECUTING ONLY SEGMENT 082, THE STOP AND END CARDS

$C * * *$ WHICH APPEAR AS COMMENT CARDS MUST HAVE THE $C=$

$C * * * *$ IN COLUMNS 1 AND 2 REMOVED.

$C=S T O P$

$C=\quad$ END

STOP

$P 0820540$

$P 0820550$

$P 0820560$

$P 0820570$

P0820580

P0820590

$P 0820600$

P0820610

$P 0820620$

$P 0820630$

P0820640

P0820650

$P 0820660$

P0820670

$P 0820680$

$P 0820690$

$P 0820700$

P0820710

$P 0820720$

P0820730

P0820740

P0820750

$P 0820760$

P0 820770

$P 0820780$

P0 820790

$P 0820800$

P $082 C 1$

END

$P 082 C 2$

$C * * * * *$
$C * * * * *$

C *****

$C * * * *$

P0830010

P0830020

P0830030

$P 0830040$

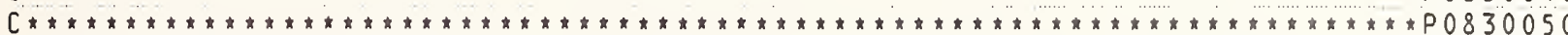

$C * * * *$ GENERAL PURPOSE

$C * * * *$. TO TEST BASIC EXTERNAL FUNCTION - ALOG -

$C * * * *$ NATURAL LOG -USED IN SIMPLE ARITHMETIC EXPRESSIONS

C**** INTRINSIC FUNCTIONS ABS,AMIN1, INT,MINO,FLOAT,

$C * * * * * \quad$ SIGN ASSUMED WORKING C**** ARGUMENTS ARE POWERS(OR SUMS) OF" 2

$P 0830060$

$C * * * * *$

ASA REF PO830070

8.3.3 P0830080

TABLE 4 PO 830090

$P 0830100$

P0830110

$P 0830120$

$P 0830130$

P 0071600

$P 0071605$

$P 0071610$

C**** WHEN EXECUTING ONLY SEGMENT 083. THE FOLLOWING STATEMENT

$C * * * * *$ NUVI $=6$ MUST HAVE THE $C=$ IN COLUMNS 1 AND 2 REMOVED. 
830 FORMAT(15H1 LOGTM - $(083) / / 32 H$ BASIC EXTERNAL FUNCTION - ALOG-

$1 / 126 \mathrm{H}$ (NATURAL LOG - TYPE REAL)

ASA REF - 8.3.3 (TABLE 4)/124H LINE I OF EACH PAIR IS/23H P0830160

3 HOLLERITH INFORMATION//9H RESULTS)

P 0830170

WRITE (NUVI, 830)

C**** HEAOER FOR SEGMENT 083 WRITTEN

AVS $=.25$

CVS $=2.0$

$M V I=-2$

$B V S "=A L O G(A V S ; 2.0)$

WRITE (NUVI, 831) BVS

$P 0830180$

$P 0830190$

$P 0830200$

P0830210

$P 0830220$

$B \vee S=A L O G$ (AVS)

P0 830230

$P 0830240$

WRITE (NUVI, 832) BVS

$P 0830250$

$B V S=A L O G(A \vee S * C V S)$

$P 0830260$

WRITE (NUVI, 833) BVS

$P 0830270$

$B V S=A L O G(A V S *$ CVS *2)

WRITE (NUVI, 834) BVS

$P 0830280$

$B V S=A L O G(A M I N 1$ (AVS $=2.0+A B S(F L O A T(M V I), C V S), C V S)$ )

$P 0830290$

$P 0830300$

WRITE (NUVI, 835) BVS

P0830310

BVS $=A L O G(S I G N(F L O A T(M I N O(M \vee I, I N T(C \vee S))), A V S))$

$P 0830320$

WRITE (NUVI, 836) BVS

831 FORMAT( $9 H 0 \quad X=0.125 .5 X, 19 H-2.0794415416798359 / 14 X, F 9.6)$

832 FORMAT( $9 H 0 X=0.25,5 X, 19 H-1.3862943611198906 / 14 X, F 9.6)$

833 FORMAT ( $9 H 0 \quad X=0.5 \quad, 5 X, 19 H-0.6931471805599453 / 14 X, F 10.7)$

$P 0830330$

P0830340

$P 0830350$

$P 0830360$

FORMAT ( $9 H 0 \quad X=1.0 \quad, 5 X .19 H \quad 0.0000000000000000 / 14 X, F 10.7)$

$P 0830370$

834

FORMAT( $9 H O X=1.5 \quad, 5 X, 19 H \quad 0.4054651081081644 / 14 X, F 10.7)$

836

FORMAT( $9 H 0 \quad X=2.0,5 X, 19 H \quad 0.6931471805599453 / 14 X, F 10.7)$

$P 0830380$

$P 0830390$

$P 0830400$

WRITE (NUVI, 837) P P0830410

837 FORMAT(//37H LINE 2 OF EACH PAIR IS THE FUNCTION/25H CALCULATIONPO830420

1 PRINTEO TO , 8HT OIGITS)

\section{$C * * * *$ END OF TEST SEGMENT 083}

C*** WHEN EXECUTING ONLY SEGMENT 083, THE STOP AND END CARDS

$C * * *$ WHICH APPEAR AS COMMENT CARDS MUST HAVE THE $C=$

$C * *$ IN COLUMNS 1 AND 2 REMOVEO.

$C=S T O P$

$C=\quad E N D$

STOP

P0 830430

P0830440

\section{ENO}

P0830450

P0830460

P0 830470

P0830480

P0 830490

P083C1

P083C2

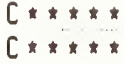

$C * * * * *$

$C * * * * *$

0840010

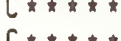

$C * * *$

$C * * * *$

$C * * * *$

GENERAL PÜRPOSE

P 0840020

P0 840030

P0 840040

C**** INTRINSIC FUNCTIONS DMINI, OABS, DBLE, FLOAT, DSIGN,

$C * * * * \quad M I N O, D I N T$, ASSUMED WORKING

$C * * *$ ARGUMENTS ARE POWERS OF 2

$C * * *$

$C * * * * *$

$C * * * *$

$S p$

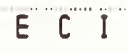

F I

C A

T $0 \mathrm{~N}$

\section{S}

SEGMENT 084

$C * * * *$ WHEN EXECUTING ONLY SEGMENT 084, THE SPECIFICATION STATEMENTS

$C * * * *$ WHICH APPEAR AS COMMENT CARDS, MUST HAVE THE $C=$

C**** IN COLUMNS 1 AND 2 REMOVED.

$C * * * *$

$C=\quad$ DOUBLE PRECISION AVD, BVD, CVD

DOUBLE PRECISION AVD, BVD, CVD

$C * \cdots * *$

$C * * * *$

$C * * * * *$

$[* * * * *$

O U T P U T

$T A P E$

ASSIGNMENT STATEMENT.

NO INPUT TAPE.

ASA REF

$P 0840060$

$C=$ NUVI $=6$

WHEN EXECUTING ONLY SEGMENT 084 . THE FOLLOWING STATEMEN
NUV! $=6$ MUST HAVE THE C IN COLUMNS 1 AND 2 REMOVED.

8.3 .3

$P 0840070$
$P 0840080$

P0840090

P0 840100

P0840110

P 0840120

P084 40130

P0840140

P0011890

P0011895

P0011900

P0011905

P0011910

P0011915

P084A1

P 0011920

P0840150

P 0071630

P0071635

P 0071640

P0071645 
$C * * * * *$

840 FORMAT(15H1 OPLOG-(084)//32H BASIC EXTERNAL FUNCTION - OLOG-

$2 / / 27 H$ ASA REF.- 8.3.3 (TABLE 4)//24H LINE 1 OF EACH PAIRIS/23H P0840180 3 HOLLERITH INFORMAT ION/19H RESULTS)

$P 0840190$ WRITE (NUVI, 840)

$P 0840200$

$C * * * * *$ HEADER FOR SEGMENT 084 WRITTEN

$A V O=.2500$

CVO $=2.000$

$P 0840210$

$M V I=-2$

$P 0840220$

$P 0840230$

$B V O=O L O G(A \vee 0 / 2.000)$

WRITE (NUVI, 841) BVO

$P 0840240$

$B \vee O=O L O G(A \vee 0)$

$P 0840250$

WRITE( NUVI, 842 ) BVD

$P 0840260$

$P 0840270$

$B \vee 0=O L O G(A \vee 0 * C \vee 0)$

WRITE (NUVI, 843 ) BVO

$B V O=O L O G(A V O * C V O * * 2)$

WRITE (NUVI, 844) BVO

$B V 0=0 L O G(D M I N 1(A V O * 2.000+D A B S(D B L E(F L O A T(M V I)) / C V D), C V O))$

WRITE (NUVI, 845 ) BVD

$B V O=O L O G(O S I G N(O B L E(F L O A T(M I N O(M V I, I O I N T(C V O)))), A V O))$

WRITE (NUVI, 846) BVD

WRITE (NUVI, 847)

841 FORMAT ( $9 H O X=0.125 .5 X .23 H-2.07944154167983590+00 / 1 P 034.13)$

842 FORMAT( $9 H O X=0.25,5 X, 23 H-1.38629436111989060+00 / 1 P 034.13)$

843 FORMAT $(9 H O X=0.5 \quad .5 X .23 H-0.69314718055994530+001 \quad 035.94)$

844 FORMAT $9 H 0 X=1.0,5 X .23 H \quad 0.000000000000000 \quad 0 \quad 035.14)$

845 FORMAT( $9 H O X=1.5,5 X, 23 H \quad 0.40546510810816440+00 / \quad 035.14)$

846 FORMAT $(9 H O \quad X=2.0 \quad .5 X .23 H \quad 0.69314718055994530+001 \quad 035.14)$

$P 0840280$

$P 0840290$

$P 0840300$

$P 0840310$

P0 840320

$P 0840330$

$P 0840340$

$P 0840350$

P0840360

$P 0840370$

P0840380

P0 840390

P0840400

P0840410

$P 0840420$

P0 840430

847 FORMAT $/ / 37 \mathrm{H}$ LINE 2 OF EACH PAIR IS THE FUNCTION/25H CALCULATI0NPO840440

A PRINTEO TO, 9 IH 14 DIGITS)

\section{$[* * * *$ END OF TEST SEGMENT 084}

$C * * * * *$ WHEN EXECUTING ONLY SEGMENT 084, THE STOP ANO END CAROS

$C * * * * *$ WHICH APPEAR AS COMMENT CAROS MUST HAVE THE $C=$

$C * * * * *$ IN COLUMNS 1 ANO 2 REMOVEO.

$C=S T O P$

$C=E N O$

STOP

$P 0840450$

$P 0840460$

$P 0840470$

$P 0840480$

P0 840490

$P 0840500$

$P 0840510$

P084C1

ENO

$P 084 C 2$

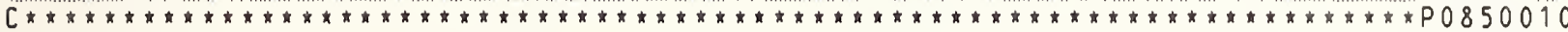

$[* * * * *$

$C * * * * *$

$[* * * * *$

$C * * * * * *$

$[* * * *$

$C * * * * *$

$C * * * * *$

$C * * * * *$

$C * * * *$

C*****

$[* * * * *$

$[* * * *$

$[* * * * *$

$[* * * *$

$C * * * *$

$C * * * * *$

$C * * * *$

$C=\quad$ COMPLEX EP1C $(30)$, AVC, BVC

$C=\quad$ OOUBLE PRECISION AVO, BVO

COMPLEX EP IC $(30)$. AVC, BVC

OOUBLE PRECISION AVD, BVO

$[* * * * *$

$[* * * * \quad 0$ U T P U T - T A P E ASSIGNMENT STATEMENT. NO INPUT TAPE.

C*******at

C***** WHEN EXECUTING ONLY SEGMENT 085. THE FOLLOWING STATEMENT

$C * * * *$ NUVI $=6$ MUST HAVE THE $C=I N$ COLUMNS 1 ANO 2 REMOVEO.

$C=\quad$ NUVI $=6$
$P 0850020$

$P 0850030$

$P 0850040$

ASA REF P0850060

ASA REF PO 850070

$8.3 .3 \quad P 0850080$

TABLE 4 PO 850090

$P 0850100$

P0 850110

$P 0850120$

P0850130

P0011930

$P 0011935$

P0011940

P0011945

P0011950

$P 0011955$

$P 0011960$

P085A1

P085A2

$P 0011965$

$P 0850140$

$P 0071660$

$P 0071665$

$P 0071670$

$P 0071675$ 
FORMAT $(15 H 1$ CXLOG - $(085) / 132 \mathrm{H}$ BASIC EXTERNAL FUNCTION - CLOG- P0850160 $1 / 129 \mathrm{H}$ (NATURAL LOG -TYPE COMPLEX)//27H ASA REF.- 8.3.3 (TABLE 4P0850170 2) $/ / 20 \mathrm{H}$ (COMPLEX ARGUMENT)/ 8X,15HEXPECTED RESULT /8X, 15HFUNCTION PO 850180 3RESULT)

$C * *: \quad L O G \quad O F \quad 10$ $B V D=2.302585092994000$

C*\#: SINE OF 60 DEGREES

$A V D=.8660254037844400$

$[* * * *$ INITIALI2E EP1C (EXPECTED VALUES)

EP1C(1) $=$ CMPLX(0.5E-7.SNGL $(-A V D * 1 . D-7))$

$E P 1 C(2) .=C M P L X(2.5 E-7, S N G L(-A V D * 5 . D-7))$

$E P 1 C(3)=(1 . E-6,0.0)$

$E P 1 C(4)=(5 . E-6,0.0)$

$E P 1 C(5)=C M P L X(0.5 E-5, S N G L(A V D * 1 . D-5))$

$E P 1 C(6)=C M P L X(2.5 E-5, S N G L(A V D * 5 . D-5))$

$E P 1 C(7)=C M P L X(-.5 E-4, S N G L(A V D * 1.0-4))$

$P 0850190$

P0850200

P0850210

$P 0850220$

P0850230

$P 0850240$

$P 0850250$

$P 0850260$

P0850270

$P 0850280$

$P 0850290$

$P 0850300$

$E P 1 C(8)=C M P L X(-2.5 E-4, S N G L(A V D * 5.0-4))$

$P 0850310$

$E P 1 C(9)=(-1 . E-3,0.0)$

$E P 1 C(10)=(-5 . E-3,0.0)$

$E P 1 C(11)=C M P L X(-0.5 E-2 . S N G L(-A V D * 1.0-2))$

$P 0850320$

$P 0850330$

$P 0850340$

$E P 1 C(12)=C M P L X(-2.5 E-2 . S N G L(-A V D * 5 . D-2))$

$P 0850350$

$E P 1 C(13)=C M P L X(0.5 E-1, S N G L(-A V D * 1.0-1))$

P0850360

$E P 1 C(14)=C M P L X(2.5 E-1, S N G L(-A V D * 5 . D-1))$

P08 850370

$E P 1 C(15)=(1.0,0.0)$

$E P 1 C(16)=(5.0,0.0)$

EP1C(17) $=$ CMPLX(0.5E1.SNGL(AVD*1.01))

$E P 1 C(18)=C M P L X(2.5 E 1, S N G L(A V D * 5 . D 1))$

$P 0850380$

$P 0850390$

$P 0850400$

P0850410

$P 0850420$

$E P 1 C(19)=C M P L X(-0.5 E 2, S N G L(A V D * 1 . D 2))$

EP1C(20) $=$ CMPLX( $-2.5 E 2 . S N G L(A V D * 5.02))$

$P 0850430$

$E P 1 C(21)=(-1 . E 3,0.0)$

$E P 1 C(22)=(-5 . E 3,0.0)$

$E P 1 C(23)=C M P L X(-0.5 E 4 . S N G L(-A V D * 1.04))$

EP1C(24) $=$ CMPLX(-2.5E4.SNGL (-AVD*5.04))

$P 0850440$

$P 0850450$

$P 0850460$

$P 0850470$

$P 0850480$

EP1C(25) $=$ CMPLX(0.5E5,SNGL(-AVD*1.D5))

$P 0850490$

EP $1[(26)=$ CMPLX(2.5E5,SNGL(-AVD*5.D5))

$P 0850500$

$E P 1 C(27)=(1 . E 6,0.0)$

$E P 1 C(28)=(5 . E 6,0.0)$

$E P 1 C(29)=C M P L X(0.5 E 7, S N G L(A \vee D * 1.07))$

$P 0850510$

$P 0850520$

P0850530

$E P 1 C(30)=C M P L X(2.5 E 7, S N G L(A \vee D * 5.07))$

P0850540

C*** YVS COMPENSATES FOR - 2PI AND + 2PI GENERATED BY USE OF XIVS*PI/3 PO850550

C** FOR EXPECTED IMAGINARY VALUES, TAKES VALUES +6,0,-6 DURING RANGEPO850560 YVS $=6$.

$I V I=0$

$P 0850570$

P0850580

P0850590

$P 0850600$

If (MOD(IVI, 2) .EQ. O) GO TO 852

$X I V S=((I V I+1) / 2)-8$

AVS $=$ BVD*XIVS

GO TO 853

$C * * * 2.609$ IS LOG OF 5

852 XIVS $=($ IVI $/ 2)-8$

AVS $=(B \vee D * X I V S)+1.609437912434100$

$\because * 1.047$ is P1/3

$853 \quad A V C=$ CMPLX (AVS, SNGL(1.047197551196600*(XIVS+YVS))

$B V C=C L O G(E P 1 C(I V I))$

WRITE (NUVI, 854) EP1C(IVI), AVC, BVC

IF(IVI - 10) 855, 858.855

855 IF (IVI - 20) 856.859.856

856 IF (IVI - 22) 857.7850,857

857 IF (IVI - 30) 851.7851.7851

858 YVS $=0.0$

859 WRITE (NUVI, 7852)

GO TO 851

7850 YVS $=-6.0$

P0850610

$P 0850620$

$P 0850630$

$P 0850640$

$P 0850650$

$P 0850660$

P0850670

P0850680

$P 0850690$

P0850700

$P 0850710$

$P 0850720$

$P 0850730$

$P 0850740$

$P 0850750$

$P 0850760$

P0850770

P0 850780

GO TO 851

7851 CONTINUE

P0850790

P0850800 
854 FORMAT $(3 H O(, E 14.7,1 \mathrm{H}, . \mathrm{E} 14.7,1 \mathrm{H}), 2(18 \mathrm{X}, 2 \mathrm{E} 16.7))$

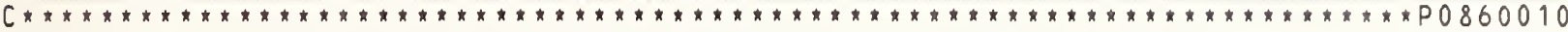

$C * * * * *$

$\operatorname{COLOG}-086$

$P 0860020$

$C * * * * *$

$P 0860030$

$C * * * * * *$

$P 0860040$

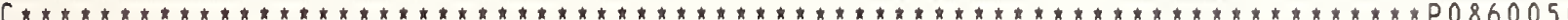

$C * * * * *$ GENERAL PURPOSE

C**** TO TEST BASIC EXTERNAL FUNCTION - ALOG10- ASA REF POS60070

$C * * * *$ COMMON LOG - TYPE REAL

$C * * *$ USED IN SIMPLE ARITHMETIC EXPRESSIONS

$C * * * * * \quad$ INTRINSIC FUNCTIONS ABS, AINT, AMAXI, SIGN, ASSUMED WORKING

$8.3 .3 \quad P 0860080$

$C * * * *$ ARGUMENT RANGE 0.5 TO 16.0 , POWERS OF 2

TABLE 4P0860090

$C * * * * *$

$C * * * * *$

0 U T $P$ U T T A P E ASSIGNMENT STATEMENT. NO INPUT TAPE.

P0860100

$P 0860110$

$P 0860120$

$C * * * * *$

$C * * * * *$

$C * * * *$ NUVI $=6$ MUST HAVE THE $C=$ IN COLUMNS 1 AND 2 REMOVED.

$C=\quad$ NUVI $=6$

$N \cup V I=6$

P0 0860130

$P 0071690$

$P 0071695$

$P 0071700$

$P 0071705$

P086B1

$C * * * * *$

860 FORMAT(15H1 COLOG - $(086) / / 34 \mathrm{H}$ BASIC EXTERNAL FUNCTION - ALOG10-

$P 0071710$

$1 / 125 \mathrm{H}$ (COMMON LOG - TYPE REAL)

$P 0860140$

$2 / 127 \mathrm{H}$ ASA REF.- 8.3 .3 (TABLE 4)//24H LINE 1 OF EACH PAIR IS/23H

3 HOLLERITH INFORMATION//9H RESULTS)

$P 0860150$

WRITE (NUVI, 860)

C***** HEADER FOR SEGMENT 086 WRITTEN

$A \vee S=-2.0$

$C V S=-4.0$

$B V S=A L O G 10$ (AVS, (VS)

P0860160

$P 0860170$

$P 0860180$

$P 0860190$

$P 0860200$

$P 0860210$

WRITE (NUVI, 861) BVS

$P 0860220$

$B \vee S=A L O G 10(A B S(A \vee S+1.0))$

00860230

WRITE (NUVI, 862) BVS

$P 0860240$

$B V S=A L O G 10(-A V S)$

$P 0860250$

WRITE (NUVI, 863) BVS

$B V S=A L O G 10(A I N T(A V S+2.0-(V S))$

$P 0860260$

P0860270

$P 0860280$

WRITE (NUVI, 864) BVS

$P 0860290$

$P 0860300$

WRITE (NUVI, 865) BVS

$P 0860310$

$B V S=A L O G 10(S I G N(C V S,(-A V S)) * 2)$

$P 0860320$

WRITE (NUVI, 866) BVS

P0860330

WRITE (NUVI, 867)

861 FORMAT $(8 H 0 X=0.5 .5 X .25 H-0.3010299956639811952137 / 8 X, F 15.7)$

862 FORMAT $(8 H 0 X=1.0 .5 X .25 H 0.0000000000000000000000 / 8 X, F 15.7)$

$P 0860340$

$P 0860350$

P0860360

863 FORMAT( $8 H O X=2.0,5 X .25 H 0.3010299956639811952137 / 8 X, F 15.7)$

864 FORMAT ( $8 H O X=4.0,5 X .25 H \quad 0.6020599913279623904275 / 8 X, F 15.7)$

P0860370

$P 0860380$

FORMAT $(8 H O X=8.0,5 X .25 H \quad 0.9030899869919435856412 / 8 X, F 15.7)$

$P 0860390$

865

FORMAT $(8 H O \quad X=16.0,5 X, 25 H \quad 1.2041199826559247808550 / 8 X, F 15.7)$

$P 0860400$

867 FORMAT $/ / 37 \mathrm{H}$ LINE 2 OF EACH PAIR IS THE FUNCTION/25H CALCULATIONP 1 PRINTED TO, 8H7 DIGITS)

$C * * *$ END OF TEST SEGMENT O 86

C***** WHEN EXECUTING ONLY SEGMENT 086, THE STOP AND END CARDS

$C * * * *$ WHICH APPEAR AS COMMENT CARDS MUST HAVE THE $C=$

$C * * * *$ IN COLUMNS 1 AND 2 REMOVED.

$C=$ STOP

$C=\quad E N D$

STOP

END

$P 0860420$

$P 0860430$

$P 0860440$

$P 0860450$

$P 0860460$

$P 0860470$

$P 0860480$

P086C 1

$P 086 C 2$

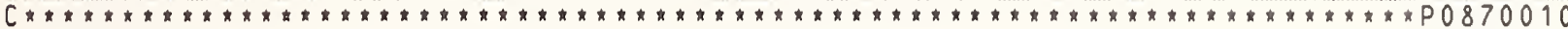




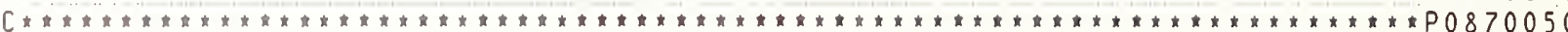

$[* * *$ GENERAL PURPOSE

C* TO TEST BASIC EXTERNAL FUNCTION-OLOG10-

$P 0870060$

C*** COMMON LOG - TYPE DOUBLE PRECISION

$C * * *$ SAME AS SEGMENT 086 EXCEPT FOR TYPE

C***\#INTRINSIC FUNCTIONS DABS, IDINT, FLOAT,DBLE,

$C *$ DMAXI,DSIGN ASSUMED WORKING

C****) ARGUMENT RANGE 0.5 TO 16.0 POWERS OF 2

ASA REFP0 870070

$C * * * * *$

$C * * * *$ S P E C I F I C A T I O N S SEGMENT 087

$C * * * * *$

8.3.3 P0870080

TABLE 4 P 0870090

$P 0870100$

$P 0870110$

$P 0870120$

$P 0870130$

$C * * * *$ WHEN EXECUTING ONLY SEGMENT 087 . THE SPECIFICATION STATEMENTS

$C * * * *$ WHICH APPEAR AS COMMENT CARDS MUST HAVE THE C $=$

$C * * * * *$ IN COLUMNS 1 AND 2 REMOVED.

$[* * * *$

$C=\quad$ DOUBLE PRECISION AVD, BVD, CVD

DOUBLE PRECISION AVD, BVD, CVD

$C * * * * *$

C***** 0 U T P U T T A P E ASSIGNMENT STATEMENT. NO INPUT TAPE

$[* * * * *$

$C * * * * *$

WHEN EXECUTING ONLY SEGMENT 087, THE FOLLOWING STATEMENT

$C * * *$ NUVI $=6$ MUST HAVE THE $C=I N$ COLUMNS 1 AND 2 REMOVED.

$C=\quad$ NUVI $=6$

NUVI $=6$

$C * * * * *$

870 FORMAT(15HI DCLOG - $(087) / / 34 \mathrm{H}$ BASIC EXTERNAL FUNCTION -DLOG1O-

$1 / 137 \mathrm{H}$ (COMMON LOG - TYPE DOUBLE PRECISION)

$2 / / 27 \mathrm{H}$ ASA REF.- 8.3.3 (TABLE 4)//24H LINE 1 OF EACH PAIR IS/23H

3 HOLLERITH INFORMATION//9H RESULTS)

WRITE (NUVI, 870)

C***** HEADER FOR SEGMENT 087 WRITTEN

$A \vee D=-2.000$

$C V D=-4.000$

$B V D=D L O G 10(A V D /$ CVD)

WRITE (NUVI, 871) BVD

$B V D=D L O G 10(D A B S(A V D+1.0 D 0))$

WRITE (NUVI, 872) BVD

$B V D=D L O G 10(-A V D)$

WRITE (NUV!, 873) BVD

$B V D=D L O G 10(D B L E(F L O A T(I D I N T(A V D+2.000-(V D))))$

$P 0870140$

$P 0011970$

P 0011975

$P 0011980$

P 0011985

$P 0011990$

$P 0011995$

P087A1

$P 0012000$

$P 0870150$

$P 0071720$

$P 0071725$

$P 0071730$

$P 0071735$

P087B1

$P 0071740$

$P 0870160$

$P 0870170$

WRITE (NUVI, 874) BVD

BVD $=$ DLOG10(DMAX1(AVD*CVD, CVD*2.000))

WRITE (NUVI, 875) BVD

$B V D=D L O G 10(D S I G N(C V D,(-A V D)) * * 2)$

00870180

$P 0870200$

P0870210

$P 0870220$

$P 0870230$

$P 0870240$

$P 0870250$

$P 0870260$

$P 0870270$

$P 0870280$

$P 0870290$

$P 0870300$

$P 0870310$

$P 0870320$

$P 0870330$

$P 0870340$

WRITE (NUVI, 876) BVD

WRITE (NUVI, 877)

871 FORMAT $8 H 0 X=0.5,5 X, 29 H-0.30102999566398119521370+00 / 034.14)$

$P 0870350$

$P 0870360$

00870370

FORMAT $(8 H 0 \quad X=1.0,5 X, 29 H \quad 0.0000000000000000000000 \quad 1034.14)$

872

FORMAT $(8 H O X=2.0,5 X, 29 H \quad 0.30102999566398119521370+00 / 034.14)$

$P 0870380$

FORMAT $(8 H O X=4.0,5 X .29 H \quad 0.60205999132796239042750+00 / 034.14)$

$P 0870390$

874

FORMAT $(8 H O X=8.0,5 X, 29 H \quad 0.70308998699194358564120+00 / 034.14)$

$P 0870400$

P0870410

876

FORMAT $(8 \mathrm{HO} \quad \mathrm{X}=16.0,5 \mathrm{X}, 29 \mathrm{H} 1.2041199826559247808550 \mathrm{D}+00 / 1 \mathrm{PD} 33.13)$

$P 0870420$

877 FORMAT(//37H LINE 2 OF EACH PAIR IS THE FUNCTION/25H CALCULATION A PRINTED TO, 9H14 DIGITS)

C**** END OF TEST SEGMENT 087

C**** WHEN EXECUTING ONLY SEGMENT 087 , THE STOP AND END CARDS

C** WHICH APPEAR AS COMMENT CARDS MUST HAVE THE C $=$

$C * * * *$ IN COLUMNS 1 AND 2 REMOVED.

$C=$ STOP

$C=\quad E N D$

STOP

$P$
$P 08704040$

$P 0870450$

$P 0870460$

$P 0870470$

P0870480

P0870490

$P 0870500$

P087C1

END

P087C2

$C * * * * * *$
$C * * * * *$

P0880010

SINUS - 088

$P 0880020$

$P 0880030$

$C * * * *$

P0880040 


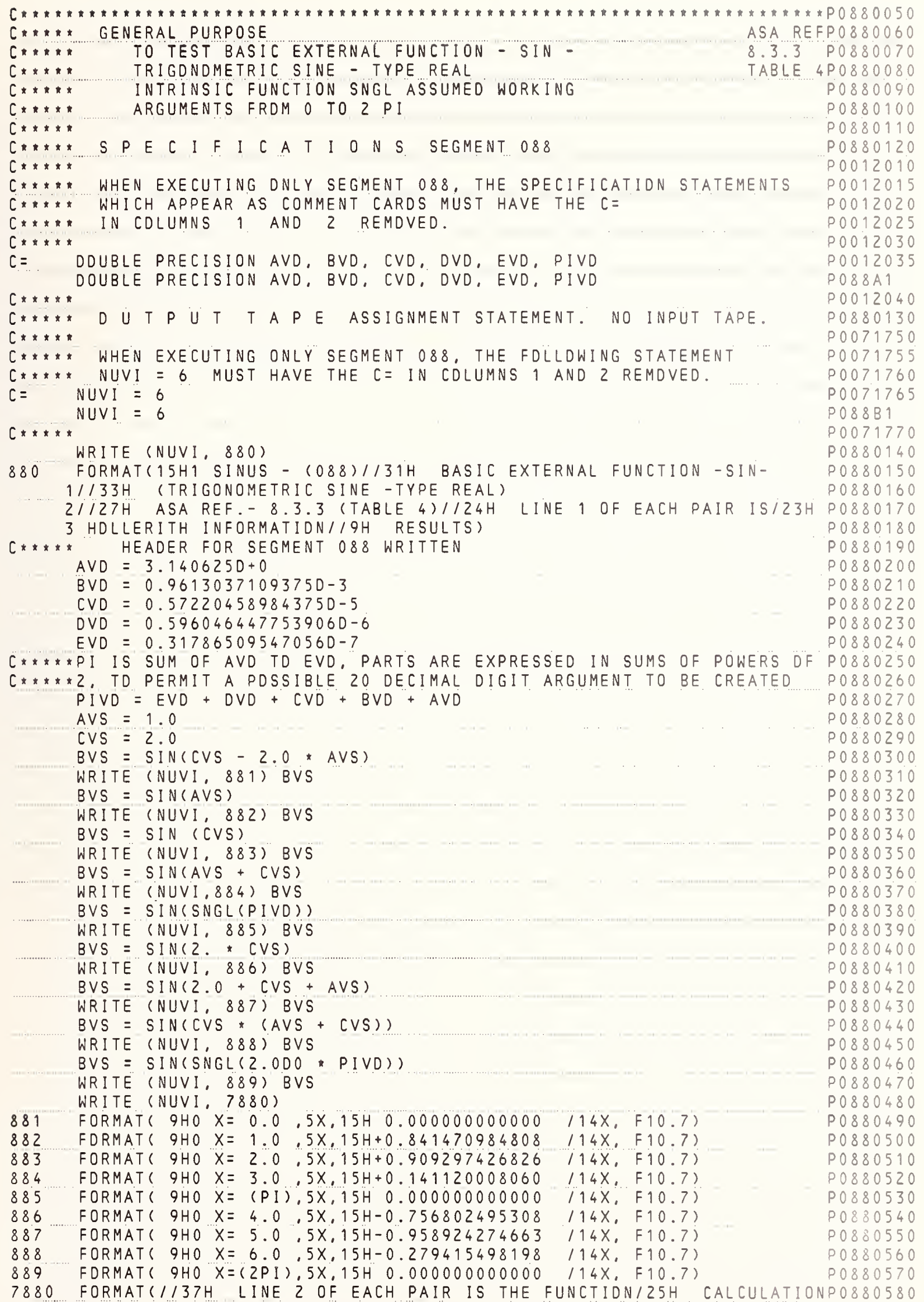


1 PRINTEO TO, 8HT DIGITS)

C*ND OF TEST SEGMENT 088
CHEN EXECUTING ONLY SEGMENT 088 . THE STOP AND END CARDS

$P 0880590$

P0880600

$C * * *$ WHICH APPEAR AS COMMENT CARDS MUST HAVE THE C $=$

P0880610

C*** IN COLUMNS $\%$ AND" 2 REMOVED.

$C=\quad S T O P$

$C=\quad E N D$

STOP

P08880620

P08880630

P0 0880640

P0880650

END

P088C1

P08 $8 C 2$

$C * * * * * * *$

$C * * \| * *$

$C * * * *$

DPSIN - 089

0010

P0890020

P0890030

$C * * * *$

P0890040

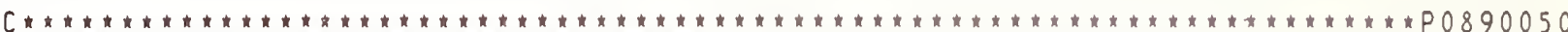

$C * * * *$ GENERAL PURPOSE

$C * * * *$ TO TEST BASIC EXTERNAL FUNCTION - DSIN -

ASA REFP0890060

$C * * * *$ TRIGONOMETRIC SINE - TYPE DOÜBLE PRECISION

8.3 .3 PO890070

$C * * * *$ SAME AS SEGMENT 088 EXCEPT D.P.

C**** INTRINSIC FUNCTION DSIGN ASSUMED WORKING

TABLE 4 PO 890080

$C * * *$ ARGUMENTS FROM 0 TO 2 P

$C * * * * *$

$C * * * *$ S P E C I F I C A T I O N S SEGMENT 089

$P 0890090$

P0890100

P0 890110

$C * * * *$

$C * * *$ WHEN EXECUTING DNLY SEGMENT 089, THE SPECIFICATION STATEMENTS

$C * * * *$ WHICH APPEAR AS CDMMENT CARDS MUST HAVE THE C

$C * * * * *$ IN COLUMNS 1 AND 2 REMOVED.

$C * * * * *$

$C=\quad$ DOUBLE PRECISIDN AVD, BVD, CVD, DVD, EVD, PIVD, XVD, FVD, GVD

DOUBLE PRECISIDN AVD, BVD, CVD, DVD, EVD, PIVD, XVD, FVD, GVD.

$C * * * * *$

$[* * * * \quad 0$ U T P U T T A P E ASSIGNMENT STATEMENT. ND INPUT TAPE.

$C * * * * *$

C***** WHEN EXECUTING ONLY SEGMENT 089, THE FDLLOWING STATEMENT

$C * * * * *$ NUVI $=6$ MUST HAVE THE C $=$ IN CDLUMNS 1 AND 2 REMDVED.

$C=\quad$ NUVI $=6$

NUVI $=6$

$C * * * *$

890 FORMAT(15HI DPSIN - $(089) / / 32 \mathrm{H}$ BASIC EXTERNAL FUNCTIDN - DSIN-

$1 / / 33 \mathrm{H}$ (TRIGONDMETRIC SINE - TYPE D.P.)

$2 / / 27 \mathrm{H}$ ASA REF.- 8.3 .3 (TABLE 4)//24H LINE 1 OF EACH PAIR IS/23H

3 HOLLERITH INFORMATION//9H RESULTS)

WRITE (NUVI, 890)

$C * * * *$ HEADER FDR SEGMENT 089 WRITTEN

$A V D=3.1406250+0$

$B V D=0.96130371093750-3$

CVD $=0.572204589843750-5$

DVD $=0.5960464477539060-6$

$P 0890120$

$P 0890130$

P0012050

$P 0012055$

$P 0012060$

$P 0012065$

$P 0012070$

P 0012075

P089A 1

$P 0012080$

P0890140

$P 0071780$

$P 0071785$

$P 0071790$

$P 0071795$

P 08981

$P 0071800$

P0890150

P0 890160

$E V D=0.317865095470560-7$

P0 890170

P0890180

P0890190

P0890200

P0890210

P0890220

P0 890230

P0 0890240

$P 0890250$

$C * * * * P I$ IS SUM OF AVD TO EVD, PARTS ARE EXPRESSED IN SUMS OF POWERS DF P0890260

$C * * * * 2$, TO PERMIT A POSSIBLE 20 DECIMAL DIGIT ARGUMENT TO BE CREATED $P I V D=E V D+D V D+C V D+B V D+A V D$

$F V D=1.000$

$G \vee D=2.000$

$X V D=D S I N(G V D-2.0 D 0 * F V D)$

WRITE (NUVI, 891) XVD

$X \vee D=D S I N(F \vee D)$

WRITE (NUVI, 892) XVD

$X \vee D=D S I N(G \vee D)$

WRI ${ }^{\top}$, UVI, 893) XVD

$X V R \quad$ IN $(G \vee D+F V D)$

W E (NUVI, 894) XVD

$\lambda \cdot D=D S I N(P I V D)$

IRITE (NUVI", 895) "XVD

KVD $=\operatorname{DSIN(2.*GVD)}$

WRITE (NUVI, 896) XVD

$X V D=D S I N(2.0+F V D+G V D)$

WRITE (NUVI, 897) XVD

$X \vee D=D S I N(G \vee D *(F \vee D+G \vee D))$

$P 0890270$

$P 0890280$

$P 0890290$

P0890300

P0 890310

P0890320

$P 0890330$

P0890340

P0890350

P0890360

P0 0890370

P0 890380

P0890390

P0890400

$P 0890410$

P0890420

$P 0890430$

P0 0890440

$P 0890450$ 
C

$[* * * *$ GENERAL PURPOSE

$C * * * * *$

$[* * * * *$

$C * * * * *$

$C * * * * *$

$[* * * * *$

$C * * * * *$

$[* * * * *$

$[* * * * *$

$C * * * * *$

$C * * * * *$

$C=$ DIMENSION L II $(10)$

$C=\quad$ COMPLEX AVC, $B V C$

DIMENSION LII (10)

COMPLEX AVC, BVC

$[* * * * *$

C********

$C * * * * *$

$[* * * * *$

$C * * * * *$

$[* * * * *$

$O U T P U T-T A P$

WHEN EXECUTING ONLY SEGMENT 090, THE FOLLOWING STATEMENT NUVI $=6$

NUVI $=6$

WRITE (NUVI, 900)

ASA REF

8.3.3 3

TABLE

FORMAT 15 H 1 CSICO

23.3 (TABLE 4) /110H FUNCTION, 10X,7HRESULTS $/ 1$ )

DATA LAZVI, LBZVI, LCZVI,LOZVI/ZHO(, 2H, ,2H1/,1H)/

DATA LII(1),LII(2),LII(3),LII(4),LII(5)/

$-2 \mathrm{H}_{1}, 2 \mathrm{H2}, 2 \mathrm{H3}, 2 \mathrm{H} 4,2 \mathrm{H} 5 \mathrm{I}$

- LII(6),LII(7),LII(8),LII(9),LII(10)/

$A V C=(1.0,1.0)$

$B \vee C=C S I N(A \vee C)$

WRITE(NUVI, 901$)$ BVC

$B \vee C=C \operatorname{Cos}(A \vee C)$

WRITE (NUVI, 902 ) BVC

$I V !=0$

905

$\| V I=I V I+1$

$A V S=I V I$
P0900010

$P 0900020$

P0900030

$P 0900040$

P0900050

$P 0900060$

P0900070

$4 P 0900080$

$P 0900090$

P0900100

P0900110

P0012090

P0012095

P0012100

P0012105

P00 12110

P0012115

P0012120

P090A 1

P090AZ

P0012125

P0900120

P0071810

$P 0071815$

$P 0071820$

$P 0071825$

P090B1

P0071830

P0900130

CPO9O0140

8.P0900150

$P 0900 \uparrow 60$

P0900170

P0900180

P0900190

$P 0900200$

P0900210

P0900220

P0900230

P0900240

P0900250

P0900260

P0900270

P0900280

$P 0900290$ 

1 ) = F10.7 F12.7 240 HO ARGUMENT RESULTS SHOULD BE $1.0,0.0$ )

\section{$C *$ END OF TEST SEGMENT 090}

$C * * * *$ HHEN EXECUTING ONLY SEGMENT 090. THE STOP AND END CARDS

$C * * * *$ WHICH APPEAR AS COMMENT CARDS MUST HAVE THE $C=$

$C * * * *$ IN COLUMNS 1 AND 2 REMOVED.

$C=\quad$ STOP

$C=\quad E N D$

STOP

CSIN $11 P 0900370$

$P 0900380$

$$
\text { END }
$$

$P 0900410$

$P 0900420$

$P 0900430$

$P 0900440$

$P 0900450$

$P 0900460$

$P 0900470$

P090C1

$P 090 C 2$

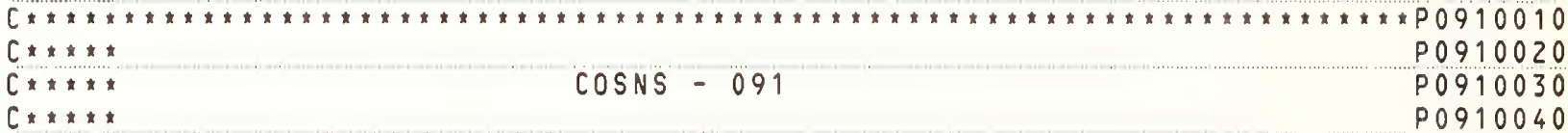

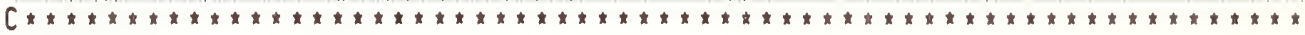

$C * * * *$ GENERAL PURPOSE

$C * * * *$ TO TEST BASIC EXTERNAL FUNCTION- COS -

$C * * * * *$ TRIGONOMETRIC COSINE - TYPE REAL

C**** SAME AS SEGMENT EXCEPT FOR COSINE

$C * * * *$ INTRINSIC FUNCTION SNGL ASSUMED WORKING

$C * * * *$ ARGUMENTS FROM 0 TO 2 PI

$C * * * * *$

$C * * * *$ S P E C I F I C A T I O N S SEGMENT 091

$C * * * * *$ WHEN EXECUTING ONLY SEGMENT 091 , THE SPECIFICATION STATEMENTS

$C * * * *$ WHICH APPEAR AS COMMENT CARDS, MUST HAVE THE $C=$

$C * * * *$ IN COLUMNS 1 AND 2 REMOVED.

$C * * * * *$

$C=$ DOUBLE PRECISION AVD, BVD, CVD, DVD, EVD, PIVD

ASA REFP0910060

8.3.3 P0910070

TABLE $4 P 0910080$

P0910090

$P 0910100$

$P 0910110$

$P 0910120$

$P 0910130$

$P 0012130$

P 0012135

P0012140

$P 0012145$

$P 0012150$

P0012155

DOUBLE PRECISION AVD, BVD, CVD, OVD, EVD, PIVD

P091A1

$C * * * * *$

$C * * * * 0 U T P U T$ T A P E ASSIGNMENT STATEMENT. NO INPUT TAPE.

P0012160

$C * * * * *$

$C * * * *$ WHEN EXECUTING ONLY SEGMENT 091, THE FOLLOWING STATEMENT

$P 0910140$

$C * * * *$ NUVI $=6$ MUST HAVE THE $C=I N$ COLUMNS 1 AND 2 REMOVED.

P0071840

$P 0071845$

$C=\quad N U V I=6$

NUVI $=6$

$C * * * * *$

910 FORMAT( $15 H 1$ COSNS - $(091) / / 31 \mathrm{H}$ BASIC EXTERNAL FUNCTION - COS-

$1 / / 35 H$ (TRIGONOMETRIC COSINE - TYPE REAL)

$2 / 1274$ ASA REF.- 8.3.3 (TABLE 4)//24H LINE 1 OF EACH PAIR IS/23H PO910170

3 HOLLERITH INFORMATION//9H RESULTS)

$P 0071850$

$P 0071855$

P09181

$P 0071860$

$P 0910150$

P0910180

WRITE (NUVI, 910 )

$C * * *$ HEADER FOR SEGMENT 091 WRITTEN

$A V D=3.1406250+0$

BVD $=0.96130371093750-3$

CVD $=0.572204589843750-5$

DVD $=0.5960464477539060-6$

$P 0910190$

$P 0910200$

PO910210

P0910220

P0910230

P0910240

$E V D=0.317865095470560-7$

$P 0910250$

C**:PI IS SUM OF AVD TO EVD, PARTS ARE EXPRESSED IN SUMS OF POWERS OF PO910Z6O

$C * * * 2$, TO PERMIT A POSSIBLE 20 DECIMAL DIGIT ARGUMENT TO BE CREATED

$P I V D=E V D+D V D+C V D+B V D+A V D$

$P 0910270$

$P 0910280$

AVS $=1.0$

CVS $=2.0$

BVS $=\operatorname{COS}($ CVS -2.0 AVS $)$

WRITE (NUVI, 911) BVS

$B \vee S=\cos (A \vee S)$

WRITE (NUVI, 912) BVS

$P 0910290$

$P 0910300$

$P 0910310$

P0910320

P0910330

$P 0910340$ 
BVS $=\cos (C V S)$

$P 0910350$

WRITE (NUVI, 913) BVS

$P 0910360$

$B \vee S=\operatorname{CDS}(A \vee S+C V S)$

WRITE (NUVI, 914) BVS

BVS $=\operatorname{COS}(S N G L(P$ IVD) $)$

WRITE (NUVI, 915) BVS

$B V S=\operatorname{CDS}(2, * C V S)$

WRITE (NUVI, 916) BVS

$B V S=\operatorname{CDS}(2.0+C V S+A V S)$

WRITE (NUVI, 917) BVS

$B V S=\operatorname{CDS}(C V S *(A V S+C V S))$

WRITE (NUVI, 918) BVS

BVS $=\operatorname{CDS}(S N G L(2.000 * P I V D))$

WRITE (NUVI, 919) BVS

WRITE (NUVI, 7910)

911 FORMAT $(9 H O X=0.0,5 X, 15 H+1.000000000000 \quad / 14 X, F 10.7)$

912

913

FORMAT 9 9HO $X=1.0,5 X, 15 \mathrm{H}+0.540302305868$

FDRMAT $(9 H O \quad X=2.0,5 X .15 H-0.416146836547$

$114 X, F 10.7$,

$(14 X, F 10.7$ )

914 FDRMAT 9 ( 00 O $X=3.0,5 X, 15 H-0.989992496600 / 14 X, F 10.7)$

915

916

FORMAT ( 9 HO $X=$ (PI) $, 5 X, 15 \mathrm{H}-1.000000000000$

$(14 X, F 10.7)$

FDRMAT ( 9 HO $X=4.0,5 X .15 H-0.653643620864 / 14 X, F 10.7$ )

917 FDRMAT $(9 H 0 \quad X=5.0,5 X, 15 H+0.283662185463,194, F 10.7)$

918 FDRMAT ( $9 H 0 X=6.0,5 X, 15 H+0.960170286650 \% / 14 X, F 10.7)$

919 FDRMAT $(9 H 0 \quad X=(2 P I), 5 X, 15 H+1.000000000000 / 14 X, F 10.7)$

7910 FDRMAT $(/ / 37 \mathrm{H}$ LINE 2 DF EACH PAIR IS THE FUNCTIDN/25H

1 PRINTED TD, $8 H 7$ DIGITS)

$C * * * * *$ END DF TEST SEGMENT 091

$C * * * * *$ WHEN EXECUTING DNLY SEGMENT 091 , THE STDP AND END CARDS

$C * * * *$ WHICH APPEAR AS CDMMENT CARDS MUST HAVE THE $c=$

$C * * * * *$ IN CDLUMNS 1 AND 2 REMDVED.

$C=\operatorname{STDP}$

$C=E N D$

STOP

END

$P 0910370$

P0910380

$P 0910390$

P0910400

P 0910410

$P 0910420$

P0910430

$P 0910440$

P0910450

$P 0910460$

$P 0910470$

P0910480

$P 0910490$

P0910500

P0910510

P0910520

P0910530

P0910540

P0910550

P0910560

P0910570

P0910580

CALCULATIONPO910590

P0910600

P0910610

P0910620

P0910630

P0910640

P0910650

P0910660

P091C1

P091C2

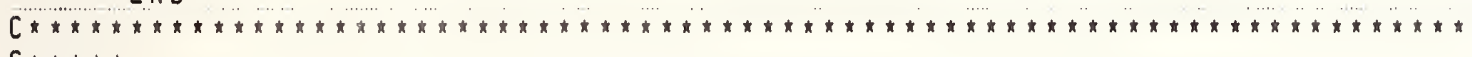

$C * * * * *$

$C * * * * *$
$C * * * * *$

$P 0920020$

P0920030

$P 0920040$

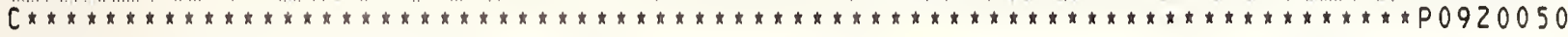

$C * * * *$ GENERAL PURPOSE

$C * * * * *$ TD TEST BASIC EXTERNAL FUNCTIDN - DCOS -

ASA REFP0920060

$C * * * * *$ TRIGDNDMETRIC CDSINE - TYPE DDUBLE PRECISIDN

$C * * * *$ SAME AS SEGMENT 091 EXCEPT D.P.

8.3 .3 P 0920070

TABLE 4P0920080

P0920090

$C * * * *$ INTRINSIC FUNCTIDN DMAXI ASSUMED WDRKING

$[* * * * *$ ARGUMENTS FRDM 0 TD 2 PI

P0920100

P0920190

$P 0920120$

$[* * * * *$ S P E C I F I C A T I D N S SEGMENT O 92

$C * * * *$

$C * * *$ WHEN EXECUTING DNLY SEGMENT 092, THE SPECIFICATIDN STATEMENTS

$C * * * *$ WHICH APPEAR AS COMMENT CAROS MUST HAVE THE $C=$

C**** IN COLUMNS 1 AND 2 REMOVED.

$C * * * *$

$C=$ DOUBLE PRECISIDN AVD, BVD, CVD, DVD, EV̈D, FVD, GVD, PIVD, XVD

DDUBLE PRECISIDN AVD, BVD, CVD, DVD, EVD, FVD, GVD, PIVD, XVD

$C * * * * *$

$C * * * * *$ D U T P U T T A P E ASSIGNMENT STATEMENT. ND INPUT TAPE.

$C * * * * *$

$C * * * *$ WHEN EXECUTING ONLY SEGMENT 092 . THE FOLLOWING STATEMENT

$C * * * *$ NUVI $=6$ MUST HAVE THE $C=$ IN COLUMNS 1 AND 2 REMDVED.

$C=\ldots$ NUVI $=6$

NUVI $=6$

$C * * * * *$

920 FORMAT( $15 H 1$ DPCDS - $(092) / / 32 \mathrm{H}$ BASIC EXTERNAL FUNCTION -DCOS-

P0920130

P0012170

P0012175

$P 0012180$

P0012185

P0012190

P0O12195

P092A1

P0012200

P0920140

P0071870

P0071875

P0071880

P 0071885

P092B 1

P0071890

P0920150

P0920160

$1 / / 35 \mathrm{H}$ (TRIGONOMETRIC COSINE - TYPE D.P.)

$2 / / 27 \mathrm{H}$ ASA REF.- 8.3.3 (TABLE 4)//24H LINE 1 DF EACH PAIR IS/23H 3 HOLLERITH INFORMATION//9H RESULTS )

P0920170

$P 0920180$

P 0920190

P0920200 
$B V D=0.96130371093750-3$

P0920210

CVO $=0.572204589843750-5$

00920220

DVD $=0.5960464477539060-6$

$P 0920230$

$E V D=0.317865095470560-7$

$P 0920240$

$C * \# * P I$ IS SUM OF AVD TO EVD, PARTS ARE EXPRESSED IN SUMS OF POWERS OF PO9ZOZ5O

$C * * * 2$, TO PERMIT A POSSIBLE 20 DECIMAL DIGIT ARGUMENT TO BE CREATED P0920260

$P I V D=E V D+D V D+C V D+B V D+A V D$

$F V D=1.000$

$G \vee D=2.000$

XVD $=$ DCOS (GVD-2.000*FVD)

WRITE (NUVI, 921) XVD

$X \vee D=D \operatorname{COS}(F \vee D)$

WRITE (NUVI, 922 ) XVD

$X \vee D=D \operatorname{COS}(G \vee D)$

WRITE (NUVI, 923) XVD

$X V D=D \operatorname{COS}(G \vee D+F V D)$

WRITE (NUVI, 924) XVD

$X \vee O=D \operatorname{COS}(P I \vee D)$

WRITE (NUVI, 925) XVD

$X V D=D \operatorname{Cos}(2, * G \vee D)$

WRITE (NUVI, 926) XVD

$X V D=D \operatorname{COS}(2.0+F V D+G V D)$

WRITE (NUVI, 927) XVD

$X V D=D C O S(G V D *(F V D+G \vee D))$

WRITE (NUVI, 928) XVD

$X V D=D \operatorname{COS}(D M A X I(2.000 * P I V D, G V D))$

WRITE (NUVI, 929) XVD

WRITE (NUVI, 7992)

921 FORMAT $(9 H 0 \quad x=0.0,31 H+0.100000000000000000000000+01 / 031.14)$

922 FORMAT(9HO $X=1.0 .31 \mathrm{H}+0.540302305868139717400940+001031.14)$

FORMAT ( $9 \mathrm{HO} X=2.0,31 \mathrm{H}-0.416146836547142386997570+00 ; 031.14)$

FORMAT (9HO $\quad X=3.0 .31 \mathrm{H}-0.989992496600445457271570+00 / 031.14)$

FORMAT (9HO $X=(P I), 31 \mathrm{H}-0.100000000000000000000000+01 / 031.14)$

FORMAT (9HO $X=4.0 .31 \mathrm{H}-0.653643620863611914639170+00 / 031.14)$

FORMAT $(9 H O \quad X=5.0,31 \mathrm{H}+0.283662185463226264466640+00 / 031.14)$

FORMAT $(9 H O \quad X=6.0,31 \mathrm{H}+0.960170286650366020545650+00 / 031.14)$

FORMAT $(9 H 0 \quad X=(2 P I) .31 H+0.100000000000000000000000+01 / 031.14)$

P 0920270

P0920280

$P 0920290$

P0920300

P0920310

$P 0920320$

P0920330

$P 0920340$

P0920350

P0920360

P0920370

P0920380

$P 0920390$

$P 0920400$

P0920410

$P 0920420$

$P 0920430$

$P 0920440$

P0920450

$P 0920460$

P0920470

P0920480

P0920490

$P 0920500$

P0920510

P0920520

P0920530

P0920540

P0920550

$P 0920560$

928

FORMAT $(/ / 37 \mathrm{H}$ LINE 2 OF EACH PAIR IS THE FUNCTION/25H

$031.14) \quad P 0920570$

A PRINTED TO, 9H 14 DIGITS )

$[* * * *$ END OF SEGMENT 092

C***** WHEN EXECUTING ONLY SEGMENT 092, THE STOP AND END CARDS

$[* * * *$ WHICH APPEAR AS. COMMENT CAROS MUST HAVE THE C $=$

$[* * * *$ IN COLUMNS 1 AND 2 REMOVED.

$C=\quad$ STOP

$C=\quad$ END

STOP

PO9 20590

$P 0920600$

P0920610

$P 0920620$

P0920630

$P 0920640$

$P 0920650$

$P 092 C 1$

END

$\mathrm{P} 092 \mathrm{C2}$

$[* * * * * *$

$[* * * * *$

$C * * * * *$

TANGH - 094

P 0940020

P09 090040

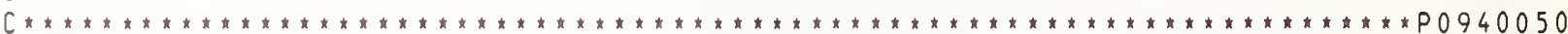

$C * * * *$ GENERAL PURPOSE

C***** TO TEST BASIC EXTERNAL FUNCTION - TANH C**** HYPERBOLIC TANGENT - TYPE REAL

ASA REFPO9 40060

8.3 .3

$P 0940070$

P 0940080

$P 0940090$

C***** USED IN SIMPLE ARITHMETIC EXPRESSIONS

$[* * * *$ INTRINSIC FUNCTIONS ABS, FLOAT, AMINO, AMAXO, INT

$[* * * * * \quad$ ASSUMED WORKING

$[* * * *$ ARGUMENTS FROM 0.0 TO 8.0

$[* * * * *$

$[* * * *$ O U T P U T T A P E ASSIGNMENT STATEMENT. NO INPUT TAPE.

$C * * * *$ WHEN EXECUTING ONLY SEGMENT 094, THE FOLLOWING STATEMENT

$C * * * *$ NUVI $=6$ MUST HAVE THE $C=$ IN COLUMNS 1 AND 2 REMOVED.

$[* * * * *$

$[=\quad$ NUVI $=6$

NUVI $=6$

940 FORMAT(15H1 TANGH - $(094) / / 32 H$ BASIC EXTERNAL FUNCTION - TANH$P 0940100$

$P 0940110$

P0940120

$P 0940130$

$P 0940140$

$P 0071900$

$P 0071905$

$P 0071910$

$P 0071915$

$P 09481$

$P 0940150$

$1 / / 33 \mathrm{H}$ (HYPERBOLIC TANGENT - TYPE REAL)

P0940160 
2//27H ASA REF.- 8.3.3 (TABLE 4)//24H LINE 1 OF EACH PAIR IS/23H P0940170

3 HOLLERITH INFORMATION//9H RESULTS)

WRITE (NUVI 940 )

P0940190

C**** HEADER FOR SEGMENT 094 WRITTEN

$P 0940200$

AVS $=2.0$

CVS $=-0.5$

$I V I=6$

$B V S=T A N H(F L O A T(I V I)-3.0 * A V S)$

WRITE (NUVI, 941) BVS

$B V S=T A N H(A \vee S)$

WRITE (NUVI, 942) BVS

$B V S=T A N H(A V S+A B S(C V S))$

WRITE (NUVI, 943) BVS

BVS = TANH(AMINO(IVI, 8) - AVS)

WRITE (NUVI, 944) BVS

BVS = TANH(AMAXO(IVI,INT(AVS)) )

WRITE (NUVI, 945) BVS

$B \vee S=T A N H(A \vee S * * 4 / A \vee S)$

WRITE (NUVI, 946) BVS

WRITE (NUVI, 947)

941 FORMAT $(7 \mathrm{HO} \quad \mathrm{X}=0.0,5 \mathrm{X}, 12 \mathrm{HO} 0.0000000000$ /F21.7)

942 FORMAT $(7 \mathrm{HO} \quad \mathrm{X}=2.0,5 \mathrm{X}, 12 \mathrm{HO} .9640275801$ /F21.7)

943 FORMAT ( 7 HO $X=2.5,5 X, 12 H O .9866142982$ /F21.7)

944 FORMAT $(7 \mathrm{HO} \quad X=4.0,5 X, 12 \mathrm{HO} .9993292997$ /F21.7)

945 FORMAT $(7 H O \quad X=6.0,5 X, 12 H 0.9999877117$ /F21.7)

946 FORMAT $(7 \mathrm{HO} \quad X=8.0,5 \mathrm{X}, 12 \mathrm{HO} .9999997749$ /F21.7)

947 FORMAT $/ / 37$ HINE 2 OF EACH PAIR IS THE FUNCTION/25H

1 PRINTED TO \&H7 DIGITS )

C***** END OF TEST SEGMENT O94

C***** WHEN EXECUTING ONLY SEGMENT 094, THE STOP AND END CARDS

$C * * * *$ WHICH APPEAR AS COMMENT CARDS MUST HAVE THE $c=$

C***** IN COLUMNS 1 AND 2 REMOVED.

$C=S T O P$

$C=E N D$

STOP

$P 0940210$

$P 0940220$

$P 0940230$

$P 0940240$

$P 0940250$

$P 0940260$

$P 0940270$

$P 0940280$

$P 0940290$

$P 0940300$

$P 0940310$

$P 0940320$

$P 0940330$

$P 0940340$

$P 0940350$

$P 0940360$

$P 0940370$

P0940380

$P 0940390$

$P 0940400$

$P 0940410$

P0940420

CALCULATI ONPO940430

$P 0940440$

$P 0940450$

P0940460

$P 0940470$

P0940480

$P 0940490$

$P 0940500$

$\mathrm{P} 094 \mathrm{C1}$

END

$P 094 C 2$

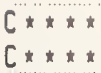

C $* x \neq * *$

SQROT - $(095)$

$P 0950020$

$P 0950030$

$P 0950040$

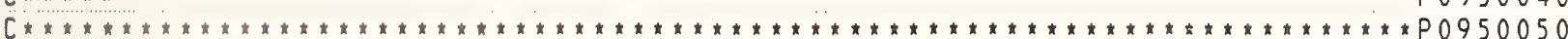

$C * * * * *$ GENERAL PURPOSE

$C * * * *$ TO TEST BASIC. EXTERNAL FUNCTION - SORT -

ASA REFP0950060

$C * * * *$ (SQUARE ROOT - TYPE REAL)

C***** USED IN SIMPLE ARITHMETIC EXPRESSIONS

C***** INTRINSIC FUNCTIONS FLOAT, INT, AMINO, MAXO

$[* * * * *$ ASSUMED WORKING C***** ARGUMENTS ARE ALL PRIME NUMBERS

$C * * * x+2$

$8.3 .3 \quad P 0950070$

TABLE $4 P 0950080$

$P 0950090$

P0950100

P0950110

P0950120

C**** O U T P U T T A P E ASSIGNMENT STATEMENT. NO INPUT TAPE.

P0950130

$C * * * * *$

C**** WHEN EXECUTING ONLY SEGMENT 095, THE FOLLOWING STATEMENT

$C * * * *$ NUVI $=6$ MUST HAVE THE $c=$ IN COLUMNS 1 AND 2 REMOVED.

$C=N U V I=6$

NUVI $=6$

$C * * * *$

950 FORMAT( 15 I SOROT - $(095) / / 32 H$ BASIC EXTERNAL FUNCTION - SORT-

P0

$P 0071920$

$P 0071925$

$P 0071930$

$P 0071935$

$\mathrm{P} 09581$

$P 0071940$

P0950150

$P 0950160$

$1 / 126 \mathrm{H}$ (SQUARE ROOT - TYPE REAL)

$2 / / 27 H$ ASA REF.- 8.3.3 (TABLE 4)//24H LINE 1 OF EACH PAIR IS/23H P0950170

3 HOLLERITH INFORMATION//9H RESULTS)

WRITE (NUVI, 950)

C**** HEADER FOR SEGMENT O95 WRITTEN

AVS $=2.0$

IVI $=3$

CVS $=17.0$

$B V S=S Q R T(F L O A T((I V I+I N T(A V S)) / 2))$

WRITE (NUVI, 951) BVS

BVS $=S G R T(A M I N O(M A X O(I V I, 2)$, INT(CVS)))

P0950180

P0950190

P0950200

$P 0950210$

$P 0950220$

$P 0950230$

$P 0950240$

P0950250

P0950260 
WRITE (NUVI, 952) BVS

$P 0950270$

$B V S=S O R T(C V S)$

WRITE (NUVI, 953) BVS

P0950280

BVS $=$ SORT $(2.0 *$ CVS - FLOAT(IVI))

P0950290

WRITE (NUVI, 954) BVS

BVS $=\operatorname{SORT}(F L O A T(I V I+1)+5.0 *$ CVS $)$

P 0950300

WRITE (NUVI, 955) BVS

$P 0950310$

WRITE (NUVI, 956)

951 FORMAT ( 8 HO $X=2.0 .4 X .16 H 1.41421356237310 \%, F 21.7$ )

952 FORMAT ( $8 H O \quad X=3.0,4 X, 16 H 1.73205080756888 / \mathrm{F} 21.7$ )

953 FORMAT ( $8 H O \quad X=17.0,4 X, 16 H 4.12310562561766 / F 21.7$ )

954 FORMAT ( $8 H O \quad X=31.0,4 X, 16 H 5.56776436283002 / \mathrm{F} 21.7)$

955 FORMAT ( 8 HO $X=89.0,4 X, 16 H 9.43398113205660 / F 21.7)$

$P 0950320$

$P 0950330$

$P 0950340$

$P 0950350$

$P 0950360$

$P 0950370$

$P 0950380$

P0950390

956..... FORMAT(//37H LINE 2 OF EACH PAIR IS THE FUNCTION/25H CALCULATIONP

9 PRINTEO TO , 8 H OIGITS )

$C * * * *$ END OF TEST SEGMENT 095

C**** WHEN EXECUTING ONLY SEGMENT 095, THE STOP ANO END CÄROS

$[* * * *$ WHICH APPEAR AS COMMENT CAROS MUST HAVE. THE $C=$

$C * * * * *$ IN COLUMNS 1 AND 2 REMOVED.

$C=$ STOP

$C=\quad E N D$

STOP

ENO

$P 0950420$

$P 0950430$

$P 0950440$

$P 0950450$

$P 0950460$

$P 0950470$

$P 095 C 1$

$P 095 C 2$

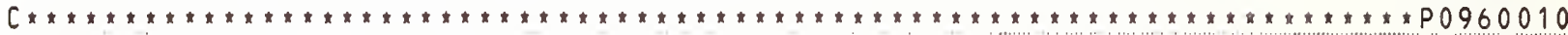

$C * * * * *$

$C * * * * *$

C*****

OSQRO - (096)

$P 0960020$

$P 0960030$

$P 0960040$

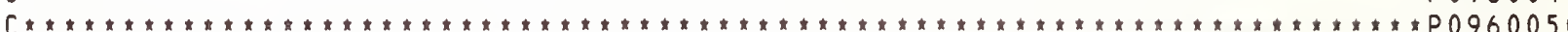

$C * * *$ GENERAL PURPOSE

C**** TO TEST BASIC EXTERNAL FUNCTION - OSQRT -

ASA REFPO960060

$C * * * *$ (SQUARE ROOT - TYPE O.P.)

$C * * * *$ USEO IN SIMPLE EXPRESSIONS

C**** INTRINSIC FUNCTIONS OBLE, IABS, FLOAT ASSUMEO WORKING

$C * * * *$ ARGUMENTS ARE ALL PRIME NUMBERS

$C * * * * *$

$[* * * *$ S P E C I F I C A T I O N S SEGMENT 096

8.3.3.P P0960070

TABLE 4 P0960080

$P 0960090$

$P 0960100$

$P 0960110$

$P 0960120$

$P 0960130$

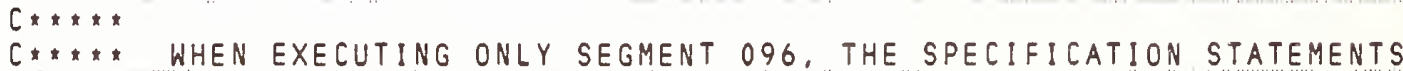

$C * * * *$ WHICH APPEAR AS COMMENT CAROS, MUST HAVE THE $C=$

$[* * * *$ IN COLUMNS 1 ANO 2 REMOVEO.

$P 0012210$

$P 0012215$

$P 0012220$

$P 0012225$

$C * * * * *$

$C=\quad$ OOUBLE PRECISION BVO

$P 0012230$

P0012235

DOUBLE PRECISION BVO

$C * * * * *$

$[* * * *$ O U T P U T T A P E ASSIGNMENT STATEMENT. NO INPUT TAPE.

P096A1

$[* * * * *$

C**** WHEN EXECUTING ONLY SEGMENT 096, THE FOLLOWING STATEMENT

$[* * * *$ NUVI $=6$ MUST HAVE THE $C=$ IN COLUMNS 9 AND 2 REMOVED.

$C=\quad$ NUVI $=6$

NUVI $=6$

$C * * * * *$

960 FORMAT( $15 H 9$ OSQRO - $(096) / / 33 H$ BASIC EXTERNAL FUNCTION -OSORT-

$P 0012240$

$P 0960140$

$P 0071950$

$P 0071955$

P0 071960

$P 0071965$

P096B1

$P 0071970$

$1 / / 26 \mathrm{H}$ (SQUARE ROOT -TYPE O.P.)

$P 0960150$

P0960160

$2 / 127 \mathrm{H}$ ASA REF. - 8.3.3 (TABLE 4)//24H LINE 1 OF EACH PAIR IS/23H P0960170

3 HOLLERITH INFORMATION//9H RESULTS)

P0960180

WRITE (NUVI, 960)

C***** HEAOER FOR SEGMENT 096 WRITTEN

AVS $=3.0$

$P 0960190$

$P 0960200$

$P 0960210$

$P 0960220$

IVI $=-2$

CVS $=17.0$

BVD $=$ DSQRT (OBLE $(F L O A T(I A B S(I V I))+A V S-3.0))$

$P 0960230$

$P 0960240$

WRITE (NUVI, 961) BVD

P0960250

$B V O=O S Q R T(0.000+A V S)$

WRITE (NUVI, 962) BVO

$B V O=O S Q R T(C V S-A V S+3.000)$

$P 0960260$

$P 0960270$

$P 0960280$

$P 0960290$

WRITE (NUVI, 963) BVD

$B V O=D S Q R T(2.000$ * CVS - OBLE(AVS))

$P 0960300$

WRITE (NUVI, 964) BVD

P0960310 
$8 V D=$ DSQRT (OBLE $(F L O A T(-I V I)+A V S) * C V S+F L O A T(I V I * 2)) \quad P 0960320$

WRITE (NUVI, 965) BVD

$P 0960330$

WRITE (NUVI, 966 )

961 FORMAT ( 8HO $x=2.0,5 x, 25 H 1.41421356237309504880+00 / 8 \times, 1 P D 24.13)$

962 FORMAT ( $8 H O X=3.0 .5 X .25 H 1.73205080756887729350+00 / 8 X .1 P D 24.13)$

963 FORMAT ( $8 H 0 \quad X=17.0,5 X, 25 H 4.12310562561766054980+00 / 8 X, 1 P D 24.13)$

964 FORMAT ( 8 HO $x=31.0 .5 \times .25 H 5.56776436283002192210+00 / 8 X, 1 P D 24.13)$

965 FORMAT ( $8 H O X=89.0,5 X, 25 H 9.43398113205660381130+00 / 8 X, 1 P D 24.13)$

966 FORMAT(//37H LINE 2 OF EACH PAIR IS THE FUNCTION/25H CALCULATIONP

A PRINTED TO, 9 H 14 DIGITS)

$C * * * * *$ END OF TEST SEGMENT 096

$C * * * *$ WHEN EXECUTING ONLY SEGMENT 096, THE STOP AND END CARDS

$C * * * *$ WHICH APPEAR AS COMMENT CAROS MUST HAVE THE $C=$

$C * * * *$ IN COLUMNS 1 AND 2 REMOVED.

$C=$ STOP

$C=E N D$

STOP

END

"P0960340

P0960350

P0960360

P0960370

P0960380

P0960390

NPO 960400

$P 0960410$

$P 0960420$

$P 0960430$

$P 0960440$

P0960450

$P 0960460$

$P 0960470$

P096C1

P096C2

$[* * * * *$
$C * * * * *$

END. P097001

$C * * * * *$
$C * * * * *$

$[* * * * *$

P0970020

C

$C * * * * *$ GENERAL PURPOSE

C******

$C * * * * *$ TO TEST BASIC EXTERNAL FUNCTION - CSQRT-

$C * * * * *$ (SQUARE ROOT OF A COMPLEX NUMBER)

$C * * * * *$ ARGUMENTS ARE EP1C(11) TO EP1C(20)

$C * * * * *$ EXPECTED RESULLTS ARE EP1C (1) TO EP1C(10)

$C * * * * * S$ S E C I F I C A T I O N S SEGMENT 097

$C * * * * *$

$[* * * * *$

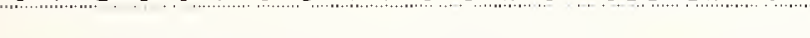

$C * * * *$ WHICH APPEAR AS COMMENT CAROS MUST HAVE THE $C=$

$C * * * * *$ IN COLUMNS 1 AND 2 REMOVED.

$C * * * * *$

$C=$

COMPLEX EP1C $(30), A V C, B \vee C$

COMPLEX EP1C $(30), A V C, B \vee C$

$C * * * * *$

C*****0 UU T P U T - T A P E ASSIGNMENT STATEMENT. NO INPUT TAPE.

$[* * * * *$

C**** WHEN EXECUTING ONLY SEGMENT 097 . 'THE FOLLOWING STATEMENT

$C * k * *$ NUVI $=6$ MUST HAVE THE $C=$ IN COLUMNS 1 AND 2 REMOVED.

$C=$ NUVI $=6$

$C * * * * *$

NUVI $=6$

$P 0970030$

$P 0970040$

*P0970050

ASA REFPO970060

$P 0970070$

8.3.3 P0970080

TABLE 4P0970090

$P 0970100$

$P 0970110$

$P 0970120$

P0012250

$P 0012255$

$P 0012260$

P0012265

P0012270

$P 0012275$

P097A1

P0012280

$P 0970130$

$P 0071980$

$P 0071985$

P0071990

P0071995

P097B1

$P 0072000$

$P 0970140$

WRITE (NUVI, 970)

970 FORMAT( $15 H 1$ CSQRO- $(097) / / 33 \mathrm{H}$ BASIC EXTERNAL FUNCTION - CSORT-

$P 0970150$

$1 / 129 \mathrm{H}$ (SOUARE ROOT -TYPE COMPLEX)//27H ASA REF. - 8.3.3 (TABLE 4)PO970160

$2 / 124 \mathrm{H}$ LINE 1 OF EACH PAIR IS /20H THE EXPECTED VALUE //9H RESULPO970170

$3 T$ )

$C * * * * *$ INITIALIZE EP1C (EXACT VALUES
EPIC $(1)=(0.9950042,0.0998334)$

$E P 1 C(2)=(0.9800666,0.1986693)$

$E P 1 C(3)=(0.9553365,0.2955202)$

$E P 1 C(4)=(0.9210610,0.3894183)$

$E P 1 C(5)=(0.8775826,0.4794255)$

$E P 1 C(6)=(0.8253356 .0 .5646425)$

$E P 1 C(7)=(0.7648422 .0 .6442177)$

$E P 1 C(8)=(0.6967067 .0 .7173561)$

$E P 1 C(9)=(0.5403023,0.8414710)$

$E P 1 C(10)=(0.4161468,-0.9092974)$

$E P 1 C(11)=(0.9800666 .0 .1986693)$

$E P 1 C(12)=(0.9210610,0.3894183)$

$E P 1 C(13)=(0.8253356,0.5646425)$

$E P 1 C(14)=(0.6967067,0.7173561)$

$E P 1 C(15)=(0.5403023,0.8414710)$

$E P 1 C(16)=(0.3623577 .0 .9320391)$

$E P 1 C(17)=(0.1699671 .0 .9854497)$

$P 0970180$

P0970190

$P 0970200$

P0970210

$P 0970220$

P0970230

$P 0970240$

$P 0970250$

$P 0970260$

$P 0970270$

P0970280

$P 0970290$

$P 0970300$

$P 0970310$

P0970320

$P 0970330$

$P 0970340$

P0970350

P0970360 
$E P 1 C(18)=(-0.0291995,0.9995736)$

$P 0970370$

$E P 1 C(19)=(-0.4161468,0.9092974)$

$P 0970380$

$E P 1 C(20)=(-0.6536436,-0.7568025)$

$P 0970390$

IVI $=0$

$P 0970400$

971 JVI $=1$

$P 0970410$

972 IVI $=I V I+1$

$J V I=J V I+1$

$A V C=\operatorname{CSQRT}(E P 1 C(I V I+10) *(10 * *((2 * J V I)-8)))$

$P 0970420$

$P 0970430$

$P 0970440$

$B \vee C=E P I C(I V I) * 10 *(J \vee I-4)$

$P 0970450$

WRITE (NUVI, 973) BVC, AVC

$P 0970460$

$P 0970470$

973 FORMAT( $2 H 02 E 14.7 / 2 \times, 2 E 14.7)$

IF (JVI - 6) 972,974,974

974 IF (IVI - 10) 971,975,975

$P 0970480$

$P 0970490$

$P 0970500$

975 WRITE (NUVI, 976)

976 FORMAT(//37H LINE 2 OF EACH PAIR IS THE FUNCTION/13H CALCULATIONPO97O51O 1)

$[* * *$ END OF TEST SEGMENT 097

C*** WHEN EXECUTING ONLY SEGMENT 097 THE STOP AND END CARDS

$P 0970520$

00970530

$P 0970540$

$C * *$ WHICH APPEAR AS COMMENT CARDS MUST HAVE THE C $=$

$C * * * *$ IN COLUMNS 1 AND 2 REMOVED.

$C=$ STOP

$C=\quad$ END

STOP

P0970550

$P 0970560$

$P 0970570$

$P 0970580$

$P 097 C 1$

END

$P 097 C 2$

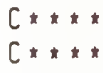

P0980010

$C * * * * *$

ARCTG - (098)

$P 0980020$

$P 0980030$

P0980040

\#P0980050

$\begin{array}{lll}C * * * & \text { GENERAL PURPOSE } & \text { ASA REFPO980060 }\end{array}$

C***** TO TEST BASIC EXTERNAL FUNCTION - ATAN -

C**** (TRIGONOMETRIC ARCTANGENT, SINGLE ARGUMENT -TYPE REAL)TABLE 4 PO980080

$[* * * *$ USED IN SIMPLE ARITHMETIC EXPRESSIONS

$C * * * *$ INTRINSIC FUNCTION ABS, FLOAT, AMAXI, INT

$[* * * *$ ASSUMED WORKING $C * * *$ ARGUMENTS ARE POWERS (OR SUMS) OF 2

$C * * * *$

$[* * * *$ O U T P U T T A P E ASSIGNMENT STATEMENT. NO INPUT TAPE.

$P 0980090$

$P 0980100$

P0980110

$P 0980120$

$P 0980130$

$P 0980140$

$P 0072010$

$C * * * *$ WHEN EXECUTING ONLY SEGMENT 098, THE FOLLOWING STATEMENT

$C * * *$ NUVI $=6$ MUST HAVE THE $C=$ IN COLUMNS 1 AND 2 REMOVED.

$C=$ NUVI $=6$

$C * * * *$ NUVI $=6$

P0072015

$P 0072020$

$P 0072025$

P098B1

$P 0072030$

WRITE (NUVI, 980)

980 FORMAT(15H1 ARCTG - $(098) / / 32 \mathrm{H}$ BASIC EXTERNAL FUNCTION - ATAN-

P0980150

P0980160

$1 / 125 \mathrm{H}$ (ARCTANGENT -TYPE REAL)
$2 / 127 \mathrm{H}$ ASA REF.- 8.3 .3 (TABLE 4)//24H LINE 1 OF EACH PAIR IS/23H PO980180

3 HOLLERITH INFORMATION//9H RESULTS) $\quad$ PO980190

C**** HEADER FOR SEGMENT 098 WRITTEN

$A V S=.125$

CVS $=-.25$

$I V I=2$

$B \vee S=A T A N(A M A X 1(A \vee S, C \vee S))$

WRITE (NUVI, 981) BVS

$B \vee S=A T A N(A \vee S * 2.0)$

WRITE(NUVI, 982) BVS

$B V S=A T A N(A B S(C V S)+A V S)$

WRITE (NUV!, 983) BVS

BVS $=$ ATAN(-CVS $A M A X O(I V I, I N T(A V S)))$

WRITE(NUVI, 984) BVS

BVS $=$ ATAN (FLOAT(IVI) * CVS - $(2.0 * A V S)$ )

WRITE (NUVI, 985) BVS

$P 0980190$
$P 0980200$

$P 0980210$

$P 0980220$

P0980230

$P 0980240$

$P 0980250$

$P 0980260$

$P 0980270$

P0980280

P0980290

P0980300

P0980310

P0980320

P0 0980330

$P 0980340$

$P 0980350$

P0980360

P0980370

WRITE (NUVI, 987)

981 FORMAT $(10 \mathrm{HO} \quad \mathrm{x}=0.125,5 \mathrm{X}, 15 \mathrm{H} 0.124354994547, / 10 \mathrm{X}, \mathrm{F} 15,7)$

982 FORMAT $(10 H O \quad X=0.250,5 X, 15 H \quad 0.244978663127,110 X, F 15.7)$

$P 0980380$ 
098, THE STOP AND END CARDS

$C * * *$ WHICH APPEAR AS CDMMENT CARDS MUST HAVE THE $C=$

$C * * * *$ IN COLUMNS 1 AND 2 REMDVED.

$C=$

STOP

$C=E N D$

STOP

$P 0980420$

CALCULATIONP0980430

$P 0980440$

$P 0980450$

$P 0980460$

$P 0980470$

$P 0980480$

P0980490

$P 0980500$

END

P098C 1

$P 098 C 2$

C

$C * * * * *$

DACTG - $(099)$

$P 0990020$

$C * * * * *$

$P 0990030$

$P 0990040$

$C * * * * *$

$C * * * *$ GENERAL PURPOSE

$C * * * *$ TO TEST BASIC EXTERNAL FUNCTION - DATAN -

$\begin{array}{ll}C * * * * * & \text { (TRIGONOMETRIC ARCTANGENT, SINGLE ARGUMENT -TYPE D.P.) } \\ C * * * * * & \text { USED IN SIMPLE ARITHMETIC EXPRESSIONS }\end{array}$

$C * * * *$ INTRINSIC FUNCTIONS DSIGN,FLOAT,OBLE ASSUMED WDRKING

$C * * * *$ ARGUMENTS ARE POWERS (DR SUMS) DF 2

$C * * * * *$

$[* * * *$ S P E C I FIC A T I ON"S SEGMENT OQ9

$C * * * *$

$C * * * *$ WHEN EXECUTING ONLY SEGMENT 099, THE SPECIFICATIDN STATEMENTS

$C * * * *$ WHICH APPEAR AS CDMMENT CARDS MUST HAVE THE C

$C * * * *$ IN COLUMNS 1 AND 2 REMDVEO.

$C * * * *$

$C=$ DOUBLE PRECISIDN AVD, BVD, CVD

DOUBLE PRECISIDN AVD, BVD, CVD

\section{$C * * * * *$}

$C * * * *$ O U T P U T T A P E ASSIGNMENT STATEMENT. ND INPUT TAPE.

$C * * * * *$

$C * * * *$

WHEN EXECUTING ONLY SEGMENT O99. THE FDLLOWING STATEMENT

$C * * * * *$ NUVI $=6$ MUST HAVE THE $C=$ IN COLUMNS 1 AND 2 REMDVED.

$C=\quad N \cup V I=6$

$C * * * *$ NUVI $=6$

C**** HEADER FOR SEGMENT 099 WRITTEN

WRITE(NUVI, 990)

990 FDRMAT( 15 H DACTG - $(099) / 133 \mathrm{H}$ BASIC EXTERNAL FUNCTIDN - DATAN-

$1 / 125 \mathrm{H}$ (ARCTANGENT -TYPE D.P.)

$2 / / 27 H$ ASA REF.- 8.3 .3 (TABLE 4)//24H LINE 1 OF EACH PAIR IS/23H

3 HDLLERITH INFDRMATIDN/ $/ 9 \mathrm{H}$ RESULTS $)$

$A \vee D=-.12500$

CVD $=.2500$

IVI $=2$

$B V D=D A T A N(D S I G N(A V D, C V D))$

WRITE (NUVI.991) BVD

$B V D=\operatorname{DATAN}(2.0 *(-A \vee D))$

WRITE(NUVI. 992) BVD

$B V D=D A T A N(C V D-A V D)$

WRITE (NUVI.993) BVD

$B V D=\operatorname{DATAN}(D B L E(F L D A T(I V I) / 4.0))$

WRITE (NUVI, 994) BVD

$B V D=D A T A N(D S I G N(1.0 D O-C V D, A V D))$

WRITE(NUVI.995) BVD

$B V D=D A T A N(D B L E(F L O A T(I V I * * 2)) * C V D)$

WRITE (NUVI, 996) BVD

WRITE (NUVI, 997)

991 FORMAT $(10 \mathrm{HO} \mathrm{x}=0.125,5 \mathrm{X}, 19 \mathrm{H} 0.1243549945470+00 / 10 \mathrm{X}, 024.12)$

992

FORMAT $(10 \mathrm{HO} \quad X=0.250,5 \mathrm{X}, 19 \mathrm{H} 0.244978663127 \mathrm{D}+00 / 10 \mathrm{X}, 024.12)$

993 FDRMAT $(10 \mathrm{HO} \quad X=0.375 .5 \mathrm{X}, 19 \mathrm{H} 0.3587706702710+00 / 10 \mathrm{X}, 024.12)$

994 FDRMAT $(10 \mathrm{HO} \quad \mathrm{X}=0.500,5 \mathrm{X}, 19 \mathrm{H} 0.4636476090010+00 / 10 \mathrm{X}, 024.12)$
ASA REFPO990060

8.3 .3 P 0990070

TABLE $4 P 0990080$

$P 0990090$

$P 0990100$

$P 0990110$

P0990120

$P 0990130$

P0012290

$P 0012295$

$P 0012300$

$P 0012305$

P0012310

P 0012315

P099A1

P0012320

$P 0990140$

$P 0072040$

$P 0072045$

P0072050

P 0072055

$P 099 B 1$

$P 0072060$

$P 0990150$

P 0990160

P0990170

P0990180

P0990190

P0990200

P0990210

P0990220

$P 0990230$

P0990240

$P 0990250$

$P 0990260$

$P 0990270$

$P 0990280$

P0990290

$P 0990300$

$P 0990310$

$P 0990320$

P0990330

$P 0990340$

$P 0990350$

P0990360

P0990370

P0990380

P0990390

$P 0990400$ 
FORMAT $/ / 137 H$ LINE 2 OF EACH PAIR IS THE FUNCTION/25H CALCULATIONPO990430

1 PRINTED TO, 9H12 OIGITS )

\section{$[* * *$ END OF TEST SEGMENT 099}

$C * * *$ WHEN EXECUTING ONLY SEGMENT 099. THE STOP ANO END CARDS

$C * * * W H I C H$ APPEAR AS COMMENT CAROS MUST HAVE THE $C=$

$C * * *$ IN COLUMNS 1 ANO 2 REMOVED.

$C=\quad S T O P$

$C=\quad E N O$

$P 0990440$

$P 0990450$

$P 0990460$

$P 0990470$

$P 0990480$

$P 0990490$

$P 0990500$

STOP

P $099 \mathrm{C} 1$

END

P099C2

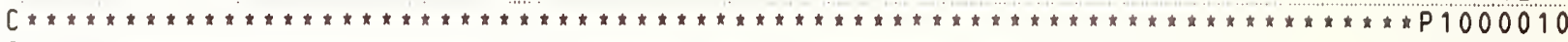

$[* * * * *$

$A C T G 2-(100)$

$P 1000020$

C******

P 1000030

C $k * k * *$

$P 1000040$

C

$[* * * *$ GENERAL PURPOSE

C***** TO TEST BASIC EXTERNAL FUNCTION - ATAN2 -

C**** (TRIGONOMETRIC ARCTANGENT, TWO ARGUMENTS -TYPE REAL) TABLE $4 P 1000080$

C***** USEO IN SIMPLE ARITHMETIC EXPRESSIONS

C*** INTRINSIC FUNCTIONS AMIN 1, FLOAT, AMAXO

$C * * * *$ ARGUMENTS ARE POWERS (OR SUMS) OF 2

ASSUMEO WORKING

$P 1000090$

C******

$C * * * * *$

O U T P U T T A P E ASSIGNMENT STATEMENTS. NO INPUT TAPE.

P1000100

P 1000110

$P 1000120$

C $* * * *$

$C * * * *$

WHEN EXECUTING ONLY SEGMENT 100, THE FOLLOWING STATEMENT

$C * * * *$ NUVI $=6$ MUST HAVE THE $C=$ IN COLUMNS 9 ANO 2 REMOVEO.

$C=\quad$ NUVI $=6$

$[* * * * *$

NUVI $=6$

P 1000130

$P 0072070$

P0072075

P0072080

P0072085

$P 100 B 1$

WRITE (NUVI, 1000)

P 0072090

$P 1000140$

1000 FORMAT(15H1 ACTG2 - (100)//33H BASIC EXTERNAL FUNCTION - ATAN2- P1000150

$1 / 137 \mathrm{H}$ (ARCTANGENT, 2 ARGUMENT - TYPE REAL)

$2 / 127 \mathrm{H}$ ASA REF. -8.3 .3 (TABLE 4)//24H LINE 1 OF EACH PAIR IS/23H P1000170

3 HOLLERITH INFORMATION//9H RESULTS)

$P 1000180$

$A V S=.125$

CVS $=-.25$

IVI $=2$

BVS $=A T A N 2(A B S(A M I N 1$ (AVS, CVS)), FLOAT(IVI))

WRITE (NUVI, 1001 ) BVS

BVS $=$ ATAN2 (CVS *2. AVS $* 2.0)$

WRITE (NUVI, 1002 ) BVS

$B V S=A T A N 2(A V S-C V S,-(4.0 * C V S))$

WRITE (NUVI, 1003) BVS

$B \vee S=A T A N 2(-C \vee S / A \vee S, A M A X O(I \vee I, 4))$

WRITE (NUVI, 1004) BVS

BVS $=$ ATAN2 $(-.09375$, AVS $)$

WRITE (NUVI, 1005 ) BVS

BVS $=$ ATAN2 (FLOAT (IVI), 2.0)

WRITE (NUVI, 1006) BVS

P 1000190

P 1000200

$P 1000210$

$P 1000220$

P 1000230

$P 1000240$

P 1000250

P 1000260

$P 1000270$

$P 1000280$

P 1000290

$P 1000300$

$P 1000310$

$P 1000320$

WRITE (NUVI, 1007)

$P 1000330$

$P 1000340$

1001 FORMAT $(10 H O X=0.125,5 X, 15 H 0.124354994547,110 X, F 15.7)$

P 1000350

FORMAT (1OHO $X=0.250,5 X .15 H 0.244978663127, / 10 X, F 15.7)$

P 1000360

P 1000370

FORMAT ( $10 \mathrm{HO} X=0.375,5 \mathrm{X}, 15 \mathrm{H} 0.358770670271,110 \mathrm{X}, \mathrm{F} 15.7)$

$P 1000380$

FORMAT ( $10 \mathrm{HO} \quad X=0.500,5 \mathrm{X}, 15 \mathrm{H} 0.463647609001, / 10 \mathrm{X}, \mathrm{F} 15.7)$

P 1000390

$P 1000400$

1005

1006

FORMAT $(10 \mathrm{HO} \quad \mathrm{X}=1.000,5 \mathrm{X}, 15 \mathrm{H} 0.785398163397,190 \mathrm{X}, \mathrm{F} 95.7)$

1007 FORMAT $/ / 37 H$ LINE 2 OF EACH PAIR IS THE FUNCTION/25H

1 PRINTEO TO, 8 H DIGITS )

C**** ENO OF TEST SEGMENT 100

C*****WHEN EXECUTING ONLY SEGMENT 100, THE STOP ANO ENO CAROS

$C * * *$ WHICH APPEAR AS COMMENT CAROS MUST HAVE THE C=

C*****IN COLUMNS 1 ANO 2 REMOVED.

STOP

ENO

STOP

NP 1000410

$P 1000420$

P 1000430

P 1000440

P 1000450

$P 1000460$

P 1000470

$P 1000480$

P $100 C 1$

ENO

P100C2 


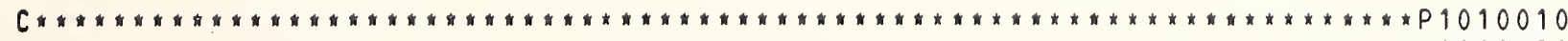

$C * * * * * *$

$C * * * * *$ GENERAL PURPOSE

$C * * * *$

$C * * * * *$

$C * * * * *$

$C * * * * *$

$C * * * * *$

$C * * * * *$

$C * * * * *$

$C * * * * *$

$C * * * * *$

$C * * * * *$

$C * * * * *$

$C * * * * *$

$C * * * * *$

$C=\quad$ DOUBLE PRECISION AVD, BVD, CVD

DOUBLE PRECISION AVD, BVD, CVD

$C * * * * *$

$C * * * * *$

$C * * * * *$

$C * * * * *$

$C * * * *$

$C=$

O U T P U T - TA P

WHEN EXECUTING

GONOMETRIC ARTERNAL FUNCTION - DATANZ

USED IN SIMPLE ARITHMETIC EXPRESSIONS

INTRINSIC FUNCTIONS DMIN1,DMAX1,DSIGN,DBLE, FLOAT

ASSUMED WORKING

ARGUMENTS ARE POWERS (OR SUMS) OF 2

$S$ P E C I F I C A T I O N S SEGMENT 101

WHEN EXECUTING ONLY SEGMENT 101, THE SPECIFICATION STATEMENTS WHICH APPEAR AS COMMENT CARDS MUST HAVE THE $C=$ IN COLUMNS

NUVI $=6$

$C * * * * *$

NUVI $=6$

WRITE (NUVI, 1010)

1010 FORMAT ( $15 H 1$ DATNZ - (101)//36H BASIC EXTERNAL FUNCTION -DATANZ$1 / 137 \mathrm{H}$ (ARCTANGENT, 2 ARGUMENT -TYPE D.P.)

$2 / 127 H$ ASA REF. 8.3 .3 (TABLE 4)//24H LINE 1 OF EACH" PAIR IS/23H 3 HOLLERITH INFORMATION//9H RESULTS)

$A \vee D=.125$

CVD $=-.25$

IVI $=2$

$B \vee D=$ DATAN2(DMINI(AVD, - CVD), 2.0DO/.DBLE(FLOAT(IVI)))

WRITE (NUVI, 1011) BVD

$B V D=$ DATANZ( AVD, FLOAT ( IVI)*(-CVD))

WRITE (NUVI, 1012) BVD

WRITE (NUVI, 1013 ) BVD

$B \vee D=$ DATAN2 (DMIN1 (AVD,.062500), DMAX1(AVD,CVD))

WRITE (NUVI, 1014) BVD

$B V D=D A T A N 2(D A B S(C V D) * D S I G N(A V D, C V D) * 6 . D 0, .25 D 0)$

WRITE (NUVI, 1015 ) BVD

$B V D=D A T A N 2$ (DBLE(FLOAT(IVI)), AVD*FLOAT(IVI**4))

WRITE (NUVI, 1016) BVD

WRITE (NUVI, 1017 )

1011 FORMAT $(10 H O \quad X=0.125,5 X, 19 H \quad 0.1243549945470+00 / 10 X, 024.12)$

1012 FORMAT $(10 \mathrm{HO} X=0.250,5 \mathrm{X}, 19 \mathrm{H} 0.244978663127 \mathrm{D}+00 / 10 \mathrm{X}, 024.12)$

1013 FORMAT $(10 H O \quad X=0.375,5 X, 19 H \quad 0.3587706702710+00 / 10 X, 024.12)$

1014 FORMAT $(10 H O \quad X=0.500,5 X, 19 H \quad 0.4636476090010+00 / 10 X, 024.12)$

1015 FORMAT $(10 H O \quad X=-0.750,5 X, 19 H-0.6435011087930+00 / 10 X, 024.12)$

1016 FORMAT $(10 \mathrm{HO} X=1.000,5 \mathrm{X}, 19 \mathrm{H} 0.7853981633970+00 / 10 \mathrm{X}, 024.12)$

1017 FORMAT $/ 137 \mathrm{H}$ LINE 2 OF EACH PAIR IS THE FUNCTION/25H CALCULATIONP 1010430

1 PRINTED TO, 9 TH2 DIGITS )

\section{$C * * * *$ END OF TEST SEGMENT 101}

$C * * * *$ WHEN EXECUTING ONLY SEGMENT 101 THE STOP AND END CARDS

$C * * * * *$ WHICH APPEAR AS COMMENT CARDS MUST HAVE THE $C=$

$C * * * * *$ IN COLUMNS 1 AND 2 REMOVED.

$C=S T O P$

$C=\quad$ END

STOP

END

$C * * * * * * * * 20$

$C * * * * *$
ASA REFP 1010060

8.3.3 P1010070

TABLE 4P1010080

P 1010090

P1010100

P 1010110

P 1010120

P1010130

P1010140

P0012330

P0012335

$P 0012340$

P0012345

P0012350

$P 0012355$

P101A1

P0012360

$P 1010150$

$P 0072100$

P0072105

P0072110

P0072115

P101B1

$P 0072120$

P1010160

P 1010170

P 1010180

010190

P1010210

P 1010220

P 1010230

P 1010240

P 1010250

P 1010260

$P 1010270$

P1010290

P 1010300

P1010310

P 1010320

P 1010330

P 1010340

P1010350

P 1010360

P 1010370

$P 1010380$

P 1010390

P 1010400

P 1010410

P1010440

P1010450

P1010460

P 1010470

P 1010480

P1010490

P1010500

P101C1

P101C2

P 1020010

P 1020020 
$P 1020090$

P1020100

$P 1020110$

$C * * * * *$

$C * * * * *$

$C * * * * *$

WHEN EXECUTING ONLY SEgment 102, THE SPECIFICATION STATEMENTS

$P 0012370$

C********t* WHICH APPEAR AS

IN COLUMNS 1 AND 2 REMOVED.

$C * * * *$

$C=\quad$ DOUBLE PRECISION AVD,BVD,CVD,DVD, EVD, FVD, GVD

DOUBLE PRECISION AVD, BVD, CVD, DVD, EVD, FVD, GVD

$P 0012375$

$P 0012380$

$P 0012385$

$P 0012390$

$P 0012395$

$[* * * * *$

$C * * * * *$

$[* * * * *$

$C * x * * *$

O U T P U T - T A P E ASSIGNMENT STATEMENT.

\section{NT.}

NO INPUT TAPE.

P102A1

$P 0012400$

$P 1020120$

$P 0072130$

$P 0072135$

C***** NUVI $=6$ MUST HAVE THE $C=I N$ CDLUMNS 1 AND 2 REMDVED.

$C=\quad$ NUVI $=6$

$C * * * * *$

$$
\text { NUVI }=6
$$

WRITE (NUVI, 1020)

1020 FORMAT( 15 HI'DMODA - (102)//32H BASIC EXTERNAL FUNCTIDN - DMOD-

$1 / 139 \mathrm{H}$ (REMAINDERING - TYPE DOUBLE PRECISION)//27H ASA REF.- $8.3 .3 P$

2 (TABLE 4)// $9 H$ RESULTS)

$A V D=16.062500$

$B V D=-4.000$

$C V D=-8.12500$

$D V D=2.500$

$E V D=-1.000$

$F V D=1.0 D O$

$F \vee D=D M O D(A \vee D, B \vee D)$

$G \vee D=F V D-0.062500$

WRITE (NUVI, 1021) GVD

$F V D=2.0 D O$

$F V D=D M O D(C V D$, DVD)

$G V D=F V D+0.62500$

WRITE (NUVI, 1021) GVD

$F V D=3.000$

$F \vee D=D M O D(B \vee D, E \vee D)$

$G V D=F V D+0.0 D 0$

WRITE (NUVI, 1021) GVD

$F V D=4.000$

$F \vee D=D M O D(B \vee D, A \vee D)$

GVD = FVD - (BVD- (DBLE (FLOAT(IDINT(BVD/AVD)))) *AVD)

WRITE (NUVI, 1021 ) GVD

WRITE (NUVI, 1022)

1021 FORMAT $(/ / D 25.14)$

1022 FORMAT ///18H END OF DMOD TEST//40H ALL ABOVE ANSWERS SHDULD BE

1 FOR THIS/32H TEST SEGMENT TD BE SUCCESSFUL.)

\section{$[* * * * *$ END DF TEST SEGMENT 102}

$C * * * * *$ WHEN EXECUTING ONLY SEGMENT 102 THE STDP AND END CARDS

$C * * * *$ WHICH APPEAR AS COMMENT CARDS MUST HAVE THE C $=$

$[* * * * *$ IN COLUMNS 1 AND 2 REMOVED.

\section{$C=$ STOP}

$C=\quad E N D$

\section{STOP}

END

P0072140

P 0072145

P $102 B 1$

$P 0072150$

$P 1020130$

$P 1020140$

$3 P 1020150$

$P 1020160$

$P 1020170$

$P 1020180$

$P 1020190$

$P 1020200$

$P 1020210$

$P 1020220$

P 1020230

P1020240

$P 1020250$

P 1020260

$P 1020270$

$P 1020280$

$P 1020290$

$P 1020300$

$P 1020310$

$P 1020320$

$P 1020330$

$P 1020340$

P 1020350

$P 1020360$

$P 1020370$

$P 1020380$

P 1020390

OP 1020400

$P 1020410$

$P 1020420$

P 1020430

P 1020440

P 1020450

$P 1020460$

P1020470

P $102 C 1$

$P 102 C 2$

C

$[* * * * *$

$P 1030020$

$C * * * * *$

CABSA - (103)

$P 1030030$

$[* * * * *$

$P 1030040$

$[* * * * * t$

$C * * * *$ GENERAL PURPOSE

C***** .TO TEST BASIC EXTERNAL FUNCTIDN - CABSASA REFP1030060 
C*****) (MODULUS OF A COMPLEX NUMBER)

C***** ARGUMENTS ARE ARRAY EPIC(30). FUNCTIONS FROM.

TABLE 4P1030080

C*****10D NUMBERED ARGUMENTS PRINTED AS SET 1 AND 2
$C * * * *$
$[* * *$ FROM EVEN NUMBERED ARGUMENTS

$P 1030090$

$\begin{array}{ll}C * * * * * & \text { ODD NUMBERED ARGUMENTS PRINTED AS SET } 1 \text { AND } 2 \\ C * * * * * & \text { FROM EVEN NUMBERED ARGUMENTS }\end{array}$

$P 1030100$

SET 1 RESULTS SHOULD BE .1 E-6 TO $11 E+8$

P 1030110

SET 2 RESULTS SHOULD BE .5 E-6 TO $.5 E+8$

P 1030120

P 1030130

P 1030140

$[* * * *$

C*\#* S P E C I F I C A T I O N S SEGMENT 103

$[* * * * *$

$[* * * * *$

WHEN EXECUTING ONLY SEgMENT 103 THE SPECIFICATION STATEMENTS

$C * * * *$ WHICH APPEAR AS COMMENT CARDS MUST HAVE THE C $=$

C*****IN COLUMNS 1 AND 2 REMOVED.

$C * * * * *$

$C=\quad$ COMPLEX EP1C(30)

COMPLEX EPIC $(30)$

$C * * * * *$

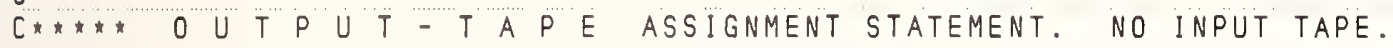

$[* * * * *$

C***** WHEN EXECUTING ONLY SEGMENT 103 THE FOLLOWING STATEMENT

C***** NUVI $=6$ MUST HAVE THE C $=$ IN COLUMNS 1 AND 2 REMOVED.

$C=\quad$ NUVI $=6$

NUVI $=6$

$[* * * * *$

WRITE (NUVI, 1030)

1030 FORMAT $\left(15 \mathrm{H}^{-1}\right.$ CABSA - $(103) / 132 \mathrm{H}$ BASIC EXTERNAL FUNCTION - CABS-

$1 / / 31 \mathrm{H}$ (MODULUS OF A COMPLEX NUMBER)//27H ASA REF. - 8.3.3 (TABLE

24)/19H RESULTS//10X,5HSET 1,15X,5HSET 2 )

[*****INITIALIZE EP1C(EXACT VALUES)

EPIC $(1)=(0.5 E-7,-0.866025 E-7)$

$E P 1 C(2)=(2.5 E-7,-4.330125 E-7)$

$E P 1 C(3)=(1 . E-6,0.0)$

$E P 1 C(4)=(5 . E-6,0.0)$

$E P 1 C(5)=(0.5 E-5,0.866025 E-5)$

$E P 1 C(6)=(2.5 E-5,4.330125 E-5)$

EP1C $(7)=(-0.5 E-4,0.866025 E-4)$

$E P 1 C(8)=(-2.5 E-4,4.330125 E-4)$

$E P 1 C(9)=(-1 . E-3,0.0)$

$E P I C(10)=(-5 . E-3,0.0)$

$E P 1 C(11)=(-0.5 E-2,-0.866025 E-2)$

$E P 1 C(12)=(-2.5 E-2,-4.330125 E-2)$

$E P 1 C(13)=(0.5 E-1,-0.866025 E-1)$

$E P 1 C(14)=(2.5 E-1,-4.330125 E-1)$

$E P 1 C(15)=(1.0 .0 .0)$

$E P 1 C(16)=(5.0,0.0)$

EPIC(17) $=(0.5 E 1,0.866025 E 1)$

$E P 1 C(18)=(2.5 E 1,4.330125 E 1)$

$E P 1 C(19)=(-0.5 E 2,0.866025 E 2)$

$E P 1 C(20)=(-2.5 E 2,4.330125 E 2)$

$E P 1 C(21)=(-1 . E 3,0.0)$

$E P 1 C(22)=(-5 . E 3,0.0)$

EP1C(23) $=(-0.5 E 4,-0.866025 E 4)$

$E P 1 C(24)=(-2.5 E 4,-4.330125 E 4)$

$E P 1 C(25)=(0.5 E 5,-0.866025 E 5)$

$E P 1 C(26)=(2.5 E 5,-4.330125 E 5)$

$E P 1 C(27)=(1 . E 6,0.0)$

$E P 1 C(28)=(5 . E 6,0.0)$

$E P 1 C(29)=(0.5 E 7,0.866025 E 7)$

$E P 1 C(30)=(2.5 E 7,4.330125 E 7)$

IVI $=-1$

1031 IVI $=I V I+2$

$A V S=C A B S(E P 1 C(I V I))$

$B V S=C A B S(E P 1 C(I V I+1))$

WRITE (NUVI, 1032 ) AVS, BVS

1032 FORMAT $(1$ HO , E17.6, 2X, E17.6)

IF (IVI - 29) 1031, 1033, 1033

1033 WRITE (NUVI, 1034)

1034 FORMAT(//39H VALUES IN EACH SET SHOULD BE POSITIVE /39H. 1 FOR SP1030600 1ET $1(.5$ FOR SET 2$)$, EXPONENT $135 \mathrm{H}$ RANGE FROM - 06 TO +08 IN SEQUEP1030610 


\section{A}

$S$ P E C I F I C A T I O

$P 1100070$
8. $1.2 P 1100080$

$P 1100090$

$P 1100100$

$C * * * * *$

$C \dot{x} \times * * *$

$\Gamma * \dot{*} ; * *$

WHEN EXECUTING ONLY SEGMENT 110, THE SPECIFICATIDN STATEMENTS

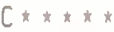

WHICH APPEAR AS CDMMENT CARDS MUST HAVE THE $C=$

$C * * * * *$

$C * * * * *$

$C=\quad$ INTEGER IFIX

$C=\quad$ REAL ABS, SQRT

INTEGER IF IX

REAL ABS, SQRT

$C * * * * *$

$C * * * * *$

$C * * * * *$

WHEN EXECUTING ONLY SEGMENT 110, THE SEGMENT O05, WHICH

$C * * * * *$

CONTAINS THE STATEMENT FUNCTIONS BEING TESTED HERE MUST BE

$10.1 .7 P 1100110$

$5.3 " P 1100120$

$P 1100130$

$P 1100140$

$P 0012450$

$P 0012455$

$P 0012460$

$P 0012465$

$P 0012470$

$P 0012475$

$P 0012480$

P $110 \mathrm{~A} 1$

$P 110 A 2$

$P 0012485$

P1100150

INSERTED AFTER THE SPECIFICATIDN STATEMENTS DF SEGMENT 110.

P 1100160

$C * * * * *$

$C * * * * *$

BSFDF - $(005)$

P0050010

C******

P0050020

P0050030

$P 0050040$

$C * * * * * *$

\section{GENERAL PURPOSE}

$C * * * * *$

DEFINING STATEMENT FUNCTIONS THAT ARE TO BE TESTED

$C * * * * *$ IN SEGMENT 110 (BASIC FORTRAN) AND 111 (FULL FORTRAN)

$C * * * * *$ HEADER FOR SEGMENT 005

$C * * * * *$ DEFINING EXPRESSION CONTAINS CONSTANTS AND VARIABLES

CMAFS (CAWVS, CBWVS) = CAWVS *2.+ CBWVS

CMBFS(MAWVI, MBWVI, MCWVI) $=($ MAWVI + MBWVI + MCWVI) $/ 3$

MCAFI (MAWVI, MBWVI) = MAWVI * MBWVI

$M C B F I$ (CAWVS, CBWVS, CCWVS ) $=$ (CAWVS + CBWVS + CCWVS $) * 2.0$

ASA REF POOSO

$P 0050070$

8.1.1.1P0050080

P0050090

P0050100

$P 0050110$

$P 0050120$

P0050130

$P 0050140$

C***** DEFINING EXPRESSION CONTAINS CONSTANTS, VARIABLES AND

$C * * * * * \quad$ INTRINSIC FUNCTIONS

CMCFS (CAWVS, CBWVS, CCWVS ) = ABS(CAWVS**2-(CBWVS+CCWVS)**2)

CMDFS(MAWVI, MBWVI) = ISIGN((MAWVI + MBWVI),(MAWVI-MBWVI))

$P 0050150$

$P 0050160$

$P 0050170$

P0050180

$M C C F I(M A W V I, M B W V I, C A W V S)=M A W V I * * 2+M B W V I * 2+I F I X(C A W V S) * 2$

MCDFI (CAWVS, CBWVS, CCWVS, CDWVS, CEWVS $)=($ CAWVS + CBWVS + CCWVS +

1 CDWVS + CEWVS) ** (ABS(CAWVS))

$C * * * * *$ DEFINING EXPRESSION CONTAINS PREVIOUSLY DEFINED STATEMENT

$C * * * *$ FUNCTIONS ANDIOR EXTERNAL FUNCTION REFERENCES

CMEFS(CAWVS, CBWVS $)=\operatorname{CMBFS}(1,2,3)+\operatorname{SORT}(($ CAWVS + CBWVS $))$

CMFFS(MAWVI, MBWVI, MCWVI) = MCCFI(MAWVI,MBWVI,3.0) + MCWVI**2

MCEFI (MAWVI, MBWVI) = MCAFI(MAWVI,MBWVI) * MCAFI(MAWVI, MBWVI)

$P 0050190$

$P 0050200$

$P 0050210$

$P 0050220$

$P 0050230$

$P 0050240$

$P 0050250$

$P 0050260$

MCFFI (CAWVS, CBWVS, CCWVS) = SQRT(CAWVS) + SORT(CBWVS) + EXP(CCWVS) P0050270

$C * * * *$ DEFINING EXPRESSION CONTAINS CONSTANTS, VARIABLES, INTRINSIC..

$C * * * * *$ OR EXTERNAL FUNCTION REFERENCES AND PREVIDUSLY DEFINED

$C * * * *$ STATEMENT FUNCTIDNS.

$P 0050280$

$P 0050290$

$P 0050300$

CMGFS (MAWVI, MBWVI, CAWVS, CBWVS) = FLDAT(MAWVI* *2) - CMAFS(CAWVS, P0050310

1 CBWVS + SQRT( (FLOAT (MAWVI + MBWVI)))

P 0050320 
MCGF!(MAWV!,MBWV!,MCWV!,CAWVS) = MCEF!(MAWV!,MBWV!) - MCEFI(MAWV!,P0050330 $1 M(W V !)+I F ! X(E X P(C A W V S))$

$C * * * * *$ END OF TEST SEGMENT 005

$P 0050340$

$P 0050350$

$C * * * * *$ O UT T P T T T A P E ASSIGNMENT STATEMENT NO INPIT TAPE

P 1100180

$P 1100190$

P 0072190

C***** WHEN EXECUTING ONLY SEGMENT 110 , THE FOLLOWING STATEMENT

$C * * * * *$ NUVI $=6$ MUST HAVE THE $c=$ IN COLUMNS 1 AND 2 REMOVED.

$C=N$ NUVI $=6$

$C * * * * *$ NUVI $=6$

$P 0072195$

$P 0072200$

$P 0072205$

P110B

$P 0072210$

WRITE (NUVI, 1100)

P 1100200

1100 FORMAT (39H1 BSFTS - (110) STATEMENT FUNCTION TEST/23X,16HINTEGER AP 1100210 IND REAL//18H ASA REF. - 8.1.2/1 9H RESULTS)

$C * * * * *$ HEADER FOR SEGMENT 110 WRITTEN

CMAVS $=9.0-\operatorname{CMAFS}(2.0,3.0+2.0)$

CMBVS $=$ CMBFS $(2 / 2,1+1,1 * 3)-2.0$

MCAVI $=\operatorname{MCAFI}(I F I X(5.0), 5)-(5 * 5)$

$M C B V I=M C B F I(1.0,2.0,3.0)-\operatorname{MCAFI}(6,2)+24$

WRITE (NUVI, 1108) CMAVS, CMBVS, MCAVI, MCBVI

CMAVS $=\operatorname{CMCFS}(4.0,2.0,2.0)$

CMBVS $=\operatorname{CMDFS}(-1,-4)-5.0$

MCAVI $=\operatorname{MCCFI}(9 * 2 / 18,(4 * 2) / 8,3.0)-14$

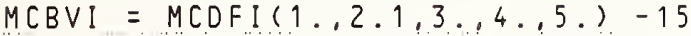

WRITE (NUVI, 1108) CMAVS, CMBVS, MCAVI, MCBVI

CMAVS $=\operatorname{CMEFS}(2.0 .1 .0 * 2.0)-4.0$

MCAVI $=3$

CMBVS $=$ CMFFS $(1,2$, MCAVI $)-23$.

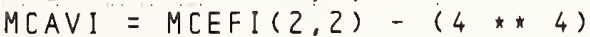

MCBVI $=$ MCFFI $(9.0,4.0$, CMBVS *CMBVS *0.0) - 6

WRITE (NUVI, 1108) CMAVS, CMBVS, MCAVI, MCBVI

CMAVS $=$ CMGFS $(3,13,2.0,5.0)-4.0$

CMBVS $=$ CMGFS(IFIX(SORT(CMAFS $(2,5))$.$) , IFIX(CMFFS (1,2,3)-10$.$) ,$

$1 \operatorname{CMBFS}(1,2,3), \operatorname{CMDFS}(-1,-4))-4.0$

$M C A V I=M C G F I(2,2,2,0,0)-1$

MCBVI $=\operatorname{MCGFI(MCAFI}(2,1), \operatorname{MCBFI}(1,0,0 \ldots 0), \operatorname{IFIXCSQRTCCMGFS}(3,13$,

$12.0,5.0))), \operatorname{EXP}(0.0)-1.0)-1$

WRITE (NUVI, 1108) CMAVS, CMBVS; MCAVI, MCBVI

WRITE (NUVI,1109)

1108 FORMAT $(12($ F $20.10 /), 2(119 /))$

1109 FORMAT ( $136 \mathrm{H}$ ALL ABOVE ANSWERS SHOULD BE O FOR I

137 H THIS TEST SEGMENT TO BE SUCCESSFUL.)

C***** END OF TEST SEGMENT 110

$C * * * * *$ WHEN EXECUTING ONLY SEGMENT 110, THE STOP AND END CARDS

$C * * * *$ WHICH APPEAR AS COMMENT CARDS MUST HAVE THE C

$C * * * * *$ IN COLUMNS 1 AND 2 REMOVED.

$C=S T O P$

$C=E N D$

STOP

END

P 1100220

P 1100230

$P 1100240$

P 1100250

P 1100260

P 1100270

P1100280

P1100290

P 1100300

P 1100310

P 1100320

P 1100330

P1100340

P 1100350

P 1100360

P 1100370

P1100380

P 1100390

P1100400

P 1100410

P 1100420

P 1100430

P11 100440

P1100450

P 1100460

P11.00470

P 1100480

P1100490

P1100500

P1100510

P1100520

P11 100530

P1100540

P1100550

P1100560

P110C1

P $110 C 2$

C

$C * * * * *$

$C * * * * *$

FSFTS - (111)

$P 1110020$

P 1110030

P1110040

$C * * * *$ GENERAL PURPOSE

C***** TEST STATEMENT FUNCTIONS THAT HAVE BEEN DEFINED IN

$C * * * * *$ SEGMENT 006 (FOR FULL FORTRAN TEST ONLY)

ASA REF P1110060

GENERAL COMMENTS

INTRINSIC AND EXTERNAL FUNCTIONS ASSUMED WORKING
$C * * * * \ldots$ INTRINSIC AND BASIC EXTERNAL FUNCTIONS DECLARED IN

$C * * * *$ TYPE STATEMENT OF SAME TYPE AS TABLES 3 AND 4

$C * * * * * *$

8.1.2P1110070

$P 1110080$

P 1110090

P1110100

$C * * * *$ S P E C I F I C A T I O N S SEGMENT 111

$C * * * * *$

C**** WHEN EXECUTING ONLY SEGMENT 111 , THE SPECIFICATION STATEMENTS

C***** WHICH APPEAR AS COMMENT CARDS MUST HAVE THE C =

$[* * * *$ IN COLUMNS 1 AND 2 REMOVED.

10.1 .7 P 1110110

5.3 P 1110120

P 1110130

P 1110140

$P 0012490$

P0012495

P0012500

P0012505 
$C=\quad$ DDUBLE PRECISIDN DPAFD,DPBFD,DPCFD,DPDFD,DPFFD,DPGFD,DPEFD,DPHFD

P0012515

$C=$ DDUBLE PRECISIDN DPAVD, DPBVD, DPCVD, DPDVD, DAWVD, DBWVD,DCWVD

P0O12520

DDUBLE PRECISIDN DPA1D(5),FC2D(5,5)

DOUBLE PRECISION DBLE, DEXP

CDMPLEX CMPLX, CEXP

CDMPLEX. CHAVC, CHBVC, CHCVC, CHDVC, CHEVC, CHFVC

CDMPLEX CHAFC, CHBFC, CHCFC, CHDFC, CAWVC, CBWVC

$P 0012525$

$P 0012530$

$P 0012535$

$P 0012540$

$P 0012545$

LDGICAL A3B $(2,2,2)$

LDGICAL MCFVB, MCHVB, ABFB, BCFB, IEFB, KLFB

$C=$

$P 0012550$

$P 0012555$

$P 0012560$

DOUBE

P $111 \mathrm{~A} 1$

DDUBLE PRECISIDN DPAFD, DPBFD,DPCFD,DPDFD,DPFFD, DPGFD,DPEFD,DPHFD DDUBLE PRECISIDN DPA1D(5), $F C 2 D(5,5)$

CDMPLEX CHAVC, CHBVC, CHCVC, CHDVC, CHEVC, CHFVC

CDMPLEX CHAFC, CHBFC, CHCFC, CHDFC, CAWVC, CBWVC

$\operatorname{LDGICAL~} A 3 B(2,2,2)$

$\angle D G I C A L$ MCFVB, MCHVB, $A B F B, B C F B$, IEFB, KLFB

- $, M C E \vee B, M C I \vee B, M C K \vee B, A T \vee B, A W \vee B, B W \vee B, C W \vee B, D W \vee B, E W \vee B, S W \vee B, T W \vee B$

CDMPLEX CMPLX, CEXP

DDUBLE PRECISIDN DBLE, DEXP

$[* * * * *$

C***** WHEN EXECUTING ONLY SEGMENT 111, THE SEGMENT 006 , WHICH

$C * * * *$ CONTAINS THE STATEMENT FUNCTIDNS BEING TESTED HERE MUST BE

$C * * * *$ INSERTED AFTER THE SPECIFICATIDN STATEMENTS DF SEGMENT 111.

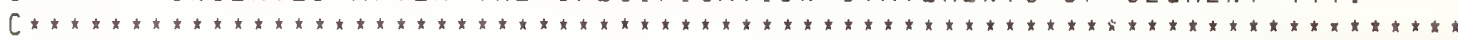

$[* * * * *$

$C * * * * *$

FSFDF - (006)

P111A2

P111A3

P111A4

P111A5

P111A6

P111A7

P111A8

P111A9

P111AA

$P 0012565$

$P 1110150$

P1110160

P1110170

$C * * * * *$

P0060010

$[* * * * * *$

$* * * * * * * * * * * * * * * * *$
GENERAL PURPDSE

$C * * * *$
$C * * * *$

\section{DEFIN ING STATE}

FSFOF - (006)

P0060020

$P 0060030$

$P 0060040$

$[ \pm t+t$

* IN SEGMENT 111 (FULL FDRTRAN STATEMENT FUNCTIDN TEST)

$C * * * *$ HEADER FDR SEGMENT 006

$C * * * *$ D.P. STATEMENT FUNCTIDNS CDNTAINING CDNSTANTS AND VARIABLES

DPAFD (DAWVD,DBWVD) $=($ DAWVD + DBWVD) **2

$D P B F D(D A W V D, D B W V D, D(W V D)=(D A W V D+D B W V D-D(W V D) * * 3$

$D P C F D(D A W V D, D B W V D, D C W V D)=3.0 D 0 *(D A W V D+D B W V D+D C W V D) / 2 . D 0$

$C * * * * \quad$ D.P. STATEMENT FUNCTIDNS CDNTAINING CDNSTANTS, VARIABLES

$C * * * * \quad$ AND INTRINSIC FUNCTIDN REFERENCES

DPDFD (DAWVD, DBWVD) = DSIGN(DAWVD, - (DBWVD))

DPEFD(DAWVD,DBWVD, CAWVC, CAWVS) = DBLE(CAWVS + AIMAG(CAWVC)

$1+$ DMAX1 (DAWVD,DBWVD + 1.D0)

C***** D.P. STATEMENT FUNCTIDNS CDNTAINING CDNSTANTS, VARIABLES.

C**** INTRINSIC FUNCTIDN AND PREVIDUSLY DEFINED STATEMENT FUNCTIDN

$C * * * * *$ REFERENCES

DPFFD (DAWVD, DBWVD, CAWVS) = DPAFD(DAWVD,DBWVD) - (2.DO* DAWVD*

1 DBWVD) + (DBLE(CAWVS)*2.DO)

$D P G F D(D A W V D, D B W V D, C A W V S, C A W V C)=D P B F D(D A W V D, D B W V D, D B L E(C A W V S))$

1 - DBLE(AIMAG(CAWVC)) + 5.000

C**** D.P. STATEMENT FUNCTIDNS CDNTAINING CDNSTANTS, VARIABLES,

$C * * * * *$ INTRINSIC FUNCTIDN, PREVIDUSLY DEFINED STATEMENT FUNCTIDN

$C * * * *$ AND EXTERNAL FUNCTIDN REFERENCES

1 DPHFD(DAWV, OBWVO, CAWVS) = DPFFO(DAWVD, DBWVD $\$ 1.00$

$C * * * * \quad$ CDMPLEX STATEMENT FUNCTIDNS CDNTAINING CDNSTANTS AND VARIABLESPOO60310

CHAFC(CAWVC, CBWVC) $=$ CAWVC $*(2.0,2.0)+C B W V C+(2.0 .2 .0) \quad P 0060320$

C***** COMPLEX STATEMENT FUNCTIDN CDNTAINING CDNSTANTS, VARIABLES,

$C * * * *$ AND INTRINSIC FUNCTIDN REFERENCES

CHBFC (CAWVC, CBWVC, CAWVS $=$ CAWVC - CBWVC + CMPLX (CAWVS, CAWVS)

$C * * * * \quad$ COMPLEX STATEMENT FUNCTIDN CDNTAINING CONSTANTS,

$C * * * * *$ VARIABLES, INTRINSIC AND EXTERNAL FUNCTIDN REFERENCES

CHCFC(CAWVC, CBWVC, CAWVS, CBWVS ) $=(C A W V C-C B W V C)+C E X P(C M P L X$

1. (CAWVS, CBWVS)) - CMPLX(CAWVS, CBWVS)

$C * * *$ CDMPLEX STATEMENT FUNCTIDN CDNTAINING CDNSTANTS, VARIABLES,

$C * * * *$ INTRINSIC, EXTERNAL AND PREVIDUSLY DEFINED STATEMENT FUNCTIDN

$[* * * * *$ REFERENCES

CHDFC(CAWVC, CBWVC, CAWVS, CBWVS) = CHCFC(CAWVC, CBWVC, CAWVS + CAWVS,

$P 0060330$

$P 0060340$

$P 0060350$

$P 0060360$

$P 0060370$

$P 0060380$

$P 0060390$

$P 0060400$

$P 0060410$

$P 0060420$

$P 0060430$ 
$12.0 *$ CBWVS $)+C M P L X(1.0 .2 .0)$

$C * * *$ STATEMENT FUNCTIDN CONTAINING LOGICAL VARIABLES

$A B F B(A W V B, B W V B, D W V B)=A W V B$. AND. BWVB.OR. FALSE. AND.DWVB

$C * * * *$ STATEMENT FUNCTIDN CONTAINING CONSTANTS, VARIABLES AND

$C * * *$ INTRINSIC FUNCTIDNS

$B C F B(E W V B, C W V B, B A W V S, B C W V S)=E W V B . A N D$. (BAWVS ABS(BCWVS). GT.

$10.5)$.AND . NOT . CWVB

$C * *$ STATEMENT FUNCTION CDNTAINING PREVIOUSLY DEFINED STATEMENT

$C * * *$ FUNCTION AND AN INTRINSIC FUNCTION REFERENCE

$I E F B(E W V B, A T V B, C W V B, B A W V S, B C W V S)=A T V B$. AND.EWVB. AND. CWVB,OR.

1 AMAX1 (BAWVS,BCWVS). GT.600. OR. BCFB (EWVB, CWVB,BAWVS, BCWVS)

$C * * * *$ STATEMENT FUNCTION CONTAINING BASIC EXTERNAL FUNCTION REFERENCE

$K L F B(S W V B, T W V B, A T V B, B A W V S)=$ SWVB. AND..NOT. TWVB.OR. (SQRT(BAWVS)

$1 . G T .9 .0) \quad . O R$. ATVB

$C * * * *$ END OF TEST SEGMENT 006

$C * * * * \pi$
$C * * * *$

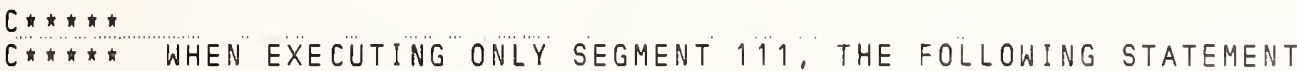

$C * * * *$ NUVI $=6$. MUST HAVE THE $C=I N$ COLUMNS 1 AND 2 REMOVED.

$C=N U V I=6$

$C * \cdots * \cdots$

$N \cup V I=6$

WRITE (NUVI, 1110)

1110 FDRMAT( $39 H 1$ FSFTS - (111) STATEMENT FUNCTION TEST/I

$139 \mathrm{H}$ DOUBLE PRECISION, COMPLEX ANO LOGICAL/I

$218 \mathrm{H}$ ASA REF. - $8.1 .21110 \mathrm{H}$ RESULTS)

$C * *$ HEADER FOR SEGMENT 111 WRITTEN

$C * * *$ CONSTANTS USED IN THIS SEGMENT

CHAVC $=(1.0,2.0)$

CHBVC $=(-2.0,3.0)$

DPATD (2) $=3.500$

$A T \ddot{B}=F \ddot{A L S E}$.

PPOVS $=18$

RRDVS $=21.0$

ATVS $=18.0$

MCFVB $=$. TRUEE.

$F C 2 D(2,2)=1.7500$

$C * * * *$ TEST OF D.P. STATEMENT FUNCTIONS

DPAVD = DPAFD $(3.500$, DPA1D(2) -49.000

DPBVD $=$ DPBFD $(1.00 .0 P A 10(2)-2.500 .0 B L E(1.0))-1.000$

$D P C V D=D P C F D(0.00,1.000,0 P A 10(2)+0.500)-7.500$

DPDVD = DPDFD(DBLE(AIMAG(CHAVC)), FC $20(2,2))+2.000$

WRITE (NUVI,1118) DPAVD, DPBVD, DPCVD, DPDVD

DPAVD = DPEFD (1.0DO, FC2D(2,2)*2.00,(1.0,-4.), $\operatorname{AMAX1}(2.0,4.0))$

$1-4.500$

$D P B V D=D P F F D(D P A 1 D(2), F(20(2,2)-1.7500,5.00)-22.2500$

$D P C V D=D P G F D(2 . D 0 / .201, D P A 1 D(2)-2.500,1.0$, CHAVC $)-4.000$

DPDVD $=$ DPHFD $(3.5 D 0, F C 2 D(2,2)-2.7500 .5 .0)-34.500$

WRITE (NUVI,1118) DPAVD, DPBVD, OPCVD, DPDVD

C* TEST OF COMPLEX STATEMENT FUNCTIONS

CHCVC $=$ CHAFC $((2.0,2)$, CHAVC $)-(3.0,12.0)$

CHDVC $=\operatorname{CHBFC}((4.0,-8.5), C H B V C, 1.0)-(7.0,-10.5)$

CHEVC $=$ CHCFC $((1.0,1.0) * 2, C H A V C, 0.000, A I M A G(C H A V C)-2.0)$

$P 0060440$

$P 0060450$

$P 0060460$

$P 0060470$

P0060480

P0060490

$P 0060500$

$P 0060510$

P0060520

P0060530

$P 0060540$

P0060550

P0060560

$P 0060570$

P0060580

$P 1110180$

$P 1110190$

$\mathrm{P} 0072220$

P0072225

P0072230

P0072235

$\mathrm{P} 111 \mathrm{~B} 1$

$P 0072240$

P1110200

P1110210

P1110220

P1110230

P1110240

P1110250

$P 1110260$

P1110270

P1110280

P1110290

P1110300

P1110310

P1110320

P 1110330

P1110340

P1110350

P 1110360

P1110370

$P 1110380$

P1110390

P1110400

P1110410

P1110420

$P 1110430$

P1110440

$P 1110450$

P1110460

P 1110470

P1110480

P1110490

$P 1110500$

CHFVC = CHDFC ( $(0.0,0.0)$,CHAVC, 0.000, SNGL (DMIN1(0.D0,4.00))P1110510

1) $-(1.0,0.0)$

WRITE (NUVI,1117) CHCVC, CHOVC, CHEVC, CHFVC

WRITE (NUVI, 1119)

C* TEST DF LDGICAL STATEMENT FUNCTION

MCEVB = PPDVS.GT. 60.0

$A 3 B(1,1,1)=A T V S . L E .20 .9$. AND. ABFB (.TRUE...TRUE. .FALSE.)

$P 1110520$

$P 1110530$

P 1110540

P 1110550

P 1110560

P 1110570

MCHVB = BCFB(.TRUE...FALSE..PPDVS,21.0).AND..NOT.PPDVS.GE.RRDVS P1110580

$M C I V B=$.NDT. (IEFB(.FALSE. ATVB, TRUE.650., -5.11). AND.ATVB)

$M C K V B=M C F V B . A N D . K L F B(. T R U E \ldots$ TRUE. . TRUE.,100.). AND..NOT.MCEVB

WRITE (NUVI,1116) A3B(1,1,1), MCHVB, MCIVB, MCKVB

1116 FDRMAT(//4(L4)//38H THE FDUR ABDVE ANSWERS SHOULD BE TRUE/

$135 H$ FOR THIS SEGMENT TD BE SUCCESSFUL)

P 1110590

P 1110600

P 1110610

P1110620

P1110630

P 1110640

1117 FORMAT(I $4(\mathrm{~F} 16.7, F 14.71))$ 
1119 FORMAT ( / $40 H$ ALL ABOVE ANSWERS SHOULD BE O FOR THIS/

P 1110660

$140 \mathrm{H}$ TEST SEGMENT TO BE SUCCESSFUL. VALUES $140 \mathrm{H}$ WITH EXPONENTS LEP 1110670

2SS THAN $10 *(-14) 122 \mathrm{H}$ ARE CONSIDERED ZERO)

\section{C**** END OF TEST SEGMENT 111}

C**** WHEN EXECUTING ONLY SEGMENT 111, THE STOP AND END CARDS

P1110680

$C * * *$ WHICH APPEAR AS COMMENT CAROS MUST HAVE THE C $=$

$C * * * * *$ IN COLUMNS 1 AND 2 REMOVED.

$C=\quad$ STOP

$C=\quad$ END

STOP

$P 1110690$

P1110700

P 1110710

P 1110720

P1110730

P11 10740

$$
\text { END }
$$

$P 111 C 1$

$P 111 C 2$

C

$C * * * * *$

C*****

CPXAD - (140)

P 1400020

1400030

$C * * * * *$

P 1400040

$C * * * * *$

GENERAL PURPOSE

C***** TO TEST ADDITION AND SUBTRACTION OF COMPLEX NUMBERS

C***** INCLUDES OPERATIONS WITH UP TO 9 TERMS

C***** DOES NOT TEST FOR ACCURACY

C*****

$C * * * *$ ADOITION AND SUBTRACTION OF 2 TERMS

$C * * * * *$

C**** S P E C I F I C A T I O N S SEGMEN̈T 140

C******W WHEN EXECUTING ONLY SEGMENT 140 , THE SPECIFICATION STATEMENTS

$C * * * *$ WHICH APPEAR AS COMMENT CARDS MUST HAVE THE $C=$

C*****IN COLUMNS 1 AND 2 REMOVED.

C*****

$C=\quad$ COMPLEX $A V C, B V C, C V C, D V C, E V C, F V C, G V C, H V C, I V C, J V C, A A V C$,

$C=\quad 1 \quad A B V C, B A V C, B B V C, C C V C, C D V C, B C V C, D C V C$

COMPLEX AVC, BVC, CVC, DVC, EVC, FVC, GVC, HVC, IVC, JVC, $A A V C$,

$1 A B V C, B A V C, B B V C, C C V C, C D V C, B C V C, D C V C$

$C * * * * *$

C*\#** OU U P U T T A P E ASSIGNMENT STATEMENT. NO INPUT TAPE.

C*\#* WHEN EXECUTING ONLY SEGMENT 140, THE FOLLOWING STATEMENT

$C * * * *$ NUVI $=6$ MUST HAVE THE $C=$ IN COLUMNS 1 AND 2 REMOVED.

$C * * * * *$

$C=\quad$ NUVI $=6$

NUVI $=6$

WRITE (NUVI, 1401)

1401 FORMAT(1H1,1X,34HCPXAD - (140) COMPLEX ADOITION AND/16X,

111 HSUBTRACTION//2X,14HASA REF - $6.1 / / 2 X, 7$ HRESULTS//)

$A \vee C=(1.467,2.560)$

$B V C=(3.568,7.480)$

$C \vee C=A \vee C+B \vee C$

$D \vee C=A V C+(3.568,7.480)$

$E V C=(1.9467 .2 .9560)+B V C$

$F \vee C=(1.467,2.560)+(3.568 .7 .480)$

$G \vee C=A \vee C-B \vee C$

$H V C=(.1467 E+1, .2560 E 1)-B V C$

$I V C=A V C-(3568 E-3, .7480 E+1)$

$J V C=(1.467 .2 .560)-(3.568 .7 .480)$

$C * * * *$ ADDITION AND SUBTRACTION OF 3 TERMS

$A A V C=A \vee C+B \vee C-C V C$

$A B \vee C=A \vee C+(3.568,7.480)-D V C$

$B A V C=(1.467 .2 .560)+B V C-C V C$

$B B V C=(1.467 .2 .560)+(3.568 .7 .480)-F V C$

$B C \vee C=A \vee C-B \vee C-G \vee C$

$C C V C=(1.467 .2 .560)-B V C-H V C$

$C D V C=A \vee C-(3.568,7.480)-I V C$

$D C V C=(1.467,2.560)-(3.568,7.480)-J V C$

WRITE(NUVI, 1402) AAVC, ABVC,BAVC,BBVC,BCVC, CCVC, CDVC,DCVC

$C * * * * A D D I T I O N$ AND SUBTRACTION OF 5 TERMS

$A A V C=A V C-(1.89 .6 .48)-A A V C-B V C+(0.0,9.830)$

$A B \vee C=A \vee C-(1.89,6.48)-A A \vee C-B \vee C+(0.0 .9 .830)$

WRITE(NUVI, 1402 ) ABVC

P 1400060

ASA REFP 1400070

6. $1 P 1400080$

P 1400090

$P 1400100$

P 1400110

$P 1400120$

P1400130

P0012570

P0012575

P0012580

P0012585

P0012590

P0012595

P0012600

P140A1

P140A2

P0012605

P 1400140

P0072250

$P 0072255$

P0072260

P0072265

P $140 B 1$

P 1400150

P 1400160

P 1400170

$P 1400180$

P1400190

P1400200

P 1400210

P 1400220

P1400230

P 1400240

P1400250

P 1400260

P1400270

P 1400280

P 1400290

P 1400300

$P 1400310$

P 1400320

P 1400330

$P 1400340$

P 1400350

P1400360

P 1400370

P 1400380

P1400390

P 1400400

P1400410 
WRITE (NUVI,1403) ABVC

$P 1400490$

$P 1400500$

$P 1400510$

1403 FORMAT $(2 X, 2 F 8.4 / / 2 X, 35 H T E S T$ IS POSITIVE IF NUMBERS PRINTEO/2X

$P 1400520$ 117 HABOVE ARE $0.0,0.0)$

\section{$C * * * * *$ ENO OF TEST SEGMENT 140}

$C * * * * *$ WHEN EXECUTING ONLY SEGMENT 140, THE STOP AND ENO CAROS

P 1400530

P 1400540

$C * * * *$ WHICH APPEAR AS COMMENT CARDS MUST HAVE THE $c=$

$C * * * * *$ IN COLUMNS 1 ANO 2 REMOVED.

$C=\quad S T O P$

$C=\quad E N D$

STOP

$P 1400550$

$P 1400560$

$P 1400570$

P1400580

$P 1400590$

ENO

P140C

P140C2

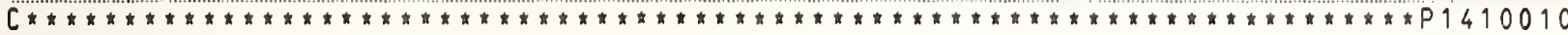

$C * * * * *$

$P 1410020$

$C * \hbar \star \star * * 10$

CPXMU $-(141)$

$P 1410030$

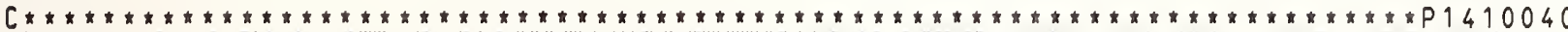

C***** GENERAL PURPOSE

$C * * * * *$ TO TEST MULTIPLICATION OF COMPLEX NUMBERS

$C * * * * *$ INCLUDES OPERATIONS WITH UP TO 10 TERMS

$P 1410050$

$C * * * * *$ DOES NOT TEST FOR ACCURACY ASA REFP 1410060

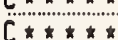

$6.1 \quad P 1410070$

$C * * * * *$

$C * * * * *$ S P E C I FI C A T I O N S SEGMENT 141

1410080

$P 1410090$

$P 1410100$

$C * * * * *$

$C * * * * *$ WHEN EXECUTING ONLY SEGMENT 141 , THE SPECIFICATION STATEMENTS

P1410110

$C * * * * *$ WHICH APPEAR AS COMMENTS MUST HAVE THE $C=$

$C * * * * *$ IN COLUMNS 1 AND 2 REMOVED.

$C * * * * *$

$C=$ COMPLEX AVC, BVC, CVC, DVC, EVC, FVC, GVC, HVC, IVC, JVC

$P 0012610$

$P 0012615$

$P 0012620$

P0012625

P0012630

$P 0012635$

$C=1 \quad A A V C, A B V C, B A V C, B B V C$

COMPLEX AVC, BVC, CVC, DVC, EVC, FVC, GVC, HVC, IVC, JVC

$P 0012640$

$1, A A \vee C, A B \vee C, B A V C, B B V C$

$C * * * *$

$C * * * * * 0$ U T 0 U T T A P E ASSIGNMENT STATEMENT. NO INPUT TAPE.

P141A1

P141A2

P0012645

$P 1410120$

$C * * * * *$

$C * * * * *$

WHEN EXECUTING ONLY SEGMENT 141, THE FOLLOWING STATEMENT

$P 0072270$

$C * * * * *$ NUVI $=6$ MUST HAVE THE $C=$ IN COLUMNS 1 ANO 2 REMOVEO.

$C * * * * *$

$C=N U V I=6$

$C * * * * *$

NUVI $=6$

P0072275

$P 0072280$

$P 0072285$

P 0072290

P $141 B 1$

WRITE (NUVI, 1411)

1411 FORMAT ( 1 H1,1 X,36HCPXMU - $(141)$ COMPLEX MULTIPLICATION//2X,

114HASA REF. - $6.1 / / 2 X, 7$ HRESULTS//1)

$C * * * * *$ MULTIPLICATION OF TWO TERMS

$A V C=(-0.5,0.86602)$

$B \vee C=(-0.5,-0.86602)$

$A A \vee C=(A \vee C * B \vee C)$

$A B \vee C=A \vee C *(-0.5,-0.86602)$

$B A V C=(-0.5,0.86602) * B V C$

$B B V C=(-0.5,0.86602) *(-0.5,-0.86602)$

WRITE (NUVI, 1412) AAVC, ABVC, BAVC, BBVC

$C * * * *$ MULTIPLICATION OF 3 TERMS

$A \vee C=(0.0,1.0)$

$B \vee C=(1.0,0.0)$

$C V C=(0.0,-1.0)$

P 0072295

$P 1410130$

$P 1410140$

$P 1410150$

$P 1410160$

$P 1410170$

$P 1410180$

P 1410190

$P 1410200$

P 1410210

$P 1410220$

$P 1410230$

P1410240

$P 1410250$

$P 1410260$

P 1410270

$A A \vee C=A \vee C * B \vee C * C \vee C$

$A B \vee C=(0.0,1.0) * B V C *(0.0,-1.0)$

WRITE (NUVI,1412) AAVC, ABVC

P 1410280

P 1410290

$P 1410300$

1412 FORMAT $(2 \times, 2 F 8.3)$

P 1410310 
$A A \vee C=A \vee C * B \vee C * C V C * D V C$

$P 1410370$

$A B \vee C=A \vee C *(-0.80901,0.58778) * C \vee C *(0.30901,-0.95105)$

P 1410380

WRITE(NUVI, 1412) AAVC, ABVC

$P 1410390$

$C * * *$ MULTIPLICATION OF 5 TERMS

P 1410400

$A V C=(0.5,0.86602)$

P 1410410

$B \vee C=(-0.5,0.86602)$

P 1410420

$C V C=(1.0,0.0)$

P 1410430

$D V C=(-0.5,-0.86602)$

P 1410440

$E V C=(0.5,-0.86602)$

P 1410450

$A A \vee C=A \vee C * B \vee C * C V C * D \vee C * E V C$

P1410460

$A B \vee C=A V C *(-0.5,0.86602) * C V C *(-0.5,-0.86602) * E V C$

P 1410470

WRITE(NUVI, 1412) AAVC, ABVC

$C * * * *$ MULTIPLICATION OF 6 TERMS

P 1410480

$A \vee C=(0.98480,0.17364)$

P 1410490

$B V C=(-0.17364,0.98480)$

P 1410500

$C V C=(-0.86602,0.5)$

$P 1410510$

$D V C=(-0.93969,-0.34202)$

P 1410520

P 1410530

$E V C=(0.34202,-0.93969)$

P 1410540

$F \vee C=(0.86602,-0.5)$

$A A \vee C=A \vee C * B \vee C * C \vee C * D V C * E V C * F \vee C$

$P 1410550$

$A B V C=A V C *(-0.17364,0.98480) * C V C *(-0.93969,-0.34202) * E V C *(0.86602$, $1-0.5)$

WRITE(NUVI,1412) AAVC, ABVC

P 1410560

$P 1410570$

P 1410580

P 1410590

$P 1410600$

P 1410610

$A \vee C=(0.70710,0.70710)$

$B \vee C=(0.0,1.0)$

$P 1410620$

$C V C=(-0.70710,0.70710)$

$P 1410630$

$D V C=(1.0,0.0)$

P 1410640

$E \vee C=(-0.70710,-0.70710)$

P 1410650

$F \vee C=(0.0,-1.0)$

P 1410660

$G \vee C=(0.70710,-0.70710)$

$A A \vee C=A \vee C * B \vee C * C \vee C * D V C * E \vee C * F \vee C * G \vee C$

$A B \vee C=A \vee C *(0.0,1.0) * C V C *(1,0,0.0) * E V C *(0.0,-1.0) * G \vee C$

$P 1410670$

$P 1410680$

WRITE(NUVI, 1412) AAVC, ABVC

$C * * * *$ MULTIPLICATION OF 8 TERMS

P1410690

P 1410700

P 1410710

$A \vee C=(0.76604,0.64278)$

P 1410720

$B V C=(0.17364,0.98480)$

P 1410730

$C V C=(-0.5,0.86602)$

$D V C=(-0.93969,0.34202)$

$P 1410740$

$E V C=(-0.93969,-0.34202)$

P 1410750

P 1410760

$F V C=(-0.5,-0.86602)$

P 1410770

$G \vee C=(0.17364,-0.98480)$

P 1410780

$H V C=(0.76604,-0.64278)$

P 1410790

$A A V C=A V C * B \vee C * C V C * D V C * E V C * F \vee C * G \vee C * H \vee C$

P 1410800

$A B \vee C=A V C *(0.17364,0.98480) * C V C * D V C *(-0.93969,-0.34202) * F V C * G \vee C * H V C P 1410810$

WRITE(NUVI, 1412) AAVC, ABVC

C*****MULTIPLICATION OF 9 TERMS

P 1410820

P 1410830

$A V C=(0.80901,0.58778)$

$B V C=(0.30901,0.95105)$

P 1410840

$C V C=(-0.94832,0.31730)$

P1410850

P1410860

$D V C=(-0.80901,0.58778)$

P 1410870

$E V C=(1.0,0.0)$

P 1410880

$F \vee C=(-0.80901,-0.58778)$

P1410890

$G \vee C=(-0.94832,-0.31730)$

P 1410900

$H V C=(0.30901,-0.95105)$

P 1410910

IVC $=(0.80901,-0.58778)$

$P 1410920$

$A A V C=A \vee C * B \vee C * C V C * D V C * E \vee C * F \vee C * G \vee C * H \vee C * I V C$

P 1410930

$A B V C=A V C *(0.30901,0.95105) * C V C *(-0.80901,0.58778) *(1.0,0.0) * F V C * P 1410940$

$1 G V C * H V C * I V C$

WRITE(NUVI, 1412) AAVC, ABVC

$C * * * *$ MULTIPLICATION OF 10 TERMS

$P 1410950$

$P 1410960$

P 1410970

$P 1410980$

$A \vee C=(0.86602,0.5)$

P 1410990 
$C V C=(0.0,1.0)$

$P 1411000$

$D \vee C=(-0.5,0.866029)$

P 1411010

$E V C=(-0.86602,0.5)$

$F V C=(-1,0,0.0)$

$P 1411020$

$P 1411030$

$G V C=(-0.86602,-0.5)$

$P 1411040$

$H V C=(-0.5,-0.86602)$

IV $C=(0.0,-1.0)$

P 1411050

$J V C=(0.0,1.0)$

P 1411060

P 1411070

P 1411080

$A A V C=A V C * B V C * C V C * D V C * E V C * F V C * G V C * H V C * I V C * J V C$

P 1411090

$10) * J \vee C$

WRITE(NUVI, 1412) AAVC, ABVC

WRITE (NUVI, 1413)

1413 FORMAT ( 1 HO, 35 HTEST IS POSITIVE IF NUMBERS PRINTEO/1X,

117 HABOVE ARE $1.0,0.0)$

WRITE (NUVI, 1414)

1414 FORMAT (//39H ERROR SHOULD NOT EXCEED + OR - .001)

C ***** END OF TEST SEGMENT 141

$C * * * * *$ WHEN EXECUTING ONLY SEGMENT 141, THE STOP AND END CARDS

$C * * * *$ WHICH APPEAR AS COMMENT CARDS MUST HAVE THE C=

$C * * * * *$ IN COLUMNS 1 AND 2 REMOVED.

$C=S T O P$

$C=\quad$ END

STOP

P 1411100

P 1411110

$P 1411120$

$P 1411130$

$P 1411140$

P 1411150

$P 1411160$

P 1411170

P1411180

$P 1411190$

$P 1411200$

P 1411210

$P 1411220$

P $141 C 1$

END

$P 141 C 2$

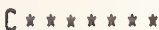

P1420010

$C * * * * *$

$P 1420020$

$C * * * * *$

CPXDV $-(142)$

P 1420030

P 1420040

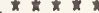

P 1420050

$C * * * *$

GENERAL PURPOSE

C******

$P 1420060$

$C * * * *$

TO TEST DIVISION OF COMPLEX NUMBERS

ASA REFP 1420070

$C * * * * *$

$C * * * * *$

$[* * * * *$

$C * * * * *$

$S P E C I F I C A T I O N S$ SEGMENT 142

6.1 P1420080

P 1420090

$P 1420100$

$P 0012650$

$P 0012655$

$P 0012660$

P 0012665

$P 0012670$

$P 0012675$

P $142 \mathrm{~A} 1$

COMPLEX NUMVC, DENVC, OAVC, OBVC, OCVC, ODVC

P0012680

$C * * * * *$

$C * * * * 0 \cup U T 0 U T$ T A P E ASSIGNMENT STATEMENT. NO INPUT TAPE.

$P 1420110$

$P 0072300$

C**** WHEN EXECUTING ONLY SEGMENT 142 , THE FOLLOWING STATEMENT

C**** NUVI $=6$ MUST HAVE THE C $=$ IN COLUMNS 1 AND 2 REMOVED.

$C * * * * *$

$C=N U V I=6$

$C * * * * *$

NUVI $=6$

$P 0072305$

$P 0072310$

$P 0072315$

$P 0072320$

$P 142 B 1$

$P 0072325$

$P 1420120$

WRITE (NUVI, 1421)

1421 FORMAT (1H1,1X,25HCPXOV - (142) DIVISION OF/16X,

115 HCOMPLEX NUMBERS//15H ASA REF.- $6.1 / 12 X, 7 H R E S U L T S / 1)$

$P 1420130$

$P 1420140$

P 1420150

P 1420160

NUMVC $=(0.36602,1.36602)$

$D E N V C=(0.86602,0.5)$

$O A V C=N U M V C / D E N V C$

$Q B \vee C=(0.36602,1.3660) / D E N \vee C$

$O C V C=N U M V C /(0.86602,0.5)$

$O O V C=(0.36602,1.36602) /(0.86602,0.5)$

WRITE (NUVI, 1422) OAVC,OBVC,OCVC, OOVC

$P 1420170$

P 1420180

P 1420190

$P 1420200$

P 1420210

P 1420220

$C * * * *$ TEST NUMBER 2

NUMVC $=(0.0,1.41420)$

$D E N \vee C=(0.70710,0.70710)$

OAVC = NUMVC/DENVC

$O B \vee C=(0.0,1.41420) / D E N V C$

$O C V C=N U M V C /(0.70710,0.70710)$

$P 1420230$

P 1420240

$P 1420250$

P 1420260

P 1420270

P 1420280

NBS FORTRAN Test Programs Version 1 
$O D V C=(0.0 .1 .41420) /(0.70710,0.70710)$

WRITE (NUVI, 1422) OAVC, OBVC, OCVC, ODVC

P 1420290

$P 1420300$

1422 FORMAT $(2 \times, 2 F 8.4)$

$C * * * *$ TEST NUMBER 3

NUMVC $=(-0.36602,1.36602)$

P1420310

P 1420320

P 1420330

$D E N V C=(0.5,0.86602)$

P 1420340

OAVC $=$ NUMVC / DENVC

OBVC $=(-0.36602,1.36602) / D E N V C$

P 1420350

$Q C V C=$ NUMVC $/(0.5,0.86602)$

P 1420360

ODVC $=(-0.36602,1.36602) /(0.5,0.86602)$

$P 1420370$

WRITE (NUVI, 1422) OAVC, OBVC,OCVC,ODVC

P1420380

P 1420390

P 1420400

C***EST NUMBER 4

NUMVC $=(0.73204,2.73204)$

$D E N V C=(1.73204,1.0)$

OAVC $=$ NUMVC / DENVC

$O B V C=(0.73204,2.73204) / D E N V C$

P 1420410

P 1420420

P 1420430

$O C V C=N U M V C /(1.73204,1.0)$

P1420440

ODVC $=(0.73204,2.73204) /(1.73204,1.0)$

P1420450

WRITE(NUVI, 1422) OAVC,OBVC,OCVC,ODVC

P 1420460

P 1420470

$C * * * *$ TEST NUMBER 5

$N U M V C=(0.0,2.82840)$

$D E N V C=(1.41420,1.41420)$

$O A V C=N U M V C / D E N V C$

$O B \vee C=(0.0 .2 .82840) / D E N V C$

$O C V C=N U M V C /(1.41420,1.41420)$

$O D V C=(0.0,2.82840) /(1.41420,1.41420)$

P 1420480

P 1420490

P 1420500

P 1420510

P 1420520

P 1420530

P 1420540

WRITE(NUVI, 1422) OAVC, OBVC, OCVC,ODVC

P 1420550

WRITE (NUVI, 1423)

P1420560

1423 FORMAT ( //2X,35HTEST IS POSITIVE IF NUMBERS PRINTEO/2X,

117 HABOVE ARE $1.0,1.0$ )

WRITE (NUVI, 1424)

1424 FORMAT (//39H ERROR SHOULD NOT EXCEED + OR - .0001)

P1420570

P 1420580

P 1420590

P1420600

C**** END OF TEST SEGMENT 142

$C * * *$ WHEN EXECUTING ONLY SEGMENT 142, THE STOP AND END CARDS

$C * * * *$ WHICH APPEAR AS COMMENT CARDS MUST HAVE THE C $=$

$C * * * *$ IN COLUMNS 1 AND 2. REMOVEO.

$C=\operatorname{STOP}$

$C=\quad E N D$

STOP

END

P1420610

P 1420620

P1420630

P 1420640

P 1420650

P 1420660

P $142 \mathrm{C} 1$

$P 142 C 2$

C

C*****

$C * * * * *$

CPXEX(143)

P 1430020

P 1430030

$[* * * * *$

P1430040

$C * * * * *$ GENERAL PURPOSE

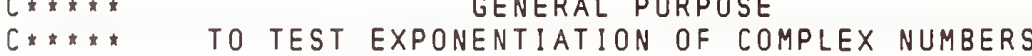

$C * * * *$ BY INTEGERS

$[* * * *$ EXPONENT VALUES VARY FROM 3 TO 100

$P 1430050$

P 1430060

$C * * * *$

$C * * * *$

S P E C I F I C A T I O N S SEGMENT 143

C***** WHEN EXECUTING ONLY SEGMENT 143, THE SPECIFICATION STATEMENTS

C*** WHICH APPEAR AS COMMENTS MUST HAVE THE C=

C****IN COLUMNS 1 AND 2 REMOVED.

$C=\ldots$ INTEGER $\frac{A V I}{C O M P L E X} \frac{A V C, B V C, C V C, D V C, E V C}{C O M}$

COMPLEX AVC,BVC,CVC, DVC,EVC

INTEGER AVI

$C * * * * 0$ U T 0 U T T A P E ASSIGNMENT STATEMENT. NO INPUT TAPE.

$C * * * * *$

$C * * * * *$

WHEN EXECUTING ONLY SEGMENT 143, THE FOLLOWING STATEMENT

$C * * * *$ NUVI $=6$ MUST HAVE THE $C=I N$ COLUMNS 1 AND 2 REMOVED.

$C * * * * *$

$C=\quad$ NUVI $=6$

$C * * * * *$

NUVI $=6$

ASA REFP 1430070

$6.1 P 1430080$

P 1430090

P 1430100

P 1430110

$P 0012690$

P 0012695

$P 0012700$

P0012705

P 0012710

P 0012715

P143A1

$P \uparrow 43 A Z^{20 .}$

P 1430120

P 0072330

P0072335

P 0072340

P0072345

P 0072350

P 14381

P0072355

WRITE (NUVI, 1431)

P1 430130 
1431 FORMAT $(1 H 1,1 \times, 36$ HCPXEX - (143) COMPLEX EXPONENTIATION $/ 1$ $12 X, 11 H A S A . R E F .6 .1 / 12 X, 29 H R E S U L T S$ BASED ON THE FUNCTION/I $22 X .25 H 1.0=\operatorname{SIN} * 2(X)+\operatorname{COS} * 2(X) / 1)$

$C * * *$ EXPONENT $=3$

$A \vee C=(-0.5,0.8660254)$

$A \vee I=3$

$B \vee C=A \vee C * * 3$

$C V C=(-0.5,0.8660254) * 3$

DVC $=(-0.5,0.8660254) * A V I$

$E \vee C=A \vee C * A \vee I$

WRITE (NUVI.1432) BVC,CVC,DVC,EVC

$C * * * *$ EXPONENT $=4$

$A \vee C=(0.0,1.0)$

$A \vee I=4$

$B \vee C=A \vee C * * 4$

$C V C=(0.0,1,0) * * 4$

$D \vee C=(0.0,1.0) * A V I$

$E \vee C=A \vee C * * A \vee I$

WRITE(NUVI,1432) BVC,CVC,OVC,EVC

1432 FORMAT $(2 \times, 2 F 8.4)$

$C * *$ EXPONENT $=6$

$A V C=(0.5,0.8660254)$

$A \vee I=6$

$B \vee C=A \vee C * * 6$

$C V C=(0.5,0.8660254) * 6$

$D V C=(0.5,0.8660254) * A V I$

$E V C=A \vee C * A \vee I$

WRITE (NUVI, 1432) BVC, CVC, DVC,EVC

$C * * *$ EXPONENT $=8$

$A V C=(0.7071068 .0 .7071068)$

$A \vee I=8$

$B \vee C=A \vee C * * 8$

$C V C=(0.7071068 .0 .7071068) * 8$

DVC $=(0.7071068 .0 .7071068) * A V I$

$E \vee C=A \vee C * A \vee I$

WRITE(NUVI, 1432) BVC,CVC, DVC, EVC

$C * * * * *$ EXPONENT $=10$

$A \vee C=(0.8090170 .0 .5877853)$

$A \vee I=10$

$B \vee C=A \vee C * 10$

$C V C=(0.8090170,0.5877853) * * 10$

DVC $=(0.8090170,0.5877853) * A V I$

$E V C=A \vee C * A V I$

WRITE (NUVI, 1432) BVC,CVC,DVC,EVC

$C * * * *$ EXPONENT $=20$

$A V C=(0.9510565 .0 .3090170)$

$A \vee I=20$

$B \vee C=A \vee C * 20$

$C V C=(0.9510565 .0 .3090170) * 20$

$D V C=(0.9510565 .0 .3090170) * A V I$

$E \vee C=A \vee C * A \vee I$

WRITE (NUVI, 1432) BVC, CVC, DVC, EVC

$C * *$ EXPONENT $=40$

$A \vee C=(0.9876883 .0 .1564345)$

$A V I=40$

$B \vee C=A \vee C * 40$

$C V C=(0.9876883 .0 .1564345) * 40$

DVC $=(0.9876883,0.1564345) * A V I$

$E \vee C=A \vee C * A V I$

WRITE(NUVI, 1432) BVC,CVC, DVC,EVC

$C * * *$ EXPONENT $=60$

$A V C=(0.9945219 .0 .1045285)$

$A V I=60$

$B \vee C=A \vee C * 60$

$C V C=(0.9945219 .0 .1045285) * 60$

$D V C=(0.9945219,0.1045285) *$ AVI

$E \vee C=A \vee C * A \vee I$

WRITE (NUVI, 1432) BVC,CVC, DVC,EVC
P 1430140

P1430150

P1430160

P1430170

P1430180

P1430190

P1 1430200

P 1430210

P1430220

$P 1430230$

P1430240

P1430250

$P 1430260$

P1430270

P1430280

P 1430290

P 1430300

$P 1430310$

P 1430320

P 1430330

$P 1430340$

$P 1430350$

$P 1430360$

P 1430370

P 1430380

P 1430390

P1430400

$P 1430410$

P 1430420

P 1430430

$P 1430440$

P 1430450

P 1430460

P1430470

P 1430480

P1430490

P 1430500

P 1430510

P1430520

P1430530

P 1430540

$P 1430550$

P1430560

P1430570

P1430580

P1 430590

$P 1430600$

P 1430610

P1430620

P1430630

P 1430640

P 1430650

P1430660

P 1430670

P 1430680

P1430690

P 1430700

P 1430710

$P 1430720$

P1430730

P 1430740

P 1430750

P 1430760

P1 1430770

P 1430780

P1430790

P1430800

P1 430810 
$C V C=(0.9969173,0.0784591) * * 80$

DVC $=(0.9969173,0.0784591) *$ * AVI

$E \vee C=A \vee C * * A \vee I$

WRITE(NUVI,1432) BVC, CVC, DVC,EVC

$C * * *$ EXPONENT $=100$

$A V C=(0.9980267,0.0627905)$

$A \vee I=100$

$B \vee C=A \vee C * * 100$

$C V C=(0.9980267 .0 .0627905) * * 100$

DVC $=(0.9980267,0.0627905) *$ AVI

$E \vee C=A \vee C * A V I$

WRITE(NUVI,1432) BVC, CVC, DVC, EVC

P 1430830

P 1430840

P 1430850

P1430860

P 1430870

P1 430880

$P 1430890$

P 1430900

P 1430910

P1430920

P 1430930

P 1430940

P 1430950

P 1430960

P 1430970

WRITE (NUVI,1433) $\quad$ P1430980

1433 FORMAT ( / / 37H TEST IS POSITIVE IF NUMBERS PRINTED/2X, P1430990

1 26HABOVE ARE CLOSE TO $1.0,0.0)$

WRITE (NUVI, 1434)

1434 FORMAT(// 39 H ERROR SHOULD NOT EXCEED + OR - .0001);

$[* * * *$ END OF TEST SEGMENT 143

C**** WHEN EXECUTING ONLY SEGMENT 143, THE STOP AND END CARDS

$C * * * *$ WHICH APPEAR AS COMMENT CARDS MUST HAVE THE C $=$

C*****IN COLUMNS 1 AND 2 REMOVEO.

$C=$ STOP

$C=\quad E N D$

STOP

P 1431000

P 1431010

P1431020

P 1431030

P 1431040

P1431050

P 1431060

P 1431070

P 1431080

P $143 C 1$

END

$P 143 C 2$

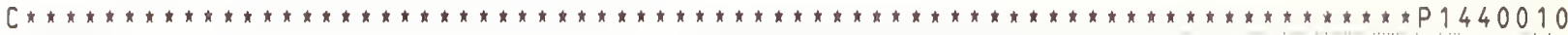

$C * * * *$

$C * * * *$

$C P X O P-(144)$

P 1440020

$C * * * * *$

P 1440030

$[* \star * \star * *$

$[* * * *$

C****t

C*****

C*****

C****

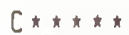

$[* * * *$

$C * * \star *$

$C * * * *$

$[* * * *$

C****

$C=\quad$ INTEGER AVI

P1440040

$C=\quad$ COMPLEX AVC, BVC, CVC, DVC, EVC, FVC, GVC,HVC,PVC, RVC, SVC, TVC,UVCPO012760

INTEGER AVI

GENERAL PURPOSE

ASA REF P 1440060

TO TEST ARITHMETIC OPERATIONS ON COMPLEX NUMBERS.

OPERATIONS INCLUDE ALL BASIC OPERATORS $(+,-, *, *)$ ACTING ON COMPLEX NUMBERS

6. 1

$P 1440070$

P 1440080

P 1440090

$P 1440100$

P 1440110

P 0012730

$P 0012735$

$P 0012740$

$P 0012745$

$P 0012750$

$P 0012755$

COMPLEX AVC, BVC, CVC, DVC, EVC, FVC, GVC,HVC,PVC, RVC, SVC, TVC, UVCP $144 A 2$

C*****

C****0 U T O U T T A P E ASSIGNMENT STATEMENT. NO INPUT TAPE.

$P 0012765$

$C * * * *$

P1440120

$P 0072360$

C**** WHEN EXECUTING ONLY SEGMENT 144, THE FOLLOWING STATEMENT

$C * * * *$ NUVI $=6$ MUST HAVE THE $C=$ IN COLUMNS 1 AND 2 REMOVED.

$C * * * * *$

$C=\quad$ NUVI $=6$

$C * * * * *$

NUVI $=6$

WRITE (NUVI, 1441)

1441 FORMAT $(1 H 1,1 \mathrm{X}, 32 \mathrm{HCPXOP}$ - (144) COMPLEX OPERATIONS//2X,

111 HASA REF $6.1 / / 2 \times$, 7HRESULTS/ $/$ )

$A V C=(0.9396926,0.3420201)$

$B V C=(1.2817127,0.5976725)$

$C V C=(0.0,1.4142136)$

$D V C=(0.7071068,0.7071068)$

$E V C=(1.0986841,0.4550899)$

$A V I=2$

$P 0072365$

$P 0072370$

P 0072375

$P 0072380$

P $144 B 1$

$P 0072385$

P 1440130

P 1440140

P 1440150

P 1440160

P 1440170

P1440180

P 1440190

P 1440200

P 1440210

$R \vee C=(A \vee C * B \vee C+(0.9396926,0.3420201) * B V C+A V C *(1.2817127 .0 .5976725)-P 1440220$ 
$1(0.9396926,0.3420201) *(1.2817127,0.5976725)+C V C / O V C+(0.0 .1 .4142136 P 1440230$ 2) $/ D V C+C V C /(0.7071068,0.7071068)-(0.0 .1 .4142136) /(0.7071068, \quad P 1440240$

$30.7071068)+E V C * * 2-E V C * * A V I+(1.0986841,0.4550899) * * 2+(1.0986841, \quad P 1440250$

$40.4550899) * A V I) * 2 /(0.0,72.0)$

$F \vee C=(0.0,4.0)$

$G \vee C=(0.43301,0.3)$

$H V C=(0.43301,0.2)$

$P V C=(1.73204,1.0)$

$S \vee C=F \vee C /((G \vee C+H \vee C) *(P \vee C * * 2))$

$T V C=(0.0,4.0) /(((0.43301,0.3)+(0.43301,0.2)) *((1.73204,1.0) * 2))$

$U V C=F \vee C /((G \vee C+(0.43301,0.2)) *(P V C * * 2))$

WRITE (NUVI,1442) RVC,SVC,TVC,UVC

P 1440260

P 1440270

P 1440280

P 1440290

P 1440300

P 1440310

P 1440320

P 1440330

P 1440340

1442 FORMAT $(4(2 \times, 2 F 8.4 /) / 37 H$ TEST IS POSITIVE IF NUMBERS PRINTED / P 1440350

$12 X, 17$ HABOVE ARE $1.0,0.0$ )

WRITE (NUVI, 1443)

1443 FORMAT(// 39H ERROR SHOULD NOT EXCEED + OR - .0001)

\section{$C * * * * *$ END OF TEST SEGMENT 144}

$C * * * *$ WHEN EXECUTING ONLY SEGMENT 144. THE STOP AND END CARDS

$C * * * * *$ WHICH APPEAR AS COMMENT CARDS MUST HAVE THE $c=$

$C * * * *$ IN COLUMNS 1 AND 2 REMOVED.

$C=\quad$ STOP

$C=E N D$

STOP

P 1440360

P1 1440370

P 1440380

P1 1440390

P1 1440400

P 1440410

P1 1440420

P 1440430

P 1440440

END

P1 144 C1

P144C2

C

$C * * * * *$

$C * * * * *$

CREAD-(145)

P 1450020

P 1450030

$[* * * * *$

P 1450040

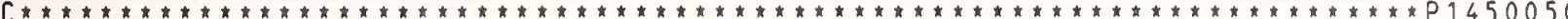

C***** GENERAL PURPOSE

$C * * * * *$ TO TEST ADDITION AND SUBTRACTION OF COMPLEX

$[* * * * *$ AND REAL NUMBERS

$C * * * * *$

$[\star \star \star \star * t$

$C * \star \star * *$

$C * * \star \star *$

C*****

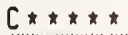

C*****

$c=$

$C=2$

S P E C I F I C A T I O N S SEGMENT 145

WHEN EXECUTING ONLY SEGMENT 145, THE SPECIFICATION STATEMENTS

WHICH APPEAR AS COMMENTS MUST HAVE THE C=

IN COLUMNS 1 AND 2 REMOVED.

COMPLEX AVC, BAVC, CAVC, DAVC, ASVC, BSVC, CSVC, AAVC

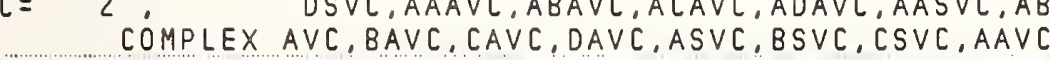

2 .

$D S \vee C, A A A \vee C, A B A \vee C, A C A \vee C, A D A \vee C, A A S \vee C, A B S \vee C, A C S \vee C, A D S \vee C$

$C * * * * *$

$[* * * * * 0$ U T 0 Ü T T A P E ASSIGNMENT STATEMENT. NO InPUT TAPE.

$C * * * * *$

$C * * * * *$

WHEN EXECUTING ONLY SEGMENT 145, THE FOLLOWING STATEMENT

$C * * * * *$ NUVI $=6$ MUST HAVE THE $C=$ IN COLUMNS 1 AND 2 REMOVED.

$C * * * * *$

$C=\quad N U V I=6$

$C * * * * *$

NUVI $=6$

WRITE (NUVI,1450)

1450 FORMAT ( 1 H $1.1 X, 38$ HCREAD - (145) ADDITION AND SUBTRACTION $/$

1 10X.27HOF COMPLEX AND REAL NUMBERS//2X。

1 12HASA REF. $6.1 / / 2 X$, 7HRESULTS//)

$A \vee C=(5.4,7.5)$

$A \vee S=4.2$

$C * * * *$ ADDITION AND SUBTRACTION OF 2 NUMBERS

$A A \vee C=A \vee C-A \vee S$

$B A \vee C=(5,4,7.5)-A V S$

$C A \vee C=A \vee C-4.2$

$D A \vee C=(5.4 .7 .5)-4.2$

$A S \vee C=A \vee C+A \vee S$

$B S V C=(5 \cdot 4,7.5)+A V S$

$C S \vee C=A \vee C+4.2$

$D S \vee C=(5.4 .7 .5)+4.2$

$C * * * \pi$ ADDITION AND SUBTRACTION OF 3 NUMBERS
ASA REF P1450060

$6.1 \quad P 1450070$

$P 1450080$

P 1450090

P 1450100

$P 0012770$

$P 0012775$

$P 0012780$

$P 0012785$

P0012790

POO 012795

P0012800

P 145A1

P1 $45 A 2$

P0012805

P 1450110

P 0072390

P0072395

P0072400

P 0072405

P 0072410

P145B1

P 0072415

P1450120

P1450130

P1 450140

P1450150

P1 1450160

P1450170

P1450180

P 1450190

P 1450200

P 1450210

P 1450220

P1 1450230

P1 450240

P 1450250

P 1450260

P1450270 
$A A A V C=A V C-A V S-A A V C$

$A B A V C=(5,4,7,5)-A V S-B A V C$

$P 1450280$

$A C A \vee C=A \vee C-4.2-(1.2,7.5)$

$P 1450290$

$A D A V C=(5,4,7,5)-4,2-(1,2,7,5)$

P 1450300

$A A S \vee C=A V C+A V S-A S \vee C$

$P\{450310$

$A B S \vee C=(5,4,7.5)+A V S-B S V C$

P 1450320

$A C S \vee C=A V C+4.2-(9.6,7.5)$

P1450330

$A D S V C=(5.4,7.5)+4.2-(9.6,7.5)$

P 1450340

WRITE (NUVI, 1451 ) ABAVC, ACAVC, ADAVC, AASVC, ABS VC, ACS C , ADSVC, AAAVC

1451 FORMAT $(2 X, 2 F 8,4)$

$C * * * *$ AOOITION AND SUBTRACTION OF 7 NUMBERS

P 1450360

P 1450370

$A D S V C=A V C-(5.4,7.5)+A V S-4.2+A S V C-3.2-(6,4,7.5)$

P1450380

WRITE (NUVI, 1452) AOSVC

1452 FORMAT (2X,2F8.4//37H TEST IS POSITIVE IF NUMBERS PRINTED/2X,

1 17HABOVE ARE $0.0,0.0$ )

P 1450390

P1 1450400

P 1450410

P1 1450420

P 1450430

$[* * *$ ENO OF TEST SEGMENT 145

$C * * * *$ WHEN EXECUTING ONLY SEGMENT 145. THE STOP AND END CARDS

$C * * * *$ WHICH APPEAR AS COMMENT CARDS MUST HAVE THE C $C=$

C*\#\#\#IN COLUMNS 1 AND" 2 REMOVED.

$C=S T O P$

$C=\quad$ END

STOP

P1 1450440

P1450450

P1 1450460

P1 1450470

P1 450480

ENO

P145C1

P1 $45 C 2$

$C * * * * *$
$C * * * *$
$C * \pi * *$

P1460010

CREMU - (146)

P1460020

P 1460030

$C * * * * *$

P 1460040

C $*$ *at? GENERAL PURPOSE

$C * * * *$ TO TEST MULTIPLICATION OF COMPLEX NUMBERS BY

$C * * * * *$ REAL NUMBERS

$[* * * * *$

$[* * * * *$

S P E C I F I C A T I O O N S SEGMENT 146

ASA REF P 1460060

$6.1 \quad P 1460070$

P1 460080

P 1460090

P 1460100

$[* * * *$

$[* * * * *$

WHEN EXECUTING ONLY SEGMENT 146. THE SPECIFICATION STATEMENTS

$C * * * *$ WHICH APPEAR AS COMMENTS MUST HAVE THE $C=$

C*\#\#:IN COLUMNS 1 AND 2 REMOVED.

P0012810

P 0012815

P 0012820

P 0012825

$P 0012830$

$\widetilde{C}=\quad C O M P L E X$ AVC,BVC, MAVC,MBVC,MCVC,MOVC

$P 0012835$

COMPLEX AVC,BVC,

$M A V C, M B V C, M C V C, M D V C$

P 146 A1

P 0012840

$C * * * *$

$C * * * * *$

$C * * * *$

$C * * * *$

O U T O U T T A P E ASSIGNMENT STATEMENT. NO INPUT TAPE.

$P 1460110$

$P 0072420$

$C * * * * *$

WHEN EXECUTING ONLY SEgMENT 146. THE FOLLOWING STATEMENT

P0072425

NUVI $=6$ MUST HAVE THE C $=$ IN COLUMNS 1 AND 2 REMOVED.

$C=\quad$ NUVI $=6$

$[* * * * *$

NUVI $=6$

$P 0072430$

P0072435

P 0072440

P 14681

P 0072445

WRITE (NUVI, 1461)

1461 FORMAT(1H1, IX,39HCREMU - (146) MULTIPLICATION OF COMPLEX/16X.

1 THBY REAL //2X,

2 11HASA.REF.6.1//2X,7HRESULTS//)

C****MULTIPLICATION OF A COMPLEX NUMBER BY A REAL NUMBER $A \vee C=(1.6,3.2)$

AVS $=0.625$

$M A \vee C=A \vee C * A \vee S$

$M B V C=(1.6,3.2) * A V S$

P1460120

P 1460130

P1460140

P 1460150

P1460160

P1460170

P 1460180

P 1460190

P1460200

P 1460210

$M C V C=A V C * 0.625$

$M D V C=(1.6,3.2) * 0.625$

P 1460220

P1460230

WRITE (NUVI, 1463) MAVC, MBVC,MCVC,MDVC

1463 FORMAT( $4(2 X, 2 F 8.4 /) / 137 \mathrm{H}$ TEST IS POSITIVE IF NUMBERS PRINTEDI, 2X,P1460240

417 HABOVE ARE 1.0.2.0)

P 1460250

P1460260

P1460270

AVS $=4.0$

P1460280

P 1460290

$A V C=(0.93969,0.34202)$

$B V C=(1.28168,0.59764)$

P1460300 
$M A V C=A V S * A V C * B V S * B \vee C$

MCVC $=4.0 * 8 V S *(0.93969 .0 .34202) * B V C$

MOVC $=4.0 * 0.25 *(0.93969,0.34202) *(1.28168,0.59764)$

WRITE (NUVI, 1462 ) MAVC,MBVC,MCVC,MDVC.

$P 1460330$

P 1460340

P 1460350

1462 FORMAT $(/ / 4(2 X, 2 F 8.4 /) / / 37 H$ TEST IS POSITIVE IF NUMBERS PRINTEO/

$12 \times .17$ HABDVE ARE 1.0 .1 .0$)$

WRITE (NUVI, 1464)

1464 FORMAT $/ / 39 H$ ERROR SHOULD NOT EXCEED + OR - .0001)

$C * * * * *$ ENO OF TEST SEGMENT 146

$C * * * *$ WHEN EXECUTING DNLY SEGMENT 146, THE STDP AND ENO CARDS

$C * * * * \quad W H I C H$ APPEAR AS CDMMENT CARDS MUST HAVE THE C

C***** IN COLUMNS 1 AND 2 REMOVEO.

$C=\operatorname{STDP}$

$C=\quad$ END

STDP

END

P1460360

P1460370

P1460380

P1460390

P 1460400

P 1460410

P 1460420

$P 1460430$

P 1460440

P1460450

P146C1

P $146 C 2$

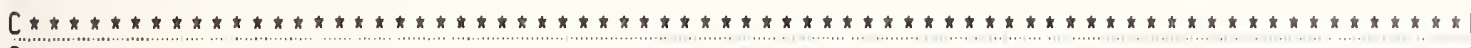

$C * * * * *$

$C * * * * *$

CREOV - $(147)$

$P 1470010$

$C * * * * *$

$P 1470020$

$P 1470030$

$P 1470040$

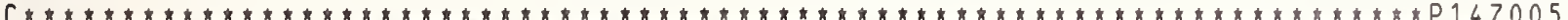

C***** GENERAL PURPDSE

$C * * * * *$ TD TEST DIVISIDN OF REAL (COMPLEX) NUMBERS BY

$C * * * *$ COMPLEX (REAL) NUMBERS

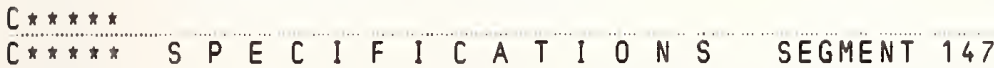

C******* WHEN EXECUTING ONLY SEGMENT 147, THE SPECIFICATIDN STATEMENTS

$C * * * *$ WHICH APPEAR AS COMMENTS MUST HAVE THE $C=$

C***** IN COLUMNS 1 ANO 2 REMOVEO.

$C * * * * *$

$C=\quad$ COMPLEX AVC, DAVC,OBVC,OCVC,OOVC

COMPLEX AVC, OAVC, OBVC, OCVC, OOVC

$C * * * * *$

$C * * * *$ OU T O U T T A P E ASSIGNMENT STATEMENT. NO INPUT TAPE.

$C * * * * *$

$C * * * *$

WHEN EXECUTING DNLY SEGMENT 147. THE FOLLOWING STATEMENT

$C * * * *$ NUV! $=6$ MUST HAVE THE $C=$ IN COLUMNS 1 AND 2 REMOVEO.

$C * * * * *$

$C=\quad N \cup V !=6$

NUVI $=6$

$C * * * * *$

WRITE (NUV!, 1471)

1471 FORMAT ( 1 H1, 1X,33HCREDV - (147) DIVISION DF CDMPLEX/16X, 16HANO REAP

1 L NUMBERS//2X,11HASA REF $6.1 / / 2 X, 7$ HRESULTS/ $/$ )

C***DIVISION OF REAL BY COMPLEX

$A \vee S=2.0$

$A \vee C=(1.0,-1.0)$

$D A \vee C=A \vee S / A V C$

$O B \vee C=2.0 / A \vee C$

$O C \vee C=A \vee S /(1.0,-1.0)$

DOVC $=2.01(1.0,-1.0)$

WRITE (NUVI, 1473) DAVC, DBVC, DCVC, DDVC

1473 FORMAT $(2 X, 2 F 8.4)$

$C * * * * *$ IVISION OF COMPLEX BY REAL

AVS $=2.5463$

$A V C=(2.5463,2.5463)$

$D A V C=A \vee C / A V S$

$D B V C=(2.5463 .2 .5463) / A V S$

$O C V C=A \vee C / 2.5463$

$O O V C=(2.5463 .2 .5463) / 2.5463$

WRITE (NUVI,1472) DAVC, DBVC, DCVC, DOVC

1472 FDRMAT $(4(2 X, 2 F 8.41) / 137 H$ TEST IS PDSITIVE IF NUMBERS PRINTEDI

$12 X .17$ HABOVE ARE 1.0.1.0)

WRITE (NUVI, 1474)

1474 FORMAT(// 39 H ERROR SHOULO NOT EXCEED + OR - .0001)

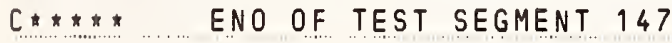

ASA REF $P 1470060$

6.1 P1470070

P 1470080

P 1470090

$P 1470100$

$P 0012850$

$P 0012855$

P0012860

P0012865

P0012870

P 0012875

P $147 \mathrm{~A} 1$

$P 0012880$

$P 1470110$

$P 0072450$

$P 0072455$

$P 0072460$

$P 0072465$

$P 0072470$

P147B1

P 0072475

$P 1470120$

$P 1470140$

$P 1470150$

$P 1470160$

$P 1470170$

P 1470180

$P 1470190$

$P 1470200$

P 1470210

P 1470220

P 1470230

$P 1470240$

P 1470250

P 1470260

P 1470270

P1470280

$P 1470290$

P 1470300

P 1470310

P 1470320

$P 1470330$

P 1470340

P 1470350

$P 1470360$ 
C* * WHEN EXECUTING ONLY SEGMENT 147. THE STOP AND END CARDS

$C * * * *$ WHICH APPEAR AS COMMENT CARDS MUST HAVE THE $c=$

$C * * * *$ IN COLUMNS I ANO 2 REMOVEO.

$C=S T O P$

$C=\quad$ END

P 1470380

$P 1470390$

P1 1470400

$P 1470410$

STOP

P147C1

END

P147C2

$[* * * *$

P1 1480010

C $* * * * *$

C $* * * * *$

$C * * * * *$

CREOP - (148)

P 1480020

P1480030

P1 480040

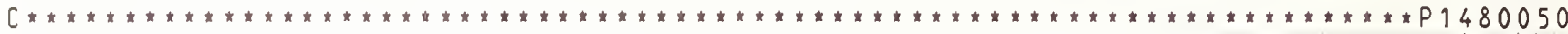

$C * * * * *$ GENERAL PURPOSE

$C * * * *$ TO TEST COMBINEO OPERATIONS ON COMPLEX AND REAL NUMBERS

$C * * * *$ OIVISION OF TWO POLYNOMIALS

ASA REF P1480060

$[* * * * *$

C**** S P E C I F I C A T I O N S SEGMENT 148

$6.1 P 1480070$

P 1480080

P 1480090

$P 1480100$

$P 0012890$

$C * * * * *$ WHEN EXECUTING ONLY SEGMENT 148, THE SPECIFICATION STATEMENTS

$[* * *$ WHICH APPEAR AS COMMENTS MUST HAVE THE C=

$P 0012895$

$[* * * *$ IN COLUMNS 1 AND 2 REMOVED.

$P 0012900$

$P 0012905$

$C * * * *$

$C=\quad$ INTEGER AVI

$C=\quad$ COMPLEX, $A V C, B V C, C V C, D V C, R V C$

INTEGER AVI

COMPLEX AVC, BVC, CVC, DVC, RVC

$[* * * * *$

[***:0 U T O U T T A P E ASSIGNMENT STATEMENT. NO INPUT. TAPE.

$C * * * * *$

$C * * * * *$

WHEN EXECUTING ONLY SEGMENT 148. THE FOLLOWING STATEMENT

$C * * *$ NUVI $=6$ MUST HAVE THE $C=$ IN COLUMNS 1 AND 2 REMOVED.

C*******1\%

$C=\quad$ NUVI $=6$

NUVI $=6$

$C * * * * *$

WRITE (NUVI, 1481)

$P 0012910$

P0012915

$P 0012920$

P $148 \mathrm{~A} 1$

P148A2

$P 0012925$

$P 1480110$

$P 0072480$

$P 0072485$

$P 0072490$

$P 0072495$

$P 0072500$

P148B1

$P 0072505$

$P 1480120$

1481 FORMAT(1H1,1X,36HCREOP - (148) OPERATIONS ON REAL AND/16X, 15HCOMPLP1480130

1EX NUMBERS// 2X,12HASA REF.6.1//2X,7HRESULTS//)

$A \vee C=(1.0,1.0)$

$A \vee S=1.0$

$B V S=2.0$

$B V C=(1.0,-1.0)$

$R \vee C=(B \vee S+A V C *(1 .+A V C *(-1 .+(1.0,1.0) *(-1 .+A \vee C)))))$

$1(4.0+B \vee C *(2.0+B \vee C *(-A V S+B V C *(0.5+B \vee C))))$

WRITE (NUVI, 1483) RVC

1483 FORMAT(

$2 \times, 2 F 8.4 / 137 \mathrm{H}$

3 NTED/2X, 18 HABOVE ARE $2.0,-1.0 / 1)$

P 1480140

P1480150

$P \uparrow 480160$

$P 1480170$

P 1480180

$P 1480190$

P 1480200

P 1480210

C****COMPLEX ARITHMETIC EXPRESSION

$A \vee C=(1.60 .3 .2)$

$A \vee S=0.625$

$B \vee S=2.0$

$B \vee C=(1.0,-1.0)$

$C \vee S=2.5$

$C V C=(2.5,2.5)$

$O V C=(1.09866,0.45508)$

$A \vee I=2$

$R \vee C=(A \vee C * A \vee S+(1.6,3.2) * A \vee S-A \vee C * 0.625-(1.6,3.2) * 0.625+B \vee S / B \vee C$

$1-B V S /(1.0,-1.0)+2.0 / B V C+2.0 /(1.0,-1.0)+C V C / C V S-(2.5,2.5) / C V S+$

$2 C V C / 2.5+(2.5 .2 .5) / 2.5+D V C * A V I-(1.09866,0.45508) * 2+D V C * 2+$

$3(1.09866,0.45508) * A V I) * 2 /(0.0,72.0)$

WRITE (NUVI, 1482) RVC

1482 FORMAT $(2 X, 2 F 8.4 / 137 H$ TEST IS POSITIVE IF NUMBERS PRINTED/2X,

1 17HABOVE ARE 1.0,0.0)

WRITE (NUVI, 1484)

1484 FORMAT (// $39 H$ ERROR SHOULO NOT EXCEEO + OR - .0001)

C***** ENO OF TEST SEGMENT 148

C*****WHEN EXECUTING ONLY SEGMENT 148, THE STOP ANO ENO CAROS

$C * * * *$ WHICH APPEAR AS COMMENT CAROS MUST HAVE THE C $=$

P 1480230

P 1480240

$P 1480250$

P 1480260

P 1480270

$P 1480280$

$P 1480290$

$P 1480300$

$P 1480310$

$P 1480320$

$P 1480330$

$P 1480340$

$P 1480350$

$P 1480360$

$P 1480370$

$P 1480380$

P 1480390

P1480400

$P 1480410$

P 1480420

P 1480430

P 1480440 


$\begin{array}{ll}C * * * * & \text { IN COLUMNS } 1 \text { AND } 2 \text { REMOVED. } \\ C= & \text { STOP } \\ C= & \text { END } \\ \text { STOP } & \text { END }\end{array}$

P 1480450

P 1480460

P1480470

P $148 C 1$

$P 148 C 2$

$[* * * * * *$

$C * * * * *$

MISC3 $-(149)$

$P 1490010$

C*****

P 1490030

P1490040

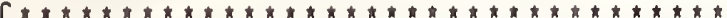

$C * * * * *$

$[* * * * *$

$C * * * * *$

$C * * * * *$

$[* * * * *$

$C * * * * *$

$C * * * * *$

$C * * * * *$

$C * * * *$

$[* * * * *$

C*******

$[\pi * * * *$

C*****

$C=$

$C=$

DIMENSION A1S(5), AZS $(2,2)$

GENERAL PURPOSE

TO TEST EFFECT OF BLANKS WITHIN STATEMENT,

CONTINUATION OF STATEMENT TO MAX.NO.OF LINES, AND USE OF SPECIAL CHARACTERS TO INDICATE CONTINUATION LINE -

FOR BASIC INTEGERS AND REAL NUMBERS

$S$ S E C I F I C A T I O N S SEGMENT 149

WHEN EXECUTING ONLY SEGMENT 149, THE SPECIFICATION STATEMENTS

WHICH APPEAR AS COMMENTS MUST HAVE THE C=

IN COLUMNS 1 AND 2 REMOVED.

INTEGER I11(5), I2I(2,2)

DIMENSION A1S(5),A2S $(2,2)$

INTEGER I11(5),121(2,2)

$C * * * *$

$C * * * *$

$C * * * * *$

$C * * * * *$

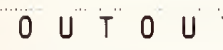

$\mathrm{T}$

A P

\section{A}

WHEN EXECUTIING "ONLY SEGMENT 149, THE FOLLOWING STATEMENT

$C * * * *$ NUVI $=6$ MUST HAVE THE $C=$ IN COLUMNS 1 AND 2 REMOVED.

$C * * * * *$

$C=$

$N \cup V I=6$

$C * * * * *$

NUVI $=6$

WRITE (NUVI.1490)

1490 FORMAT $(1 H 1.1 X .37 H M I S C 3$ - (149) EFFECT OF BLANKS WITHIN/16X, 122 HSTMNT ANO CONTINUATION $/ 16 \mathrm{X} .20 \mathrm{HOF}$ STMNT TO $20 \mathrm{LINES//}$ $239 \mathrm{H}$ ASA REFS..-3.1.4.1 3.2.4.3.3 3.2.4/12X.7HRESULTS)

-3.1 .4$.
$=$

$=1$

$+1$

$-($

$\star 2$

i)

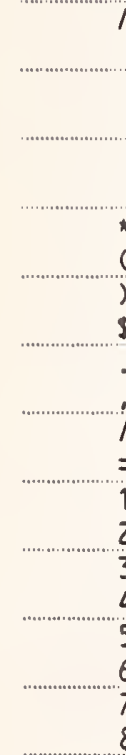

CVI

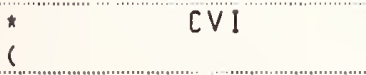

)

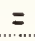

(a)

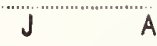

(1)

(n)

(1)

31

42

5 8
ASA REF P1490060

3.1 .4 .1 P1490070

$3.2 .4,3.3 P 1490080$

$3.2 .4 P 1490090$

$P 1490100$

P1490110

P1490120

$P 1490130$

$P 0012930$

P0012935

$P 0012940$

$P 0012945$

$P 0012950$

$P 0012955$

$P 0012960$

P149A1

P149A2

P0012965

P1490140

$P 0072510$

$P 0072515$

$P 0072520$

$P 0072525$

$P 0072530$

P149B1

$P 0072535$

P1490150

P1490160

P1 490170

P1 490180

P 1490190

P1 490200

P1 490210

P 1490220

P 1490230

P1 490240

P1490250

P 1490260

P 1490270

P 1490280

P 1490290

P 1490300

P 1490310

$P 1490320$

P 1490330

P1 1490340

P 1490350

P1490360

P 1490370

P 1490380

P 1490390

+P1490400

P 1490410

P1490420

P1490430

P1490440

P 1490450

P1490460 


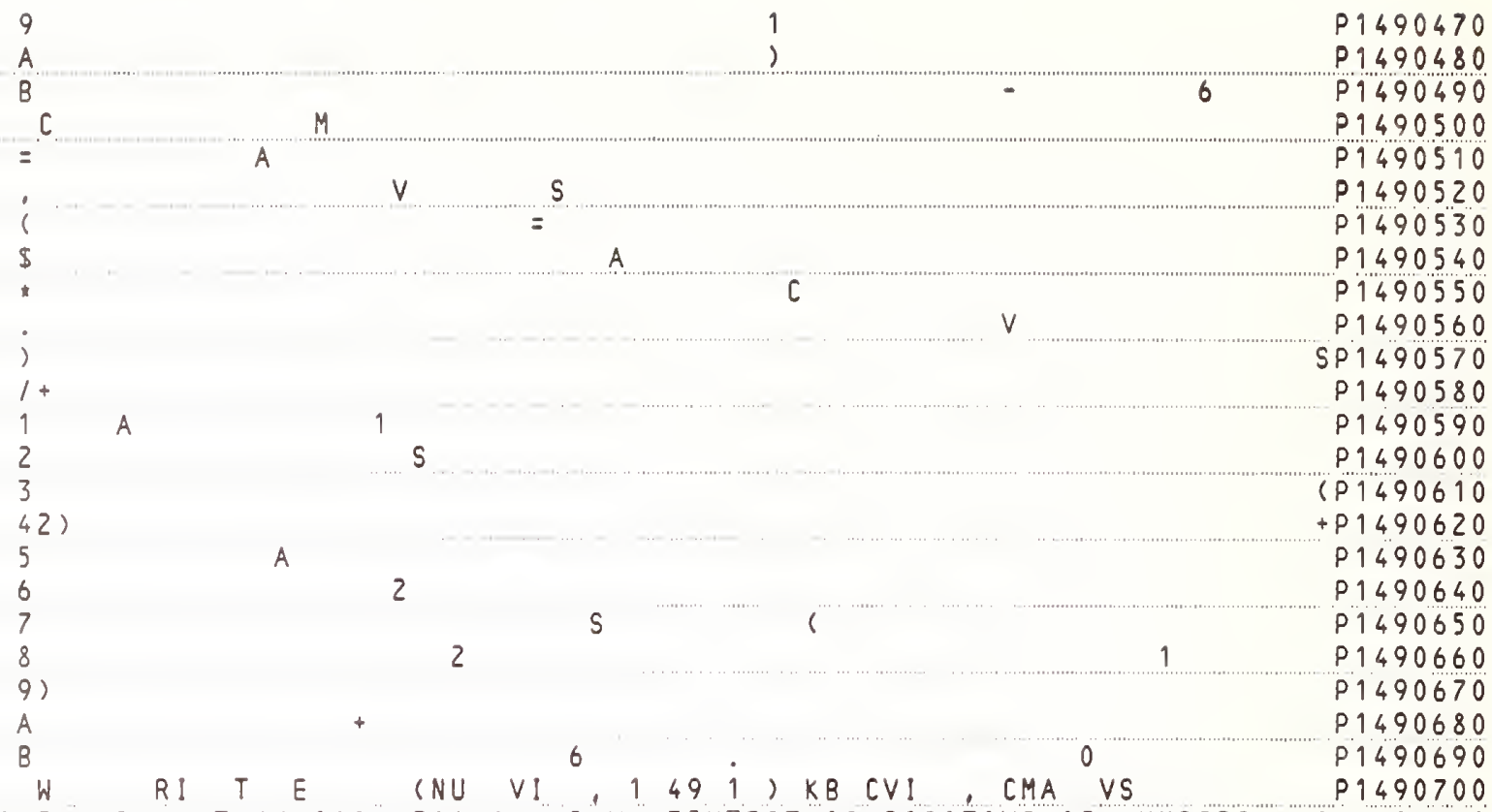

$1491 \mathrm{~F}$ O RM A T (//110//F11.1// $2 X, 35$ HTEST IS POSITIVE IF NUMBERS PRIP1490710 INTED 2 $2 \quad X, 1$ 1HABOVE ARE 0 )

C***** END OF TEST SEGMENT 149

$C * * * *$ WHEN EXECUTING ONLY SEGMENT 149, THE STOP AND END CARDS

$[* * *$ WHICH APPEAR AS COMMENT CARDS MUST HAVE THE C $c=$

$C * * * *$ IN COLUMNS 1 AND 2 REMOVED.

$C=S T O P$

$C=\quad E N D$

STOP

END

P 1490720

P 1490730

P1 490740

P1490750

P1490760

P1490770

P 1490780

P149C1

P149C2

C

$C * * * * *$

$\operatorname{MISC} 4-(150)$

$P 1500020$

$C * * * * *$

P 1500030

$C * * * * *$

$P 1500040$

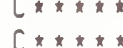
GENERAL PURPOSE

$C * * * *$ TO TEST EFFECT OF BLANKS WITHIN STATEMENT,

$C * * *$ CONTINUATION OF STATEMENT TO 20 LINES,

$C * * * *$ AND USE OF SPECIAL CHARACTERS TO INDICATE CONTINUATION

$C * * *$ CONTINUATION LINE CAN CONTAIN FORTRAN CHARACTERS

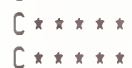
(OTHER THAN C IN COLUMN 1) IN COLUMNS 1 THRU 5 (

$C * * * * *$ S P E C I F I C A T I O N S SEgMENT 150

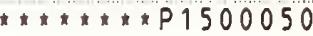

$C * * * * *$

$C * * * * *$

WHEN EXECUTING ONLY SEgMENT 150. THE SPECIFICATION STATEMENTS

$C * *$ WHICH APPEAR AS COMMENTS MUST HAVE THE C $C=$

$C * \| *$ IN COLUMNS 1 AND 2 REMOVED.

$C * * * * *$

$C=\quad$ INTEGER AV!

$C=\quad$ COMPLEX AVC,BVC, CVC, DVC, RVC

ASA REF P1500060

$3.1 .4 .1 P 1500070$

$3.2 .4 .3 .3 P 1500080$

$3.2 .4 P 1500090$

$P 1500100$

3) $P 1500110$

P1500120

P1500130

$P 0012970$

P 0012975

P0012980

P0012985

P0012990

POO12995

$P 0013000$

INTEGER AVI

COMPLEX AVC, BVC, CVC, DVC, RVC

P150A1

P150A2

$C * * * * *$

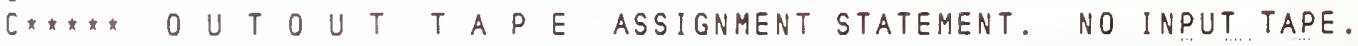

P0013005

P 1500140

$P 0072540$

C*******t*

C $* * *$ WHEN EXECUTING ONLY SEgMENT 150 . THE FOLLOWING STATEMENT

$C * * * *$ NUVI $=6$ MUST HAVE THE $c=$ IN COLUMNS 1 AND 2 REMOVED.

$C * * * * *$

$C=$ NUVI $=6$

$P 0072545$

$P 0072550$

P0072555

$P 0072560$

P150B1

NUVI $=6$

PO072565

WRITE (NUVI, 1500)

$1500 \mathrm{~F} O$ RM A TS 1 HI, 1 X 13 HMISC4-1950)

P 1500150

$X, 1 X, 23$ HEFFECT OF BLANKS WITHIN 
YTINUATION/ 16X, 2OHOF STMNT TO 20 LINES/I

C****COMPLEX ARITHMETIC EXPRESSION

$C * * * *$ STATEMENT LABEL NOT REFERENCED

$1503 \mathrm{~A}$

$V C=1 \cdot+V$
$-C$

- $=$

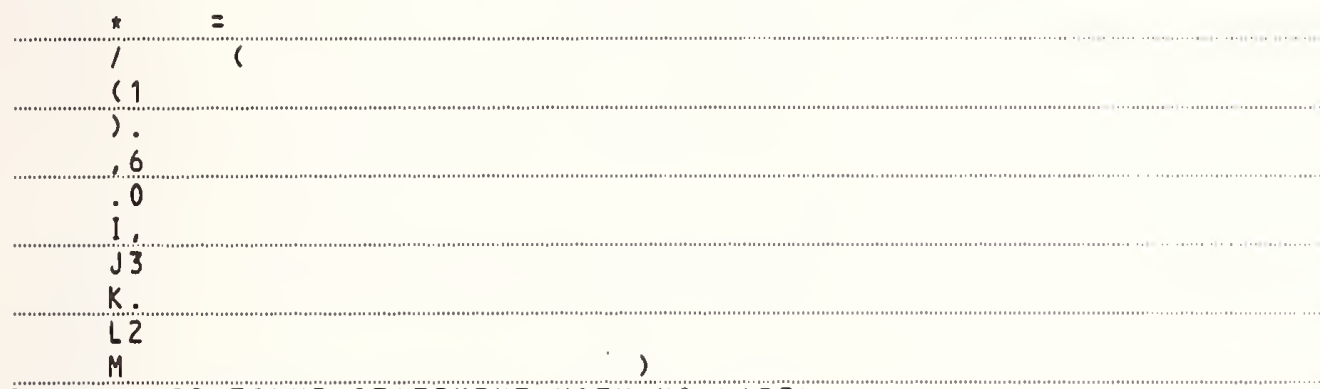

$C * *$ CONTINUE STATEMENT WITH NO LABEL

CONTINUE

AVS $=0.625$

BVS $=2.0$

$B \vee C=(1.0,-1.0)$

CVS $=2.5$

$C V C=(2.5 .2 .5)$

$D V C=(1.0986841,0.4550899)$

$A V I=2$

RVC

$B(A \vee C * A \vee S$

$C+(1,6,3.2)$

D*AVS-AVC

$E * 0.625$

$F-(1.6,3.2)$

$G * 0.625$

$\mathrm{H}+\mathrm{B} \vee \mathrm{S} / \mathrm{B} \vee \mathrm{C}$

$J-B \vee S /(1,0,-1.0)$

$J+2.0 / B \vee C+2.01$

$\mathrm{K}(1.0,-1.0)+\mathrm{CVC} / \mathrm{CVS}$

$L-(2.5,2.5) / C V S+C V C / 2.5$

$M+(2.5,2.5) / 2.5+D V C * A V !$

$N-(1.0986841,0.4550899) * 2$

$0+D V C * 2$

$0(1.0986841,0.4550899)$

$R * A \vee I)$

$S * * 21(0,0,72,0)$

$T \quad-(1.0,0.0)$

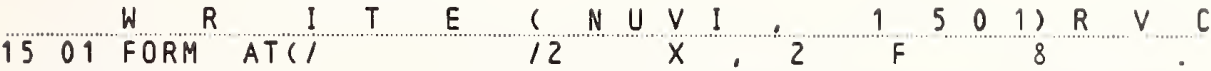

$150121 / 3$ TH TEST IS POSITIVE IF NUMBERS PRINTED/ $2 X$

$$
=.117 \text { HABOVE ARE } 0.0 .0 .0
$$

C***** END OF TEST SEGMENT 150
$C * * * *$ WHEN EXECUTING ONLY SEGMENT 150 , THE STOP AND END CARDS

$C * * * *$ WHICH APPEAR AS COMMENT CARDS MUST HAVE THE $C=$

C**** IN COLUMNS 1 AND 2 REMOVED.

$C=S T O P$

p 1500210

P 1500220

P1500230

"P1500240

P1500250

P 1500260

P 1500270

P 1500280

P 1500290

P1500300

P1500310

P 1500320

$3.4 P 1500330$

P 1500340

P1500350

P1500360

P1500370

P1500380

P 1500390

P1500400

P 1500410

P1 1500420

P 1500430

P1500440

P 1500450

$P 1500460$

P1500470

$3.4 P 1500480$

P 1500490

P1500500

P1500510

P1500520

$P 1500530$

P1500540

P1500550

P1500560

P 1500570

P 1500580

P1500590

P1500600

P1500610

$P 1500620$

P1500630

P 1500640

P1500650

P1500660

P 1500670

P1500680

P1500690

P1500700

$P 1500710$

P 1500720

P 1500730

P 1500740

P 1500750

P 1500760

P1500770

F 1500780

P1500790

P 1500800

P 1500810

$P 1500820$

P 1500830

P1500840

P1500850 
$C * * * *$ 2. OUMMY ARGUMENTS ARE REAL OR INTEGER VARIABLES,OR ARRAY NAMES

$C * * * *$

C*****

C*****

$[* * * *$

$[* * * * *$

$C * * * * *$

$[* * * * *$

$C * * * * *$

$C * * * * *$

$C * * * * *$

$C * * * * *$

$C * * * * *$

C******

$C * * * * *$

$C * * * * *$

. FUNCTIONS CONTAIN UP TO 20 ARGUMENTS

4. IN REFERENCE, ACTUAL ARGUMENTS ARE VARIABLE NAME, ARRAY NAME, ARRAY ELEMENT NAME, OR AN ARITHMETIC EXPRESSION

RESTRICTIONS OBSERVEO

$$
\text { 1. ITEMS ( } 2),(3),(4),(5),(6) \text { OF PARAGRAPH } 8.3 .1
$$

P 1600030

$P 1600040$

2. LAST SENTENCE OF PARAGRAPH 3.2

THIS SEGMENT IS TO BE RUN WITH SEGMENTS

$$
400,420,430,440,450,460
$$

CONTAINS ALL FUNCTIONS BEING TESTEO HERE.

WHI CH

ASA REFP 1600060

$8.3 .1 P 1600070$

$P 1600080$

$P 1600090$

P1600100

P 1600110

P 1600120

\section{$S P E C I F I C A T I O N$ I SEGMENT 160}

$8.3 .2 P 1600130$

$P 1600140$

$P 1600150$

$P 1600160$

P 1600170

P 1600180

$P 1600190$

P 1600200

$P 1600210$

$P 0013010$

WHEN EXECUTING ONLY SEGMENT 160, THE SPECIFICATION STATEMENTS

$C * * *$ WHICH APPEAR AS COMMENT CAROS MUST HAVE THE $C=$

C*****IN IN COLUMNS 1 ANO 2 REMOVEO.

$C \star * \star * *$

$C=\quad$ OIMENSION A1S $(5), \operatorname{AZS}(2,2)$

DIMENSION AIS (5), AZS $(2,2)$

$C * * * *$

C**** 0 U U T P U T T A P E ASSIGNMENT STATEMENT. NO INPUT TAPE.

C**** WHEN EXECUTING ONLY SEGMENT 160, THE FOLLOWING STATEMENT

$C * * * *$ NUVI $=6$ MUST HAVE THE $C=$ IN COLUMNS 1 ANO 2 REMOVEO.

$C * * * * *$

$C=\quad$ NUVI $=6$

NUVI $=6$

WRITE (NUVI, 1604)

1604 FORMAT ( 1 H1, 1X,37HBRFCP - (160) REAL EXTERNAL FUNCTIONS/

$1 / 2 X, 16 H A S A$ REF. $-8.3 .1 / / 28 H$ RESULTS SHOULO BE POSITIVE)

$I A \vee I=2$

$A 1 S(1)=1.0$

$A 1 S(2)=1.0$

$P 0013015$

P 0013020

$P 0013025$

P 0013030

$P 0013035$

P $160 \mathrm{~A} 1$

$P 0013040$

P 1600220

$P 0072570$

P 0072575

$P 0072580$

$P 0072585$

P160B1

$P 1600230$

$P 1600240$

P 1600250

P1600260

P 1600270

P 1600280

$A 2 S(2,2)=1.0$

P 1600290

$\operatorname{AZS}(2,1)=1.0$

P 1600300

$A \vee S=1.0$

$P 1600310$

$B \vee S=2.0$

$P 1600320$

$C V S=1.0$

DVS $=1.0$

P 1600330

$P 1600340$

$P 1600350$

$E V S=1.0$

$I V I=\operatorname{AFS}(2.0)-8.0$

P 1600360

$M A \vee I=1$

P 1600370

$P 1600380$

IF(IVI) $1600,1601,1600$

P 1600390

P 1600400

P 1600410

$M A \vee !=2$

IF (IVI) $1600,1601,1600$

1606 IVI CFS(2) -16.0

MAV

If $v 1600,1601,1600$

$P 1600420$

P 1600430

$P 1600440$

$P 1600450$

$P 1600460$

$A V I=4$

IF(IVI) $1600,1601,1600$

160 IVI $=E F S(A 1 S)-2.0$

$P 1600470$

P1600480

$M A V I=5$

IF(IVI) $1600,1601,1600$

P 1600490

P1600500

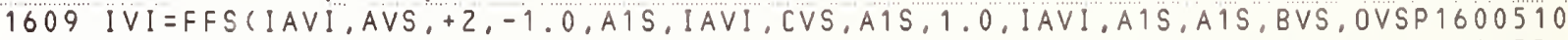
$1, A 1 S(1), A 2 S, A 2 S, A 2 S, E V S+1.0, I A V I-1)+1.0$

P1600520 
$M A V I=6$

IF (IVI) $1600,1601,1600$

1600 WRITE (NUVI.1602) MAVI

GO TO 7001

1601 WRITE (NUVI.1603) MAVI

1602 FORMAT $(1 / 2 x, 5 H T E S T$ I $11,12 H$ IS NEGATIVE)

1603 FORMAT (//2X,5HTEST.11,12H IS POSITIVE)

7001 GO TO (1605,1606,1607,1608,1609,7000), MAVI

7000 CONTINUE

\section{$C * * * *$ END OF TEST SEGMENT 160}

C***WHEN EXECUTING ONLY SEGMENT 160., THE STOP AND END CARDS

$C * * * *$ WHICH APPEAR AS COMMENT CARDS MUST HAVE THE $C=$ IN

$C * * *$ COLUMNS 1 AND 2 REMOVED.

$C=\quad S T O P$

$C=E N D$

STOP

$P 1600550$

$P 1600560$

$P 1600570$

$P 1600580$

$P 1600590$

$P\{600600$

P1600610

$P 1600620$

$P 1600630$

P1600640

$P 1600650$

$P 1600660$

P1600670

END

P160C

P160C2

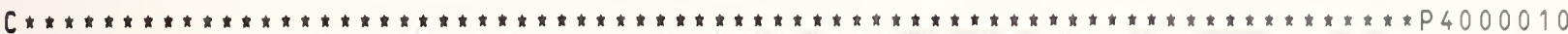

$C * * * t$

$A F S-(400)$

$P 4000020$

$C * * * * * 10$

AF

$P 4000030$

$C * * * * *$

$P 4000040$

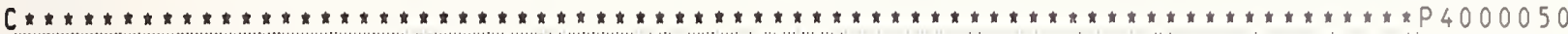

$C * * *$ REAL FUNCTION OF REAL ARGUMENT (TEST 1)

FUNCTION AFS(AWVS)

$A F S=4.0 * A W V S$

RETURN

END

$P 4000070$

$P 4000080$

$P 4000090$

$P 4000100$

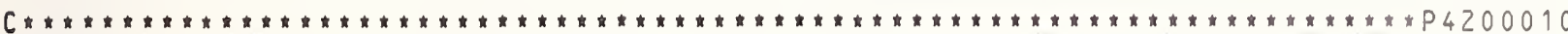

$C * * * * *$

$B F S-(420)$

$P 4200020$

$C * * * * *$

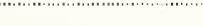

$P 4200030$

$P 4200040$

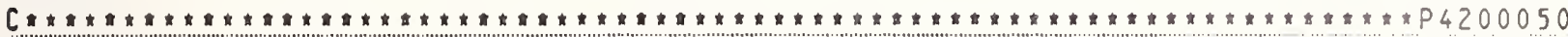

$C * * * *$ REAL FUNCTION OF REAL ARGUMENTS (TEST 2 )

FUNCTION BFS (AWVS, BWVS)

$B F S=A W V S+B W V S$

RETURN

END

P4200060

$P 4200070$

$P 4200080$

$P 4200090$

P4200100

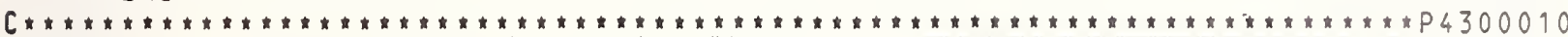

$C * * * * *$

$C F S-(430)$

$P 4300020$

$C * * * * *$

CES $-(430)$

$P 4300030$

P4300040

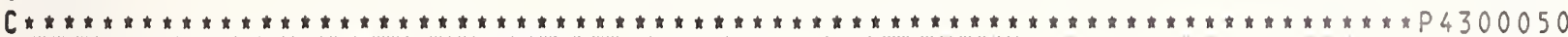

C****REAL FUNCTION OF INTEGER ARGUMENT (TEST 3 )

FUNCTION CFS(IWVI) $\quad P 4300070$

CFS $=4.0 *$ IWVI

$\begin{array}{ll}\text { RETURN } & P 4300090\end{array}$

END

$P 4300100$

C

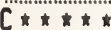

$D F S-(440)$

$P 4 \dddot{4000020}$

$C * * * * *$

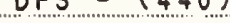

$P 4400030$

$P 4400040$

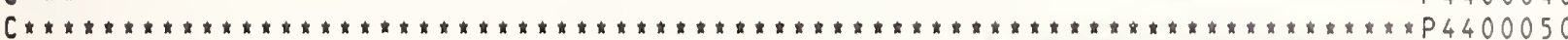

$C * * * *$ REAL FUNCTION OF INTEGER ARGUMENTS (TEST 4 )

FUNCTION DFS(IWVI, JWVI)

$K V I=I W V I-J W V !$

DFS $=4.6 * K V I$

RETURN

END

$P 4400060$

$P 4400070$

$P 4400080$

$P 4400090$

$P 4400100$

P4400110

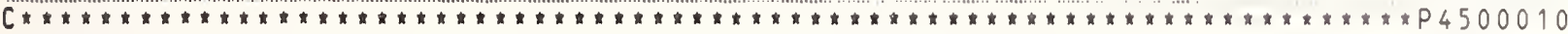

$C * * * *$

$E F S-(450)$

$P 4500020$

P4500030

$P 4500040$

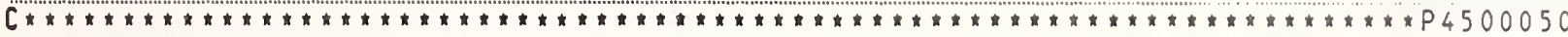

C****REAL FUNCTION OF ARRAY NAME (TEST 5)

FUNCTION EFS(AWIS)

$P 4500060$

DIMENSION AW1S(2)

EFS =AW1S(1)+AH1S(2)

RETURN

$P 4500070$

$P 4500080$

$P 4500090$

$P 4500100$ 


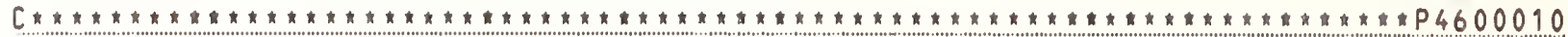

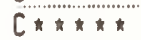

$F F S-(460)$

$P 4600020$

$C * * * * *$

44600030

$C * * * * *$

44600040

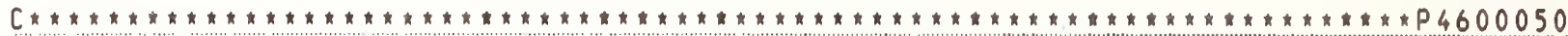

C**REAL FUNCTION OF DIFFERENT TYPES OF ARGUMENTS(TEST 6 )

FUNCTION FFSCIWVI, AWVS, JWVI, BWVS, AW1S, KWVI,CWVS, BW1S, OWVS, LWVI, P4600070

1CW1S, DW1S, EWVS, FWVS, GWVS, BW2S, CW2S, DW2S, HWVS, MWVI) 14600080

DIMENSION AWIS(2), BW1S(2),CW1S(2), OW1S(2),BW2S(2,2),CW2S(2,2), P4600090

$10 W 2 S(2,2)$

FFS = AWVS* IWVI-BWVS* JWVI+AW1S(1)-CWVS* KWVI+BW1S(2)-DWVS+CW1S(1) 04600110

$1 *$ LWVI +DW IS (1)-EWVS +FWVS-GWVS +BW2S $(2,1)-$ CW2S $(2,2)+D W 2 S(2,2)-H W V S * P 4600120$

2MWVI

RETURN

END

$P 4600130$

44600140

$P 4600150$

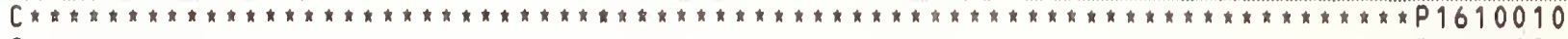

$C * * * * *$

$B I F C P-(161)$

P 1610020

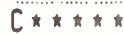

$B I F C P-(161)$

1610030

C $\# * * *$

$P 1610040$

C

$C * * * *$ GENERAL PURPOSE

$C * \#$ 1-TO TEST INTEGER FUNCTIONS

C**** 2-DUMMY ARGUMENTS ARE REAL OR INTEGER VARIABLES OR

C*** ARRAY NAMES

$C * * * *$ 3-FUNCTIONS CONTAIN UP TO 20 ARGUMENTS

C**** 4-IN REFERENCE, ACTUAL ARGUMENTS ARE VARIABLE NAME,

ASA REFP1610060

8.3.1P1610070

P1610080

$C *$ ARRAY NAME, ARRAY ELEMENT NAME,OR AN ARITHMETIC

C**** EXPRESSION

$[* * * *$ RESTRICTIONS OBSERVED

8.3.1P1610090

P1610100

P 1610110

P1610120

$C * * * \pi$ 1-ITEMS (2), (3), (4)

C**** 2-LAST SENTENCE OF PARAGRAPH 3.2

C*****THIS SEGMENT IS TO BE RUN WITH SEGMENTS

$C * * * 401,421,431,441,451,461 \ldots$ WHICH

C* CONTAINS ALL FUNCTIONS BEING TESTED HERE.

8.3 .1

8.3.2P1610130

P 1610140

P1610150

P1610160

P 1610170

P 1610180

$C * * \cdots * *$

$C * * * * *$

$C * * * *$

$C * * * *$

S P E I I F I C A T I ON S SEGMENT 161

P 1610190

$P 1610200$

P 1610210

$P 0013050$

WHEN EXECUTING ONLY SEGMENT 161, THE SPECIFICATION STATEMENTS

P0013055

$C * * * *$ WHICH APPEAR AS COMMENT CARDS MUST HAVE THE $C=$

$C * * *$ IN COLUMNS 1 AND 2 REMOVED.

$C * * * * *$

$C=$ DIMENSION A1S(5)

$C=\quad$ INTEGER I1\(5)

DIMENSION A1S( 5 )

$P 0013060$

P0013065

$P 0013070$

P0013075

$P 0013080$

INTEGER I11(5)

P161A1

P161A2

$C * * *$

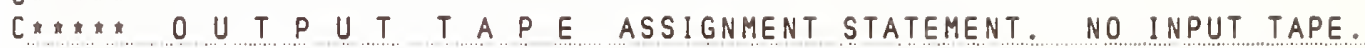

P 0013085

P 1610220

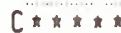

$[* * * * *$

WHEN EXECUTING ONLY SEGMENT 161, THE STATEMENT NUVI = 6

$C *$ MUST HAVE THE $C=$ IN COLUMNS 1 AND 2 REMOVED.

$P 0072590$

$C * * * * *$

$C=\quad$ NUVI $=6$

$C * * * * *$

NUVI $=6$

$P 0072595$

P0072600

$P 0072605$

P0072610

P161B1

WRITE (NUVI, 1614)

P0072615

P 1610230

1614 FORMAT(1H1,1X,40HBIFCP - (161) INTEGER EXTERNAL FUNCTIONSI

P1610240

$116 \mathrm{X}, 26 \mathrm{HWITH}$ INTEGER AND REAL ARGS//2X,16HASA REF.- $8.3 .1 / 1$

228 RESULTS SHOULD BE POSITIVE)

P1610250

$\mid A V I=2$

$A 1 S(1)=1.0$

$P 1610260$

P 1610270

$A 1 S(2)=1.0$

P1610280

P1610290

$111(1)=1$

$111(2)=$ ?

$P 1610300$

P1610310

A $Y S=1.0$

P1610320

$B V S=2.0$

P1610330

CVS $=1.0$

P 1610340

DV $S=1.0$

P.1610350 
$E V S=1.0$

$I V I=I \operatorname{AFI}(2.0)-8$

P1610360

MAVI $=1$

IF (IVI) $1610,1611,1610$

P 1610370

P 1610380

$P \$ 610390$

1615 IVI =IBFI(2.0.BVS $)-4$

MAVI $=2$

IF (IVI) 1610.1611 .1610

P 9610400

P1610410

P 1610420

P 1610430

CFI(2)- 16

MAVI $=3$

IF (IVI) $1610,1611,1610$

1617 IVI $=$ IDFI $(2$, IAVI $)-1$

MAVI $=4$

$P 1610440$

P1610450

IF (IVI) $1610,1611,1610$

1618 IVI $=I E F I(111)-2$

MAVI $=5$

IF (IVI) $1610,1611,1610$

1619 IVI = IFFI(IAVI, AVS, 2, - 1.0,A1S, IAVI, CVS,A1S, 1.0, IAVI, A 1S,A1S, BVS, $10 V S, A 1 S(1), A 1 S, A 1 S, A 1 S, E V S+1.0, I A V I-1)+1$

MAVI $=6$

IF(IVI) $1610,1611,1610$

P 1610460

P 1610470

P 1610480

P 1610490

P 1610500

P1610510

P 1610520

P 1610530

P 1610540

P 1610550

1610 WRITE(NUVI, 1612 ) MAVI

GO TO 7002

1611 WRITE(NUVI, 1613 ) MAVI

1612 FORMAT (//2X,5HTEST, I $1,12 \mathrm{H}$ IS NEGATIVE)

1613 FORMAT ( $/ / 2 X, 5$ HTEST, $11.12 \mathrm{H}$ IS POSITIVE)

7002 GO TO $(1615,1616,1617,1618,1619,7003)$, MAVI

7003 CONTINUE

$C * * * * *$ END OF TEST SEGMENT 161

$C * * * * *$ WHEN EXECUTING ONLY SEGMENT 161 , THE STOP AND END CARDS

$C * * * *$ WHICH APPEAR AS COMMENT CARDS MUST HAVE THE $C=$

$C * * * *$ IN COLUMNS 1 AND 2 REMOVED.

$C=S T O P$

$C=\quad E N D$

STOP

END

$P 1610560$

P 1610570

P 1610580

P 1610590

P1610600

P 1610610

P 1610620

P1610630

P 1610640

P 1610650

P1610660

P1610670

P 1610680

P161C1

$P 161 C 2$

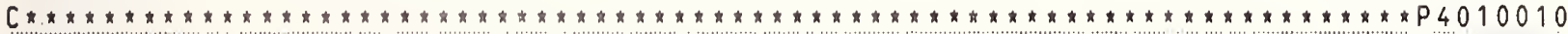

$C * * * * *$

$I A F I-(401)$

$P 4010020$

$[* * * * *$

$P 4010030$

$C * * * * *$

$P 4010040$

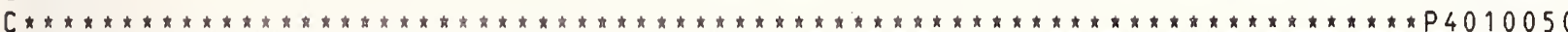

C*****INTEGER FUNCTION" OF REAL ARGUMENT (TEST 1 )

$P 4010060$

FUNCTION IAFI (AWVS)

P4010070

I AFI $=4.0$ * AWVS

$P 4010080$

RETURN

$P 4010090$

END

$P 4010100$

$C * * * *$

$C * * * * *$

P4210010

$C * * * * *$

$I B F I-(421)$

$P 4210020$

$P 4210030$

$C * * * * *$

$P 4210040$

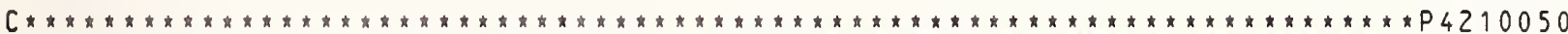

C*****INTEGER FUNCTION OF TWO REAL ARGUMENTS (TEST 2 )

$P 4210060$

FUNCTION IBF! (AWVS, BWVS)

P 4210070

$I B F !=A W V S+B W V S$

RETURN

$P 4210080$

P4210090

END

P4210100

C********

$P 4310010$

$C * * * * *$

$P 4310020$

$C * * * * *$

$I C F I-(431)$

P4310030

P4310040

$C * * * *$

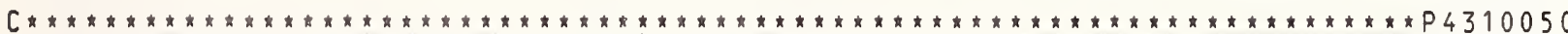

$C * * *$ INTEGER FUNCTION OF INTEGER ARGUMENT(TEST 3 )

$P 4310060$ FUNCTION ICFI (IWVI)

I CFI $=4.0 * *$ I WVI

RETURN

P4310070

P 4310080

$P 4310090$

END

$P 4310100$

$C * * * * * * * *$

$C * * * * *$

$C * * * * *$

IDFI-(441)

P4410020

$P 4410030$

NBS FORTRAN TESt Programs Version 1 


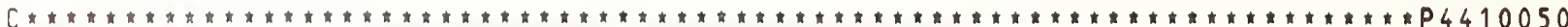

C**** *INTEGER FUNCTION OF INTEGER ARGUMENTS (TEST 4)

$P 4410060$

INTEGER FUNCTION IDFI (IWVI, JWVI)

P44 10070

REAL KUVS

$I D F I=I W V I-J W V I$

P4410080

$I D F I=$ KUVS * IDFI

P4410090

RETURN

P 4410100

DATA KUVS $14.6 /$

$P 4410110$

E N D

$P 4410120$

$P 4410130$

C

$P 4510020$

C *******

IEFI - (451)

$P 4510030$

C******

$P 4510040$

C

C*** *INTEGER FUNCTION OF ARRAY NAME (TEST 5) P4510060

FUNCTION IEFI (IAWII)

DIMENSION IAW II ( 2$)$

$P 4510070$

$I E F I=I A W I I(1)+I A W 1 I(2)$

$P 4510080$

$P 4510090$

RETURN

$P 4510100$

END

$P 4510110$

C

$[* * * * *$

C*****) IFFI $-(461)$

$P 4610020$

$P 4610030$

$[* * * * *$

04610040

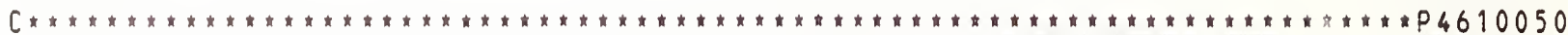

C**** * INTEGER FUNCTION OF DIFFERENT TYPES OF ARGÜMENTS(TEST 6)

FUNCTION IFFI(IWVI, AWVS, JWVI, BWVS, AW1S, KWVI, CWVS, BW1S, OWVS, LWVI, P4610070

ICW1S, OW1S, EWVS, FWVS, GWVS, EWIS, GW 1 S, HW1S, HWVS, MWVI)

OIMENSION AW1S(2), BW1S(2), CWIS(2), OW1S(2), EW1S(5), GW1S(5),

$P 4610080$

$P 4610090$

1 HWIS 15 )

$P 4610100$

$I F F I=A W V S *$ * IWVI-BWVS * JWVI+AW1S(1)-CWVS* *WVI+BW1S(2)-OWVS+CW1S(1)P4610110

$1 *$ LWVI+DW1S(1)-EWVS + FWVS -GWVS+EW1S(1) -GW1S(2) +HW1S(2) - HWVS**P4610120

2MWVI

RETURN

$P 4610130$

END

$P 4610140$

$P 4610150$

C

$C * * * * *$

FRFCP - (162)

$P 1620020$

$[* * * * *$

P 1620030

$P 1620040$

$C * * * * *$

GENERAL PURPOSE

$C * * * *$

GENERAL PURPOSE

C***** 1.TO TEST REAL FUNCTIONS IN FULL FORTRAN

P1620050

C**** 2.THIS SEgMENT COMPLETES SEGMENT (160) IN ORDER TO TEST

C***** FOR ALL FEATURES REQUIRED IN FULL FORTRAN

ASA REF P1620060

P 1620070

$[* * * * *$

3. DUMMY ARGUMENTS CAN BE INTEGER(TESTED IN 160), REAL(TESTED

C****160), ARRAY NAME(TESTED IN 160), DOUBLE PRECISION, COMPLEX,

C***** LOGICAL OR EXTERNAL PROCEOURE

C*****4.0UMMY ARGUMENTS MAY BE REDEFINEO IN SUBPROGRAM(ITEM 4)

C*****5. IN REFERENCE, ACTUAL ARGUMENTS MAY BE AS IN (160) AND

C**** BESIDES EXTERNAL PROCEDURE. IN THIS CASE, EXTERNAL

$C * * * *$ PROCEDURE IS REFERENCED BY AN EXTERNAL STATEMENT

C*****6. 6. USE CAN BE MADE OF ADJUSTABLE OIMENSION

C*****RESTRICTIONS OBSERVED

C*****1. ITEMS (1), (2), (3), (5) OF 8.3 .1

C****2.PARAGRAPH 8.3 .2 , LINE 18 TO END OF PARAGRAPH

C***** THIS SEGMENT USES 5 REAL FUNCTIONS

C***** THIS SEGMENT IS TO BE RUN WITH SEGMENTS

$C * * * * \quad 402,422,432,442,452$ WHICH

C******t)

WHICH CONTAINS ALL FUNCTIONS BEING TESTED HERE

P 1620080

$8.3 .1 P 1620090$

$C * * * * *$

$C * * * *$

$C * * * * *$

$C * * * * *$

S P E C I F I C A T I O N S SEGMENT 162

$P 1620100$

P 1620110

$8.3 .1 P 1620120$

$8.3 .1 P 1620130$

$P 1620140$

$C * *$ WHICH APPEAR AS COMMENT CARDS MUST HAVE THE C $=$

$C * * * *$ IN COLUMNS 1 AND 2 REMOVED.

$C * * * * *$

8.3.2P1620150

$P 1620160$

$P 1620170$

$P 1620180$

P 1620190

P 1620200

$P 1620210$

$P 1620220$

$P 1620230$

$P 1620240$

$P 1620250$

P 1620260

P0013090

$P 0013095$

$P 0013100$

$P 0013105$

$P 0013110$

P0013115 
$C=\quad$ INTEGER I1I(5), I2I(2,2),I3I(2,2,2)

P 0013120

REAL JRFS, IRFS

$C=\quad \operatorname{LGICALAIB(2),AZB(2,2),A3B(2,2,2),A\vee B,B\vee B}$

$C=\quad$ DOUBLE PRECISION AVD,A1D(4),AZD(2,2),A3D(2,2,2)

$C=\quad$ COMPLEX AVC,BVC,A1C $(12), A 2 C(2,2), A 3 C(2,2,1)$

$C=\quad C O M M O N$ AXVS, CXVS

$C=\quad$ EXTERNAL GFS

DIMENSION A1S(5),AZS $(2,2), A 3 S(3,3,3)$

P 0013125

$P 0013130$

P 0013135

$P 0013140$

$P 0013145$

$P 0013150$

INTEGER I I $(5)$, I I $(2,2)$, I 3 I $(2,2,2)$

REAL JRFS, IRFS

LOGICAL A1B(2),A2B (2,2), A $3 B(2,2,2), A \vee B, B \vee B$

DOUBLE PRECISION AVD,A1D(4),A2D(2,2), A3D(2,2,2)

P162A1

P162A2

P $162 \mathrm{A3}$

P162A4

P162A5

P162A6

COMPLEX ÄVC,BVC,A1C(12),AZC (2,2),A3C(2,2,1)

P162A7

EXTERNAL GFS

P162A8

P 0013155

C****:OU TP U T T A P E ASSIGNMENT STATEMENT. NO INPUT TAPE.

P 1620270

P 0072620

C*:* WHEN EXECUTING ONLY SEGMENT 162 , THE STATEMENT NUVI = 6

$C *$ MUST HAVE THE $C=$ IN COLUMNS 1 AND 2 REMOVED.

$P 0072625$

$P 0072630$

$P 0072635$

$C * * * * *$

$C=\quad$ NUVI $=6$

$C * * * *$

NUVI $=6$

$P 0072640$

P $162 B 1$

WRITE (NUVI, 1624)

$P 0072645$

P 1620280

1624 FORMAT(1H1,1X,33HFRFCP - (162) REAL FUNCTIONS WITH/10X,31HLOGICAL,P1620290

1 D.P. AND COMPLEX ARGS//16H ASA REF. $8.3 .1 / 1$

$228 H$ RESULTS SHOULD BE POSITIVE)

$P 1620300$

P 1620310

P 1620320

$A \vee D=1.000$

MAVI $=1$

IVI $=1.0-G F S(A \vee D)$

IF (IVI) $1620,1621,1620$

$C * * *$ TEST 2

1625 MAVI $=2$

$A \vee C=(1,0,-1.0)$

$B \vee C=(1.0,1.0)$

$I V I=\operatorname{HFS}(A \vee C, B \vee C)$

IF (IVI) $1620,1621.1620$

C*** *TEST 3

1626 MAVI $=3$

$A V B=. T R U E$.

$I V I=I R F S(A \vee B) * 2.0$

$A \vee B=. F A L S E$.

$J V I=I R F S(A \vee B) * 4.0$

$L V I=I V I+J V I-4$

IF (LVI) 1620.1621 .1620

P 1620330

P1620340

$P 1620350$

P1620360

$P 1620370$

P 1620380

$P 1620390$

P1620400

P1620410

P1620420

$P 1620430$

$P 1620440$

P 1620450

P 1620460

P1620470

P 1620480

P 1620490

$P 1620500$

$P 1620510$

$C * * *$ TEST 4

1627 MAVI $=4$

$I V I=J R F S(A \vee D, G F S)$

IF (IVI-1) $1620,1621,1620$

C***TEST $5,6,7$

1628 AXVS $=1.0$

AVS $=1.0$

$A 1 S(1)=1.0$

$\operatorname{AZS}(1,1)=1.0$

$A 3 S(1,1,1)=1.0$

$A V B=. F A L S E$.

$A 1 B(1)=. F A L S E$.

$A Z B(1,1)=$ FALSE.

$A 3 B(1,1,1)=$. FALSE.

$\triangle A V I=1$

$111(1)=1$

$12 I(1,1)=1$

$13 I(1,1,1)=1$

$A 1 C(1)=(1.0,1.0)$

$A 2 C(1,1)=(1,0,1.0)$

$A 3 C(1,1,1)=(-2,0,-2.0)$

P 1620520

$P 1620530$

$P 1620540$

$P 1620550$

P1620560

P 1620570

$P 1620580$

$P 1620590$

P 1620600

$P 1620610$

P 1620620

$P 1620630$

$P 1620640$

P1620650

P1620660

$P 1620670$

$P 1620680$

$P 1620690$

$P 1620700$

$P 1620710$ 
$A \vee D=1.000$

$P 1620720$

$A 1 D(1)=1.000$

$P 1620730$

$A 2 D(1,1)=1.000$

P 1620740

$A 3 D(1,1,1)=1.0 D 0$

$P 1620750$

IVI = RFS(AVS, IAVI, AVB,AVC,AVD,A1S,A2S,A3S,111,121,131,A1B,A2B,A3B,P 1620760

$1 A 1 C, A 2 C, A 3 C, A 10, A 2 D, A 3 D, G F S)$

$P 1620770$

MAVI $=5$

IF (IVI) $1620,1621,1620$

$P 1620780$

$P 1620790$

1629 MAVI $=6$

$B \vee B=A \vee B \cdot A N D \cdot A 1 B(1) \cdot A N D \cdot A 2 B(1,1) \cdot A N D \cdot A 3 B(1,1,1)$

IF (BVB) GD TD 1621

GD TO 1620

$P 1620800$

$P 1620810$

P 1620820

7010 IVI = REAL (AVC)

$J \vee I=A I M A G(A \vee C)$

P 1620830

$M A V I=7$

$P 1620840$

$B V B=I V I . E O \cdot 0 . A N D . J V I . E O .0$

$P 1620850$

IF (BVB) GD TD 1621

1620 WRITE (NUVI,1622) MAVI

GO TO 7011

1621 WRITE (NUVI, 1623) MAVI

1622 FDRMAT $(/ / 2 X, 5 H T E S T, 11,13 H$ IS NEGATIVE.)

1623 FDRMAT (//2X,5HTEST, I1,13H IS POSITIVE..)

7011 GO TO $(1625,1626,1627,1628,1629,7010,7012)$, MAVI

7012 CONTINUE

$[* * * *$ END DF TEST SEGMENT 162

C*** WHEN EXECUTING DNLY SEGMENT 162, THE STDP AND END CARDS

$[* *$ WHICH APPEAR AS CDMMENT CARDS MUST HÄVE THE C

$[* * *$ IN CDLUMNS 1 AND 2 REMDVED.

$C=\quad S T D P$

$C=\quad E N D$

STDP

$P 1620860$

$P 1620870$

P1620880

P1620890

P1620900

P1620910

P1620920

$P 1620930$

P1620940

P1620950

P 1620960

P1620970

P1620980

$P 1620990$

P1621000

P1621010

END

P162C1

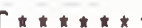

$P 162 C 2$

$[* * * * *$

$[* * * * *$

$C * * * * *$

GFS - $(402)$

P 4020020

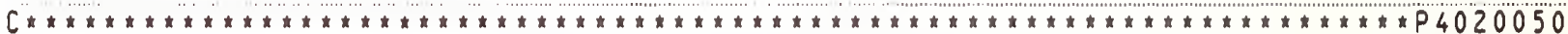

$C * * *$ REAL FUNCTION OF DOUBLE PRECISION ARGUMENT (TEST 1 )

FUNCTIDN GFS(AWVD)

DOUBLE PRECISIDN AWVD

$P 4020060$

$G F S=A W V D$

RETURN

$P 4020070$

$P 4020080$

P4020090

END

$P 4020100$

P 4020110

C

$[* * * *$

$P 4220010$

C *****

$H F S-(422)$

P4220020

$P 4220030$

C * * * *

P4220040

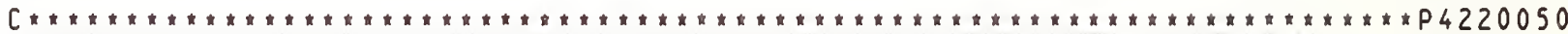

$C * * *$ REAL FUNCTION OF COMPLEX ARGUMENT (TEST 2)

FUNCTIDN HFS (AWVC, BWVC)

$P 4220060$

$P 4220070$

CDMPLEX AWVC, BWVC, CVC

$P 4220080$

$C V C=A W V C * B W V C$

P4220090

$H F S=A I M A G(C V C)$

P 4220100

RETURN

$P 4220110$

END

$P 4220120$

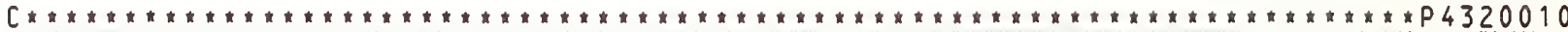

$C * \| *$

$[* * * *$

IRFS - (432)

$P 4320020$

$P 4320030$

$[* * * *$

$P 4320040$

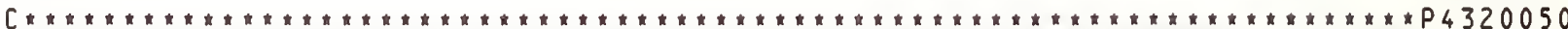

C****REAL FUNCTIDN DF LDGICAL ARGUMENT (TEST 3)

$P 4320060$

REAL FUNCTION IRFS (AWVB)

$P 4320070$

LOGICAL AWVB

$P 4320080$

$P 4320090$

IF (AWVB) GD TD 4321

4320 IF (.NOT. AWVB) GO TD 4322

RETURN

$P 4320100$

$P 4320110$

$P 4320120$

4321 IRFS $=2.0$

GD TD 4320

$P 4320130$ 
$C * * *$ REAL FUNCTION OF EXTERNAL PROCEDURE (TEST 4 )

$P 4420050$

REAL FUNCTION JRFS ( BWVD, BWFS)

$P 4420060$

DOUBLE PRECISION BWVD

$P 4420070$

JRFS $=B W F S(B W V D)$

$P 4420080$

RETURN

P 4420090

END

$P 4420100$

$P 4420110$

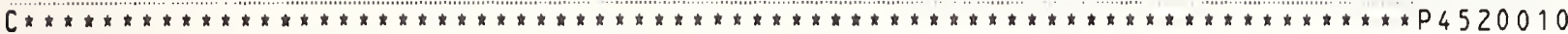

$C * * * * *$

$R F S-(452)$

$P 4520020$

$C * * * * *$

$P 4520030$

$P 4520040$

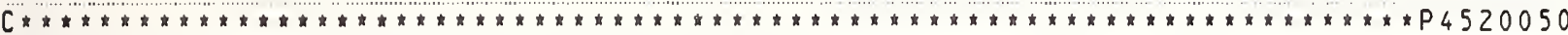

$C * * *$ REAL FUNCTION OF DIFFERENT TYPES OF ARGUMENTS. USE IS MADE OF

$P 4520060$

$C * * * * A D J U S T A B L E$ DIMENSION (TEST $5,6,7$ )

FUNCTION RFS (AWVS, IWVI, AWVB,AWVC, AWVD,AW1S,AW2S, AW3S, IW1 I, IW I ,

$P 4520070$

1IW3I, AW1B,AW2B, AW3B, AW1C,AW2C, AW3 C, AW1D,AW2D, AW 3D, AWFS )

LOGICAL AWVB, AW1B, AW2B, AW3B

COMPLEX AWVC, AW 1C, AW2C, AW3C

DOUBLE PRECISION AWVD, AW1D, AW2D, AW3D

DIMENSION AW1S(IWVI), AWZS(IWVI, IWVI),AW3S(IWVI, IWVI, IWVI)

$P 4520080$

$P 4520090$

$P 4520100$

$P 4520110$

$P 4520120$ 1

2

3

COMMON BXVS

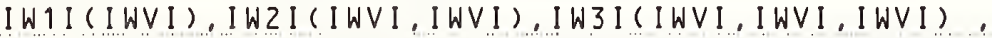

$P 4520130$

$P 4520140$ $A W 1 B($ IWVI),AW2B(IWVI, IWVI),AW3B(IWVI,IWVI,IWVI)

P4520150

$P 4520160$ AWID(IWVI), AW2D(IWVI, IWVI), AW3D(IWVI, IWVI, IWVI)

$P 4520170$

$P 4520180$

RFS =AWVS* IWVI+AW1S(IWVI)* IWII(IWVI)-AWZS(IWVI, IWVI)* IWZI

P4520190

1 (IWVI, IWVI) +AW3S (IWVI, IWVI, IWVI)**IW3I(IWVI, IWVI, IWVI)-AWVD+

$P 4520200$

2 AW1D(IWVI)-AW2D(IWVI,IWVI)-AW3D(IWVI,IWVI,IWVI) +AWFS(AWVD)-BXVS $A W V B=1 W V I . E Q .1$

$A W 1 B(I W V I)=I W V I . E O \cdot{ }^{\prime} 1$

$P 4520210$

$P 4520220$

$P 4520230$

$A W 2 B(I W V I, I W V I)=I W V ! . E 0 \cdot 1$

$A W 3 B(I W V I, I W V I, I W V I)=I W V I . E Q .1$

$A W V C=A W 1 C(I W V I)+A W 2 C(I W V I, I W V I)+A W 3 C(I W V I, I W V I, I W V I)$

$P 4520240$

$P 4520250$

$P 4520260$

RETURN

$C * \hbar * * *$ END END OF TEST SEGMENT 402

P4520270

P 4520280

$P 4520290$

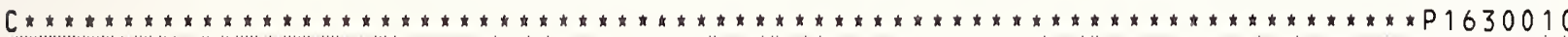

$C * * * * *$

$C * * * * *$

FIFCP - (163)

$P 1630020$

$C * * * * *$

$P 1630030$

P 1630040

$C * * * * *$

$C * * * * *$

$C * * * * *$

1.TO TEST INTEGER FUNCTIONS IN FULL FORTRAN

* 2.THIS SEGMENT COMPLETES SEGMENT (161) IN ORDER TO TEST

$[* * * * *$ FOR ALL FEATURES REQUIRED IN FULL FORTRAN.

C****3.DUMMY ARGUMENTS CAN BE INTEGER(TESTED IN 161), REAL (TESTED

$C * * * * *$ IN 161), DOUBLE PRECISION, COMPLEX, LOGICAL, OR EXTERNAL PROCE

$C * * *$ 4.DUMMY ARGUMENTS MAY BE REDIFINED IN SUBPROGRAM (ITEM 4)

$C * * * *$ 5. IN REFERENCE, ACTUAL ARGUMENTS MAY BE AS IN (161) AND BESIDES

$C * * * *$ EXTERNAL PROCEDURE. IN THIS CASE,EXTERNAL PROCEDURE IS

$C * * * *$ REFERENCED BY AN EXTERNAL STATEMENT.

$C * * * *$ 6. USE CAN BE MADE OF ADJUSTABLE DIMENSION.

$C * * * *$ RESTRICTIONS OBSERVED

C****1.ITEMS $(1),(2),(3),(5)$, OF 8.3 .1

$C * * * 2$ PARAGRAPH 8.3 .2 . LINE 18 TO END OF PARAGRAPH

C***** THIS SEGMENT USES 5 INTEGER FUNCTIONS

C***** THIS SEGMENT IS TO BE RUN WITH SEGMENTS

$C * * * * * 403,423,433,443,453$ $C * * * * \quad$ WHICH CONTAINS ALL FUNCTIONS BEING TESTED HERE

WHICH

$C * \# * * *$

$C * * * *$ S P E C I F I C A T I O N S SEGMENT 163
$P 1630050$

ASA REF P1630060

$P 1630070$

P 1630080

8.3.1P1630090

$P 1630100$

P 1630110

$P 1630120$

$P 1630130$

$P 1630140$

P1630150

P1630160

P 1630170

P1630180

P1630190

P 1630200

P 1630210

P 1630220

$P 1630230$

P1630240

P1630250 


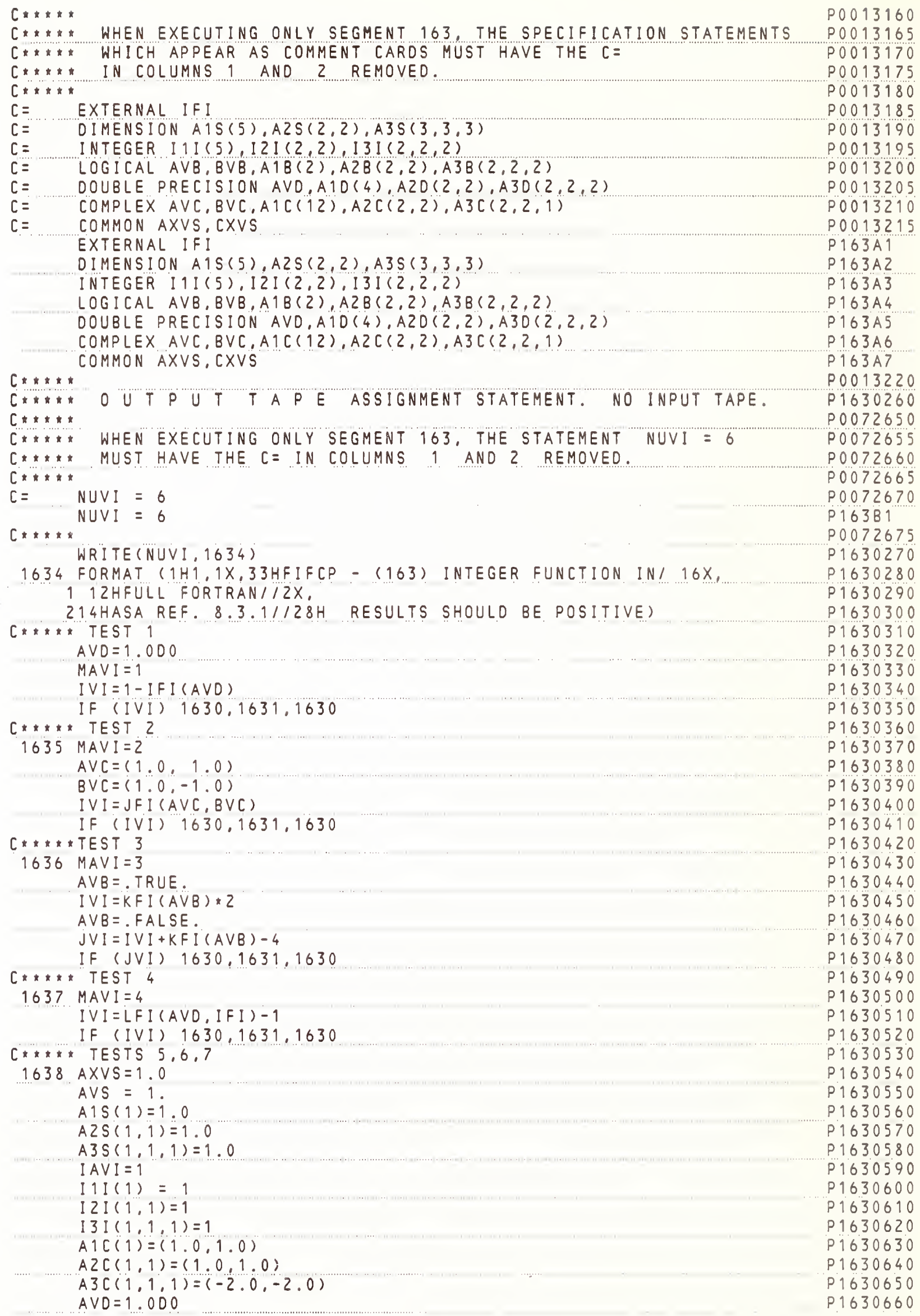


$A 10(1)=1.000$

P 1630670

$A 2 D(1,1)=1.000$

P 1630680

$A 3 D(1,1,1)=1.000$

$P 1630690$

$I V I=M F 1$ (AVS, IAVI, AVB, AVC,AVD,A1S,A2S,A3S, I11, I21, I3I, A1B,A2B,A3B, P1630700

$1 A 1 C, A 2 C, A 3 C, A 1 D, A 2 D, A 3 D, I F I)$

MAVI $=5$

1639 MAVI $=6$

$B \vee B=A \vee B$. $A N D \cdot A 1 B(1) \cdot A N D \cdot A 2 B(1,1)$. AND.A3B $(1,1,1)$

P1630710

$P\{630720$

P1630730

P1630740

P1630750

IF (BVB) GO TO 1631

IF (.NOT.BVB) GO TO 1630

7007 IVI $=$ REAL (AVC)

$J \vee I=A I M A G(A \vee C)$

MAVI = ?

IF (IVI+JVI) $1630,1631,1630$

1630 WRITE (NUVI, 1632) MAVI

GO TO 7008

1631 WRITE (NUVI.1633) MAVI

1632 FORMAT ( / / $2 X$, SHTEST .I2.12H IS NEGATIVE)

1633 FORMAT ( / /2X,5HTEST I $12,12 \mathrm{H}$ IS POSITIVE)

7008 GOTO(1635.1636.1637.1638.1639.7007.7009), MAVI

7009 CONTINUE

$C * * * * *$ END OF TEST SEGMENT 163

$C * * * *$ WHEN EXECUTING ONLY SEGMENT 163, THE STOP AND END CARDS

$C * * *$ WHICH APPEAR AS COMMENT CAROS MÜST HAVE THE $C=$

$C * * * *$ IN COLUMNS 1 AND 2 REMOVED.

$C=S T O P$

$C=\quad$ END

STOP

$P 1630760$

$P 1630770$

$P 1630780$

P 1630790

P1630800

$P 1630810$

P1630820

$P 1630830$

$P 1630840$

$P 1630850$

P1630860

$P 1630870$

$P 1630880$

P1630890

$P 1630900$

P1630910

P 1630920

P1630930

P1630940

P163C1

END

$P 163 C 2$

$C * * * * * * *$

$P 4030010$

$C \neq \pm \geqslant * *$

$I F I-(403)$

$P 4030020$

$P 4030030$

$P 4030040$

C*****

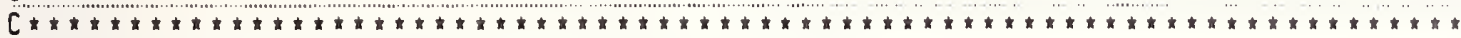

$C * * * *$ INTEGER FUNCTION OF DOUBLE PRECISION ARGUMENT(TEST 1 ).

FUNCTION IFI (AWVO)

DOUBLE PRECISION AWVD

I $F I=A W V D$

RETURN

END

$P 4030050$

$P 4030060$

$P 4030070$

$P 4030080$

$P 4030090$

$P 4030100$

$P 4030110$

C

$C * * * * *$

$J F I-(423)$

$P 4230020$

$C * * * *$

$P 4230030$

$P 4230040$

C*x***

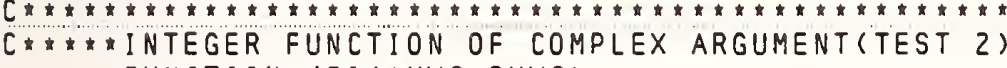

FUNCTION JFI (AWVC,BWVC)

COMPLEX AWVC,BWVC, CVC

$C V C=A W V C * B W V C$

$J F I=A I M A G(C \vee C)$

RETURN

END

$P 4230050$

$P 4230070$

$P 4230080$

$P 4230090$

$P 4230100$

$P 4230110$

$P 4230120$

$C * * \star * * * *$

$P 4330010$

$C * \pm \pm * *$

$C * \pm * * *$

$K F I-(433)$

$P 4330020$

$P 4330030$

$P 4330040$

$C \star \star \star * \star *$

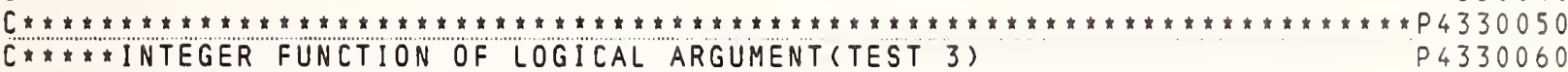

FUNCTION KFI (AWVB)

LOGICAL AWVB

$P 4330070$

$P 4330080$

$P 4330090$

IF (AWVB) GO TO 4331

4330 IF (.NOT.AWVB) GO TO 4332

RETURN

$4331 \mathrm{KFI}=2$

GO TO 4330

$4332 \mathrm{KFI}=0$

RETURN

P4330100

$P 4330110$

$P 4330120$

$P 4330130$

$P 4330140$

$P 4330150$ 


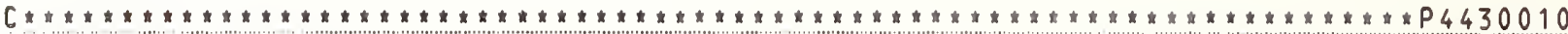

$C * * *$ $L F I-(443)$

$P 4430020$

$C * * * t$

$P 4430030$

$C * * * *$

$P 4430040$

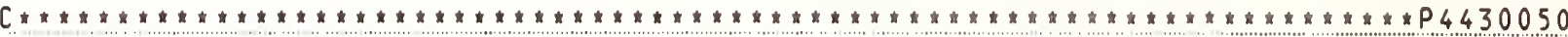

C***INTEGER FUNCTION OF EXTERNAL PROCEDURE(TEST 4 )

FUNCTION LFI (BWVD, IWFI)

$P 4430070$

DOUBLE PRECISION BWVD

$P 4430080$

$L F I=I W F I(B W V D)$

$P 4430090$

RETURN

$P 4430100$

END

$P 4430110$

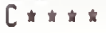

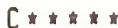

$[* * * *$

$M F I-(453)$

P 4530020

$C * * * *$

$P 4530030$

C****INTEGER FUNCTION OF DIFFERENT TYPES OF ARGUMENTS. USE IS MADE OF P 4530060

$C * * *$ ADJUSTABLE DIMENSION (TEST $5,6,7)$

FUNCTION MFI (AWVS, IWVI, AWVB, AWVC, AWVD,AW1S, AW2S, AW3S, IW1I, IWZI,

$P 4530070$

$P 4530080$

1 IW3 I, AW1B, AW2B, AW3B, AW1C, AW2C, AW3C, AW 1D, AW2D, AW3D, IWFI)

DOUBLE PRECISION AWVD, AW 1D, AW2D, AW3D

$L O G I C A L$ AWVB, AW1B,AW2B,AW3B

COMPLEX AWVC,AW1C, AW2C, AW 3 C

$P 4530090$

$P 4530100$

$P 4530110$

$P 4530120$

DIMENSION AW1S(IWVI), AWZS(IWVI, IWVI),AW3S(IWVI, IWVI,IWVI)

$P 4530130$

2

IWII(IWVI), IWZI(IWVI, IWVI), IWJI(IWVI, IWVI, IWVI)

$P 4530140$

AWIB (IWVI),AWZB (IWVI, IWVI),AW3B (IWVI, IWVI, IWVI)。

$P 4530150$

$P 4530160$

COMMON BXVS

AW1C(IWVI), AW2C(IWVI, IWVI),AW3C(IWVI, IWVI,IWVI)

P4530170

$P 4530180$

MFI =AWVS IWVI +AWIS(IWVI)*IWII(IWVI)-AWZS(IWVI,IWVI)*IWZI

$P 4530190$

1 (IWVI,IWVI)+AW3S(IWVI,IWVI,IWVI)*\#IW3I(IWVI,IWVI,IWVI) - AWVD+

$P 4530200$

2 AW 10(IWVI)-AW2D(IWVI, IWVI)-AW3D(IWVI, IWVI, IWVI) +BXVS* IWFI(AWVD)

$3-1.0$

$A W V B=I W V I . E 0.1$

$A W 1 B(I W V I)=I W V I . E Q .1$

$A W 2 B(I W V I, I W V I)=I W V I . E 0.1$

$P 4530210$

$P 4530220$

$P 4530230$

$P 4530240$

$P 4530250$

$P 4530260$

$A W 3 B$ (IWVI, IWVI, IWVI) = IWVI.EQ.1

$P 4530270$

$A W V C=A W 1 C(I W V I)+A W 2 C(I W V I, I W V I)+A W 3 C(I W V I, I W V I$,IWVI)

RETURN

END

$P 4530280$

$P 4530290$

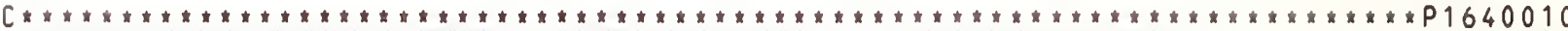

$C \div * *$

$C F C C P-(164)$

$P 1640020$

$C * * * *$

$P 1640030$

P1640040

C GENERAL̈ PURPOSE

$[* * * *$

C**** 1.TO TEST COMPLEX FUNCTIONS IN FULL FORTRAN

$C * * * *$

$C * * * *$

2. DUMMY ARGUMENTS ARE REAL, INTEGER, COMPLEX, LOGICAL

DOUBLE PRECISION, EXTERNAL PROCEDURE, ARRAY NAME.

C*:*:3.FUNCTIONS CONTAIN UP TO 20 ARGUMENTS

C***** 4. IN REFERENCE ACTUAL ARGUMENTS ARE VARIABLE NAME

$C * * *$ ARRAY NAME, ARRAY ELEMENT NAME, ARITHMETIC EXPRESSION

C**** EXTERNAL PROCEDURE

C*** 6. USE CAN BE MADE OF AOJUSTABLE DIMENTION

C*\#* 7.ARGUMENTS CAN BE PASSED THROUGH COMMON

C*****RESTRICTIONS OBSERVED

C***** 1.ITEMS (2), (3), (4), (5), (6) OF PARAGRAPH

C**** 2.LAST SENTENCE OF PARAGRAPH 3.2

$C * * * *$ THIS SEGMENT USES \& COMPLEX FUNCTIONS

C**** THIS SEGMENT IS TO BE RUN WITH SEGMENTS

$C * * * * 404,414,424,434,444,454,464$

C**** WHICH CONTAIN ALL FUNCTIONS BEING TESTED HERE

$C * * * *$

Cथा in

$[* * * *$

C *\#\#*

SP E C I F I C A T I ON S SEGMENT 164

ASA REFP 1640060 8.3.1 P1640070

P1640080

P1640090

P1640100

P1640110

P1640120

P1640130

P 1640140

$P 1640150$

P1640160

P1640170

P1640180

P1640190

P1640200

P 1640210

P 1640220

$P 1640230$

P1640240

$P 0013230$

WHEN EXECUTING ONLY SEGMENT 164, THE SPECIFICATION STATEMENTS

$P 0013235$

$P 0013240$ 
$C=\quad \quad$ OGICAL AVB,A1B(2),A3B(2,2,2),AZB(2,2), BVB

$C=\quad$ DOUBLE PRECISION AVD,AID (4),AZD $(2,2), A 3 D(2,2,2)$

$C=\quad C O M P L E X A F C, B F C, C F C, D F C, E F C, F F C, H F C, A V C, B \vee C$

$C=1, A 1 C(12), A Z C(2,2), A 3 C(2,2,1)$

$C=\quad$ COMMON AXVS, CXVS

$C=\quad$ EXTERNAL BFC

DIMENSION A1S $(5), A Z S(2,2), A 3 S(3,3,3)$

$P 0013260$

$P 0013265$

$P 0013270$

$P 0013275$

$P 0013280$

$P 0013285$

$P 0013290$

INTEGER I1I(5), I I $(2,2), I 3 I(2,2,2)$

$L O G I C A L A \cup B, A\} B(2), A 3 B(2,2,2), A Z B(2,2), B \vee B$

DOUBLE PRECISION AVD,A1D(4),A2D(2,2),A3D(2,2,2)

COMPLEX AFC,BFC, CFC, DFC, EFC, FFC, HFC, AVC, BVC

P $164 \mathrm{~A} 1$

P $164 \mathrm{AZ}$

P $164 \mathrm{A3}$

P $164 A 4$

1, $A 1 C(12), A 2 C(2,2), A 3 C(2,2,1)$

COMMON AXVS, CXVS

EXTERNAL BFC

\section{$C * * * * *$}

EXTERNAL BFC

$C * * * * 0 \cup T P U T$ T A P E ASSIGNMENT STATEMENT.. NO INPUT TAPE.

P 164 A5

$P 164 A 6$

P $164 \mathrm{~A} 7$

P164A8

$P 0013295$

$C * * * * *$

$C * * * * *$

WHEN EXECUTING ONLY SEGMENT 164. THE STATEMENT NUV! $=6$

P1640250

$C * * * * *$

MUST HAVE THE C = IN COLUMNS 1 AND 2 REMOVED.

$C * * * * *$

$C=\quad$ NUVI $=6$

NUVI $=6$

$C * * * * *$

WRITE (NUVI, 1641)

1641 FORMAT $(1 H 1,1 X, 31 H C F C C P$ - (164) COMPLEX FUNCTIONS//2X,

1 21HASA REFS. 8.3.1.8.3.21/2X,7HRESULTS)

$C * * *$ TEST 1

$B \vee C=A F C(1,0)$

$M A V I=1$

WRITE (NUVI, 1642) BVC, MAVI

1642 FORMAT( 1 HO, $2 F 5.1 .9 \mathrm{H}$ - TEST.12.2OH POSITIVE IF $0.0,0.0)$

$C * * * *$ TEST 2

MAVI $=2$

$B \vee C=B F C(1)-(1.0,1.0)$

WRITE (NUVI, 1642 ) BVC, MAVI

$C * * * * *$ TEST 3

MAVI $=3$

A $1 S(1)=1.0$

$A 1 S(2)=1.0$

$B \vee C=C F C(A 1 S)$

WRITE (NUVI, 1642)BVC, MAVI

$C * * * *$ TEST 4

$M A V I=4$

$B V C=D F C(1.00)$

WRITE (NUVI, 1642 ) BVC, MAVI

$C * * * *$ TEST 5

MAVI $=5$

$A \vee C=(1.0,1.0)$

$B \vee C=E F C(A \vee C)$

WR ITE (NUVI, 1642 ) BVC, MAVI

$C * * * * *$ TEST 6

$M A V I=6$

$A \vee B=. T R U E$.

$B \vee C=F F C(A \vee B)-(1,0,1.0)$

WRITE(NUVI, 1642) BVC, MAVI

$C * * *$ TEST 7

MAVI $=7$

$A \vee B=. F A L S E$.

$B \vee C=F F C(A \vee B)$

WR ITE (NUVI, 1642) BVC, MAVI

$C * \#$ TEST $8,9,10$

I $V I=1$

$A \vee D=1.000$

$A 10(1)=1.000$

P0072685

P 0072690

$P 0072695$

$P 0072700$

P $164 \mathrm{~B} 1$

$P 0072705$

$P 1640260$

$P 1640270$

$P 1640280$

$P 1640290$

$P 1640300$

P1640310

$P 1640320$

P1640330

P1640340

$P 1640350$

P1640360

$P 1640370$

$P 1640380$

P1640390

P1640400

P1640410

P 1640420

P1640430

P1640440

P1640450

P1640460

P1640470

P1640480

P1640490

P1640500

P1640510

P1640520

P1640530

$P 1640540$

P1640550

P1640560

P1640570

$P 1640580$

P1640590

P1640600

P1640610

P 1640620

P1640630

P1640640

P1640650

P1640660 
$A \vee S=1.0$

P1640690

A $1 S(1)=1.0$

P1640700

$\operatorname{AZS}(1,1)=1.0$

$\operatorname{A} 3 S(1,1,1)=1.0$

P1640710

$A 1 C(1)=(1,0,1.0)$

P1640720

$A Z C(1,1)=(1.0,1.0)$

P1640730

$A 3 C(1,1,1)=(1.0,1.0)$

P1640740

I 1 I $(1)=1$

I 2 I $(1,1)=1$

I $3 I(1,1,1)=1$

$A V C=(0.0,0.0)$

P1640750

P1640760

P1640770

$B \vee C=H F C(A \vee S, I V I, A V B, A V C, A \vee D, A 1 S, A 2 S, A 3 S, I 11,12 I, I 3 I, A 1 B, A 2 B, A 3 B$,

$1 A 1 C, A Z C, A 3 C, A 1 D, A Z D, A 3 D, B F C)$

MAVI $=8$

WRITE (NUVI, 1642) BVC, MAVI

MAVI $=9$

IF(AXVS) $1643,1644,1643$

1648 MAVI $=10$

$B \vee B=A \vee B, A N D, A 1 B(1), A N D, A Z B(1,1), A N D \cdot A 3 B(1,1,1)$

IF (BVB) GO TO 1644

1643 WRITE(NUVI, 1645 ) MAVI

GO TO 1647

1644 WRITE(NUVI, 1646)MAVI

1645 FORMAT (/15X,5HTEST, I2,12H IS NEGATIVE)

1646 FORMAT(/15X,5HTEST, I2,12H IS POS!TIVE)

1647 IF (MAVI - 9) $1649,1648,1649$

P 1640780

P 1640790

P 1640800

P 1640810

P1640820

P 1640830

P1640840

P 1640850

$P 1640860$

P1640870

P 1640880

P1640890

P1640900

$P 1640910$

P1640920

P1640930

P1640940

CONTINUE

C**** END OF TEST SEGMENT 164

$[* * * *$ WHEN EXECUTING ONLY SEGMENT 164, THE STOP AND END CARDS

$C * * * *$ WHICH APPEAR AS COMMENT CARDS MUST HAVE THE C $=$

C*****IN COLUMNS 1 AND 2 REMOVED.

$C=\operatorname{STOP}$

$c=\quad$ END

STOP

P1640950

P1640960

P1640970

P1640980

P 1640990

P 1641000

P1641010

P164C1

END

P164C2

$[* * * * *$
$[* * * *$

C*****

C*****

$P 4040010$

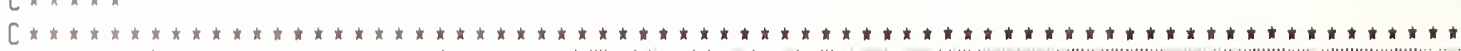

$[* * * *$ COMPLEX FUNCTION OF REAL ARGUMENT (TEST 1 )

COMPLEX FUNCTION AFC(AWVS)

$A F C=(-1.0,0.0)+A W V S$

RETURN

P4040020

P4040030

P4040040

END

P4040060

P4040070

P4040080

P4040090

P4040100

C

$[* * * *$

P4140020

$C * * * * \quad$ BFC - (414)

$[* * * * *$

P4140040

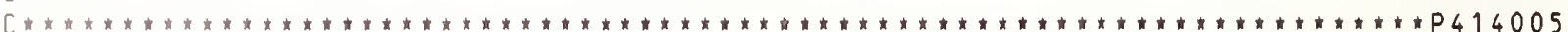

C**** *OMPLEX FUNCTION OF INTEGER ARGUMENT (TEST "2)

$P 4140060$

COMPLEX FUNCTION BFC(IWVI)

$P 4140070$

$B F C=(1.0,1.0) *$ IWVI

P 4140080

$P 4140090$

RETURN

$P 4140100$

END

-P4240010

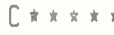

$P 4240020$

C*****

$C F C-(424)$

$P 4240030$

$[\star * * * *$

$P 4240040$

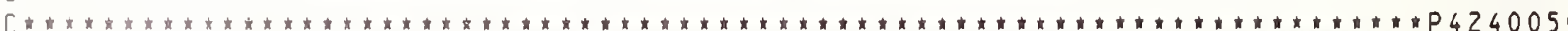

C****COMPLEX FUNCTION OF ARRAY NAME (TEST 3)

P4240060

COMPLEX FUNCTION CFC(AW1S)

P4240070

DIMENSION AW1S(Z)

$C F C=(2.0,0.0)-A W 1 S(1)-A W 1 S(2)$

RETURN

P 4240080

P4240090

$P 4240100$

END

$P 4240110$ 


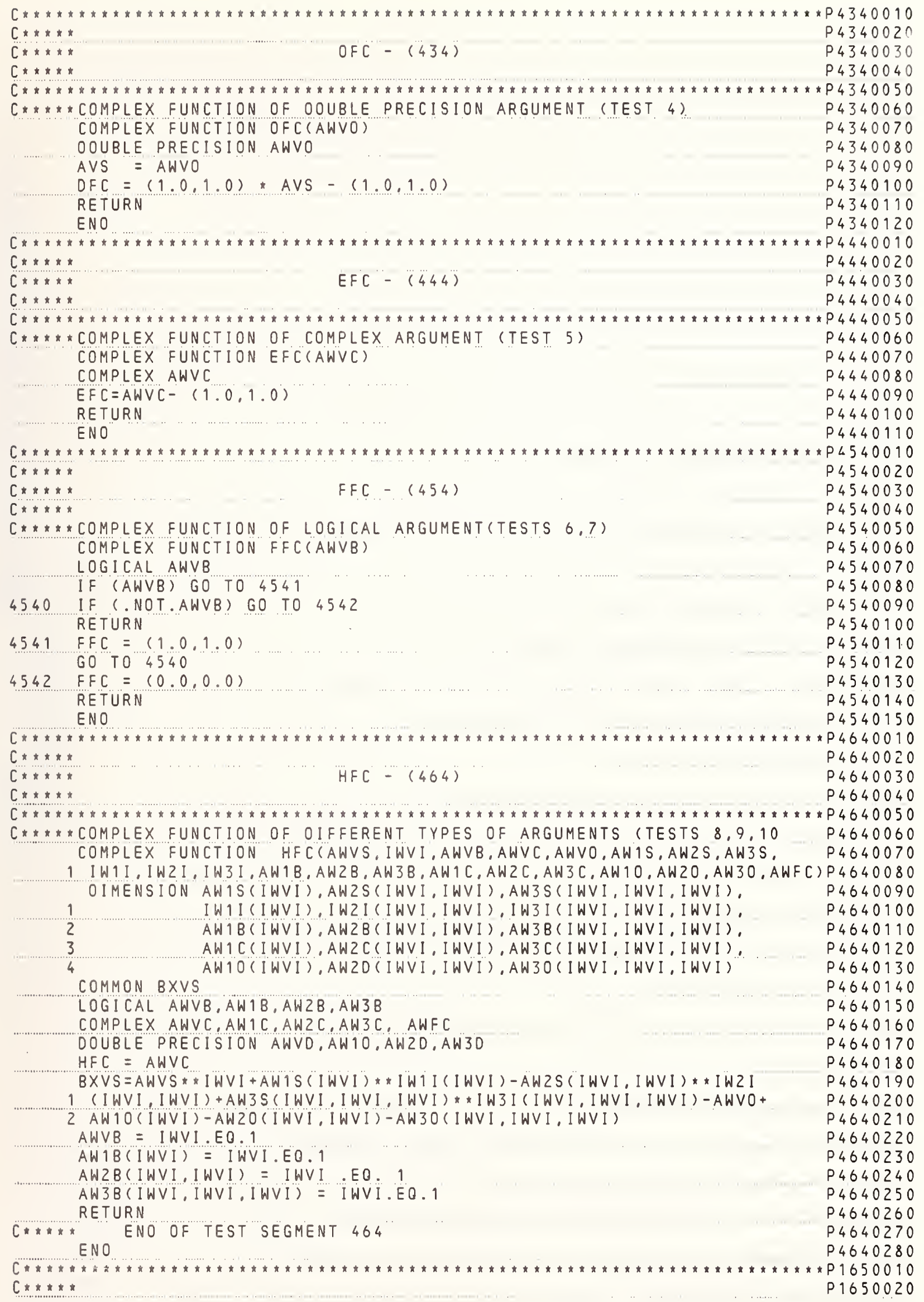


C***** DOUBLE PRECISION,EXTERNAL PROCEOURE, ARRAY NAME

C***** 3. FUNCTIONS CONTAIN UP TO 20 ARGUMENTS

C***** 4. IN REFERENCE, ACTUAL ARGUMENTS ARE VARIABLE1NAME。

8.3 .191650070

P1650080

$P 1650090$

P1650100

C*****RESTRICTIONS OBSERVED

C***** 1. ITEMS $(2),(3),(4),(5),(6)$ OF PARAGRAPH 8.3 .1

$2 P 1650120$

$C * * * * \quad 2$ LAST SENTENCE OF PARAGRAPH 3.2

$[* * * *$

$$
\text { TH }
$$

$C * * * * *$

$C * \star * * *$

$[* * * *$

$C * * * *$

$C * * * *$

$C * * * * *$

$C * * * *$

$C * * * * *$

$[* * * *$

$C=$ DIMENSION A1S( 5$), \operatorname{AZS}(2,2), \operatorname{A3S}(3,3,3)$

$C=\quad$ INTEGER I I (5), IZI(2,2), I I $I(2,2,2)$

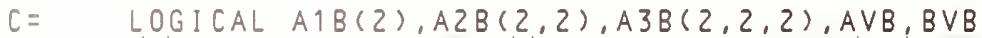

$C=$ DOUBLE PRECISION AFD, BFD, CFD, DFD, EFD, FFD, GFD, HFD,AVD

$C=1, A 1 D(4), A 2 D(2,2), A 3 D(2,2,2)$

$C=\quad$ COMPLEX AVC, A1C (12), AZC (2,2), A3C (2,2,1)

$C=\quad$ COMMON AXVS, CXVS

$C=\quad$ EXTERNAL CFD, AFD

DIMENSION A1S(5), AZS $(2,2), \operatorname{ABS}(3,3,3)$

$P 1650130$

P1650140

P 1650150

P1650160

$P 1650170$

P 1650180

P1650190

$P 1650200$

$P 0013300$

$P 0013305$

$P 0013310$

$P 0013315$

$P 0013320$

$P 0013325$

$P 0013330$

$P 0013335$

$P 0013340$

$P 0013345$

POO13350

$P 0013355$

INTEGER I I I (5), I I I $(2,2), I 3 I(2,2,2)$

$P 0013360$

$L O G I C A L A 1 B(2), A 2 B(2,2), A 3 B(2,2,2), A \vee B, B \vee B$

P $165 \mathrm{~A} 1$

DOUBLE PRECISION AFD, BFD, CFD, DFD, EFD, FFD, GFD, HFD,AVD

$P 165 A 2$

P $165 A 3$

1. $A 1 D(4), A 2 D(2,2), A 3 D(2,2,2)$

COMPLEX AVC, $A 1 C(12), A Z C(2,2), A 3 C(2,2,1)$

COMMON AXVS, CXVS

EXTERNAL , CFD, AFD

P165A4

P 165 A5

P165A6

P165A7

P165A8

P0013365

$C * * * *$

O U T P U T T A P E ASSIGNMENT STATEMENT. NO INPUT TÄ TAPE.

$P 1650210$

C**** WHEN EXECUTING ONLY SEGMENT 165, THE FOLLOWING STATEMENT

C***** NUVI $=6$ MUST HAVE THE $C=$ IN COLUMNS 1 AND 2 REMOVED.

$[* * * * *$

$C=\quad$ NUVI $=6$

NUVI $=6$

WRITE (NUVI, 1650)

$P 0072710$

$P 0072715$

$P 0072720$

$P 0072725$

P 165 B 1

$P 1650220$

1650 FORMAT(1H1,1X,30HDPFCP - (165) DOUBLE PRECISION! 16X, 9HFUNCTIONS P1650230

$1 / / 2 X, 21 H A S A$ REFS. $8=3.1,8.3 .2 / / 2 X, 7$ HRESULTS)

$C * * *$ TEST 1

$P 1650240$

P1650250

MAVI $=1$

$I V I=A F D(1.0)-1.000$

P 1650260

P 1650270

IF (IVI) $1652,1653,1652$

P 1650280

$[* * * *$ TEST 2

P 1650290

1657 MAVI $=2$

P1650300

$I V I=B F D(1)-1.000$

$P 1650310$

IF (IVI) 1652,1653,1652

$C * * * * *$ TEST 3

$P 1650320$

P1650330

1658 MAVI $=3$

P $1650340^{\circ}$

$A \vee D=1.000$

$I V I=C F D(A \vee D)-1.000$

IF (IVI) $1652,1653,1652$

C*\#** TEST 4. ONE ARGUMENT IS ARRAY ELEMENT NAME

P1650350

P1650360

$P 1650370$

P1650380

1659 MAVI $=4$

P1650390

$A V C=(1.0,1.0)$

$A 1 C(1)=(1.0,-1.0)$

P 1650400

P1650410

$I V I=D F D(A \vee C, A 1 C(1))$

$P 1650420$

IF (IVI) $1652,1653,1652$

P1650430 
7015 MAVI $=6$

$A \vee B=. F A L S E$.

$I V I=E F D(A \vee B)$

P1650490

P 1650500

IF(IVI) $1652.1653,1652$

C***** TEST ?

P1650510

$P 1650520$

$P 1650530$

7016 MAVI $=7$

$P 1650540$

$I V I=F F D(1 . E 0, A F D)-1.000$

IF (IVI) $1652,1653,1652$

\section{$C * * * *$ TEST 8}

$P 1650550$

$P 1650560$

P1650570

7017 MAVI $=8$

P1650580

$A 10(1)=1.000$

$A 10(2)=-1.000$

IVI $=G F D(A 1 D)$

IF (IVI) $1652,1653.1652$

$C * \approx *$ TESTS $9,10,11,12$

7018 IAVI $=1$

$A \vee O=1.000$

$A 10(1)=1.000$

$A 20(1,1)=1.000$

$A 30(1,1,1)=1.000$

$A V S=1.0$

$A 1 S(1)=1.0$

$A Z S(1,1)=1.0$

A $3 S(1,1,1)=1.0$

$A 1 C(1)=(1.0,1.0)$

$A 2 C(1,1)=(1.0,1.0)$

$A 3 C(1,1,1)=(1,0,1,0)$

II $(1)=1$

I $2 I(1,1)=1$

$13 I(1,1,1)=1$

MAVI $=9$

$P 1650590$

$P 1650600$

P1650610

$P 1650620$

$P 1650630$

$P 1650640$

$P 1650650$

$P 1650660$

$P 1650670$

$P 1650680$

P1650690

$P 1650700$

$P 1650710$

$P 1650720$

P 1650730

$P 1650740$

$P 1650750$

P1650760

P1650770

P 1650780

$P 1650790$

$I V I=H F D(A \vee S, I A V I, A \vee B, A V C, A V D, A 1 S, A 2 S, A 3 S, I 1 I, I 21, I 3 I, A 1 B, A 2 B, A 3 B, P 1650800$

$1 A 1 C, A 2 C, A 3 C, A 10, A 2 D, A 3 D, C F D)$

IF (IVI) $\quad 9652.1653,1652$

7019 MAVI $=10$

IVI $=A X V S$

IF (IVI) $1652,1653,1652$

7020 MAVI $=11$

WRITE (NUVI, 1656) AVC, MAVI

1656 FORMAT(//2F5.1//2X,5HTEST, 12,31H IS POSITIVE IF NUMBERS PRINTEOI $12 X, 17 H A B O V E$ ARE $0.0,0.0)$

7021 MAVI $=12$

$B \vee B=A \vee B \cdot A N D \cdot A 1 B(1) \cdot A N D \cdot A 2 B(1,1) \cdot A N D \cdot A 3 B(1,1,1)$

IF (BVB) GO TO 1653

$P 1650810$

$P 1650820$

$P 1650830$

P1650840

P 1650850

$P 1650860$

$P 1650870$

WRITE (NUVI, 1654 ) MAVI

GO TO 1651

1653 WRITE (NUVI, 1655 ) MAVI

1654 FORMAT ( $/ 2 X, 5$ HTEST .I2,12H IS NEGATIVE)

1655 FORMAT ( $/ 2 X, 5 H T E S T$. I2, $12 \mathrm{H}$ IS POSITIVE)

1651 GO TO (1657,1658,1659,7014,7015,7016,7017,7018,7019,7020,7021, $17022), M A V I$

7022 CONTINUE

\section{$C * * * * *$ END OF TEST SEGMENT 165}

C***** WHEN EXECUTING ONLY SEGMENT 165. THE STOP AND END CARDS

$C * * * *$ WHICH APPEAR AS COMMENT CAROS MUST HAVE THE $C=$

$C * *:$ IN COLUMNS 1 AND 2 REMOVEO.

$C=$ STOP

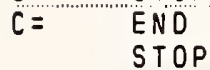

END

P1650890

P1650900

P 1650910

P 1650920

P 1650930

$P 1650940$

P 1650950

P 1650960

P1650970

P1650980

$P 1650990$

$P 1651000$

$P 1651010$

P1651020

P 1651030

P1651040

P1651050

P1651060

P165C1

P $165 \mathrm{CZ}$

$C * * * * *$
$[* * * * *$
$[* * * * *$

$\star \star \star *$

$P 4050010$

P4050020

$P 4050030$ 


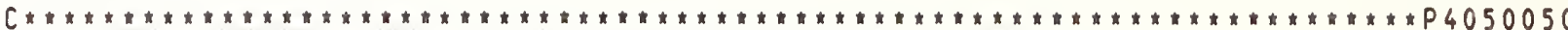

C***0OUBLE PRECISION FUNCTION OF REAL ARGUMENT (TEST 1) DOUBLE PRECISION FUNCTION AFO(AWVS) $\quad P 4050070$ $A F D=A W V S$

44050080

RETURN $P 4050090$

END

$P 4050100$

$C * * * * * * *$

$[* * * * *$

$[* * * * *$

$B F D-(415)$

$P 4150010$

$C * * * * *$

$P 4150020$

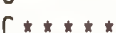
$P 4150030$

$C * * * *$ OOUBLE PRECISION FUNCTION OF INTEGER ARGUMENT(TESTZ)

P4150040

DOUBLE PRECISION FUNCTION BFD(IWVI)

$B F D=1.000 * \because$ IWVI

RETURN

$P 4150050$

$P 4150060$

$P 4150070$

P 4150080

$P 4150090$

END

P4150100

$[* * * * * *$

$C * * * * *$

$C F O-(425)$

$P 4250020$

C******

$P 4250030$

C $* * * *$

P4250040

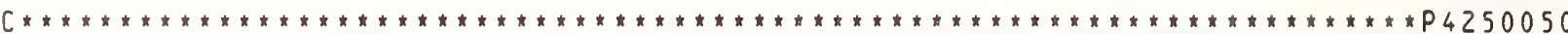

C**** DOUBLE PRECISION FUNCTION OF DOUBLE PRECISION ARGUMENT(TEST 3 ) P4250060 DOUBLE PRECISION FUNCTION CFO(AWVD)

DOUBLE PRECISION AWVD

$C F D=A W V D$

$P 4250070$

$P 4250080$

$P 4250090$

RETURN

$P 4250100$

END

$P 4250110$

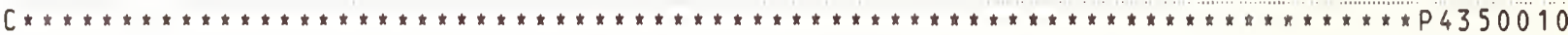

$[* * * *$

DFO $-(435)$

$P 4350020$

$[* t * * *$

$P 4350030$

$P 4350040$

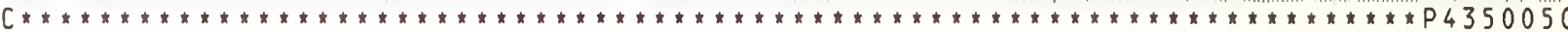

$C * * * *$ DOUBLE PRECISION FUNCTION OF COMPLEX ARGUMENT(TEST 4 )

DOUBLE PRECISION FUNCTION DFD(AWVC, BWVC)

$P 4350060$

COMPLEX AWVC, BWVC, CVC

$P 4350070$

$P 4350080$

$C V C=B W V C * A W V C$

$D F D=A I M A G(C V C)$

P4350090

RETURN

$P 4350100$

$P 4350110$

END

$P 4350120$

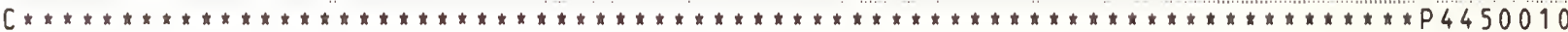

$C * * * * *$

$P 4450020$

$C * * * * *$

$E F D-(445)$

$P 4450030$

$C * * * * *$

$P 4450040$

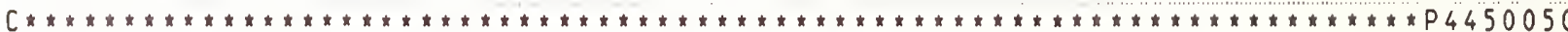

$C * * * *$ DOUBLE PRECISION FUNCTION OF LOGICAL ARGUMENT(TEST 5,6) P4450060

DOUBLE PRECISION FUNCTION EFD(AWVB)

$P 4450070$

LOGICAL AWVB

I $F$ (AWVB) GO TO 4451

4450 IF (.NOT.AWVB) GO TO 4452

RETURN

$4451 \quad E F O=1.000$

GO TO 4450

4452 EFD $=0.000$

$P 4450080$

P4450090

$P 4450100$

P 4450110

$P 4450120$

P4450130

$P 4450140$

RETURN

$P 4450150$

END

$P 4450160$

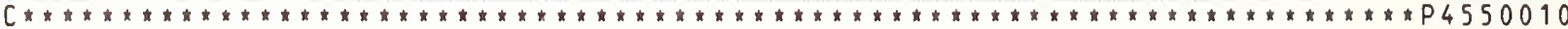

$[* * * * *$

$C * * * *$

$F F D-(455)$

$P 4550020$

P4550030

$P 4550040$

$C * * * *$

$P 4550050$

$C * * * *$ DOUBLE PRECISION FUNCTION OF EXTERNAL PROCEDURE (TEST 7 )

$P 4550060$

DOUBLE PRECISION FUNCTION FFO(BWVS, BWFD)

$P 4550070$

DOUBLE PRECISION BWFD

P 4550080

$F F D=B W F D$ (BWVS)

$P 4550090$

RETURN

$P 4550100$

END

P4550110

$C * t+* * t * t$

84550010 
$C * *$ DOUBLE PRECISION FUNCTION OF ARRAY NAME (TEST 8) DOUBLE PRECISION FUNCTION GFD(AWID) $\quad$ P4650070

OIMENSION AWID(2)

$P 4650070$

OOUBLE PRECISION AW 10

$P 4650090$

$G F O=A W 10(1)+A W 10(2)$

RETURN

$P 4650100$

ENO

$P 4650110$

$P 4650120$

$C * * * * * *$

$P 4750010$

$C * * * * *$

$P 4750020$

$C * * * *$

$H F O-(475)$

$P 4750030$

$P 4750040$

$C * \|$

$P 4750050$

C***DOUBLE PRECISION FUNCTION OF OIFFERENT TYPES OF ARGUMENTS.USE CAN P4750060

$C * * *$ BE MAOE OF AOJUSTABLE OIMENSION.SOME ARGUMENTS CAN BE PASSED

$P 4750070$

$C * * *$ THROUGH A COMMON STATEMENT.

$P 4750080$

DOUBLE PRECISION FUNCTION HFDLAWVS, IWVI, AWVB, AWVC, AWVD, AW1S,AW2S, P4750090

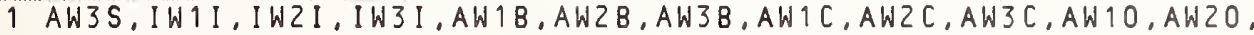

$2 A W 3 D, C W F O)$

DIMENSION AWIS(IWVI), AWZS(IWVI, IWVI), AWZS(IWVI, IWVI, IWVI),

$P 4750100$

1

IWI!(IWVI), IWZ!(IWVI, IWVI), IW3I(IWVI, IWVI, IWVI),

$P 4750110$

$P 4750120$

$P 4750130$

AW1C(IWVI), AW2C(IWVI, IWVI), AW3C(IWVI, IWVI, IWVI),

$P 4750140$

AW10(IWVI), AW20(IWVI, IWVI),AW30(IWVI, IWVI, IWVI),

$P 4750150$

AW 18(IWVI), AW2B(IWVI, IWVI), AW3B(IWVI, IWVI, IWVI)

$P 4750160$

OOUBLE PRECISION AWVO,AW10, AW20, AW30, CWFO

COMPLEX AWVC, AW $1 C, A W Z C, A W Z C$

REAL AW1S, AWZS, AW3S

LOGICAL. AWVB, AW1B, AW2B,AW3B

COMMON BXVS

HFO = AWVD - AW1D(IWVI) + AW20(IWVI, IWVI) - AW3D(IWVI, IWVI, IWVI)

$1+C W F D(A W V O)-1.000$

$A W V C=A W 1 C(I W V I)+A W 2 C(I W V I, I W V I)-A W 3 C(I W V I, I W V I, I W V I)-(1.0,1.0)$

BXVS = AWVS* *IWVI-AW1S(IWVI)* IWII(IWVI)+AW2S(IWVI,IWVI)* IWZI

1 (IWVI, IWVI)-AW3S(IWVI, IWVI, IWVI)* IWJI(IWVI, IWVI, IWVI)

$A W \vee B=I W V I . E 0.1$

$A W 1 B(I W V I)=I W V I . E O .1$

$A W 2 B(I W V I, I W V I)=I W V I . E 0.1$

$A W 3 B(I W V I, I W V I, I W V I)=I W V I . E 0.1$

RETURN

P 4750170

$P 4750180$

$P 4750190$

$P 4750200$

$P 4750210$

$P 4750220$

$P 4750230$

$P 4750240$

$P 4750250$

$P 4750260$

$P 4750270$

$P 4750280$

$P 4750290$

$P 4750300$

$P 4750310$

ENO

$P 4750320$

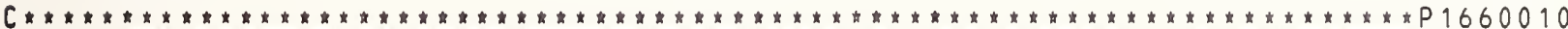

\section{$C * * * *$}

$C * * * *$

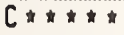

$B F C C P-(166)$
$C * \star \star \star *$

$C * * * *$

C****t

$C * * \star *$

$C * * * *$

$C \star \star \star \star *$

$C * * * *$

$C * * * *$

$C * * * *$

$C * * *$

$C * * * *$

$C * * *$

$C * * *$

$C * * *$

$C * * *$

$C * * * *$

$C * * *$

$C * * * *$

$C * * * *$

$C * \star * *$
$P 1660020$

P 1660030

P 1660040

GENERAL PURPOSE

P1660060

P 1660070

P 1660080

P 1660090

P 1660100

$P 1660110$

P 1660120

$P 1660130$

$P 1660140$

P 1660150

P 1660160

$P 1660170$

P 1660180

$P 1660190$

WHICH

$406,416,426,436,446,456,466,476$

CONTAINS ALL FUNCTIONS BEING TESTEO HERE.

$P 1660200$

$P 1660210$

$P 1660220$

$P 1660230$

$P 1660240$

$P 0013370$ 
C**** WHEN EXECUTING ONLY SEGMENT 166. THE SPECIFICATION STATEMENTS

C*** WHICH APPEAR AS COMMENTS MUST HAVE THE $C=$

C**** IN COLUMNS 1 AND 2 REMOVED.

$C * * * *$

$C=$ DIMENSIONATS(5),AZS $(2,2)$, A3S $(3,3,3)$

$C=\quad$ INTEGER III(5), I2I(2,2), I $I(2,2,2)$

$C=\quad L O G \perp C A L A V B, A F B, B F B, C F B, O F B, E F B, F F B, G F B, H F B$

$C=1, A 1 B(2), A Z B(2,2), A 3 B(2,2,2)$

$C=$ DOUBLE PRECISION AVD, A1D (4),AZD $(2,2), A 3 D(2,2,2)$

$C=\quad$ COMPLEX AVC,A\}C $(12), A Z C(2,2), A 3 C(2,2,1)$

$C=\quad$ COMMON AXVS, CXVS

$C=\quad$ EXTERNAL AFB

DIMENSION A1S(5), AZS (2,2), A3S $(3,3,3)$

INTEGER I1 $1(5), 121(2,2), 131(2,2,2)$

LOGICAL AVB, AFB,BFB, CFB, DFB, EFB, FFB, GFB, HFB

1, $A 1 B(2), A 2 B(2,2), A 3 B(2,2,2)$

DOUBLE PRECISION AVD,A1O(4),A2D(2,2),A30(2,2,2)

COMPLEX $A \vee C, A 1 C(12), A 2 C(2,2), A 3 C(2,2,1)$

COMMON AXVS, CXVS

EXTERNAL AFB

$C * * * * *$

$C * * * * *$

C******?

$C * * * * *$

$C * * * *$

$C=$

C $=$ NUVI $=6$

NUVI $=6$

MAVI $=1$

WR ITE (NUVI, 1662)

1662 FORMAT $(1 H 1,1 X, 31$ HBFCCP - (166) LOGICAL FUNCTIONS/ $/ 2 X$,

1 13HASA REF $8.3 .1 / / 2 X, 7$ HRESULTS $)$

$A \vee B=A F B(1.0)$

If (AVB) GO TO 1664

WRITE (NUVI,1661) MAVI

GO TO 1665

1660 FORMAT (17H TEST, I2,12H IS POSITIVE)

1661 FORMAT (17H TEST. I2,12H IS NEGATIVE)

1664 WRITE (NUVI,1660) MAVI

GO TO $(1665,1666,1667,1668,1669,7030,7031,7032,7033,7034)$, MAVI

C**** LOGICAL FUNCTION OF INTEGER ARGUMENT (TEST 2)

1665 MAVI $=2$

$A \vee B=B F B(1)$

IF (AVB) GO TO 1664

WRITE (NUVI, 1661) MAVI

C****LOGICAL FUNCTION OF DOUBLE PRECISION ARGUMENT(TEST 3 )

1666 MAVI $=3$

$A \vee D=1.000$

$A \vee B=C F B(A \vee D)$

IF (AVB) GO TO 1664

WRITE (NUVI, 1661) MAVI

C** LOGICAL FUNCTION OF LOGICAL ARGUMENT(TEST 4 )

1667 MAVI $=4$

$A \vee B=O F B$ (.TRUE.)

IF (AVB) GO TO 1664

WRITE(NUVI, 1661) MAVI

C*\# LOGICAL FUNCTION OF COMPLEX ARGUMENT(TEST 5)

$1668 \quad M A V I=5$

$A \vee B=E F B((1.0,1.0))$

IF (AVB) $G 0$ TO 1664

WRITE(NUVI, 1661) MAVI

C*** LOGICAL FUNCTION OF ARRAY NAME (TEST 6)

1669 MAVI $=6$

$A 1 S(1)=1.0$

$A 1 S(Z)=0.0$

$A \vee B=F F B(A 1 S)$

If ( $A \vee B$ ) $G 0$ TO 1664

WRITE (NUVI, 1661) MAVI

C***** LOGICAL FUNCTION OF EXTERNAL PROCEDURE(TEST 7)
$P 0013375$

$P 0013380$

P0013385

POO 013390

P0013395

$P 0013400$

P0013405

$P 0013410$

P0013415

$P 0013420$

P0013425

$P 0013430$

P166A1

P166A2

P166A3

P166A4

P166A5

P166A6

P166A7

P166A8

P0013435

P1660250

P0072730

$P 0072735$

P0072740

P 0072745

P16681

P 9660260

P 1660270

P1660280

P1660290

$P 1660300$

P 1660310

$P 1660320$

P 1660330

$P 1660340$

P 1660350

$P 1660360$

P1660370

P 1660380

P 1660390

P1660400

P1660410

P 1660420

P 1660430

P1660440

P 1660450

P1660460

P1660470

P1660480

P1 1660490

P1660500

P1660510

P1660520

P 1660530

P1660540

P1660550

$P 1660560$

P1660570

P 1660580

P1660590

P1660600

P 1660610

P 1660620

P1660630

P 1660640

P1660650

P1660660 
$C * * *$ * LOGICAL FUNCTION OF DIFFERENT TYPES OF ARGUMENTS

7031 MAVI $=8$

$A \vee D=1.000$

$A V C=(1.0,1.0)$

$I A V I=1$

$A V B=. T R U E$.

$A 1 B(1)=$.TRUE.

$A Z B(1,1)=$.TRUE.

$A 3 B(1,1,1)=$. TRUE.

$A 1 C(1)=(1.0, \uparrow .0)$

$A 2 C(1,1)=(1,0,1,0)$

$A 3 C(1,1,1)=(-2.0,-2.0)$

$A 1 D(1)=1.000$

$A 2 D(1,1)=1.0 D 0$

$A 3 D(1,1,1)=-2.000$

I 1 I $(1)=1$

$121(1,1)=1$

$13 I(1,1,1)=1$

$\operatorname{Ais}(1)=1.0$

$\operatorname{AZS}(1,1)=1.0$

A $3 S(1,1,1)=1.0$

$A X V S=1.0$

$A V B=H F B(A \vee S, I A V I, A \vee B, A V D, A V C, A 1 S, A 2 S, A 3 S, 111,121,131, A 1 B, A 2 B$,

$1 A 3 B, A 1 C, A 2 C, A 3 C, A 10, A 2 D, A 3 D, A F B)$

IF (AVB) GO TO 1664

WRITE (NUVI, 1661) MAVI

7032 MAVI $=9$

$I A \vee I=A \vee D$

IF (IAVI.EO.O) GO TO 1664

WRITE (NUVI, 1661) MAVI

$7033 \quad$ IAVI $=1$

$M A V I=10$

$I A V I=A V S$

IF(IAVI.EO.0) GO TO 1664

WRITE (NUVI.1661) MAVI

$7034 \quad \mathrm{MAVI}=11$

WRITE(NUVI, 1663) AVC, MAVI

$P 1660700$

P1660710

P1660720

P 1660730

P 1660740

P 1660750

$P 1660760$

P1660770

P 1660780

P 1660790

P 1660800

$P 1660810$

P 1660820

P1660830

P1660840

P1660850

P1660860

P 1660870

P 1660880

P1660890

P1660900

P1660910

P1660920

P 1660930

P 1660940

P 1660950

P 1660960

P1660970

P1 1660980

P1660990

P 1661000

P1661010

P1661020

P1661030

P 1661040

P1661050

P 1661060

P 1661070

1663 FORMAT (//2F8.4//7H TEST, 12,31H IS POSITIVE IF NUMBERS PRINTEO/ P1661080

$119 \mathrm{H}$ ABOVE ARE $0.0,0.0112 \mathrm{X}, 12 \mathrm{HEND}$ OF (166)) $\quad$ P1661090

$[* * * * \quad$ END OF TEST SEGMENT 166

C**** WHEN EXECUTING ONLY SEGMENT 166 . THE STOP AND END CAROS

$C * * *$ WHICH APPEAR AS COMMENT CARDS MUST HAVE THE $C=$ IN

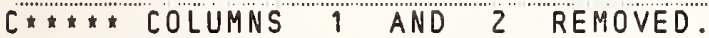

$C=S T O P$

$C=E N D$

STOP

P 1661100

P1661110

P1661120

P1661130

P1661140

P1661150

P166C1

END

P166C2

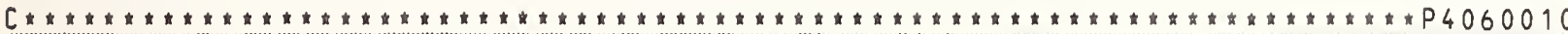

$C * * * *$

$A F B=(406)$

$P 4060020$

C $* * * *$

$P 4060030$

$P 4060040$

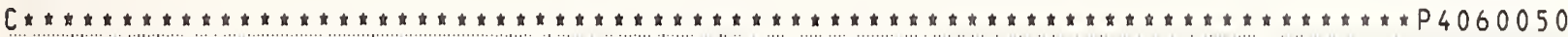

C*\#**LOGICAL FUNCTION OF REAL ARGUMENT" (TEST 1)

$P 4060060$

LOGICAL FUNCTION AFB(AWVS)

$P 4060070$

$A F B=A W V S . G T .0 .0$

$P 4060080$

RETURN

END

P4060090

$P 4060100$

C

$C * * * *$

$B F B-(416)$

$P 4160020$

$C * * * *$

$P 4160030$

$C * * * * *$

$P 4160040$

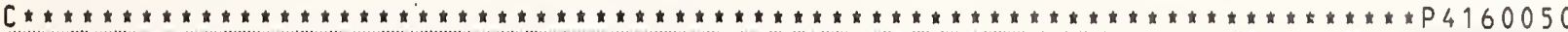

C*\#LOGICAL FUNCTION OF INTEGER ARGUMENT (TEST 2 )

$P 4160060$

LOGICAL FUNCTION BFB(IWVI)

$P 4160070$ 
$B F B=$ IWVI.GT.0

$P 4160080$

RETURN

$P 4160090$

END

P4 460100

$C * * * * *$
$C * * * *$

$C * * * *$

$C F B-(426)$

$P 4260020$

C*****

$P 4260030$

$C * * * *$

$P 4260040$

C****LOGICAL FUNCTION OF DOUBLE PRECISION ARGUMENT(TEST 3 )

LOGICAL FUNCTION CFB(AWVD)

DOUBLE PRECISION AWVD

$C F B=$ AWVD.GT.0.ODO

$P 4260050$

RETURN

END

$P 4260060$

$P 4260070$

$P 4260080$

P 4260090

$P 4260100$

$P 4260110$

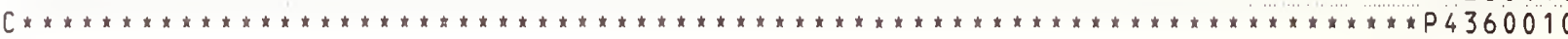

$C * \star \star * *$

$C * * * *$

$D F B-(436)$

P4360020

$C * * * *$

P4360030

$C * * * *$

$P 4360040$

$C * * * *$ LOGICAL FUNCTION OF LOGICAL ARGUMENT (TEST 4)

LOGICAL FUnCTION DFB(AWVB)

LOGICAL AWVB

$D F B=A W V B$

RETURN

END

$P 4360050$

$P 4360060$

$P 4360070$

P4360080

$P 4360090$

$P 4360100$

$P 4360110$

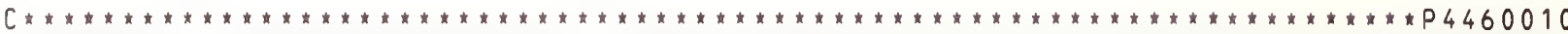

$C * * * *$

$E F B-(446)$

$P 4460020$

$C * * * *$

$C * * * *$

$P 4460030$

$P 4460040$

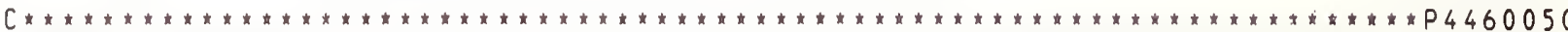

$C * * * *$ LOGICAL FUNCTION OF COMPLEX ARGUMENT (TEST 5)

$P 4460060$

LOGICAL FUNCTION EFB(AWVC)

$P 4460070$

COMPLEX AWVC

$P 4460080$

$A \vee S=A \amalg M A G(A W \vee C)$

$P 4460090$

$E F B=A \vee S . G T .0 .0$

$P 4460100$

RETURN

P 4460110

END

$P 4460120$

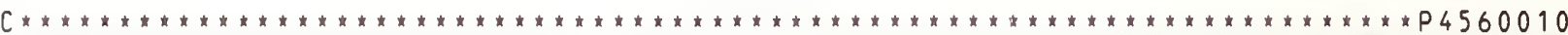

$C \star \star \star \star \star *$

$C * \star * *$

$F F-(456)$

$P 4560020$

$P 4560030$

$P 4560040$

$C * * * *$

FFB

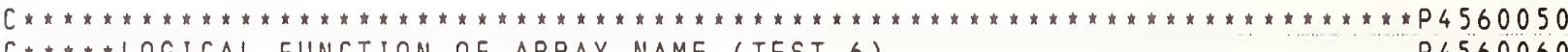

$C * * * *$ LOGICAL FUNCTION OF ARRAY NAME (TEST 6)

$P 4560070$

LOGICAL FUNCTION FFB (AW 1S)

$P 4560080$

DIMENSION AW1S(Z)

$B V S=A W 1 S(1)+A W 1 S(2)$

$P 4560090$

$F F B=B \vee S . G T .0 .0$

$P 4560100$

RETURN

$P 4560110$

END

$P 4560120$

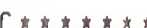

$P 4660010$

$C \star * * *$

P 4660020

$C * * * *$

$G F B-(466)$

$P 4660030$

$P 4660040$

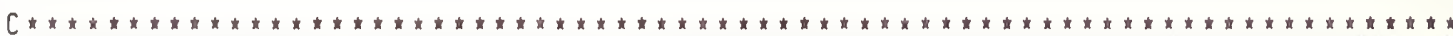

C**** LOGICAL FUNCTION OF EXTERNAL PROCEDURE (TEST 7)

LOGICAL FUNCTION GFB(AWFB, AWVS)

LOGICAL AWFB

$G F B=A W F B$ (AWVS)

RETURN

END

$P 4660060$

$P 4660070$

$P 4660080$

$P 4660090$

$P 4660100$

$P 4660110$

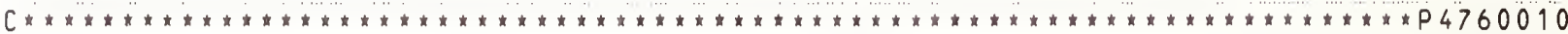

$C \star \star \star \star *$

$H F B-(476)$

$P 4760020$

$P 4760030$

$P 4760040$

$C * * * * *$

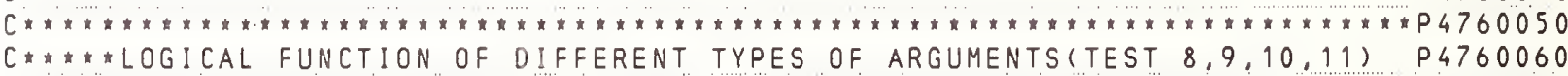

LOGICAL FUNCTION HFB (AWVS, IWVI, AWVB, AWVD, AWVC, AW $1 S, A W Z S, A W 3 S$

$P 4760070$

1 IW1 I, IW2I, IW3!, AW1B,AW2B,AW3B,AW1C,AW2C,AW3C,AW1D,AW2D,AW3D, AWFB)

$P 4760080$ 
1 IWVI, IWVI). AND.AWFB( 1.0$)$

$A W V C=A W 1 C(I W V I)+A W 2 C(I W V I, I W V I)+A W 3 C(I W V I, I W V I, I W V I)$

$P 4760180$

$P 4760190$

$A W V D=A W I D(I W V I)+A W Z D(I W V I, I W V I)+A W 3 D(I W V I, I W V I, I W V I)$

$P 4760200$

P4760210

AWVS =BXVS+AW1S (IWVI)* \#IWII(IWVI)-AW2S (IWVI, IWVI)* \#IWII (IWVI, IWVI) P4760220

1 -AW3S(IWVI,IWVI,IWVI)* *IW3I(IWVI,IWVI,IWVI)

$P 4760230$

RETURN

$P 4760240$

END

$P 4760250$

$C * * * * * *$

$C * * * * *$ SBRTN - $(167)$

P 1670020

$C * * * * *$

P1670030

$C * * * * *$

P1670040

$C * * * * * *$

C***** GENERAL PURPOSE

$C * * * *$ TO TEST SUBROUTINE SUBPROGRAMS

$C * * *$ RESTRICTIONS OBSERVED

$C * * * *$ SYMBOLIC NAME OF A SUBROUTINE MAY NOT APPEAR IN ANY $8.4 .1 .1119 P 1670090$

$C *$ STATEMENT IN THIS SUBROUTINE EXCEPT IN THE

$C * * * *$ SUBROUTINE STATEMENT ITSELF

* SYMBOLIC NAMES OF DUMMY ARGUMENTS MAY NOT APPEAR $8.4 .1 .1123 P 1670120$

C**** IN EOUIVALENCE OR COMMON STATEMENTS IN THE SUBPROGRAM P

$C * * * *$ SUBROUTINES MAY NOT CONTAIN A FUNCTION STATEMENT, 8:4.1.1129P1670140

$C * * * *$ ANOTHER SUBROUTINE STATEMENT, OR ANY STATEMENT THAT P P 1670150

C**** DIRECTLY OR INDIRECTLY REFERENCES THE SUBROUTINE

$C * * * *$ BEING DEFINED.

$C * * * *$ AT LEAST ONE RETURN STATEMENT MUST BE IN A SUBROUTINE

$P 1670160$

$P \uparrow 670170$

$C * * * * *$

$C * * * *$ GENERAL COMMENTS

8.4.1.1/33P1670190

$C * * * *$ THIS SEGMENT IS TO BE RUN WITH SEGMENT $407,417,427 \quad$ P 1670210

$C * * * * *$

$C * * * *$ S P E C I F I C A T I O N S SEGMENT $167 \quad$ P 1670230

$C * * * * *$

$C * * * *$ WHEN EXECUTING ONLY SEGMENT 167 . THE SPECIFICATION STATEMENTS

$C * * * *$ WHICH APPEAR AS COMMENT CARDS MUST HAVE THE C

$C * * * *$ IN COLUMNS I AND 2 REMOVED.

$C * * * * *$

$C=\quad$ DIMENSION IABII(4), IABZI 3,3$), A B 1 S(4), A B 2 S(3,3)$

$P 1670230$

$C=\quad$ COMMON AXVS, CXVS, IXVI, IAX11(4), IAX2I(3,3), IAX3I $(2,2,2)$,

$C=\quad 1 \quad B \times V S, \quad A X 1 S(4), A \times 2 S(3,3)$

$C=$ EXTERNAL SQRT

DIMENSION IABII(4), IABZI $(3,3), A B 1 S(4), A B 2 S(3,3)$

$P 0013445$

$P 0013450$

$P 0013455$

$P 0013460$

$P 0013465$

$P 0013470$

$P 0013475$

P 0013480

P167A1

COMMON AXVS, CXVS, IXVI, IAX1I(4), IAX2I(3,3), IAX3I (2,2,2),

$B \times V S, A \times 1 S(4), A \times 2 S(3,3)$

P $167 A 2$

P167A3

EXTERNAL SORT

P167A4

$C * * * *$

C**** O U T P U T T A P E ASSIGNMENT STATEMENT. NO INPUT TAPE.

$P 0013485$

$C * * * * *$

$C * * * * *$

WHEN EXECUTING ONLY SEGMENT 167. THE FOLLOWING STATEMENT

$C * * * *$ NUVI $=6$ MUST HAVE THE $C=I N$ COLUMNS 1 AND 2 REMOVED.

$C=N$ NUVI $=6$

NUVI $=6$

$P 1670240$

$P 0072750$

P 0072755

$P 0072760$

$P 0072765$

P 16781

$C * * * *$

$C * * * *$ WRITE HEADING

$P 0072770$

WRITE (NUVI, 1670)

1670 FORMAT $(1 H 1,1 X, 35 H S B R T N-(167)$ SUBROUTINE SUBPROGRAMI

P 1670250

P 1670260

$P 1670270$

$1 / 2 X .16$ HASA REF. - 8.4.1/12X,7HRESULTS)

$C * * * * \quad S E T$ ALL VARIABLES AND SOME ELEMENTS IN ARRAYS TO ZERO

P 1670280

$P 1670290$

$I A V I=4$

P 1670300

AVS $=0.0$

P 1670310 
P1670390

P1670400

P1670410

P 1670420

P1670430

P1670440

$P 1670450$

$A \times 1 S(2)=0.0$

P1670460

$A \times 2 S(1.2)=0.0$

P1670470

P 1670480

$C * * * *$

C*** SET ELEMENTS IN INTEGER ANO REAL ARRAY TO 1 TO TEST

P 1670490

$C *$ EXPRESSIONS IN SUBROUTINE ARGUMENT

\section{$\lfloor A B \perp \mid(2)=1$ \\ $\lfloor A B 1 \mid(4)=1$ \\ $\triangle A B Z \llbracket(2,1)=1$

$$
|A B Z|(2,2)=1
$$

$C * * *$

$P 1670500$

P 1670510

P 1670520

$P 1670530$

P 1670540

P 1670550

$A B 1 S(2)=1.0$

$A B 1 S(3)=1.0$

P 1670560

P 1670570

$A B Z S(1,2)=1.0$

P 1670580

$A B Z S(2,2)=1.0$

P1670590

$C * * * *$

CALL AAQ(\AVI, AVS, \AB\!, IABZ!, AB 1S, ABZS, SORT,

$P 1670600$

P 1670610

$1 ! A B 1 !(2)+\lfloor A B 1 !(4) *\lfloor A B 2 !(2,1)-\lfloor A B 2 !(2,2)$,

P1670620

$2 A B 1 S(2)+A B 1 S(3): A B 2 S(1,2)-A B 2 S(2,2), 1.0$ :

P1670630

CALL ACO

C*\#\# WRITE RESULTS

WRITE (NUVI, 1671) IAVI, AVS, IABII(1), IABII(3), IABZI(1,2),

P 1670640

P 1670650

$P 1670660$ $A$

A

$\lfloor A B 2\lfloor(3,3), A B 1 S(1), A B \backslash S(4)$

$A B 2 S(1,3), A B 2 S(2,3),\{X V 1, B \times V S$,

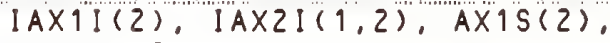

$\operatorname{Ax} 2 S(1,2)$

$P 1670670$

P 1670680

P1670690

P1670700

P 1670710

1671 FORMAT (//I10/F11.1/4(I10/),4(F11.1/),I10/F11.1/2(110/), 2(F11.1/P1670720

A) )

WRITE (NUVI, 1672)

1672 FORMAT ( $/ / 2 X, 38$ HTEST SUCCESSFUL IF ALL RESULTS EOUAL $1 / 1$ )

\section{C*\#\#\# END OF TEST SEGMENT 167}

C**** WHEN EXECUTING ONLY SEGMENT 167. THE STOP AND END CARDS

$C * *:$ WHICH APPEAR AS COMMENT CARDS, MUST HAVE THE $C=$

C**** IN COLUMNS 1 AND 2 REMOVED.

$C=S T O P$

$C=E N D$

STOP

P 1670730

P 1670740

P 1670750

P1670760

P 1670770

P1670780

P 1670790

P 1670800

P 1670810

P167C1

END

P167C2

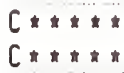

C $A A Q-(407)$

04070020

$P 4070030$

$P 4070040$

C

AAQ $-(407)$

$P 4070050$

C****THIS SUBROUTINE IS TO BE RUN WITH SEGMENT 167

$P 4070060$

SUBROUTINE AAO (IWVI, AWVS, IAWII, IAWZI, AW1S, AWZS, SOFI,

1MWVI, BWVS, (WVS)

DIMENSION IAWII(4), IAWZI $(3,3), \operatorname{AWIS}(4)$,

$1 \quad \operatorname{AHZS}(3,3)$

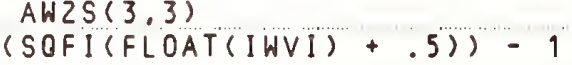

AWVS $=$ AWVS +1.0

$|A V|=5$

IAWII(1) = MHVI

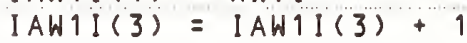

$\operatorname{IAWZI}(3,3)=\operatorname{IAWZI}(3,3)+1$

$P 4070070$

P4070080

$P 4070090$

$P 4070100$

P4070110

$P 4070120$

P4070130

$P 4070140$

$P 4070150$

P4070160 
AHIS(1) = BWVS

AH2S $(1,3)=$ CWVS

$C * * * * *$

$C * * * * *$

$A B Q-(417)$

$C * * * * *$

$P 4170010$

$P 4170020$

$P 4170030$

$P 4170040$

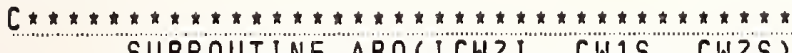

DIMENSION ICWZI $(3,3), C W 1 S(4), \operatorname{CWZS}(3,3)$

$C * * * *$

ICWZI $(1,2)=I \operatorname{CWZI}(1,2)+1$

$P 4170060$

$P 4170070$

$P 4170080$

$P 4170090$

$\operatorname{CW1S}(4)=\operatorname{CW} 15(4)+1.0$

$\operatorname{CWZS}(2,3)=\operatorname{CWZS}(2,3)+1.0$

$P 4170100$

$P 4170110$

RETURN

END

$P 4170120$

$P 4170130$

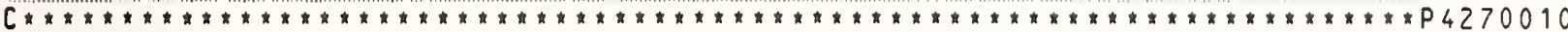

$C * * * * *$

$A C O-(427)$

$P 4270020$

$C * * * * *$

$P 4270030$

$P 4270040$

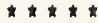

$P 4270050$

SUBROUTINE ACO

DIMENSION IOXII(4), IOX2!(3,3), I0X3!(2,2,2)

$P 4270060$

$P 4270070$

$1 \quad \operatorname{AAX} 1 S(4), \operatorname{AAX} 2 S(3,3)$

COMMON ABXVS, ACXVS, IAXVI, IOX1I, IDXZI, IDX3I,

$1 \quad A A X V S, A A X 1 S, A A X Z S$

$I A X V I=\mid A X V I+1$

$A A X V S=A A X V S+1.0$

$10 \times 1 I(2)=I 0 \times 1 I(2)+1$

$C * * * * *$

$10 \times 2 I(1,2)=10 \times 2 I(1,2)+1$

$P 4270080$

$P 4270090$

$P 4270100$

$P 4270110$

$P 4270120$

$P 4270130$

$P 4270140$

$P 4270150$

$\operatorname{AAX} 1 S(2)=\operatorname{AAX} 1 S(2) * 2+1.0$

$C * * * * *$

$A A X Z S(1,2)=A A X Z S(1,2)+4.0-3.0$

$P 4270160$

P4270170

P4270180

P4270190

$P 4270200$

$C * * * *$ END OF TEST SEGMENT 427

$P 4270210$

FSBRT - (168)

P 1680020

$C * * * * *$

$C * * * * *$

P 1680030

P1680040

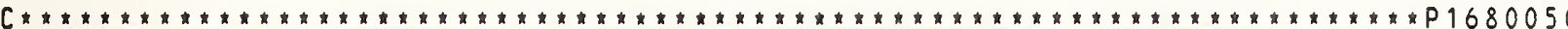

C**** GENERAL PURPOSE

ASA REFSP 1680060

$C * * * *$ TO TEST SUBROUTINE SUBPROGRAM IN FORTRAN

$C * * * * *$ RESTRICTIONS OBSERVEO

8.4.1P1680070

P 1680080

$C * * * *$ SYMBOLIC NAME OF A SUBROUTINE MAY NOT APPEAR IN ANY 8.4.1.1/56P1680090

$C * * * *$ STATEMENT IN THIS SUBROUTINE EXCEPT IN THE

P 1680100

$C * * * * *$ SUBROUTINE STATEMENT ITSELF.

P1680110

$C * * * *$

* SYMBOLIC NAME OF DUMMY ARGUMENTS MAY NOT APPEAR IN EQUIVALENCE OR COMMON STATEMENTS IN THE SUBPROGRAM

$C * * * * *$

$C * * * * *$

$C * * * * *$

* SUBROUTINES MAY NOT CONTAIN A FUNCTION STATEMENT. ANOTHER SUBROUTINE STATEMENT, OR ANY STATEMENT THAT

$C * * * *$ OIRECTLY OR INDIRECTLY REFERENCES THE SUBROUTINE

$C * * * * *$ BEING DEFINED.

$C * * *$ AT LEAST ONE RETURN STATEMENT MUST BE IN A SUBROUTINE

$C * * * * *$

$C * * * * *$

$C * * * * *$

$C * * * * *$

GENERAL COMMENTS

THIS SEGMENT IS TO BE RUN WITH SEGMENT $408,418,428$

$C * * * *$ S P E C I F I C A T I O N S SEGMENT 168

$C * * * * *$

$C * * * * *$

WHEN EXECUTING ONLY SEGMENT 168, THE SPECIFICATION STATEMENTS

$C * *$ WHICH APPEAR AS COMMENTS MUST HAVE THE $C=$

$C * * * * *$ IN COLUMNS 1 AND 2 REMOVED.

4.1.1/39P1680120

$P 1680130$

$8.4 .1 .1 / 45 P 1680140$

$P 1680150$

P1680160

P 1680170

P 1680180

$.4 .1 .1 / 49 P 1680190$

$P 1680200$

P 1680210

P 1680220

P 1680230

P0013490

$P 0013495$

$P 0013500$

P0013505 


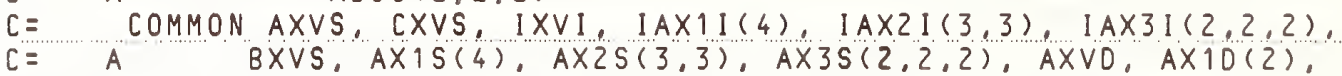

(3,3), P0013515

$P 0013525$

$B \quad A \times 2 D(2,2), A \times 30(2,2,2), A \times V C, A \times 1 C(2), A \times 2 C(2,2)$,

$C=C \quad A \times 3 C(2,2,2), A \times V B, A \times 1 B(2), A \times 2 B(2,2), A \times 3 B(2,2,2)$

$P 0013530$

DOUBLE PRECISION AXVD, AX1D, AX2D, $A \times 30$

DOUBLE PRECISION AVD,A1D(4),A2D(2,2),A3D(2,2,2)

COMPLEX AXVC, $A X 1 C, A X 2 C, A X 3 C$

COMPLEX AVC, A $1 C(12), A Z C(2,2), A 3 C(2,2,1)$

LOGICAL $A X V B, A X 1 B, A \times 2 B, A \times 3 B$

$\operatorname{LOGICAL~} A 1 B(2), A 2 B(2,2), A 3 B(2,2,2), A \vee B$

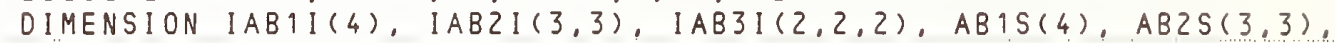
A $\quad A B 3 S(2,2,2)$

COMMON AXVS, CXVS, IXVI, IAX1I(4), IAXZI(3,3), IAX3I(2,2,2),

$P 0013535$

$P 0013540$

$P 0013545$

$P 0013550$

$P 0013555$

$P 0013560$

$P 0013565$

$P 0013570$

$A \quad B X V S, A X 1 S(4), A X Z S(3,3), A \times 3 S(2,2,2), A X V D, A X 1 D(2)$,

$B \quad A \times 2 D(2,2), A \times 3 D(2,2,2), A \times V C, A \times 1 C(2), A \times 2 C(2,2)$,

C $A \times 3 C(2,2,2), A \times \vee B, A \times 1 B(2), A \times 2 B(2,2), A \times 3 B(2,2,2)$

DOUBLE PRECISION AXVD, AX10, AX2D, AX3D

DOUBLE PRECISION AVD,A10(4), AZO (2,2), A3D (2,2,2)

COMPLEX AXVC, AX1C, AXZC, AX3C

COMPLEX AVC, $A 1 C(12), A Z C(2,2), A 3 C(2,2,1)$

LOGICAL $A X V B, A \times 1 B, A \times 2 B, A \times 3 B$

LOGICAL $A 1 B(2), A Z B(2,2), A 3 B(2,2,2), A \vee B$

P168A1

$P 168 \mathrm{AZ}$

$P 168 A 3$

P168A4

P 168 A 5

$P 168 A 6$

$P 168 A 7$

P $168 \mathrm{AB}$

P168A9

P168 AA

P $168 \mathrm{AB}$

$C * * * * *$

$C * * * *$

o U t P U t T a P E assignment statement. nO inPut tape.

$P 168 A C$

$P 0013575$

$C * * * * *$

$C * * * * *$

WHEN EXECUTING ONLY SEGMENT 168. THE FOLLOWING STATEMENT

$C * * * *$ NUVI $=6$ MUST HAVE THE $C=I N$ COLUMNS 1 AND 2 REMOVED.

$C=$ NUVI $=6$

NUVI $=6$

$C * * * *$ SET INTEGER VARIABLES AND SOME ELEMENTS IN ARRAYS TO ZERO

$C * * * *$ WRITE HEAOING

WRITE (NUVI, 1680)

1680 FORMAT ( 1 H $1,1 \mathrm{X}, 36 \mathrm{HFSBRT}$ - (168) SUBROUTINE SUBPROGRAMS/

A/ $18 H$ ASA REF. $-8.4 .1 / 12 X, 7$ HRESULTS)

$I A V I=0$

$\triangle A B 1 \mid(1)=0$

$\triangle A B Z I(1,2)=0$

$I A B 3 I(1,1,2)=0$

$I X V I=0$

$1 A \times 1 \mid(1)=0$

$1 A \times 2 I(1,2)=0$

$I A X 3 I(1,1,2)=0$

$C * * * *$ SET REAL VARIABLES AND SOME ELEMENTS IN ARRAYS TO ONE $A V S=1$.

$A B 1 S(1)=1$.

$A B Z S(1,2)=1$.

$A B 3 S(1,1,2)=1$.

$B X V S=1$.

$A \times 1 S(2)=1$.

$\operatorname{AXZS}(1,2)=1$.

$\operatorname{AX3S}(1,1,2)=1$.

$C * * *$ SET OP VARIABLES AND SOME ELEMENTS IN ARRAY TO TWO

$A \vee D=2.000$

$A 10(1)=2.000$

$A 2 D(1,2)=2.000$

$A 30(1,1,2)=2.000$

$A \times \vee D=2.000$

$A \times 10(1)=2.000$

$A \times 2 D(1,2)=2.00$

$A \times 3 D(1,1,2)=2.000$

$P 1680240$

$P 0072780$

$P 0072785$

$P 0072790$

$P 0072795$

P168B1

P 1680250

$P 1680260$

P 1680270

P 1680280

P 1680290

$P 1680300$

P 9680310

$P 1680320$

P 1680330

$P 1680340$

P1680350

P 1680360

P1680370

P 1680380

P 1680390

P1680400

P 1680410

P1680420

P1 1680430

P1680440

P1680450

P1680460

P 1680470

P1680480

P 1680490

P1680500

P1680510

$P 1680520$

P1680530

$P 1680540$

P1680550

C*\#"** SET COMPLEX VARIABLES AND SOME ELEMENTS IN ARRAYS TO $(3.0,3.0)$ P 1680560 $A V C=(3.0 .3 .0)$

$A 1 C(1)=(3.0,3.0)$

$A 2 C(1,2)=(3.0,3.0)$

$P 1680570$

P 1680580

P 1680590

$A 3 C(1,2,1)=(3,0,3.0)$

P1680600 
$A X V C=(3.0,3.0)$

$A \times 1 C(1)=(3,0,3,0)$

$A \times 2 C(1.2)=(3.0,3.0)$

$A \times 3 C(1,1,2)=(3,0,3,0)$

$C * * * *$ SET LOGICAL VARIABLES AND SOME ELEMENTS IN ARRAYS TO.FALSE.

$A \vee B=$.FALSE.

$A 1 B(1)=$. FALSE.

$A 2 B(1,2)=$.FALSE.

$A 3 B(1,1,2)=$. FALSE.

$A X V B=$. FALSE.

$A \times 1 B(1)=$.FALSE.

$A \times 2 B(1,2)=$.FALSE.

$A \times 3 B(1,1,2)=$.FALSE.

$C * * * * * \quad S E T$ INTEGER AND REAL VARIABLES FOR EXPRESSION USAGE IN

\section{$C * * * *$ DUMMY ARGUMENT}

$\lfloor A B 1 \mid(4)=0$

$I A B 1 I(2)=0$

$A B 1 S(4)=0.0$

$A B 1 S(2)=0.0$

JAVI = 1

KAVI = 1

LAVI = 1

MAVI = 1

NAVI $=1$

$A B \vee S=1$.

$A C V S=1$

ADVS $=2$.

$A E V S=2$.

$A F V S=2$.

CALL ADOCIAVI, IABII, IABZI, IAB I, AVS, AB1S, ABZS, AB 3S, AVD,

P 1680640

P 1680650

P 1680660

P 1680670

P 1680680

P 1680690

P1680700

P1680710

P1680720

$P 1680730$

P1680740

$P 1680750$

P1680760

P 1680770

P 1680780

P 1680790

P1680800

P 1680810

P 1680820

P1680830

P 1680840

P 1680850

P1680860

P1680870

P 1680880

P 1680890

P 1680900

P 1680910

$B$

WRITE (NUVI, 1681)

$A 10, A 2 D, A 30, A \vee C, A 1 C, A 2 C, A 3 C, A \vee B, A 1 B, A 2 B, A 3 B$,

P1680920

P 1680930

P 1680940

P 1680950

P 1680960

FORMAT ( $128 \mathrm{H}$ TEST IS SUCCESSFUL IF EACHI

AZ $8 H$ GROUP CONTAINS SAME VALUES)

WRITE (NUVI, 1682) IAVI, IABII(1), IABII(2), IABII(4), IABZI $(1,2)$,

P 1680970

A

B $C 2), A B 1 S(2), A B 1 S(4), \quad B \times V S, A \times 1 S(2), A X 2 S(1,2), A \times 3 S(1,1,2), A \vee D$,

$I A B 3 I(1,1,2), I X V I, I A X 1 I(1), I A X 2 I(1,2)$

IAX3I $(1,1,2)$ AVS $A B 1 S(1), A B 2 S(1,2)$ AB3S(1,1, 1680990

$D \quad A 1 D(1), A 2 D(1,2), A 3 D(1,1,2), A X V D, A \times 1 D(1)$,

$A \times 2 D(1,2), A X 3 D(1,1,2), A \cup C, A 1 C(1), A 2 C(1,2)$ $A 3 C(1,2,1), A X \vee C, A X 1 C(1), A X 2 C(1,2)$,

$A \times 3 C(1,1,2), A \vee B, A 1 B(1), A 2 B(1,2), A 3 B(1,1,2)$

G

$\begin{array}{cc}1682 \text { FORMAT ( } 10(110 /) / \\ 1 & 10(F 11.1 /) / \\ 2 & 8(1 P D 15.1 /) 1 \\ 3 & 8(O P F 5.1, F 5.1 /) 1 \\ 4 & 8(L O /))\end{array}$

END OF TEST SEGMENT 168

C***** WHEN EXECUTING ONLY SEGMENT 168. THE STOP AND END CARDS

$C * * * *$ WHICH APPEAR AS COMMENT CAROS MUST HAVE THE $C=$ IN

$C * * * * *$ COLUMNS 1 AND 2 REMOVED.

$C=S T O P$

$C=E N D$

STOP

END

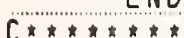

$C * * * * *$

$C * * * * *$

$C * * * * *$

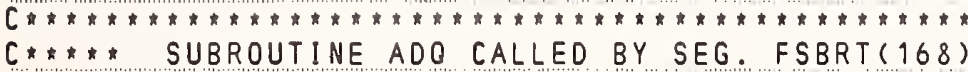

$A D O-(408)$

SUBROUTINE ADOCIWVI, IAWII, IAWZI, IAW3 I, AWVS, AW1S, AWZS, AW3S, A AWVD, AW 10, AW2D, AW3D, AWVC, AW 1C, AW2 C, AW 3 C,

B AWVB,AW1B, AWZB,AW3B, KWVI, MWVI, BWVS, CWVS)

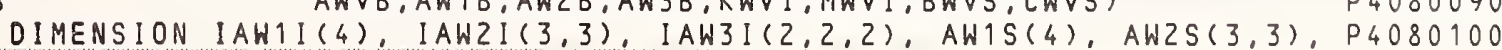

P 1681020

P 1681030

P 1681040

$P 1681050$

P 1681060

P 1681070

P 1681080

P 1681090

P 1681100

P 1681110

P 1681120

P 1681130

P 1681140

P1681150

P 1681160

P $168 \mathrm{C1}$

P168C2

$P 4080010$

P4080020

P 4080030

P4080040

$P 4080050$

$P 4080060$

P 4080070

$P 4080080$

$P 4080090$ 
$A W 3 S(2,2,2), A W 1 D(2), A H 2 D(2,2), A W 3 D(2,2,2), A W 1 C(2), P 4080110$ $A W 2 C(2,2), A W 3 C(2,2,1), \quad A W 1 B(2), A W 2 B(2,2)$

$P 4080120$

C $A W 3 B(2,2,2)$

DOUBLE PRECISION AWVO, AW10, AW2O, AW3O

$P 4080130$

COMPLEX

LOGICAL

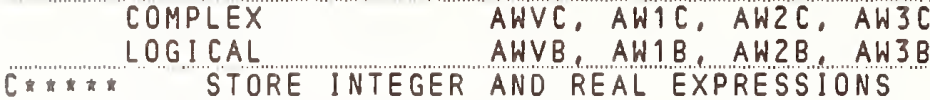

P4080140

$P 4080150$

$P 4080160$

IAWII $(4)=$ KWVI

$P 4080170$

IAWII $(2)=$ MWVI

P4080180

AW1S(4) = BWVS

$P 4080190$

AW1S(2) $=$ CWVS

CALL AEO (IWVI, IAW1I, IAW2I, IAW3I, AWVS, AW1S, AW2S, AW3S)

C*\#\# INCREMENT DOUBLE PRECISION

P4080200

$P 4080210$

$P 4080220$

$A W V D=A W V D+A W V D$

$A W 10(1)=A W 1 D(1)+A W 10(1)$

$A W 2 D(1,2)=A W 2 D(1,2)+A W 2 D(1,2)$

$P 4080230$

$P 4080240$

$P 4080250$

$\operatorname{AW} 30(1,1,2)=\operatorname{AW} 30(1,1,2)+\operatorname{AW} 30(1,1,2)$

$P 4080260$

$P 4080270$

$C * * *$ INCREMENT COMPLEX

$A W V C=A W V C+A W V C$

$A W 1 C(1)=A W 1 C(1)+A W 1 C(1)$

$A W 2 C(1,2)=A W 2 C(1,2)+A W 2 C(1,2)$

$A W 3 C(1,2,1)=A H 3 C(1,2,1)+A H 3 C(1,2,1)$

$C \# \# \quad$ CHANGE LOGICAL

$A W V B=$.NOT. AWVB

$P 4080280$

$P 4080290$

$P 4080300$

$P 4080310$

$P 4080320$

$P 4080330$

$A W 1 B(1)=$.NOT. $A W 1 B(1)$

$A W 2 B(1,2)=$.NOT. $A W 2 B(1,2)$

$P 4080340$

$P 4080350$

$A H 3 B(1,1,2)=$.NOT. $A H 3 B(1,1,2)$

$P 4080360$

RETURN

END

$P 4080370$

$P 4080380$

$P 4080390$

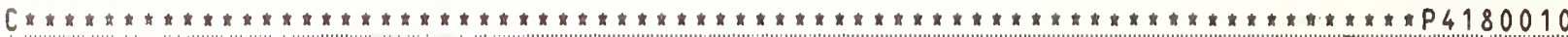

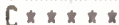

$A E O-(418)$

$P 4180020$

$C * * * * *$

$P 4180030$

$[* * * * *$

$P 4180040$

C

C***: SUBROUTINE AEO CALLED BY SEG ADO(408) WHICH IS P

$C * *$ CALLED BY SEG. FSBRT(168)

SÜBROUTINE AEO(KWVI, KAWII, KAWZI, KAW3I, AAWVS, AAWIS, AAWZS,

P4180070

$P 4180080$

A $\quad$ AAW3S)

DIMENSION KAWII (4), KAW2 I $(3,3)$, KAW3 I $(2,2,2)$, AAWIS $(4), \operatorname{AAW} 2 S(3,3)$,

P4180090

$P 4180100$

A AAW3S $(2,2,2)$

P4180110

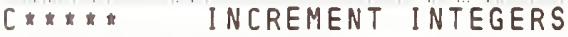

P 4180120

KWVI $=$ KWVI +1

P4180130

KAWII $(1)=K A W I I(1)+1$

P4180140

$\operatorname{KAWZ} I(1,2)=\operatorname{KAW} 2 I(1,2)+1$

P4180150

$\operatorname{KAW3}[(1,1,2)=\operatorname{KAW} 3](1,1,2)+1$

$C * * *$ INCREMENT REAL

P4180160

AAWVS = AAWVS + 1 .

AAW1S(1) = AAW1S(1) + 1 .

$\operatorname{AAWZS}(1,2)=\operatorname{AAW} 2 S(1,2)+1$.

$\operatorname{AAW} 3 S(1,1,2)=\operatorname{AAW} 3 S(1,1,2)+1$.

P4180170

$P 4180180$

P4180190

$P 4180200$

$P 4180210$

RETURN

$P 4180220$

END

$P 4180230$

C

$C * * * *$

$A F O=(428)$

P4280020

$C * * \cdots *$

$P 4280030$

P4280040

C

C*\#\# SUBROUTINE AFO CALLED BY SEG. FSBRT(168) $\quad$ P4280060

SUBROUTINE AFO

P4280070

COMMON ABXVS, ACXVS, IAXVI, IAXII(4), IAXZI(3,3), IAX $I(2,2,2)$,

P4280080

A AXVS, AX1S(4), AXZS(3,3), AX3S(2,2,2), AXVD, AX1D(2), P 4280090

$2 \quad A \times 20(2,2), A \times 3 D(2,2,2), A \times V C, A \times 1 C(2), A \times 2 C(2,2), A \times 3 C(2,2,2) P 4280100$

$3 \quad A X \vee B, A X 1 B(2), A \times 2 B(2,2), A \times 3 B(2,2,2)$

DOUBLE PRECISION AXVD, AX10, AX2D, AX3D

COMPLEX AXVC, $A X 1 C, A \times 2 C, A \times 3 C$

$L O G I C A L A X V B, A X 1 B, A \times 2 B, A \times 3 B$

$P 4280110$

$P 4280120$

P 4280130

$P 4280140$

$C *$ SET INTEGERS TO

$P 4280150$ $I$ AXVI $=1$

P4280160 
$\mid A \times 1 I(1)=1$

GENERAL PURPOSE

$C * * * *$ TD TEST BLDCK DATA SUBPRDGRAM

$C * * * * *$ GENERAL CDMMENTS

$C * * * * *$

$C * * * *$

$C * * * * *$

$C * * * * *$

$C * * * * *$

$C * * * * *$ THIS SEGMENT IS TO BE RUN WITH SEGMENT 409. THIS

$C * * * * *$ SEGMENT WRITES DUT THE DATA FORMED IN SEGMENT 409.

$C * * * * *$ $C * * * * *$

$C=$ COMMON/BLK1/JXVI, JAXII $(2), J A X 2 I(3,3)$

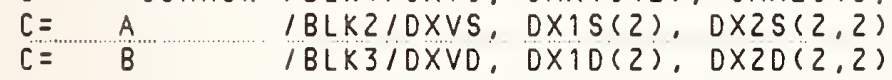

$C=\quad C \quad / B L K 4 / D \times V C, D \times 1 C(2), D \times 2 C(2,2)$

$C=D \quad / B L K S / D \times V B, D \times 1 B(2), D \times 2 B(2,2)$

$C=E$

$C=F$

$c=$

$c=$

$c=$

| $B L K 6 / \operatorname{JAX} 3 \mathrm{I}(2,2,2), \operatorname{DX} 3 \mathrm{~S}(2,2,2), \operatorname{DX} 30(2,2,2)$, DZ3C(2,2,2), DX3B(2,2,2)

DOUBLE PRECISIDN DXVD, DX1D, DX2D, DX3D

COMPLEX DXVC, DX1C, DXZC, DZZC

LOGICAL $D \times V B, D \times 1 B, D \times 2 B, D \times 3 B$

COMMON /BLK1/JXVI, JAXII(2), JAXZI $(3,3)$

\section{A \\ B \\ C \\ D \\ F}

DOUBLE PRECISIDN DXVD, DX1D, DX2D, DX3D

CDMPLEX DXVC, DX1C, DXZC, DZZC LDGICAL

/ BLK2 IDXVS, DX1S(2), DX2S(2,2)

/ BLK3/DXVD, DX1D(2), DX2D(2,2)

/BLK4/DXVC, DX1C(2), DXZC(2,2)

/ BLK5/DXVB, DX1B(2), DX2B(2,2)

/ BLK6/JAX3I $(2,2,2), D \times 3 S(2,2,2), D \times 3 D(2,2,2)$, $D X V C, D \times 1 C, D \times 2 C, D Z 3 C$
$D X V B, D \times 1 B, D \times 2 B, D \times 3 B$

$C * * * * *$

$C * * * * *$

$C * * * * *$

C***** WHEN EXECUTING DNLY SEGMENT 169. THE FOLLOWING STATEMENT

$c=$

NUVI $=$ NUVI $=6$
$P 1690040$

ASA REFSP 1690060

$8.5 \quad P 1690070$

$P 1690080$

P1690090

P1690100

P1690110

P 1690120

P 0013580

P0013585

$P 0013590$

P0O13595

P0013600

P0013605

$P 0013610$

P0013615

P0013620

P0013625

P 0013630

POO 013635

P0013640

$P 0013645$

P0013650

P169A1

P169A2

P169A3

P169A4

P169A5

P169A6

P169A7

P169A8

P169A9

P169AA

P0013655

P 1690130

P0072800

P0072805

P 0072810

P0072815 
NUVI $=6$

C**** WRITE HEADING FOR SEGMENT 169

P 16981

WRITE (NUVI, 1690)

1690 FDRMAT ( 1 H $1,1 \times, 35 H B L K D T$ - (169) BLDCK DATA SUBPRDGRAMII

P1690140

P 1690150

A $16 \mathrm{H}$ ASA REF. $-8.5 / 12 \times, 7$ HRESULTS)

WRITE (NUVI, 1691)

1691 FDRMAT ( $128 \mathrm{H}$ TEST IS SUCCESSFUL IF EACHI

$P 1690160$

P 1690170

P 1690180

A28H GRDUP CDNTAINS SAME VALUES)

P 1690190

WRITE (NUVI, 1692) JAXZI(1,1), JAX1I(2), JAXZI(2,1), JAX3I (2,2,1) P1690210

$A \quad, D X 3 S(1,2,1), D \times 1 S(1), D \times 2 S(1,1), D \times 3 S(2,2,1), D \times 2 D(2,2), D \ldots \ldots 1690220$

$B \quad D \times 1 D(2), D \times 2 D(2,1), D \times 3 D(2,2,1), D \times 2 C(2,2), D \times 1 C(2), D 1690230$

C $\quad D \times 2 C(2,1), D Z 3 C(2,1,1), D \times 2 B(2,2), D \times 1 B(2), D \times 2 B(2,1) \quad P 1690240$

$D \quad, D \times 3 B(2,2,1), \operatorname{JAX} I(3,1)$,

E $\quad D \times 3 B(2,1,2), D \times 2 S(2,2)$

1692 FDRMAT $(/ / 4(110 /) / /$

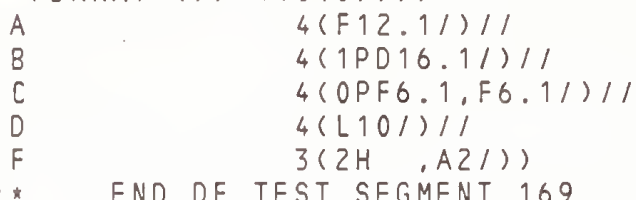

$4(F 12.1 /) / 1$

$4(1 P D 16.1 /) / /$

$4(\mathrm{~L} 10 /) / 1$

$3(2 \mathrm{H}, \mathrm{AZ} /))$

$C * * * *$ END DF TEST SEGMENT 169

$C * * * *$ WHEN EXECUTING DNLY SEGMENT 169. THE STDP AND END CARDS

$C * * * *$ WHICH APPEAR AS CDMMENT CARDS MUST HAVE THE $C=I N$

$C * * * * *$ CDLUMNS 1 AND 2 REMDVED.

$C=\operatorname{STDP}$

$C=\quad E N D$

STDP

$P 1690240$

P 1690260

P1690270

P 1690280

P 1690290

P1690300

P 1690310

P 1690320

P 1690330

P 1690340

P 1690350

P 1690360

P 1690370

$P 1690380$

END

P 16901

$P 969 C 2$

$C * * * *$
$C * * * *$

$C * * * *$

END

$C * * * * *$

$P 4090020$

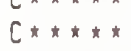

$C * * * *$ GENERAL PURPDSE

$P 4090030$

$P 4090040$

$C * * * *$

$P 4090050$

$P 4090060$

$P 4090070$

IT IS TD BE RUN WITH SEGMENT 169

C***** GENERAL CDMMENTS

C***** THIS SEgMENT USES ALL THE PERMISSIBLE STATEMENTS IN A

$P 4090080$

$P 4090090$

$P 4090100$

$C * * * *$ BLDCK DATA SUBPRDGRAM. THE DATA STATEMENT CDNSISTS DF ALL

$C * * * *$ TYPES DF VARIABLES AND ARRAYS. A HDLLERITH CDNSTANT

$C * * * *$ IS ASSIGNED TD INTEGER, REAL AND LDGICAL

BLDCK DATA

CDMMDN / BLK1/JXVI, JAXII (2), JAXZI $(3,3)$

A I BLK2/DXVS, DX1S(2), DX2S(2,2)

$B \quad$ IBLK3/DXVD, DX1D(2), DX2D(2,2)

C IBLK4/DXVC, DX1C(2), DX2C(2,2)

/ BLK5/DXVB, DX1B(2), DX2B(2,2)

$/ B L K 6 / J A X 3 I(2,2,2), \operatorname{DX} 3 S(2,2,2), \operatorname{DX} B(2,2,2)$,

$P 4090110$

$P 4090120$

$P 4090130$

$P 4090140$

$P 4090150$

$P 4090160$

$P 4090170$

$P 4090180$

$P 4090190$

$P 4090200$ $D 23 C(2,2,2), D \times 3 B(2,2,2)$

$P 4090210$

DIMENSIDN CY3C $(2,2,2)$

$P 4090220$

DDUBLE PRECISIDN DXVD, DX1D, DX2D, DX3D

CDMPLEX

LDG!CAL

INTEGER JXV!

DXVC, DX1C, DXZC, DZZC, CY $3 C$

P4090230

$P 4090240$

$P 4090250$

$P 4090260$

REAL DXVS

EOUIVALENCE (DZ3C(1, 1, 1), CY3C(1,1,1))

DATA JAXZI $(1,1), \operatorname{JAX} 1 \mathrm{I}(2), \operatorname{JAXZI}(2,1), \operatorname{JAX} 3 I(2,2,1), D \times 3 S(1,2,1)$,

$P 4090270$

$P 4090280$

$P 4090290$

$P 4090300$

A DX1S(1), DX2S(1,1), DX3S(2,2,1), DX2D(2,2), DX1D(2),

$D \times 2 D(2,1), D \times 3 D(2,2,1), D \times 2 C(2,2), D \times 1 C(2), D \times 2 C(2,1)$,

$P 4090310$

$D 23 C(2,1,1), D \times 2 B(2,2), D \times 1 B(2), D \times 2 B(2,1), D \times 3 B(2,2,1)$,

$P 4090320$

$J A X 2 I(3,1), D \times 3 B(2,1,2), D \times 2 S(2,2) / 4 * 2,4 * 3,0,4 * 4.0 D 0,4 *(4,5),, P 4090330$

$4 *$. TRUE. ,

$C * * * *$ END DF TEST SEGMENT 409

$2 H A B, 2 H A B, 2 H A B /$

$P 4090340$

END

$P 4090350$

$P 4090360$

C******

C******1*

$C * * * * *$

$B L K D A-(179)$

P 1790020

P 1790030

P 1790040 


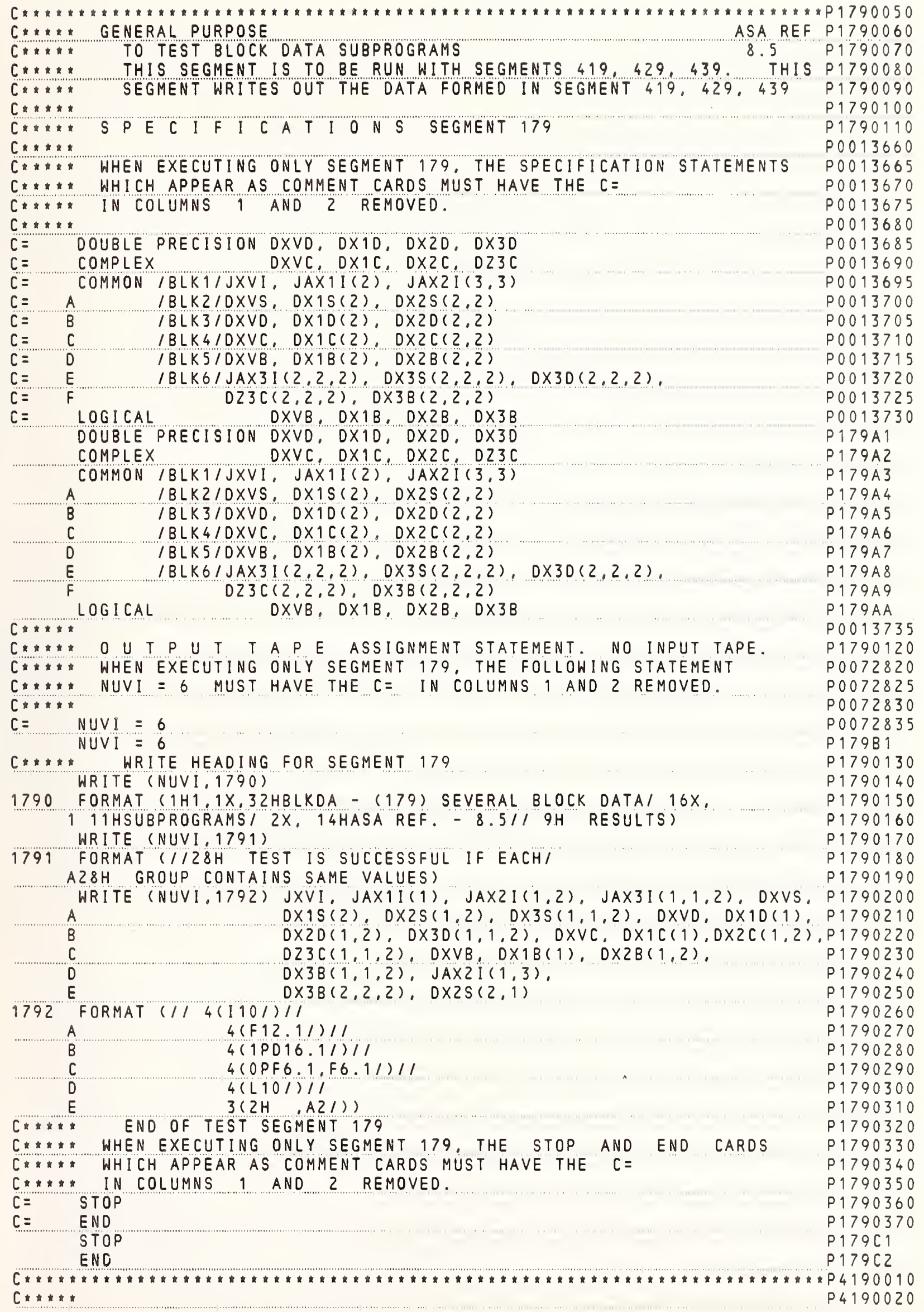




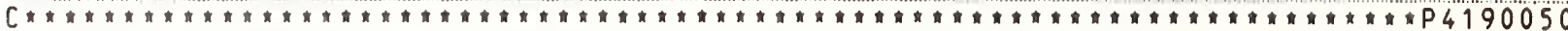

C**** GENERAL PURPOSE

$P 4190060$

C*\# THIS SEGMENT CONTAINS THE FIRST OF THREE BLOCK OATA SUBPROGRAMSP 4190070

C*\#*\# TO BE RUN WITH SEGMENT $179 \quad$ P4190080

$C * * * *$ THESE SEGMENTS USE ALL THE PERMISSIBLE STATEMENTS IN A P P

C**** BLOCK OATA SUBPROGRAM. THE DATA STATEMENTS CONSIST OF ALL P

C***: TYPES OF VARIABLES ANO ARRAYS. A HOLLERITH CONSTANT IS

$C * * * *$ ASSIGNEO TO INTEGER , REAL, ANO LOGICAL

BLOCK DATA

DOUBLE PRECISION OXVD, OX10, DX20

COMMON / BLK1/JXVI, JAXII(2), JAX2I $(3,3)$

$A \quad / B L K 2 / D X V S, D X 1 S(2), D \times 2 S(2,2)$

B /BLK3/ OXVO, DX10(2), DX20 (2, 2)

$P 4190120$

P4190130

$P 4190140$

$P 4190150$

INTEGER JXVI

$P 4190160$

$P 4190170$

$P 4190180$

REAL OXVS

DATA JXVI, JAX1!(1), JAXZI(1,2), DXVS, DX1S(2)

$P 4190190$

$P 4190200$

$A \quad, 0 \times 2 S(1,2)$, OXVD, OX1D(1), DX2O(1,2), 3,1

$P 4190210$

$B \quad 3 * 2.0 .3 * 4.000 /, J A X 2 I(1,3) .0 \times 2 S(2,1) / 2 \mathrm{HHP}, 2 \mathrm{HHP} /$

$P 4190220$

END

$P 4190240$

C

$C * * * *$

$[* * * *$

$B L B K D-(429)$

$P 4290020$

$C * * * * *$

$P 4290030$

C**** TO BE RUN WITH SEGMENT 179

$P 4290050$

C**** THIS SEGMENT CONTAINS THE 2ND OF THREE BLOCK DATA SUBPROGRAMS P4290070

$C * * * *$ TO BE RUN WITH SEGMENT 179

BLOCK DATA

COMPLEX OXVC, OX1C, OXZC

COMMON / BLK4/ OXVC, OX1C(2), DX2C(2,2)

C /BLK5/DXVB, OX1B(2), DX2B(2,2)

$P 4290080$

$P 4290090$

$P 4290100$

$P 4290110$

$P 4290120$

LOGICAL OXVB, OX1B, OX2B

DATA DXVC, DX1C(1), OXZC(1,2), DXVB, $D \times 1 B(1), D \times 2 B(1,2) /$

$0 \quad 3 *(3 \ldots 4),. 3 *$ FALSE.

$P 4290130$

$P 4290140$

$P 4290150$

P4290160

END

P4290170

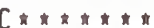

END OF TEST SEGMENT 429

$C * * * * *$

C*****

BLCKD - (439)

$P 4390010$

$P 4390020$

$P 4390030$

P 4390040

C*****

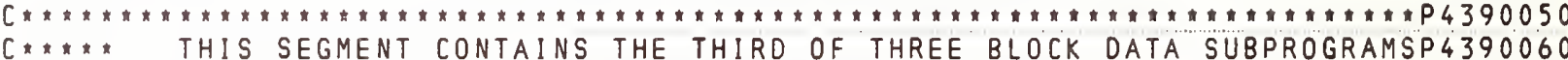

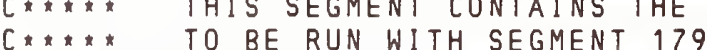

$P 4390070$

BLOCK OATA

COMMON /BLK6/JAX3I $(2,2,2), 0 \times 3 S(2,2,2), 0 \times 30(2,2,2)$

$P 4390080$ $.023 C(2,2,2), 0 \times 3 B(2,2,2)$

$P 4390090$

DOUBLE PRECISION DX30

84390100

$P 4390110$

DIMENSION CY3C $(2,2,2)$

$P 4390120$

COMPLEX OZ3C, CY3C

EQUIVALENCE $(D Z 3 C(1,1,1), \quad C Y 3 C(1,1,1))$

$P 4390130$

LOGICAL DX3B

$P 4390140$

$P 4390150$

DATA JAX3I $(1,1,2), D \times 3 S(1,1,2), D \times 3 D(1,1,2), C Y 3 C(1,1,2), 0 \times 3 B(1,1,2) / P 4390160$

$F 1,2.0,4.000,(3 ., 4)$, FALSE.1, DX3B(2,2,2)/, P4390170

G $2 \mathrm{HHP} /$

$C * * * *$ ENO OF TEST SEGMENT 439 END

P 4390180

$P 4390190$

$P 4390200$

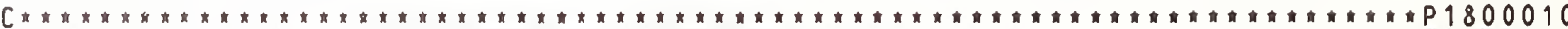

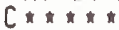

$C * * * * *$

UNFRW - (180)

$P 1800020$

P1800030

$[* * * * *$

$P 1800040$

C**** GENERAL PURPOSE

$C * * * *$ TEST OF UNFORMATTED READ AND WRITE STATEMENTS

ASA REF P1800060

C $* * * *$

$7.1 .3 .2 .4 P 1800070$

C****

$S P$

C A T

SEGMENT 180

$7.1 .3 .2 .5 P 1800080$

P 1800090 
C*** HHEN EXECUTING ONLY SEGMENT 180, THE SPECIFICATION STATEMENTS

$C * * *$ WHICH APPEAR AS COMMENT CARDS. MUST HAVE THE $c=$

$C * * *$ IN COLUMNS 1 AND 2 REMOVED.

$C * * * * *$

$C=\quad$ DIMENSION CMA1S(5), CMB1S(5), AC1S(25)

DIMENSION CMA1S(5), CMB1S(5), AC1S(25)

\section{$C * * * *$}

C*****0 0 U T P U T T A P E ASSIGNMENT STATEMENTS. NO INPUT TAPE.

$C * * * * *$

C**** WHEN EXECUTING ONLY SEGMENT 180, THE FOLLOWING STATEMENTS

$C * * * *$ NUVI $=6$ AND INVI $=9$ MUST HAVE THE $C=I N$ COLUMNS 1 AND 2 REMOVED.

$[* * * * * \pi$

$C=N U V I=6$

$C=\quad$ INVI $=9$

NUVI $=6$

$C * * * *$

INVI $=9$

WRITE (NUVI,0180)

180 FORMAT $(1 H 1,1 \times, 30 H U N F R W$ - (180) UNFORMATTED READI $14 X$,

$P 0013750$

$P 0013755$

$P 0013760$

$P 0013765$

P180A1

$P 0013770$

$P 1800100$

$P 0072840$

$P 0072845$

$P 0072850$

$P 0072855$

$P 0072860$

$P 0072865$

P 18081

P $180 B 2$

$P 0072870$

P1800110

$P 1800120$

$122 \mathrm{H}$ AND WRITE STATEMENTS//36H ASA REFS - 7.1.3.2.4 AND7.1.3.2.5P1800130 $2 / 110 \mathrm{H}$ RESULTS )

$C * * * *$ HEADER FOR SEGMENT 180 WRITTEN

CMAVS $=1.5 E 01$

CMBVS $=-2.75 E-0$

MCAVI $=5$

MCBVI $=-10$

DPAVS $=1.02 E 0$

DPBVS $=9876.0 E-2$

CMA1S(1) $=1.0 E O$

CMA1S(2) $=2.0 E 0$

CMA $1 S(3)=3.0 E O$

CMA1S(4) $=4.0 E O$

CMA $1 S(5)=5.0 E O$

$C * * * *$ WRITE AND READ VARIABLES OF THE SAME TYPE

REWIND INVI

WRITE (INVI) CMAVS, CMBVS

WRITE (INVI) MCAVI, MCBVI

WRITE (INVI) DPAVS, DPBVS

WRITE (INVI) CMAIS

WRITE (INVI) (CMAIS(IVI), IVI $=1,5,1$ )

REWIND INVI

READ (INVI) CMCVS, CMDVS

READ (INVI) MCCVI, MCDVI

READ (INVI) DPCVS, DPDVS

READ (INVI) CMBIS

READ (INVI) (ACIS(IVI), IVI $=1,5,1$ )

$C * * *$ CHECK RECORDS BY SÜBTRACTING CORRESPONDING VALUES.

CMEVS = CMAVS - CMCVS

CMFVS $=$ CMBVS - CMDVS

MCEVI = MCAVI - MCCVI

MCFVI = MCBVI - MCDVI

DPEVS = DPAVS - DPCVS

DPFVS $=$ DPBVS - DPDVS

$A C V S=C M A 1 S(1)-C M B 1 S(1)$

BCVS $=$ CMA1S(2) - CMB1S(2)

CCVS $=$ CMA1S(3)-CMB1S(3)

DCVS $=$ CMA1S(4) - CMB1S(4)

FFCVS $=$ CMA1S(5)-CMB1S(5)

CMGVS $=$ CMA1S(1) $-A C 1 S(1)$

CMHVS $=$ CMA1S(2)-AC1S(2)

CMIVS $=$ CMA1S(3) $-A C 1 S(3)$

CMJVS $=$ CMÄ1S(4) $-A C 1 S(4)$

CMKVS $=$ CMA1S(5) $-A C 1 S(5)$

WRITE (NUVI,181) CMEVS, CMFVS, MCEVI, MCFVI, DPEVS, DPFVS,

$P 1800140$

P1800150

P1800160

P 1800170

P1800180

P $1800 \uparrow 90$

P 1800200

P 1800210

P 1800220

P 1800230

P1800240

P1800250

P1800260

$P 1800270$

P 1800280

P1800290

P1800300

P 1800310

$P 1800320$

P1 1800330

P1 800340

$P 1800350$

$P 1800360$

$P 1800370$

P1 800380

P1800390

$P \uparrow 800400$

P 1800410

P1800420

$P 1800430$

P $\{800440$

P1 1800450

P 1800460

P1800470

P1800480

P1 1800490

P1 1800500

P 1800510

P1800520

P1800530

P1800540

$P 1800550$

P1800560

P1800570

1 ACVS, BCVS, CCVS, DCVS, FFCVS, CMGVS, CMHVS, CMIVS, CMJVS,P1800580 2 CMKVS 
C*: READ AND WRITE VARIABLES OF DIFFERENT TYPES

P 1800610 REWIND INV!

WRITE (INVI) CMAVS, MCAVI

P1800620

WRITE (INVI) CMA1S(1), CMA1S(2), CMBVS, MCBVI

WRITE (INVI) CMA1S(3), CMA1S(4), CMA1S(5), DPAVS, DPBVS

P1800630

REWIND INV!

READ (INVI) CMCVS, "MCCVI

READ (INVI) CMBIS(1), CMB1S(2), CMDVS, MCDVI

READ (INVI) CMBIS(3), CMB IS(4), CMBIS(5), DPCVS, DPDVS

CMEVS = CMAVS - CMCVS

CMFVS $=$ CMBVS - CMDVS

MCEVI = MCAVI - MCCVI

MCFVI = MCBVI - MCDVI

DPEVS = DPAVS - DPCVS

DPFVS = DPBVS - DPDVS

CMGVS $=$ CMA1S(1)-CMB1S(1)

CMHVS $=$ CMA1S(2)-CMB1S(2)

CMIVS $=$ CMA1S(3)-CMB1S(3)

CMJVS $=$ CMA1S(4)-CMB1S $(4)$

$P 9800640$

P1800650

P1800660

$P 1800670$

$P 1800680$

P1800690

P1 1800700

P 1800710

P 1800720

P. 1800730

$P 1800740$

P1800750

P1 1800760

P1 800770

P1800780

CMKVS $=$ CMA1S(5)-CMB1S(5)

P1 1800790

WRITE (NUVI,0182) CMEVS, CMFVS, MCEVI, MCFVI, DPEVS, DPFVS, CMGVS,P1800810

1 CMHVS, CMIVS, CMJVS, CMKVS

0182 FORMAT (//2(F20.10/), 2(119/),7(F20.10/))

$P 1800820$

$[* * * *$ TEST UNFORMATTED READ WITH NO LIST

P 1800830

REWIND INVI

WRITE (INVI) CMAVS, MCAVI

WRITE (INVI) CMAIS

WRITE (INVI) CMBVS, MCBVI

WRITE (INVI) CMA1S(5), CMAIS(4), CMA1S(3), CMA1S(2), CMA1S(1)

P1800840

P 1800850

P1 1800860

$P 1800870$

$P 1800880$

P 1800890

$[* * * *$ ENDFILE CAN NOT BE TESTED, BUT INCLUDED FOR ACCEPTANCE AS

P 1800900

$[* * * *$ A STATEMENT.

ENDF!LE INV!

REWIND INV!

$P 1800910$

P 1800920

P 1800930

C****CHECK THAT A RECORD IS READ WHEN NO LIST IS SUPPLIED BY COMPARING P1800940

$C * * * *$ VALUES OF THE THIRD RECORD

$P 1800950$

READ (INVI) CMCVS, MCCV!

$P 1800960$

READ (INVI)

READ (INVI) CMDVS, MCDV!

CMEVS $=$ CMAVS - CMCVS

CMFVS $=$ CMBVS - CMDVS

MCEVI = MCAVI - MCCVI

MCFVI = MCBVI- MCDVI

WRITE (NUVI, 0183) CMEVS, CMFVS, MCEVI, MCFVI

183 FORMAT $(/ / 2(F 20.10 /), 2(119 /))$

WRITE (NUVI,0184)

184 FORMAT 37 HO ALL ABOVE ANSWERS SHOULD BE ZERO IF I

1 37H THE READ AND WRITE RECORDS COMPARE.

REWIND INV!

$C * * * *$ END OF TEST SEGMENT 180

$C * * * *$ WHEN EXECUTING ONLY SEGMENT 180, THE STOP AND END

$C * * * *$ CARDS WHICH APPEAR AS COMMENT CARDS, MUST HAVE THE $C=$

$C * * * *$ IN COLUMNS 1 AND 2 REMOVED.

$C=$ STOP

$C=\quad E N D$

STOP

P1800970

P 1800980

P 1800990

$P 1801000$

P 1801010

$P 1801020$

P 1801030

P1801040

P1801050

P 1801060

P 1801070

P1801080

P 1801090

P1801100

P1801110

$P 1801120$

$P 1801130$

P1801140

P $1800^{\circ}$

END

P180C2

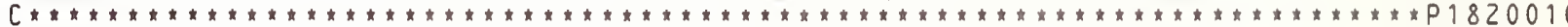

$C * * * *$

$C * * * *$

$[* * * * *$

$C * * * * *$

$C * * * *$ LENERAL PURPOSE

C**** WRITE A BLOCK, 1024 WORDS IN LENGTH, UNFORMATTED,

$C * * *$

$C * * * * *$

$C * * * * *$

$C * * * * *$

$C * * * * *$

S P E C I F I C A T I O N S SEGMENT 182

BACUP ( 182$)$

P1820020

$P 1820030$

P1820040

P 1820050

ASA REF P1820060

7.1.3.2.5P1820070

7.1.3.3.2P1820080

$7.1 .3 .2 .4 P 1820090$

P1820100

P 0013780

P 0013785 
$C * * * *$

C**** OU T P U T T A P E ASSIGNMENT STATEMENTS. NO INPUT TAPE.

P0013805

$C * * * *$

WHEN EXECUTING ONLY SEGMENT' 182, THE FOLLOWING STATEMENTS

P $182 \mathrm{~A}$

$P 0013810$

$C * * *$ NUVI $=6$ AND IRVI $=9$ MUST HAVE THE $C=$ IN COLUMNS 1 ANO 2 REMOVED.

$C * * * *$

$C * * * *$

$C=\quad$ NUVI $=6$

$C=\quad$ INVI $=9$

NUVI $=6$

INVI $=9$

P 1820110

$P 0072880$

$P 0072885$

$P 0072890$

$P 0072895$

$P 0072900$

$P 0072905$

P $182 B$

P $182 B 2$

$C * * * * *$

1820 FORMAT (1H1.1X.28HBACUP - (182) BACKSPACE TAPE//2X.18HASA REF.7.1.P1820120 $33.3 .2119 H \quad$ RESULTS)

WRITE (NUVI 1820)

$C * * * *$ HEAOER FOR SEGMENT 182 WR!TTEN

$C * * * * *$

REW!NO INV!

$C * *$ CREATE A LIST, 1024 WOROS IN LENGTH, CONTAINING

$C * * * *$ THE INTEGERS 1 TO 1024, ONE INTEGER PER WORO.

ISVI $=0$

MRRVI $=1$

1821 ISVI = ISVI + 1

IVII (ISVI) $=$ ISVI

IF (ISVI - 1024) 1821, 1822, 1823

$C * * * *$ WRITE THE LIST TO AN INTERMEOIATE TAPE

1822 WRITE (INVI) IVII

WRITE(NUVI, 1828) MRRVI, (IV1I (JCVI), JCVI =1,9),

1 (IVII (KCVI), KCVI $=1016,1024$ )

$C * * * *$ CHANGE MEMORY VALUES TO 5 TIMES THE ORIGINAL VALUES

MRRVI $=2$

ISVI $=0$

1825 ISVI $=$ ISVI +1

$I V 1 I(I S V I)=5 * \llbracket S V I$

IF (ISVI- 1024$) 1825.1826 .1823$

1826 BACKSPACE INVI

C**** WRITE THE CHANGEO VALUES

WRITE (NUVI, 1828) MRRVI, (IV1! (JCV!), JCVI=1,9),

1 (IVII(KCVI),KCVI $=1016,1024)$

MRRVI $=3$

$C * * * *$ READ INTERMEOIATE TAPE WHICH HAS BEEN BACKSPACEO READ (【NVI) IVII

REWINO INVI

$C * * * *$ WRITE INITIAL VALUES FROM BACKSPACED TAPE.

WRITE (NUVI,1828) MRRVI,(IVII (LVI), LVI =1,9), (IVII (KVI),KVI =

$1 \quad 1016,1024)$

1823 WRITE (NUVI.1829)

1828 FORMAT $(1 / 7 H$ GROUP, I $3,3(12 \times, 3(16)), 3(12 X, 3(16)))$

1829 FORMAT $(/ / 2 \times, 33$ HGROUPS 1 ANO 3 SHOULO BE THE SAME /

$130 \mathrm{H}$ ANO GROUP 2, 5 TIMES GROUP 1)

C**** ENO OF TEST SEGMENT 182

C*****WHEN EXECUTING ONLY SEGMENT 182, THE STOP ANO ENO

$C * * * *$ CAROS WHICH APPEAR AS COMMENT CAROS, MUST HAVE THE $C=$

$C * * * *$ IN COLUMNS 1 ANO 2 REMOVEO.

$C=$ STOP

$C=E N D$

STOP

P 1820130

P 1820140

P 1820150

P1820160

P 1820170

P 1820180

P 1820190

P 1820200

P 1820210

P 1820220

P 1820230

P 1820240

P 1820250

P 1820260

P 1820270

P 1820280

P 1820290

P1820300

P 1820310

P 1820320

P 1820330

P 1820340

P 1820350

P 1820360

P 1820370

P 1820380

P 1820390

$P 1820400$

P 1820410

P1 1820420

P 1820430

P 1820440

P 1820450

P 1820460

P 1820470

P 1820480

P 1820490

P1820500

P 1820510

P 1820520

P1820530

P 1820540

P1820550

P182C1

END

P $182 C 2$

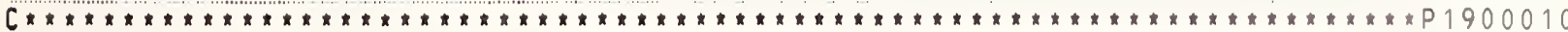

$C * * * * * *$

P 1900020

$C * * * * *$

OOTRM - (190)

P 1900030

$C * * * * *$

P 1900040

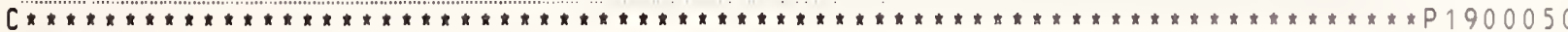

$C * * * *$

P 1900060 

P1900140

$C * * * * *$

$[* * * * *$ DO STATEMENT

$C * \hbar * * *$

* M1, M2 AND M3 aRE nOT REDEFINED WITHIN DO

$C * * * * *$

* bRanches to terminal STATEMENT FOR MORE THAN ONE DO ARE CONTAINED IN INNERMOST DO OF A NEST

$C * * * * *$ CONTROL IS NEVER PASSED INTO RANGE OF DO FROM OUTSIDE ITS RANGE

$7.1 .2 .8 / 07 P 1900150$

$7.1 .2 .8 / 10 P 1900160$

$C * * * * *$

$C * * * * *$

$C * * * * *$

$C * * * * *$

$C * * * *$

$S$ S E C I F I C A T I I O N S SEGMENT 190

$C * * * * *$

WHEN EXECUTING ONLY SEGMENT 190, THE SPECIFICATION STATEMENTS

$C * * * *$ IN COLUMNS 1 AND 2 REMOVED.

$C * * * * *$

$C=\quad$ DIMENSION IACII(5)

DIMENSION IACII(5)

$7 \cdot 1.2 .8 .210191900190$ P1900170

$C * * * * *$

$C * * * * *$

$C * * * * *$

$C * * * *$

O U T T P U T T T P E

ASS IGNMENT ST

TATEMENT

WHEN EXECUTING ONLY SEGMENT 190,"THE FOLLOWING STATEMENT

$C * * * *$ NUVI $=6$ MUST HAVE THE $C=$ IN COLUMNS 1 AND 2 REMOVED.

$C * * * * *$

$C=\quad$ NUVI $=6$

$C * * * * *$

$$
\text { NUVI }=6
$$

WRITE (NUVI, 8906)

8906 FORMAT (1H1,1X,25HDOTRM - (190) DO TERMINAL//2X,

-17HASA REF - 7.1.2.8/12X.7HRESULTS)

$C * * * *$ HEADER FOR SEGMENT 190

P 1900200

P 1900220

P 1900230

P 1900240

P0013820

$P 0013825$

$P 0013830$

$P 0013835$

P0013840

$P 0013845$

P $190 \mathrm{~A} 1$

$P 0013850$

P1900250

$P 0072920$

P 0072925

$P 0072930$

P 0072935

$P 0072940$

P $190 \mathrm{~B} 1$

$P 0072945$

$P 1900260$

$P 1900270$

P 1900280

P 1900290

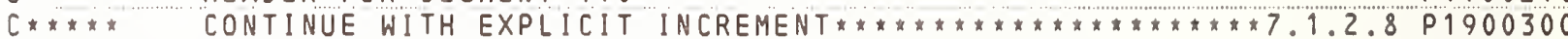
WRITE (NUVI.8905)

8905 FORMAT ( / / 2 X, 23HTESTI CONTINUE EXPLICIT)

C***** HEADER FOR CONTINUE EXPLICIT TEST

1900310

P 1900320

P 1900330

DO 1901 JACVI $=1,4,1$

$I A C 1](J A C V I)=J A C V I$

1901 CONTINUE

IF $(I A C 1 I(1)-1) \quad 1909,1902,1909$

P1900340

P 1900350

P 1900360

$P 1900370$

P 1900380

$P 1900390$

P 1900400

P 1900410

P 1900420

$P 1900430$

WRITE (NUVI,8904)MRRVI

8904 FORMAT (/2X,6H**TEST,I $1,1 \mathrm{X}, 17 \mathrm{HINOICATES} \mathrm{ERROR**)}$

P 1900440

P1900450

P 9900460 GO TO 8909

$C * * * * *$ NO ERROR

C*** WRITE OUT CONTINUE EXPLICIT TEST IS SUCCESS

P 1900470

$P 1900480$

P 1900490

MRRVI $=1$

WRITE (NUVI,8903)MRRVI

8903 FORMAT ( $2 X, 6 H * *$ TEST, 1 1, 1X,12HSUCCESSFUL**)

C*** SUCCESS FOR CONTINUE EXPLICIT TEST

P 1900500

P 1900510

P 1900520

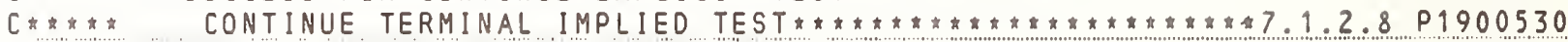
WRITE (NUVI, 8902)

8902 FORMAT (//2X, 22HTEST2 CONTINUE IMPLIED)

1900540

P 1900550

P1900560

$P 1900570$

$8909 \quad$ LCCVI $=2$

DO 7900 KBCVI $=$ LCCVI.4

P 1900580

P 1900590

7900 IACII (KBCVI) = KBCVI + 1 
9908 FORMAT (//2X,12HTEST3 ASSIGN)

$C * * * *$ HEADER FOR ASSIGN TEST

8908 MOCVI $=0$

ASSIGN 7904 TO JFCVI

DO 7902 NECVI $=2,5,2$ MDCVI = MDCVI +1

$P 1900720$

$P 1900730$

$P 1900740$

P 1900750

P 1900760

$P 1900770$

P 1900780

P1900790

7902 ASSIGN 7903 TO JFCVI

P1900800

GO TO JFCVI, (7903,7904,7904)

$C * * * *$ AN ERROR IN ASSIGN TEST

7904 MRRVI $=3$

WRITE (NUVI.8904) MRRVI

$P 1900810$

P 1900820

P1900830

P1900840

$C * * *$ ERROR FOR ASSIGN TEST

GO TO 8907

7903 IF (MOCVI-2) $7904,7905,7904$

C***** ASSIGN TEST IS SUCCESS

7905 MRRVI $=3$

WRITE (NUVI, 8903 ) MRRVI

P 1900850

P 1900860

P 1900870

$P 1900880$

P 1900890

P 1900900

C $* * * *$ SUCCESS FOR ASSIGN TEST

P 1900910

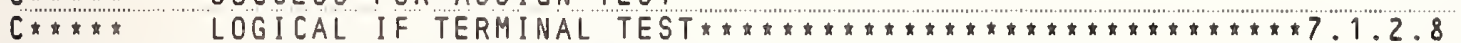
WRITE (NUVI,9905)

9905 FORMAT (//2X,16HTEST4 LOGICAL IF)

$P 1900920$

P1900930

$P 1900940$

$C * * *$ HEADER FOR LOGICAL IF TEST

P 1900950

8907 KGCVI $=1$

$P 1900960$

LHCVI $=3$

ASSIGN 7908 TO XCVI

P 1900970

DO 7906 JCVI $=1.3$

P 1900980

P 1900990

KGCVI $=$ KGCVI +1

P1901000

7906 IF (KGCVI .EQ. LHCVI) ASSIGN 7907 TO KCVI

GO TO KCVI $(7908,7907,7908)$

P 1901010

P 1901020

P 1901030

$C * * * * *$ TEST IS SUCCESS

7907 MRRVI $=4$

WRITE (NUVI, 8903) MRRVI

$C * * * *$ SUCCESS FOR LOGICAL IF TEST

P 1901040

P 1901050

P 1901060

60 TO 9902

C*** LOGICAL IF IS NOT SUCCESS

P1901070

$P 1901080$

7908 MRRVI $=4$

WRITE (NUVI,8904)MRRVI

$C * * * * \quad$ ERROR FOR LOGICAL IF TEST

9902 CONTINUE

$P 1901090$

P 1901100

P 1901110

P 1901120

P 1901130

P 1901140

$C * \# \#$ WHEN EXECUTING ONLY SEGMENT 190. THE STOP AND END CARDS

P1901150

$C * * * *$ WHICH APPEAR AS COMMENT CAROS MUST HAVE THE C

$C * * * * *$ IN COLUMNS 1 AND 2 REMOVEO.

$C=S T O P$

$C=\quad$ END

1901160

P 1901170

P 1901180

STOP

P190C1

P190C2

ENO

$P 1910010$

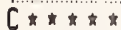

$C * * * * *$

DOLMT - (191)

P1910020

$C * * * * *$

P1910030

$P 1910040$

$C * * * * * * t)$

C***** GENERAL PURPOSE

$C * * * *$ TEST DO LOOPS WHERE 


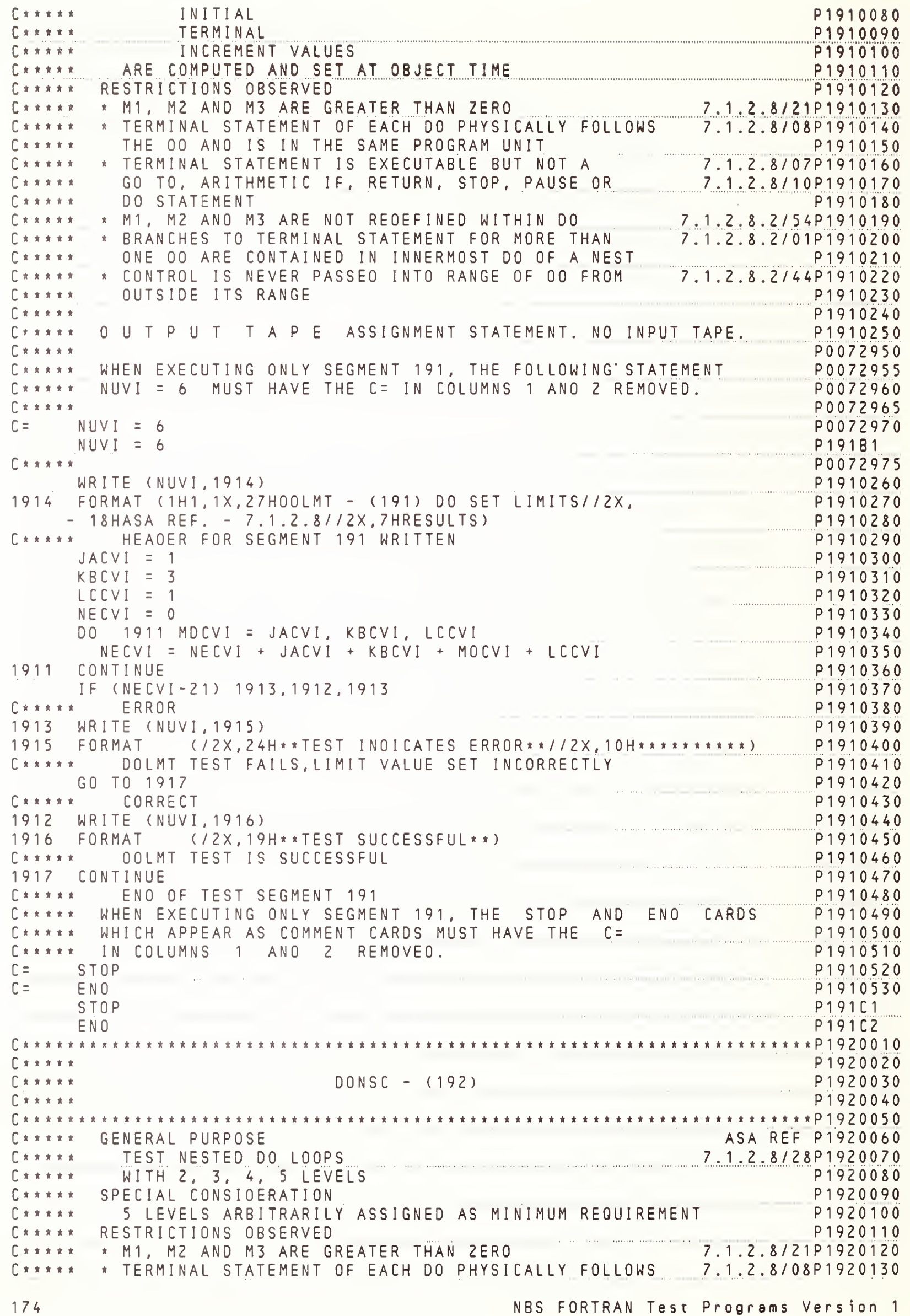


C*****M1, M2 AND M3 ARE NOT REDEFINED WITHIN DO

$C * * *$ BRANCHES TO TERMINAL STATEMENT FOR MORE THAN

C*** ONE DO ARE CONTAINED IN INNERMOST DO OF A NEST

$C * * * * *$

C*****SPECIF I C A T I O N S SEGMENT 192

$C * * * * *$

$C * * * *$ WHEN EXECUTING ONLY SEGMENT 192. THE SPECIFICATION STATEMENTS

$C * * * *$ WHICH APPEAR AS COMMENT CARDS MUST HAVE THE $C=$

C***:IN COLUMNS 1 AND 2 REMOVED.

$[* * * * *$

$C=$ INTEGER MCA3I $(2,3,3)$

INTEGER $\operatorname{MCA} 3$ I $(2,3,3)$

C*k***

$C * * * * \quad 0 U T P U T T$ T A P E ASSIGNMENT STATEMENT. NO INPUT TAPE.

$C * * * * *$

$C * * * *$

WHEN EXECUTING ONLY SEGMENT 192. THE FOLLOWING STATEMENT

C***** NUVI $=6$ MUST HAVE THE $C=I N$ COLUMNS 1 AND 2 REMOVED.

$C * * * *$

$C=N U V I=6$

$C * * * * *$

NUVI $=6$

WRITE (NUVI.8920)

8920 FORMAT (1H1,1X,26HDONSC - (192) NESTED LOOPSI/ $2 X$,

- 18 HASA REF. - 7.1.2.8/12X,7HRESULTS)

$C * * * *$ HEADER FOR SEGMENT 192 WRITTEN

$P 1920170$

$7 \cdot 1 \cdot 2 \cdot 8.2154 P 1920180$

$7.1 .2 .8 .2101 P 1920190$

P 1920200

P1920210

P1920220

P 0013860

$P 0013865$

P0013870

P0013875

P0013880

P0013885

P192A1

P0013890

P1920230

P0072980

P0072985

$P 0072990$

$P 0072995$

$P 0073000$

P192B1

P 0073005

P1920240

P1920250

$P 1920260$

P1920270

MRRVI $=2$

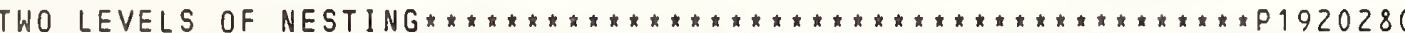

WRITE (NUVI, 8921) MRRVI

8921 FORMAT ( / / 2 . 11, 1X,17HLEVELS OF NESTING)

$C * * * *$ HEADER FOR TWO LEVELS

JACVI $=0$

$D O \quad 1922 \mathrm{KBCVI}=1,2,1$

JACVI $=$ KBCVI*3+JACVI DO 1921 LCCVI $=1,5,2$

JACVI $=$ JACVI + LCCVI

1921 CONTINUE

1922 CONTINUE

C*****TEST JACVI FOR VALUE OF 27

IF (JACVI-27) 1924.1923.1924

$C *: * *$ CORRECT

1923 WRITE (NUVI.8922)

8922 FORMAT (2X,19H**TEST SUCCESSFUL**)

$C * * * * *$ TWO LEVELS OF NESTING IS CORRECT GO TO 7927

C***** ERROR

1924 WRITE (NUVI.8923)

8923 FORMAT (2X,24H**TEST INDICATES ERROR**)

$C * * *$ TWO LEVELS OF NESTING IN ERROR

P1920290

P1920300

P 1920310

P 1920320

P1920330

P 1920340

p 1920350

P 1920360

P1920370

P 1920380

P 1920390

P1920400

P1920410

p 1920420

P1920430

P 1920440

P1920450

P1920460

P 1920470

P1920480

P 1920490

P1920500

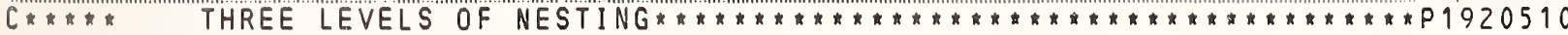

7927 MRRVI $=3$

WRITE (NUVI, 8921) MRRVI

$C * * * * \quad H E A D E R \quad F O R$ THREE LEVELS

$M D C V I=0$

DO 1927 LCCVI $=6,7$

$$
001926 \text { KBCVI }=8,10,2
$$

$D O 1925$ JACVI $=1,3,1$

1925

MDCVI $=$ MDCVI + JACVI + KBCVI + LCCVI

1926 CONTINUE

1927 CONTINUE

C***** TEST MOCVI FOR VALUE OF 210

IF (MOCVI - 210) 1928,1929,1928

ERROR

1928 WRITE (NUVI, 8923)

P1920520

P 1920530

P1920540

P1920550

P 1920560

P1920570

P1920580

P1920590

P1920600

P1920610

P 1920620

P1920630

P1920640

P1920650

P 1920660 
C**** THREE LEVELS OF NESTING IN ERROR GO TO 7928

\section{$C * * * *$ CORRECT}

1929 WRITE (NUVI, 8922)

$C * * * *$ THREE LEVELS OF NESTING IS CORRECT

7928 MRRVI $=4$

FOUR LEVELS OF NESTING

P 1920730

P 1920740

IHOV! $=0$

P1920750

$|\mathrm{GOV}|=0$

$|F O V|=0$

$P 1920760$

IEOVI $=0$

P 1920770

P 1920780

$I C V I=1$

P 1920790

P 1920800

007920 MDCVI $=2,3$

P 1920810

$\lfloor$ HOVI = IHOVI + MOCVI + IEDVI

$P 1920820$

007920 LCCVI $=3,5,3$

IGOVI $=1$ GOVI + LCCVI+ IHOVI

$007920 \mathrm{KBCVI}=1,2$, ICVI

$I F O V I=I F O V I+K B C V I+I G D V I$

007920 JACVI $=4,5,2$

IEOV! = IEOVI + JACVI + IFDVI

7920 CONTINUE

$C * * *$ TEST IEOVI FOR VALUE OF 185

IF (IEOVI - 185) 7921,7922,7921

$P 1920830$

P 1920840

P 1920850

P 1920860

p 1920870

P 1920880

P 1920890

P 1920900

P 1920910

P 1920920

7921 WRITE (NUVI, 8923)

$C * * *$ FOUR LEVELS OF NESTING IN ERROR

GO TO 7929

C**** CORRECT

7922 WRITE (NUVI, 8922)

C*** FOUR LEVELS OF NESTING IS CORRECT

p 1920930

P 1920940

P 1920950

P1920960

P 1920970

$P 1920980$

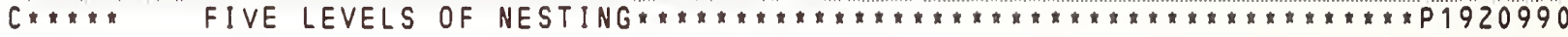

7929 MRRVI $=5$

WRITE (NUVI,8921) MRRV!

P 1921000

P 1921010

$[* * * *$ HEAOER FOR FIVE LEVELS

P 1921020

IGOVI $=0$

007923 NECVI $=10,11,1$

p 1921030

$007923 \mathrm{MOCVI}=4,5,1$

007924 LCCVI $=1,2,3$

$007924 \mathrm{KBCVI}=6,8,4$

007924 JACVI $=1,3,2$

IGOVI =IGOVI+JACVI-KBCVI+LCCVI-MOCVI+NECVI

P 1921040

P 1921050

P 1921060

P 1921070

$P 1921080$

7924 CONTINUE

7923 CONTINUE

C**** TEST IGOVI FOR VALUE OF 24

$C *$ IF (IGOVI - 24) 7925,7926.7925

P 1921090

P 1921100

P 1921110

P 1921120

P 1921130

P 1921140

7925 WRITE (NUVI,8923)

$C * *$ FIVE LEVELS IN ERROR

P1921150

P 1921160

GO TO 9923

7926 WRITE (NUVI, 8922)

C*** FIVE LEVELS CORRECT

$C * * *$ CONTROL VARIABLES FOR 3 DO LOOPS USED IN SUBSCRIPT EXPRESSIONS

$[* * *$ FOR A 3 OIMENSIONAL ARRAY

9923 WRITE(NUVI, 9920)

9920 FORMAT(//2X,34HCONTROL VARIABLE USEO IN SUBSCRIPT)

P 1921170

$P 1921180$

P 1921190

P 1921200

P 1921210

P 1921220

P 1921230

P 1921240

$I V I=1$

$K V I=0$

$8924 K V I=K V I+1$

JVI $=0$

$8925 \mathrm{JVI}=J V I+1$

$M C A 3 I(I V I, J V I, K V I)=I V I+2 *(J V I-1)+6 *(K V I-1)$

$M C A 3 I(I V I+1, J V I, K V I)=\lfloor V I+1+2 *(J V I-1)+6 *(K V I-1)$

IF (JVI-3) $8925,8926,8929$

P 1921250

P 1921260

P 1921270

P 1921280

P 1921290

P 1921300

P 1921310

P 1921320

8926 IF (KVI-3)8924,8927,8929

P 1921330

IIVI $=1$
$008928 \mathrm{KVI}=1.3$

P 1921340 
DO 8928 JVI $=1,3$

008928 IVI $=1,2$

IAVI $=M C A 3 I(I V I, J V I, K V I)-I I V I$

IF (IAVI) $8929,8928,8929$

$P 1921350$

$P 1921360$

$P 1921370$

P 1921380

P 1921390

WRITE (NUVI, 8922)

GO TO 9921

8929 WRITE (NUVI, 8923)

P 1921400

P 1921410

$P 1921420$

9921 CONTINUE

C**** END OF TEST SEGMENT 192

$C * * *$ WHEN EXECUTING ONLY SEGMENT. 192, THE STOP AND END CARDS

$C * * * *$ WHICH APPEAR AS COMMENT CARDS MUST HAVE THE C $C$

$C * * *$ IN COLUMNS 1 AND 2 REMOVED.

$C=S T O P$

$C=E N D$

STOP

END

$P 1921430$

P1921440

P 1921450

P 1921460

P 1921470

P 1921480

P 1921490

P192C1

P192C2

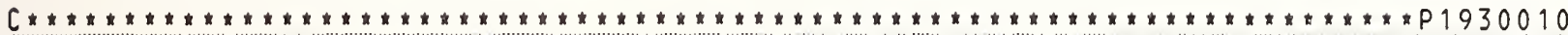

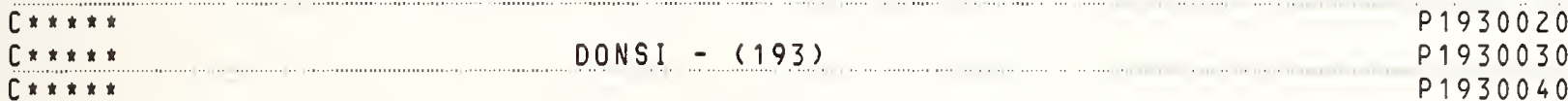

$C * * *$ GENERAL PURPOSE

C*****TESTS INCOMPLETE DO LOOP

$C * * * *$ RESTRICTIONS OBSERVED

C******M1, MZ ANO MZ ARE GREATER THAN ZERO

C****** TERMINAL STATEMENT OF EACH DO PHYSICALLY FOLLOWS

C***** THE DO AND IS IN THE SAME PROGRAM UNIT

C****** TERMINAL STATEMENT IS EXECUTABLE BUT NOT A

C***** GO TO, ARITHMETIC IF, RETURN, STOP, PAUSE OR

$C * * * *$ DO STATEMENT

$C * * * *$ M1, M2 AND M3 ARE NOT REDEFINED WITHIN DO

$C * * * *$ * BRANCHES TO TERMINAL STATEMENT FOR MORE THAN

$C * * * * *$ ONE DO ARE CONTAINED IN INNERMOST DO OF A NEST

$C * * * *$
$C * * * *$ U U T U T T A P E ASSIGNMENT STATEMENT. NO INPUT TAPE.

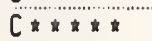

C***** WHEN EXECUTING ONLY SEGMENT 193, THE FOLLOWING STATEMENT

$C * * * *$ NUVI $=6$ MUST HAVE THE $c=$ IN COLUMNS 1 AND 2 REMOVED.

$[* * * * *$

$C=\quad$ NUVI $=6$

$C * * * * *$

NUVI $=6$

WRITE (NUVI, 1935)

1935 FORMAT ( 1 H1,1X,27HDONSI - (193) INCOMPLETE DO/12X,

- 18HASA REF. - 7.1.2.8/12X,7HRESULTS)

$C * * * *$ HEADER FOR SEGMENT 193 WRITTEN

$K B C V I=0$

DO 1931 JACVI $=1,5,1$

$K B C V I=K B C V I+J A C V I$

IF (KBCVI - 6) 1931, 1930, 1931

$1930 \quad$ GO TO 1932

1931 CONTINUE

C***** ERROR EXIT

WRITE (NUVI,1936)

1936 FORMAT ( 1 HO, 2X,28H* INCOMPLETE LOOP IN ERROR**)

$C * * * *$ INCOMPLETE LOOP TEST IN ERROR

GO TO 1937

C***** TEST JACVI FOR VALUE OF 3

1932 IF (JACVI - 3) 1933,1934,1933

ASA REF P 1930060

$7.1 .2 .8 .1 / 19 P 1930070$

$P 1930080$

$7.1 .2 .8 / 21 P 1930090$

$7.1 .2 .8108 P 1930100$

$P 1930110$

$7.1 .2 .8107 P 1930120$

$7.1 .2 .8 / 10 P 1930130$

$P 1930140$

$7.1 .2 .8 .1 / 54 P 1930150$

$7.1 .2 .8 .1 / 01 P 1930160$

P1930170

$P 1930180$

P1930190

$P 0073010$

POO 073015

$P 0073020$

$P 0073025$

$P 0073030$

P193B1

$P 0073035$

$P 1930200$

$P 1930210$

$P 1930220$

$P 1930230$

$P 1930240$

$P 1930250$

$P 1930260$

$P 1930270$

P 1930280

P 1930290

P 1930300

P 1930310

$P 1930320$

P 1930330

$P 1930340$

$7.1 .2 .8 .1 / 21 P 1930350$

$P 1930360$

P 1930370

P 1930380

P 1930390

P1930400

P 1930410

$P 1930420$

P 1930430

P 1930440

1939 FORMAT (1HO,1X,30H* I NCOMPLETE LOOP SUCCESSFUL**)

$C * * * *$ INCOMPLETE LOOP TEST SUCCESS 
$C * * *$ WHEN EXECUTING ONLY SEGMENT 193, THE STOP AND END CARDS P1930470

$[* * *$ WHICH APPEAR AS COMMENT CAROS MUST HAVE THE $C=\quad$ P 1930480

$C * * * * *$ IN COLUMNS 1 AND 2 REMOVED.

$C=\operatorname{STOP}$

$C=\quad$ ENO

STOP

P 1930490

P 1930500

ENO

P1930510

P193C1

P $993 C 2$

$C * * * *$

$C * * * * *$

DONSX - (194)

*P1940010

$C * * *$

P1940020

C********

$C * * *$ GENERAL PURPOSE

$C * * * *$ TESTS EXTENOEO RANGE OF 00 LOOP VARIABLE

$C * * * *$ RESTRICTIONS OBSERVEO

$C * * * *$ M1, M2 ANO M3 ARE GREATER THAN ZERO

C**** *TERMINAL STATEMENT OF EACH OO PHYSICALLY FOLLOWS

$C * * * *$ THE 00 AND IS IN THE SAME PROGRAM UNIT

C**** * TERMINAL STATEMENT IS EXECUTABLE BUT NOT A

$C * * * *$ GO TO, ARITHMETIC IF, RETURN, STOP, PAUSE OR

$[* * * * * 00$ STATEMENT

$C * * * *$ M1, M2 ANO M3 ARE NOT REOEFINEO WITHIN DO

$C * * * *$ BRANCHES TO TERMINAL STATEMENT FOR MORE THAN

$C * * * * *$ ONE DO ARE CONTAINEO IN INNERMOST OO OF A NEST

$C * * * *$ *THE EXTENOED RANGE OF A OO DOES NOT CONTAIN A

$[* * * * *$ DO OF THE SAME PROGRAM UNIT THAT HAS AN

$C * * * *$ EXTENOEO RANGE.

P 1940030

$P 1940040$

$C * * * * *$

$C * * * * *$

S P E C I F I C A T I 0 N S SEGMENT 194

$C * * * * *$

$C * * * * *$

WHEN EXECUTING ONLY SEGMENT 194, THE SPECIFICATION STATEMENTS

ASA REF P 1940060

$[* * * * *$

WHICH APPEAR AS COMMENT CAROS, MUST HAVE THE $C=$

$7.1 .2 .8 .2 P 1940070$

$C * * * *$ IN COLUMNS 1 ANO 2 REMOVEO.

$C * * * * *$

$C=$ DIMENSION IACII(5)

$C=\quad$ INTEGER $13 !(2,2,2)$

DIMENSION IACII (5)

INTEGER I3! $(2,2,2)$

$7.1 .2 .8 / 21 P 1940090$

$7.1 .2 .8 / 08 P 1940100$

$P 1940110$

$7.1 .2 .8 / 07 P 1940120$

$7.1 .2 .8 / 10 P 1940130$

$P 1940140$

$7.1 .2 .8 .2 / 54 P 1940150$

$7.1 .2 .8 .2101 P 1940160$

$P 1940170$

$7.1 .2 .8 .2 / 48 P 1940180$

P 1940190

$P 1940200$

$P 1940210$

$P 1940220$

$P 0013900$

$P 0013905$

$P 0013910$

P0013915

$P 0013920$

$P 0013925$

$P 0013930$

P194A1

P194A2

$C * * * * *$

$[* * * * *$ O U T P U T T A P E ASSIGNMENT STATEMENT. NO INPUT TAPE.

P 0013935

$C * * * *$

$C * * * * *$

WHEN EXECUTING ONLY SEGMENT 194, THE FOLLOWING STATEMENT

$C * * * *$ NUVI $=6$ MUST HAVE THE $C=$ IN COLUMNS 1 ANO 2 REMOVEO.

$C * * * * *$

$C=\quad$ NUVI $=6$

$C * * * * *$

NUVI $=6$

$P 1940230$

P0073040

$P 0073045$

$P 0073050$

$P 0073055$

P 0073060

$P 19481$

P 0073065

WRITE (NUVI,8944)

8944 FORMAT ( 1 H $1,1 \mathrm{X}, 31$ HOONSX - (194) EXTENDEO OO RANGE/I $2 X$.

$P 1940240$

$P 1940250$

120HASA REF. $-7.1 .2 .8 .21 / 2 X, 7$ HRESULTS)

P1940260

C***** HEADER FOR SEGMENT 194 WRITTEN

$P 1940270$

C***** EXTENOED RANGE FROM SINGLE LEVEL******************************P1940280 MRRVI $=1$

WRITE (NUVI,8942) MRRVI

$P 1940290$

$P 1940300$

P 1940310

8942 "FORMAT" ( / /2X, 26HEXTENOEO RANGE FROM LEVEL , I1)

P 1940320

P 1940330

DO $194 . \quad$ JACVI $=1,4,2$

IACII (JACVI) = JACVI

GO TO 1942

1943 IF (JACVI-1) $1945,1941,1945$

1941 CONTINUE

GO TO 1949

C**** TEST JACVI FOR VALUE OF 1

1942 IF (JACVI - 1) 1946.1943.1946

C*** TEST IAC1I(1) ANO IAC1I(3) FOR VALUES OF 1 ANO 3

1946 If $(I A C I I(1)-1) \quad 1947,7946,1947$

P 1940340

P 1940350

$P 1940360$

$P 1940370$

P 1940380

$P 1940390$

P 1940400

P 9940410

P 1940420 
1947 WRITE (NUVI.7947)

P 1940450

7947 FORMAT ( $/ 2 X, 24 \mathrm{H} *$ TEST INDICATES ERROR**)

C***** ERROR IN SETTING OF IACII ARRAY, LOOP NOT WORKING

P 1940460 GO TO 8940

C**** TEST JACVI FOR VALUE OF 3

P 1940470

P 1940480

P 1940490

1945 IF (JACVI - 3) 1948,1941,1948

P 1940500

$C * * * * \quad$ ERROR

P1940510

1948 HRITE (NUVI,7947)

C***** ERROR IN SETTING OF INDUCTION VARIABLE

$P 1940520$

GO TO 8940

P1940530

$P 1940540$

1949 WRITE (NUVI.7949)

7949 FORMAT ( $/ 2 X, 19 H *$ *TEST SUCCESSFUL**)

C***** EXTENDED RANGE SUCCESS FOR SINGLE LEVEL

P1940550

P 1940560

P1940570

8940 MRRVI $=2$

P1940580

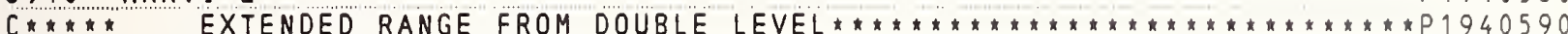

WRITE (NUVI.8942)MRRVI

$C * * * * *$ HEADER FOR DOUBLE LEVEL WRITTEN

$P 1940600$

$D O 7940 \quad K B C V I=3,4$

007940 JACVI $=1,2,3$

GO TO 7941

8947

I GOVI $=1$

7940 CONTINUE

C***** TEST JACVI FOR VALUE OF 1

7941 IF $(J A C V I-1) \quad 7942,7943,7942$

$C * * * * \quad$ ERROR

7942 HRITE (NUVI.7947)

C**** DOUBLE LEVEL NESTING IN ERROR

GO TO 8946

C***** TEST KBCVI FOR VALUE OF 3 OR 4

7943 If $(K B C V I-3) \quad 7942,8947,7944$

7944 If (KBCVI-4) 7942.7945 .7942

$C * * * * \quad$ CORRECT

$P 1940610$

P 1940620

$P 1940630$

$P 1940640$

$P 1940650$

P 1940660

$P 1940670$

$P 1940680$

$P 1940690$

$P 1940700$

$P 1940710$

$P 1940720$

$P 1940730$

$P 1940740$

$P 1940750$

P1940760

$P 1940770$

7945 WRITE (NUVI,7949)

$C * * * * *$ DOUBLE LEVEL TEST CORRECT

8946 CONTINUE

I3! $(1,1,1)=2$

$131(2,1,1)=4$

$13 !(1,2,1)=1$

$131(2,2,1)=2$

$131(1,1,2)=-2$

$131(2,1,2)=0$

$131(1,2,2)=-3$

$131(2,2,2)=-2$

8952 FORMAT( $/ 12 X .40 H E X T E N D E D$ RANGE CONTAINING A DO STATEMENT)

WRITE (NUVI. 8952 )

008948 IVI $=1,2$

$131(1,1,1 \vee 1)=131(1,1,1 \mathrm{VI})+1$

DO $8948 \quad J V I=1,2$

$131(1 . \mathrm{JVI}, 1 \mathrm{VI})=131(1 . \mathrm{JVI}, 1 \mathrm{VI})+2$

GO TO 8949

8959 CONTINUE

$8948 \quad$ CONTINUE

WRITE (NUVI.8950) I3I

8950 FORMAT $(8(115) / 30 H$ THE ABOVE 8 VALUES SHOULO BE $/$

$133 \mathrm{H}$ IN DESCENDING ORDER FROM 8 TO 1 )

GO TO 8953

$8949008954 \mathrm{KVI}=1.2$

$I 3 I(K V I, J V I, I V I)=13 I(K V I, J V I, I V I)+3$

8954 CONTINUE

GO TO 8951

8953 CONTINUE

$[* * * * *$ END OF TEST SEGMENT 194

$C * * * *$ WHEN EXECUTING ONLY SEGMENT 194, THE STOP AND END CARDS

$C * * * * *$ WHICH APPEAR AS COMMENT CARDS MUST HAVE THE $C=$

$C * * * * *$ IN COLUMNS 1 AND 2 REMOVED.

$C=S T O P$

$P 1940780$

$P 1940790$

$P 1940800$

P 1940810

P 1940820

P 1940830

P 1940840

$P 1940850$

$P 1940860$

P 1940870

P 1940880

$P 1940890$

P 1940900

P 1940910

$P 1940920$

P1940930

P 1940940

$P 1940950$

$P 1940960$

$P 1940970$

P1940980

P 1940990

P1941000

P 1941010

P1941020

P 1941030

$P 1941040$

P1941050

P1941060

$P 1941070$

P 1941080

P 1941090

P 1941100 


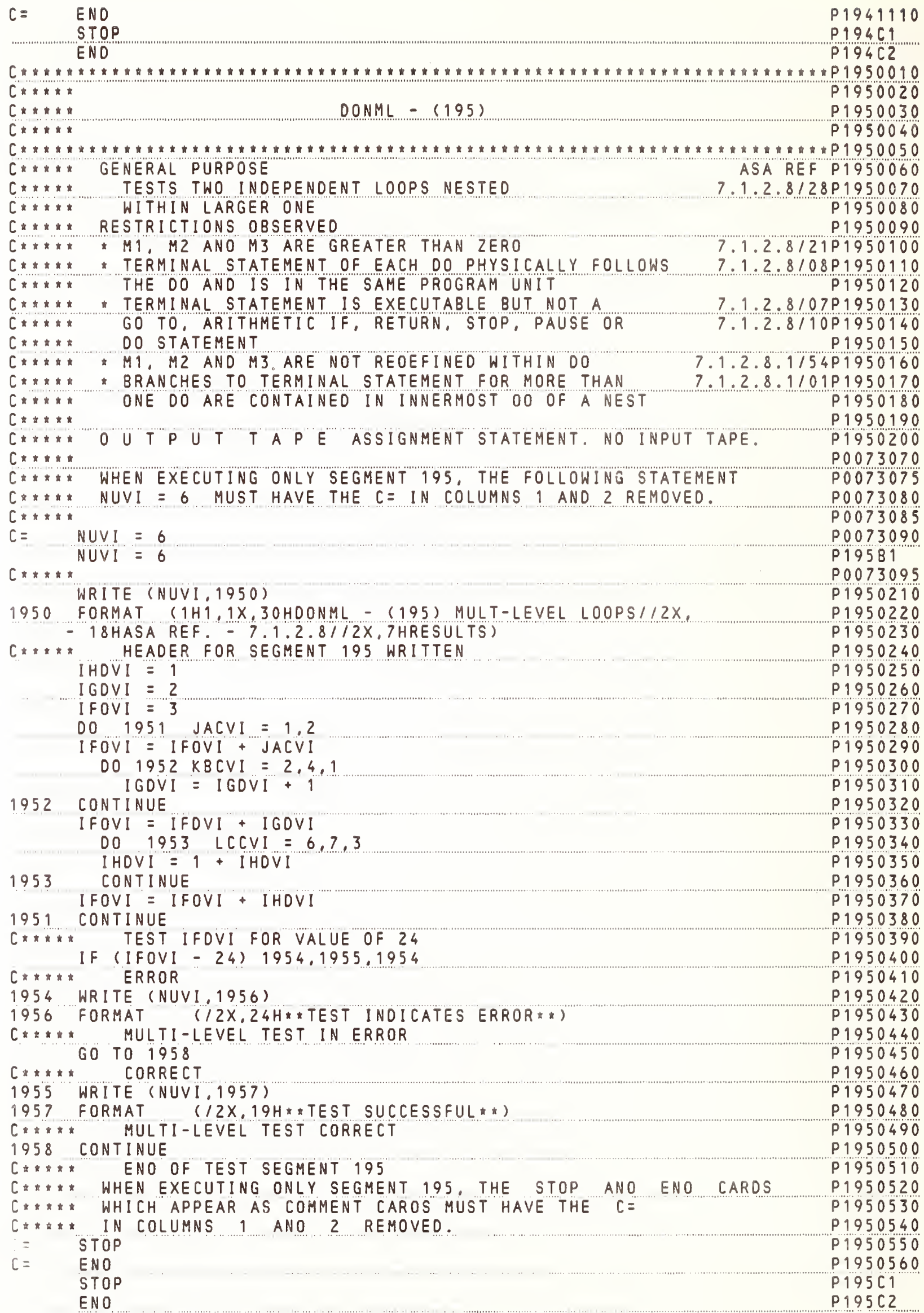




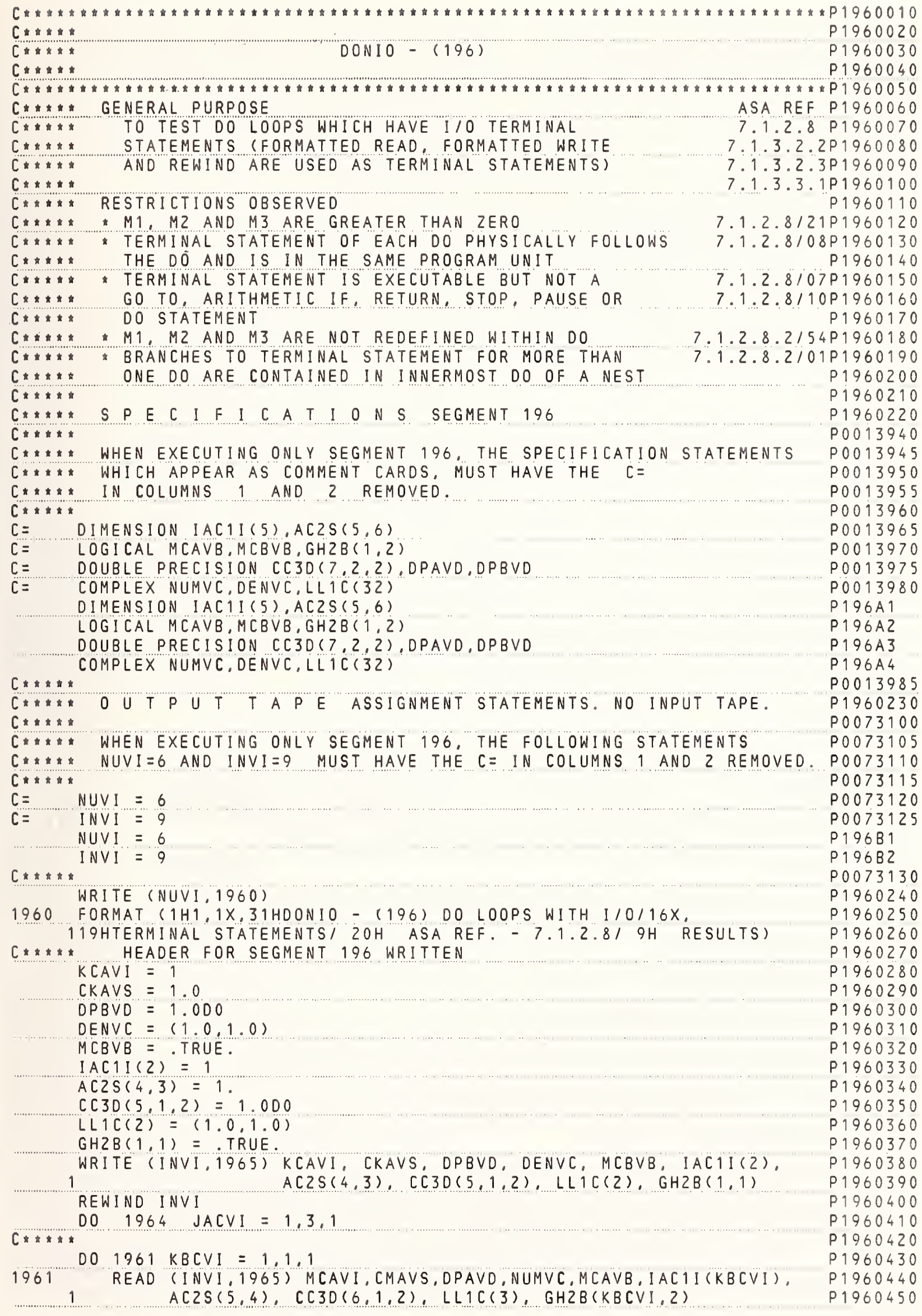


1964 CONTINUE

WRITE (NUVI, 1967)

C*\#*\# FORMAT STATEMENTS FOR THIS SEGMENT

$P 1960520$

$P \uparrow 960530$

P1960540

P 1960550

FORMAT (2(I5,F5.1,D8.1,2(F5.1), L5))

P 1960560

1965

1966

FORMAT ( $/ / 2(110 /), 2$ (F11.1/), 2(D 15

P 1960570

1967

FORMAT (1/30H THIS TEST IS SUCCESSFUL IF $3 / 38 \mathrm{H}$ IDENTICAL ORO

P 9960580

IS OF OUTPUT HAVE BEEN/12H GENERATED.)

$P P 9960590$

C***** END OF SEGMENT 196

C**** WHEN EXECUTING ONLY SEGMENT 196. THE STOP AND END CARDS

$C * * * *$ WHICH APPEAR AS COMMENT CARDS MUST HAVE THE C=

C*****IN COLUMNS 1 AND 2 REMOVED.

$C=S T O P$

$C=\quad E N D$

$P \uparrow 960600$

P 1960610

STOP

END

P1960620

$P 1960630$

P 1960640

P1960650

P1960660

P196C1

P $196 C 2$

$[\star * * *$
$C * * * *$

*P1970010

$C \star \star \star \star *$

$P 1970020$

$C * \star \star \star * t$

P 1970030

C $* * * * * * *$

MORDO - (197)

P 1970040

$[* * * * *$

GENERAL PURPOSE

C*t***t

$C * * * * *$

C*****

$C * * * *$

$C * * * *$

$C * * * * *$

$[* * * * *$

$C * * * *$

$C=$ DIMENSION IACII (5), MCAII (5)

A MORE COMPLicated SEgment testing the do statement

*P1970050

s P E C I F I C A T I O N S SEgment 197

ASA REF P1970060

7.1.2.8P1970070

P 1970080

WHEN EXECUTING ONLY SEGMENT 197 THE SPECIFICATION STATEMENTS

P 1970090

P 0013990

WHICH APPEAR AS COMMENT CARDS MUST HAVE THE $C=$

IN COLUMNS 1 AND 2 REMOVED.

$P 0013995$

$P 0014000$

P0014005

P 0014010

P0014015

DIMENSION IACII $(5), \operatorname{MCA} I I(5)$

P 197 A 1

$C * \pi * * *$

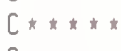

$[* * * * *$

$C * * * *$

$[* * * *$

WHEN EXECUTING ONLY SEgMENT 197, THE SEgMENT 005, WHICH

CONTAINS THE STATEMENT FUNCTIONS BEING USED HERE, MUST BE

P0014020

P1970100

INSERTED AFTER THE SPECIFICATION STATEMENTS OF SEGMENT 197.

P1970110

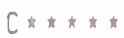

$C * * * *$

$[* * * * *$

$[* * * * *$

P 1970120

$P 1970130$

$C * * * * * * *$

$[* * * * *$

$C * * * * *$

$C * * * * *$

$[* * * * *$

$C * * * * *$

general purpose
defining statement functions that are to be tested

$B S F D-(005)$

PO

P0 050520

P0050530

IN SEGMENT 197

HEADER FOR SEGMENT 005

DEFINING EXPRESSION CONTAINS CONSTANTS AND VARIABLES

ASA REF POO50550

P0050560

CMAFS (CAWVS, CBWVS) = CAWVS*2.+ CBWVS

CMBFS(MAWVI, MBWVI, MCWVI) $=(M A W V I+M B W V I+M(W V I) / 3$

$M C A F I(M A W V I, M B W V I)=M A W V I * M B W V I$

$M C B F I$ (CAWVS,CBWVS, CCWVS ) $=($ CAWVS + CBWVS + CCWVS $) * 2.0$

$C * * * \#$ DEFINING EXPRESSION CONTAINS CONSTANTS, VARIABLES AND

C*****INTRINSIC FUNCTIONS

CMCFS (CAWVS, CBWVS, CCWVS ) = ABS (CAWVS*2-(CBWVS+CCWVS)*2)

CMOFS(MAWVI,MBWVI) = ISIGN((MAWVI+MBWVI),(MAWVI-MBWVI))

MCCFI(MAWVI, MBWVI, CAWVS $=$ MAWVI* $* 2+M B W V I * 2+I F I X(C A W V S) * 2$

MCDFI (CAWVS, CBWVS, CCWVS, CDWVS, CEWVS $)=($ CAWVS + CBWVS + CCWVS +

1 CDWVS + CEWVS )** (ABS (CAWVS))

C*****DEFINING EXPRESSION CONTAINS PREVIOUSLY DEFINED STATEMENT

C**** FUNCTIONS ANDIOR EXTERNAL FUNCTION REFERENCES

CMEFS(CAWVS, CBWVS) $=\operatorname{CMBFS}(1,2,3)+\operatorname{SORT}(($ CAWVS + CBWVS)

8.1.1P0050570

P0050580

P0050590

P0050600

P0050610

P0050620

P0050630

P0050640

P0050650

P0050660

P0050670

P0050680

P0050690

P0050700

P0050710

P0050720

P 0050730 
CMFFS(MAWVI, MBWVI, MCWVI) = MCCFI(MAWVI, MBWVI,3.0) + MCWVI * 2

MCEFI (MAWVI, MBWVI) = MCAFI (MAWVI, MBWVI) * MCAFI (MAWVI, MBWVI)

P0050740

MCFFI (CAWVS, CBWVS, CCWVS) = SORT (CAWVS) + SORT (CBWVS) + EXP(CCWVS) P0050760

C**** DEFINING EXPRESSION CONTAINS CONSTANTS, VARIABLES, INTRINSIC

C***** OR EXTERNAL FUNCTION REFERENCES ANO PREVIOUSLY OEFINEO

$C * * * *$ STATEMENT FUNCTIONS.

(MAWVI, MBWVI, CAWVS, CBWVS) = FLOAT(MAWVI**2) - CMAFS(CAWVS,P0050800

1 CBWVS) + SORT((FLOAT (MAWVI + MBWVI))) P P0050810

MCGFI(MAWVI, MBWVI, MCWVI, CAWVS) = MCEFI (MAWVI, MBWVI) - MCEFI (MAWVI,P0050820

$1 M(W V I)+I F I X(E X P(C A W V S))$

$C * * * *$ END OF TEST SEGMENT 005

$C * * * * \quad 0 \quad U T P U T$ T A P E ASSIGNMENT STATEMENTS. NO INPUT TAPE.

$P 0050830$

P0050840

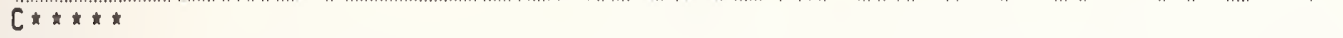

$C * * * * *$ WHEN EXECUTING ONLY SEGMENT 197, THE FOLLOWING STATEMENTS

$C * * *$ NUVI $=6$ AND INVI $=9$ MUST HAVE THE C $=$ IN COLUMNS 1 AND 2 REMOVED.

$C * * * *$

$C=N U V I=6$

$C=\quad I N V I=9$

NUVI $=6$

INVI $=9$

$C * \cdots * *$

WRITE (NUVI, 1970)

1970 FORMAT ( 1 H1, 1X.37HMORDO- (197) A MORE COMPLICATED SEG. $116 \mathrm{X}$.

$1 \quad 16$ HOF DO STATEMENTS/

$235 \mathrm{H}$ ASA REFS -7.1 .2 .8 AND $7.1 .2 .8 .1 / 19 \mathrm{H}$ RESULTS)

$C * * * *$ HEAOER FOR SEGMENT 197 WRITTEN

$C * \#$ TEST OF DO WITH STATEMENT FUNCTIONS AND INTRINSIC FUNCTIONS

$C * * * *$ REFERENCED WITHIN ITS RANGE. TO BE RUN WITH SEG. 005 AND 412 ASSIGN 9190 TO MVI

$M C B V I=0$

MCHVI $=1971$

DO 1971 MCAVI $=4,8,4$

CMAVS $=$ CMAFS $(1.0$, FLOAT(MCAVI) $)$

$1971 M C B V I=$ MCBVI + MCAFI (MCAVI,IFIX(CMAVS) $-(M C A V I+2))$

IF (MCBVI - 2) 9966,9190,9966

9190 MCHVI $=1973$

$C * * * *$ TEST OF 00 WITH CALL STATEMENTS REFERENCED WITHIN ITS RANGE $I V I=0$

ASSIGN 9968 TO MVI

001973 MCAVI $=1,3$

1973 CALL MOQ( MCAVI, IVI)

$I F(I V I-6) 9966,9968,9966$

C*** TEST OF OO WITH THE FOLLOWING FEATURES COMBINED -

$C * * * \quad 1$. AN EXIT FROM THE RANGE OF A OO BY THE EXECUTION OF A

C*** GO-TO STATEMENT, THE CONTROL VARIABLE OF THE OO IS

$C * * * \quad$ OEFINEO

C***** 2. A GO TO STATEMENT CAUSES CONTROL TO PASS FROM AN

$C * * * *$ INNER DO TO THE OUTER OO (WITHIN THE NESTEO RANGE)

$P 1970140$

$P 0073140$

P0073145

PO073150

$P 0073155$

$P 0073160$

$P 0073165$

P $197 B 1$

P $197 B 2$

$P 0073170$

$P 1970150$

P1970160

P 1970170

$P 1970180$

P 1970190

P1970200

P 1970210

P 1970220

P 1970230

P 1970240

P 1970250

P 1970260

P 1970270

P 1970280

P 1970290

$P 1970300$

P 1970310

P 1970320

P 1970330

P 1970340

$P 1970350$

P 1970360

$P 1970370$

$P 1970380$

9968 MCHVI $=1976$

ASSIGN 9191 TO MVI

MCBVI $=0$

DO 1976 MCAVI $=1.1,1$

9192 MCBVI $=$ MCBVI +1 .

$D O 1975 M C C V I=1,3,1$

$M C B V I=M C B V I+1$

IF(MCBVI - 4) 9197.9192.91975

9197 GOTO $(1975,9975,9966)$. MCCVI

1975 CONTINUE

1976 CONTINUE

IF (MCBVI - 8) 9966,9191,9966

$C * * * *$ TEST THAT THE STATEMENT LABEL OF THE TERMINAL STATEMENT

$C * * * * \quad$ OF MORE THAN ONE OO CAN BE USEO IN ANY GO TO OR ARITHMETIC

$C * *$ IF STATEMENT THAT OCCURS IN THE RANGE OF THE MOST OEEPLY

$3 P 1970390$

P1970400

P 1970410

$P 1970420$

$P 9970430$

$P 1970440$

$P 1970450$

$P 1970460$

$P 1970470$

$P 1970480$

$P 1970490$

$P 1970500$

P 1970510

$P 1970520$

$P 1970530$

P 1970540

P1970550

$C * * * *$ CONTAINED DO WITH THAT TERMINAL STATEMENT. $7.1 .2 .8 .2 / 1-6$

$C * * *$ ALSO THE CONTROL VARIABLE IS DEFINED WHEN EXIT IS MAOE BY THE

$C * * * *$ EXECUTION OF AN ARITHMETIC IF STATEMENT.

P 1970560

$P 1970570$

9191

ASSIGN 9194 TO MVI

$P 1970580$
$P 1970590$

$P 1970600$

$P 1970610$

NBS FORTRAN Test Programs Version 1

183 
MCEVI $=-24$

P 1970620

DO 1977 MCAVI $=1,2$

P 1970630

MCEVI $=$ MCEVI +1

DO 1977 MCBVI $=1,2$

P1970640

$M C E V I=M C E V I+1$

DO 1977 MCCVI $=1,5,1$

970650

MCEVI = MCEVI + 1

IF (MCEVI) $\quad 1977,1977,1978$

P 1970660

$P 1970670$

P 1970680

P 1970690

1977 CONTINUE

CONTINUE IF loOP tERMINATES thru CONTINUE

P 1970700

GO TO 9966

P 1970710

P 1970720

C***** CONTROL VARIABLE DEFINED ON FIRST LEVEL ON ARITH. IF P1970730

1978 MCEVI = MCAVI + MCBVI + MCCVI

MCHVI $=1978$

P 1970740

P 1970750

IF (MCEVI - 8) 9966.9194 .9966

P 1970760

9194 MCHVI $=1974$

P 1970770

MCEVI $=0$

P 1970780

ASSIGN 9961 TO MVI

P 1970790

DO 1974 MCAVI $=1.2$

P 1970800

DO 1974 MCBVI $=1,2,1$

DO 1974 MCCVI $=4,5,1$

P 1970810

DO 1974 MCDVI $=2,3$

$P 1970820$

GO TO 9193

$9195 \quad$ GO TO 1974

P 1970830

P 1970840

P 1970850

$9193 \quad$ MCEVI = MCAVI + MCBVI + MCCVI + MCDVI + MCEVI

GO TO 9195

P 1970870

CONTINUE

P 1970880

IF(MCEVI - 160) 9966,9961,9966

P 1970890

C***** TEST OF DO WITH I/O STATEMENTS REFERENCED WITHIN ITS RANGE.

C***** REWIND, UNFORMATTED READ AND WRITE ARE REFERENCED. THE

C**** FOLLOWING 3 DOS MUST BE KEPT TOGETHER FOR SELF-CHECKING

P 1970900

P 1970910

C***** PURPOSES

$9961^{\circ}$ MCHVI $=1972$

ASSIGN 9196 TO MVI

P 1970920

P 1970930

P 1970940

P 1970950

REWIND INVI

P1970960

DO 9963 MCAVI $=1,4$

P 1970970

$M C A I I(M C A V I)=M C A V I$

$P 1970980$

WRITE ( INVI) (MCAII (MCBVI), MCBVI $=1, M C A V I, 1)$

P 1970990

9963 CONTINUE

DO 9964 MCCVI $=1,4$

P 1971000

P 1971010

9964 REWIND INVI

DO 1972 MCDVI $=1,4$

READ (INVI) (IACII (MCEVI), MCEVI $=1, M C D V I)$

P 1971020

P 1971030

P 1971040

DO 1972 MCFVI = 1, MCDVI

P 1971050

MCGVI = IACII(MCFVI) - MCAII(MCFVI)

$P 1971060$

IF (MCGVI) 9966, 1972,9966

1972 CONTINUE

9196 WRITE(NUVI, 9969)

P 1971070

P 1971080

P 1971090

GO TO 9198

C*** ERROR MESSAGES IF DO STATEMENT IS EXECUTED IN ERROR

P 1971100

P 1971110

9966 WRITE (NUVI,9967) MCHVI

9967 FORMAT (// 36H DO RANGE ENDING AT STATEMENT LABEL, 15, $114 \mathrm{H}$ IS IN ERROR.)

P 1971120

$P 1971130$

$P 1971140$

P 1971150

9969 FORMAT // 35 H THIS SEGMENT SUCCESSFULLY TESTED /

P1971160

$222 \mathrm{H}$ IF NO ERROR MESSAGES)

GO TO MVI, (9190,9968,9191,9194,9961,9196)

$P 1971170$

9198 REWIND INVI

C ***** END OF TEST SEGMENT 197

C***** WHEN EXECUTING ONLY SEGMENT 197, THE STOP AND END CARDS

$C * *$ WHICH APPEAR AS COMMENT CARDS MUST HAVE THE $C=$

C****IN COLUMNS 1 AND 2 REMOVED.

$C=S T O P$

$C=\quad$ END

STOP

P 1971180

P 1971190

P 1971200

P1971210

P 1971220

P 1971230

P 1971240

P $197 C 1$

END

P $197 C 2$

$[* * * * * *$
$C * * * * *$

P4120010

MDO

- (412)

P4120020

P4120030 


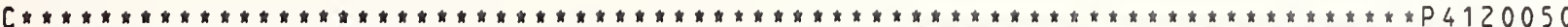

$C * * *$ GENERAL PURPOSE

C*** THIS SUBROUTINE IS USEO WITH SEGMENT 197 TO

$C * *$ SHOW THAT SUBROUTINES MAY BE CALLEO FROM OO LOOPS

SUBROUTINE MOO(MWVI, IWVI)

$I W V I=M W V I+I W V I$

RETURN

$C * * *$ ENO OF TEST SEGMENT 412

END
ASA REF P 4120060

$P 4120070$

$P 4120080$

$P 4120090$

$P 4120100$

P4120110

$P 4120120$

$P 4120130$

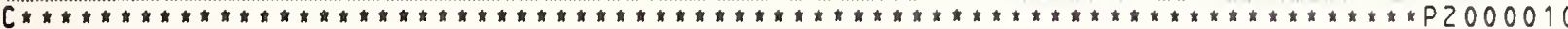

$C * * * *$

$C * * * * *$

SUBR $1-(200)$

$P 2000020$

$P 2000030$

$P 2000040$

$C * * * *$

$C * * * * *$

$C * * * * *$

GENERAL PURPOSE

ASA REF P2000060

C**** TO TEST SUBROUTINE SUBPROGRAM WITHOUT AN ARGUMENT LIST $8.4 .1 .1 P 2000070$

$C * * *$ GENERAL COMMENTS

C*** IT IS TO BE RUN WITH SEGMENT 410

$P 2000080$

$P 2000090$

$P 2000100$

$C * * * *$ RESTRICTIONS OBSERVEO

$C * *$ SYMBOLIC NAME OF A SUBROUTINE MAY NOT APPEAR IN ANY $8.4 .1 .1 / 56 P 2000110$

$C * * * *$

$C * * * *$ STATEMENT IN THIS SUBROUTINE EXCEPT IN THE

$P 2000120$

$P 2000130$

- SYMBOLIC NAMES OF OUMMY ARGUMENTS MAY NOT APPEAR 8.4.1.1/39P2000140

C*** IN EOUIVALENCE OR COMMON STATEMENTS IN THE SUBPROGRAM

C:**: SUBROUTINES MAY NOT CONTAIN A FUNCTION STATEMENT,
$C * *:$ ANOTHER SUBROUTINE STATEMENT, OR ANY STATEMENT THAT

$8.4 .1 .1 / 45 P 2000160$

OIRECTLY OR INOIRECTLY REFERENCES THE SUBROUTINE

$C * * *$ BEING OEFINEO

$C * * * *$ aT LEAST ONE RETURN STATEMENT MUST BE IN A SUBROUTINE

8.4.1.1/49P2000210

$C * * * *$

$C * * *$ S P E C I F I C A T I O N S SEGMENT 200

$C * * * * *$

$C * * * *$

WHEN EXECUT!NG ONLY SEGMENT 200, THE SPECIFICATION STATEMENTS

C***WHICH APPEAR AS COMMENT CAROS MUST HAVE THE $C=$

$C * * * *$ IN COLUMNS 1 ANO 2 REMOVEO.

$C=$ COMMON AXVS, CXVS, IXVI, IAXII(4)

$C * * * *$

COMMON AXVS, CXVS, IXVI, IAXII (4)

$C * \because \quad 0 \quad T P U T$ T A P E ASSIGNMENT STATEMENT. NO INPUT TAPE.

$C * * *$ WHEN EXECUTING ONLY SEGMENT 200 . THE FOLLOWING STATEMENTS

C*** NUVI=6 ANO INVI=9 MUST HAVE THE $C=$ IN COLUMNS 1 AND 2 REMOVEO.

$C * * * * *$

$C=$ NUVI $=6$

$C=\quad$ INVI $=9$

NUVI $=6$

INVI $=9$

WRITE (NUVI, 0200 )

200 FORMAT $(39 H 1$ SUBR 1 - $(200)$ SUBROUTINE SUBPROGRAM / $15 X$,

$124 \mathrm{HWITHOUT} \mathrm{AN} \mathrm{ARGUMENT} \mathrm{LIST} \mathrm{//18H} \mathrm{ASA} \mathrm{REF.} \mathrm{-} \mathrm{8.4.1//9H}$

$I X V I=N U V I$

$I A X 1|(1)=| N \vee I$

$P 2000170$

$P 2000180$

$P 2000190$

$P 2000200$

$P 2000220$

$P 0014030$

$P 0014035$

$P 0014040$

$P 0014045$

P0014050

P $200 A 1$

$P 0014055$

$P 2000230$

$P 0073180$

$P 0073185$

$P 0073190$

$P 0073195$

$P 0073200$

$P 200 B 1$

$\mathrm{P} 200 \mathrm{B2}$

$P 2000240$

$P 2000250$

RESULTS ) $P 2000260$

$P 2000270$

$P 2000280$

$P 2000290$

$P 2000300$

$P 2000310$

$P 2000320$

$P 2000330$

$P 2000340$

$P 2000350$

$P 2000360$

P200C1

$P 200 C 2$

ENO

$C * * *$

$C * * * *$

SUBRO - (410)

$P 4100020$

$P 4100030$

$P 4100040$

$C * * * *$

$C * * *$ THIS SEGMENT TESTS THAT A VARIETY OF FORTRAN STATEMENTS

$P 4100060$

NBS FORTRAN Test Programs Version 1

185 
C***** CAN BE USED IN A SUBROUTINE. IT IS TO BE RUN WITH SEGMENT 200 P4100070 SUBROUTINE SUBRO $\quad P 4100080$

8867 FORMAT (//36H DO RANGE ENDING AT STATEMENT LABEL, 15, 14H IS IN ERP4100090 IROR.)

DIMENSION KCAII(5), KACII(5)

$P 4100100$

COMMON BXVS, DXVS, NXVI, IXVI

$C * * * *$ DEFINE ARITHMETIC STATEMENT FUNCTION

$P 4100110$

$P 4100120$

CKAFS (CEWVS, CFWVS) = CEWVS*2.+CFWVS

8868 FORMAT ( //35H THIS SEGMENT SUCCESSFULLY TESTED $/$

$P 4100130$

$123 \mathrm{H}$ IF NO ERROR MESSAGES.)

KCAFI(KEWVI,KFWVI) = KEWVI*KFWVI

$C * * * *$ TEST OF DO WITH STATEMENT FUNCTIONS

KCHVI $=4101$

ASSIGN 4102 TO MVI

$P 4100140$

P4100150

$P 4100160$

P 4100170

$P 4100180$

P4100190

$P 4100200$

KCBVI $=0$

DO 4101 KCAVI $=4,8,4$

$P 4100210$

CKAVS $=$ CKAFS $(1.0$, FLOAT (KCAVII) $)$

$4101 K C B V I=K C B V I+K C A F I(K C A V I, I F I X(C K A V S)-(K C A V I+2))$

$P 4100220$

$P 4100230$

IF (KCBVI - 2) $8866,4102,8866$

C**** TEST OF DO WITH THE FOLLOWING FEATURES COMBINED -

$P 4100240$

$P 4100250$

$P 4100260$

C**** 1. AN EXIT FROM THE RANGE OF A DO BY THE EXECUTION OF A

$C * * * *$ GO-TO STATEMENT, THE CONTROL VARIABLE OF THE DO IS P P

$C * * * *$ DEFINED A GO P P

C**** 2. A GO TO STATEMENT CAUSES CONTROL TO PASS FROM AN P

C***** INNER DO TO THE OUTER DO (WITHIN THE NESTED RANGE)

$4102 \mathrm{KCHVI}=4106$

ASSIGN 8870 TO MVI

$K C B V I=0$

DO 4106 KCAVI $=1,1,1$

$8872 K C B V I=K C B V I+1$

$D O 4105 \mathrm{KCCVI}=1,3,1$

KCBVI = KCBVI +1

IF (KCBVI - 4) 8873,8872, 4105

8873 GO TO $(4105,4105,8866)$, KCCVI

4105 CONTINUE

4106 CONTINUE

IF (KCBVI - 8) $8866,8870,8866$

C**** TEST THAT THE STATEMENT LABEL OF THE TERMINAL STATEMENT

P4 100320

$P 4100330$

P4100340

P4100350

P 4100360

$P 4100370$

P 4100380

P 4100390

P4100400

P4100410

$P 4100420$

$P 4100430$

$P 4100440$

C**** OF MORE THAN ONE DO CAN BE USED IN ANY GO TO OR ARITHMETIC P4100450

$C * * * *$ IF STATEMENT THAT OCCURS IN THE RANGE OF THE MOST DEEPLY

$C * * * *$ CONTAINED DO WITH THAT TERMINAL STATEMENT

8870 ASSIGN 8876 TO MVI

KCHVI $=4107$

KCEVI $=-24$

DO 4107 KCAVI $=1.2$

KCEVI $=$ KCEVI + 1

DO 4107 KCBVI $=1,2$

KCEVI $=$ KCEVI +1

DO $4107 \mathrm{KCCVI}=1.5 .1$

$K C E V I=K C E V I+1$

IF (KCEVI) $\quad 4107.4107 .4104$

4107 CONTINUE

C****ERROR IF LOOP TERMINATES THRU CONTINUE

GO TO 8866

$C * * *$ CONTROL VARIABLE DEFINED ON FIRST LEVEL ON ARITH. IF

4104 KCEVI $=$ KCAVI + KCBVI + KCCVI

KCHVI $=4104$

IF (KCEVI - 8) $8866,8876,8866$

0876 KCHVI $=4103$

KCEVI $=0$

ASSIGN 8871 TO MVI

DO 4103 KCAVI $=1,2$

$D O 4103$ KCBVI $=1,2,1$

DO 4103 KCCVI $=4,5,1$

DO 4103 KCDV! $=2,3$

GO TO 8878

P4100460

$P 4100470$

P4100480

$P 4100490$

P4100500

P 4100510

$P 4100520$

P4100530

P4100540

$P 4100550$

P4100560

P 4100570

$P 4100580$

P4 100590

$P 4100600$

P4100610

$P 4100620$

$P 4100630$

$P 4100640$

P4100650

P4100660

P4 4100670

$P 4100680$

P4100690

P 4100700

P4100710

$P 4100720$

$P 4100730$

P4100740 
$\begin{array}{ll}4103 & \text { GOTO } 8877 \\ \text { CONTINUE }\end{array}$

$C * * *$ TEST OF DO WITH I/O STATEMENTS

8871 ASSIGN 8860 TO MVI

KCHVI $=4108$

REW IND I XVI

DO $8863 \mathrm{KCAVI}=1,4$

$K C A 1 I(K C A V I)=K C A V I$

WRITE (IXVI) (KCA I (KCBVI),KCBVI $=1, K C A \vee I, 1)$

8863 CONTINUE

DO 8864 KCCVI $=1,4$

8864 REWIND IXVI

DO 4108 KCDVI $=1.4$

READ (IXVI) (KACII(KCEVI),KCEVI $=1, K C D V I)$

DO 4108 KCFVI $=1, K C D V I$

$K C G V I=K A C I I(K C F V I)-K C A 1 I(K C F V I)$

IF (KCGVI) $8866.4108,8866$

4108 CONTINUE

8860 WRITE (NXVI.8868)

GO TO 8869

8866 WRITE(NXVI,8867) KCHVI

GO TO MVI, $(8860,4102,8870,8871,8876)$

8869 REWIND IXVI

RETURN

$C * *$ END OF TEST SEGMENT 410

END
IF(KCEVI - 160$) 8866.8871 .8866$

P4100750

$P 4100760$

$P 4100770$

$P 4100780$

$P 4100790$

P4100800

$P 4100810$

$P 4100820$

$P 4100830$

$P 4100840$

$P 4100850$

$P 4100860$

$P 4100870$

P4 100880

$P 4100890$

$P 4100900$

$P 4100910$

$P 4100920$

P4100930

P4100940

$P 4100950$

$P 4100960$

$P 4100970$

$P 4100980$

P4 100990

P4101000

P4101010

$P 3000010$

$P 3000020$

P 3000030

P3000040

C $x * x *$

LOGIF - $(300)$

P3000050

ASA REF P3000060

7.1.2.3P3000070

P3000080

P3 3000090

P3000100

P3000110

P3000120

$P 0014060$

$P 0014065$

P0014070

$P 0014075$

P0014080

P0014085

P0014090

P300A1

P3OOAZ

P0014095

$P 3000130$

$P 0073210$

$P 0073215$

$P 0073220$

$P 0073225$

P300B1

P3000140

P3000150

P3000160

P3000170

P3000180

P3 3000190

P3 3000200

P3 3000210

P3000220

P3000230

4.2111193000240

P3000250

P3000260 
IIVI $=-8$

$J V I=+0$

P3000270

JJVI $=-0$

$K V I=8$

KKVI $=8$

AVS $=-0.5$

P3000280

P3000290

$P 3000300$

AAVS $=-0.5$

P3000390

P3000320

BVS $=+0.0$

P3000330

BBVS $=-0.0$

P3000340

CVS $=0.5$

P 3000350

CCVS $=0.5$

P3000360

DPAVD $=-0.500$

$P 3000370$

DPBVD $=-0.500$

P3000380

$D P C V D=+0.0 D 0$

P3 3000390

DPOVD $=-0.000$

P3000400

DPEVD $=0.500$

P 3000410

DPFVD $=0.500$

P3000420

P3 300430

P3000440

IF( (JVI) .EO. (JJVI)) MACVI = MACVI + 1

$I F((J J V I) . E O$. (JVI)) MACVI = MACVI + 1

P 3000450

$I F((+0) \cdot E Q,(-0))$ MACVI $=$ MACVI + 1

P3 3000460

$I F((-0) \cdot E Q .(+0))$ MACVI $=$ MACVI +1

P 3000470

IF (MACVI - 4) 9951,9954,9951

9951 WRITE (NUVI, 9953)

P 3000480

P3000490

GO TO 9955

9952 FORMAT $(14 \mathrm{H}$ +O EOUALS - 0$)$

9953 FORMAT $(17 \mathrm{H}+0$ NOT EQUAL -0$)$

9954 WRITE (NUVI, 9952)

9955 MACVI $=0$

C***** TEST EXPLICITLY WRITTEN + 0.0 EQUALS - 0.0

$I F((B V S) . E O .(B B V S))$ MACVI = MACVI + 1

$I F((B B V S) . E Q .(B V S))$ MACVI $=M A C V I+1$

$I F((+0.0)$.EQ. $(-0.0))$ MACVI $=$ MACVI +1

$I F((-0.0) . E Q .(0.0))$ MACVI $=$ MACVI +1

IF (MACVI - 4) 9944,9947,9944

P3000500

P3000510

P3000520

$P 3000530$

P3000540

$P 3000550$

P3000560

P 3000570

83000580

P3000590

P3000600

$P 3000610$

9944 WRITE (NUVI, 9946)

P3000620

GO TO 9948

9945 FORMAT $(18 \mathrm{H}+0.0$ EQUALS -0.0$)$

9946 FORMAT $(21 \mathrm{H}+0.0$ NOT EQUAL -0.0$)$

9947 WRITE (NUVI, 9945)

C**** TEST EXPLICITLY WRITTEN +0.0DO EOUALS - 0.000

P 3000630

P3000640

P3000650

P3 3000660

P 3000670

P3000680

9948 MACVI $=0$

IF ( (DPCVD) .EQ. (DPDVD)) MACVI = MACVI + 1

$C * * * * *$

IF ((DPDVD).EQ. (DPCVD)) MACVI = MACVI +

$P 3000690$

P3000700

P3000710

IF $((+0.000)$.EQ. $(-0.000))$ MACVI $=$ MACVI +1

IF $((-0.000)$.EO. $(0.000))$ MACVI $=$ MACVI +9

$P 3000720$

IF (MACVI - 4) 9949, 9957,9949

9949 WRITE (NUVI; 9960)

GO TO 9958

9959 FORMAT (22H +0.0DO EQUALS - 0.0DO)

9960 FORMAT $(25 \mathrm{H}+0.000$ NOT EQUAL -0.000$)$

9957 WRITE (NUVI, 9959)

9958 MACVI $=0$

WRITE (NUVI, 7950)

7950 FORMAT ( 33 HO TEST COMPUTATIONAL SIGN OF ZERO/2X)

C*** $*$ TEST FOR COMPUTATIONALLY CREATED +0 AND - 0

IF ( (IVI * JVI).EQ. (JVI))MACVI = MACVI + 1

$I F((J V I) . E Q$. (JVI *IIVI))MACVI = MACVI + 1

$I F((J V I) I V I) . E Q .(+0)) M A C V I=M A C V I+1$

$I F((I V I+K V I) . E O$. (JVI) )MACVI = MACVI + 1

$I F((K K V I+I I V I) \cdot E O \cdot(J V I)) M A C V I=M A C V I+1$

IF ( (IIVI - IVI) .EO." (JVI) )MACVI = MACVI + 1

$I F((K V I-K K V I) \cdot E Q \cdot(J V I)) M A C V I=M A C V I+1$

P 3000730

P3000740

$P 3000750$

P3000760

P 3000770

P3000780

$P 3000790$

P 3000800

P3000810

P 3000820

P 3000830

$P 3000840$

P 3000850

P3 3000860

P 3000870

P3 3000880

P3000890

P3 3000900

IF (MACVI - 7)9956,9940,9956

9956 WRITE (NUVI.9953)

$P 3000910$

P3000920

GO TO 7955

P 3000930

9940 WRITE (NUVI.9952)

03000940 
C*\#\# TEST FOR COMPUTATIONALLY CREATED + 0.0 ANO -0.0

7955 MACVI $=0$

IF ((AVS"BVS) $\cdot E O \cdot(B V S))$ MACVI $=$ MACVI +1

$P 3000960$

IF ((BVS) .EO. (BVS*AAVS)) MACVI $=M A C V I+1$

P3000970

IF ( (BVS / AVS $) . E O .(0.0))$ MACVI $=$ MACVI +1

P3000980

$I F((A V S+C V S) \cdot E Q \cdot(B V S)) \cdot M A C V I=M A C V I+1$

P3 3000990

IF ( (CCVS + AAVS ) $\cdot E O \cdot($ BVS $))$ MACVI = MACVI + 1

P3 3001000

IF ( (AAVS - AVS) $\cdot E O \cdot(B V S))$ MACVI = MACVI + 1

$P 3001010$

IF ((CVS - CCVS) .EO. (BVS)) MACVI = MACVI + 1

P3001020

P3001030

IF (MACVI - 7) 7951,7952,7951

7951 WRITE (NUVI,9946)

GO TO 7953

7952 WRITE (NUV.I, 9945)

C*\#\# TEST FOR COMPUTATIONALLY CREATED + 0.000 AND - 0.000

7953 MACVI $=0$

$I F((D P A V D * D P C V D) \cdot E O \cdot(D P C V D)) M A C V I=M A C V I+q$

IF ( (DPCVD) .EO. (OPCVD* DPBVD)) MACVI = MACVI + 1

IF $((D P C V D$ I DPAVD) ..EQ. $(0.000))$ MACVI $=$ MACVI + 1

IF ( (DPAVD + DPEVD) .EO. (DPCVD) ) MACVI $=$ MACVI + 1

$I F((D P F V D+D P B V D) \cdot E Q .(D P C V D)) M A C V I=M A C V I+1$

IF ( (DPBVD - DPAVD) .EO. (DPCVD)) MACVI = MACVI + 1

IF ( (DPEVD - DPFVD) .EO. (DPCVD)) MACVI = MACVI + 1

IF (MACVI - 7) 7954, 9939, 7954

7954 WRITE (NUVI, 9960)

GO TO 9941

9939 WRITE (NUVI 9959)

9941 MCAVI $=0$

WRITE (NUVI, 9942)

9942 FORMAT 31 HO TEST - LOGICAL IF- FOLLOWED BYI

$131 \mathrm{H}$ DIFFERENT KINDS OF STATEMENTS )

C****: TEST 1

C**** LOGICAL IF FOLLOWEO BY SIMPLE ASSIGNMENT STATEMENT

$C * * * * \quad$ CORRECT RESULT $=0$, OTHERWISE RESULT $=1$

$P 3001040$

P3001050

P3001060

P3001070

P3001080

P3001090

P3001100

P3 3001110

P3001120

P3001130

P3001140

P3001150

P3001160

P3001170

P3 3001180

P3001190

P3001200

$P 3001210$

P3001220

P3001230

P3001240

P 3001250

P3001260

IF (MCA1B(2)) MCAVI = 1

P3001270

P3 3001280

WRITE (NUVI,3009) MCAVI

C*****TEST 2

C***** LOGICAL IF FOLLOWED BY USE OF INTRINSIC FUNCTION

$P 3001290$

P3001300

P3001310

$P 3001320$

CORRECT RESULT $=0$, OTHERWISE RESULT $=2$

$M C A V I=2$

IF (MCAVB) MCAVI $=I F I X(5.0-4.0-1.0)$

WRITE (NUVI, 3009 ) MCAVI

$M C A V I=0$

C***** TEST 3

C**** LOGICAL IF FOLLOWED BY ARITHMETIC STATEMENT

$C * * * *$ CORRECT RESULT $=0$, OTHERWISE RESÜLT $=3$

IF (MCAVB.AND. MCBVB) MCAVI $=3 * 212$

WRITE (NUVI,3009) MCAVI

C**** TEST 4

C*\#\# LOGICAL IF FOLLOWED BY GO TO STATEMENT

$C * * * \quad$ CORRECT RESULT $=0$, OTHERWISE RESULT $=4$

MCAVI $=0$

IF (MCAVB. AND. MCBVB .OR. MCAIB (1)) GO TO 3001

$M C A V I=4$

3001 WRITE (NUVI, 3009) MCAVI

$C * * * *$ TEST 5

C**** LOGICAL IF FOLLOWED BY CALL STATEMENT

C**** CORRECT RESULT $=0$, OTHERWISE RESULT $=5$

$M C A V I=0$

IF (MCBVB .OR. (1 . GE. 2) .AND..FALSE.) CALL SMCO(MCAVI)

$P 3001330$

P3001340

P3 3001350

P3001360

$P 3001370$

P3001380

$P 3001390$

P3001400

$P 3001410$

$P 3001420$

$P 3001430$

$P 3001440$

P3 3001450

P3001460

$P 3001470$

P3001480

P3 3001490

P3001500

P3001510

$P 3001520$

P3001530

WRITE (NUVI, 3009) MCAVI

C***** TEST 6

C**** LOGICAL IF FOLLOWED BY NESTED USE OF INTRINSIC FUNCTIONS

P3001540

P3001550

P3001560 CORRECT RESULT $=0$, OTHERWISE RESULT $=6$

$M C A V I=6$

P 3001570

P 3001580

IF (.TRUE. .OR. ( (1. .LE. $(0.1+1.5))$. AND. (MCA1B(1) .OR. . TRUE P3001590 1.)) . AND. MCBVB) MCAVI $=I F I X(R E A L((0.0,1.0)))$

WRITE (NUVI, 3009) MCAVI

$C * \#$ TEST 7

$P 3001600$

P3001610

P3001620 
IF (.NOT. (MCAVB.AND. MCBVB.AND. FALSE. .OR. (.NOT..TRUE.) )) P3001670

$1 G 0$ TO MCBV!, $(3001,3002,3003)$

33001680

GO TO 3003

3002 MCAVI $=0$

P 3001690

3003 WR!TE (NUV!,3009) MCAV!

$C * * * *$ TEST 8

C**** LOGICAL IF FOLLOWED BY ARITHMETIC IF STATEMENT

$P 3001700$

P 3001710

C***** CORRECT RESULT $=0$, OTHERWISE RESULT $=8$

P 3001720

$M C A V I=0$

IF (.NOT. (.NOT.(.TRUE. .OR.MCAVB.AND. (8. .NE. 7.))) )

P 3001730

P3001740

1IF (MCAVI) $3004,3005,3004$

3004 MCAVI $=8$

3005 WRITE (NUVI,3009) MCAVI

$C * * * *$ TEST 9

C***** LOGICAL IF FOLLOWED BY I /O STATEMENT

$P 3001750$

P3001760

P 3001770

$P 3001780$

$P 3001790$

$P 3001800$

P 3001810

$C * * *$ CORRECT RESULT $=0$, OTHERWISE RESULT $=9$

P3001820 $M C A V I=0$

IF $((8.000 . E Q .(1 .+7)$.$) . AND. (.NOT. (3.NE. 3 ) ))$

P 3001830

1 WRITE (NUVI, 3009 ) MCAVI

$C * * * * *$ TEST 10

C**** LOGICAL IF FOLLOWED BY COMPÜTED GO TO STATEMENT

P3001840

P3001850

P3001860

P 3001870

$C * * *$ CORRECT RESULT $=0$, OTHERWISE RESULT $=10$

MCAVI $=2$

IF (.TRUE. AND. (8.GE.6) .OR. (.FALSE.)) GO TO $(9950,3006)$,

$P 3001880$

$1 M C A V I$

$9950 \quad$ MCAVI $=10$

GO TO 3007

$P 3001890$

P3001900

P 3001910

P3001920

p 3001930

3006 MCAVI $=0$

P3001940

3007 WRITE (NUVI.3009) MCAVI

WRITE (NUVI, 3008 )

$C * * * *$ TEST EXPRESSIONS IN LOGICAL IF STATEMENTS

$C * * * *$ TEST 11 . LT. EXPRESSION, RELATION, EXPRESSION (TRUE)

P3001950

P3001960

P3001970

MCAVI $=1 \uparrow$

IF ((SNGL(DABS(-DSIGN(DBLE(2.0), 1.0D0)))). LT.AMIN 1((FLOAT(IDIM

P3001980

P3001990

P3002000

$1(1+2,0))),(A I M A G(C M P L X(1.0,2.0))))+1.0)$ MCAVI $=0$

WRITE (NUVI, 3009 ) MCAVI

C**** TEST 12 .LT. EXPRESSION, RELATION, CONSTANT (TRUE)

P3002010

$P 3002020$

MACVI $=12$

P3002030

P3002040

IF( (AMINI(FLOAT(IDIM(4-1,0)), AIMAG(CMPLX(1.0,2.0)))).LT.4.0 4 P3002050

1 MACVI $=0$

WRITE (NUVI, 3009 ) MACVI

03002060

P3002070

C**** TEST 13 . LT. CONSTANT(D.P.), RELATION, EXPRESSION (REAL) (TRUE)P3OOZOSO

MACVI = $13 \quad$ P3002090

$C * * * * I F(1 .(D 0) \cdot L T .(S N G L(D A B S(D S I G N(D B L E(4.0), 1.000)))))$ MACVI = 0

$C * * *$ WRITE (NUVI, 3009 ) MACVI

C***** TEST 14 . LE...AND...LE. (SHOULD BE LESS AND EOUAL) (TRUE) $M A C V I=14$

P 3002110

P 3002120

p 3002130

P 3002140

IF( (REAL $(C O N J G((1.0,-2.0)))+\operatorname{AIMAG}((16.0,-4.0)) . L E$.

$1 \quad \operatorname{AIMAG}(\operatorname{CONJG}((1.0,-2.0)))+\operatorname{REAL}((-4.0 .16 .0))+1.0)$.AND.

$2 \quad(A I M A G(C O N J G((2.0,-4.0)))+\operatorname{REAL}((-8.0,16.0)) . L E$.

P 3002150

P3002160

REAL(CONJG((4.0,-2.0)) ) + AIMAG $((16.0,-8.0)))) M A C V I=0$

WRITE (NUV!, 3009 ) MACVI

C**** TEST I 5 . LE. (FALSE)

$P 3002170$

P3002180

P 3002190

P3002200

MACVI $=0$

IF $(\operatorname{MAX} 1((\operatorname{AMAXO}(4,2,-(1 * 4))), 16.0)$.LE. 2*3)MACVI $=15$

WRITE (NUVI, 3009 ) MACVI

$C * * *$ TEST 16 .NE. AND. EO. (TRUE)

P3002210

P 3002220

P 3002230

MACVI $=16$
IF $((A I N T(A I N T(A I N T(1.4+2.9)+1.6)-8.1)) \cdot N E .(-8.0)) . A N D .(-1.0 . E 0 . P 3002250$

IAINT(AINT(AINT $(2.6+4.8)+1.4)-9.2))) M A C V I=0 \quad P 3002260$

WRITE (NUVI, 3009 ) MACVI

C*** TEST 17 . GT. (TRUE)

P 3002270

P3002280

P 3002290

$\operatorname{IF}((F L O A T(\operatorname{IABS}(\operatorname{IFIX(ABS}(-5.0+\operatorname{SIGN}(-1.0,2.0)))))) . G T .2 .0 D 0)$

P3002300 
$1 M A C V I=0$

WRITE (NUVI, 3009) MACVI

C*** TEST 18 .GE. EOUAL (TRUE)

P 3002320

$M A C V I=18$

P3002330

$P 3002340$

IF((8.0).GE.(FLOAT(IABS(IFIX(ABS(-4.0+SIGN(4.0.-2.0))))))) MACVI $=0$ P3002350

WRITE (NUVI, 3009 ) MACVI

$C * * * *$ TEST 19 .GE. GREATER (TRUE)

P 3002360

P3002370

MACVI $=19$

IF( (MACVI) . GE. (IABS(IFIX(ABS(-4.0+SIGN(8.0.4.0))))))MACVI=0 P3002390 WRITE (NUVI, 3009 ) MACVI

$C * * *$ TEST 20 .GT. (FALSE).OR. .EO. (TRUE)

P3002400

P3002410

MACVI $=20$

IF( (-MACVI) . GT. (MAXI (AMAXO $(8,-(2 * 4), 4), 16.0)) .0 R$. NOT. (IABS P300Z430

$1(-20)$. NE. MACVI) M MACVI $=0$

WRITE (NUVI, 3009 ) MACVI

WRITE (NUVI, 9943)

9943 FORMAT 28 HO ALL VALUES SHOULD BE ZERO.I

$137 \mathrm{H}$ A VALUE OTHER THAN ZERO WILL BE THE ।

$234 \mathrm{H}$ NUMBER OF THE TEST WHICH FAILED.)

P 3002430

P3 3002450

$P 3002460$

$P 3002470$

P3002480

$P 3002490$

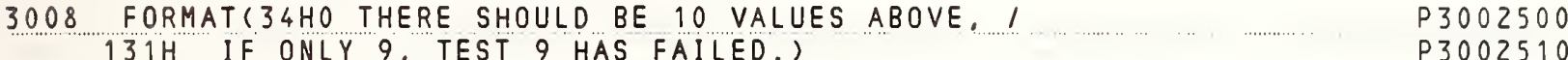

$131 \mathrm{H}$ IF ONLY 9, TEST 9 HAS FAILED.)

3009 FORMAT $(12 X$, I 10$)$

C**** END OF TEST SEGMENT 300

C***** WHEN EXECUTING ONLY SEGMENT 300. THE STOP AND END CARDS P P

$P 3002520$

$P 3002530$

$C * * *$ WHICH APPEAR AS COMMENT CAROS MUST HAVE THE $C=$ P 3002550

$C * * * *$ IN COLUMNS 1 AND 2 REMOVED.

$C=S T O P$

$C=\quad$ END

STOP

$P 3002560$

$P 3002570$

$P 3002580$

P3OOC 1

END

$P 300 C 2$

$C * * * * * * *$

$C * * * * *$

$C * m * * *$

SMCO- $-(411)$

110010

$C * * * * *$

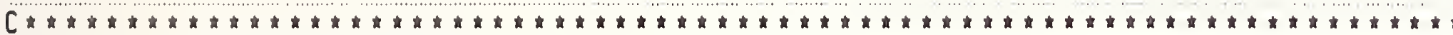

$C * * * *$ GENERAL PURPOSE

$C * * * *$ TO DEFINE SUBROUTINE SMCO WHICH IS USED IN SEGMENT 300 SUBROUTINE SMCO(MWVI)

$M W V I=M W V I+5$

RETURN

$C * * *$ END OF TEST SEGMENT 411 END

$P 4110020$

$P 4110030$

P4110040

110050

$P 4110060$

$P 4110070$

P4110080

P4110090

P4110100

P4110110

P4110120

C

$C * * * * *$

$B A R I F-(301)$

P3010020

$C * \pi * * *$

BARIF - $(301)$

P3010030

P3010040

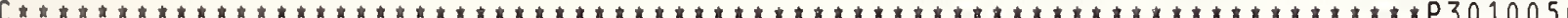

$C * * * *$ GENERAL PURPOSE

$C * * * *$ TEST BASIC FORTRAN ARITHMETIC IF STATEMENT

ASA REF P3010060

$C * * * *$ GENERAL COMMENTS

$C * * *$ BASIC INTRINSIC FUNCTIONS ASSUMED WORKING

7.1.2.2P3010070

P3010080

P3010090

P3010100

$C * \# * \# *$ S P E C I FI C A T I O N S SEGMENT 301

P3010110

$P 0014100$

C****WHEN EXECUTING ONLY SEGMENT 301 , THE SPECIFICATION STATEMENTS

$C * * * *$ WHICH APPEAR AS COMMENT CARDS. MUST HAVE THE $C=$

$C * \# * \#$ IN COLUMNS 1 AND 2 REMOVED.

$C=$ DIMENSION LII (10)

$C=$ DIMENSION MCAII (5), CMA1S(5)

DIMENSION LII $(10)$

DIMENSION MCA1I(5), CMA1S(5)

$C * * * * *$

$C * * * * \quad 0$ U TPU T T A P E ASSIGNMENT STATEMENT. NO INPUT TAPE.

$C * * * *$

$C * \| \cdots * *$

WHEN EXECUTING ONLY SEGMENT 301. THE FOLLOWING STATEMENT

$C * * * *$ NUVI $=6$ MUST HAVE THE $C=I N$ COLUMNS 1 AND 2 REMOVED.

$C * * * * *$

$C=$

NUVI $=6$

$P 0014105$

$P 0014110$

$P 0014115$

$P 0014120$

$P 0014125$

P301A1

P301AZ

P0014130

P3010120

$P 0073230$

P 0073235

$P 0073240$

P0073245

P 0073250

NBS FORTRAN TESt Programs Version 1 
WRITE (NUVI, 3010)

$P 0073255$

P 3010130

3010 FORMAT (1H1,1X,27H8ARIF - (301) BASIC FORTRAN/15X.

$24 \mathrm{HP} 3010140$

1 ARITHMETIC IF STATEMENT/2X,18HASA REF. - 7.1.2.2/2X,7HRESULTS) P3010150

$C * *$ HEADER FOR SEGMENT 301 WRITTEN

$M C A 1 I(1)=5$

P3010160

MCAVI $=0$

$M C B V I=21$

JACVI $=-0$

CMA1S(1) $=10.5$

CMAVS $=-0.0$

CMBVS $=-15 \cdot E 0$

$C * * * *$ TEST FOR SIGN OF ZERO - TYPE INTEGER

DO 8335 IVI $=1.9$

$P 3010170$

P3010180

P3010190

P 3010200

P 3010210

P3010220

P 3010230

8335 LII (IVI) $=0$

$4.2 / 11$ P3010240

P3010250

MV̈I $=1$

P 3010260

$K V I=0$

P $3010270^{\circ}$

$P 3010280$

JVI $=-0$

$8 V S=-0.0$

P3010290

NVI $=1$

P 3010300

WRITE (NUVI, 8300)

IF $(-0) 8311,8314,8317$

8320 IF (0) $8312,8315,8318$

$P 3010310$

P3010320

P 3010330

P 3010340

P3010350

IF $(+0) 8313,8316,8319$

8322 NVI $=10$

IF (JVI + (-0)) $8311,8314,8317$

P3010360

8323 IF (-IABS(JVI)) $8312,8315,8318$
8324 IF (-JVI+(+0)) $8313,8316,8319$

P 3010370

P3010380

P 3010390

8325 WRITE (NUVI, 8303 )(L1!(IVI), IVI $=1,9$ )

$C * * * *$ TEST FOR SIGN OF $2 E R O$ - TYPE REAL

P 3010400

P 3010410

MVI $=2$

P 3010420

KVI $=" 0$

P3010430

NVI $=1$

DO 8336 IVI $=1,9$

8336 L 1 I (IVI) $=0$

WRITE (NUVI, 8304)

P3010440

P3010450

P3010460

P 3010470

IF $(-0.0) 8311,8314,8317$

8326 If $(0.0) 8312,8315,8318$

8327 IF $(+0.0) 8313,8316,8319$

8328 NVI $=10$

IF $(B V S+(-0.0)) 8311,8314,8317$

8329 If (-ABS(BVS)) $8312,8315,8318^{\circ}$

8330 IF $(-B V S+(+0.0)) 8313,8316,8319$

8331 WRITE (NUVI, 8303) (LII(IVI), IVI $=1,9$ )

WRITE (NUVI, 8337)

P3010480

$P 3010490$

P3010500

P3010510

P3010520

P3010530

P 3010540

P3010550

P3010560

GO TO 8305

C***** SWITCH FOR INTEGER AND REAL TESTS

$8332 \mathrm{KVI}=\mathrm{KVI}+1$

GO TO $(8333,8334)$, MVI

C***** RETURNS FOR TEST SIGN OF INTEGER ZERO

8333 GO TO $(8320,8321,8322,8323,8324,8325), \mathrm{KVI}$

$C * * * *$ RETURNS FOR TEST SIGN OF REAL ZERO

8334 GO TO $(8326,8327,8328,8329,8330,8331), \mathrm{KVI}$

C**** TALLY RESULTS OF CONTROL TRANSFERS

8311 LII (1) $=$ LII (1) + NVI GO TO 8332

P 3010570

P3010580

P3010590

P3010600

P3010610

P3010620

P 3010630

P3010640

P3010650

P3010660

P 3010670

P3010680

$8312 L 1 I(2)=L 1 I(2)+N V I$

GO TO 8332

$8313 L 1 I(3)=L 1 I(3)+N V I$ GO TO 8332

P3010690

P3010700

P3010710

P3010720

P 3010730

P3 310740

P 3010750

P 3010760

P 3010770

P3010780

8317 L1I(7) = LII(7)+NVI 
8300 FORMATS/ 38 H TEST FOR SIGN OF 2ERO - TYPE INTEGER/I 29H PATH * FP3010840 10RM OF EXPRESSION *I 29H OF IF"

8303 FORMAT $(1 \mathrm{H}, 7(4 \mathrm{H} * * *) / 1 \mathrm{H}, 4(6 \mathrm{X}, 1 \mathrm{H} *) / 8 \mathrm{H}$ NEG, *,3(14,3H*)/1H,4P3010860 $1(6 X, 1 H *) / 8 H$ ZERO *,3(14,3H*)/1H, 4(6X,1H*)/8H POS.*,3614, P3010870 $23 \mathrm{H}, 2) / 1 \mathrm{H}, 4(6 \mathrm{X}, 1 \mathrm{H} *) / 1 \mathrm{H})$

P3 3010880

8304 FORMAT(/135H TEST FOR SIGN OF ZERO - TYPE REAL // 29H PATH" FOP3010890 1RM OF EXPRESSION $/ 29 H$ OF IF* $-0.0 * 0.0 *+0.0 *$ ) P3010900

8337 FORMAT $/ 34 \mathrm{H}$ ALL ENTRIES SHOULD BE O EXCEPT /36H THE ZERO PATH,P3010910 1 WHICH SHOULD BE $11 / 33 \mathrm{H}$ IN EACH COLUMN. OTHER TESTS MAY I 31 HP3010920 2 FAIL IF THESE RESULTS DIFFER.//137H TEST EXPRESSIONS IN IF STP3010930 3ATEMENTS /1H,

$C * * * * *$ ARITHMETIC IF WITH EXPRESSIONS OF TYPE INTEGER

C**** TEST 1 - SHOULD TAKE ZERO BRANCH

8305 IF (MCA1I(1) - S) $9981,3011,9981$

C**** TEST 2 - SHOULD TAKE ZERO BRANCH

3011 IF (MCAII (1) +5-IFIX(CMAIS(1))) $9982.3012,9982$

P3010940

C*\#\# TEST 3 - SHOULD TAKE MINUS BRANCH

P3010950

P 3010960

P3010970

P3010980

$P 3010990$

$P 3011000$

3012 IF ( (MCBVI*2/ 7) - IABS(IFIX(10.5-10.4)) - 7) 3013,9983.9983P3011010

$C * * * *$ TEST 4 - SHOULD TAKE PLUS BRANCH

3013 IF ((MCAII(1) - 4)* *99/(MCBVI - 4*MCAII(1))) 9984,9984,3014

$C * * * *$ ARITHMETIC IF WITH EXPRESSION OF TYPE REAL

C*\#** TEST 5 - SHOULD TAKE ZERO BRANCH

3014 IF (CMA1S(1) - 10.5) 9985,3015.9985

C***** TEST 6 - SHOULD TAKE MINUS BRANCH

3015 IF (CMA1S(1)*2.0 - (FLOAT(MCBVI)*1)-1.0) 3016.9986 .9986

C**** TEST 7 - SHOULD TAKE PLUS BRANCH

P 3011020

P 3011030

P3011040

P 3011050

P3011060

$P 3011070$

P 3011080

3016 IF (CMBVS*(-2.0)* (MCBVI - 4*MCA1I(1)) - 29.0)9987.9987.3017P3011100

C*\#** TEST 8 - SHOULD TAKE ZERO BRANCH

3017 IF (MCAVI) 9988,3018,9980

3018 WRITE (NUVI, 3019 )

GO TO 9980

3019 FORMAT ( 18 H TESTS SÜCCESSFUL)

9981 MCAVI = 1

IF (IABS(MCA1I(1)-5)) 8301.8302 .8301

8301 WRITE (NUVI,9989) MCAVI

GO TOO 3011

8302 WRITE (NUVI,8306) MCAVI

8306 FORMAT (//2X,14HERROR IN TEST, 12.23H BECAUSE MINUS ZERO WAS/ $13 O H$ TREATED AS A NEGATIVE NUMBER) GO TO 3011

9982 MCAVI $=2$ IF (IABS(MCAII(1) + 5 - IFIX(CMAIS(1)))) $8307,8308,8307$

8307 WRITE (NUVI,9989) MCAVI

GO TO 3012

8308 WRITE (NUVI, 8306)。MCAVI

GO TO 3012

9983 MCAVI $=3$

WRITE (NUVI,9989) MCAVI

GO TO 3013

9984 MCAVI $=4$

WRITE (NUVI,9989) MCAVI

GO TO 3014

9985 MCAVI $=5$

IF (ABS(CMA1S(1) - 10.5)) 8309.8310 .8309

8309 WRITE (NUVI,9989) MCAVI

GO TO 3015

8310 WRITE (NUVI,8306) MCAVI

GO TO 3015

9986 MCAVI $=6$

WRITE (NUVI,9989) MCAVI

GO TO 3016

9987 MCAVI $=7$

WRITE (NUVI.9989) MCAVI

$P 3011110$

P3011120

P 3011130

P3011140

P 3011150

$P 3011160$

P 3011170

P3011180

P3011190

P3011200

P3011210

P 3011220

P3011230

P3011240

P3011250

P3011260

P3011270

P 3011280

P3011290

P3011300

P 3011310

P 3011320

$P 3011330$

P3011340

P3011350

P 3011360

$P 3011370$

$P 3011380$

$P 3011390$

P3011400

$P 3011410$

P3011420

P3011430

P 3011440

$P 3011450$

P3011460 
GO TO 3017

P 3011470

MCAVI $=8$

P 3011480

WRITE (NUVI,9989) MCAVI

9989 FORMAT ( $6 \mathrm{H}$ TEST, $12,7 \mathrm{H}$ FAILED)

P3019490

$P 3011500$

9980 CONTINUE

C**** WHEN EXECUTING ONLY SEGMENT 301 , THE STOP AND END CARDS

P3011510

P3011520

$C * * *$ WHICH APPEAR AS COMMENT CARDS MUST HAVE THE C $\quad$ P 3011540

$[* * * *$ IN COLUMNS 1 ANO 2 REMOVEO.

$C=\quad$ STOP

$C=\quad$ ENO

STOP

END

$P 3011550$

P3011560

P3011570

P301C1

P301C2

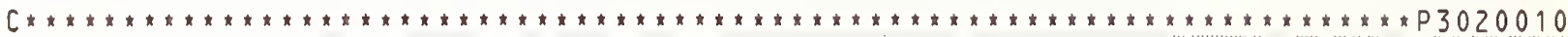

$C * * * * *$

$F A R I F-(302)$

$P 3020020$

$[* * * *$

$P 3020030$

$P 3020040$

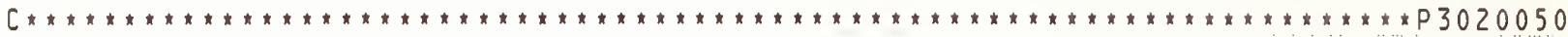

$[* * *$ GENERAL PURPOSE

C***** TEST OF FULL FORTRAN ARITHMETIC IF STATEMENT

$[* * * *$ GENERAL COMMENTS

$C * * * *$ INTRINSIC FUNCTIONS ASSUMEO WORKING

ASA REF P 3020060

7.1.2.2P3020070

$P 3020080$

$P 3020090$

P3020100

P3020110

S P E C I F I C A T I O N S SEGMENT 302

P0014140

C***** W**** WHEN EXECUTING ONLY SEGMENT 302 , THE SPECIFICATION STATEMENTS

$C * * * *$ WHICH APPEAR AS COMMENT CAROS, MUST HAVE THE $C=$

C****:IN COLUMNS 1 AND 2 REMOVEO.

C******1\%

$C=\quad$ OIMENSION MCAII(5), ACZS $(5,6)$

$C=\quad$ OOUBLE PRECISION MCAVD, MCBVD

$C=\quad$ COMPLEX CHAVC

DIMENSION MCA1I(5), ACZS $(5,6)$

DOUBLE PRECISION MCAVO, MCBVD

COMPLEX CHAVC

$[* * * * *$

O U T P U T T A P E ASSignMENT STATEMENT. NO INPUT tAPE.

$P 0014145$

$P 0014150$

$P 0014155$

$P 0014160$

$P 0014165$

$P 0014170$

$P 0014175$

P302A1

P $302 A 2$

P302A3

P0014180

$C * * * *$

WHEN EXECUTING ONLY SEGMENT 302, THE FOLLOWING STATEMENT

$[* * *$ NUVI $=6$ MUST HAVE THE C $=$ IN COLUMNS 1 ANO 2 REMOVEO.

P 3020120

$P 0073260$

$P 0073265$

$P 0073270$

$C * * * * *$

$C=\quad$ NUVI $=6$

$C * * * * *$

NUVI $=6$

$P 0073275$

$P 0073280$

P 30281

$P 0073285$

$P 3020130$

3020 FORMAT (1H1,1X,26HFARIF - (302) FULL FORTRAN/ 16X,24HARITHMETIC IP3O20140

1 F STATEMENTS / $\quad$ P3020150

$220 \mathrm{H}$ ASA REF. - 7.1 .2 .2 (2X,7HRESULTS)

C**** HEAOER FOR SEGMENT 302 WRITTEN

$M C A 1 I(1)=5$

$M C A V I=0$

$A C 2 S(1,1)=10.5$

$M C A \vee O=-15.000$

CHAVC $=(1.0,2.0)$

$P 3020150$
$P 3020160$

P3020170

P 3020180

P 3020190

$P 3020200$

P 3020210

P3 3020220

P3020230

$M C B \vee O=-0.000$

C**** ARITHMETIC IF WITH EXPRESSION OF TYPE OOUBLE PRECISION

$[* * * *$ TEST THAT MINUS ZERO IS TREATEO AS ZERO

P3020240

IF (MCBVO) $9301,9303,9301$

$4.2111 P 3020250$

$P 3020260$

P3020270

P 3020280

P 3020290

P3020300

P 3020310

P 3020320

P 3020330

P 3020340

$P 3020350$

P 3020360 
3022 IF (MCAVD/(-15.000) +6.0D0-2.0D0* *3) 3023.9971.9971

$C * * * *$ TEST 5 - SHOULD TAKE ZERO BRANCH

3024 IF $(2.000 * 2-4.00011 .000) 9973.3025 .9973$

P3020390

3025 IF (MCAVI) $9974,3026,9970$

P3020400

$P 3020410$

$P 3020420$

3026 WRITE (NUVI.3027)

GO TO 9970

3027 FORMAT ( $1 / 34$ H SEGMENT 302 TESTED SUCCESSFULLŸ.)

$P 3020430$

$P 3020440$

$P 3020450$

$3028 \quad M C A V I=1$

IF (DABS(MCAVD+15.000))9304,9305,9304

9304 WRITE (NUVI.9975) MCAVI

GO TO 3021

9305 WRITE (NUVI,9306) MCAVI

9306 FORMAT (1/2X,14HERROR IN TEST, 12,23 H BECAUSE MINUS ZERO WAS/

$130 \mathrm{H}$ TREATED AS A NEGATIVE NUMBER) GO TO 3021

3029 MCAVI $=2$

WRITE (NUVI.9975) MCAVI

GO TO 3022

9971 MCAVI $=3$

WRITE (NUVI,9975) MCAVI

GO TO 3023

$P 3020460$

P3020470

P3020480

P3020490

P3020500

P3020510

$P 3020520$

P3020530

P3020540

P3020550

P3020560

P3020570

P3020580

P3020590

9972 MCAVI $=4$

WRITE (NUVI.9975) MCAVI

P 3020600

GO TO 3024

9973 MCAVI $=5$

IF (DABS(2.000**2-4.000/1.000)) 9307.99308.9307

9307 WRITE (NUVI,9975) MCAVI

GO TO 3025

9308 WRITE (NUVI.9306) MCAVI

GO TO 3025

9974 MCAVI $=6$

WRITE (NUVI,9975) MCAVI

P3020610

$P 3020620$

$P 3020630$

$P 3020640$

$P 3020650$

$P 3020660$

$P 3020670$

$P 3020680$

P3020690

$P 3020700$

P 3020710

9975 FORMAT (1/6H TEST,I3.8H FAILED.)

9970 CONTINUE

$C * * * *$ END OF TEST SEGMENT 302

$C * * * *$ WHEN EXECUTING ONLY SEGMENT 302, THE STOP AND END CARDS

$C * * * *$ WHICH ÄPPEAR AS COMMENT CARDS MUST HAVE THE C $=$

$C * * * *$ IN COLUMNS 1 AND 2 REMOVED.

$C=$ STOP

$C=\quad$ END

STOP

END

P3020720

P 3020730

P3020740

P3020750

$P 3020760$

$P 3020770$

P3020780

P $302 C 1$

P302C2

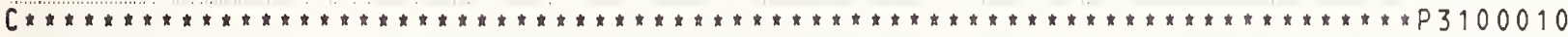

$C * * * *$

$P 3100020$

$C * * * * *$

IOFMT $-(310)$

$P 3100030$

$C * * * * *$

P3 3100040

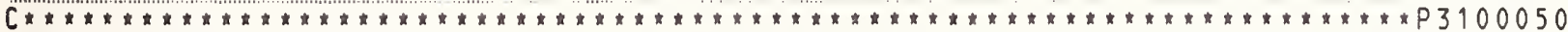

$C * * * *$ GENERAL PURPOSE

$C * * *$ TO TEST ADDITIONAL FEATURES OF FORMATTED READ

$C * * * *$ AND WRITE STATEMENTS AND FORMAT STATEMENTS

$C * * * *$ RESTRICTIONS OBSERVED

$C * * * * *$ ALL FORMAT STATEMENTS ARE LABELED

$C * * * * *$ H ANO X DESCRIPTORS ARE NEVER REPEATED

$C * * * * * *$ FOR W.D DESCRIPTORS, D IS ALWAYS SPECIFIED AND

C*\#** W IS EQUAL TO OR GREATER THAN D

$C * * * * *$ FIELD WIDTH IS NEVER ZERO

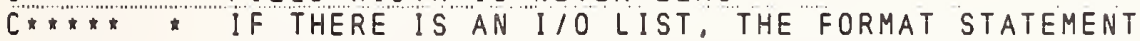

$C * * * *$ CONTAINS AT LEAST ONE FIELD DESCRIPTOR (OTHER

$(* * * *$ THAN HOR $X)$

$C * * *$ ITEMS IN I/O LIST CORRESPOND TO FORMAT DESCRIPTORS

C**** *NEGATIVE OUTPUT VALUES ARE SIGNED

$C * * * * *$ FIELD WIDTH NEVER EXCEEDED BY OUTPUT

$C * * * *$ FOR I CONVERSION, EXTERNAL INPUT FIELOS ARE

$C * * * *$ INTEGER CONSTANTS

C INPUT DATA TO THIS SEGMENT CONSISTS OF 38 CARD IMAGES IN COL. 1 - 80 P3100230

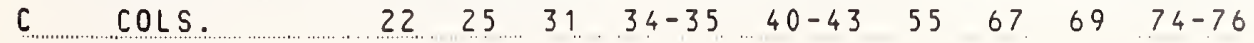

NBS FORTRAN Test Programs Version 1
ASA REFSP 3100060

7.1.3.2.2P3100070

7.1.3.2.3P3100080

$P 3100090$

$7.2 .3157 P 3100100$

$7.2 .3 .3 / 54 P 3100110$

7.2.3.1/31P3100120

7.2.3.1/33P3100130

7.2.3 $118 P 3100140$

7.2.3.4/22P3100150

$P 3100160$

$P 3100170$

$7.2 .3 .4 / 36 P 3100180$

$7.2 .3 .6 / 56 P 3100190$

7.2.3.6/01P3100200

$7.2 .3 .6 .1 / 07 P 3100210$

$P 3100220$
$P 3100230$

P3100240 
CARD 1

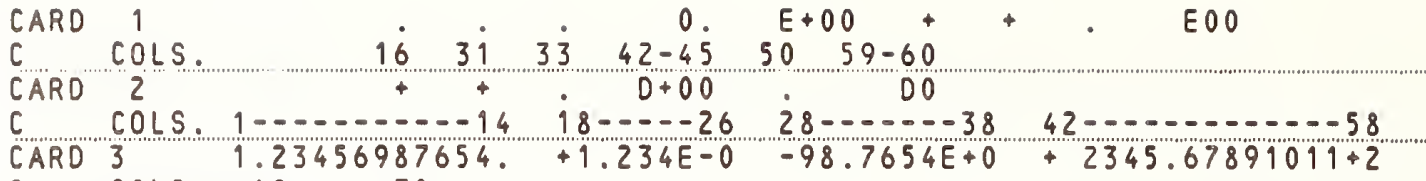

C COLS, $69-\cdots---78$

CARD $3 \quad-.109876-4$

C COLS $1---5$

CAROS $4.5,6.7 .8 \quad 12345$

C COLS. $1-3$

CARDS $9,10,11,12 \cdots 1.1$

P3100250

P3100260

P3100270

P3100280

P 3100290

P3100300

P 3100310

P3100320

P 3100330

P3100340

P 3100350

C COLS. 1-.

CARD $13+0.339567 E+02$

P3100360

CARD $14+.339567+2$

P 3100370

CARO $15+3.395670 E 1$

P 3100380

CARD 16

CARO 17

CARD 18

CARO 19

CARO 20

CARO 21

CARD 22

CARD 23

CARD 24

CARD 25

CARD 26

CARD 27

$0.962951342440+04$

.96295134244004

$0.96295134244+4$

P 3100390

P3100400

$0.962951342440+04$

P3100410

P3100420

$31.23+0.14 E+04+0.20+02$

P3100430

$31.23 .14 E+04+.2+2$

P3100440

$-0.13579 E+054444$

P 3100450

4444

4444

4444

4444

$-3335.555+0.4545 E-04$

P 3100460

P3100470

P3100480

P 3100490

$P 3100500$

$-6.666 \quad .9989 E+12$

CARD $29 \quad 7.77-0.747 E-02+0.549 E 022$

P 3100510

P3100520

$+0.662 E-00 \quad 0.468-1011$

$0.595420+04-44.6666-0.12345678900-03$

P 3100530

CARD 30

CARD 31

CARD 32

$54.9327-0.13956245340+00$

P3100540

P3100550

65432.1

CARD 33

$+0.848 E+03$

$.848 E 3+.129007+0.1290+07 \quad 0.412021$

P3100560

P3100570

CARD 35

CARD 36

222222222222222222222
$-.987 E 0-0.987 E+00$

22222222222222222222
$+0.600+0.60+00$

2222222

P3100580

P 3900590

P3100600

CARD 37

5555

$987654 \quad 8647.86 \quad 987.654$

P3100610

P3100620

CARD COLS. NOT MENTIONED ARE BLANK

C ******

$C * * * *$

READ AND WRITE STATEMENTS FOR ENTIRE SEGMENT FOLLOW"

P3100630

P3100640

$C * * * *$

TEST THAT COMPLETELY BLANK FIELDS IN THE INPUT

C*****ARE TREATED AS ZEROS. CALL VARIABLES AND ARRAY

P 3100650

P3100660

C*** ELEMENTS USED IN THIS TEST ARE FIRST SET TO

$C * * *$ NON-ZERO VALUES. I, E, F AND D DESCRIPTORS

$C * * *$ APPEAR IN THE CORRESPONDING FORMAT STATEMENT

P3100670

P3100680

P3100690

P3100700

P 3100710

$C * k * * *$

$C * * * * S P E C$ I F I C A T I O N S SEGMENT 310

P3100720

P3 100730

$C * * * * *$

C*:** WHEN EXECUTING ONLY SEGMENT 310, THE SPECIFICATION STATEMENTS

$C \# \#$ WHICH APPEAR AS COMMENT CARDS, MUST HAVE THE $C=$

C*AB: IN COLUMNS 1 AND 2 REMOVEO.

$C * * * *$

$C=$ DIMENSION AIS (5), AZS (2,2),A3S(3,3,3),EP1S(33)

$C=$ DIMENSION IAC11(5), IACZI(2,7), AC1S(25),AC2S(5,6)

$C=\quad$ INTEGER MCA3 I $(2,3,3)$

$C=\quad$ REAL MVS

$C=$ DOUBLE PRECISION MCAVD,MCBVD,MCCVD,A10(4),A20(2,2),A30(2,2,2)

$P 0014190$

P 0014195

$P 0014200$

P 0014205

P0 014210

POO14215

P0014220

P0014225

P0014230

P 0014235

$C=$ DOUBLE PRECISION OPAVD,OPBVD.DPCVD,DPOVD.DPEVD,DPFVD,DPHVD. AAAVD

DIMENSION A1S (5), AZS $(2,2), A 3 S(3,3,3)$, EP IS $(33)$

P0014240

P $310 \mathrm{~A} 1$

P310A2

DIMENSION IAC1 I (5), IACZI $(2,7), A C 1 S(25), A C 2 S(5,6)$

INTEGER MCA3! $(2,3,3$;

REAL MVS

DOUBLE PRECISION MCAVD,MCBVD,MCCVD,A1O(4),AZD $(2,2), A 3 D(2,2,2)$

DOUBLE PRECISION DPAVD, OPBVD, OPCVD, DPOVD, DPEVD, OPFVD, DPHVD, AAAVD

$C * \pi * \pi$ IN P U T - 0 U T P U T TAPE ASSIGNMENT STATEMENTS

P $310 A 3$

P $310 A 4$

P 310 A5

P310A6

$P 0014245$

P 3100740 
C $* * * *$

C***** HEADER FORMAT STATEMENT

3100 FORMAT (1H1.1X,38HIOFMT - (310) ADOITIONAL FORMATTED I/O

1 1/2X.38HASA REFS $-7.1 .3 .2 .27 .1 .3 .2 .37 .2 .3 / 12 \times, 7$ HRESULTS)

WRITE (NUVI, 3100)

JACVI $=11111$

$\triangle A C 1 I(1)=-2345$

$\operatorname{IAC21(1,1)=9999}$

$\operatorname{MCA} 3[(1,1,1)=2$

ACVS $=1.2$

$B C V S=-.34 E-3$

$A 1 S(1)=34.56$

$A 1 S(2)=456.789 E+02$

$A Z S(1.1)=-7899.3$

$A Z S(2.1)=+9876.543 E-01$

$A 3 S(1,1,1)=.543$

$A 3 S(2,1,1)=4.33 E+1$

AAAVD $=+2.220+01$

$A 1 D(1)=-.334560-01$

$A 2 D(1,1)=9987.760+2$

$A 3 D(1,1,1)=44 . D-2$

C**** FORMATS TO TEST THAT BLANK INPUT FIELDS ARE

$C * * *$ TREATED AS ZEROS.I.E,F AND D FIELDS ARE TESTED

$C * \pi *$ CARDS 1 AND 2

3101 FORMAT $(4(15), 4(F 3.1), 4(E 11.4) / 4(015.8))$

P 0073305

$P 0073310$

P 31081

P310B2

P0073315

P3100750

P3100760

P3100770

P 3100780

P3100790

P3100800

P3100810

P 3100820

P3100830

P3100840

P3100850

P3100860

P3100870

P3100880

P 3100890

P3100900

P3100910

P3100920

P3100930

P3100940

$7.2 .3 .6 / 45 P 3100950$

P3100960

P 3100970

P 3100980

READ (IRVI,3101) JACVI, IACII(1), IACZI(1,1), MCA3I (1,1,1), ACVS, P3100990 $1 \quad A 1 S(1), A Z S(1,1), A 3 S(1,1,1), B C V S, A 1 S(2), A Z S(2,1)$,

$2 \quad A 3 S(2,1,1), A A A V D, A 1 D(1), A 2 D(1,1), A 3 D(1,1,1)$

$P 3101000$

P 3101010

3102 FORMAT ( I 2X,16HTEST BLANK INPUTI2X,26HEACH ANSWER SHOULD BE 2ERO,P3101020

$14(1 / 6), 4(/ F 8.1) / 4(/ E 12.1) / 4(1012.1))$

$P 3101030$

WRITE (NUVI,3102) JACVI, IACII(1), IACZI(1,1), $\operatorname{MCA} I(1,1,1), A C V S, P 3101040$

$1 \quad A 1 S(1), A 2 S(1,1), A 3 S(1,1,1), B C V S, A 1 S(2), A 2 S(2,1), \quad P 3101050$

$2 \quad A 3 S(2,1,1), A A A V D, A 1 D(1), A Z D(1,1), A 3 D(1,1,1)$

P3101060

$C * * * *$ TEST THAT DECIMAL POINTS APPEARING IN INPUT FIELDS

$C * * * *$ OVERRIDE THE SPECIFICATIONS SUPPLIED BY E. F AND

$7 \cdot 2 \cdot 3 \cdot 6 / 47 P 3101070$

C*****DEIELD DESCRIPTORS

P3101080

3103 FORMAT (/34H TEST DEC. PT. SPECIFIED BY INPUT/ $36 H$ LINES IN EAP3101100 $1 \mathrm{CH}$ GROUP SHOULD MATCH / $26 \mathrm{H}$ "LINE IS HOLLERITH OATA)

WRITE (NUVI, 3103)

CMAVS $=1.23456$

CMBVS $=987654$.

CMEVS $=0.1234 E+01$

CMFVS $=-0.987654 E+02$

DPAVD $=0.2345678910110+06$

DPBVD $=-0.1098760-04$

$C * * *$ CARD 3

3104 FORMAT $(2(F 7.3), 2(E 12.5), 2(020.11))$

READ (IRVI, 3104) ACVS, BCVS, FFCVS, GGCVS, MCAVD, MCBVD

3105 FORMAT $(/ 12 \mathrm{H} * 1.23456,2(/ F 12.5) / / 13 \mathrm{H} * 987654.0 .2(/ F 13.1)$

P3101110

P3101120

P3101130

P3101140

P3101150

P3101160

P3101170

P3101180

P3101190

P3101200

P3101210

P3101220

$1 / 15 H * 0.1234 E+01.2(/ E 15.4) / / 17 H *-0.987654 E+02.2(/ E 17.6) / \% 3101230$

$2 / 23 H * 0.2345678910110+06,2(1023.12) / 1 / 7 H *-0.1098760-04, \quad P 3101240$

$32(1017.6))$

WRITE (NUVI,3105) CMAVS, ACVS, CMBVS, BCVS, CMEVS, FFCVS, CMFVS,

1 GGCVS, DPAVD, MCAVD, DPBVD. MCBVD

C* TEST SIMPLE REPETITION OF FORMAT DESCRIPTORS

C**** WHEN ADDITIONAL ITEMS REMAIN IN AN I/O LIST

$C * * * *$ AND THE LAST RIGHT PARENTHESIS HAS BEEN REACHED

C***** IN THE CORRESPONDING FORMAT STATEMENT

3106 FORMAT ( $35 \mathrm{H} 1$ TEST FORMAT DESCRIPTOR REPETITION/ $32 \mathrm{H}$

IIN EACH GROUP SHOULD/ $14 \mathrm{H}$ BE IDENTICAL)

WRITE (NUVI, 3106)
$7 \cdot 2 \cdot 3 \cdot 41$

7.1 .3 .2 .11

P3101250

P3101260

P 3101270

P3101280

P3101290

P3101300

P 3101310

ALL LINES P3101320

P3101330

P3101340 
$J A C V I=+12345$

KBCVI $=3$

CMAVS $=1.1$

P 3101370

CMBVS $=1.23$

P3101380

CMEVS $=33.9567$

$P 3101390$

CMGVS $=1.4 E+03$

P3101400

DPAVD $=962951342.440-5$

P 3101410

DPBVD $=2.001$

P 3101420

P3101430

P3101440

$P 3101450$

P3101460

P 3101470

P 3101480

P 3101490

P 3101500

P3101510

READ (IRVI,9320) A1S(1), HHCVS, A1S(2)

P3101520

P 3101530

$P 3101540$

READ (IRVI.9321) A2D

P3101550

$P 3101560$

9322 FORMAT (I 1,F4.2,E9.2,D8.1)

READ (IRVI,9322) LCCVI, DCVS, ACZS $(5,6), A 30(1,2,2), M D C V I, F F C V S, P 3101570$ 1 GGCVS, AAAVD

9323 FORMAT $(110 \mathrm{H} * 12345)$

WRITE (NUVI,9323)

P3101580

FORMAT (I 10)

WRITE (NUVI,9324) JACVI, IAC1I

P3101590

P 3101600

P3 3101610

P3101620

9325 FORMAT ( / 8H * 1.1)

WR!TE (NUV!,9325)

P 3101630

$P 3101640$

FORMAT (F8.1)

WRITE (NUVI,9326) CMAVS, AZS

9329 FORMAT ( /17H * $0.339567 \mathrm{E}+02)$

P3101650

P3101660

WRITE (NUVI, 9329)

$P 3101670$

P3101680

P3101690

WRITE (NUVI,9330) CMEVS, A1S(1), HHCVS, A1S(2)

P3101700

P3101710

$P 3101720$

WRITE (NUVI,9331)

$P 3101730$

P3 101740

WRITE (NUVI,9332) DPAVD, AZD

9333 FORMAT $\left(\begin{array}{llllll}131 \mathrm{H} & * 3 & 1.23 & 0.14 E+04 & 0.2 \mathrm{D}+02\end{array}\right)$

$P 3101750$

WRITE (NUVI,9333)

P3101760

P3 101770

P3101780

WRITE (NUVI,9334) KBCVI, CMBVS, CMGVS, DPBVD, LCCVI, DCVS,

P3101790

1 ACZS $(5,6), A 3 D(1,2,2), M D C V I, F F C V S, G G C V S, A A A V D$

C**** TEST THAT FORMAT CONTROL PASSES TO THE GROUP

C***** ENCLOSED BY THE LAST PRECEDING RIGHT PAREN.

C***** WHEN THE $1 / 0$ LIST CONTAINS MORE ELEMENTS THAN

$7.2 \cdot 3.4103 P 3101800$

C**** THE NUMBER OF DESCRIPTORS IN THE FORMAT STMNT.

7.1.3.2.1/39P3101810

P3101820

P 3101830

P3101840

JACVI $=+4444$

$K B C V I=-333$

$L C C V I=22$

P 3101850

MDCVI $=11$

ACVS $=5.555$

P3 101860

P3101870

P3101880

$B C V S=-6.666$

P3101890

CCVS $=+7.77$

P3101900

DCVS $=65432.1$

P3101910

CMAVS $=-0.13579 E+5$

P3101920

CMBVS $=0.4545 E-04$

P3101930

CMCVS $=0.9989 E 12$

P3101940

CMDVS $=-0.747 E-2$

P3101950

P3101960

CMEVS $=+0.549 E+00$

$P 3101970$

CMFVS $=0.662 E-0$

CMGVS $=0.468 E-10$

P 3101980

DPAVD $=+59.542002$

P 3101990

DPBVD $=-0.01234567890-2$

P3 3102000

DPCVD $=-1395624534 . D-10$

P3102010

DPDVD $=+129.04$

P3 102020 
READ (IRVI,9336) MRRVI, AC1S(1), EP1S(1), A3S(1,1,1), ACZS(2, 2)

$C * * * *$ CARDS 29,30

9337 FORMAT (F4.2, (2(E10.3)), I2)

$R E A D(1 R V 1,9337)$ A2S $(2,2), A 3 S(2,1,1), E P 1 S(2), \quad M C A 31(1,1,1)$, 1 BVS, $A C 2 S(2,1), N E C V I$

$C * * * *$ CARDS 31,32

9338 FDRMAT (D12.5, (F8.4, D17.10))

READ (IRVI,9338) MCAVD, EP1S(3), $A 1 D(1), A 2 S(1,2), A 2 D(2,1)$

P3102120

$P 3102130$

P3102140

P3102150

P3102160

P3102170

P 3102180

P3102190

P 3102200

$P 3102210$

$P 3102220$

P3102230

P 3102240

$C * * * *$ CARDS $33,34,35,36$

$C * * * *$ THIS READ CAUSES AN INPUT DATA CARD TO BE SKIPPED

9339 FORMAT( F7.1, (/2(E10.3), 2(D10.3)), 010.3)

P3102250

P3102260

P 3102270

READ (IRVI,9339) CVS, $A 2 S(2,1), A 3 S(1,2,2), A 3 D(1,1,1)$,

P 3102280 1

2

$A 30(1,2,1), A 2 D(2,2), A 3 S(1,2,1), E P 1 S(4)$. $A 10(2), M C B \vee D, M C C V D$

9340 FDRMAT (/16H * $-0.13579 E+05.2(1 \mathrm{E} 16.5) / 19 \mathrm{H} * 4444.6(1 / 9))$

P 3102290

P3102300

P3102310

WRITE (NUVI,9340) CMAVS, A1S(2), JACVI, IAC1I

9341 FDRMAT (I 8H*-333,2(1/18)/10H1*5.555,2(/F10.3)//

$115 \mathrm{H} * 0.4545 \mathrm{E}-04,2(/ E 15.4) / 110 \mathrm{H} *-6.666,2(/ F 10.3) / 1$

P3102320

$215 H * 0.9989 E+12,2(/ E 15.4))$

WRITE (NUVI,9341) KBCVI, MRRVI, ACVS, AC1S(1), CMBVS, EP1S(1),

1 BCVS, A3S $(1,1,1)$, CMCVS, ACZS $(2,2)$

9342 FDRMAT $(19 \mathrm{H} * 7.77,2(1 \mathrm{F9} .2) 1114 \mathrm{H} *-0.747 \mathrm{E}-02,2(1 \mathrm{E} 14.3) 11$

P3102330

P3102340

P3102350

P3 3102360

$P 3102370$

P 3102380

$114 \mathrm{H} * 0.549 \mathrm{E}+00,2(1 \mathrm{E} 14.3) 1 / 7 \mathrm{H} * 22,2(117) 11$

$214 \mathrm{H} * 0.662 \mathrm{E}+00,2(/ E 14.3) / 114 \mathrm{H} * 0.468 \mathrm{E}-10.2(/ E 14.3) / 1$

$37 \mathrm{H} * 11,2(117))$

WRITE (NUVI,9342) CCVS, AZS $(2,2)$, CMDVS, A3S $(2,1,1)$, CMEVS,

1 EP1S(2), LCCVI, MCA3I $1,1,1)$, CMFVS, BVS, CMGVS, AC2S $(2,1)$, 2 MDCVI, NECVI

9343 FORMAT (116H $0.595420+0402(1016.5)(112 \mathrm{H}$

$1 / 21 \mathrm{H}-0.12345678900-032(1021.0 j / 12 H 1 * 54.9327,2(1 \mathrm{~F} 12.4)(1 / \mathrm{P} 3102460$

$221 \mathrm{H} *-0.13956245340+00,2(1021.10))$

WRITE (NUVI,9343) DPAVD, MCAVD, FFCVS, EP1S(3), DPBVD, A1D(1),

1 GGCVS, AZS $(1,2)$, DPCVD, AZD $(2,1)$

9344 FDRMAT $(/ 12 \mathrm{H} * 65432.1 / 2(\mathrm{~F} 12.1 /) / 14 \mathrm{H} * 0.848 \mathrm{E}+03 /$

$13(\mathrm{E} 14.3 /) / 14 \mathrm{H} * 0.129 \mathrm{D}+07 / 3(\mathrm{D} 14.3 /) / 14 \mathrm{H} * 0.412 \mathrm{D}+21 /$

$22(014.3 /) / 14 \mathrm{H} *-0.987 \mathrm{E}+00 / 3(\mathrm{E} 14.3 /) / 12 \mathrm{H} * 0.6 \mathrm{D}+00 /$

$33(012.1 /), 14 \mathrm{H} * 0.368 \mathrm{D}-05,2(1014.3))$

P3102390

$P 3102400$

P 3102410

P3 102420

P 3102430

P3102440 WRITE (NUVI,9344) DCVS, CVS, HHCVS, AZS $(2,1), A 3 S(1,2,2)$, DPDVD,

$$
1
$$

2

3

9345

FORMAT $(/ 14 \mathrm{H}$

$A 3 D(1,1,1), A 3 D(1,2,1), D P E V D, A 2 D(2,2)$

MVS, A3S $(1,2,1), E P 1 S(4), D P H V D, A 1 D(2), M C B V D$, DPFVD, MCCVD

WRITE (NUVI,9345) CCVS, A2S $(2,2)$

9346 FORMAT ( / 22H * $-333 \quad 0.595420+04 / 18,014.5)$

WRITE (NUVI,9346) KBCVI, DPAVD, MRRVI, MCAVD

9347 IF (MRRVI - 5) 9348,9349,9348

$C * * * *$ CARD 37

9348 READ (IRVI, 9336) MRRVI

GO TD 9347

$C * * * * * *$ ADDITIONAL SCALE FACTOR ON INPUT-OUTPUT

$C * * * *$ CARO 38

9349 READ (IRVI, 9327) A1S(3), A1S(4), A1D(4)

9327 FORMAT ( IPE10.3,-1PE10.2, D10.3)

WRITE (NUVI, 9328$)$ A1S(3), A1S(4), A1D (4)
P 3102470

P 3102480

P3 102490

$P 3102500$

P3102510

P3 3102520

P3102530

P3102540

P3102550

P3102560

P3102570

P3102580

P 3102590

P3102600

P3102610

P3102620

P3102630

P3102640

P3102650

P3102660

P 3102670

P3102680

P3102690

P 3102700 
9328 FORMAT///22H1 SCALE FACTOR ON READ/ $31 \mathrm{H}$ IN ORDER OF FORMAT OCCURRP 3102710 2ENCE $128 \mathrm{H}$ NO EXPONENT ON INPUT DATA II

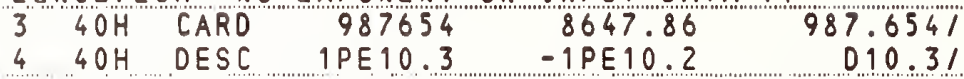

P3102720

$4 \quad 40 H$ DESC 1 PE10.3 $\quad-1$ PE 10.2 $\quad 010.31$

P 3102730

$540 \mathrm{H}$ TO BE .988E+02 .8648E+05 .98770+041

33102740

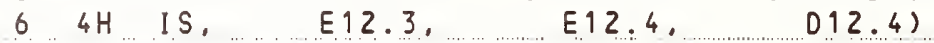

P 3102750

C**** END OF TEST SEGMENT 310
C**** WHEN EXECUTING ONLY SEGMENT 310 . THE STOP AND END CARDS

$C * * * *$ WHICH APPEAR AS COMMENT CARDS MUST HAVE THE C=

P3102760

C***** IN COLUMNS 1 AND 2 REMOVED.

$C=$ STOP

$C=\quad$ END

STOP

END

P 3102770

P3102780

P3102790

P3102800

P 3102810

P3102820

P310C1

P3 $10 C 2$

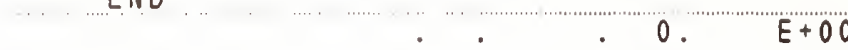

$1.23456987654+2+234 E-0-98.7654 E+0+2345.67891019+200$

$+\quad+\quad E 0$

12345

12345

12345

12345

12345

1.1

1.1

1.1

1.1

$+0.339567 E+02$

$+.339567+2$

$+3.395670 E 1$

$0.962951342440+04$

.96295134244004

$0.96295134244+4$

$0.962951342440+04$

$31.23+0.14 E+04+0.20+02$

$31.23 .14 E+04+.2+2$

$-0.13579 E+054444$

4444

4444

4444

4444

$-3335.555+0.4545 E-04$

$-6.666 \quad .9989 E+12$

$7.77-0.747 E-02+0.549 E 022$

$+0.662 E-00 \quad 0.468-1011$

$0.595420+04-44.6666-0.12345678900-03$

$54.9327-0.13956245340+00$

65432.1

$+0.848 E+03 \quad .848 E 3+.129007+0.1290+07 \quad 0.412021$

22222222222222222222222222222222222222222222222222

$-.987 E 0-0.987 E+00+0.600+0.60+00 \quad .3680-5$

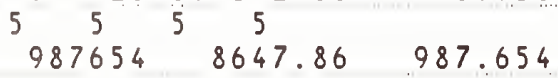

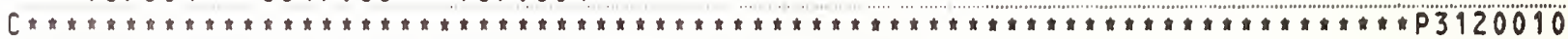

$C * * * * *$

$[* * * *$

RDFMT - $(312)$

P3120020

$C * * * *$

C***** GENERAL PURPOSE

$C * * *$ TO TEST FORMATTED READ AND WRITE STATEMENTS

$C * * *$ IN WHICH THE FORMAT STATEMENT IS CONTAINED IN

P3120030

$P 3120040$

$C * * * *$ AN ARRAY

$C * * *$ RESTRICTIONS OBSERVED

C*\#*** "AN H DESCRIPTOR MAY NOT BE PART OF A FORMAT

$\because P 3120050$

$[* * *$ STATEMENT IN AN ARRAY

C******ALL FORMAT STATEMENTS ARE LABELED

C***** *H AND X DESCRIPTORS ARE NEVER REPEATED

$C * * *$ \# FOR W.D DESCRIPTORS, D IS ALWAYS SPECIFIED AND

C*****WIS EOUAL TO OR GREATER THAN D

ASA REFSP 3120060

7.2.3.10P3120070

P3120080

P3120090

P3120100

$7.2 .3 .10148 P 3120110$

$P 3120120$

$7.2 .3 \quad 157 P 3120130$

$7.2 .3 .3154 P 3120140$

$7.2 .3 .1 / 31 P 3120150$

$7.2 .3 .1 / 33 P 3120160$ 
$C * * * * *$ FIELD WIDTH IS NEVER ZERO

$C * * * *$ IF THERE IS AN I/O LIST, THE FORMAT STATEMENT

$C * * *$ CONTAINS AT LEAST ONE FIELD DESCRIPTOR (OTHER

$(* * * * * \quad T H A N \quad H O R \quad X)$

$C * * * *$ ITEMS IN I/0 LIST CORRESPONO TO FORMAT DESCRIPTORS

$C * * * * *$ NEGATIVE OUTPUT VALUES ARE SIGNEO

C******FIELD WIDTH NEVER EXCEEOED BY OUTPUT

$C * * * * *$ FOR I CONVERSION, EXTERNAL INPUT FIELDS ARE

C***** INTEGER CONSTANTS

$C * * * * *$ TEST HOLLERITH IN ARGUMENT OF A CALL

$C * * *$ ARRAY NAME IN ARGUMENT LIST USEO AS FORMAT SPECIFIER

$C * * * *$ SUBROUTINE FMTO ALSO TESTS THE EMPTY FORMAT STATEMENT

$C * * * *$ THE FOLLOWING DATA STATEMENTS INITIALIZE SOME

C*****aRRAYS WITH FORMAT STATEMENTS TO BE USED FOR

$C * * *$ READING WITHA, F ANO 0 CONVERSION AND FOR

$C * * * *$ WRITING WITH I, E ANO L CONVERSION

$[* * * * * 2$

C INPUT DATA TO THIS SEG. CONSISTS OF 13 CARD IMAGES IN COLS. $1-80$

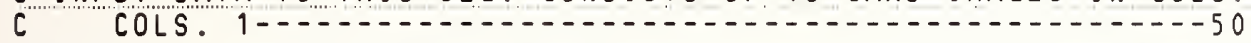

CARO $1 \quad(15.6 x, 14,2(13)$, I2)

CARO 2 (E $9.2,3(E 13.6))$

CARO $3 \quad(L 1,2(L 2), L 3)$

CARD $4 \quad(2 X, A 2,5(A 2))$

CARD $5 \quad(2 X, F 5.3, \ldots 4.0,2(F 7.2))$

CARD $6 \quad(2 \times, 016.9,09.2)$

CARD $7 \quad 4756 \quad-867224+39-6$

CARD $8 \quad 23498 .-77.27547 .18$

CARD $9 \quad-.0076+11+08.93421 E-13 \quad 893.421 E-15+08.93421 E-13$

CARO $10-0.3579012460+00+0.520-2$

CARD 11 TTA FF9\$

CARD $12 \quad A B$

CARD $13 \quad C O E+*=123$

CARO COLS. NOT MENTIONED ARE BLANK

$C * * * * *$

$C * * *$ S P E C I F I C A T I O N S SEGMENT 312

$C * * * * *$

$C * * *$ WHEN EXECUTING ONLY SEGMENT 312 , THE SPECIFICATION STATEMENTS

$C * * *$ WHICH APPEAR AS COMMENT CARDS, MUST HAVE THE $C=$

$C * * *$ IN COLUMNS 1 AND 2 REMOVED.

$C * * * * *$

$C=$ DIMENSION L1I(10), A3S(3,3,3), YER1S(7), IAC1I(5), AC1S(25)

$C=\quad$ DIMENSION ZU3S(3,2,2), ZT1S(4),2U1S(12),2U2S(4,2), IACZI(2,7)

$C=\quad$ INTEGER AVI, IUZI $(4,2), I T 3 I(4,2,2), I \cup 3 I(2,3,3), \operatorname{MCA} I(2,3,3)$

$C=\quad L O G I C A L \quad A \vee B, B \vee B, C \vee B, G G 1 B(2), A 1 B(2)$

$C=$ OOUBLE PRECISION OPAVO,OPBVD,DPCVD,A10(4)

$C=\quad$ COMPLEX CHAVC,CHBVC

DIMENSION L1I(10),A3S(3,3,3), YER1S(7), IAC1I(5),AC1S(25)

OIMENSION IAC2I $(2,7), 2 U 1 S(13), 2 U 3 S(3,2,2), 2 U 2 S(4,2), 2 T 1 S(4)$

INTEGER AVI, IUZI $(4,2), I T 3 I(4,2,2), I \cup 3 I(2,3,3), \operatorname{MCA} I(2,3,3)$

$\angle O G I C A L \quad A \vee B, B \vee B, C V B, G G 1 B(2), A 1 B(2)$

DOUBLE PRECISION DPAVO, OPBVD,OPCVO,A1D(4)

COMPLEX CHAVC,CHBVC

$C * * * * *$

$C * * * * I N P U T-0 U T P U T$ TAPE ASSIGNMENT STATEMENTS

$C * * * * *$

$C * * * * *$ WHEN EXECUTING ONLY SEGMENT 312, THE FOLLOWING STATEMENTS

$C * * \#$ NUVI $=6$ AND IRVI $=5$ MUST HAVE THE $C=$ IN COLUMNS 1 ANO 2 REMOVEO.

$C * * * * \pi$

$C=\quad$ NUVI $=6$

$C=\quad I R \vee I=5$

NUVI $=6$

IRVI $=5$

$C * \because * *$

OATA IUZI $(1,1), I \cup Z I(2,1), I \cup Z I(3,1), I \cup Z I(4,1), I \cup Z I(1,2), I \cup 2 I(2,2)$, IUZI $(3,2) / 2 \mathrm{H}(\mathrm{A}, 2 \mathrm{H} 2 /, 2 \mathrm{H} 2 \mathrm{X}, 2 \mathrm{H}, 5,2 \mathrm{H}(\mathrm{A}, 2 \mathrm{HZ}), 1 \mathrm{H})$

DATA $2 U 1 S(1), 2 U 1 S(2), 2 U 1 S(3), 2 U 1 S(4), 2 U 1 S(5), 2 U 1 S(6), 2 U 1 S(7)$,

$1 \quad 2 U 1 S(8), 2 \cup 1 S(9), 2 U 1 S(10), 2 U 1 S(11), 2 U 1 S(12)$

$2 \mathrm{HC}, 2 \mathrm{H}, 2 \mathrm{HF}, 2 \mathrm{H} .3,1 \mathrm{H}, 2 \mathrm{2HF}, 2 \mathrm{H}, 0,2 \mathrm{H}, .2 \mathrm{H} 2(, 2 \mathrm{HF} 6,2 \mathrm{H} .2,2 \mathrm{H}))$

$18 P 3120170$

P 3120190

$P 3120200$

$2.3 .4 / 36 P 3120210$

$3.6 / 56 P 3120220$

$.2 .3 .6101 P 3120230$

P3120240

P3120250

P3120260

P3120270

P3120280

SOP3120290

P3120300

P3120310

P 3120320

P3120330

P3120340

P3120350

P3120360

P3120370

P 3120380

P3120390

P3120400

P 3120410

P3120420

P3120430

P3120440

P3120450

P3120460

P 3120470

P3120480

P3120490

P3120500

P3120510

P0014250

P0014255

P0014260

P0014265

P0014270

$P 0014275$

P0014280

$P 0014285$

P0O14290

$P 0014295$

$P 0014300$

P312A1

P312A2

P312A3

P312A4

P312A5

P312A6

P0014305

P3120520

$P 0073320$

$P 0073325$

P 0073330

$P 0073335$

P0073340

P 0073345

P312B1

P312B2

P 0073350

P3120530

P3120540

P3120550

P3120560

P3120570 
DATA IU3I $(1,1,1), I \cup 3 I(2,1,1), I \cup 3 I(1,2,1), I \cup 3 I(2,2,1), I \cup 3 I(1,3,1), P 3120580$

$1 \quad[\cup 3 !(2,3,1), I \cup 3 !(1,1,2), I \cup 3 !(2,1,2)$,

$P 3120590$

$2 \quad 2 H(, 2 H D, 2 H 16,2 H .9,2 H, 1 H D, 2 H 9,2 H 2)$,

P 3120600

DATA IT3 I $(1,1,1)$, IT $3 I(2,1,1), I T 3 !(3,1,1), I T 3 I(4,1,1), I T 3 I(1,2,1), P 3120610$

$1 \quad$ IT3I(2,2,1), IT3I(3,2,1), IT3! $(4,2,1), 1 T 3 !(1,1,2), I T 3 !(2,1,2)$,

P3120620

$2 \quad I T 3 I(3,1,2), I T 3 I(4,1,2), I T 3 I(1,2,2) \quad 12 \mathrm{H}(2,2 \mathrm{HX}, 2 \mathrm{H} I 5,2 \mathrm{H}, 1, \ldots$

P3120630

$32 \mathrm{HX}, 2 \mathrm{H} 14,2 \mathrm{H}, 1,2 \mathrm{H} 4,2 \mathrm{H} 1 \mathrm{X}, 2 \mathrm{H}, \mathrm{I}, 2 \mathrm{H} 2,2 \mathrm{HI}, 9 \mathrm{H})$ ।

$P 3120640$

DATA ZT1S(1), ZT1S(2), ZT1S(3), ZT1S(4)/2H(E, 2H11,2H.2,1H) I

P3120650

DATA $Z \cup 3 S(1,1,1), Z \cup 3 S(2,1,1), 2 \cup 3 S(3,1,1), 2 \cup 3 S(1,2,1), 2 \cup 33(2,2,1), P 3120660$

$1 \quad 2 U 3 S(3,2,1) / 2 H(4,2 H(E, 2 H 14,2 H, 6,2 H /), 1 H)$,

P3120670

DATA ZUZS(1,1),ZUZS(2,1),ZUZS(3,1),ZUZS(4,1),ZUZS(1,2),ZUZS(2,2), P3120680

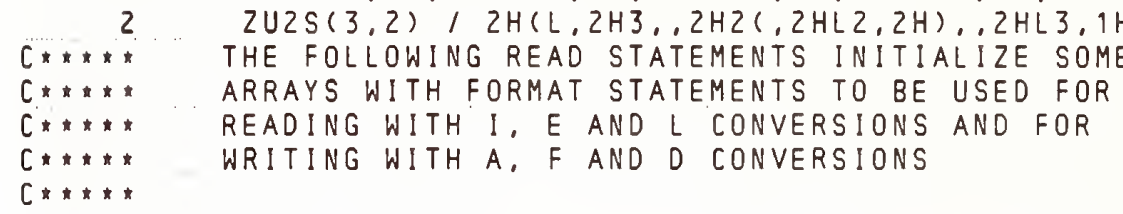

P3120690

WRITE (NUVI, 3120)

$C * * *$ CARD 1

READ (IRVI,3121) AC1S(1), AC1S(2), AC1S(3), AC1S(4), AC1S(5),

$1 \quad A C 15(6), A C 15(7), A C 15(8), A C 1 S(9), A C 15(10), A C 1 S(11), A C 15(12)$

7.2.3.10/51P3120700

P3 3120710

P3 120720

P3 120730

P3 320740

P3 320750

P3120760

P3120770

P3120780

$C * * * *$ CARD 2

READ (IRVI, 3122) L1I

$C * * *$ CARD 3

READ (IRVI, 3121) A3S

$[* * * *$ CARD 4

READ (IRVI.3123) yER1S

$C * * * *$ CARD 5

READ (IRVI.3124) MCA3I

$[* * * *$ CARD 6

READ (IRVI,3124) IAC2I

P3120790

P3 120800

P3 120810

P3 320820

P3120830

P3 320840

P3 3120850

P3 120860

P 3120870

P3120880

$C * * * * *$

$[* * * * *$

$[* * * *$

THE FOLLOWING STATEMENTS MAKE USE OF THE FORMATS

P3 320890

P3 120900

P3 320910

P3 3120920

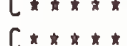

$C * * * * *$

CONTAINED IN THE ARRAYS

P3120930

READ AND WRITE WITH I CONVERSION USING FORMATS IN ARRAYS

P3120940

$J A C V I=4756$

$K B C V I=-867$

$L C C V I=224$

$M D C V I=+39$

NECVI $=-6$

$C * * * *$ CARD 7 WITH CARD 1 AS FORMAT

READ (IRVI,AC1S) AVI, MRRV!, IAC1!(1), IAC1I(2), IAC1I(3)

WR!TE (NUV!,3125)

WRITE(NUVI, ITII) JACVI, KBCVI, LCCVI, MDCVI, NECVI, AVI, MRRVI,

P3 320950

P3 3120960

P3120970

P3 3120980

P3120990

P3121000

P3 321010

P 3121020

1 IAC1 I (1), IACII(2), IACII(3)

$C * * *$ READ AND WRITE WITH F CONVERSION USING FORMATS IN ARRAYS AVS $=.234$

BVS $=98$.

CHAVC $=(-77.27,+547.18 E 0)$

C***** CARD 8 FORMAT IS (F3.3.F3.0.2(F6.2))

READ (IRVI,ZU1S) CVS, DVS, CHBVC

WRITE (NUVI,3127)

WRITE (NUVI, MCA3I) AVS, BVS, CHAVC

WRITE (NUVI, MCA3I) CVS, DVS, CHBVC

C**** READ AND WRITE WITH E CONVERSION USING FORMATS IN ARRAYS

AVS $=-0.76 E+9$

BVS $=+08.93421 \mathrm{E}-13$

C***** CARC 9 WITH CARD 2 AS FORMAT

R_AD (IRVI,L1I) ZU3S (2,2,2), CVS,DVS, ZU3S(1,2,2)

WR!TE (NUV!,3128)

WRITE(NUVI, ZT 1S) AVS, ZU3S $(2,2,2)$

WRITE (NUVI,3129)

WRITE (NUVI, 2U3S) BVS, $2 U 3 S(1,2,2)$, CVS, DVS

C**** READ AND WRITE WITH O CONVERSION USING FORMATS IN ARRAYS DPAVD $=-0.3579012460+00$

P 3121030

P3121040

P3121050

P 3121060

P 3121070

P3121080

P3121090

P 3121100

P3121110

P3121120

P3121130

P3121140

P3121150

P3121160

P3121170

P3121180

P 3121190

P3121200

P 3121210

P 3121220

P3121230

P3121240

DPBVD $=+.000520+1$

P3121250 
C**** CARD 10 FDRMAT IS (D16.9.09.2)

READ (IRVI, IU3 I) A1D(1), OPCVD

WRITE (NUVI.9930)

WRITE (NUVI, IAC2I) DPAVD, DPBVD, A1D (1), DPCVD

$C * * *$ READ AND WRITE WITH L CDNVERSIDN USING FDRMATS IN ARRAYS

$A \vee B=$.TRUE.

$B \vee B=. F A L S E$.

C**** CARD 11 WITH CARD 3 AS FDRMAT

READ (IRVI,A3S) A1B(1), AIB(2), CVB, GG1B(2)

WRITE (NUVI,9931)

WRITE (NUVI, ZU2S) AVB, AVB, BVB, BVB

WRITE (NUVI, ZU2S) A1B(1), AIB(2), CVB, GG1B(2)

$C * * *$ READ AND WRITE WITH A CDNVERSIDN USING FDRMATS IN ARRAYS

C***** CARDS 12 AND 13 FDRMAT IS (AZ/2X,5(AZ))

READ (IRVI, IULI) JACVI, AVS, IACII(1), GGIB, BVB

WRITE (NUVI, 3126)

WRITE (NUVI, YER1S) JACVI, AVS, IACII(1), GG1B, BVB

$C * * * *$

CALL FMTO CNUVI, ZT1S,0.9999, 2HHO, 2HLL, 2HER, 2HIT, 2HH, 2HCD,2HNS,

$12 \mathrm{HTA}, 2 \mathrm{HNT}, 2 \mathrm{HS}, 2 \mathrm{HAS}, 2 \mathrm{H}$ C, $2 \mathrm{HAL}, 2 \mathrm{HL}, 2 \mathrm{HAR}, 2 \mathrm{HGU}, 2 \mathrm{HME}, 2 \mathrm{HNT}, 1 \mathrm{HS}$ )

\section{$C * * * *$ \\ $C * * * *$ \\ $C * m * *$ \\ $C * * * *$ \\ $C * * * * *$ \\ 3121 \\ RMAT (27(A2)) \\ FORMAT $(10(A 2))$ \\ 3123 FORMAT $(7(A 2))$ \\ 3124 FORMAT $(18(A 2))$}

C*:** THE FOLLDWING ARRAYS ARE USED TO WRITE OUT ALL

$C * * \#$ HDLLERITH INFORMATION, SINCE H FIELD DESCRIPTDRS

$C *$ MAY NOT BE PART DF A FORMAT WITHIN AN ARRAY

3120 FORMAT (1H1,1X,31HRDFMT-(312) FDRMATS IN ARRAYS/)

$7,2,101 / 34 \mathrm{H}$

3125 FORMAT (i) $22 \mathrm{H} \quad 4756-86722439-6)$

3126 FORMAT (1 $13 \mathrm{H} \quad$ ABCDE $+*=123)$

3127 FORMAT ( $\left.25 H^{0} 0.23498 .-77.27547 .18\right)$

3128 FORMAT $(111 \mathrm{H}-0.76 E+09)$

3129 FORMAT (114H $0.893421 \mathrm{E}-12)$

9930 FDRMAT ( $/ 27 \mathrm{H}-0.3579012460+00 \quad 0.520-02)$

9931 FDRMAT ( $10 \mathrm{H}$ T T F F)

$C * *$ END DF TEST SEGMENT 312

C*WHEN EXECUTING DNLY SEGMENT 312 , THE STOP ANO END CARDS

$C * *$ WHICH APPEAR AS CDMMENT CARDS MUST HAVE THE $C=$

C\#\#\# IN COLUMNS 1 AND 2 REMOVEO.

$C=S T O P$

$C=\quad E N D$

STDP

END

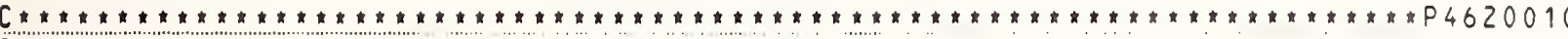

$[* * * *$

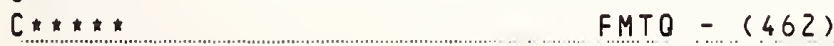

$C * \cdots *$

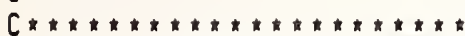

$\because *$ GENERAL PURPOSE

C*****TD DEFINE SUBRDUTINE FMTO WHICH IS USED IN SEGMENT 312

$C * * *$ TO TEST FORMAT IN AN ARRAY PASSED AS AN ARGUMENT, AN

$C * * * \quad$ EMPTY FORMAT STATEMENT, AND

C*\#\#\#HOLLERITH IN A CALL ARGUMENT

SUBROUTINE FMTOCNWVI, ZTW1S,AWVS, IWVH, JWVH, KWVH, LWVH, MWVH, NWVH,

2

DIMENSION ZTWIS(4)

I I WVH, J JWVH, KKWVH, LLWVH, MMWVH, NNWVH, I JWVH, IKWVH,

I LWVH, IMWVH, INWVH, J IWVH, JKWVH)

WRITE (NWVI, 4620)

4620 FORMAT $(1111 \mathrm{H}+.10 \mathrm{H}+01)$

$C * * * *$ FORMAT LABELED ZTWIS PASSED AS ARGUMENT IS (E11.2)

WRITE (NWVI, ZTWIS) AWVS

WRITE (NWVI, 4621)
P3 121260

P3121270

P3121280

P3 121290

$P 3121300$

P3121310

P3121320

P 3121330

$P 3121340$

$P 3121350$

P3121360

P 3121370

P3121380

P3121390

P3121400

P3121410

$P 3121420$

$P 3121430$

$P 3121440$

P 3121450

P3121460

P 3121470

P3121480

$P 3121500$

$P 3121510$

P3121520

$P 3121530$

$P 3121540$

$7.2 .3 .10 / 48 P 3121550$

$P 3121560$

$P 3121570$

$P 3121580$

$P 3121590$

$P 3121600$

$P 3121610$

P3121620

P3121630

P3121640

$P 3121650$

P3121660

P3121670

P3121680

P3121690

P 3121700

P3121710

P3121720

P312C1

$P 4620020$

$P 4620030$

$P 4620040$

P4620050

$P 4620060$

$P 4620070$

$P 4620080$

$P 4620090$

$P 4620100$

$P 4620110$

$P 4620120$

P4620130

$P 4620140$

$P 4620150$

$P 4620160$

$P 4620170$

P4620180

$P 4620190$ 
4621 FORMAT ( $/ 39 H$ HOLLERITH CONSTANTS AS CALL ARGUMENTS )

4623 FORMAT(//29H TEST EMPTY FORMAT STATEMENT /

$P 4620260$

$136 \mathrm{H}$ THE FOLLOWING LINE SHOULO BE BLANK)

$P 4620270$

WRITE(NWVI, 4624)

$P 4620280$

4624 FORMAT( )

$P 4620290$

WRITE (NWVI,4625)

4625 FORMAT $23 \mathrm{H}$ ENO EMPTY FORMAT TEST/122H ENO SEGMENT 312 TEST) RETURN

P 4620300

$P 4620310$ ENO

$P 4620320$

$P 4620330$

$(15,6 x, 14,2(13)$,

( $\quad 9.2 .3(E 13.6)$ )

12)

$(L 1,2(L 2), L 3)$

$(2 X, A 2,5(A Z))$

$(2 \times, F 5.3, \quad F 4.0,2(F 7.2))$

$(2 X, 016.9,09.2)$

$4756 \quad-867224+39-6$

$23498 .-77.27547 .18$

$-.0076+11+08.93421 E-13 \quad 893.421 E-15+08.93421 E-13$

$-0.3579012460+00+0.520-2$

TTA FFO\$

$A B$

COE $+*=123$

$C * * * * * * * * * * 2)$

$C * * * * *$

$C * * * *$

$M ! S C 5-(350)$

P3500010

P3500020

$C * * * * *$

M.

P3500030

$C * * * *$ GENERAL PURPOSE

C**** TO TEST SPECIFICATIONS FOR PROGRAM FORM

P3500040

*P3500050

$C * * \hbar * *$

$C * * * * *$

$C * * * *$ GENERAL COMMENTS

C*** * AMONG OTHER THINGS, THIS SEGMENT TESTS THAT COMMENTS ARE

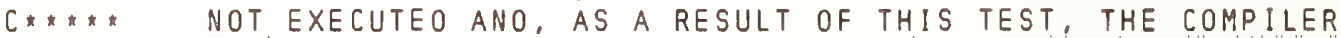

$C * * * *$ MAY GENERATE SOME WARNING MESSAGES.

$C * * *$ * BECAUSE OF THE NATURE OF THE TESTS BEING PERFORMEO, SOME

$C * * * *$ LABELS ANO NAMES OO NOT FOLLOW THE CONVENTIONS

$C * * * *$ SPECIFIED IN THE USERS MANUAL.

$C * * * * *$

$C * * * * *$

$S$ S $E$ E $C$ I $F$ I $C$ C A $T$ T I $O 0$ N $S$ S SEGMENT 350

ASA REFP 3500060

$3.2 \quad P 3500070$

$3.2 .1 \quad P 3500080$

$3.4 \quad P 3500090$

$3.5 \quad P 3500100$

P3500110

P3500120

P3500130

$P 3500140$

$P 3500150$

P3500160

P3500170

P3500180

P3500190

C***** WHEN EXECUTING ONLY SEGMENT 350 , THE SPECIFICATION STATEMENTS

$C * * *$ WHICH APPEAR AS COMMENT CARDS MUST HAVE THE C $=$

$P 0014310$

$P 0014315$

P 0014320

$C * * * *$ IN COLUMNS 1 ANO 2 REMOVEO.

$P 0014325$

P0014330

$C=\quad$ OIMENSION J(2), JJ(1,1),JJJ(1,1,1),JJJJ(1,1),JJJJJ(1),JJJJJJ(1)P0014335 OIMENSION J(2), JJ(1,1), JJJ(1,1,1), JJJJ(1,1),JJJJJ(1), JJJJJJ(1)P350A1

C*****

C****OU U T U T T A P E ASSIGNMENT STATEMENT. NO INPUT TAPE.

$P 0014340$

$C * * *$ WHEN EXECUTING ONLY SEGMENT 350. THE FOLLOWING STATEMENT

$C * * * *$ NUVI $=6$ MUST HAVE THE $C=$ IN COLUMNS 1 ANO 2 REMOVED.

$C * * * * *$

$C=\quad$ NUVI $=6$

NUVI $=6$

P3500200

$P 0073360$

$P 0073365$

$P 0073370$

$P 0073375$

P350B1

WRITE (NUV!, 3500)

3500 FORMAT ( 1 H $1,1 \mathrm{X}, 32 \mathrm{HMISC5}$ - $(350)$ SPECIFICATIONSFOR/ $16 \mathrm{X}$, P3500210

1GRAM FORM//2X.32HASA REFS. $-3.23 .2 .13 .43 .5 / 1$

$22 \times, 35$ HTEST THAT COMMENTS ARE NOT EXECUTEO)

$C * * * *$ HEAOER FOR SEGMENT 350 WRITTEN

$C * * * *$ TEST THAT COMMENTS ARE NOT EXECUTEO

C*****WRITE (NUVI,3501)

3501 FORMAT ( $X, 34$ HERROR - COMMENT STATEMENT EXECUTEO)

P 3500220

P3500230

P3500240

P3500250

$3.2 .1136 P 3500260$

P 3500270

P3500280 
3504 WRITE (NUVI.3501)

P3500350

3505 WRITE (NUVI,3506)

P3500360

3506 FORMAT (2X,35HTEST SUCCESSFUL IF NO ERROR MESSAGE)

P 3500370 GO TO 3509

$C * * * *$ TEST THAT ALL 72 CHARACTERS IN A LINE MAY BE USED

3509 WRITE (NUVI,8100)

P 3500380

8100 FORMAT ( ///2X,22HTEST 72 CHARACTER LINE)

WRITE (NUVI,8101)

$3.2124 P 3500390$

P3500400

P 3500410

$P 3500420$

8101 OFORMAT ( I XX,29H123456789101111213141516171819/2X,29H123456789101112P3500430 $113141516171819)$

WRITE (NUV1,8102)

$P 3500440$

P3500450

8102 FORMAT ( $12 X, 36$ HTEST SUCCESSFUL IF 2 LINES ABOVE ARE $12 X, 19 H D I G I T S$ P 3500460 11 THROUGH 19 )

$C * * * *$ TEST THAT STATEMENT LABELS MAY BE 1, 2, 3, 4 OR 5

\section{$C * * * *$ DIGITS LONG}

P3500470 WRITE (NUVI,8112)

$3.4 / 12 P 3500480$

8112 FORMAT (//2X,37HTEST $1,2,3,4,5$ CHARACTER STMNT. LABEL/)

8113 GO TO 22

$8114 \quad G O \quad T 0 \quad 333$

8115 GO TO 8099

8097 GO TO 22255

P 3500490

P3500500

P3500510

P3500520

P3500530

P3500540

1 MRRVI $=1$

P3500550

P3500560

WRITE (NUVI.8118) MRRVI

GO TO 8113

P3500570

P3500580

P3500590

P3500600

$22 \quad$ MRRVI $=2$

WRITE (NUVI.8118) MRRVI

P 3500610 GO TO 8114

P3500620

P 3500630

333 MRRVI $=3$

WRITE (NUVI,8118) MRRVI

P3500640 GO TO 8115

8099 MRRVI $=4$

P3500650

P3500660

WRITE (NUVI. 8118) MRRVI

P3500670

GO TO 8097

22255 MRRVI $=5$

P3500680

P 3500690

WRITE (NUVI,8118) MRRVI

P3500700

8118 FORMAT ( $2 X_{1} 11,1 \times, 24 H C H A R A C T E R$ LABEL ACCEPTED)

P 3500710

$C * * * *$ TEST THAT VARIABLE AND ARRAY NAMES MAY BE

$C * * * * * \quad 1,2,3,4$ OR 5 CHARACTERS LONG

$3.5121 P 3500720$

WRITE (NUVI,8098)

8098 FORMAT (//2X,36HTEST $1,2,3,4,5,6$ CHARACTER VARIABLES/2X。

P 3500730

115 HAND ARRAY NAMES)

$M=1$

$M M=1$

$M M M=1$

MMMM $=1$

P3500740

P3500750

P3500760

P3500770

P 3500780

P3500790

P3500800

MMMMM $=1$

MMMMMM $=1$

P3500810

P3500820

$J(1)=1$

P3500830

$J J(1,1)=1$

$J J J(1,1,1)=1$

P3500840

$\operatorname{JJJJ}(1,1)=1$

JปJJJ $(1)=1$

J」JJJJ(1) $=1$

P3500850

P3500860

P3500870

P 3500880

IF $(M-1) 8119,8103,8119$

P 3500890

8103 IF $(M M-1) \quad 8119,8104,8119$

8104 IF (MMM-1) 8119.8105 .8119

P3500900

P3500910

P3500920

8105 IF (MMMM-1) $8119,8106,8119$

8106 IF (MMMMM-1) $8119,8096,8119$

P3500930

P3500940

8096 IF (MMMMMM-1) $8119,8107,8119$

$P 3500950$

8107 IF $(J(1)-1) 8119.8108 .8119$

8108 IF $(J J(1.1)-1) \quad 8119.8109 .8119$

$P 3500960$ 
8109 IF (JJJ $(1,1,1)-1) \quad 8119,8110,8119$

P3500970

8110 IF (JJJJ $(1,1)-1) \quad 8119,8111,8119$

P3500980

IF (JJJJJ(1)-1) $8119,8095,8119$

P3500990

IF (JJJJJJ(1)-1) $8119,8121,8119$

P3501000

P 3501010

WRITE (NUVI, 8120)

P3501020

FORMAT ( / 2X, $21 H *$ *TEST UNSUCCESSFUL*

P3501030

GO TO 8123

8121 WRITE (NUVI, 8122)

P3501040

8122 FORMAT (/ $2 X, 38 H *$ TEST SUCCESSFUL-ALL NAMES ACCEPTED**)

P3501050

$C * * *$ TEST THAT STATEMENT LABELS MAY BE PLACED

$C * * * *$ ANYWHERE IN COLUMNS 1 TO 5 AND THAT LEADING

$3.4 / 13 P 3501060$

C***** ZEROS ON STATEMENT LABELS ARE NOT SIGNIFICANT

$3.4 / 1793501070$

8123 WRITE (NUVI, 8116)

8116 FORMAT (//2X,34HTEST PLACEMENT OF STATEMENT LABELS/2X,

P3501080

1 29HAND LABELS WITH LEADING ZEROS $($ )

MRRVI = 1

GO TO 10

$2 \ldots$ MRRVI $=2$

GO TO 010

3 MRRVI $=3$

GO TO 0010

4 MRRVI $=4$

GO TO 0010

5 MRRVI $=5$

GO TO 0010

P3501090

P3501100

P3501110

P3501120

P3501130

P3501140

P3501150

P3501160

P3501170

P3501180

P3501190

P3501200

P3501210

06 MRRVI $=6$

P3501220

GO TO 00010

P3501230

P3501240

007 MRRVI $=$ ?

P3501250

GO TO 0010

P3501260

0008 MRRVI $=8$

P 3501270

GO TO 0010

P3501280

0009 MRRVI $=9$

P3501290

0010 WRITE (NUVI, 11) MRRVI

P3501300

FORMAT ( I 10$)$

$P 3501310$

GO TO $(02,3,004,0005,6,7,8,009,8117)$, MRRVI

8117 WRITE (NUVI,012)

12 FORMAT (//2X,28HTEST SUCCESSFUL IF 9 NUMBERS/2X,

P3501320

P 3501330

131 HIN SEQUENTIAL ORDER FROM 1 TO $9 / 2 X$,

P3501340

2 17HARE WRITTEN ABOVE///2X,18HEND OF SEGMENT 350)

P 3501350

C***** END OF TEST SEGMENT 350

$C * * *$ WHEN EXECUTING ONLY SEGMENT 350 , THE STOP AND END CARDS

P3501360

$C * * * *$ WHICH APPEAR AS COMMENT CARDS MUST HAVE THE $c=$

P3501370

C****IN COLUMNS 1 AND 2 REMOVED.

$C=S T O P$

$C=E N D$

STOP

P3501380

P3501390

P 3501400

P3501410

END

P $350 C 1$

P $350 C 2$

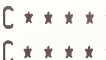

P3510010

C*k*k*n,

FUNMX - (351)

P3510020

C******)

P3510030

$P 3510040$

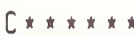

C***** GENERAL PURPOSE

C***** THIS SEGMENT FURTHER TESTS SOME

$C * * * *$ BASIC EXTERNAL FUNCTIONS BY USING TRIGONOMETRIC

$P 3510050$

$C * * * * *$

FORMULAE

ASA REF P 3510060

8.3 .3

P3510070

P3510080

P3510090

P3510100

$[* * * *$ O U T P U T T A P E ASSIGNMENT STATEMENT. NO INPUT TAPE.

P3510110

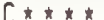

C******)

WHEN EXECUTING ONLY SEGMENT 351. THE FOLLOWING STATEMENT

P0073380

P0073385

C*****NUV!=6 MUST HAVE THE $C=$ IN COLUMNS 1 AND 2 REMOVED.

$C * * * * *$

$C=N U V I=6$

$C * * * * *$

NUVI $=6$

$P 0073390$

$P 0073395$

$P 0073400$

P 35181

P 0073405

WRITE (NUVI, 3510 )

P3510120

3510 FORMAT ( 1 H $1,2 X, 13$ HFUNMX - $(351) / 11 X, 22 H$ THIS SEGMENT FURTHER

P3510130

1 SHTESTS

$121 \mathrm{H}$ SOME BASIC EXTERNAL.

P3510140 
$210 \mathrm{H}$ FUNCTIONS $133 \mathrm{H}$ BY USING TRIGONOMETRIC FORMULAE//

P3510150

$319 \mathrm{H}$ ASA REFS. - 8.3.3//2X,7HRESULTS)

C***** HEADER FOR SEGMENT 351 WRITTEN

$C * * * *$ TEST STATEMENTS USING ORDINARY TRIGONOMETRIC FUNCTIONS

P3510160

P3510170

P3510180

CMAVS $=1.75$

CMCVS $=$ ALOG(EXP(CMAVS) $)-1.75$

CMDVS $=\operatorname{EXP}($ ALOG (CMAVS $))-1.75$

CMEVS $=(\operatorname{SIN}(2.0)) * 2+(\operatorname{COS}(2.0)) * 2-1.0$

CMFVS $=(1.0 / \cos (1.2)) * 2-((\operatorname{SIN}(1.2) / \cos (1.2)) * 2)-1.0$

WRITE (NUVI, 3511 ) CMCVS, CMOVS, CMEVS,.CMFVS

CMCVS $=\operatorname{SIN}(.78)-\operatorname{SORT}(1 .-\operatorname{COS}(0.78) * 2)$

CMDVS $=\operatorname{COS}(1.57)-\operatorname{SORT}(1.0-\operatorname{SIN}(1.57) * 2)$

CMEVS $=\operatorname{SQRT}((1.0 / \operatorname{COS}(0.5236)) * 2-1.0)-\operatorname{SIN}(0.5236) / \operatorname{COS}(0.5236)$

CMFVS $=\operatorname{ATAN2}(S ! N(0.5) \operatorname{COS}(0.5))-0.5$

WRITE (NUVI,3511) CMCVS, CMDVS, CMEVS, CMFVS

C***** TEST STATEMENTS USING HYPERBOLIC FUNCTIONS

CMAVS $=\operatorname{EXP}(1.85)$

CMBVS $=\operatorname{EXP}(-1.85)$

CMCVS = TANH $(1.85)-($ (CMAVS - CMBVS),$($ CMAVS + CMBVS))

CMEVS $=2.1(\operatorname{EXP}(1.05)+\operatorname{EXP}(-1.05))-\operatorname{SORT}(1.0-$ TANH $(1.05) * 2)$

CMFVS $=\operatorname{TANH}(2.01))(\operatorname{SORT}(1.0-\operatorname{TANH}(2.01) * 22))-.5 *(\operatorname{EXP}(2.01)$

$1 \operatorname{EXP}(-2.01))$

WRITE (NUVI, 3512) "CMCVS, CMEVS, CMFVS

WRITE (NUVI, 3513)

3511 FORMAT $(/ / 4($ F $15.5 /))$

3512 FORMAT $(/ / 3(F 15.5 /))$

3513 FORMAT (//39H ALL ABOVE ANSWERS SHOULD BE O PLUS OR /

$140 \mathrm{H}$ MINUS AN ERROR FACTOR OF NOT MORE THAN I

$212 \mathrm{H} 10 *(-4))$

C**** END OF TEST SEGMENT 351

C*** WHEN EXECUTING ONLY SEGMENT 351, THE STOP AND END CARDS

$C * * * *$ WHICH APPEAR AS COMMENT CARDS MUST HAVE THE C $=$

$C * * \#$ IN COLUMNS 1 AND 2 REMOVED.

$C=S T O P$

$C=E N D$

STOP

END

P3 510190

P3510200

P3510210

P3510220

P3510230

P3510240

P3510250

P3510260

P 3510270

P3510280

P3510290

P3510300

P3510310

P3510320

P3510330

P3510340

P3510350

P3510360

P3510370

P3510380

P3510390

P3510400

P3510410

P3510420

P3510430

P3510440

P3510450

P3510460

P3510470

P3 510480

P35 $10490^{\circ}$

P351C1

P351C2

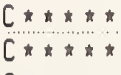

C $* * * *$

NAMES - $(352)$

P3520010

P3520020

P3520030

P3520040

$C * * * * *$

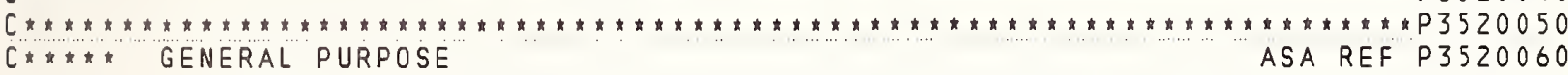

C*****TO TO TEST THE CAPABILITY OF COMPILERS TO IDENTIFY DATA $10.1 .7 / 54$ P 3520070

$C * * * * \quad$ NAMES THAT RESEMBLE FORTRAN VERBS ANDIOR PREDEFINED $\quad$ P3520080

$C * * * *$ FUNCTION NAMES.

$C * * * * *$ GENERAL COMMENTS

$C * * * *$ BECAUSE OF THE NATURE OF THIS TEST SEGMENT, NAMING

C***** CONVENTIONS THAT EXISTED IN OTHER SEGMENTS WILL NOT

$C * * * *$ BE OBSERVED.

$C * * \ldots * x$

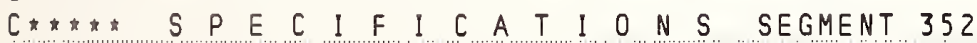

$C * * * * *$

S P E C I F I C A T I O N S SEGMENT 352

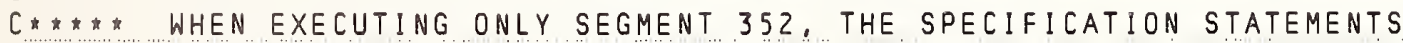

$C * * * * *$ WHICH APPEAR AS COMMENT CARDS, MUST HAVE THE C=

C**** IN COLUMNS 1 AND 2 REMOVED.

$C * * * * *$

$C=\quad$ DIMENSION GOTO $(2,2), I F(5)$

DIMENSION GOTO $(2,2)$, IF $(5)$

$C * * * * *$

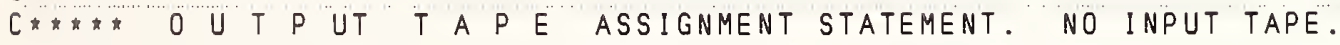

$C * * * *$ *

$C * * * * *$

WHEN EXECUTING ONLY SEGMENT 352, THE FOLLOWING STATEMENT

$C * * * *$ NUVI $=6$ MUST HAVE THE $C=I N$ COLUMNS 1 AND 2 REMOVED.

$C * \pi * \pi *$

$C=N U V I=6$

$C * * * *$

$$
\text { NUVI }=6
$$

P3520090

P3520100

P3520110

P3520120

P3520130

P3520140

P3520150

PO0 14350

P0014355

PO0 14360

P0014365

P0014370

P 0014375

P352A1

P0014380

P3520160

P0073410

P 0073415

P0073420

P 0073425

P 0073430

P352B1

P0073435 
3520 FORMAT (1H1,1X,13HNAMES - (352)/12X,36HTEST DF THE CDMPILERS CAPAP3520180

$1 B$ ILITY DF /2X,37HIDENTIFYING DATA NAMES THAT RESEMBLE / $2 X$. P 3520190

232 HFORTRAN VERBS ANDIOR PREDEFINED /2X, 15HFUNCTION NAMES II P P

$322 H$ ASA REFS. $-10.9 .7 / 41 / 2 \times$. 7 HRESULTS)

C**** HEADER FOR SEGMENT 352 WRITTEN

03520210

INTEG $=0$

P3520220

REAL $=2.0$

P3520230

GDTD5 $=$ REAL -2.0

$\operatorname{GDTD}(1,2)=10.0-5.0 * 2.0$

P3520240

DD13! = INTEG

P3520250

P 3520260

$130 D 14 \mathrm{~J}=I N T E G+0$

P3520270

$14 \prod F(2)=5-5$

$C A L L=0$

STDPT = REAL -2.0

P3520280

P 3520290

PAUSE $=$ REAL, $2.0-1.0$

P3520300

READG $=0.0 * 5$

03520310

WRITE $=7.0-7.0$

P3520320

WRITE (NUVI,3521) GDTD5, GOTO(1,2), DD13I, DD14J, IF(2), CALL, P3520350

1 STOP7, PAUSE, READ6, WRITE

3521 FORMAT $(/ / 10(F 10.5 /))$

C*** TEST THAT THE SAME INTRINSIC FUNCTIDN NAMES OF

P3520360

C*: A PROGRAM UNIT OF AN EXECUTABLE PROGRAM CAN BE

P 3520370

P 3520380

C**** USED TO IDENTIFY SDME DTHER ENTITY IN A DIFFERENT

$C * \#$ PROGRAM UNIT DF THAT EXECUTABLE PROGRAM

P3520390

$M C A V I=I A B S(-5)$

P3520400

P 3520410

P 3520420

CALL MAOO(MCAVI, IVI)

$M C C V I=I V !$

P3520430

$M C B \vee I=I S I G N(1,-2)$

P3520440

CALL MBOO(MCBVI, IVI)

P 3520450

MCOVI $=$ IVI

P3520460

CMAVS $=$ FLOAT $(5+7)$

03520470

CALL AMOO(CMAVS,AVS)

P3520480

CMCVS $=A \vee S$

P3520490

CMBVS $=A B S(-10.0-8.00)$

P3520500

CALL BMOO(CMBVS, AVS)

P 3520510

$P 3520520$

CMDVS = AVS

P3520530

WRITE (NUVI,3522) MCCVI, MCDVI, CMCVS, CMDVS

$P 3520540$

3522 FORMAT (/2(I10/)//2(F10.5/)//35H ALL ABOVE ANSWERS SHDULD BE O FDP352055O $1 R / 36 \mathrm{H}$ THIS TEST SEGMENT TO BE SUCCESSFUL)

P3520560

$C * * *$ ENO OF TEST SEGMENT 352

C**** WHEN EXECUTING ONLY SEGMENT 352, THE STOP AND END CARDS

P 3520570

$C * *$ WHICH APPEAR AS COMMENT CAROS MUST HAVE THE $C=$

$C * * * *$ IN COLUMNS 1 ANO 2 REMOVEO.

$C=S T O P$

$C=\quad E N D$

P 3520600

P3520610

STOP

P3520620

ENO

P $352 \mathrm{C1}$

P352C2

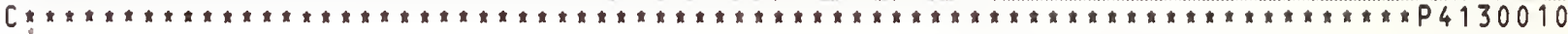

$[* * * * *$

MAOO- (413)

$P 4130020$

$C * * * *$

$P 4130030$

$C * * * * *$

$P 4130040$

$C * * * *$

GENERAL PURPDSE

C*** THIS SEGMENT CONTAINS A SUBROUTINE WHICH IS CALLED

$P 4130060$

C***:BY BEGMENT 352 .

P4130070

$C * *$ GENERAL COMMENTS
SUBRDUTINE MAQO BEING OEFINEO

$P 4130080$

$P 4130090$

P4 130100

$P 4130110$

SUBROUTINE MAOO(MHVI,IHVI)

$I A B S=$ MWVI

IWVI $=$ IABS + ISIGN(MWVI, -MWVI)

RETURN

$P 4130120$

P4130130

$P 4130140$

ENO

$P 4130150$

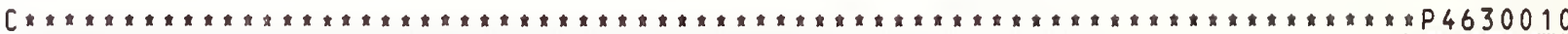

$[* * * * \pi$

$C * \geqslant * 2$

$M B O Q-(463)$

$P 4630020$

P4630030

$P 4630040$

C 
C*\#*" GENERAL PURPOSE

C***** THIS SEGMENT CONTAINS A SUBROUTINE WHICH IS CALLED

$P 4630060$

$C * * *$ BY SEGMENT 352

C**** GENERAL COMMENTS

$P 4630070$

$P 4630080$

$P 4630090$

SUBROUTINE MBOO BEING DEFINED

$P 4630100$

SUBROUTINE MBOO(MWVI, IWVI)

$P 4630110$

ISIGN $=-M W V I$

IWVI = ISIGN + MWVI

RETURN

P4630120

$P 4630130$

$P 4630140$

END

$P 4630150$

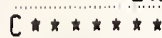

$P 4730010$

$C * * * * *$

C******

$A M O O-(473)$

$P 4730020$

$[* * * * *$

$P 4730030$

$P 4730040$

C

C***** GENERAL PURPOSE

C***** THIS SEGMENT CONTAINS A SUBROUTINE WHICH IS CALLED

$P 4730060$

$C * * * *$ BY SEGMENT 352

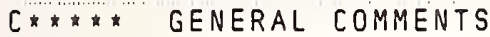

$C * * * *$ SUBROUTINE AMOO BEING DEFINED

C**** * STATEMENT FUNCTION NAME IS THE SAME AS SUBROUUTINE NAME CALLED BY P 4730110

$C * * * *$ SEGMENT 352 . STAT. FUNCTION DUMMY ARGUMENT NAME SAME AS SUBROUTINEP 4730120

$C * * *$ DUMMY ARGUMENT NAME, VARIABLE IS REFERENCED IN STAT. FUNCTION

SUBROUTINE AMOO(CWVS, AWVS)

$B M O O(C W V S)=$ CWVS + BVS

FLOAT = AVS

BVS = CWVS

AWVS $=B M O Q(F L O A T)-(B V S+1.0)$

DATA AVS 11.01

RETURN

$P 4730070$

$P 4730080$

$P 4730090$

P4730100

END

$P 4730130$

$P 4730140$

$P 4730150$

$P 4730160$

P4730170

$P 4730180$

P4730190

$P 4730200$

$P 4730210$

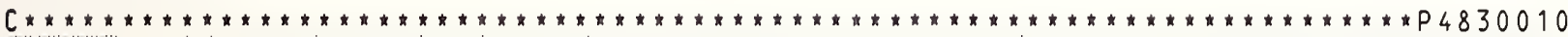

$[* * * * *$

$C * * * * *$

$B M Q Q-(483)$

$P 4830020$

$C * \| * *$

$P 4830030$

$P 4830040$

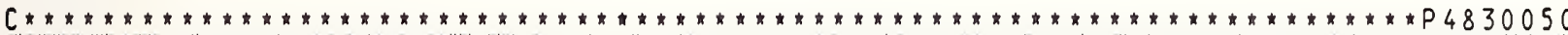

$C * * * *$ GENERAL PURPOSE

$C * * * *$ THIS SEGMENT CONTAINS A SUBROUTINE WHICH IS CALLED

$P 4830060$

$C * * * *$ BY SEGMENT 352

$C * * * *$ GENERAL COMMENTS

$P 4830070$

$P 4830080$

$P 4830090$

$C * * * *$ SUBROUTINE BMOO BEING DEFINED

SUBROUTINE BMOO(CWVS, AWVS)

$A B S=$ CWVS

AWVS $=$ FLOAT(ISIGN(IFIX(ABS), -2$))+18.0$

RETURN

C***** END OF TEST SEGMENT 483 END

$P 4830100$

$P 4830110$

$P 4830120$

$P 4830130$

$P 4830140$

$P 4830150$

$P 4830160$

$C * * * * * * * * *$

P3600010

$C * * * * *$

$C * * * * *$

$C * * * * *$

$P 3600020$

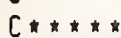

SPEC2 - $(360)$

$P 3600030$

P3600040

$C * * * * *$ GENERAL PURPOSE

$C * * * *$ * TO TEST COMMON, DIMENSION AND EOUIVALENCE

C*****) STATEMENTS

C****** TO TEST THAT VARIABLES AND ARRAYS WHICH ARE

$C * * * *$ EOUATED AND/OR IN COMMON MAY BE USED IN A

$C * * * *$ VARIETY OF FORTRAN STATEMENTS

$C * * * *$ RESTRICTIONS OBSERVED

$C * * * * *$ NO DUMMY ARGUMENTS APPEAR IN COMMON OR EOUIVALENCE

$C * * * *$ STATEMENTS

$C * * * * *$ NUMBER OF SUBSCRIPTS IN EOUIVALENCE STATEMENTS

$C * * * *$ CORRESPONDS TO ARRAY DIMENSIONALITY OR IS ONE

$C * * * *$ * COMMON NEVER LENGTHENED BY EQUIVALENCE IN A

C**** BACKWARD DIRECTION

$[* * * * *$ ONLY ONE OF AN EOUATED PAIR OF ITEMS APPEARS

$C * * * * *$ IN COMMON

C***** * VARIABLES ARE NEVER EOUATED TO MORE THAN ONE

*P3600050

ASA REFS P3600060

$7.2 .1 .2 P 3600070$

$7.2 .1 .3 P 3600080$

?.2.1.4P3600090

$P 3600100$

P3600110

$P 3600120$

$7.2 \cdot 1.4 / 40 P 3600130$

$8.4 .1 .1 / 23 P 3600140$

$P 3600150$

$7.2 \cdot 1.4109 P 3600160$

$7.2 .1 .4 / 31 P 3600170$

P3600180

$7.2 .1 .4 / 36 P 3600190$

$P 3600200$

$7.2 .1 .4 / 42 P 3600210$ 


\begin{tabular}{|c|c|c|}
\hline$[* * * * * x$ & $\begin{array}{l}\text { ELEMENT OF THE SAME ARRAY } \\
\text { GENERAL COMMENTS }\end{array}$ & $\begin{array}{l}P 3600220 \\
P 3600230\end{array}$ \\
\hline \multirow{3}{*}{$\begin{array}{l}C * * * * * \\
C * * * * * \\
C * * * * * \\
C * * * * *\end{array}$} & THIS SEGMENT FOLLOWS THE ORDER OF SPECIFICATION STATEMENTS & $P 3600240$ \\
\hline & REQUIRED IN BASIC FORTRAN (SEE $9.1 .2 / 56$ IN BASIC ASA BOOK) & $P 3600250$ \\
\hline & & \\
\hline$[* * * * *$ & I $F$ I C A T I O N S SEGMENT 360 & 0 \\
\hline \multirow{2}{*}{$\begin{array}{l}C * * * * * \\
C * * * * *\end{array}$} & & $\mathrm{PO}$ \\
\hline & WHEN EXECUTING ONLY SEGMENT 360, THE SPECIFICATION STATEMENTS & 0143 \\
\hline $\begin{array}{l}C * * * * * \\
C * * * * * \ldots\end{array}$ & WHICH APPEAR AS COMMENT CARDS, MUST HAVE THE $C=$ & P0014400 \\
\hline$C * * * * *$ & IN COLUMNS 1 AND 2 REMOVED. & $P 001440$ \\
\hline \multirow{2}{*}{\multicolumn{2}{|c|}{$C * * * *$ OIMENSION }} & P001441 100100 \\
\hline \multirow{2}{*}{$\begin{array}{c}c= \\
c=\ldots\end{array}$} & & P001441 \\
\hline & OIMENSION MXZI $(2,3), \operatorname{TXZS}(2,2), \operatorname{WAZZS}(3,2), \operatorname{RVY} 1 S(2), \operatorname{RVYZS}(1,2)$ & P001442 \\
\hline & OIMENSION JYZI $(2,2)$, JYII $(5)$, NZII(4), NZZI $(4,2)$, WAZ IS(2) & $P 0014425$ \\
\hline \multirow{2}{*}{$\begin{array}{l}C= \\
C=\end{array}$} & OIMENSION MMY II $(400)$, NNY3 I $(20,10,2)$ & P0014430 \\
\hline & EQUIVALENCE (MMY II (1), NNY3I $(1,1,1)),(N Z 1$ I (1),NNY3I(1)) & 14435 \\
\hline & COMMON MX1I, MX2I, N21I, N2VI, N22I & P0014440 \\
\hline & COMMON MXVI & P0014445 \\
\hline & COMMON IAXVI & 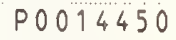 \\
\hline & COMMON WAZ1S & P0014455 \\
\hline & COMMON TX1S, TXZS, JBZVI, WAZZS & 14460 \\
\hline & EQUIVALENCE (MYVI, NZVI), (IYVI,NZ1I(1)), (NZZI (4, 1), JYVI) & 1446 \\
\hline & EQUIVALENCE (NZZI(3), KYVI), (AAYVS, JBZVI,JYZI(1), RVY1S(2)) & P0014470 \\
\hline & EQUIVALENCE (RVYZS $(1,1)$, WAZ1S(2)) & 014475 \\
\hline & EQUIVALENCE (JY1I (3), RVY 1S( 2$)$ ) & P0014480 \\
\hline & EQUIVALENCE (WAZZS (1), BBYVS, CCYVS), (WAZZS $(2,1)$, DDYVS) & 14485 \\
\hline & OIMENSION MXII $(3), \operatorname{TXIS(3)}$ & $O A 1$ \\
\hline & 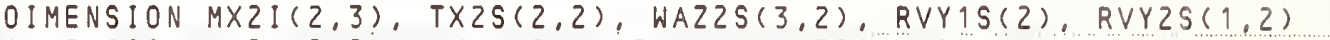 & P360A2 \\
\hline & OIMENSION JYZI $(2,2), J Y I I(5), N Z 1 I(4), N Z 2 I(4,2), W A Z I S(2)$ & $P 360 A 3$ \\
\hline & OIMENSION MMY 1 I $(400)$, NNY 3 I $(20,10,2)$ & 0.044 \\
\hline & EQUIVALENCE (MMY1I ( 1$), N N Y 3 I(1,1,1)),(N Z 1$ I $(1), N N Y 3 I(1))$ & P360A5 \\
\hline & COMMON MX1I, MX2I, NZ1I, NZVI, NZ2I & OAG \\
\hline & COMMON MXVI & $0 A 7$ \\
\hline & COMMON IAXVI & P360A8 \\
\hline & COMMON WAZIS & OA9 \\
\hline & COMMON TX1S, TXZS, JBZVI, WAZZS & $O A A$ \\
\hline & EQUIVALENCE (MYVI,NZVI), (IYVI,NZ1I(1)), (NZZI(4,1), JYVI) & $P 360 A B$ \\
\hline & EQUIYALENCE (NZZI(3), KYVI), (AAYVS,JBZVI,JYZI(1), RVY1S(2)) & $O A C$ \\
\hline & EQUUIVALENCE (RVYZS $(1,1)$,WAZ1S(2)) & P360AO \\
\hline & EQUIVALENCE (JY II (3), RVY $1 S(2))$ & $O A E$ \\
\hline & (1), BBYVS, CCYVS), (WAZZS $(2,1)$, ODYVS) & \\
\hline$c * * * * *$ & & 00280 \\
\hline$[* * * * *$ & SOME OF THE ITEMS OEFINED ABOVE ARE USED IN A VARIETY & 00290 \\
\hline$* * * \pi *$ & & \\
\hline$[* * * * *$ & OF FORTRAN STATEMENTS & P3600310 \\
\hline$* * * * *$ & 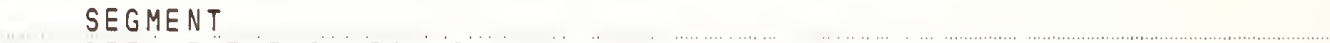 & \\
\hline$[* * * * *$ & DEFINE THE SYMBOLIC OUTPUT UNIT FOR USE IN THIS 7.1. & 00330 \\
\hline$c * * * * *$ & OUTPU T - T A P E ASSIGNMENT STATEMENT. NO INPUT TAPE. & 00340 \\
\hline$* * * * *$ & & \\
\hline$* * * * *$ & WHEN EXECUTING ONLY SEGMENT 360, THE FOLLOWING STATE & $P 0073440$ \\
\hline $2 * * * a *$ & NUVI $=6$ MUST HAVE THE $C=$ IN COLUMNS " ANO 2 REMOVEO. & P0073445 \\
\hline$* * * * *$ & & \\
\hline & NUVI $=6$ & P0073455 \\
\hline & NUVI $=6$ & 081 \\
\hline$* * * * x$ & & 3460 \\
\hline & $J Y Z I(1,1)=N U V !$ & 0360 \\
\hline$C * * * * *$ & WRITE HEAOER FOR THIS SEGMENT & $P 3600370$ \\
\hline & WRITE (JBZVI, 3600) & \\
\hline 3600 & FORMAT $(1 \mathrm{H} 1,1 \mathrm{X}, 36 \mathrm{HSPECZ}-(360)$ COMMON AND EQUIVALENCE / & 00390 \\
\hline & 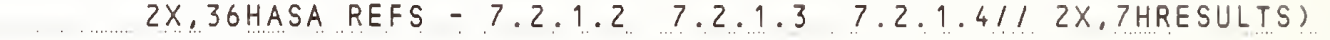 & \\
\hline$* * * * *$ & & \\
\hline$C * * * * *$ & TEST THAT EQUIVALENCE WORKS - ASSOCIATED ITEM OF & \\
\hline$[* * * * *$ & SAME TYPE BECOMES DEFINEO WHEN EQUATED ITEM IS & P3600430 \\
\hline 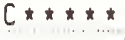 & 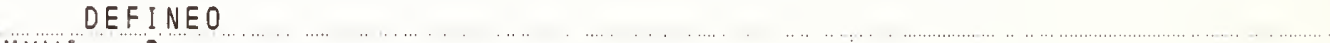 & $P 3600440$ \\
\hline & $M Y V I=2$ & $P 3600450$ \\
\hline & WAZ1S $(2)=2.0$ & \\
\hline 601 & $\begin{array}{l}\text { WRITE ( JBZVI, 3601) NZVI, RVYZS }(1,1) \\
\text { FORMAT }(/ / 27 \mathrm{H} \text { LINE } 1 \text { BELOW IS HOLLERITH }\end{array}$ & $\begin{array}{l}00470 \\
00480\end{array}$ \\
\hline
\end{tabular}


7368 MMYII IVI) = IVI

IVI $=0$

007369 LVI $=1,2$

$007369 \mathrm{KVI}=1,10$

007369 JVI $=1,20$

IF (NNY3I(JVI,KVI, LVI) - (JVI+20*(KVI+10*LVI) - 220))7369,8360,7369

8360 IVI $=$ IVI +1

7369 CONTINUE

IF $(I V I-400) 8363,8361,8363$

8363 WRITE (NUVI, 8364)

8364 FORMAT ( 13 HO TEST FAILEO)

P3601020

P3601030

P3601040

P3601050

P3601060

P3601070

P 3601080

P3601090

P3601100

P3601110

P3601120

$P 3601130$

$P 3601140$

P3601150

P3601160 
8365 CONTINUE

$C * * *$ END OF TEST SEGMENT 360

C**** WHEN EXECUTING ONLY SEGMENT 360 , THE STOP AND END CARDS

P3601190

$P 3601200$

$c * * * *$ WHICH APPEAR AS COMMENT CARDS MUST HAVE THE $c=$

P3601210

P3601220

C***** IN COLUMNS 1 AND 2 REMOVED.

P3601230

P3601240

$C=$ STOP 77777

$C=E N D$

$P 3601250$

STOP 77777

P3601260

END

P360C1

$P 360 C 2$ 
NBS.114A (REV. 7-73)

\begin{tabular}{|c|c|c|}
\hline $\begin{array}{c}\text { U.S. DEPT. OF COMM. } \\
\text { BIBLIOGRAPHIC DATA } \\
\text { SHEET }\end{array}$ & $\begin{array}{c}\text { 1. PUBLICATION OR REPORT NO. } \\
\text { NBS-SP- 399 VO1.2 }\end{array}$ & $\begin{array}{l}\text { 2. Gov't Accession } \\
\text { No. }\end{array}$ \\
\hline
\end{tabular}

4. TITLE AND SUBTITLE

3. Recipient's Accession No.

5. Publication bate

October 1974

NBS FORTRAN TEST PROGRAMS

6. Performing Organization Code

8. Performing Organ. Report No.

Frances E. Holberton Elizabeth G. Parker

9. PERFORMING ORGANIZATION NAME AND ADDRESS

NATIONAL BUREAU OF STANDARDS

DEPARTMENT OF COMMERCE

WASHINGTON, D.C. 20234

10. Project/Task/Work Unit No. 6401123

11. Contract/Grant No.

12. Sponsoring Organization Name and Complete Address (Street, City, State, ZIP)

Same as 9

Library of Congress Catalog Card Number: 74-12314

13. Type of Report \& Period Covered

Final

14. Sponsoring Agency Code

15. SUPPLEMENTARY NOTES

Volumes 1, 2, and 3 contain the documentation, Version 1 program listing, Version 3 program listing, respectively. The magnetic tape containing the NBS FORTRAN Test Programs is available in 7-track BCD, 9-track ASCII or EBCDIC recording.

16. ABSTRACT (A 200-word or less factual summary of most significant information. If document includes a significant bibliography or literature survey, mention it here.)

The NBS FORTRAN test programs, written in Standard FORTRAN, are designed to test whether a FORTRAN compiler accepts the forms and interpretations of the FORTRAN language as described in the American National Standard FORTRAN document X3.9-1966. The test programs, comprised of 116 test units, are structured into two versions, each containing approximately 14,500 punch card images. The test units may be used as separate executable FORTRAN programs, or may be linked end to end with other test units, with a minimum of user effort, to improve operating efficiency. Version 1 is structured into 116 executable FORTRAN programs, and Version 3, containing the same 116 test units, is structured into 14 executable FORTRAN programs for use on large FORTRAN processors.

The test program design criteria was to:

- Constrain all test programs to the FORTRAN Standard X3.9-1966.

- Reduce the effect of those areas in which the FORTRAN Standard does not prescribe a method or solution, e.g., range, precision, size of computer, etc.

- Simplify the use of the FORTRAN test programs.

- Test FORTRAN language elements before they are used in support of other tests.

- Maintain an open ended system so that tests may be changed or added.

The test programs require the use of a card reader, printer and one intermediate tape unit.

17. KEY WORDS (six to twelve entries; alphabetical order; capitalize only the first letter of the first key word unless a proper name; separated by semicolons)

Computer Programing language; FORTRAN; FORTRAN validation; language validation; standard FORTRAiv; test program design.
18. AVAILABILITY
E Unlimited

$\square$ For Official Distribution. Do Not Release to NTIS

X. Order From Sup. of Doc., U.S. Government Pfinting 8 ffice 2
Washington, D.C. 20402, SD Cat. No.C13.10:399.2

Order From National Technical Information Service (NTIS) Springfield, Virginia 22151

\begin{tabular}{|l|c|}
\hline $\begin{array}{l}\text { 19. SECURITY CLASS } \\
\text { (THIS REPURT) } \\
\text { UNCL ASSIFIED }\end{array}$ & 21. NO. OF PAGES \\
\hline $\begin{array}{l}\text { 20. SECURITY CLASS } \\
\text { (THIS PAGE) }\end{array}$ & 22. Price \\
UNCLASSIFIED & $\$ 2.85$ \\
\hline
\end{tabular}







\section{NBS TECHNICAL PUBLICATIONS}

\section{PERIODICALS}

JOURNAL OF RESEARCH reports National Bureau of Standards research and development in physics, mathematics, and chemistry. Comprehensive scientific papers grive complete details of the work, including laboratory data, experimental procedures, and theoretical and mathematical analyses. Illustrater with photographs. drawings, and charts. Includes listings of other NBS papers as issued.

Published in two sections, available separately:

- Physics and Chemistry (Section A)

Papers of interest primarily to scientists working in these fields. This section cover's a broad range of physical and chemical research, with major emphasis on standards of physical measurement, fundamental constants, and properties of matter. Issued six times a year. Annual subscription: Domestic, $\$ 17.00$; Foreign, $\$ 21.25$

- Mathematical Sciences (Section B)

Studies and compilations designed mainly for the mathematician and theoretical physicist. Topics in mathematical statistics, theory of experiment design, numerical analysis, theoretical physics and chemistry, logical design and programming of computers and computer systems. Short numerical tables. Issued quarterly. Annual subscription: Domestic, $\$ 9.00 ;$ Foreign, $\$ 11.25$.

DIMENSIONS/NBS (formerly Technical News Bulletin)-This monthly magazine is published to inform scientists, engineer's, businessmen, industry, teachers, students, and consumers of the latest advances in science and technology, with primary emphasis on the work at NBS.

DIMENSIONS/NBS highlights and reviews such issues as energy research, fire protection, building technology, metric conversion, pollution abatement, health and safety, and consumer product performance. In addition, DIMENSIONS/NBS reports the results of Bureau programs in measurement standards and techniques, properties of matter and materials, engineering standards and services, instrumentation, and automatic data processing.

Annual subscription: Domestic, $\$ 6.50$; Foreign, $\$ 8.25$.

\section{NONPERIOOICALS}

Monographs-Major contributions to the technical literature on various subjects related to the Bureau's scientific and technical activities.

Handbooks-Recommended codes of engineering and industrial practice (including safety codes) developed in cooperation with interested industries, professional organizations, and regulatory bodies.

Special Publications-Include proceedings of high-level national and international conferences sponsored by NBS, precision measurement and calibration volumes, NBS annual reports, and other special publications appropriate to this grouping such as wall charts and bibliographies.

Applied Mathematics Series-Mathematical tables, manuals, and studies of special interest to physicists, engineers, chemists, biologists, mathematicians, computer programmers, and others engaged in scientific and technical work.
National Standard Reference Data Series-Provides quantitative data on the physical ancl chemical properties of materials, compiled from the world's literature and critically evaluated. Developed under a world-wide program coordinated by NBS. Program under authority of National Standard I)ata Act (Public Law 90-396). See also Section 1.2.3.

Building Science Series--Disseminates technical information developed at the Bureau on building materials, components, systems, and whole structures. The series presents research results, test methods, and performance criteria related to the structural and environmental functions and the durability and safety characteristics of building elements and systems.

Technical Notes-Studies or reports which are complete in themselves but restrictive in their treatment of a subject. Analogous to monographs but not so comprehensive in scope or definitive in treatment of the subject area. Often serve as a vehicle for final reports of work performed at NBS under the sponsorship of other government agencies.

Voluntary Product Standards--Developed under procedures published by the Department of Commerce in Part 10, Title 15, of the Code of Federal Regulations. The purpose of the standards is to establish nationally recognized requirements for products, and to provide all concerned interests with a basis for common understanding of the characteristics of the products. The National Bureau of Standards administers the Voluntary Product Standards program as a supplement to the activities of the private sector standardizing organizations.

Federai Information I'rocessing Standards Publications (FIPS PUBS)-Publications in this series collectively constitute the Federal Information Processing Standards Register. The purpose of the Register is to serve as the official source of information in the Federal Government regarding standards issued by NBS pursuant to the Federal Property and Administrative Services Act of 1949 as amended, Public Law 89-306 (79 Stat. 1127), and as implemented by Executive Order 11717 (38 FR 12315, dated May 11, 1973) and Part 6 of Title 15 CFR (Code of Federal Regulations). FIPS PUBS will include approved Federal information processing standards information of general interest, and a complete index of relevant standards publications.

Consumer Information Series-Practical information, based on NBS research and experience, covering areas of interest to the consumer. Easily understandable language and illustrations provide useful background knowledge for shopping in today's technological marketplace.

NBS Interagency Reports-A special series of interim or final reports on work performed by NBS for outside sponsols (both government and non-government). In general, initial distribution is handled by the sponsor; public distribution is by the National Technical Information Service (Springfield, Va. 22151) in paper copy or microfiche form.

Order NBS publications (except Bibliographic Subscription Services) from: Superintendent of Documents, Government Printing: Office, Washington, D.C. 20402.

\section{BIBLIOGRAPHIC SUBSCRIPTION SERVICES}

The following current-awareness and literature-survey bibliographies are issued periodically by the Bureau:

Cryogenic Data Center Current Awareness Service (Publications and Reports of Interest in Cryogenics). A literature survey issued weekly. Annual subscription: Domestic, $\$ 20.00$; foreign, $\$ 25.00$.

Liquefied Natural Gas. A literature survey issued quarterly. Annual subscription: $\$ 20.00$.

Superconducting Devices and Materials. A literature survey issued quarterly. Annual subscription: $\$ 20.00$. Send subscription orders and remittances for the pre- ceding bibliographic services to the U.S. Department of Commerce, National Technical Information Service, Springfield, Va. 22151.

Electromagnetic Metrology Current Awareness Service (Abstracts of Selected Articles on Measurement Techniques and Standards of Electromagnetic Quantities from D-C to Millimeter-Wave Frequencies). Issued monthly. Annual subscription: $\$ 100.00$ (Special rates for multi-subscriptions). Send subscription order and remittance to the Electromagnetic Metrology Information Center, Electromagnetics Division, National Bureau of Standards, Boulder, Colo. 80302. 
U.S. DEPARTMENT OF COMMERCE

National Bureau of Standards

Washington, OC. 20234

POSTAGE AND FEES PAIO U.S. OEPARTMENT OF COMMERCE $C O M-215$

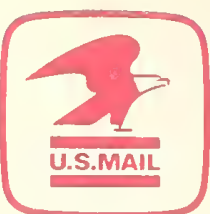

Penalty for Private Use, $\$ 300$

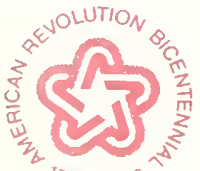

$1>6-197^{6}$ 




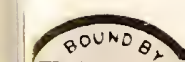

HM 
